RIMS-1757

INTER-UNIVERSAL TEICHMÜLLER THEORY II:

HODGE-ARAKELOV-THEORETIC EVALUATION

By

Shinichi MOCHIZUKI

August 2012

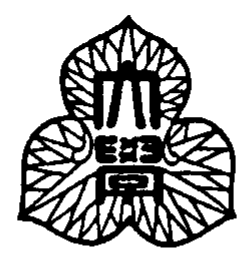

京都大学 数理解析研究所

RESEARCH INSTITUTE FOR MATHEMATICAL SCIENCES

KYOTO UNIVERSITY, Kyoto, Japan 


\title{
INTER-UNIVERSAL TEICHMÜLLER THEORY II: HODGE-ARAKELOV-THEORETIC EVALUATION
}

\author{
SHINICHI MOCHIZUKI
}

August 2012

\begin{abstract}
.
In the present paper, which is the second in a series of four papers, we study the Kummer theory surrounding the Hodge-Arakelovtheoretic evaluation - i.e., evaluation in the style of the scheme-theoretic HodgeArakelov theory established by the author in previous papers - of the [reciprocal of the $l$-th root of the] theta function at $l$-torsion points, for $l \geq 5$ a prime number. In the first paper of the series, we studied "miniature models of conventional scheme theory", which we referred to as $\Theta^{ \pm \text {ell }} N F$-Hodge theaters, that were associated to certain data, called initial $\Theta$-data, that includes an elliptic curve $E_{F}$ over a number field $F$, together with a prime number $l \geq 5$. These $\Theta^{ \pm \text {ell } N F-H o d g e ~ t h e a t e r s ~ w e r e ~}$ glued to one another by means " $\Theta$-links", that identify the [reciprocal of the $l$-th root of the] theta function at primes of bad reduction of $E_{F}$ in one $\Theta^{ \pm \text {ell }} \mathrm{NF}$-Hodge theater with [2l-th roots of] the q-parameter at primes of bad reduction of $E_{F}$ in another $\Theta^{ \pm \text {ell }} \mathrm{NF}$-Hodge theater. The theory developed in the present paper allows one to construct certain new versions of this " $\Theta$-link". One such new version is the $\Theta_{\text {gau }}^{\times \boldsymbol{\mu}}$ link, which is similar to the $\Theta$-link, but involves the theta values at l-torsion points, rather than the theta function itself. One important aspect of the constructions that underlie the $\Theta_{\mathrm{gau}}^{\times \boldsymbol{\mu}}$-link is the study of multiradiality properties, i.e., properties of the "arithmetic holomorphic structure" — or, more concretely, the ring/scheme structure - arising from one $\Theta^{ \pm \text {ell }} \mathrm{NF}$-Hodge theater that may be formulated in such a way as to make sense from the point of the arithmetic holomorphic structure of another $\Theta^{ \pm \text {ell }} \mathrm{NF}$-Hodge theater which is related to the original $\Theta^{ \pm e l l} \mathrm{NF}$-Hodge

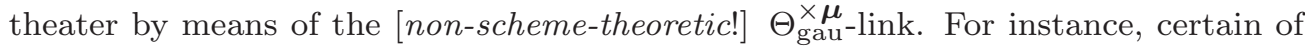
the various rigidity properties of the étale theta function studied in an earlier paper by the author may be intepreted as multiradiality properties in the context of the theory of the present series of papers. Another important aspect of the constructions

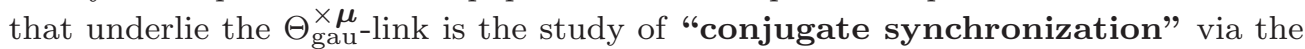
$\mathbb{F}_{l}^{\rtimes \pm}$-symmetry of a $\Theta^{ \pm \text {ell }} \mathrm{NF}$-Hodge theater. Conjugate synchronization refers to a certain system of isomorphisms — which are free of any conjugacy indeterminacies! - between copies of local absolute Galois groups at the various $l$-torsion points at which the theta function is evaluated. Conjugate synchronization plays an important role in the Kummer theory surrounding the evaluation of the theta function at $l$ torsion points and is applied in the study of coricity properties of [i.e., the study of objects left invariant by] the $\Theta_{\text {gau }}^{\times \boldsymbol{\mu}}$-link. Global aspects of conjugate synchronization require the resolution, via results obtained in the first paper of the series, of certain technicalities involving profinite conjugates of tempered cuspidal inertia groups.
\end{abstract}

\section{Contents:}

Introduction

$\S 1$. Multiradial Mono-theta Environments

Typeset by $\mathcal{A} \mathcal{M S}-\mathrm{T}_{\mathrm{EX}}$ 
§2. Galois-theoretic Theta Evaluation

§3. Tempered Gaussian Frobenioids

§4. Global Gaussian Frobenioids

\section{Introduction}

In the following discussion, we shall continue to use the notation of the Introduction to the first paper of the present series of papers [cf. [IUTchI], §I1]. In particular, we assume that are given an elliptic curve $E_{F}$ over a number field $F$, together with a prime number $l \geq 5$. In the present paper, which forms the second paper of the series, we study the Kummer theory surrounding the HodgeArakelov-theoretic evaluation [cf. Fig. I.1 below] — i.e., evaluation in the style of the scheme-theoretic Hodge-Arakelov theory of [HASurI], [HASurII] — of the reciprocal of the $l$-th root of the theta function

$$
\underline{\underline{\Theta}}_{\underline{v}} \stackrel{\text { def }}{=}\left\{q_{\underline{v}}^{-\frac{1}{8}} \cdot \sum_{n \in \mathbb{Z}}(-1)^{n} \cdot q_{\underline{\underline{2}}}^{\frac{1}{2}\left(n+\frac{1}{2}\right)^{2}} \cdot U_{\underline{v}}^{n+\frac{1}{2}}\right\}^{-\frac{1}{l}}
$$

[cf. [EtTh], Proposition 1.4; [IUTchI], Example 3.2, (ii)] at $\boldsymbol{l}$-torsion points in the context of the theory of $\Theta^{ \pm \text {ell }} \mathbf{N F - H o d g e}$ theaters developed in [IUTchI]. Here, relative to the notation of [IUTchI], $\S \mathrm{I} 1, \underline{v} \in \underline{\mathbb{V}}^{\text {bad }} ; q_{\underline{v}}$ denotes the $q$-parameter at $\underline{v}$ of the given elliptic curve $E_{F}$ over a number field $F$; $U_{\underline{v}}$ denotes the standard multiplicative coordinate on the Tate curve obtained by localizing $E_{F}$ at $\underline{v}$. Let $\underline{\underline{q}} \underline{\underline{v}}$ be a $2 l$-th root of $q_{\underline{v}}$. Then these "theta values at l-torsion points" will, up to a factor given by a $2 \bar{l}$-th root of unity, turn out to be of the form [cf. Remark 2.5.1, (i)]

$$
\underline{\underline{q}} \underline{\underline{\underline{j}}} \underline{\underline{\underline{v}}}
$$

— where $\underline{\underline{j}} \in\{0,1, \ldots, l$ * $\stackrel{\text { def }}{=}(l-1) / 2\}$, so $\underline{\underline{j}}$ is uniquely determined by its image $j \in\left|\mathbb{F}_{l}\right| \stackrel{\text { def }}{=} \mathbb{F}_{l} /\{ \pm 1\}=\{0\} \cup \mathbb{F}_{l}^{*}[$ cf. the notation of [IUTchI]].

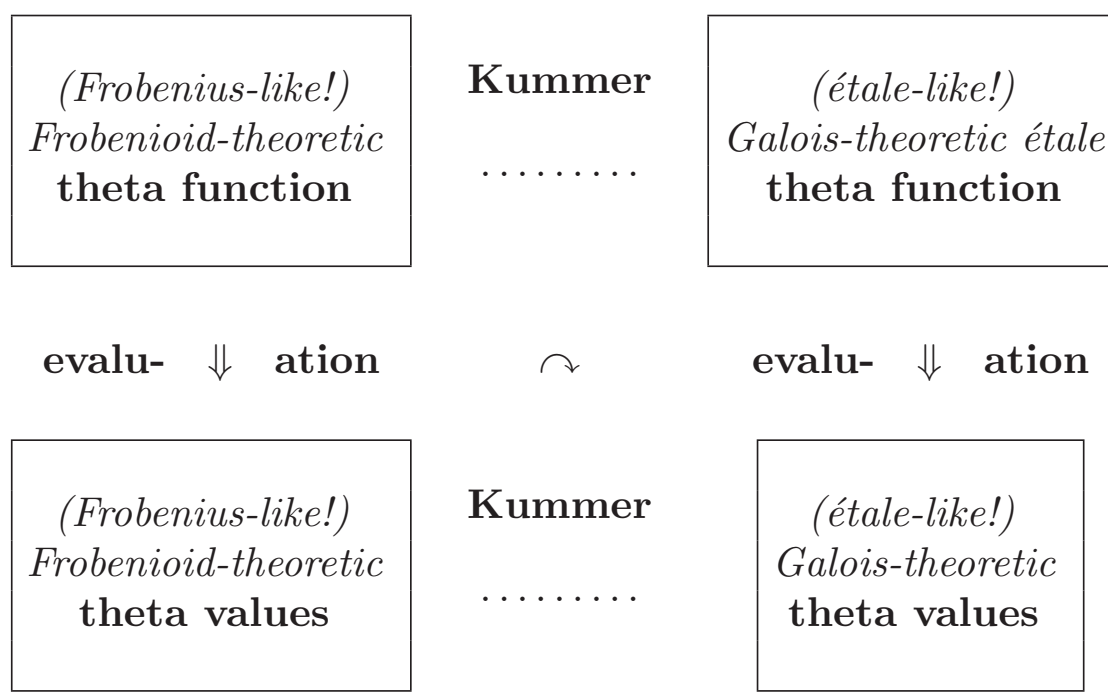

Fig. I.1: The Kummer theory surrounding Hodge-Arakelov-theoretic evaluation 
In order to understand the significance of Kummer theory in the context of Hodge-Arakelov-theoretic evaluation, it is important to recall the notions of "Frobenius-like" and "étale-like" mathematical structures [cf. the discussion of [IUTchI], §I1]. In the present series of papers, the Frobenius-like structures constituted by [the monoidal portions of] Frobenioids - i.e., more concretely, by various monoids - play the important role of allowing one to construct gluing isomorphisms such as the $\Theta$-link which lie outside the framework of conventional scheme/ring theory [cf. the discussion of [IUTchI], §I2]. Such gluing isomorphisms give rise to Frobenius-pictures [cf. the discussion of [IUTchI], §I1]. On the other hand, the étale-like structures constituted by various Galois and arithmetic fundamental groups give rise to the canonical splittings of such Frobeniuspictures furnished by corresponding étale-pictures [cf. the discussion of [IUTchI], $\S I 1]$. In [IUTchIII], absolute anabelian geometry will be applied to these Galois and arithmetic fundamental groups to obtain descriptions of alien arithmetic holomorphic structures, i.e., arithmetic holomorphic structures that lie on the opposite side of a $\Theta$-link from a given arithmetic holomorphic structure [cf. the discussion of [IUTchI], §I3]. Thus, in light of the equally crucial but substantially different roles played by Frobenius-like and étale-like structures in the present series of papers, it is of crucial importance to be able

to relate corresponding Frobenius-like and étale-like versions of various objects to one another.

This is the role played by Kummer theory. In particular, in the present paper, we shall study in detail the Kummer theory that relates Frobenius-like and étalelike versions of the theta function and its theta values at $l$-torsion points to one another [cf. Fig. I.1].

One important notion in the theory of the present paper is the notion of multiradiality. To understand this notion, let us recall the étale-picture discussed in [IUTchI], $\S \mathrm{I} 1$ [cf., [IUTchI], Fig. I1.6]. In the context of the present paper, we shall be especially interested in the étale-like version of the theta function and its theta values constructed in each $\mathcal{D}-\Theta^{ \pm \text {ell }} \mathrm{NF}$-Hodge theater ${ }^{(-)} \mathcal{H} \mathcal{T}^{\mathcal{D}-\Theta^{ \pm \text {ell }} \mathrm{NF}}$; thus, one can think of the étale-picture under consideration as consisting of the diagram given in Fig. I.2 below. As discussed earlier, we shall ultimately be interested in applying various absolute anabelian reconstruction algorithms to the various arithmetic fundamental groups that [implicitly] appear in such étale-pictures in order to obtain descriptions of alien holomorphic structures, i.e., descriptions of objects that arise on one spoke that make sense from the point of view of another spoke. In this context, it is natural to classify the various algorithms applied to the arithmetic fundamental groups lying in a given spoke as follows [cf. Example 1.7]:

- We shall refer to an algorithm as coric if it in fact only depends on input data arising from the mono-analytic core of the étale-picture, i.e., the data that is common to all spokes.

- We shall refer to an algorithm as uniradial if it expresses the objects constructed from the given spoke in terms that only make sense within the given spoke. 
- We shall refer to an algorithm as multiradial if it expresses the objects constructed from the given spoke in terms of corically constructed objects, i.e., objects that make sense from the point of view of other spokes.

Thus, multiradial algorithms are compatible with simultaneous execution at multiple spokes [cf. Example 1.7, (v); Remark 1.9.1], while uniradial algorithms may only be consistently executed at a single spoke. Ultimately, in the present series of papers, we shall be interested - relative to the goal of obtaining "descriptions of alien holomorphic structures" - in the establishment of multiradial algorithms for constructing the objects of interest, e.g., [in the context of the present paper] the étale-like versions of the theta functions and the corresponding theta values discussed above. Typically, in order to obtain such multiradial algorithms, i.e., algorithms that make sense from the point of view of other spokes, it is necessary to allow for some sort of "indeterminacy" in the descriptions that appear in the algorithms of the objects constructed from the given spoke.

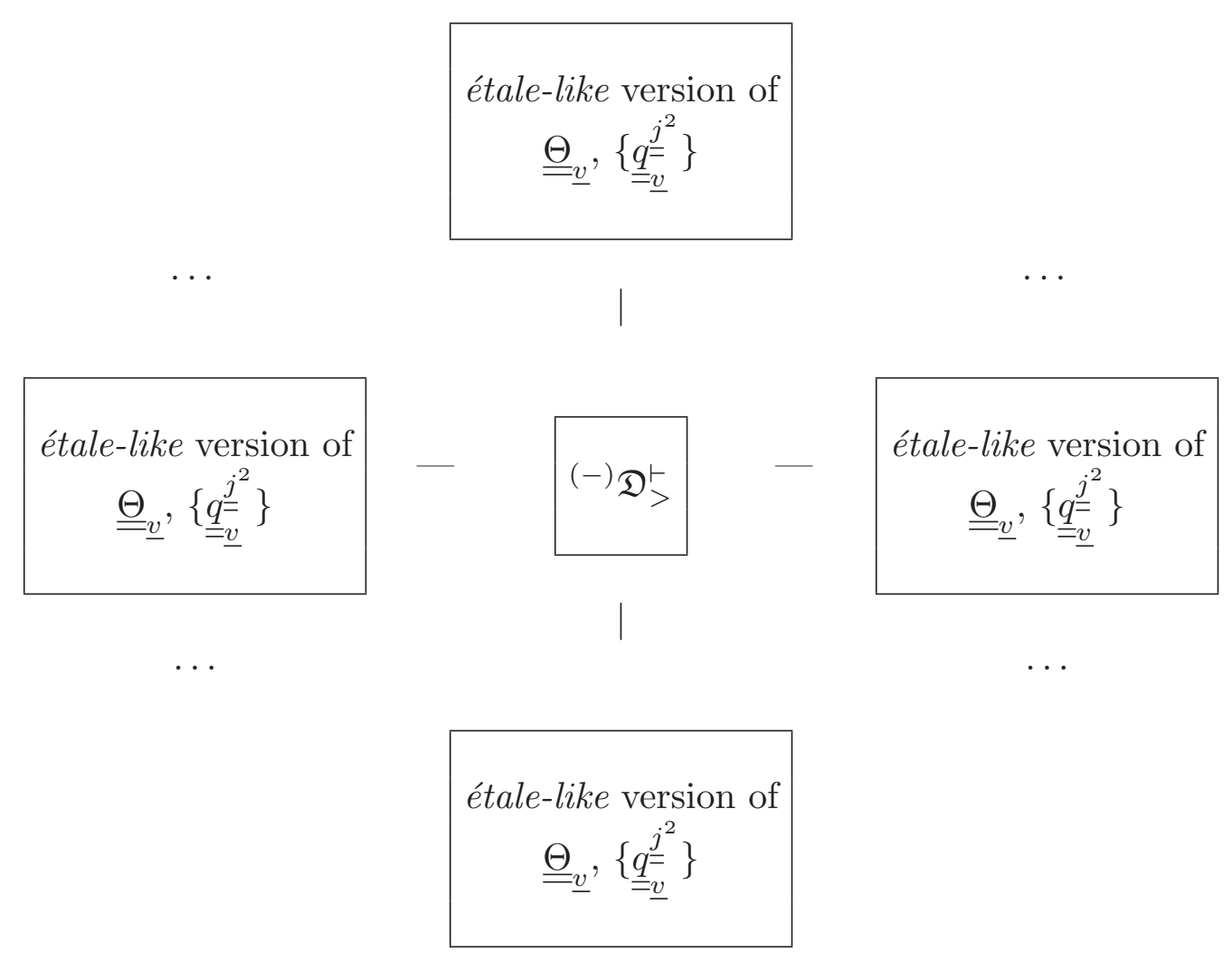

Fig. I.2: Étale-picture of étale-like versions of theta functions, theta values

Relative to the analogy between the inter-universal Teichmüller theory of the present series of papers and the classical theory of holomorphic structures on Riemann surfaces [cf. the discussion of [IUTchI], §I4], one may think of coric algorithms as corresponding to constructions that depend only on the underlying real analytic structure on the Riemann surface. Then uniradial algorithms correspond to constructions that depend, in an essential way, on the holomorphic structure of the given Riemann surface, while multiradial algorithms correspond 
to constructions of holomorphic objects associated to the Riemann surface which are expressed [perhaps by allowing for certain indeterminacies!] solely in terms of the underlying real analytic structure of the Riemann surface - cf. Fig. I.3 below; the discussion of Remark 1.9.2. Perhaps the most fundamental motivating example in this context is the description of "alien holomorphic structures" by means of the Teichmüller deformations reviewed at the beginning of [IUTchI], $\S \mathrm{I} 4$, relative to "unspecified/indeterminate" deformation data [i.e., consisting of a nonzero square differential and a dilation factor]. Indeed, for instance, in the case of once-punctured elliptic curves, by applying well-known facts concerning Teichmüller mappings [cf., e.g., [Lehto], Chapter V, Theorem 6.3], it is not difficult to formulate the classical result that

"the homotopy class of every orientation-preserving homeomorphism between pointed compact Riemann surfaces of genus one 'lifts' to a unique Teichmüller mapping"

in terms of the "multiradial formalism" discussed in the present paper [cf. Example 1.7]. [We leave the routine details to the reader.]

\begin{tabular}{|c|c|c|}
\hline$\underline{\text { abstract }}$ & $\underline{\text { Teichter-universal }}$ & $\frac{\text { classical complex }}{\text { Teichmüller theory }}$ \\
\hline $\begin{array}{l}\text { uniradial } \\
\text { algorithms }\end{array}$ & $\begin{array}{l}\text { arithmetic holomorphic } \\
\text { structures }\end{array}$ & $\begin{array}{l}\text { holomorphic } \\
\text { structures }\end{array}$ \\
\hline $\begin{array}{l}\text { multiradial } \\
\text { algorithms }\end{array}$ & $\begin{array}{l}\text { arithmetic holomorphic } \\
\text { structures described in } \\
\text { terms of underlying } \\
\text { mono-analytic structures }\end{array}$ & $\begin{array}{l}\text { holomorphic structures } \\
\text { structures described in } \\
\text { terms of underlying } \\
\text { real analytic structures }\end{array}$ \\
\hline $\begin{array}{l}\text { coric } \\
\text { algorithms }\end{array}$ & $\begin{array}{l}\text { underlying mono-analytic } \\
\text { structures }\end{array}$ & $\begin{array}{c}\text { underlying real analytic } \\
\text { structures }\end{array}$ \\
\hline
\end{tabular}

Fig. I.3: Uniradiality, Multiradiality, and Coricity

One interesting aspect of the theory of the present series of papers may be seen in the set-theoretic function arising from the theta values considered above

$$
\underline{j} \quad \mapsto \quad \underline{\underline{q}} \underline{\underline{\underline{j}}} \underline{\underline{v}}
$$

- a function that is reminiscent of the Gaussian distribution $(\mathbb{R} \ni) x \mapsto$ $e^{-x^{2}}$ on the real line. From this point of view, the passage from the Frobeniuspicture to the canonical splittings of the étale-picture [cf. the discussion of [IUTchI], 
$\S I 1]$, i.e., in effect, the computation of the $\Theta$-links that occur in the Frobeniuspicture by means of the various multiradial algorithms that will be established in the present series of papers, may be thought of [cf. the diagram of Fig. I.2!] as a sort of global arithmetic/Galois-theoretic analogue of the computation of the classical Gaussian integral

$$
\int_{-\infty}^{\infty} e^{-x^{2}}=\sqrt{\pi}
$$

via the passage from cartesian coordinates, i.e., which correspond to the Frobeniuspicture, to polar coordinates, i.e., which correspond to the étale-picture - cf. the discussion of Remark 1.12.5.

One way to understand the difference between coricity, multiradiality, and uniradiality at a purely combinatorial level is by considering the $\mathbb{F}_{l}^{*}$ - and $\mathbb{F}_{l}^{\rtimes \pm}$ symmetries discussed in [IUTchI], §I1 [cf. the discussion of Remark 4.7.4 of the present paper]. Indeed, at a purely combinatorial level, the $\mathbb{F}_{l}^{*}$-symmetry may be thought of as consisting of the natural action of $\mathbb{F}_{l}^{*}$ on the set of labels $\left|\mathbb{F}_{l}\right|=$ $\{0\} \cup \mathbb{F}_{i}^{*}$ [cf. the discussion of [IUTchI], §I1]. Here, the label 0 corresponds to the [mono-analytic] core. Thus, the corresponding étale-picture consists of various copies of $\left|\mathbb{F}_{l}\right|$ glued together along the coric label 0 [cf. Fig. I.4 below]. In particular, the various actions of copies of $\mathbb{F}_{l}^{*}$ on corresponding copies of $\left|\mathbb{F}_{l}\right|$ are "compatible with simultaneous execution" in the sense that they commute with one another. That is to say, at least at the level of labels, the $\mathbb{F}_{l}^{*}$-symmetry is multiradial.

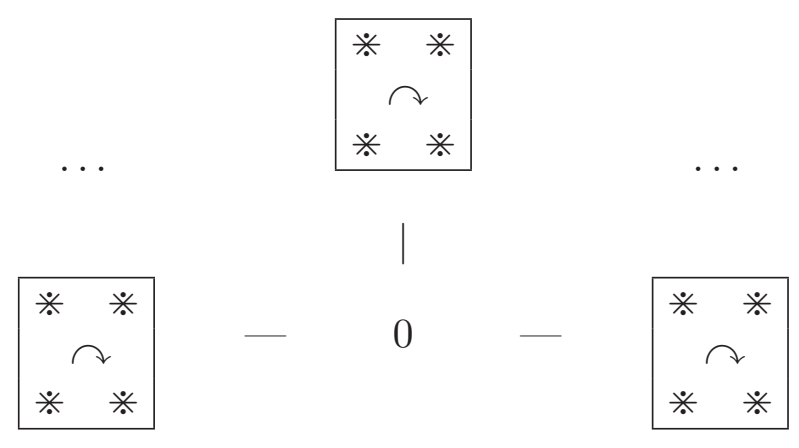

Fig. I.4: Étale-picture of $\mathbb{F}_{i}^{*}$-symmetries

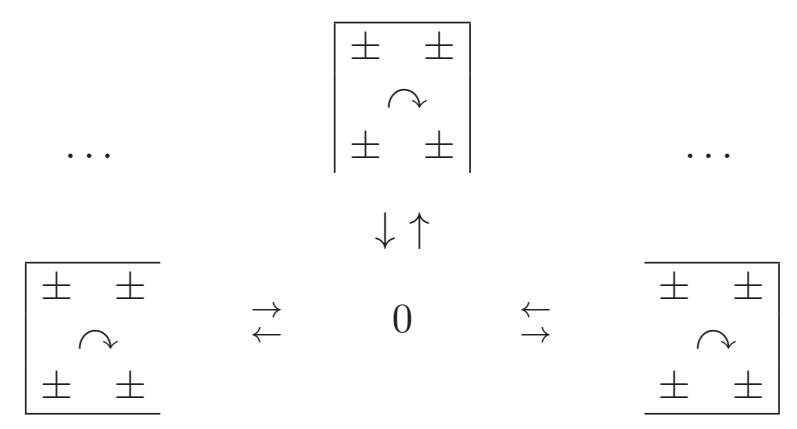

Fig. I.5: Étale-picture of $\mathbb{F}_{l}^{\rtimes \pm}$-symmetries 
In a similar vein, at a purely combinatorial level, the $\mathbb{F}_{l}^{\rtimes \pm}$-symmetry may be thought of as consisting of the natural action of $\mathbb{F}_{l}^{\rtimes \pm}$ on the set of labels $\mathbb{F}_{l}$ [cf. the discussion of [IUTchI], §I1]. Here again, the label 0 corresponds to the [mono-analytic] core. Thus, the corresponding étale-picture consists of various copies of $\mathbb{F}_{l}$ glued together along the coric label 0 [cf. Fig. I.5 above]. In particular, the various actions of copies of $\mathbb{F}_{l}^{\rtimes \pm}$ on corresponding copies of $\mathbb{F}_{l}$ are "incompatible with simultaneous execution" in the sense that they clearly fail to commute with one another. That is to say, at least at the level of labels, the $\mathbb{F}_{l}^{\rtimes \pm}$-symmetry is uniradial.

Since, ultimately, in the present series of papers, we shall be interested in the establishment of multiradial algorithms, "special care" will be necessary in order to obtain multiradial algorithms for constructing objects related to the a priori uniradial $\mathbb{F}_{l}^{\rtimes \pm}$-symmetry [cf. the discussion of Remark 4.7.3 of the present paper; [IUTchIII], Remark 3.11.2, (i), (ii)]. The multiradiality of such algorithms will be closely related to the fact that $\mathbb{F}_{l}^{\rtimes \pm}$-symmetry is applied to relate the various copies of local units modulo torsion, i.e., " $\mathcal{O}^{\times \mu}$ " [cf. the notation of [IUTchI], §1] at various labels $\in \mathbb{F}_{l}$ that lie in various spokes of the étale-picture [cf. the discussion of Remark 4.7.3, (ii)]. This contrasts with the way in which the a priori multiradial $\mathbb{F}_{l}^{*}$-symmetry will be applied, namely to treat various "weighted volumes" corresponding to the local value groups and global realified Frobenioids at various labels $\in \mathbb{F}_{l}^{*}$ that lie in various spokes of the étale-picture [cf. the discussion of Remark 4.7.3, (iii)]. Relative to the analogy between the theory of the present series of papers and p-adic Teichmüller theory [cf. [IUTchI], §I4], various aspects of the $\mathbb{F}_{l}^{\rtimes \pm}$-symmetry are reminiscent of the additive monodromy over the ordinary locus of the canonical curves that occur in $p$-adic Teichmüller theory; in a similar vein, various aspects of the $\mathbb{F}_{l}^{*}$-symmetry may be thought of as corresponding to the multiplicative monodromy at the supersingular points of the canonical curves that occur in $p$-adic Teichmüller theory - cf. the discussion of Remark 4.11.4, (iii); Fig. I.7 below.

Before discussing the theory of multiradiality in the context of the theory of Hodge-Arakleov-theoretic evaluation theory developed in the present paper, we pause to review the theory of mono-theta environments developed in [EtTh]. One starts with a Tate curve over a mixed-characteristic nonarchimedean local field. The mono-theta environment associated to such a curve is, roughly speaking, the Kummer-theoretic data that arises by extracting $N$-th roots of the theta trivialization of the ample line bundle associated to the origin over appropriate tempered coverings of the curve [cf. [EtTh], Definition 2.13, (ii)]. Such mono-theta environments may be constructed purely group-theoretically from the [arithmetic] tempered fundamental group of the once-punctured elliptic curve determined by the given Tate curve [cf. [EtTh], Corollary 2.18], or, alternatively, purely categorytheoretically from the tempered Frobenioid determined by the theory of line bundles and divisors over tempered coverings of the Tate curve [cf. [EtTh], Theorem 5.10, (iii)]. Indeed, the isomorphism of mono-theta environments between the monotheta environments arising from these two constructions of mono-theta environments - i.e., from tempered fundamental groups, on the one hand, and from tempered Frobenioids, on the other [cf. Proposition 1.2 of the present paper] - may be thought of as a sort of Kummer isomorphism for mono-theta environments [cf. Proposition 3.4 of the present paper, as well as [IUTchIII], Proposition 2.1, (iii)]. One important consequence of the theory of [EtTh] asserts that mono-theta 
environments satisfy the following three rigidity properties:

\section{(a) cyclotomic rigidity, \\ (b) discrete rigidity, and \\ (c) constant multiple rigidity}

— cf. the Introduction to [EtTh].

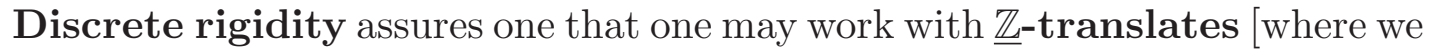
write $\underline{\mathbb{Z}}$ for the copy of " $\mathbb{Z}$ " that acts as a group of covering transformations on the tempered coverings involved], as opposed to $\underline{\mathbb{Z}}$-translates [i.e., where $\widehat{\mathbb{Z}} \cong \widehat{\mathbb{Z}}$ denotes the profinite completion of $\underline{\mathbb{Z}}]$, of the theta function, i.e., one need not contend with $\widehat{\mathbb{Z}}$-powers of canonical multiplicative coordinates [i.e., "U"], or q-parameters [cf. Remark 3.6.5, (iii); [IUTchIII], Remark 2.1.1, (v)]. Although we will certainly "use" this discrete rigidity throughout the theory of the present series of papers, this property of mono-theta environments will not play a particularly prominent role in the theory of the present series of papers. The $\widehat{\mathbb{Z}}$-powers of " $U$ " and " $q$ " that would occur if one does not have discrete rigidity may be compared to the PDformal series that are obtained, a priori, if one attempts to construct the canonical parameters of p-adic Teichmüller theory via formal integration. Indeed, PD-formal power series become necessary if one attempts to treat such canonical parameters as objects which admit arbitrary $\widehat{\mathcal{O}}$-powers, where $\widehat{\mathcal{O}}$ denotes the completion of the local ring to which the canonical parameter belongs [cf. the discussion of Remark 3.6.5, (iii); Fig. I.6 below].

Constant multiple rigidity plays a somewhat more central role in the present series of papers, in particular in relation to the theory of the log-link, which we shall discuss in [IUTchIII] [cf. the discussion of Remark 1.12 .2 of the present paper; [IUTchIII], Remark 1.2.3, (i); [IUTchIII], Proposition 3.5, (ii); [IUTchIII], Remark 3.11.2, (iii)]. Constant multiple rigidity asserts that the multiplicative monoid

$$
\mathcal{O}_{\bar{F}_{\underline{v}}} \cdot \underline{\underline{\Theta}}_{\underline{v}}
$$

- which we shall refer to as the theta monoid - generated by the reciprocal of the l-th root of the theta function and the group of units of the ring of integers of the base field $\bar{F}_{\underline{v}}$ [cf. the notation of [IUTchI], §I1] admits a canonical splitting, up to $2 l$-th roots of unity, that arises from evaluation at the [2-Jtorsion point corresponding to the label $0 \in \mathbb{F}_{l}$ [cf. Corollary 1.12, (ii); Proposition 3.1, (i); Proposition 3.3, (i)]. Put another way, this canonical splitting is the splitting determined, up to $2 l$-th roots of unity, by $\underline{\underline{\Theta}}_{\underline{v}} \in \mathcal{O}_{\bar{F}_{\underline{v}}}^{\times} \cdot \underline{\underline{\Theta}}_{\underline{v}}$. The theta monoid of the above display, as well as the associated canonical splitting, may be constructed algorithmically from the mono-theta environment [cf. Proposition 3.1, (i)]. Relative to the analogy between the theory of the present series of papers and $p$-adic Teichmüller theory, these canonical splittings may be thought of as corresponding to the canonical coordinates of p-adic Teichmüller theory, i.e., more precisely, to the fact that such canonical coordinates are also completely determined without any constant multiple indeterminacies — cf. Fig. I.6 below; Remark 3.6.5, (iii); [IUTchIII], Remark 3.12.4, (i). 


\begin{tabular}{|c|c|}
\hline $\begin{array}{l}\text { Mono-theta-theoretic rigidity property } \\
\text { in inter-universal Teichmüller theory }\end{array}$ & $\frac{\text { Corresponding phenomenon in }}{\text { p-adic Teichmüller theory }}$ \\
\hline $\begin{array}{c}\text { mono-theta-theoretic } \\
\text { constant } \\
\text { multiple } \\
\text { rigidity }\end{array}$ & $\begin{array}{l}\text { lack of constant multiple } \\
\text { indeterminacy of } \\
\text { canonical coordinates } \\
\text { on canonical curves }\end{array}$ \\
\hline $\begin{array}{l}\text { mono-theta-theoretic } \\
\text { cyclotomic } \\
\text { rigidity }\end{array}$ & $\begin{array}{c}\text { lack of } \widehat{\mathbb{Z}}^{\times} \text {-power indeterminacy } \\
\text { of canonical coordinates } \\
\text { on canonical curves, } \\
\text { Kodaira-Spencer } \\
\text { isomorphism }\end{array}$ \\
\hline $\begin{array}{l}\text { multiradiality of } \\
\text { mono-theta-theoretic } \\
\text { constant multiple, } \\
\text { cyclotomic } \\
\text { rigidity }\end{array}$ & $\begin{array}{l}\text { Frobenius-invariant } \\
\text { nature of } \\
\text { canonical coordinates }\end{array}$ \\
\hline $\begin{array}{l}\text { mono-theta-theoretic } \\
\text { discrete } \\
\text { rigidity }\end{array}$ & $\begin{array}{c}\text { formal = "non-PD-formal" } \\
\text { nature of canonical coordinates } \\
\text { on canonical curves }\end{array}$ \\
\hline
\end{tabular}

Fig. I.6: Mono-theta-theoretic rigidity properties in inter-universal Teichmüller theory and corresponding phenomena in $p$-adic Teichmüller theory

Cyclotomic rigidity consists of a rigidity isomorphism, which may be constructed algorithmically from the mono-theta environment, between

- the portion of the mono-theta environment — which we refer to as the exterior cyclotome - that arises from the roots of unity of the base field and

• a certain copy of the once-Tate-twisted Galois module " $\widehat{\mathbb{Z}}(1)$ ” — which we refer to as the interior cyclotome - that appears as a subquotient of the geometric tempered fundamental group

[cf. Definition 1.1, (ii); Proposition 1.3, (i)]. This rigidity is remarkable - as we shall see in our discussion below of the corresponding multiradiality property - 
in that unlike the "conventional" construction of such cyclotomic rigidity isomorphisms via local class field theory [cf. Proposition 1.3, (ii)], which requires one to use the entire monoid with Galois action $G_{\underline{v}} \curvearrowright \mathcal{O} \bar{F}_{\underline{v}}$, the only portion of the monoid $\mathcal{O}{\frac{\triangleright}{F_{\underline{v}}}}^{\triangleright}$ that appears in this construction is the portion [i.e., the "exterior cyclotome"] corresponding to the torsion subgroup $\mathcal{O}_{F_{\underline{v}}}^{\mu} \subseteq \mathcal{O}{\frac{\triangleright}{F_{\underline{v}}}}$ [cf. the notation of [IUTchI], §I1]. This construction depends, in an essential way, on the commutator structure of theta groups, but constitutes a somewhat different approach to utilizing this commutator structure from the "classical approach" involving irreducibility of representations of theta groups [cf. Remark 3.6.5, (ii); the Introduction to [EtTh]]. One important aspect of this dependence on the commutator structure of the theta group is that the theory of cyclotomic rigidity yields an explanation for the importance of the special role played by the first power of [the reciprocal of the l-th root of] the theta function in the present series of papers [cf. Remark 3.6.4, (iii), (iv), (v); the Introduction to [EtTh]]. Relative to the analogy between the theory of the present series of papers and $p$-adic Teichmüller theory, monotheta-theoretic cyclotomic rigidity may be thought of as corresponding either to the fact that the canonical coordinates of p-adic Teichmüller theory are completely determined without any $\widehat{\mathbb{Z}}^{\times}$-power indeterminacies or [roughly equivalently] to the Kodaira-Spencer isomorphism of the canonical indigenous bundle - cf. Fig. I.6; Remark 3.6.5, (iii); Remark 4.11.4, (iii), (b).

\section{The theta monoid}

$$
\mathcal{O}_{\bar{F}_{\underline{v}}} \cdot \underline{\underline{\Theta}}_{\underline{v}}
$$

discussed above admits both étale-like and Frobenius-like [i.e., Frobenioid-theoretic] versions, which may be related to one another via a Kummer isomorphism [cf. Proposition 3.3, (i)]. The unit portion, together with its natural Galois action, of the Frobenioid-theoretic version of the theta monoid

$$
G_{\underline{v}} \curvearrowright \mathcal{O}_{\bar{F}_{\underline{v}}}^{\times}
$$

forms the portion at $\underline{v} \in \underline{\mathbb{V}}^{\text {bad }}$ of the $\mathcal{F}^{\vdash \times}$-prime-strip " $\mathfrak{F}_{\text {mod }}^{\vdash \times}$ " that is preserved, up to isomorphism, by the $\Theta$-link [cf. the discussion of [IUTchI], §I1; [IUTchI], Theorem A, (ii)]. In the theory of the present paper, we shall introduce modified versions of the $\Theta$-link of [IUTChI] [cf. the discussion of the " $\Theta^{\times \boldsymbol{\mu}_{-}}, \Theta_{\mathrm{gau}}^{\times \boldsymbol{\mu}}$-links" below], which, unlike the $\Theta$-link of [IUTchI], only preserve [up to isomorphism] the $\mathcal{F}^{\vdash \times \mu}$-prime-strips - i.e., which consist of the data

$$
G_{\underline{v}} \curvearrowright \mathcal{O}_{\bar{F}_{\underline{v}}}^{\times \mu}=\mathcal{O}_{\bar{F}_{\underline{v}}}^{\times} / \mathcal{O}_{\bar{F}_{\underline{v}}}^{\mu}
$$

[cf. the notation of [IUTchI], §I1] at $\underline{v} \in \underline{\mathbb{V}}^{\text {bad }}$ — associated to the $\mathcal{F}^{\vdash \times}$-primestrips preserved [up to isomorphism] by the $\Theta$-link of [IUTchI]. Since this data is only preserved up to isomorphism, it follows that the topological group " $G_{\underline{v}}$ " must be regarded as being only known up to isomorphism, while the monoid $\mathcal{O}_{\overline{F_{\underline{w}}}}^{\times \boldsymbol{\mu}}$ must be regarded as being only known up to [the automorphisms of this monoid determined by the natural action of] $\widehat{\mathbb{Z}}^{\times}$. That is to say, one must regard the data $G_{\underline{v}} \curvearrowright \mathcal{O}_{\bar{F}_{\underline{v}}}^{\times \mu}$ as subject to $\operatorname{Aut}\left(G_{\underline{v}}\right)-, \widehat{\mathbb{Z}}^{\times}$-indetermnacies. 
These indeterminacies will play an important role in the theory of the present series of papers - cf. the indeterminacies "(Ind1)", "(Ind2)" of [IUTchIII], Theorem 3.11, (i).

Now let us return to our discussion of the various mono-theta-theoretic rigidity properties. The key observation concerning these rigidity properties, as reviewed above, in the context of the $\operatorname{Aut}\left(G_{\underline{v}}\right)-, \widehat{\mathbb{Z}}^{\times}$-indeterminacies just discussed, is the following:

the canonical splittings, via "evaluation at the zero section", of the theta monoids, together with the construction of the mono-theta-theoretic cyclotomic rigidity isomorphism, are compatible with, in the sense that they are left unchanged by, the $\operatorname{Aut}\left(G_{\underline{v}}\right)$-, $\widehat{\mathbb{Z}}^{\times}$-indeterminacies discussed above

- cf. Corollaries 1.10, 1.12; Proposition 3.4, (i). Indeed, this observation constitutes the substantive content of the multiradiality of mono-theta-theoretic constant multiple/cyclotomic rigidity [cf. Fig. I.6] and will play an important role in the statements and proofs of the main results of the present series of papers [cf. [IUTchIII], Theorem 2.2; [IUTchIII], Corollary 2.3; [IUTchIII], Theorem 3.11, (iii), (c); Step (ii) of the proof of [IUTchIII], Corollary 3.12]. At a technical level, this "key observation" simply amounts to the observation that the only portion of the monoid $\mathcal{O}_{\overline{F_{v}}}^{\times}$that is relevant to the construction of the canonical splittings and cyclotomic rigidity isomorphism under consideration is the torsion subgroup $\mathcal{O}_{\overline{F_{\underline{v}}}}^{\mu}$, which [by definition!] maps to the identity element of $\mathcal{O}_{\bar{F}_{\underline{v}}}^{\times \mu}$, hence is immune to the various indeterminacies under consideration. That is to say, the multiradiality of mono-theta-theoretic constant multiple/cyclotomic rigidity may be regarded as an essentially formal consequence of the triviality of the natural homomorphism

$$
\mathcal{O}_{\bar{F}_{\underline{v}}}^{\mu} \rightarrow \mathcal{O}_{\bar{F}_{\underline{v}}}^{\times \mu}
$$

[cf. Remark 1.10.2].

After discussing, in $\S 1$, the multiradiality theory surrounding the various rigidity properties of the mono-theta environment, we take up the task, in $\S 2$ and $\S 3$, of establishing the theory of Hodge-Arakelov-theoretic evaluation, i.e., of passing [for $\underline{v} \in \underline{\mathbb{V}}^{\text {bad }}$ ]

$$
\mathcal{O}_{\bar{F}_{\underline{v}}}^{\times} \cdot \underline{\underline{\Theta}}_{\underline{v}} \rightsquigarrow \mathcal{O} \bar{F}_{\underline{v}}^{\times} \cdot\{\underline{\underline{q} \underline{\underline{v}}}\}_{\underline{j}}^{j^{2}}=1, \ldots, l *
$$

from theta monoids as discussed above [i.e., the monoids on the left-hand side of the above display] to Gaussian monoids [i.e., the monoids on the right-hand side of the above display] by means of the operation of "evaluation" at $\boldsymbol{l}$-torsion points. Just as in the case of theta monoids, Gaussian monoids admit both étale-like versions, which constitute the main topic of $\S 2$, and Frobenius-like [i.e., Frobenioidtheoretic] versions, which constitute the main topic of $\S 3$. Moreover, as discussed at the beginning of the present Introduction, it is of crucial importance in the theory of the present series of papers to be able to relate these étale-like and Frobenius-like versions to one another via Kummer theory. One important observation in this 
context — which we shall refer to as the "principle of Galois evaluation" - is the following: it is essentially a tautology that

this requirement of compatibility with Kummer theory forces any sort of "evaluation operation" to arise from restriction to Galois sections of the [arithmetic] tempered fundamental groups involved

[i.e., Galois sections of the sort that arise from rational points such as $l$-torsion points!] — cf. the discussion of Remarks 1.12.4, 3.6.2. This tautology is interesting both in light of the history surrounding the Section Conjecture in anabelian geometry [cf. [IUTchI], §I5] and in light of the fact that the theory of [SemiAnbd] that is applied to prove [IUTchI], Theorem B - a result which plays an important role in the theory of $\S 2$ of the present paper! [cf. the discussion below] - may be thought of as a sort of "Combinatorial Section Conjecture".

At this point, we remark that, unlike the theory of theta monoids discussed above, the theory of Gaussian monoids developed in the present paper does not, by itself, admit a multiradial formulation [cf. Remarks 2.9.1, (iii); 3.4.1, (ii); 3.7.1]. In order to obtain a multiradial formulation of the theory of Gaussian monoids which is, in some sense, the ultimate goal of the present series of papers! - it will be necessary to combine the theory of the present paper with the theory of the log-link developed in [IUTchIII]. This will allow us to obtain a multiradial formulation of the theory of Gaussian monoids in [IUTchIII], Theorem 3.11.

One important aspect of the theory of Hodge-Arakelov-theoretic evaluation is the notion of conjugate synchronization. Conjugate synchronization refers to a collection of "symmetrizing isomorphisms" between the various copies of the local absolute Galois group $G_{\underline{v}}$ associated to the labels $\in \mathbb{F}_{l}$ at which one evaluates the theta function [cf. Corollaries 3.5, (i); 3.6, (i); 4.5, (iii); 4.6, (iii)]. We shall also use the term "conjugate synchronization" to refer to similar collections of "symmetrizing isomorphisms" for copies of various objects [such as the monoid $\mathcal{O} \bar{F}_{v}$ ] closely related to the absolute Galois group $G_{\underline{v}}$. With regard to the collections of isomorphisms between copies of $G_{\underline{v}}$, it is of crucial importance that these isomor-

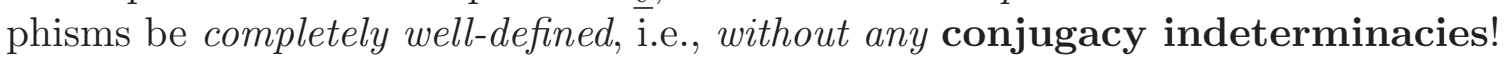
Indeed, if one allows conjugacy indeterminacies [i.e., put another way, if one allows oneself to work with outer isomorphisms, as opposed to isomorphisms], then one must sacrifice either

- the distinct nature of distinct labels $\in\left|\mathbb{F}_{l}\right|$ — which is necessary in order to keep track of the distinct theta values " $\underline{\underline{p^{j}}}=$ " for distinct $\underline{\underline{j}}$ - or

- the crucial compatibility of étale-like and Frobenius-like versions of the symmetrizing isomorphisms with Kummer theory

— cf. the discussion of Remark 3.8.3, (ii); [IUTchIII], Remark 1.5.1; Step (vii) of the proof of [IUTchIII], Corollary 3.12. In this context, it is also of interest to observe that it follows from certain elementary combinatorial considerations that one must require that 
- these symmetrizing isomorphisms arise from a group action, i.e., such as the $\mathbb{F}_{l}^{\rtimes \pm}$-symmetry

— cf. the discussion of Remark 3.5.2. Moreover, since it will be of crucial importance to apply these symmetrizing isomorphisms, in [IUTchIII], $\S 1$ [cf., especially, [IUTchIII], Remark 1.3.2], in the context of the log-link — whose definition depends on the local ring structures at $\underline{v} \in \underline{\mathbb{V}}^{\text {bad }}$ [cf. the discussion of [AbsTopIII], $\S \mathrm{I} 3]$ - it will be necessary to invoke the fact that

- the symmetrizing isomorphisms at $\underline{v} \in \underline{\mathbb{V}}^{\text {bad }}$ arise from conjugation operations within a certain [arithmetic] tempered fundamental group - namely, the tempered fundamental group of $\underline{X}_{v}$ [cf. the notation of [IUTchI], §I1] — that contains $\Pi_{\underline{v}}$ as an open subgroup of finite index

- cf. the discussion of Remark 3.8.3, (ii). Here, we note that these "conjugation operations" related to the $\mathbb{F}_{l}^{\rtimes \pm}$-symmetry may be applied to establish conjugate synchronization precisely because they arise from conjugation by elements of the geometric tempered fundamental group [cf. Remark 3.5.2, (iii)].

The significance of establishing conjugate synchronization - i.e., subject to the various requirements discussed above! - lies in the fact that the resulting symmetrizing isomorphisms allow one to

$$
\text { construct the crucial coric } \mathcal{F}^{\vdash \times \mu} \text {-prime-strips }
$$

- i.e., the $\mathcal{F}^{\vdash \times \boldsymbol{\mu}}$-prime-strips that are preserved, up to isomorphism, by the modified versions of the $\Theta$-link of [IUTchI] [cf. the discussion of the " $\Theta^{\times \boldsymbol{\mu}_{-}}, \Theta_{\mathrm{gau}}^{\times \boldsymbol{\mu}}$-links" below] that are introduced in $\S 4$ of the present paper [cf. Corollary 4.10, (i), (iv); [IUTchIII], Theorem 1.5, (iii); the discussion of [IUTchIII], Remark 1.5.1, (i)].

In $\S 4$, the theory of conjugate synchronization established in $\S 3$ [cf. Corollaries 3.5 , (i); 3.6, (i) $]$ is extended so as to apply to arbitrary $\underline{v} \in \underline{\mathbb{V}}$, i.e., not just $\underline{v} \in \underline{\mathbb{V}}^{\text {bad }}$ [cf. Corollaries 4.5, (iii); 4.6, (iii)]. In particular, in order to work with the theta value labels $\in \mathbb{F}_{l}$ in the context of the $\mathbb{F}_{l}^{\rtimes \pm}$-symmetry, i.e., which involves the action

$$
\mathbb{F}_{l}^{\rtimes \pm} \curvearrowright \mathbb{F}_{l}
$$

on the labels $\in \mathbb{F}_{l}$, one must avail oneself of the global portion of the $\Theta^{ \pm \text {ell }}$-Hodge theaters that appear. Indeed, this global portion allows one to synchronize the $a$ priori independent indeterminacies with respect to the action of $\{ \pm 1\}$ on the various $\underline{\underline{X}}_{\underline{v}}\left[\right.$ for $\left.\underline{v} \in \underline{\mathbb{V}}^{\text {bad }}\right], \underline{X}_{\underline{v}}\left[\right.$ for $\left.\underline{v} \in \underline{\mathbb{V}}^{\text {good }}\right]$ — cf. the discussion of Remark 4.5.3, (iii). On the other hand, the copy of the arithmetic fundamental group of $\underline{X}_{K}$ that constitutes this global portion of the $\Theta^{ \pm \text {ell }}$-Hodge theater is profinite, i.e., it does not admit a "globally tempered version" whose localization at $\underline{v} \in \underline{\mathbb{V}}^{\text {bad }}$ is naturally isomorphic to the corresponding tempered fundamental group at $\underline{v}$. One important consequence of this state of affairs is that

in order to apply the global \pm -synchronization afforded by the $\Theta^{ \pm \text {ell }}$ Hodge theater in the context of the theory of Hodge-Arakelov-theoretic evaluation at $\underline{v} \in \underline{\mathbb{V}}^{\text {bad }}$ relative to labels $\in \mathbb{F}_{l}$ that correspond to conjugacy classes of cuspidal inertia groups of tempered fundamental groups at 
$\underline{v} \in \underline{\mathbb{V}}^{\text {bad }}$, it is necessary to compute the profinite conjugates of such tempered cuspidal inertia groups

— cf. the discussion of [IUTchI], Remark 4.5.1, as well as Remarks 2.5.2 and 4.5.3, (iii), of the present paper, for more details. This is precisely what is achieved by the application of [IUTchI], Theorem B [i.e., in the form of [IUTchI], Corollary 2.5] in $\S 2$ of the present paper.

As discussed above, the theory of Hodge-Arakelov-theoretic evaluation developed in $\S 1, \S 2, \S 3$ is strictly local [at $\underline{v} \in \underline{\mathbb{V}}^{\text {bad }}$ ] in nature. Thus, in $\S 4$, we discuss the essentially routine extensions of this theory, e.g., of the theory of Gaussian monoids, to the "remaining portion" of the $\Theta^{ \pm \text {ell }}$-Hodge theater, i.e., to $\underline{v} \in \underline{\mathbb{V}}^{\text {good }}$, as well as to the case of global realified Frobenioids [cf. Corollaries 4.5, (iv), (v); 4.6, (iv), (v)]. We also discuss the corresponding complements, involving the theory of [IUTchI], §5, for $\Theta N F-H o d g e$ theaters [cf. Corollaries 4.7, 4.8]. This leads naturally to the construction of modified versions of the $\Theta$-link of [IUTchI] [cf. Corollary 4.10, (iii)]. These modified versions may be described as follows:

- The $\Theta^{\times \boldsymbol{\mu}}$-link is essentially the same as the $\Theta$-link of [IUTchI], Theorem A, except that $\mathcal{F}^{\Vdash}$-prime-strips are replaced by $\mathcal{F}^{\Vdash} \triangleright \times \boldsymbol{\mu}$-prime-strips [cf. [IUTchI], Fig. I1.2] — i.e., roughly speaking, the various local " $\mathcal{O}^{\times}$" are replaced by " $\mathcal{O}^{\times \mu}=\mathcal{O}^{\times} / \mathcal{O}^{\mu} "$.

- The $\Theta_{\text {gau }}^{\times \boldsymbol{\mu}}$-link is essentially the same as the $\Theta^{\times \boldsymbol{\mu}}$-link, except that the theta monoids that give rise to the $\Theta^{\times \mu}$-link are replaced, via composition with a certain isomorphism that arises from Hodge-Arakelov-theoretic evaluation, by Gaussian monoids [cf. the above discussion!] — i.e., roughly speaking, the various " $\underline{\underline{\Theta}}_{\underline{v}}$ " at $\underline{v} \in \underline{\mathbb{V}}^{\text {bad }}$ are replaced by " $\left.\{\underline{\underline{q} \underline{\underline{\underline{v}}}}\}_{\underline{\underline{j}}=1, \ldots, l *}\right\}^{*}$ ".

The basic properties of the $\Theta^{\times \boldsymbol{\mu}_{-}}, \Theta_{\text {gau }}^{\times \boldsymbol{\mu}}$-links, including the corresponding Frobeniusand étale-pictures, are summarized in Theorems A, B below [cf. Corollaries 4.10, 4.11 for more details]. Relative to the analogy between the theory of the present series of papers and $p$-adic Teichmüller theory, the passage from the $\Theta^{\times \mu}$-link to the $\Theta_{\mathrm{gau}}^{\times \boldsymbol{\mu}}$-link via Hodge-Arakelov-theoretic evaluation may be thought of as corresponding to the passage

\section{$\mathcal{M F}^{\nabla}$-objects $\rightsquigarrow$ Galois representations}

in the case of the canonical indigenous bundles that occur in $p$-adic Teichmüller theory - cf. the discussion of Remark 4.11.4, (ii), (iii). In particular, the corresponding passage from the Frobenius-picture associated to the $\Theta^{\times} \boldsymbol{\mu}_{\text {-link }}$ to the Frobenius-picture associated to the $\Theta_{\mathrm{gau}}^{\times \boldsymbol{\mu}}$-link — or, more properly, relative to the point of view of [IUTchIII] [cf. also the discussion of [IUTchI], §I4], from the log-theta-lattice arising from the $\Theta^{\times \mu}$-link to the log-theta-lattice arising from the $\Theta_{\text {gau }}^{\times \mu}$-link - corresponds [i.e.., relative to the analogy with $p$-adic Teichmüller theory] to the passage

from thinking of canonical liftings as being determined by canonical $\mathcal{M F}^{\nabla}$-objects to thinking of canonical liftings as being determined by canonical Galois representations [cf. Fig. I.7 below]. 
In this context, it is of interest to note that this point of view is precisely the point of view taken in the absolute anabelian reconstruction theory developed in [CanLift], $\S 3$ [cf. Remark 4.11.4, (iii), (a)]. Finally, we observe that from this point of view, the important theory of conjugate synchronization via $\mathbb{F}_{l}^{\rtimes \pm}$-symmetry may be thought of as corresponding to the theory of the deformation of the canonical Galois representation from " $\bmod p^{n}$ " to " $\bmod p^{n+1}$ " [cf. Fig. I.7 below; the discussion of Remark 4.11.4, (iii), (d)].

\begin{tabular}{|c|c|}
\hline $\begin{array}{l}\frac{\text { Property related to }}{\text { Hodge-Arakelov-theoretic }} \\
\frac{\text { evaluation in inter-universal }}{\text { Teichmüller theory }}\end{array}$ & $\begin{array}{l}\frac{\text { Corresponding phenomenon }}{\underline{\text { in }}} \\
\text { p-adic Teichmüller theory }\end{array}$ \\
\hline $\begin{array}{c}\text { passage from } \\
\Theta^{\times \boldsymbol{\mu}} \text {-link } \\
\text { to } \\
\Theta_{\mathrm{gau}}^{\times \mu} \text {-link }\end{array}$ & $\begin{array}{c}\text { passage from } \\
\text { canonicality via } \mathcal{M F}^{\nabla} \text {-objects } \\
\text { to canonicality via } \\
\text { crystalline Galois representations }\end{array}$ \\
\hline $\begin{array}{l}\mathbb{F}_{l}^{\rtimes \pm}-, \mathbb{F}_{l}^{*}- \\
\text { symmetries }\end{array}$ & $\begin{array}{c}\text { ordinary, supersingular monodromy } \\
\text { of canonical Galois representation }\end{array}$ \\
\hline $\begin{array}{l}\text { conjugate } \\
\text { synchronization } \\
\text { via } \mathbb{F}_{l}^{\rtimes \pm} \text {-symmetry }\end{array}$ & $\begin{array}{l}\text { deformation of } \\
\text { canonical Galois representation } \\
\text { from "mod } p^{n} " \text { to " } \bmod p^{n+1} "\end{array}$ \\
\hline
\end{tabular}

Fig. I.7: Properties related to Hodge-Arakelov-theoretic evaluation in inter-universal Teichmüller theory and corresponding phenomena in $p$-adic Teichmüller theory

Certain aspects of the various constructions discussed above are summarized in the following two results, i.e., Theorems $A, B$, which are abbreviated versions of Corollaries 4.10, 4.11, respectively. On the other hand, many important aspects — such as multiradiality! — of these constructions do not appear explicitly in Theorems A, B. The main reason for this is that it is difficult to formulate "final results" concerning such aspects as multiradiality in the absence of the framework that is to be developed in [IUTchIII].

Theorem A. (Frobenioid-pictures of $\Theta^{ \pm \text {ell }}$ NF-Hodge Theaters) Fix a collection of initial $\Theta$-data $\left(\bar{F} / F, X_{F}, l, \underline{C}_{K}, \underline{\mathbb{V}}, \underline{\epsilon}\right)$ as in [IUTchI], Definition 3.1. Let ${ }^{\dagger} \mathcal{H} \mathcal{T}^{\Theta^{ \pm \text {ell }} \mathrm{NF}} ; \quad \ddagger \mathcal{H} \mathcal{T}^{\Theta^{ \pm \text {ell }} \mathrm{NF}}$ be $\Theta^{ \pm \text {ell }} \mathbf{N F}$-Hodge theaters [relative to the given initial $\Theta$-data] - cf. [IUTchI], Definition 6.13, (i). Write ${ }^{\dagger} \mathcal{H} \mathcal{T}^{\mathcal{D}-\Theta^{ \pm \mathrm{ell}} \mathrm{NF}}$, 
$\ddagger \mathcal{H} \mathcal{T}^{\mathcal{D}-\Theta^{ \pm \mathrm{ell}} \mathrm{NF}}$ for the associated $\mathcal{D}$ - $\Theta^{ \pm \mathrm{ell}} \mathrm{NF}$-Hodge theaters - cf. [IUTchI], Definition 6.13, (ii). Then:

(i) (Constant Prime-Strips) By applying the symmetrizing isomorphisms, with respect to the $\mathbb{F}_{l}^{\rtimes \pm}$-symmetry, of Corollary 4.6, (iii), to the data of the underlying $\Theta^{ \pm \text {ell }}$-Hodge theater of ${ }^{\dagger} \mathcal{H} \mathcal{T}^{\Theta^{ \pm e l l} \mathrm{NF}}$ that is labeled by $t \in \operatorname{LabCusp}^{ \pm}\left({ }^{\dagger} \mathfrak{D}_{\succ}\right)$, one may construct, in a natural fashion, an $\mathcal{F}^{\Vdash}$-prime-strip

$$
{ }^{\dagger} \mathfrak{F}_{\triangle}^{\vdash}=\left({ }^{\dagger} \mathcal{C}_{\triangle}^{\Vdash}, \operatorname{Prime}\left({ }^{\dagger} \mathcal{C}_{\triangle}^{\Vdash}\right) \stackrel{\sim}{\rightarrow} \underline{\mathbb{V}},{ }^{\dagger} \mathfrak{F}_{\triangle}^{\vdash},\left\{{ }^{\dagger} \rho_{\triangle, \underline{v}}\right\}_{\underline{v} \in \underline{\mathbb{V}}}\right)
$$

that is equipped with a natural identification isomorphism of $\mathcal{F}^{\Vdash}$-prime-strips ${ }^{\dagger} \mathfrak{F}_{\triangle} \stackrel{\sim}{\rightarrow} \dagger \mathfrak{F}_{\text {mod }}^{\vdash}$ between ${ }^{\dagger} \mathfrak{F}_{\triangle}^{\Vdash}$ and the $\mathcal{F}^{\Vdash}$-prime-strip ${ }^{\dagger} \mathfrak{F}_{\text {mod }}^{\Vdash}$ of [IUTchI], Theorem $A$, (ii); this isomorphism induces a natural identification isomorphism of $\mathcal{D}^{\vdash}$. prime-strips ${ }^{\dagger} \mathfrak{D}_{\triangle}^{\vdash} \stackrel{\sim}{\rightarrow}{ }^{\dagger} \mathfrak{D}_{>}^{\vdash}$ between the $\mathcal{D}^{\vdash}$-prime-strip ${ }^{\dagger} \mathfrak{D}_{\triangle}^{\vdash}$ associated to ${ }^{\dagger} \mathfrak{F}_{\triangle}^{\vdash}$ and the $\mathcal{D}^{\vdash}$-prime-strip ${ }^{\dagger} \mathfrak{D}_{>}^{\vdash}$ of [IUTchI], Theorem A, (iii).

(ii) (Theta and Gaussian Prime-Strips) By applying the constructions of Corollary 4.6, (iv), ( $v)$, to the underlying $\Theta$-bridge and $\Theta^{ \pm \text {ell }}$-Hodge theater of ${ }^{\dagger} \mathcal{H} \mathcal{T}^{\Theta^{ \pm \mathrm{ell}} \mathrm{NF}}$, one may construct, in a natural fashion, $\mathcal{F}^{\Vdash}$-prime-strips

$$
\begin{aligned}
& { }^{\dagger} \mathfrak{F}_{\text {env }}^{\Vdash}=\left({ }^{\dagger} \mathcal{C}_{\text {env }}^{\Vdash}, \operatorname{Prime}\left({ }^{\dagger} \mathcal{C}_{\text {env }}^{\Vdash}\right) \stackrel{\sim}{\rightarrow} \underline{\mathbb{V}},{ }^{\dagger} \mathfrak{F}_{\text {env }}^{\vdash}, \quad\left\{{ }^{\dagger} \rho_{\text {env }, \underline{v}}\right\}_{\underline{v} \in \underline{\mathbb{V}}}\right) \\
& { }^{\dagger} \mathfrak{F}_{\text {gau }}^{\Vdash}=\left({ }^{\dagger} \mathcal{C}_{\text {gau }}^{\Vdash}, \operatorname{Prime}\left({ }^{\dagger} \mathcal{C}_{\text {gau }}^{\Vdash}\right) \stackrel{\sim}{\rightarrow} \underline{\mathbb{V}},{ }^{\dagger} \mathfrak{F}_{\text {gau }}^{\vdash}, \quad\left\{{ }^{\dagger} \rho_{\text {gau }, \underline{v}}\right\}_{\underline{v} \in \underline{\mathbb{V}}}\right)
\end{aligned}
$$

that are equipped with a natural identification isomorphism of $\mathcal{F}^{\Vdash}$-prime-strips ${ }^{\dagger} \mathfrak{F}_{\text {env }}^{\Vdash} \stackrel{\sim}{\rightarrow}{ }^{\dagger} \mathfrak{F}_{\text {tht }}^{\Vdash}$ between ${ }^{\dagger} \mathfrak{F}_{\text {env }}^{\Vdash}$ and the $\mathcal{F}^{\Vdash}$-prime-strip ${ }^{\dagger} \mathfrak{F}_{\text {tht }}^{\Vdash}$ of [IUTchI], Theorem $A$, (ii), as well as an evaluation isomorphism

$$
\dagger \mathfrak{F}_{\text {env }}^{\vdash} \stackrel{\sim}{\rightarrow}+\mathfrak{F}_{\text {gau }}^{\Vdash}
$$

of $\mathcal{F}^{\Vdash}$-prime-strips.

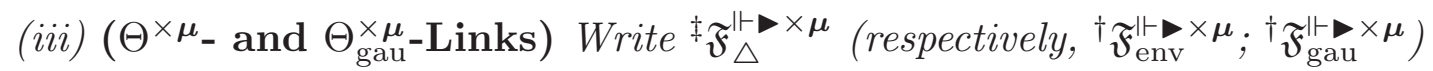
for the $\mathcal{F}^{\Vdash} \triangleright \times \boldsymbol{\mu}$-prime-strip associated to the $\mathcal{F}^{\Vdash}$-prime-strip ${ }^{\ddagger} \mathfrak{F}_{\triangle}$ (respectively, ${ }^{\dagger} \mathfrak{F}_{\text {env }}^{\Vdash} ;{ }^{\dagger} \mathfrak{F}_{\text {gau }}^{\Vdash}$ ). We shall refer to the full poly-isomorphism ${ }^{\dagger} \mathfrak{F}_{\text {env }}^{\Vdash \bullet} \times \stackrel{\sim}{\rightarrow} \ddagger \mathfrak{F}_{\triangle}^{\Vdash \bullet} \times \boldsymbol{\mu}$ as the $\Theta^{\times \mu}$-link

$$
\dagger \mathcal{H} \mathcal{T}^{\Theta}{ }^{ \pm \mathrm{ell}} \mathrm{NF} \quad \stackrel{\Theta^{\times \mu}}{\longrightarrow} \ddagger \mathcal{H} \mathcal{T}^{\Theta^{ \pm \mathrm{ell}} \mathrm{NF}}
$$

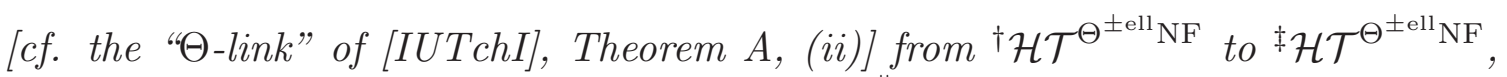

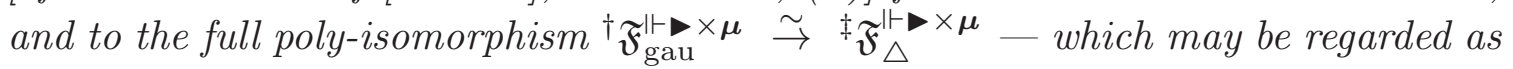
being obtained from the full poly-isomorphism ${ }^{\dagger} \mathfrak{F}_{\text {env }}^{\Vdash} \times \boldsymbol{\mu} \stackrel{\sim}{\rightarrow}{ }^{\ddagger} \mathfrak{F} \mathfrak{F}_{\triangle} \times \times \boldsymbol{\mu}$ by composition with the inverse of the evaluation isomorphism of (ii) - as the $\Theta_{\mathrm{gau}}^{\times \boldsymbol{\mu}}$-link

$$
\dagger \mathcal{H} \mathcal{T}^{\Theta^{ \pm \text {ell }} \mathrm{NF}} \stackrel{\Theta_{\text {gau }}^{\times \mu}}{\longrightarrow} \ddagger \mathcal{H} \mathcal{T}^{\Theta^{ \pm \text {ell }} \mathrm{NF}}
$$

from ${ }^{\dagger} \mathcal{H} \mathcal{T}^{\Theta^{ \pm \mathrm{ell}} \mathrm{NF}}$ to ${ }^{\ddagger} \mathcal{H} \mathcal{T}^{\Theta^{ \pm \mathrm{ell}} \mathrm{NF}}$.

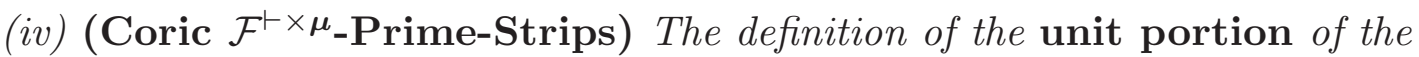
theta and Gaussian monoids that appear in the construction of the $\mathcal{F}^{\Vdash}$-primestrips ${ }^{\dagger} \mathfrak{F}_{\text {env }}^{\Vdash},{ }^{\dagger} \mathfrak{F}_{\text {gau }}^{\Vdash}$ of (ii) gives rise to natural isomorphisms

$$
\dagger \mathfrak{F}_{\triangle}^{\vdash \times \mu} \stackrel{\sim}{\rightarrow} \dagger \mathfrak{F}_{\text {env }}^{\vdash \times \mu} \stackrel{\sim}{\rightarrow} \dagger \mathfrak{F}_{\text {gau }}^{\vdash \times \mu}
$$


of the $\mathcal{F}^{\vdash \times \boldsymbol{\mu}}$-prime-strips associated to the $\mathcal{F}^{\Vdash}$-prime-strips ${ }^{\dagger} \mathfrak{F} \mathfrak{F}_{\triangle}^{\Vdash},{ }^{\dagger} \mathfrak{F}_{\text {env }}^{\Vdash},{ }^{\dagger} \mathfrak{F}_{\text {gau }}^{\Vdash}$. Moreover, by composing these natural isomorphisms with the poly-isomorphisms induced on the respective $\mathcal{F}^{\vdash \times \boldsymbol{\mu}}$-prime-strips by the $\Theta^{\times \boldsymbol{\mu}}$ - and $\Theta_{\mathrm{gau}}^{\times \boldsymbol{\mu}}$-links of (iii), one obtains a poly-isomorphism

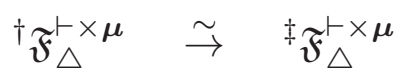

which coincides with the full poly-isomorphism between these two $\mathcal{F}^{\vdash \times \boldsymbol{\mu}}$-prime-

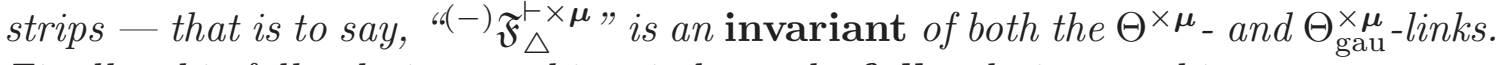
Finally, this full poly-isomorphism induces the full poly-isomorphism

$$
{ }^{\dagger} \mathfrak{D}_{\triangle}^{\vdash} \stackrel{\sim}{\rightarrow}{ }^{\ddagger} \mathfrak{D}_{\triangle}^{\vdash}
$$

between the associated $\mathcal{D}^{\vdash}$-prime-strips; we shall refer to this poly-isomorphism as the $\mathcal{D}$ - $\Theta^{ \pm \text {ell }} \mathbf{N F}$-link from ${ }^{\dagger} \mathcal{H} \mathcal{T}^{\mathcal{D}-\Theta^{ \pm \mathrm{ell}} \mathrm{NF}}$ to ${ }^{\ddagger} \mathcal{H} \mathcal{T}^{\mathcal{D}-\Theta^{ \pm \mathrm{ell}} \mathrm{NF}}$.

(v) (Frobenius-pictures) Let $\left\{{ }^{n} \mathcal{H} \mathcal{T}^{\Theta^{ \pm e l l} \mathrm{NF}}\right\}_{n \in \mathbb{Z}}$ be a collection of distinct $\Theta^{ \pm e l l}$ NF-Hodge theaters indexed by the integers. Then by applying the $\Theta^{\times \mu_{-}}$ and $\Theta_{\text {gau }}^{\times \mu}$-links of (iii), we obtain infinite chains

$$
\begin{aligned}
& \ldots \stackrel{\Theta^{\times \mu}}{\longrightarrow}(n-1) \mathcal{H} \mathcal{T}^{\Theta^{ \pm \mathrm{ell}} \mathrm{NF}} \stackrel{\Theta^{\times \mu}}{\longrightarrow}{ }^{n} \mathcal{H} \mathcal{T}^{\Theta^{ \pm \mathrm{ell}} \mathrm{NF}} \stackrel{\Theta^{\times \mu}}{\longrightarrow}{ }^{(n+1)} \mathcal{H} \mathcal{T}^{\Theta^{ \pm \mathrm{ell}} \mathrm{NF}} \stackrel{\Theta^{\times \mu}}{\longrightarrow} \ldots \\
& \ldots \stackrel{\Theta_{\text {gau }}^{\times \mu}}{\longrightarrow}(n-1) \mathcal{H} \mathcal{T}^{\Theta^{ \pm e l l}} \mathrm{NF} \stackrel{\Theta_{\text {gau }}^{\times \mu}}{\longrightarrow} n \mathcal{H} \mathcal{T}^{\Theta^{ \pm e l l}} \mathrm{NF} \stackrel{\Theta_{\text {gau }}^{\times \mu}}{\longrightarrow}(n+1) \mathcal{H} \mathcal{T}^{\Theta^{ \pm e l l}} \mathrm{NF} \stackrel{\Theta_{\text {gau }}^{\times \mu}}{\longrightarrow} \ldots
\end{aligned}
$$

of $\Theta^{\times \mu}-/ \Theta_{\text {gau }}^{\times \mu}$-linked $\Theta$-Hodge theaters - cf. Fig. I.8 below, in the case of the $\Theta_{\mathrm{gau}}^{\times \mu}$-link. Either of these infinite chains may be represented symbolically as an oriented graph $\vec{\Gamma}$

- i.e., where the arrows correspond to either the " $\stackrel{\Theta^{\times \mu}}{\longrightarrow}$ 's" or the " $\stackrel{\Theta_{\text {gau }}^{\times \mu}}{\longrightarrow}$ 's", and the "•'s" correspond to the "n $\mathcal{H} \mathcal{T}^{\Theta^{ \pm \mathrm{ell}} \mathrm{NF}}$ ". This oriented graph $\vec{\Gamma}$ admits a natural action by $\mathbb{Z}$ - i.e., a translation symmetry - but it does not admit arbitrary permutation symmetries. For instance, $\vec{\Gamma}$ does not admit an automorphism that switches two adjacent vertices, but leaves the remaining vertices fixed.

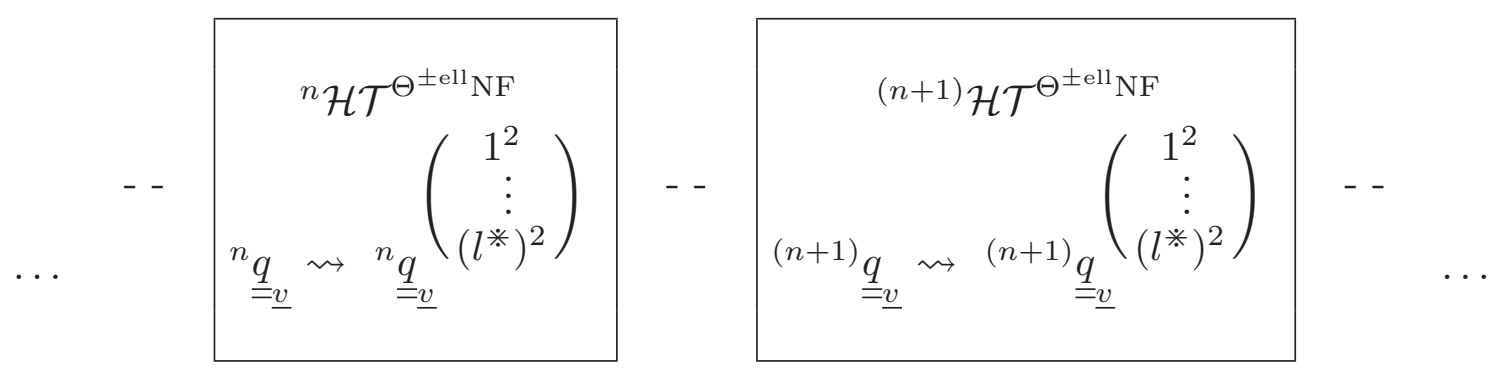

Fig. I.8: Frobenius-picture associated to the $\Theta_{\mathrm{gau}}^{\times \mu}$-link 
Theorem B. (Étale-pictures of Base- $\Theta^{ \pm \text {ell }}$ NF-Hodge Theaters) Suppose that we are in the situation of Theorem $A,(v)$.

(i) Write

$$
\quad \stackrel{\mathcal{D}}{\longrightarrow} n^{n} \mathcal{H} \mathcal{T}^{\mathcal{D}-\Theta^{ \pm \mathrm{ell}} \mathrm{NF}} \stackrel{\mathcal{D}}{\longrightarrow} \quad{ }^{(n+1)} \mathcal{H} \mathcal{T}^{\mathcal{D}-\Theta^{ \pm \mathrm{ell}} \mathrm{NF}} \stackrel{\mathcal{D}}{\longrightarrow} \quad \ldots
$$

- where $n \in \mathbb{Z}$ - for the infinite chain of $\mathcal{D}-\Theta^{ \pm \text {ell }} \mathbf{N F}$-linked $\mathcal{D}$ - $\Theta^{ \pm \text {ell }} \mathbf{N F}$ Hodge theaters [cf. Theorem A, (iv), (v)] induced by either of the infinite chains of Theorem A, $(v)$. Then this infinite chain induces a chain of full polyisomorphisms

$$
\ldots \stackrel{\sim}{\rightarrow} n^{\circ} \mathfrak{D}_{\triangle}^{\vdash} \stackrel{\sim}{\rightarrow}(n+1) \mathfrak{D}_{\triangle}^{\vdash} \stackrel{\sim}{\rightarrow} \ldots
$$

[cf. Theorem A, (iv)]. That is to say, “(-) $\mathfrak{D}_{\triangle}^{\vdash}$ ” forms a constant invariant i.e., a "mono-analytic core" [cf. the discussion of [IUTchI], §I1] - of the above infinite chain.

(ii) If we regard each of the $\mathcal{D}-\Theta^{ \pm \mathrm{ell}} N F$-Hodge theaters of the chain of (i) as a spoke emanating from the mono-analytic core " (-) $\mathfrak{D}_{\triangle}^{\vdash}$ " discussed in (i), then we obtain a diagram - i.e., an étale-picture of $\mathcal{D}-\Theta^{ \pm \text {ell }} \mathbf{N F}-H o d g e$ theaters - as in Fig. I.9 below [cf. the situation discussed in [IUTchI], Theorem A, (iii)]. Thus, each spoke may be thought of as a distinct "arithmetic holomorphic structure" on the mono-analytic core. Finally, [cf. the situation discussed in [IUTchI], Theorem A, (iii)] this diagram satisfies the important property of admitting arbitrary permutation symmetries among the spokes [i.e., the labels $n \in \mathbb{Z}$ of the $\mathcal{D}-\Theta^{ \pm e l l} N F$-Hodge theaters].

(iii) The constructions of (i) and (ii) are compatible, in the evident sense, with the constructions of [IUTchI], Theorem A, (iii), relative to the natural identification isomorphisms ${ }^{(-)} \mathfrak{D}_{\triangle}^{\vdash} \stackrel{\sim}{\rightarrow}\left({ }^{(-)} \mathfrak{D}_{>}^{\vdash}[c f\right.$. Theorem $A,(i)]$.

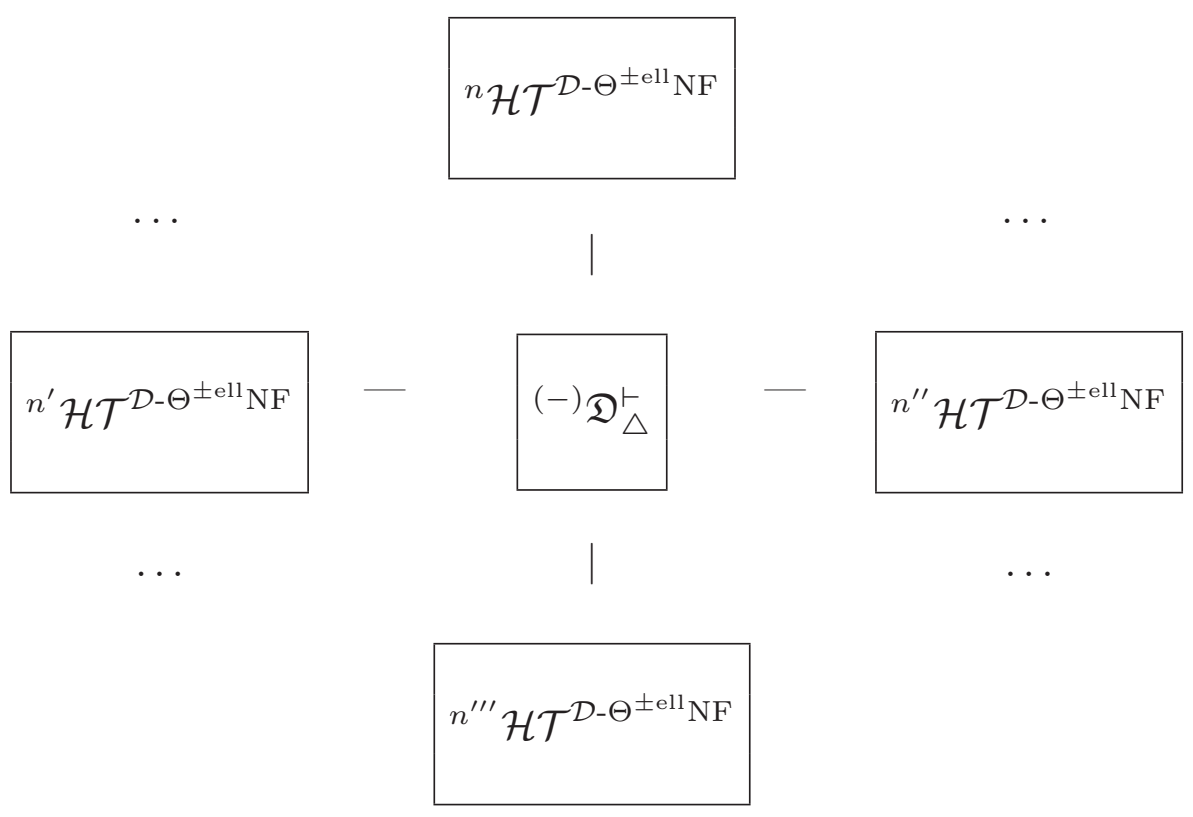

Fig. I.9: Étale-picture of $\mathcal{D}-\Theta^{ \pm \text {ell }} \mathrm{NF}-$ Hodge theaters 


\section{Acknowledgements:}

I would like to thank Fumiharu Kato and Akio Tamagawa for many helpful discussions concerning the material presented in this paper.

\section{Notations and Conventions:}

We shall continue to use the "Notations and Conventions" of [IUTchI], $\S 0$. 


\section{Section 1: Multiradial Mono-theta Environments}

In the present $\S 1$, we review the theory of mono-theta environments developed in [EtTh] and give a "multiradial" interpretation of this theory which will be of substantial importance in the present series of papers. Roughly speaking, in the language of [AbsTopIII], §I3, this interpretation consists of the computation of which portion of the various objects constructed from the "arithmetic holomorphic structures" of various $\Theta$-Hodge theaters may be glued together, in a consistent fashion, via a "mono-analytic" [i.e., "arithmetic real analytic"] core. Put another way, this computation may be thought of as the computation of

what one arithmetic holomorphic structure looks like from the point of view of a distinct arithmetic holomorphic structure that is only related to the orginal arithmetic holomorphic structure via the mono-analytic core.

In fact, this sort of computation forms one of the central themes of the present series of papers.

Let $N \in \mathbb{N}_{\geq 1}$ be a positive integer; $l$ a prime number; $k$ an MLF of odd residue characteristic $p$ that contains a primitive $4 l$-th root of unity; $\bar{k}$ an algebraic closure of $k$;

$$
\underline{\underline{X}} k
$$

a hyperbolic curve of type $\left(1, l\right.$-tors $\left.{ }^{\Theta}\right)$ over $k$ that admits a stable model over the ring of integers $\mathcal{O}_{k}$ of $k ; \underline{\underline{X}}_{k} \rightarrow C_{k}$ the $k$-core determined by $\underline{\underline{X}}_{k}$ [cf. the discussion preceding [EtTh], Definition 1.7]. Write $\Pi_{\underline{\underline{X}}_{k}}^{\mathrm{tp}}$ for the tempered fundamental group of $\underline{\underline{X}}_{k} ; G_{k} \stackrel{\text { def }}{=} \operatorname{Gal}(\bar{k} / k) ; \Delta_{\underline{\underline{X}}_{k}}^{\mathrm{tp}} \stackrel{\text { def }}{=} \operatorname{Ker}\left(\Pi_{\underline{\underline{X}}_{k}}^{\mathrm{tp}} \rightarrow G_{k}\right) \subseteq \Pi_{\underline{\underline{X}}_{k}}^{\mathrm{tp}}$ for the geometric tempered fundamental group of $\underline{\underline{X}}_{k}$. We shall use similar notation for objects associated to $C_{k}$.

\section{Definition 1.1. $\quad$ Let}

$$
\mathbb{M}^{\Theta}
$$

be a $\bmod N$ mono-theta environment [cf. [EtTh], Definition 2.13, (ii)] which is isomorphic to the mod $N$ model mono-theta environment determined by $\underline{\underline{X}}_{k}$; write $\Pi_{\mathbb{M} \Theta}$ for the underlying topological group of $\mathbb{M}^{\Theta}$ [cf. [EtTh], Definition 2.13, (ii), (a)]. Then:

(i) There exist functorial algorithms

$$
\begin{gathered}
\mathbb{M}^{\Theta} \mapsto \prod_{\underline{\underline{Y}}}\left(\mathbb{M}^{\Theta}\right) ; \quad \mathbb{M}^{\Theta} \mapsto \Pi_{\underline{\underline{X}}}\left(\mathbb{M}^{\Theta}\right) ; \quad \mathbb{M}^{\Theta} \mapsto G\left(\mathbb{M}^{\Theta}\right) ; \quad \mathbb{M}^{\Theta} \mapsto \Delta_{\mathbb{M}^{\Theta}} ; \\
\mathbb{M}^{\Theta} \mapsto \Delta_{\underline{\underline{Y}}}\left(\mathbb{M}^{\Theta}\right) ; \quad \mathbb{M}^{\Theta} \mapsto \Delta_{\underline{\underline{X}}}\left(\mathbb{M}^{\Theta}\right) ; \quad \mathbb{M}^{\Theta} \mapsto\left(l \cdot \Delta_{\Theta}\right)\left(\mathbb{M}^{\Theta}\right) ; \quad \mathbb{M}^{\Theta} \mapsto \Pi_{\mu}\left(\mathbb{M}^{\Theta}\right)
\end{gathered}
$$

for constructing from $\mathbb{M}^{\Theta}$ a quotient $\Pi_{\mathbb{M} \Theta} \rightarrow \Pi_{\underline{Y}}\left(\mathbb{M}^{\Theta}\right)$ [cf. [EtTh], Corollary 2.18, (iii)]; a topological group $\Pi_{\underline{\underline{X}}}\left(\mathbb{M}^{\Theta}\right)$ which is isomorphic to $\Pi_{\underline{\underline{X}}_{k}}^{\text {tp }}$ and contains $\Pi_{\underline{\underline{Y}}}\left(\mathbb{M}^{\Theta}\right)$ as a normal open subgroup [cf. [EtTh], Corollary 2.18, (iii)]; a 
quotient $\Pi_{\underline{X}}\left(\mathbb{M}^{\Theta}\right) \rightarrow G\left(\mathbb{M}^{\Theta}\right)$ corresponding to $G_{k}$ [cf., [EtTh], Corollary 2.18, (i)], which may also be thought of as a quotient $\Pi_{\mathbb{M} \Theta} \rightarrow \Pi_{\underline{Y}}\left(\mathbb{M}^{\Theta}\right) \rightarrow G\left(\mathbb{M}^{\Theta}\right)$; a closed normal subgroup $\Delta_{\mathbb{M}^{\Theta}} \stackrel{\text { def }}{=} \operatorname{Ker}\left(\Pi_{\mathbb{M}^{\Theta}} \rightarrow G\left(\mathbb{M}^{\Theta}\right)\right) \subseteq \Pi_{\mathbb{M}^{\Theta}}$; a closed normal subgroup $\Delta_{\underline{\underline{Y}}}\left(\mathbb{M}^{\Theta}\right) \stackrel{\text { def }}{=} \operatorname{Ker}\left(\Pi_{\underline{\underline{Y}}}\left(\mathbb{M}^{\Theta}\right) \rightarrow G\left(\mathbb{M}^{\Theta}\right)\right) \subseteq \Pi_{\underline{\underline{Y}}}\left(\mathbb{M}^{\Theta}\right)$; a closed normal subgroup $\Delta_{\underline{\underline{X}}}\left(\mathbb{M}^{\Theta}\right) \stackrel{\text { def }}{=} \operatorname{Ker}\left(\Pi_{\underline{\underline{X}}}\left(\mathbb{M}^{\Theta}\right) \rightarrow G\left(\mathbb{M}^{\Theta}\right)\right) \subseteq \Pi_{\underline{\underline{X}}}\left(\mathbb{M}^{\Theta}\right)$ corresponding to $\Delta_{\underline{\underline{X}}_{k}}^{\text {tp }}[$ cf., [EtTh], Corollary 2.18, (i)]; a subquotient $\left(l \cdot \Delta_{\Theta}\right)\left(\mathbb{M}^{\Theta}\right)$ of $\Pi_{\underline{Y}}\left(\mathbb{M}^{\Theta}\right)$ which admits a natural $\Pi_{\underline{\underline{X}}}\left(\mathbb{M}^{\Theta}\right)$-action [hence also a $\Pi_{\underline{\underline{Y}}}\left(\mathbb{M}^{\Theta}\right)$-action, as well as, by composition, a

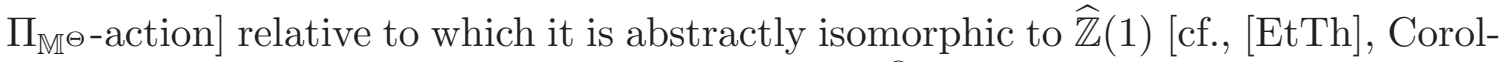
lary 2.18, (i)]; a closed normal subgroup $\Pi_{\boldsymbol{\mu}}\left(\mathbb{M}^{\Theta}\right) \subseteq \Pi_{\mathbb{M} \Theta}$ [cf., [EtTh], Corollary 2.19, (i)] which admits a natural $\Pi_{\underline{\underline{X}}}\left(\mathbb{M}^{\Theta}\right)$-action [hence also a $\Pi_{\underline{\underline{Y}}}\left(\mathbb{M}^{\Theta}\right)$-action, as

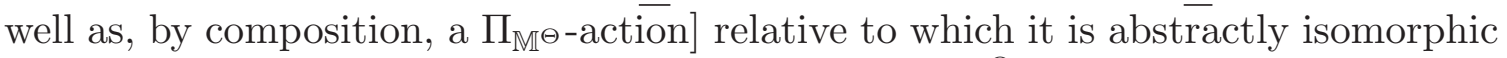
to $(\mathbb{Z} / N \mathbb{Z})(1)$. Also, we recall that the structure of $\mathbb{M}^{\Theta}$ determines a lifting of the natural outer action of

$$
(l \cdot \underline{\mathbb{Z}})\left(\mathbb{M}^{\Theta}\right) \stackrel{\text { def }}{=} \Pi_{\underline{\underline{X}}}\left(\mathbb{M}^{\Theta}\right) / \Pi_{\underline{\underline{Y}}}\left(\mathbb{M}^{\Theta}\right) \cong \Delta_{\underline{\underline{X}}}\left(\mathbb{M}^{\Theta}\right) / \Delta_{\underline{\underline{Y}}}\left(\mathbb{M}^{\Theta}\right)
$$

on $\Delta_{\underline{Y}}\left(\mathbb{M}^{\Theta}\right)$ to an outer action of $(l \cdot \underline{\mathbb{Z}})\left(\mathbb{M}^{\Theta}\right)$ on $\Delta_{\mathbb{M}} \Theta[$ cf. [EtTh], Definition 2.13, (i), (ii), and the preceding discussion; [EtTh], Proposition 2.14, (i)].

(ii) We shall refer to $\left(l \cdot \Delta_{\Theta}\right)\left(\mathbb{M}^{\Theta}\right)$ (respectively, $\Pi_{\boldsymbol{\mu}}\left(\mathbb{M}^{\Theta}\right)$ ) as the interior (respectively, exterior) cyclotome associated to $\mathbb{M}^{\Theta}$. By [EtTh], Corollary 2.19, (i), there is a functorial algorithm for constructing from $\mathbb{M}^{\Theta}$ a cyclotomic rigidity isomorphism

$$
\left(l \cdot \Delta_{\Theta}\right)\left(\mathbb{M}^{\Theta}\right) \otimes(\mathbb{Z} / N \mathbb{Z}) \stackrel{\sim}{\rightarrow} \Pi_{\boldsymbol{\mu}}\left(\mathbb{M}^{\Theta}\right)
$$

between the reductions modulo $N$ of the interior and exterior cyclotomes associated to $\mathbb{M}^{\Theta}$.

One key property of mono-theta environments is that they may be constructed either group-theoretically from $\Pi_{\underline{\underline{X}}_{k}}^{\mathrm{tp}}$ or category-theoretically from certain tempered Frobenioids related to $\underline{\underline{X}}_{k}$.

\section{Proposition 1.2. (Group- and Frobenioid-theoretic Constructions of Mono-theta Environments)}

(i) Let $\Pi$ be a topological group isomorphic to $\Pi_{\underline{\underline{X}}_{k}}^{\mathrm{tp}}$. Then there exists a functorial group-theoretic algorithm

$$
\Pi \mapsto \mathbb{M}^{\Theta}(\Pi)
$$

for constructing from the topological group $\Pi$ a $\bmod N$ mono-theta environment "up to isomorphism" [cf. [EtTh], Corollary 2.18, (ii)] such that the composite of this algorithm with the algorithm $\mathbb{M}^{\Theta}(\Pi) \mapsto \Pi_{\underline{X}}\left(\mathbb{M}^{\Theta}(\Pi)\right)$ discussed in Definition 1.1, (i), admits a functorial isomorphism $\Pi \stackrel{\widetilde{\sim}}{\rightarrow} \Pi_{\underline{\underline{X}}}\left(\mathbb{M}^{\Theta}(\Pi)\right)$. Here, 
the "isomorphism indeterminacy" of $\mathbb{M}^{\Theta}(\Pi)$ is with respect to a group of " $\boldsymbol{\mu}_{N^{-}}$ conjugacy classes" of automorphisms which is of order 1 (respectively, 2) if $N$ is odd (respectively, even) [cf. [EtTh], Corollary 2.18, (iv)].

(ii) Let $\mathcal{C}$ be a category equivalent to the tempered Frobenioid determined by $\underline{\underline{X}}_{k}$ [i.e., the Frobenioid denoted "C" in the discussion at the beginning of [EtTh],

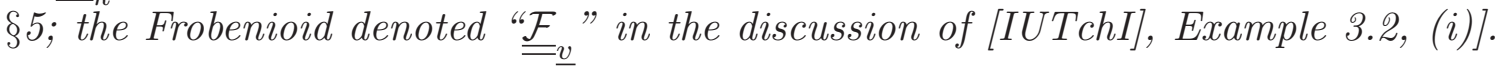
Thus, $\mathcal{C}$ admits a natural Frobenioid structure over a base category $\mathcal{D}$ equivalent to $\mathcal{B}^{\text {temp }}\left(\Pi_{\underline{\underline{X}}_{k}}^{\mathrm{tp}}\right)^{0} \quad$ [cf. [FrdI], Corollary 4.11, (ii), (iv); [EtTh], Proposition 5.1].

Then there exists a functorial algorithm

$$
\mathcal{C} \mapsto \mathbb{M}^{\Theta}(\mathcal{C})
$$

for constructing from the category $\mathcal{C}$ a $\bmod N$ mono-theta environment [cf. [EtTh], Theorem 5.10, (iii)] such that the composite of this algorithm with the algorithm $\mathbb{M}^{\Theta}(\mathcal{C}) \mapsto \Pi_{\underline{X}}\left(\mathbb{M}^{\Theta}(\mathcal{C})\right)$ discussed in Definition 1.1, (i), admits a functorial

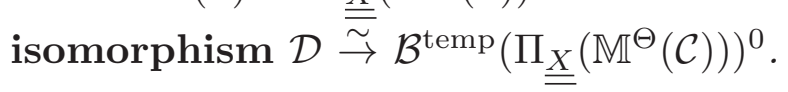

Proof. The assertions of Proposition 1.2 follow immediately from the results of [EtTh] that are quoted in the statements of these assertions.

The cyclotomic rigidity isomorphism of Definition 1.1, (ii), that arises in the case of the mono-theta environment $\mathbb{M}^{\Theta}(\mathcal{C})$ constructed from the tempered Frobenioid $\mathcal{C}$ [cf. Proposition 1.2, (ii)] is compatible with a certain cyclotomic rigidity isomorphism that arises in the theory of [AbsTopIII] [cf. also [FrdII], Theorem 2.4, (ii)] in the following sense.

Proposition 1.3. (Compatibility of Cyclotomic Rigidity Isomorphisms) In the situation of Proposition 1.2, (ii):

(i) (Mono-theta Environments Associated to Tempered Frobenioids) For a suitable object $S \in \mathrm{Ob}(\mathcal{C})$ [cf. [EtTh], Lemma 5.9, (v)], whose image in $\mathcal{D}$ we denote by $S^{\mathrm{bs}} \in \mathrm{Ob}(\mathcal{D})$, the interior cyclotome $\left(l \cdot \Delta_{\Theta}\right)\left(\mathbb{M}^{\Theta}(\mathcal{C})\right) \otimes(\mathbb{Z} / N \mathbb{Z})$ corresponds to a certain subquotient of $\operatorname{Aut}\left(S^{\mathrm{bs}}\right)$, which we denote by $\left(l \cdot \Delta_{\Theta}\right)_{S} \otimes$ $(\mathbb{Z} / N \mathbb{Z})$, while the exterior cyclotome $\Pi_{\boldsymbol{\mu}}\left(\mathbb{M}^{\Theta}(\mathcal{C})\right) \otimes(\mathbb{Z} / N \mathbb{Z})$ corresponds to the subgroup $\boldsymbol{\mu}_{N}(S) \subseteq \mathcal{O}^{\times}(S) \subseteq \operatorname{Aut}(S)$. In particular, the cyclotomic rigidity isomorphism of Definition 1.1, (ii), takes the form of an isomorphism

$$
\left(l \cdot \Delta_{\Theta}\right)_{S} \otimes(\mathbb{Z} / N \mathbb{Z}) \stackrel{\sim}{\rightarrow} \boldsymbol{\mu}_{N}(S)
$$

[cf. [EtTh], Proposition 5.5; [EtTh], Lemma 5.9, (v)].

(ii) (MLF-Galois Pairs) Relative to the formal correspondence between $p$ adic Frobenioids [such as the base-field-theoretic hull $\mathcal{C}^{\text {bs-fld }}$ associated to $\mathcal{C}$ - cf. [EtTh], Definition 3.6, (iv)] and "MLF-Galois TM-pairs" in the theory of [AbsTopIII] [cf. [AbsTopIII], Remark 3.1.1], " $\mu_{N}(S)$ " [cf. (i)] corresponds to " $\boldsymbol{\mu}_{\widehat{\mathbb{Z}}}\left(M_{\mathbb{T M}}\right) \otimes(\mathbb{Z} / N \mathbb{Z})$ " in the theory of $\left[\right.$ Abs TopIII], §3, while " $\left(l \cdot \Delta_{\Theta}\right)_{S} \otimes(\mathbb{Z} / N \mathbb{Z})$ " [cf. (i)] corresponds to " $\boldsymbol{\mu}_{\widehat{\mathbb{Z}}}\left(\Pi_{X}\right) \otimes(\mathbb{Z} / N \mathbb{Z})$ " in the theory of [AbsTopIII], $\S 1$ [cf. 
[IUTchI], Remark 3.1.2, (iii)]. In particular, by composing the inverse of the isomorphism " $\boldsymbol{\mu}_{\widehat{\mathbb{Z}}}\left(G_{k}\right) \stackrel{\sim}{\rightarrow} \boldsymbol{\mu}_{\widehat{\mathbb{Z}}}\left(\Pi_{X}\right)$ " of [AbsTopIII], Corollary 1.10, (c), with the inverse of the isomorphism " $\boldsymbol{\mu}_{\widehat{\mathbb{Z}}}\left(M_{\mathbb{T M}}\right) \stackrel{\sim}{\rightarrow} \boldsymbol{\mu}_{\widehat{\mathbb{Z}}}(G)$ " of [AbsTopIII], Remark 3.2.1, we obtain another cyclotomic rigidity isomorphism

$$
\left(l \cdot \Delta_{\Theta}\right)_{S} \otimes(\mathbb{Z} / N \mathbb{Z}) \stackrel{\sim}{\rightarrow} \boldsymbol{\mu}_{N}(S)
$$

[cf. the various identifications/correspondences of notation discussed above].

(iii) (Compatibility) The cyclotomic rigidity isomorphisms $\left(*^{\text {mono- } \Theta}\right),\left(*^{\text {bs-Gal }}\right)$ of [EtTh], [AbsTopIII] [cf. (i), (ii)] coincide.

Proof. Assertions (i), (ii) follow immediately from the results and definitions of [EtTh], [AbsTopIII] that are quoted in the statements of these assertions. Assertion (iii) follows immediately from the fact that in the situation where the Frobenioid $\mathcal{C}$ involved is not just "some abstract category", but rather arises from familiar objects of scheme theory [cf. the theory of [EtTh], $\S 1$ !], both isomorphisms $\left(*^{\text {mono- } \Theta}\right)$, $\left(*^{\text {bs-Gal }}\right)$ coincide with the conventional identification between the cyclotomes involved that arises from conventional scheme theory.

Proposition 1.4. (Étale Theta Functions of Standard Type) Let $\Pi$ be as in Proposition 1.2, (i). Then there are functorial group-theoretic algorithms [cf. [EtTh], Corollary 2.18, (i)]

$$
\Pi \mapsto \Pi_{\underline{\underline{Y}}}(\Pi) ; \quad \Pi \mapsto\left(l \cdot \Delta_{\Theta}\right)(\Pi)
$$

for constructing from $\Pi$ the open subgroup $\Pi_{\underline{\underline{Y}}}(\Pi) \subseteq \Pi$ corresponding to the tempered covering " $\underline{\underline{Y}}$ " [cf. [EtTh], §2] and a certain subquotient $\left(l \cdot \Delta_{\Theta}\right)(\Pi)$ of $\Pi$ [cf. the subquotient " $\left(l \cdot \Delta_{\Theta}\right)\left(\mathbb{M}^{\Theta}\right)$ " of Definition 1.1, (i)], as well as a functorial group-theoretic algorithm

$$
\Pi \quad \mapsto \quad \underline{\underline{\theta}}(\Pi) \subseteq H^{1}\left(\Pi_{\underline{\underline{Y}}}(\Pi),\left(l \cdot \Delta_{\Theta}\right)(\Pi)\right)
$$

- cf. the constant multiple rigidity property of [EtTh], Corollary 2.19, (iii) - for constructing from $\Pi$ the set $\underline{\underline{\theta}}(\Pi)$ of $\boldsymbol{\mu}_{l}$-multiples [i.e., where $\boldsymbol{\mu}_{l}$ denotes the group of l-th roots of unity] of the reciprocal of the " $\left(l \cdot \underline{\mathbb{Z}} \times \boldsymbol{\mu}_{2}\right)$-orbit $\ddot{\ddot{\eta}}^{\Theta, l \cdot \underline{\mathbb{Z}} \times \boldsymbol{\mu}_{2}}$ of an $l$-th root of the étale theta function of standard type" of [EtTh $\overline{\bar{j}}$, Definition 2.7. In this context, we shall write

$$
\infty \underline{\underline{\theta}}(\Pi) \subseteq \varliminf_{J} H^{1}\left(\left.\Pi_{\ddot{\underline{Y}}}(\Pi)\right|_{J},\left(l \cdot \Delta_{\Theta}\right)(\Pi)\right)
$$

- where $\infty \underline{\underline{\theta}}(\Pi)$ denotes the subset of elements of the direct limit of cohomology modules in the display for which some [positive integer] multiple [i.e., some [positive integer] power, if one writes these modules "multiplicatively"] belongs to $\underline{\underline{\theta}}(\Pi)$; $J$ ranges over the finite index open subgroups of $\Pi$. 
Proof. The assertions of Proposition 1.4 follow immediately from the results and definitions of [EtTh] that are quoted in the statements of these assertions.

Remark 1.4.1. Before proceeding, let us recall from [EtTh], $\S 1, \S 2$, the theory surrounding the "étale theta functions of standard type" that appeared in Proposition 1.4 .

(i) Write

$$
\underline{\underline{X}}_{k} \rightarrow \underline{X}_{k} \rightarrow \underline{C}_{k}
$$

for the hyperbolic orbicurves of type (1,l-tors), (1,l-tors) $)_{ \pm}$determined by $\underline{\underline{X}}_{k}[\mathrm{cf}$. [EtTh], Proposition 2.4]. Thus, $\underline{X}_{k}$ has a unique zero cusp [i.e., unique cusp that lies over precisely one cusp of $\left.\underline{C}_{k}\right]$. Write

$$
\mu_{-} \in \underline{X}_{k}(k)
$$

for the unique torsion point of order 2 whose closure in any stable model of $\underline{X}_{k}$ over $\mathcal{O}_{k}$ intersects the same irreducible component of the special fiber of the stable model as the zero cusp [cf. the discussion of [IUTchI], Example 4.4, (i)].

(ii) The unique order two automorphism $\iota_{\underline{\underline{X}}}$ of $\underline{\underline{X}}_{k}$ over $k$ [cf. [EtTh], Remark 2.6.1] corresponds [cf., e.g., [SemiAnbd], Theorem 6.4] to the unique order two $\Delta_{\underline{\underline{X}}_{k}}^{\text {tp }}$-outer automorphism of $\Pi_{\underline{\underline{X}}_{k}}^{\text {tp }}$ over $G_{k}$, which, by abuse of notation, we shall also denote by $\iota_{\underline{\underline{X}}}$. Write

$$
\underline{\underline{Y}}_{k} \rightarrow \underline{\underline{Y}}_{k} \rightarrow \underline{\underline{X}}_{k}
$$

for the tempered coverings of $\underline{\underline{X}}_{k}$ that correspond, respectively, to the open subgroups $\Pi_{\underline{\underline{\underline{Y}}}_{k}}^{\mathrm{tp}} \stackrel{\text { def }}{=} \Pi_{\underline{\underline{Y}}_{\underline{\underline{Y}}}}\left(\Pi_{\underline{\underline{X}}_{k}}^{\mathrm{tp}}\right) \subseteq \Pi_{\underline{\underline{X}}_{k}}^{\mathrm{tp}}\left[\mathrm{cf} . \quad\right.$ Proposition 1.4], $\Pi_{\underline{\underline{Y}}_{k}}^{\mathrm{tp}} \stackrel{\text { def }}{=} \Pi_{\underline{\underline{Y}}}\left(\Pi_{\underline{\underline{X}}_{k}}^{\mathrm{tp}}\right) \stackrel{\text { def }}{=}$ $\Pi_{\underline{\underline{Y}}}\left(\mathbb{M}^{\Theta}\left(\Pi_{\underline{\underline{X}}_{k}^{\mathrm{tp}}}\right)\right) \subseteq \Pi_{\underline{\underline{X}}_{k}}^{\mathrm{tp}} \quad$ cf. Definition 1.1, (i); Proposition 1.2, (i)]. Since $k$ contains a primitive $4 l$-th root of unity, it follows from the definition of an "étale theta function of standard type" [cf. [EtTh], Definition 1.9, (ii); [EtTh], Definition 2.7] that there exists a rational point

$$
\left(\mu_{-}\right)_{\underline{\underline{Y}}} \in \underline{\underline{Y}}_{k}(k)
$$

that lifts $\mu_{-}$. Moreover, there exists an order two automorphism $\iota_{\underline{\underline{Y}}}$ of $\underline{\underline{\underline{Y}}}_{k} \operatorname{lifting} \iota^{\iota_{\underline{X}}}$ which is uniquely determined up to $l \cdot \underline{\mathbb{Z}}$-conjugacy and composition with an element $\in \operatorname{Gal}\left(\underline{\underline{\underline{Y}}}_{k} / \underline{\underline{Y}}_{k}\right)$ by the condition that it fix the $\operatorname{Gal}\left(\underline{\underline{\underline{Y}}}_{k} / \underline{\underline{Y}}_{k}\right)$-orbit of $\left(\mu_{-}\right)_{\underline{\underline{Y}}}$. Here, we think of $l \cdot \underline{\mathbb{Z}}, \operatorname{Gal}\left(\underline{\underline{Y}}_{k} / \underline{\underline{Y}}_{k}\right)(\cong \mathbb{Z} / 2 \mathbb{Z})$ as the subquotients appearing in the natural exact sequence

$$
1 \rightarrow \operatorname{Gal}\left(\underline{\underline{Y}}_{k} / \underline{\underline{Y}}_{k}\right) \rightarrow \operatorname{Gal}\left(\underline{\underline{Y}}_{k} / \underline{\underline{X}}_{k}\right) \rightarrow l \cdot \underline{\underline{Z}} \rightarrow 1
$$

determined by the coverings $\underline{\underline{Y}}_{k} \rightarrow \underline{\underline{Y}}_{k} \rightarrow \underline{\underline{X}}_{k}$. Again, by abuse of notation, we shall also denote by $\iota_{\ddot{\underline{Y}}}$ the corresponding $\Delta_{\underline{\underline{\underline{Y}}}_{k}}^{\text {tp }}\left(=\Delta_{\underline{\underline{X}}_{k}}^{\text {tp }} \cap \prod_{\underline{\underline{\underline{Y}}}_{k}}^{\text {tp }}\right)$-outer automorphism of $\Pi_{\underline{\underline{\underline{Y}}}_{k}}^{\text {tp }}$. We shall refer to the various automorphisms $\iota_{\underline{\underline{X}}}, \iota_{\underline{\underline{Y}}}$ as inversion automorphisms [cf. [EtTh], Proposition 1.5, (iii)]. Write

$$
D_{\mu_{-}} \subseteq \Pi_{\underline{\underline{Y}}_{k}}
$$


for the decomposition group of $\left(\mu_{-}\right)_{\underline{\underline{\underline{Y}}}}$ [which is well-defined up to $\Delta_{\underline{\underline{\underline{Y}}}_{k}}^{\text {tp }}$-conjugacy] - so $D_{\mu_{-}}$is determined by $\iota_{\underline{\underline{Y}}}$ up to $\Delta_{\underline{\underline{Y}}_{k}}^{\mathrm{tp}}\left(=\Delta_{\underline{\underline{X}}_{k}}^{\mathrm{tp}} \cap \Pi_{\underline{\underline{Y}}_{k}}^{\mathrm{tp}}\right)$-conjugacy. We shall refer to either of the pairs

$$
\left(\iota_{\underline{\underline{\underline{Y}}}} \in \operatorname{Aut}\left(\underline{\underline{\underline{Y}}}_{k}\right),\left(\mu_{-}\right)_{\underline{\underline{\underline{Y}}}}\right) ; \quad\left(\iota_{\underline{\underline{Y}}} \in \operatorname{Aut}\left(\Pi_{\underline{\underline{\underline{Y}}}_{k}}^{\mathrm{tp}}\right) / \operatorname{Inn}\left(\Delta_{\underline{\underline{\underline{Y}}}_{k}^{\mathrm{tp}}}\right), D_{\mu_{-}}\right)
$$

as a pointed inversion automorphism. Again, we recall from [EtTh], Definition 1.9, (ii); [EtTh], Definition 2.7, that

an "étale theta function of standard type" is defined precisely by the condition that its restriction to $D_{\mu_{-}}$be a $\mathbf{2 l}$-th root of unity.

Proposition 1.5. (Projective Systems of Mono-theta Environments) In the notation of the above discussion, let

$$
\mathbb{M}_{*}^{\Theta}=\left\{\ldots \rightarrow \mathbb{M}_{M^{\prime}}^{\Theta} \rightarrow \mathbb{M}_{M}^{\Theta} \rightarrow \ldots\right\}
$$

be a projective system of mono-theta environments - where $\mathbb{M}_{M}^{\Theta}$ is a mod $M$ mono-theta environment [which is isomorphic to the mod $M$ model mono-theta determined by $\left.\underline{\underline{X}}_{k}\right]$, and the index $M$ of the projective system varies multiplicatively among the elements of $\mathbb{N}_{\geq 1}$ [cf. [EtTh], Corollary 2.19, (ii), (iii)]. Then:

(i) Such a projective system is uniquely determined, up to isomorphism, by $\underline{\underline{X}}_{k}$ [cf. Remark 1.5.1 below; the discrete rigidity property of [EtTh], Corollary 2.19, (ii)].

(ii) The transition morphisms of the resulting projective system of topological groups $\left\{\ldots \rightarrow \Pi_{\underline{\underline{X}}}\left(\mathbb{M}_{M^{\prime}}^{\Theta}\right) \rightarrow \Pi_{\underline{\underline{X}}}\left(\mathbb{M}_{M}^{\Theta}\right) \rightarrow \ldots\right\}$ [cf. the notation of Definition 1.1, (i)] are all isomorphisms. $\overline{\bar{M}}$ oreover, any isomorphism of topological groups $\Pi_{\underline{X}}\left(\mathbb{M}_{M^{\prime}}^{\Theta}\right) \stackrel{\sim}{\rightarrow} \Pi_{\underline{X}}\left(\mathbb{M}_{M}^{\Theta}\right)$, where $M$ divides $M^{\prime}$, lifts to a morphism of mono-theta environments $\mathbb{M}_{M^{\prime}}^{\bar{\Theta}} \rightarrow \mathbb{M}_{M}^{\Theta}$ [cf. [EtTh], Corollary 2.18, (iv)]. Thus, to simplify the notation, we shall identify these topological groups via these transition morphisms and denote the resulting topological group by the notation $\Pi_{\underline{\underline{X}}}\left(\mathbb{M}_{*}^{\Theta}\right)$. In particular, we have an open subgroup $\Pi_{\underline{\underline{Y}}}\left(\mathbb{M}_{*}^{\Theta}\right) \subseteq \Pi_{\underline{\underline{X}}}\left(\mathbb{M}_{*}^{\Theta}\right)$, a subquotient $\left(l \cdot \Delta_{\Theta}\right)\left(\mathbb{M}_{*}^{\Theta}\right)$ of $\Pi_{\underline{X}}\left(\mathbb{M}_{*}^{\Theta}\right)$, and a quotient $\Pi_{\underline{X}}\left(\overline{\overline{\mathbb{M}_{*}}} \Theta^{\Theta}\right) \rightarrow G\left(\overline{\mathbb{M}}_{*}^{\Theta}\right)$ [cf. Definition 1.1, (i); Proposition $1 . \overline{\overline{4}}]$.

(iii) The projective system of exterior cyclotomes $\left\{\ldots \rightarrow \Pi_{\boldsymbol{\mu}}\left(\mathbb{M}_{M^{\prime}}^{\Theta}\right) \rightarrow\right.$ $\left.\Pi_{\boldsymbol{\mu}}\left(\mathbb{M}_{M}^{\Theta}\right) \rightarrow \ldots\right\}$ [cf. the notation of Definition 1.1, (i)] determines a projective limit exterior cyclotome $\Pi_{\boldsymbol{\mu}}\left(\mathbb{M}_{*}^{\Theta}\right)$ which is equipped with a uniquely determined cyclotomic rigidity isomorphism

$$
\left(l \cdot \Delta_{\Theta}\right)\left(\mathbb{M}_{*}^{\Theta}\right) \stackrel{\sim}{\rightarrow} \Pi_{\boldsymbol{\mu}}\left(\mathbb{M}_{*}^{\Theta}\right)
$$

[i.e., obtained by applying the cyclotomic rigidity isomorphisms of Definition 1.1, (ii), to the various members of the projective system $\mathbb{M}_{*}^{\Theta}$ ]. In particular, [cf. Proposition 1.4] we obtain a functorial algorithm

$$
\mathbb{M}_{*}^{\Theta} \mapsto \underline{\theta}_{\text {env }}\left(\mathbb{M}_{*}^{\Theta}\right) \subseteq H^{1}\left(\Pi_{\underline{\underline{Y}}}\left(\mathbb{M}_{*}^{\Theta}\right), \Pi_{\mu}\left(\mathbb{M}_{*}^{\Theta}\right)\right)
$$


- where one may think of the "env" as an abbreviation of the term "[mono-theta] environment" - for constructing from $\mathbb{M}_{*}^{\Theta}$ an exterior cyclotome version $\underline{\underline{\theta}}_{\text {env }}\left(\mathbb{M}_{*}^{\Theta}\right)$ of $\underline{\underline{\theta}}(\Pi)$ [i.e., by transporting $\underline{\underline{\theta}}(\Pi)$ via the above cyclotomic rigidity isomorphism] - cf. [EtTh], Corollary 2.19, (iii). In this context, we shall write

$$
\infty \underline{\underline{\theta}}_{\mathrm{env}}\left(\mathbb{M}_{*}^{\Theta}\right) \subseteq \underset{J}{\underline{\lim }} H^{1}\left(\left.\Pi_{\ddot{\underline{Y}}}\left(\mathbb{M}_{*}^{\Theta}\right)\right|_{J}, \Pi_{\boldsymbol{\mu}}\left(\mathbb{M}_{*}^{\Theta}\right)\right)
$$

- where $\infty \underline{\underline{\theta}}_{\text {env }}\left(\mathbb{M}_{*}^{\Theta}\right)$ denotes the subset of elements of the direct limit of cohomology modules in the display for which some [positive integer] multiple [i.e., some [positive integer] power, if one writes these modules "multiplicatively"] belongs to $\infty \underline{\underline{\theta}}_{\text {env }}\left(\mathbb{M}_{*}^{\Theta}\right) ; J$ ranges over the finite index open subgroups of $\Pi_{\underline{\underline{X}}}\left(\mathbb{M}_{*}^{\Theta}\right)$.

(iv) Suppose that $\mathbb{M}_{*}^{\Theta}$ arises from a tempered Frobenioid $\mathcal{C}$ [cf. Propositions 1.2, (ii); 1.3]. Then this construction of $\underline{\underline{\theta}}_{\text {env }}\left(\mathbb{M}_{*}^{\Theta}\right)[c f$. (iii)] is compatible with the Kummer-theoretic construction of the étale theta function - i.e., by considering Galois actions on roots of the Frobenioid-theoretic theta function [cf. the theory of [EtTh], §5]. In particular, it is compatible with the Kummer theory of the base-field-theoretic hull $\mathcal{C}^{\text {bs-fld }}$ [cf. [FrdII], Theorem 2.4; [AbsTopIII], Proposition 3.2, (ii); [AbsTopIII], Remark 3.1.1].

Proof. The assertions of Proposition 1.5 follow immediately from the results and definitions of [EtTh] [as well as [FrdII], [AbsTopIII]] that are quoted in the statements of these assertions.

Remark 1.5.1. We recall in passing that one important consequence of the discrete rigidity property established in [EtTh], Corollary 2.19, (ii) - which, in effect, allows one to restrict one's attention to $l \cdot \underline{\mathbb{Z}}$-translates [i.e., as opposed to $l \cdot \underline{\mathbb{Z}}$-translates] of the usual theta function - is the resulting compatibility of projective systems of mono-theta environments [as in Proposition 1.5] with the discrete structure inherent in the various isomorphs of the monoid $\mathbb{N}$ that appear in the structure of the tempered Frobenioids that arise in the theory [cf., [EtTh], Remark 2.19.4; [EtTh], Remark 5.10.4, (i), (ii)].

Remark 1.5.2. Note that, in the notation of Proposition 1.5, (iii), by considering "tautological Kummer classes" of elements of $\Pi_{\boldsymbol{\mu}}\left(\mathbb{M}_{*}^{\Theta}\right)$, one obtains a natural Galois-equivariant injection

$$
\Pi_{\boldsymbol{\mu}}\left(\mathbb{M}_{*}^{\Theta}\right) \otimes \mathbb{Q} / \mathbb{Z} \hookrightarrow \underline{\lim _{J}} H^{1}\left(\left.\Pi_{\underline{\underline{Y}}}\left(\mathbb{M}_{*}^{\Theta}\right)\right|_{J}, \Pi_{\boldsymbol{\mu}}\left(\mathbb{M}_{*}^{\Theta}\right)\right)
$$

whose image may is equal to the torsion subgroup of the codomain of the injection. Indeed, it follows immediately from the fact that $\Pi_{\boldsymbol{\mu}}\left(\mathbb{M}_{*}^{\Theta}\right)$ is torsion-free that the torsion subgroup of the codomain of the displayed injection may be identified with the torsion subgroup of

$$
\underset{J}{\varliminf_{J}} H^{1}\left(J_{G}, \Pi_{\mu}\left(\mathbb{M}_{*}^{\Theta}\right)\right)
$$

— where $J$ ranges over the finite index open subgroups of $\Pi_{\underline{\underline{X}}}\left(\mathbb{M}_{*}^{\Theta}\right)$; we write $J_{G}$ for the image of $J$ in $G\left(\mathbb{M}_{*}^{\Theta}\right)$. The desired conclusion thus follows immediately from 
the well-known Kummer theory of MLF's, i.e., the fact that the Kummer map $\left.\left(\Pi_{\boldsymbol{\mu}}\left(\mathbb{M}_{*}^{\Theta}\right)\right) \otimes \mathbb{Q} / \mathbb{Z}\right)^{J} \rightarrow H^{1}\left(J_{G}, \Pi_{\boldsymbol{\mu}}\left(\mathbb{M}_{*}^{\Theta}\right)\right)$ [where the superscript " $J$ " denotes the submodule of $J$-invariants] is injective with image equal to the torsion subgroup of the codomain.

Before proceeding, we review a certain portion of the theory of [AbsTopII] that is relevant to the content of the present $\S 1$.

Proposition 1.6. (Cores and Cuspidalizations) Let $\Pi$ be as in Proposition 1.2, (i). Write $\Delta \subseteq \Pi$ for the [group-theoretic! - cf., e.g., [AbsAnab], Lemma 1.3.8] subgroup corresponding to $\Delta_{\underline{\underline{X}}_{k}}^{\mathrm{tp}}$. Then:

(i) (Cores) There exists a functorial group-theoretic algorithm $[c f$. [AbsTopII], Corollary 3.3, (i); [AbsTopII], Remark 3.3.3]

$$
\Pi \quad \mapsto \quad\left\{(\Pi \subseteq) \Pi_{C}(\Pi) \rightarrow \Pi / \Delta\right\}
$$

for constructing from $\Pi$ a topological group $\Pi_{C}(\Pi)$ equipped with an augmentation [i.e., a surjection] $\Pi_{C}(\Pi) \rightarrow \Pi / \Delta$ - whose kernel we denote by $\Delta_{C}(\Pi)$ - that contains $\Pi$ as an open subgroup in a fashion that is compatible with the respective surjections to $\Pi / \Delta$ and which satisfies the property that when $\Pi=\Pi_{\underline{\underline{X}}_{k}}^{\text {tp }}$, the inclusion $\Pi \subseteq \Pi_{C}(\Pi)$ may be naturally identified with the inclusion $\Pi_{\underline{\underline{X}}_{k}}^{\mathrm{tp}} \subseteq \Pi_{C_{k}}^{\mathrm{tp}}$.

(ii) (Elliptic Cuspidalizations) Let $N$ be a positive integer. Then there exists a functorial group-theoretic algorithm [cf. [AbsTopII], Corollary 3.3, (iii); [AbsTopII], Remark 3.3.3]

$$
\left.\Pi \mapsto \quad \mapsto \Pi_{U_{N}}(\Pi) \rightarrow \Pi\right\}
$$

for constructing from $\Pi$ a topological group $\Pi_{U_{N}}(\Pi)$ equipped with a surjection $\Pi_{U_{N}}(\Pi) \rightarrow \Pi$ [so the augmentation $\Pi \rightarrow \Pi / \Delta$ determines, by composition, an aug-

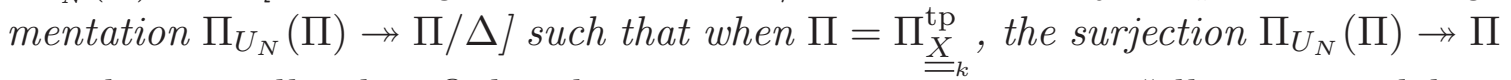
may be naturally identified with a certain surjection - i.e., "elliptic cuspidalization" - that arises from a certain open immersion determined by the $N$-torsion points of a once-punctured elliptic curve that forms a double covering of $C_{k}[c f$. [AbsTopII], Corollary 3.3, (iii)].

Proof. The assertions of Proposition 1.6 follow immediately from the results of [AbsTopII] that are quoted in the statements of these assertions [cf. also Remark 1.6.1 below].

Remark 1.6.1. We recall in passing that the construction of Proposition 1.6, (i), amounts, in effect, to the computation of various centralizers of the image of various open subgroups of $G$ in the outer automorphism groups of various open subgroups of $\Delta$. In a similar vein, the construction of Proposition 1.6, (ii), amounts to the computation of various outer isomorphisms between various subquotients of 
$\Delta$ that are compatible with the outer actions of various open subgroups of $G$. More generally, although in Proposition 1.6, we restricted our attention to the construction of cores and elliptic cuspidalizations, an analogous result may be obtained for more general functorial group-theoretic algorithms involving "chains of elementary operations", as discussed in [AbsTopI], 33 - e.g., for Belyi cuspidalizations, as discussed in [AbsTopII], Corollary 3.7.

Next, we proceed to discuss the "multiradial" interpretation of the theory of [EtTh] that is of interest in the context of the present series of papers. We begin by examining various examples of the sort of situation that gives rise to such an interpretation.

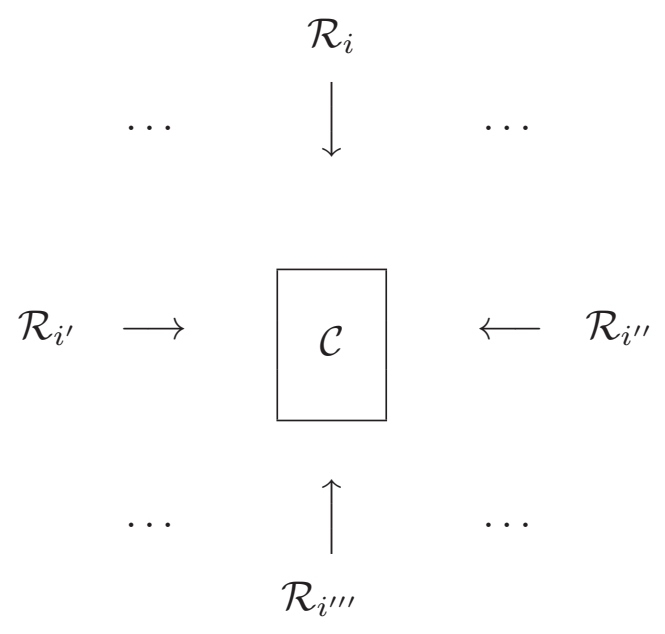

Fig. 1.1: Radial functors valued in a single coric category

\section{Example 1.7. Radial and Coric Data I: Generalities.}

(i) In the following discussion, we would like to consider a certain "type of mathematical data", which we shall refer to as radial data. This notion of a "type of mathematical data" may be formalized - cf. [IUTchIV], §3, for more details. From the point of view of the present discussion, one may think of a "type of mathematical data" as the input or output data of a "functorial algorithm" [cf. the discussion of [IUTchI], Remark 3.2.1]. At a more concrete level, we shall assume that this "type of mathematical data" gives rise to a category

$$
\mathcal{R}
$$

- i.e., each of whose objects is a specific collection of radial data, and each of whose morphisms is an isomorphism. In the following discussion, we shall also consider another "type of mathematical data", which we shall refer to as coric data. Write

\section{$\mathcal{C}$}

for the category obtained by considering specific collections of coric data and isomorphisms of collections of coric data. In addition, we shall assume that we are given a functorial algorithm - which we shall refer to as radial - whose input data consists of a collection of radial data, and whose output data consists of a collection of coric data. Thus, this functorial algorithm gives rise to a functor $\Phi: \mathcal{R} \rightarrow \mathcal{C}$. In 
the following discussion, we shall assume that this functor is essentially surjective. We shall refer to the category $\mathcal{R}$ and the functor $\Phi$ as radial and to the category $\mathcal{C}$ as coric. Finally, if $I$ is some nonempty index set, then we shall often consider collections

$$
\left\{\Phi_{i}: \mathcal{R}_{i} \rightarrow \mathcal{C}\right\}_{i \in I}
$$

of copies of $\Phi_{i}$ and $\mathcal{R}_{i}$, such that the various copies of $\Phi_{i}$ have the same codomain $\mathcal{C}$ - cf. Fig. 1.1. Thus, one may think of each $\mathcal{R}_{i}$ as the category of radial data equipped with a label $i \in I$, and isomorphisms of such data.

(ii) We shall refer to a triple $(\mathcal{R}, \mathcal{C}, \Phi: \mathcal{R} \rightarrow \mathcal{C})$ [or to the triple consisting of the corresponding "types of mathematical objects" and "functorial algorithm"] of the sort discussed in (i) as a radial environment. If $\Phi$ is full, then we shall refer to the radial environment under consideration as multiradial. We shall refer to a radial environment which is not multiradial as uniradial. Suppose that the radial environment $(\mathcal{R}, \mathcal{C}, \Phi: \mathcal{R} \rightarrow \mathcal{C})$ under consideration is uniradial. Then an object of $\mathcal{R}$ may, in general, lose a certain portion of its rigidity - i.e., may be subject to a certain additional indeterminacy - when it is mapped to $\mathcal{C}$. Put another way, in general, an object of $\mathcal{C}$ is imparted with a certain additional rigidity - i.e., loses a certain portion of its indeterminacy - when one fixes a lifting of the object to $\mathcal{R}$. Thus, in summary, the condition that $(\mathcal{R}, \mathcal{C}, \Phi: \mathcal{R} \rightarrow \mathcal{C})$ be multiradial may be thought of as a condition to the effect that the application of the radial algorithm does not result in any loss of rigidity.

(iii) In passing, we pause to observe that one way to think of the significance of the multiradiality of a radial environment $(\mathcal{R}, \mathcal{C}, \Phi: \mathcal{R} \rightarrow \mathcal{C})$ is as follows: Write

$$
\mathcal{R} \times \times_{\mathcal{C}} \mathcal{R}
$$

for the category whose objects are triples $\left(R_{1}, R_{2}, \alpha\right)$ consisting of a pair of objects $R_{1}, R_{2}$ of $\mathcal{R}$ and an isomorphism $\alpha: \Phi\left(R_{1}\right) \stackrel{\sim}{\rightarrow} \Phi\left(R_{2}\right)$ between the images of $R_{1}$, $R_{2}$ via $\Phi$, and whose morphisms are the morphisms [in the evident sense] between such triples [cf. the discussion of the "categorical fiber product" given in [FrdI], §0]. Write $\mathfrak{s w}: \mathcal{R} \times_{\mathcal{C}} \mathcal{R} \stackrel{\sim}{\rightarrow} \mathcal{R} \times_{\mathcal{C}} \mathcal{R}$ for the functor $\left(R_{1}, R_{2}, \alpha\right) \mapsto\left(R_{2}, R_{1}, \alpha^{-1}\right)$ obtained by switching the two factors of $\mathcal{R}$. Then

one formal consequence of the multiradiality of a radial environment $(\mathcal{R}, \mathcal{C}, \Phi: \mathcal{R} \rightarrow \mathcal{C})$ is the property that the switching functor $\mathfrak{s w}:$ $\mathcal{R} \times{ }_{\mathcal{C}} \mathcal{R} \stackrel{\sim}{\rightarrow} \mathcal{R} \times{ }_{\mathcal{C}} \mathcal{R}$ preserves the isomorphism class of objects of $\mathcal{R} \times{ }_{\mathcal{C}} \mathcal{R}$.

Indeed, one verifies immediately that this multiradiality is, in fact, equivalent to the condition that every object $\left(R_{1}, R_{2}, \alpha\right)$ of $\mathcal{R} \times_{\mathcal{C}} \mathcal{R}$ be isomorphic to the object $\left(R_{1}, R_{1}\right.$, id $\left.: \Phi\left(R_{1}\right) \stackrel{\sim}{\rightarrow} \Phi\left(R_{1}\right)\right)$ [which is manifestly left unchanged by the switching functor].

(iv) Next, suppose that we are given another radial environment $\left(\mathcal{R}^{\dagger}, \mathcal{C}^{\dagger}, \Phi^{\dagger}\right.$ : $\left.\mathcal{R}^{\dagger} \rightarrow \mathcal{C}^{\dagger}\right)$. We shall refer to the "type of mathematical object" / "functorial algorithm" that gives rise to $\mathcal{R}^{\dagger}$ (respectively, $\mathcal{C}^{\dagger} ; \Phi^{\dagger}$ ) as daggered radial data (respectively, daggered coric data; the daggered radial functorial algorithm). Also, let us 
suppose that we are given a 1-commutative diagram

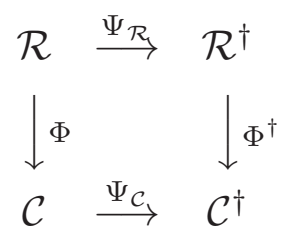

- where $\Psi_{\mathcal{R}}$ and $\Psi_{\mathcal{C}}$ arise from "functorial algorithms". If $(\mathcal{R}, \mathcal{C}, \Phi: \mathcal{R} \rightarrow \mathcal{C})$ is multiradial (respectively, uniradial), then we shall refer to $\Psi_{\mathcal{R}}$ as multiradially defined (respectively, uniradially defined), or [when there is no fear of confusion between $\Phi$ and $\Psi_{\mathcal{R}}$ ] as multiradial (respectively, uniradial). If $\Psi_{\mathcal{R}}$ admits a 1factorization $\Xi_{\mathcal{R}} \circ \Phi$ for some $\Xi_{\mathcal{R}}: \mathcal{C} \rightarrow \mathcal{R}^{\dagger}$ that arises from a functorial algorithm, then we shall say that $\Psi_{\mathcal{R}}$ is corically defined, or [when there is no fear of confusion] coric. Thus, by considering the case where $\mathcal{R}=\mathcal{C}, \Phi=\mathrm{id}_{\mathcal{R}}$, one may think of the notion of a corically defined $\Psi_{\mathcal{R}}$ as a sort of special case of the notion of a multiradial $\Psi_{\mathcal{R}}$.

(v) Suppose that we are in the situation of (iv), and that $\Psi_{\mathcal{R}}$ is multiradially defined. Then one way to think of the significance of the multiradiality of $\Psi_{\mathcal{R}}$ is as follows:

The multiradiality of $\Psi_{\mathcal{R}}$ renders it possible to consider the simultaneous execution of the functorial algorithm corresponding to $\Psi_{\mathcal{R}}$ relative to various collections of radial input data indexed by the set $I[\mathrm{cf}$. Fig. 1.1] in a fashion that is compatible with the identification of the coric portions [i.e., corresponding to $\Phi$ ] of these collections of radial input data

— cf. Remark 1.9.1 below for more on this point of view. That is to say, at a more technical level, if one implements this identification of the various coric portions by means of various gluing isomorphisms in $\mathcal{C}$, then the multiradiality of $\Psi_{\mathcal{R}}$ implies that one may lift these gluing isomorphisms in $\mathcal{C}$ to gluing isomorphisms in $\mathcal{R}$; one may then apply $\Psi_{\mathcal{R}}$ to these gluing isomorphisms in $\mathcal{R}$ to obtain gluing isomorphisms of the output data of $\Psi_{\mathcal{R}}$. Put another way, if one assumes instead that $\Psi_{\mathcal{R}}$ is uniradial, then the output data of $\Psi_{\mathcal{R}}$ depends, a priori, on the "additional rigidity" [cf. (ii)] of objects of $\mathcal{R}$ relative to these images in $\mathcal{C}$; thus, if one attempts to identify these images in $\mathcal{C}$ via arbitrary gluing isomorphisms in $\mathcal{C}$, then one does not have any way to compute the effect of such gluing isomorphisms on the output data of $\Psi_{\mathcal{R}}$.

Remark 1.7.1. One way to understand the significance of the fullness condition in the definition of a multiradial environment is as a condition that allows one to execute a sort of parallel transport operation between "fibers" of the radial functor $\Phi: \mathcal{R} \rightarrow \mathcal{C}$ [cf. the notation of Example 1.7, (iv)] — i.e., by lifting isomorphisms in $\mathcal{C}$ to isomorphisms in $\mathcal{R}$ [cf. the discussion of Example 1.7, (v)]. Here, it is perhaps of interest to make the tautological observation that, up to an indeterminacy arising from the extent that $\Phi$ fails to be faithful, such liftings are unique. That is to say, whereas a uniradial environment may be thought of as a sort of abstraction of the geometric notion of a "fibration that is not equipped with a connection", 
a multiradial environment may be thought of as a sort of abstraction of the geometric notion of a "fibration equipped with a connection" i.e., that allows one to execute parallel transport operations between the "fibers".

Relative to this point of view, one may think of the coric data as the portion of the radial data of a multiradial environment that is horizontal with respect to the "connection structure". We refer to Remarks 1.9.1, 1.9.2 below for more on the significance of multiradiality.

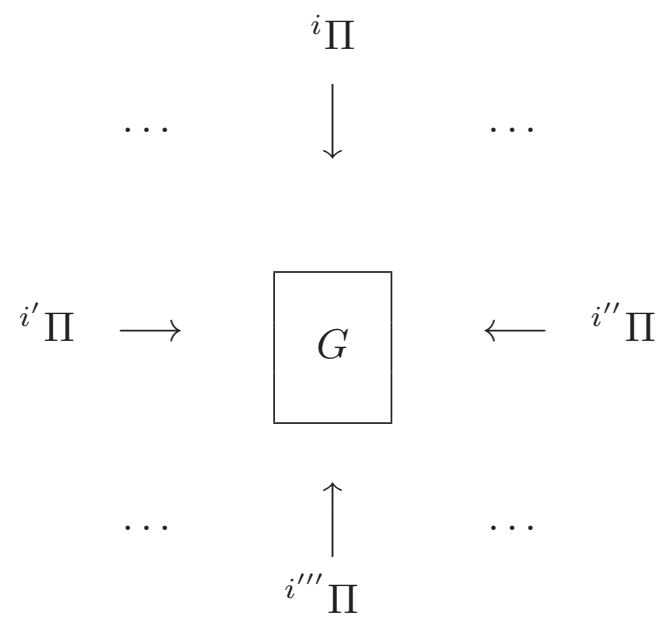

Fig. 1.2: Different arithmetic holomorphic structures on a single coric $G$

\section{Example 1.8. Radial and Coric Data II: Concrete Examples.}

(i) From the point of view of the theory to be developed in the remainder of the present $\S 1$, perhaps the most basic example of a radial environment is the following. We define a collection of radial data

$$
(\Pi, G, \alpha)
$$

to consist of a topological group $\Pi$ isomorphic to $\Pi_{\underline{X}_{k}}^{\mathrm{tp}}$, a topological group $G$ isomorphic to $G_{k}$, and the full poly-isomorphism [cf. [IUTchI], §0] of topological groups $\alpha: \Pi / \Delta \stackrel{\sim}{\rightarrow} G$, where we write $\Delta \subseteq \Pi$ for the [group-theoretic! - cf., e.g., [AbsAnab], Lemma 1.3.8] subgroup corresponding to $\Delta_{\underline{\underline{X}}_{k}}^{\mathrm{tp}}$. An isomorphism of collections of radial data $(\Pi, G, \alpha) \stackrel{\sim}{\rightarrow}\left(\Pi^{*}, G^{*}, \alpha^{*}\right)$ is defined to be a pair of isomorphisms of topological groups $\Pi \stackrel{\sim}{\rightarrow} \Pi^{*}, G \stackrel{\sim}{\rightarrow} G^{*}$ [which are necessarily compatible with $\left.\alpha, \alpha^{*} !\right]$. A collection of coric data is defined to be a topological group isomorphic to $G_{k}$; an isomorphism of collections of coric data is defined to be an isomorphism of topological groups. The radial algorithm is the algorithm given by the assignment

$$
(\Pi, G, \alpha) \mapsto G
$$

- whose associated radial functor is full and essentially surjective, hence determines a multiradial environment. Note that this example may be thought of as a sort of formalization in the present context of the situation depicted in [IUTchI], Fig. 3.2, 
at $\underline{v} \in \underline{\mathbb{V}}^{\text {bad }}$ - cf. Fig. 1.2. Here, we recall that the topological group " $G$ " [which is isomorphic to $G_{k}$ ] that appears in the center of Fig. 1.2 is regarded as being known only up to isomorphism, and that the various isomorphs of $\Pi_{\underline{X}_{k}}$ that appear in the "spokes" of Fig. 1.2 may be regarded as various "arithmetic holomorphic structures" on "G" [cf. [IUTchI], Remark 3.8.1, (iii)].

(ii) Recall the functorial group-theoretic algorithm

$$
\Pi \mapsto \quad\left(\Pi \curvearrowright M_{\mathbb{T M}}(\Pi)\right)
$$

of [AbsTopIII], $\S 3$ [cf., especially, the functors $\kappa_{\mathfrak{A} \mathfrak{n}}, \phi_{\mathfrak{A} \mathfrak{n}}$ of $[$ AbsTopIII], Definition 3.1, (vi); [AbsTopIII], Corollary 3.6, (ii); [IUTchI], Remark 3.1.2] that assigns to a topological group $\Pi$ isomorphic to $\Pi_{\underline{\underline{X}}_{k}}^{\text {tp }}$ an MLF-Galois TM-pair, which we shall denote $\Pi \curvearrowright M_{\mathbb{T M}}(\Pi)$, and which is isomorphic to the "model" MLF-Galois TM-pair determined by the natural action of $\Pi_{\underline{\underline{X}}_{k}}^{\mathrm{tp}}$ on the topological monoid $\mathcal{O}_{\bar{k}}^{\triangleright}$. In fact, [the union with $\{0\}$ of] the underlying topological monoid $M_{\mathbb{T M}}(\Pi)$ is also equipped with a natural ring structure [cf. [AbsTopIII], Proposition 3.2, (iii)]. On the other hand, if one is willing to sacrifice this ring structure, then there exists a functorial group-theoretic algorithm

$$
G \mapsto\left(G \curvearrowright \mathcal{O}^{\triangleright}(G)\right)
$$

[cf. [AbsTopIII], Proposition 5.8, (i)] that assigns to a topological group $G$ isomorphic to $G_{k}$ an MLF-Galois TM-pair, which we shall denote $G \curvearrowright \mathcal{O}^{\triangleright}(G)$, and which is isomorphic to the MLF-Galois TM-pair determined by the natural action of $G_{k}$ on the topological monoid $\mathcal{O} \frac{\triangleright}{k}$. Moreover, by [AbsTopIII], Proposition 3.2, (iv) [cf. also Remark 1.11.1, (i), (a), below], there is a [uniquely determined] functorial tautological isomorphism of MLF-Galois TM-pairs

$$
\left.\left(\Pi \curvearrowright M_{\mathbb{T M}}(\Pi)\right) \stackrel{\sim}{\rightarrow}\left(\Pi / \Delta \curvearrowright \mathcal{O}^{\triangleright}(\Pi / \Delta)\right)\right|_{\Pi}
$$

$(* \mathbb{M M} \triangleright)$

- where $\Delta \subseteq \Pi$ is as in (i), and the notation " $\mid \Pi$ " denotes the restriction of the action of $\Pi / \Delta$ to an action of $\Pi$. Then another important example of a radial environment is the following. We define a collection of radial data

$$
\left(\Pi \curvearrowright M_{\mathbb{T M}}(\Pi), G \curvearrowright \mathcal{O}^{\triangleright}(G), \alpha_{\triangleright}\right)
$$

to consist of the output data of the algorithm $\left(*_{\mathbb{T M}}\right)$ associated to a topological group $\Pi$ isomorphic to $\Pi_{\underline{\underline{X}}_{k}}^{\text {tp }}$, the output data of the algorithm $\left(*_{\triangleright}\right)$ associated to a topological group $G$ isomorphic to $G_{k}$, and the poly-isomorphism [cf. [IUTchI], §0] of MLF-Galois TM-pairs

$$
\alpha_{\triangleright}:\left.\left(\Pi \curvearrowright M_{\mathbb{T M}}(\Pi)\right) \stackrel{\sim}{\rightarrow}\left(G \curvearrowright \mathcal{O}^{\triangleright}(G)\right)\right|_{\Pi}
$$

determined [in light of [AbsTopIII], Proposition 3.2, (iv)] by the composite of the natural surjection $\Pi \rightarrow \Pi / \Delta$ with the full poly-isomorphism of topological groups $\Pi / \Delta \stackrel{\sim}{\rightarrow} G$ [where $\Delta \subseteq \Pi$ is as in (i)]. An isomorphism of collections of radial data $\left(\Pi \curvearrowright M_{\mathbb{T M}}(\Pi), G \curvearrowright \mathcal{O}^{\triangleright}(G), \alpha_{\triangleright}\right) \stackrel{\sim}{\rightarrow}\left(\Pi^{*} \curvearrowright M_{\mathbb{T M}}\left(\Pi^{*}\right), G^{*} \curvearrowright \mathcal{O}^{\triangleright}\left(G^{*}\right), \alpha_{\triangleright}^{*}\right)$ is defined to be a pair of isomorphisms of MLF-Galois TM-pairs $\left(\Pi \curvearrowright M_{\mathbb{T M}}(\Pi)\right) \stackrel{\sim}{\rightarrow}\left(\Pi^{*} \curvearrowright\right.$ 
$\left.M_{\mathbb{T M}}\left(\Pi^{*}\right)\right),\left(G \curvearrowright \mathcal{O}^{\triangleright}(G)\right) \stackrel{\sim}{\rightarrow}\left(G^{*} \curvearrowright \mathcal{O}^{\triangleright}\left(G^{*}\right)\right)$ [which are necessarily compatible with $\left.\alpha_{\triangleright}, \alpha_{\triangleright}^{*} !\right]$. A collection of coric data is defined to be the output data of the algorithm $\left(*_{\triangleright}\right)$ for some topological group isomorphic to $G_{k}$; an isomorphism of collections of coric data is defined to be the isomorphism between collections of output data of $\left(*_{\triangleright}\right)$ associated to an isomorphism of topological groups. The radial algorithm is the algorithm given by the assignment

$$
\left(\Pi \curvearrowright M_{\mathbb{T M}}(\Pi), G \curvearrowright \mathcal{O}^{\triangleright}(G), \alpha_{\triangleright}\right) \mapsto\left(G \curvearrowright \mathcal{O}^{\triangleright}(G)\right)
$$

- whose associated radial functor is full and essentially surjective, hence determines a multiradial environment

(iii) Let

$$
\Gamma \subseteq \widehat{\mathbb{Z}}^{\times}
$$

be a closed subgroup [cf. Remark 1.11.1, (i), (ii), below, for more on the significance of $\Gamma$. Then by considering the subgroups of invertible elements of the various topological monoids that appeared in (ii), one obtains functorial group-theoretic algorithms

$$
\Pi \mapsto\left(\Pi \curvearrowright M_{\mathbb{T M}}^{\times}(\Pi)\right) ; \quad G \mapsto\left(G \curvearrowright \mathcal{O}^{\times}(G)\right)
$$

defined, respectively, on topological groups $\Pi$ isomorphic to $\Pi_{\underline{\underline{X}}_{k}}^{\mathrm{tp}}$ and $G$ isomorphic to $G_{k}$. Here, we note that we may think of $\Gamma$ as acting on the output data of the second algorithm of $\left(*_{\times}\right)$by means of the trivial action on $G$ and the natural action of $\widehat{\mathbb{Z}}^{\times}$on $\mathcal{O}^{\times}(G)$. Then one obtains another example of a radial environment as follows. We define a collection of radial data

$$
\left(\Pi \curvearrowright M_{\mathbb{T M}}^{\times}(\Pi), G \curvearrowright \mathcal{O}^{\times}(G), \alpha_{\times}\right)
$$

to consist of the output data of the first algorithm of $\left(*_{\times}\right)$associated to a topological group $\Pi$ isomorphic to $\Pi_{\underline{X}_{p}}^{\mathrm{tp}}$, the output data of the second algorithm of $\left(*_{\times}\right)$ associated to a topological group $G$ isomorphic to $G_{k}$, and the poly-isomorphism [cf. [IUTchI], §0] of topological modules equipped with topological group actions

$$
\alpha_{\times}:\left.\left(\Pi \curvearrowright M_{\mathbb{T M}}^{\times}(\Pi)\right) \stackrel{\sim}{\rightarrow}\left(G \curvearrowright \mathcal{O}^{\times}(G)\right)\right|_{\Pi}
$$

determined by the $\Gamma$-orbit of the poly-isomorphism " $\left.\alpha_{\triangleright}\right|_{\times}$" induced by the polyisomorphism $\alpha_{\triangleright}$ of (ii). An isomorphism of collections of radial data $\left(\Pi \curvearrowright M_{\mathbb{T M}}^{\times}(\Pi)\right.$, $\left.G \curvearrowright \mathcal{O}^{\times}(G), \alpha_{\times}\right) \stackrel{\sim}{\rightarrow}\left(\Pi^{*} \curvearrowright M_{\mathbb{M} M}^{\times}\left(\Pi^{*}\right), G^{*} \curvearrowright \mathcal{O}^{\times}\left(G^{*}\right), \alpha_{\times}^{*}\right)$ is defined to consist of the isomorphism of topological modules equipped with topological group actions $\left(\Pi \curvearrowright M_{\mathbb{T M}}^{\times}(\Pi)\right) \stackrel{\sim}{\rightarrow}\left(\Pi^{*} \curvearrowright M_{\mathbb{T M}}^{\times}\left(\Pi^{*}\right)\right)$ induced by an isomorphism of topological groups $\Pi \stackrel{\sim}{\rightarrow} \Pi^{*}$, together with a $\Gamma$-multiple of the isomorphism of topological modules equipped with topological group actions $\left(G \curvearrowright \mathcal{O}^{\times}(G)\right) \stackrel{\sim}{\rightarrow}\left(G^{*} \curvearrowright \mathcal{O}^{\times}\left(G^{*}\right)\right)$ induced by an isomorphism of topological groups $G \stackrel{\sim}{\rightarrow} G^{*}$ [so one verifies immediately that these isomorphisms are compatible with $\alpha_{\times}, \alpha_{\times}^{*}$ in the evident sense]. A collection of coric data is defined to be the output data of the second algorithm of $\left(*_{\times}\right)$for some topological group isomorphic to $G_{k}$; an isomorphism of collections of coric data is defined to be a $\Gamma$-multiple of the isomorphism between collections 
of output data of $\left(*_{x}\right)$ associated to an isomorphism of topological groups. The radial algorithm is the algorithm given by the assignment

$$
\left(\Pi \curvearrowright M_{\mathbb{M} \mathbb{M}}^{\times}(\Pi), G \curvearrowright \mathcal{O}^{\times}(G), \alpha_{\times}\right) \mapsto\left(G \curvearrowright \mathcal{O}^{\times}(G)\right)
$$

- whose associated radial functor is full and essentially surjective, hence determines a multiradial environment.

(iv) By considering the subgroups of torsion elements of the various topological monoids that appeared in (ii), one obtains functorial group-theoretic algorithms

$$
\Pi \quad \mapsto \quad\left(\Pi \curvearrowright M_{\mathbb{T M}}^{\mu}(\Pi)\right) ; \quad G \mapsto \quad\left(G \curvearrowright \mathcal{O}^{\mu}(G)\right) \quad\left(*_{\mu}\right)
$$

defined, respectively, on topological groups $\Pi$ isomorphic to $\Pi_{\underline{\underline{X}}_{k}}^{\mathrm{tp}}$ and $G$ isomorphic to $G_{k}$ - i.e., a "cyclotomic version" of the algorithms of $\left(*_{x}\right)[$ [cf. (iii)]. Moreover, by forming the quotients $M_{\mathbb{T M}}^{\times}(-) / M_{\mathbb{T M}}^{\mu}(-), \mathcal{O}^{\times}(-) / \mathcal{O}^{\mu}(-)$, one obtains functorial group-theoretic algorithms

$$
\Pi \mapsto\left(\Pi \curvearrowright M_{\mathbb{T M}}^{\times \mu}(\Pi)\right) ; \quad G \mapsto \quad\left(G \curvearrowright \mathcal{O}^{\times \mu}(G)\right) \quad\left(*_{\times}\right)
$$

defined, respectively, on topological groups $\Pi$ isomorphic to $\Pi_{\underline{\underline{X}}_{k}}^{\mathrm{tp}}$ and $G$ isomorphic to $G_{k}$ - i.e., a "co-cyclotomic version" of the algorithms of $\left(_{*}\right)$ [cf. (iii)]. Now one verifies easily that

by replacing the symbol " $\times$ " in (iii) by the symbol " $\boldsymbol{\mu}$ " or, alternatively, by the symbol " $\times \boldsymbol{\mu}$ ",

one obtains, respectively, "cyclotomic" and "co-cyclotomic" versions of the example treated in (iii). In the case of " $\times \boldsymbol{\mu}$ ", let us write

$$
\operatorname{Ism}(G)
$$

for the compact topological group of $G$-isometries of $\mathcal{O}^{\times \boldsymbol{\mu}}(G)$, i.e., $G$-equivariant automorphisms of the topological module $\mathcal{O}^{\times \boldsymbol{\mu}}(G)$ that, for each open subgroup $H \subseteq G$, preserve the "lattice" in $\mathcal{O}^{\times \boldsymbol{\mu}}(G)^{H}$ determined by the image of $\mathcal{O}^{\times}(G)^{H}$ [i.e., where the superscript " $H$ " denotes the submodule of $H$-invariants]. Let

$$
\Gamma^{\times \mu} \subseteq \operatorname{Ism}(-)
$$

be a closed subgroup, i.e., a collection of closed subgroups of each $\operatorname{Ism}(G)$ that is preserved by arbitrary isomorphisms of topological groups $G_{1} \stackrel{\sim}{\rightarrow} G_{2}$. Then one verifies easily that, in the "co-cyclotomic" version discussed above of the example treated in (iii),

$$
\text { one may replace the " } \Gamma^{\prime} \text { in (iii) by such a " } \Gamma^{\times \mu} " \text {. }
$$

Finally, we observe that one example of such a " $\Gamma^{\times \mu}$ " — which we shall denote by means of the notation 
- is the case where one takes $\Gamma^{\times \boldsymbol{\mu}}$ to be the entire group "Ism(-)"; another example of such a " $\Gamma^{\times \mu}$ " is the image $\operatorname{Im}\left(\widehat{\mathbb{Z}}^{\times}\right)$of the natural homomorphism $\widehat{\mathbb{Z}}^{\times} \rightarrow$ $\mathbb{Z}_{p}^{\times} \hookrightarrow$ Ism.

(v) Another example of a radial environment may be obtained as follows. We define a collection of radial data

$$
\left(\Pi \curvearrowright M_{\mathbb{T M}}^{\boldsymbol{\mu}}(\Pi), G \curvearrowright \mathcal{O}^{\times \boldsymbol{\mu}}(G), \alpha_{\boldsymbol{\mu}, \times \boldsymbol{\mu}}\right)
$$

to consist of the output data of the first algorithm of $\left(*_{\boldsymbol{\mu}}\right)$ associated to a topological group $\Pi$ isomorphic to $\Pi_{\underline{X}_{k}}^{\text {tp }}$, the output data of the second algorithm of $\left(*_{\times} \boldsymbol{\mu}\right)$ associated to a topological group $G$ isomorphic to $G_{k}$, and the poly-morphism [cf. [IUTchI], §0] of topological modules equipped with topological group actions

$$
\alpha_{\boldsymbol{\mu}, \times \boldsymbol{\mu}}:\left.\left(\Pi \curvearrowright M_{\mathbb{T M}}^{\boldsymbol{\mu}}(\Pi)\right) \rightarrow\left(G \curvearrowright \mathcal{O}^{\times \boldsymbol{\mu}}(G)\right)\right|_{\Pi}
$$

determined by the full poly-isomorphism $\Pi / \Delta \stackrel{\sim}{\rightarrow} G$ [cf. (i)] and the trivial homomorphism $M_{\mathbb{T M}}^{\boldsymbol{\mu}}(\Pi) \rightarrow \mathcal{O}^{\times \mu}(G)$ - i.e., the composite of the natural homomorphisms $M_{\mathbb{T M}}^{\mu}(\Pi) \subseteq M_{\mathbb{M} M}^{\times}(\Pi) \stackrel{\sim}{\rightarrow} \mathcal{O}^{\times}(G) \rightarrow \mathcal{O}^{\times}(G)$ [where the " $\stackrel{\sim}{\rightarrow}$ " arises from the poly-isomorphism $\alpha_{\times}$of (iii)]. An isomorphism of collections of radial data $\left(\Pi \curvearrowright M_{\mathbb{T M}}^{\boldsymbol{\mu}}(\Pi), G \curvearrowright \mathcal{O}^{\times \boldsymbol{\mu}}(G), \alpha_{\boldsymbol{\mu}, \times \boldsymbol{\mu}}\right) \stackrel{\sim}{\rightarrow}\left(\Pi^{*} \curvearrowright M_{\mathbb{T M}}^{\boldsymbol{\mu}}\left(\Pi^{*}\right), G^{*} \curvearrowright \mathcal{O}^{\times \boldsymbol{\mu}}\left(G^{*}\right), \alpha_{\boldsymbol{\mu}, \times \boldsymbol{\mu}}^{*}\right)$ is defined to consist of the isomorphism of topological modules equipped with topological group actions $\left(\Pi \curvearrowright M_{\mathbb{T M}}^{\mu}(\Pi)\right) \stackrel{\sim}{\rightarrow}\left(\Pi^{*} \curvearrowright M_{\mathbb{T M}}^{\mu}\left(\Pi^{*}\right)\right)$ induced by an isomorphism of topological groups $\Pi \stackrel{\sim}{\rightarrow} \Pi^{*}$, together with a $\Gamma^{\times \boldsymbol{\mu}}$-multiple of the isomorphism of topological modules equipped with topological groups actions $(G \curvearrowright$ $\left.\mathcal{O}^{\times \boldsymbol{\mu}}(G)\right) \stackrel{\sim}{\rightarrow}\left(G^{*} \curvearrowright \mathcal{O}^{\times \boldsymbol{\mu}}\left(G^{*}\right)\right)$ induced by an isomorphism of topological groups $G \stackrel{\sim}{\rightarrow} G^{*}$ [so one verifies immediately that these isomorphisms are compatible with $\alpha_{\boldsymbol{\mu}, \times \boldsymbol{\mu}}, \alpha_{\boldsymbol{\mu}, \times \boldsymbol{\mu}}^{*}$ in the evident sense]. A collection of coric data is defined to be the output data of the second algorithm of $(* \times \mu)$ for some topological group isomorphic to $G_{k}$; an isomorphism of collections of coric data is defined to be a $\Gamma^{\times \boldsymbol{\mu}}$-multiple of the isomorphism between collections of output data of $\left(*_{\times \mu}\right)$ associated to an isomorphism of topological groups. [That is to say, the definition of the coric data is the same as in the "co-cyclotomic" version discussed in (iv).] The radial algorithm is the algorithm given by the assignment

$$
\left(\Pi \curvearrowright M_{\mathbb{T M}}^{\boldsymbol{\mu}}(\Pi), G \curvearrowright \mathcal{O}^{\times \boldsymbol{\mu}}(G), \alpha \boldsymbol{\mu}, \times \boldsymbol{\mu}\right) \mapsto\left(G \curvearrowright \mathcal{O}^{\times \boldsymbol{\mu}}(G)\right)
$$

- whose associated radial functor is full and essentially surjective, hence determines a multiradial environment.

(vi) By replacing the notation " $M_{\mathbb{T M}}^{\boldsymbol{\mu}}(\Pi)$ " in the discussion of (v) by the notation " $\Pi_{\mu}\left(\mathbb{M}_{*}^{\Theta}(\Pi)\right) \otimes \mathbb{Q} / \mathbb{Z}$ " [cf. Propositions 1.2, (i); 1.5, (i), (iii)], one verifies immediately that one obtains an "exterior-cyclotomic version" of the multiradial environment constructed in (v).

(vii) In the discussion to follow, we shall also consider the functorial grouptheoretic algorithms

$$
\Pi \mapsto\left(\Pi \curvearrowright M_{\mathbb{T M}}^{\mathrm{gp}}(\Pi)\right) ; \quad G \mapsto \quad\left(G \curvearrowright \mathcal{O}^{\mathrm{gp}}(G)\right)
$$


obtained by passing to the respective groupifications of the monoids $M_{\mathbb{T M}}(\Pi)$, $\mathcal{O}^{\triangleright}(G)$, as well as the functorial group-theoretic algorithms

$$
\Pi \mapsto\left(\Pi \curvearrowright M_{\mathbb{T M}}^{\widehat{\mathrm{gp}}}(\Pi)\right) ; \quad G \mapsto\left(G \curvearrowright \mathcal{O}^{\widehat{\mathrm{gp}}}(G)\right) \quad\left(*_{\widehat{\mathrm{gp}}}\right)
$$

obtained by passing to the respective inductive limits of the profinite completions of $M_{\mathbb{T M}}^{\mathrm{gp}}(\Pi)^{J}, \mathcal{O}^{\mathrm{gp}}(G)^{J}$ [i.e., where the superscript " $J$ " denotes the submodule of $J$-invariants], as $J$ ranges over the open subgroups of $\Pi$ or $G$. Thus, there is a natural action of $\Gamma$ on the underlying topological modules of $M_{\mathbb{T M}}^{\widehat{\mathrm{gp}}}(\Pi), \widehat{\mathcal{O}} \widehat{\mathrm{gp}}(G)$; by considering the $\Gamma$-orbit of the poly-isomorphism induced by the poly-isomorphism $\alpha_{\triangleright}$ of (ii), one obtains a poly-isomorphism

$$
\alpha_{\widehat{\mathrm{gp}}}:\left.\left(\Pi \curvearrowright M_{\mathbb{T M}}^{\widehat{\mathrm{gp}}}(\Pi)\right) \stackrel{\sim}{\rightarrow}\left(G \curvearrowright \mathcal{O}^{\widehat{\mathrm{gp}}}(G)\right)\right|_{\Pi}
$$

that is compatible [in the evident sense] with the poly-isomorphism $\alpha_{\times}$of (iii).

(viii) The following example of a radial environment is another variant of the example of $(\mathrm{v})$. We define a collection of radial data

$$
\left(\Pi \curvearrowright M_{\mathbb{T M}}(\Pi), G \curvearrowright \mathcal{O}^{\widehat{g p}}(G), \alpha_{\triangleright, \times \boldsymbol{\mu}}\right)
$$

to consist of the output data of the algorithm of $\left(*_{\mathbb{M M}}\right)$ associated to a topological group $\Pi$ isomorphic to $\Pi_{\underline{\underline{X}}_{k}}^{\mathrm{tp}}$, the output data of the second algorithm of $(* \widehat{\mathrm{gp}})[\mathrm{cf}$. (vii)] associated to a topological group $G$ isomorphic to $G_{k}$, and the following $d i$ agram $\alpha_{\triangleright, \times \mu}$ of poly-morphisms of topological monoids equipped with topological group actions

$$
\begin{aligned}
& \left(\Pi \curvearrowright M_{\mathbb{T M}}(\Pi)\right) \hookrightarrow \quad\left(\Pi \curvearrowright M_{\mathbb{T M}}^{\widehat{\mathrm{gp}}}(\Pi)\right) \\
& \left.\left.\stackrel{\sim}{\rightarrow}\left(G \curvearrowright \mathcal{O}^{\widehat{\mathrm{gp}}}(G)\right)\right|_{\Pi} \hookleftarrow\left(G \curvearrowright \mathcal{O}^{\times}(G)\right)\right|_{\Pi} \\
& \left.\rightarrow\left(G \curvearrowright \mathcal{O}^{\times \boldsymbol{\mu}}(G)\right)\right|_{\Pi}
\end{aligned}
$$

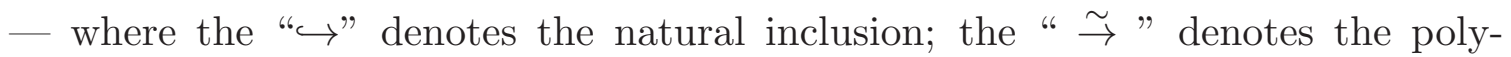
isomorphism $\alpha_{\widehat{g p}}$ of (vii); the " $\hookleftarrow$ " denotes the natural inclusion; the " $\rightarrow$ " denotes the natural surjection. An isomorphism of collections of radial data $(\Pi \curvearrowright$ $\left.M_{\mathbb{T M}}(\Pi), G \curvearrowright \widehat{\mathcal{O}^{\mathrm{gp}}}(G), \alpha_{\triangleright, \times \boldsymbol{\mu}}\right) \stackrel{\sim}{\rightarrow}\left(\Pi^{*} \curvearrowright M_{\mathbb{T M}}\left(\Pi^{*}\right), G^{*} \curvearrowright \widehat{\mathcal{O}^{g p}}\left(G^{*}\right), \alpha_{\triangleright, \times \boldsymbol{\mu}}^{*}\right)$ is defined to consist of the isomorphism of topological monoids equipped with topological group actions $\left(\Pi \curvearrowright M_{\mathbb{T M}}(\Pi)\right) \stackrel{\sim}{\rightarrow}\left(\Pi^{*} \curvearrowright M_{\mathbb{T M}}\left(\Pi^{*}\right)\right)$ induced by an isomorphism of topological groups $\Pi \stackrel{\sim}{\rightarrow} \Pi^{*}$, together with a $\Gamma$-multiple of the isomorphism of topological modules equipped with topological group actions $(G \curvearrowright$ $\left.\mathcal{O}^{\widehat{\mathrm{gp}}}(G)\right) \stackrel{\sim}{\rightarrow}\left(G^{*} \curvearrowright \mathcal{O}^{\widehat{\mathrm{gp}}}\left(G^{*}\right)\right)$ induced by an isomorphism of topological groups $G \stackrel{\sim}{\rightarrow} G^{*}$ [so one verifies immediately that these isomorphisms are compatible with $\alpha_{\triangleright, \times \boldsymbol{\mu}}, \alpha_{\triangleright, \times \boldsymbol{\mu}}^{*}$ in the evident sense]; here, we note that any such isomorphism $\left(G \curvearrowright \mathcal{O}^{\widehat{g p}}(G)\right) \stackrel{\sim}{\rightarrow}\left(G^{*} \curvearrowright \widehat{\mathcal{O}^{\mathrm{gp}}}\left(G^{*}\right)\right)$ induces isomorphisms $\left(G \curvearrowright \mathcal{O}^{\times}(G)\right) \stackrel{\sim}{\rightarrow}\left(G^{*} \curvearrowright\right.$ $\left.\mathcal{O}^{\times}\left(G^{*}\right)\right),\left(G \curvearrowright \mathcal{O}^{\times \boldsymbol{\mu}}(G)\right) \stackrel{\sim}{\rightarrow}\left(G^{*} \curvearrowright \mathcal{O}^{\times \boldsymbol{\mu}}\left(G^{*}\right)\right)$ in a fashion compatible with $\alpha_{\triangleright, \times \boldsymbol{\mu}}$, $\alpha_{\triangleright, \times \mu}^{*}$. The definition of coric data and isomorphisms of collections of coric data is 
the same as in (v) [i.e., where one takes " $\Gamma^{\times \mu}$ " to be the image $\operatorname{Im}(\Gamma)$ of $\Gamma \subseteq \widehat{\mathbb{Z}}^{\times}$]. The radial algorithm is the algorithm given by the assignment

$$
\left(\Pi \curvearrowright M_{\mathbb{T M}}(\Pi), G \curvearrowright \mathcal{O}^{\widehat{g p}}(G), \alpha_{\triangleright, \times \boldsymbol{\mu}}\right) \mapsto\left(G \curvearrowright \mathcal{O}^{\times \mu}(G)\right)
$$

- whose associated radial functor is full and essentially surjective, hence determines a multiradial environment.

(ix) Note that if $G$ is a topological group isomorphic to $G_{k}$, then, in addition to $G \curvearrowright \mathcal{O}^{\times}(G), G \curvearrowright \mathcal{O}^{\times \boldsymbol{\mu}}(G)$, one may also construct the log-shell $\mathcal{I}(G) \subseteq \mathcal{O}^{\times \boldsymbol{\mu}}(G)$ [i.e., the image of the $G$-invariants of $\mathcal{O}^{\times}(G)$ in $\mathcal{O}^{\times \boldsymbol{\mu}}(G)$ - cf. [AbsTopIII], Proposition 5.8, (ii)]. In particular, if one replaces the notation " $G \curvearrowright \mathcal{O}^{\times \boldsymbol{\mu}}(G)$ " in the discussion of (v), (vi), and (viii) by the notation " $\left(G \curvearrowright \mathcal{O}^{\times \boldsymbol{\mu}}(G), \mathcal{I}(G) \subseteq \mathcal{O}^{\times \boldsymbol{\mu}}(G)\right)$ " [i.e., " $G \curvearrowright \mathcal{O}^{\times \boldsymbol{\mu}}(G)$ equipped its associated log-shell"], then one verifies immediately that one obtains a "log-shell version" of the multiradial environments constructed in (v), (vi), and (viii).

Remark 1.8.1. In the context of the various examples given in Example 1.8, (iii), (iv), (v), (vi), (vii), (viii), and (ix), it is useful to note that

no automorphism of $\mathcal{O}^{\times \boldsymbol{\mu}}(G)$ induced by an element of $\operatorname{Aut}(G)$ [e.g., an element of $G$, regarded as an inner automorphism of $G$ ] coincides with an automorphism of $\mathcal{O}^{\times \boldsymbol{\mu}}(G)$ induced by an element of $\Gamma$ that has nontrivial image in $\mathbb{Z}_{p}^{\times}$.

Indeed, this follows immediately by observing that the cyclotomic character of $G$ determines [in light of the definition of $\mathcal{O}^{\times}(G)$, in terms of abelianizations of open subgroups of $G$ - cf. [AbsTopIII], Proposition 5.8, (i)] a natural surjection $\mathcal{O}^{\times \boldsymbol{\mu}}(G) \rightarrow \mathbb{Q}_{p}$, which [cf., e.g., [AbsAnab], Proposition 1.2.1, (vi)] is $\operatorname{Aut}(G)$ equivariant, relative to the trivial action of $\operatorname{Aut}(G)$ on $\mathbb{Q}_{p}$, and $\Gamma$-equivariant, relative to the natural action of $\Gamma \subseteq \widehat{\mathbb{Z}}^{\times}$[via the natural surjection $\widehat{\mathbb{Z}}^{\times} \rightarrow \mathbb{Z}_{p}^{\times}$] on $\mathbb{Q}_{p}$.

\section{Example 1.9. Radial and Coric Data III: Graphs of Functorial Group- theoretic Algorithms.}

(i) Let $\mathcal{E}$ and $\mathcal{F}$ be categories that arise from "types of mathematical data" [cf. the discussion of Example 1.7, (i)]; $\Xi: \mathcal{E} \rightarrow \mathcal{F}$ a functor that arises from a "functorial algorithm" [cf. the discussion of Example 1.7, (i)]. Then one may define a new category $\mathcal{G}$ - that also arises from a "type of mathematical data" — as follows: the objects of $\mathcal{G}$ are pairs $(E, \Xi(E))$, where $E \in \mathrm{Ob}(\mathcal{E})$, and $\Xi(E) \in \mathrm{Ob}(\mathcal{F})$ is the image of $E$ via $\Xi$; the morphisms of $\mathcal{G}$ are the pairs of arrows $(f: E \rightarrow$ $\left.E^{\prime}, \Xi(f): \Xi(E) \rightarrow \Xi\left(E^{\prime}\right)\right)$. We shall refer to $\mathcal{G}$ [or the "type of mathematical data" that gives rise to $\mathcal{G}$ ] as the graph of $\Xi$. Note that this construction was applied, in effect, in the discussion of the various radial environments constructed in Example 1.8. Finally, we observe that we have natural functors $\mathcal{E} \rightarrow \mathcal{G}$ [given by $E \mapsto(E, \Psi(E))], \mathcal{G} \rightarrow \mathcal{E}$ [given by $(E, \Psi(E)) \mapsto E], \mathcal{G} \rightarrow \mathcal{F}$ [given by $(E, \Psi(E)) \mapsto$ $\Psi(E)]$. 
(ii) In the notation of (i), suppose that $\mathcal{E}$ is the category of topological groups isomorphic to $\Pi_{\underline{\underline{X}}_{k}}^{\mathrm{tp}}$ and isomorphisms of topological groups, and that $\Xi$ is some "functorial group-theoretic algorithm" [whose input data consists of a topological group isomorphic to $\left.\Pi_{\underline{\underline{X}}_{k}^{\mathrm{p}}}^{\mathrm{t}}\right]$. Let $\left(\mathcal{R}, \mathcal{C}, \Psi_{\mathcal{R}}\right)$ be the radial environment of Example 1.8, (i). Then composing the functor $\mathcal{R} \rightarrow \mathcal{E}$ given by the assignment $(\Pi, G, \alpha) \mapsto \Pi$ with $\Xi: \mathcal{E} \rightarrow \mathcal{F}$ yields a functor $\mathcal{R} \rightarrow \mathcal{F}$, whose graph we denote by $\mathcal{R}^{\dagger}$. Thus, by considering the natural functors $\Psi_{\mathcal{R}}: \mathcal{R} \rightarrow \mathcal{R}^{\dagger}$ [cf. (i)], $\mathcal{R}^{\dagger} \rightarrow \mathcal{R} \rightarrow \mathcal{C}$, and taking $\mathcal{C}^{\dagger} \stackrel{\text { def }}{=} \mathcal{C}$, we obtain a diagram as in the display of Example 1.7, (iv). Since $\left(\mathcal{R}, \mathcal{C}, \Psi_{\mathcal{R}}\right)$ is a multiradial environment, it thus follows that $\Psi_{\mathcal{R}}$ is multiradially defined [cf. Example 1.7, (iv)]. That is to say, by using the radial environment of Example 1.8, (i), one concludes that

any "functorial group-theoretic algorithm" whose input data consists of a topological group isomorphic to $\Pi_{\underline{\underline{X}}_{k}}^{\mathrm{tp}}$ gives rise - in a tautological fashion - to a multiradially defined functor.

This approach will be discussed further in Remark 1.9.1 below.

(iii) On the other hand, one may also construct a radial environment as follows. We define a collection of radial data to be a topological group $\Pi$ isomorphic to $\Pi_{\underline{\underline{X}}_{k}}^{\mathrm{tp}}$, and an isomorphism of collections of radial data to be an isomorphism of topological groups. The definitions of coric data and isomorphisms of collections of coric data are the same as in Example 1.8, (i). The radial functor $\Psi: \mathcal{R} \rightarrow \mathcal{C}$ is defined via the assignment $\Pi \mapsto \Pi / \Delta$ [cf. the notation of Example 1.8, (i)]. Thus, $\Psi$ fails to be full [cf., e.g., [AbsTopIII], §I3; [AbsTopIII], Remark 1.9.1]. That is to say, $\left(\mathcal{R}, \mathcal{C}, \Psi_{\mathcal{R}}\right)$ is a uniradial environment. Now suppose that $\Xi: \mathcal{E} \rightarrow \mathcal{F}$ is as in (ii). Then since $\mathcal{R}$ may be identified with $\mathcal{E}$, the graph of $\Xi: \mathcal{R}=\mathcal{E} \rightarrow \mathcal{F}$ yields a category $\mathcal{R}^{\dagger}$ equipped with natural functors $\Psi_{\mathcal{R}}: \mathcal{R} \rightarrow \mathcal{R}^{\dagger}, \Phi^{\dagger}: \mathcal{R}^{\dagger} \rightarrow \mathcal{R} \rightarrow \mathcal{C}^{\dagger} \stackrel{\text { def }}{=} \mathcal{C}$. In particular, we obtain a diagram as in the display of Example 1.7, (iv). Since $\left(\mathcal{R}, \mathcal{C}, \Psi_{\mathcal{R}}\right)$ is a uniradial environment, it thus follows that $\Psi_{\mathcal{R}}$ is uniradially defined [cf. Example 1.7, (iv)]. That is to say, by using the radial environment just defined, one concludes that

any "functorial group-theoretic algorithm" whose input data consists of a topological group isomorphic to $\Pi_{\underline{\underline{X}}_{k}}^{\mathrm{tp}}$ also gives rise - in a tautological fashion - to a uniradially defined functor.

This approach will be discussed further in Remark 1.9.1 below.

(iv) Let $\Pi$ be a topological group isomorphic to $\Pi_{\underline{\underline{X}}_{k}}^{\mathrm{tp}} ; \Delta \subseteq \Pi$ the subgroup of Example 1.8, (i). Recall the isomorphism " $\boldsymbol{\mu}_{\widehat{\mathbb{Z}}}\left(G_{k}\right) \stackrel{\sim}{\rightarrow} \boldsymbol{\mu}_{\widehat{\mathbb{Z}}}\left(\Pi_{X}\right)$ " of [AbsTopIII], Corollary 1.10, (c), which is constructed by means of a "functorial group-theoretic algorithm". The inverse of this isomorphism yields a cyclotomic rigidity isomorphism

$$
\left(l \cdot \Delta_{\Theta}\right)(\Pi) \stackrel{\sim}{\rightarrow} \boldsymbol{\mu}_{\widehat{\mathbb{Z}}}(\Pi / \Delta)
$$

[cf. the discussion of Proposition 1.3, (ii)] — where we write " $\left(l \cdot \Delta_{\Theta}\right)(\Pi)$ " for the [group-theoretic!] subquotient of $\Pi$ discussed in [EtTh], Corollary 2.18, (i). 
Thus, in summary, one has a "functorial group-theoretic algorithm" whose input data consists of the topological group $\Pi$ and whose output data may be thought of as consisting of $\Pi$, the two topological $\Pi$-modules " $\left(l \cdot \Delta_{\Theta}\right)(\Pi)$ ", " $\boldsymbol{\mu}_{\widehat{\mathbb{Z}}}(\Pi / \Delta)$ ", and the above isomorphism of $\Pi$-modules $\left(l \cdot \Delta_{\Theta}\right)(\Pi) \stackrel{\sim}{\rightarrow} \boldsymbol{\mu}_{\widehat{\mathbb{Z}}}(\Pi / \Delta)$. Thus, if one takes this "functorial group-theoretic algorithm" to be the algorithm that gives rise to the functor $\Xi$ in the discussion of (ii) and (iii), then one concludes that the above cyclotomic rigidity isomorphism $\left(l \cdot \Delta_{\Theta}\right)(\Pi) \stackrel{\sim}{\rightarrow} \boldsymbol{\mu}_{\widehat{\mathbb{Z}}}(\Pi / \Delta)$ may be thought of as giving rise to either

(a) a multiradially defined functor, via the approach of (ii), or

(b) a uniradially defined functor, via the approach of (iii).

On the other hand, there is also another way to obtain a multiradially defined functor from this cyclotomic rigidity isomorphism, as follows. Let $\left(\mathcal{R}, \mathcal{C}, \Psi_{\mathcal{R}}\right)$ be the multiradial environment of Example 1.8, (i). Now define a collection of daggered radial data

$$
\left(\Pi, G, \alpha,\left(l \cdot \Delta_{\Theta}\right)(\Pi) \stackrel{\sim}{\rightarrow} \boldsymbol{\mu}_{\widehat{\mathbb{Z}}}(G)\right)
$$

to consist of radial data $(\Pi, G, \alpha)$ as in Example 1.8, (i), together with the polyisomorphism $\left(l \cdot \Delta_{\Theta}\right)(\Pi) \stackrel{\sim}{\rightarrow} \boldsymbol{\mu}_{\widehat{\mathbb{Z}}}(G)$ obtained by composing the above cyclotomic rigidity isomorphism " $\left(l \cdot \Delta_{\Theta}\right)(\Pi) \stackrel{\sim}{\rightarrow} \boldsymbol{\mu}_{\widehat{\mathbb{Z}}}(\Pi / \Delta)$ " with the poly-isomorphism $\boldsymbol{\mu}_{\widehat{\mathbb{Z}}}(\Pi / \Delta)$ $\stackrel{\sim}{\rightarrow} \boldsymbol{\mu}_{\widehat{\mathbb{Z}}}(G)$ induced by the poly-isomorphism $\alpha: \Pi / \Delta \stackrel{\sim}{\rightarrow} G$. Thus, the polyisomorphism $\left(l \cdot \Delta_{\Theta}\right)(\Pi) \stackrel{\sim}{\rightarrow} \boldsymbol{\mu}_{\widehat{\mathbb{Z}}}(G)$ consists not of a single isomorphism of topological modules, but rather of an $\operatorname{Aut}(G)$-orbit - or, more precisely, a $\Gamma$-orbit, where $\Gamma \subseteq \widehat{\mathbb{Z}}^{\times}$is the image of $\operatorname{Aut}(G)$ via the cyclotomic character on $\operatorname{Aut}(G)[\mathrm{cf}$. [AbsAnab], Proposition 1.2.1, (vi)] — of isomorphisms of topological modules. An isomorphism of collections of daggered radial data is defined to be an isomorphism between the underlying collections of radial data [which is necessarily compatible with the poly-isomorphism of topological modules that constitutes the final member of the collections of daggered radial data in question]. Thus, if we take $\mathcal{C}^{\dagger} \stackrel{\text { def }}{=} \mathcal{C}$, then the "functorial group-theoretic algorithm" that gives rise to the cyclotomic rigidity isomorphism " $\left(l \cdot \Delta_{\Theta}\right)(\Pi) \stackrel{\sim}{\rightarrow} \boldsymbol{\mu}_{\widehat{\mathbb{Z}}}(\Pi / \Delta)$ " yields a functor $\Psi_{\mathcal{R}}: \mathcal{R} \rightarrow \mathcal{R}^{\dagger}$ [that arises from a "functorial algorithm"], together with a diagram as in the display of Example 1.7, (iv). That is to say,

(c) this multiradially defined functor $\Psi_{\mathcal{R}}: \mathcal{R} \rightarrow \mathcal{R}^{\dagger}$ yields an alternative [i.e., relative to (a)] multiradial approach to representing the "functorial grouptheoretic algorithm" that gives rise to the cyclotomic rigidity isomorphism " $\left(l \cdot \Delta_{\Theta}\right)(\Pi) \stackrel{\sim}{\rightarrow} \boldsymbol{\mu}_{\widehat{\mathbb{Z}}}(\Pi / \Delta)$ ".

This is the approach taken in Corollary 1.11, (b), below.

Remark 1.9.1. In general, the portion of the "functorial group-theoretic algorithm" that appears in the discussion of Example 1.9, (ii), (iii), and (iv), which involves the quotient $\Pi / \Delta$ of $\Pi$ will depend not only on the structure of the abstract topological group underlying $\Pi / \Delta$, but also on the structure of $\Pi / \Delta$ as a quotient of $\Pi$ - i.e., from the point of view of the discussion of Example 1.8, (i), on 
the "arithmetic holomorphic structure" on the topological group $\Pi / \Delta$ determined by this quotient structure. In fact, the original motivation for the introduction of the "multiradial terminology" of Example 1.7 was precisely to study the extent to which such "functorial group-theoretic algorithms" could be formulated in such a way as to compute

which portions of the output data of such algorithms do indeed depend in an essential way on the "arithmetic holomorphic structure" and which portions are "mono-analytic" [cf. [AbsTopIII], §I3], i.e., depend only on the structure of the topological group $\Pi / \Delta$ [which one thinks of as a sort of "underlying arithmetic real analytic structure" of the "arithmetic holomorphic structures" involved.

From this point of view, the tautological approach of Example 1.9, (ii) [i.e., Example 1.9, (iv), (a)], may be thought of as expressing the idea that if one thinks of each of the quotients " $\Pi / \Delta$ " in the "spokes" of Fig. 1.2 as being equipped with a fixed "arithmetic holomorphic structure" and hence only related to the coric "G" via some indeterminate isomorphism of topological groups, then one obtains a multiradially defined functor, i.e., a functor that is tautologically compatible with mono-analytic deformations of the various "arithmetic holomorphic structures" that one might impose on the coric " $G$ ". Put another way, a multiradially defined algorithm is an algorithm that is compatible with simultaneous execution on multiple spokes of Fig. 1.2. By contrast, the tautological approach of Example 1.9, (iii) [i.e., Example 1.9, (iv), (b)], may be thought of as expressing the idea that if one tries to identify the various quotients " $\Pi / \Delta$ " in the "spokes" of Fig. 1.2 via arbitrary mono-analytic isomorphisms, then one only obtains a uniradially defined functor, i.e., a functor that fails to be compatible with mono-analytic identifications [i.e., gluing isomorphisms], of the various "arithmetic holomorphic structures" on the coric " $G$ ". Put another way, a uniradially defined algorithm is an algorithm that can only be consistently executed on one spoke at a time. Finally, the approach of Example 1.9, (iv), (c), expresses the idea that, in the case of the particular cyclotomic rigidity isomorphism under consideration, if one weakens the rigidity of this isomorphism by working with this isomorphism up to a certain indeterminacy, then one may construct a multiradially defined functor, i.e., a functor that is indeed compatible with mono-analytic identifications [i.e., gluing isomorphisms] of the various "arithmetic holomorphic structures" on the coric " $G$ ", albeit up to a certain specified indeterminacy. Thus, the multiradiality obtained in Example 1.9, (iv), (c), depends, in an essential way, on the content of the "functorial group-theoretic algorithm" involved. This approach taken in Example 1.9, (iv), (c), is representative of the approach taken in Corollaries 1.10, 1.11, and 1.12 below, which may be thought of as "computations" of the "certain indeterminacy" that one must allow in order to construct a multiradially defined functor as in Example 1.9, (iv), (c).

\section{Remark 1.9.2.}

(i) One way to summarize the discussion of Remark 1.9.1 is as follows. If uniradially defined functors correspond to constructions that depend, in a strict sense, on the "arithmetic holomorphic structure", while corically defined functors 
correspond to constructions that only depend on the underlying mono-analytic structure [i.e., "arithmetic real analytic structure"], then multiradially defined functors correspond to constructions that depend on the "arithmetic holomorphic structure", but only in a fashion that is

compatible with a given description of how this arithmetic holomorphic structure is related to - e.g., "embedded in" - the underlying monoanalytic structure.

For instance, in the various multiradial environments of Example 1.8, this description of the relation to the underlying mono-analytic structure is given, at a concrete level, by the various poly-morphisms [or diagrams of poly-morphisms] " $\alpha_{(-)}$" that appear in the radial data of these multiradial environments. This point of view is summarized in Fig. 1.3 below.

\begin{tabular}{|c|c|c|}
\hline $\begin{array}{c}\text { abstract general } \\
\text { nonsense }\end{array}$ & $\begin{array}{c}\text { inter-universal } \\
\text { Teichmüller theory }\end{array}$ & $\begin{array}{c}\text { classical complex } \\
\text { Teichmüller theory }\end{array}$ \\
\hline $\begin{array}{c}\text { uniradially } \\
\text { defined functors }\end{array}$ & $\begin{array}{c}\text { arithmetic holomorphic } \\
\text { structures }\end{array}$ & $\begin{array}{c}\text { holomorphic } \\
\text { structures }\end{array}$ \\
\hline $\begin{array}{c}\text { multiradially } \\
\text { defined functors }\end{array}$ & $\begin{array}{c}\text { arithmetic holomorphic } \\
\text { structures described in } \\
\text { terms of underlying } \\
\text { mono-analytic structures }\end{array}$ & $\begin{array}{c}\text { holomorphic structures } \\
\text { structures described in } \\
\text { terms of underlying } \\
\text { real analytic structures }\end{array}$ \\
\hline $\begin{array}{c}\text { corically } \\
\text { defined functors }\end{array}$ & $\begin{array}{c}\text { underlying mono-analytic } \\
\text { structures }\end{array}$ & $\begin{array}{c}\text { underlying real analytic } \\
\text { structures }\end{array}$ \\
\hline
\end{tabular}

Fig. 1.3: Uniradiality, Multiradiality, and Coricity

(ii) From the point of view of the analogy with connections discussed in Remark 1.7.1, one may think of a multiradial environment as a structure that allows one to execute parallel transport operations between distinct arithmetic holomorphic stuctures, i.e., to describe what one arithmetic holomorphic structure looks like from the point of view of a distinct arithmetic holomorphic structure that is only related to the orginal arithmetic holomorphic structure via the mono-analytic core.

(iii) From the point of view of the analogy with connections discussed in Remark 1.7.1, it is also interesting to observe that one may think of the different approaches to multiradiality discussed in Example 1.9, (iv), (a), (c), as being roughly analogous to the phenomenon of distinct connection structures on a single 
fibration. Moreover, of these different approaches, the tautological, "general nonsense" approach of Example 1.9, (iv), (a), is, in some sense, [not surprisingly!] the "least interesting" [although it will at times be of use in the theory of the present series of papers!]. This sort of "general nonsense" approach is reminiscent of the tautological approach to constructing connections that occurs in the p-adic theory of the crystalline site, i.e., by simply forming the tensor product with

the ring of functions of the PD-envelope along the diagonal of the fiber product of two copies of the space under consideration.

From the point of view of the issue of "describing what one arithmetic holomorphic structure looks like from the point of view of another" [cf. (ii)], the "tautological" approach is not very interesting precisely because it involves working, in effect, with

the "tautological" collection of "labels of all possible arithmetic holomorphic structures" - i.e., corresponding to the various choices of one particular arrow among the arrows that constitute the poly-morphism denoted " $\alpha$ " in Example 1.8, (i) — without describing in further, more explicit terms what these various "alien" arithmetic holomorphic structures look like relative to structures determined by a given arithmetic holomorphic structure.

By contrast, the "non-tautological" approach to multiradiality of Example 1.9, (iv), (c), by means of the explicit computation of indeterminacies is much more interesting in that it yields a more detailed, explicit description of a structure [e.g., a cyclotomic rigidity isomorphism] associated to an "alien" arithmetic holomorphic structure in terms of the structure associated to a given arithmetic holomorphic structure.

We now proceed to discuss our main results concerning multiradiality.

Corollary 1.10. (Multiradial Mono-theta Cyclotomic Rigidity Isomorphisms) Write $(\mathcal{R}, \mathcal{C}, \Phi: \mathcal{R} \rightarrow \mathcal{C})$ - i.e., in the notation of Example 1.8, (v), (vi),

$$
\left(\Pi \curvearrowright \Pi_{\boldsymbol{\mu}}\left(\mathbb{M}_{*}^{\Theta}(\Pi)\right) \otimes \mathbb{Q} / \mathbb{Z}, G \curvearrowright \mathcal{O}^{\times \boldsymbol{\mu}}(G), \alpha_{\boldsymbol{\mu}, \times \boldsymbol{\mu}}\right) \mapsto\left(G \curvearrowright \mathcal{O}^{\times \boldsymbol{\mu}}(G)\right)
$$

- for the multiradial environment constituted by the exterior-cyclotomic version [cf. Example 1.8, (vi)] of the multiradial environment discussed in Example $1.8,(v)$. Consider the cyclotomic rigidity isomorphism

$$
\left(l \cdot \Delta_{\Theta}\right)(\Pi) \stackrel{\sim}{\rightarrow} \Pi_{\boldsymbol{\mu}}\left(\mathbb{M}_{*}^{\Theta}(\Pi)\right)
$$

[where we identify $\left(l \cdot \Delta_{\Theta}\right)\left(\mathbb{M}_{*}^{\Theta}(\Pi)\right)$ with $\left(l \cdot \Delta_{\Theta}\right)(\Pi)$ ] obtained by composing the functorial algorithm $\Pi \mapsto \mathbb{M}_{*}^{\Theta}(\Pi)$ of Proposition 1.2, (i) [cf. also Proposition 1.5, (i)], with the functorial algorithm for constructing a cyclotomic rigidity isomorphism of Proposition 1.5, (iii). Then the data consisting of the topological group $\Pi$, the topological $\Pi$-modules constituted by the domain and codomain of $\left(* \frac{\text { mono- } \Theta}{\Pi}\right)$, 
and the isomorphism $\left(*{ }_{\Pi}^{\text {mono- } \Theta}\right)$ determines a functor $\mathcal{R} \rightarrow \mathcal{F}$ which arises from a functorial algorithm in the topological group $\Pi$; denote the corresponding graph [cf. Example 1.9, (i)] by $\mathcal{R}^{\dagger}$. In particular, the resulting natural functor $\Psi_{\mathcal{R}}: \mathcal{R} \rightarrow \mathcal{R}^{\dagger}$ [cf. Example 1.9, (i)] is multiradially defined.

Proof. The various assertions of Corollary 1.10 follow immediately from the definitions involved.

Remark 1.10.1. We recall in passing that the domain and codomain of the isomorphism $\left(* \frac{\text { mono- } \Theta}{\Pi}\right)$, as well as the isomorphism $\left(*_{\Pi}^{\text {mono- } \Theta}\right)$ itself, are constructed from various subquotients of $\Delta_{\mathbb{M}_{*}^{\Theta}(\Pi)}$ which are completely determined by the structure of $\Delta_{\mathbb{M}_{*}^{\Theta}(\Pi)}$ as a topological group, the subgroup of $\Delta_{\mathbb{M}_{*}^{\Theta}(\Pi)}$ determined by the image of the "theta section" portion of the system of mono-theta environments $\mathbb{M}_{*}^{\Theta}(\Pi)$, and the images [arising from the natural outer actions involved - cf. Definition 1.1, (i)] of $(l \cdot \underline{\mathbb{Z}})\left(\mathbb{M}_{*}^{\Theta}(\Pi)\right)$ and $G\left(\mathbb{M}_{*}^{\Theta}(\Pi)\right)$ in $\operatorname{Out}\left(\Delta_{\mathbb{M}_{*}^{\Theta}(\Pi)}\right)$. Indeed, the algorithms described in the proofs of [EtTh], Corollary 2.18, (i), (ii), (iii); [EtTh], Corollary 2.19, (i), for constructing the various subquotients of $\Delta_{\mathbb{M}_{*}^{\Theta}}(\Pi)$ corresponding to the domain and codomain of $\left(*_{\Pi}^{\text {mono- } \Theta}\right)$, as well as to the graph of the isomorphism $\left(* *_{\Pi}^{\text {mono- } \Theta}\right)$ itself, depend only on the structure of the topological group $\Delta_{\mathbb{M}_{*}^{\Theta}(\Pi)}$ [cf., e.g., [EtTh], Proposition 2.11, (i)], the subgroup of $\Delta_{\mathbb{M}_{*}^{\Theta}(\Pi)}$ determined by the image of the "theta section" portion of the system of monotheta environments $\mathbb{M}_{*}^{\Theta}(\Pi)$ [cf. [EtTh], Definition 2.13, (ii), (c)], and the construction of the group $\Delta_{C}(\Pi)$ [which was reviewed in Proposition 1.6, (i)] containing $\left(\Delta_{\mathbb{M}_{*}^{\Theta}(\Pi)} \rightarrow\right) \Delta_{\underline{\underline{Y}}}\left(\mathbb{M}_{*}^{\Theta}(\Pi)\right) \subseteq \Delta_{\underline{\underline{X}}}\left(\mathbb{M}_{*}^{\Theta}(\Pi)\right) \cong \Delta$, which is used to construct the various subquotients that appear in the crucial [EtTh], Proposition 2.12; [EtTh], Proposition 2.14, (i).

Remark 1.10.2. In words, the content of Corollary 1.10 may be understood as follows:

Since $\mathcal{O}^{\times \boldsymbol{\mu}}(G)$ is constructed by forming the quotient of $\mathcal{O}^{\times}(G)$ by the roots of unity [i.e., $\left.\mathcal{O}^{\mu}(G)\right]$ - recall the triviality of the homomorphism $\Pi_{\boldsymbol{\mu}}\left(\mathbb{M}_{*}^{\Theta}(\Pi)\right) \otimes \mathbb{Q} / \mathbb{Z} \rightarrow \mathcal{O}^{\times \boldsymbol{\mu}}(G)[\mathrm{cf}$. Example $1.8,(\mathrm{v}),(\mathrm{vi})]$ ! — any rigidification of the cyclotome $\Pi_{\boldsymbol{\mu}}\left(\mathbb{M}_{*}^{\Theta}(\Pi)\right)$ will be tautologically compatible with the coricity of $\mathcal{O}^{\times \boldsymbol{\mu}}(G)$, i.e., with the "sharing of $\mathcal{O}^{\times \boldsymbol{\mu}}(G)$ " by distinct arithmetic holomorphic structures [cf. the discussion of Remark 1.9.1; Fig. 1.4 below].

This contrasts sharply with the situation to be considered in Corollary 1.11 below — cf. Remarks 1.11.3, 1.11.4, below. A similar statement may be made concerning the subquotient $\left(l \cdot \Delta_{\Theta}\right)(\Pi)$ of $\Delta \subseteq \Pi$, which maps trivially to $\Pi / \Delta \stackrel{\sim}{\rightarrow} G$.

Remark 1.10.3. In the context of Corollary 1.10, it is useful to recall the following [cf. the discussion of [EtTh], Remark 1.10.4, (ii)]. Although at first glance, it might appear as though it might be possible to develop a similar theory to the theory developed in the present series of papers based on a more general 
sort of meromorphic function than the theta function, it is by no means clear that such a more general meromorphic function satisfies the crucial cyclotomic rigidity, discrete rigidity, and constant multiple rigidity properties studied in [EtTh]. Of these properties, the cyclotomic rigidity property, which forms the basis of Corollary 1.10, depends most explicitly [cf. [EtTh], Remark 2.19.2] on the structure of the theta quotient $1 \rightarrow \Delta_{\Theta} \rightarrow \Delta_{X}^{\Theta} \rightarrow \Delta_{X}^{\text {ell }} \rightarrow 1$ reviewed in [IUTchI], Remark 3.1.2, (iii), i.e., which corresponds to the "theta group" in more classical treatments of the theta function. Since the theta function is, roughly speaking, essentially characterized among meromorphic functions by the property that it satisfies the "theta symmetries" arising from the theta group, it is thus difficult to see how to generalize the theory of the present series of papers so as to treat more general meromorphic functions than the theta function. Also, in this context, it is useful to recall that unlike the theta function itself, which is strictly local in nature, the theta quotient $\Delta_{X}^{\Theta}$, hence, in particular, the subquotient $\Delta_{\Theta}$, is defined globally [cf. the discussion of [IUTchI], Remark 3.1.2], hence may be applied to the execution of various global anabelian reconstruction algorithms via

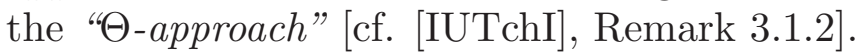

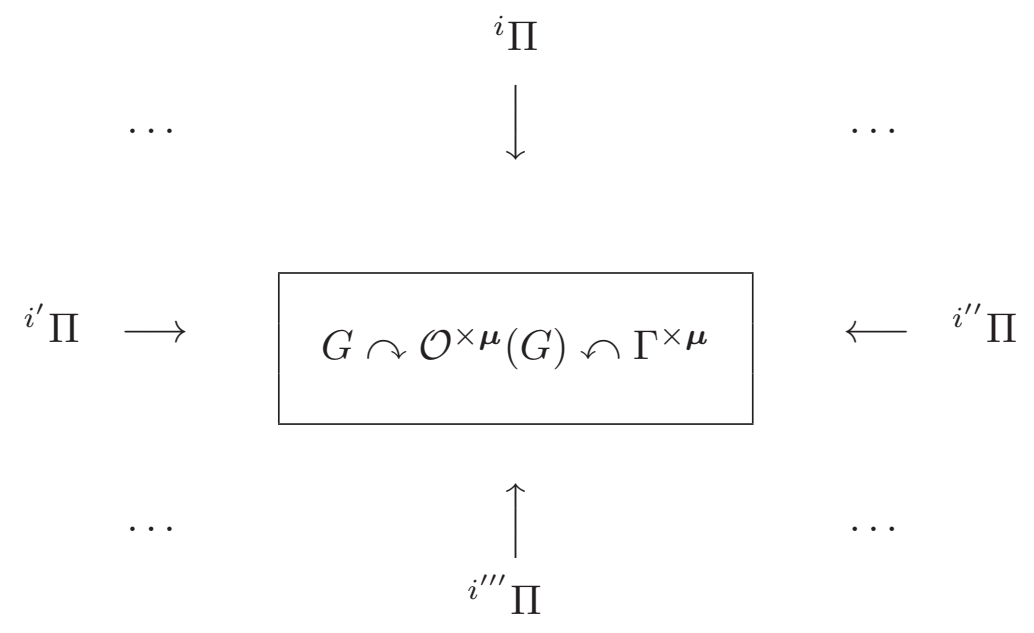

Fig. 1.4: A single coric pair $G \curvearrowright \mathcal{O}^{\times \boldsymbol{\mu}}(G)$, regarded up to the action of $\Gamma^{\times \boldsymbol{\mu}}$

Corollary 1.11. (Multiradial MLF-Galois Pair Cyclotomic Rigidity Isomorphisms with Indeterminacies) Write $(\mathcal{R}, \mathcal{C}, \Phi: \mathcal{R} \rightarrow \mathcal{C})$ - i.e., in the notation of Example 1.8, (viii),

$$
\left(\Pi \curvearrowright M_{\mathbb{T M}}(\Pi), G \curvearrowright \mathcal{O}^{\widehat{\mathrm{gp}}}(G), \alpha_{\triangleright, \times \boldsymbol{\mu}}\right) \mapsto\left(G \curvearrowright \mathcal{O}^{\times \boldsymbol{\mu}}(G)\right)
$$

- for the multiradial environment discussed in Example 1.8, (viii). Consider

(a) the $\Gamma$-orbit [where we recall that $\Gamma \subseteq \widehat{\mathbb{Z}}^{\times}$is a closed subgroup]

$$
\boldsymbol{\mu}_{\widehat{\mathbb{Z}}}(G) \stackrel{\sim}{\rightarrow} \boldsymbol{\mu}_{\widehat{\mathbb{Z}}}\left(\mathcal{O}^{\times}(G)\right) \stackrel{\text { def }}{=} \operatorname{Hom}\left(\mathbb{Q} / \mathbb{Z}, \mathcal{O}^{\times}(G)\right) \quad\left(*_{G, \triangleright}^{\text {bs-Gal }}\right)
$$

of the cyclotomic rigidity isomorphism obtained by applying to the $M L F-G a l o i s$ pair determined by $G \curvearrowright \mathcal{O}^{\triangleright}(G)$ the algorithm applied to 
construct [the inverse of] the isomorphism " $\boldsymbol{\mu}_{\widehat{\mathbb{Z}}}\left(M_{\mathbb{T M}}\right) \stackrel{\sim}{\rightarrow} \boldsymbol{\mu}_{\widehat{\mathbb{Z}}}(G)$ " in [AbsTopIII], Remark 3.2.1 [cf. the discussion of Proposition 1.3, (ii)]; and

(b) the $\operatorname{Aut}(G)$-orbit [where we recall from [AbsAnab], Proposition 1.2.1, (vi), that $\operatorname{Aut}(G)$ admits a natural cyclotomic character] of isomorphisms

$$
\boldsymbol{\mu}_{\widehat{\mathbb{Z}}}(G) \stackrel{\sim}{\rightarrow}\left(l \cdot \Delta_{\Theta}\right)(\Pi) \quad\left(*_{G, \Pi}^{\text {bs-Gal }}\right)
$$

obtained by composing the poly-isomorphism induced by applying " $\boldsymbol{\mu}_{\widehat{\mathbb{Z}}}(-)$ " to the [inverse of the] full poly-isomorphism of topological groups $\alpha$ : $\Pi / \Delta \stackrel{\sim}{\rightarrow} G$ [cf. Example 1.8, (i)] with the cyclotomic rigidity isomorphism “ $\boldsymbol{\mu}_{\widehat{\mathbb{Z}}}\left(G_{k}\right) \stackrel{\sim}{\rightarrow} \boldsymbol{\mu}_{\widehat{\mathbb{Z}}}\left(\Pi_{X}\right)$ " of [AbsTopIII], Corollary 1.10, (c) [cf. the discussion of Proposition 1.3, (ii)].

Then the data consisting of the triple $(\Pi, G, \alpha)$ [cf. Example 1.8, (i)], the topological $G$-modules constituted by the domain and codomain of $\left(*_{G, \triangleright}^{\mathrm{bs}-\mathrm{Gal}}\right)$, the topological $\Pi$ module constituted by the codomain of $\left(*_{G, \Pi}^{\mathrm{bs}-G \mathrm{Gal}}\right)$, and the poly-isomorphisms $\left(*_{G, \triangleright}^{\mathrm{bs}-\mathrm{Gal}}\right)$ and $\left(*_{G, \Pi}^{\text {bs-Gal }}\right)$ determines a functor $\mathcal{R} \rightarrow \mathcal{F}$ which arises from a functorial algorithm in the triple $(\Pi, G, \alpha)$; denote the corresponding graph [cf. Example 1.9, (i)] by $\mathcal{R}^{\dagger}$. In particular, the resulting natural functor $\Psi_{\mathcal{R}}: \mathcal{R} \rightarrow \mathcal{R}^{\dagger}$ [cf. Example 1.9, (i)] is multiradially defined.

Proof. The various assertions of Corollary 1.11 follow immediately from the definitions involved.

\section{Remark 1.11.1.}

(i) In the context of Corollary 1.11, it is useful to recall that:

(a) the group of automorphisms of the underlying topological monoid equipped with a topological group action - i.e., in the terminology of [AbsTopIII], Definition 3.1, (ii), MLF-Galois TM-pair - of

$$
G \curvearrowright \mathcal{O}^{\triangleright}(G)
$$

maps bijectively [i.e., by forgetting " $\mathrm{O}^{\triangleright}(G)$ "] onto the group of automorphisms of the topological group $G$ [cf. [AbsTopIII], Proposition 3.2, (iv)];

(b) the group of automorphisms of the underlying topological module equipped with a topological group action - i.e., in the terminology of [AbsTopIII], Definition 3.1, (ii), MLF-Galois $\mathbb{T} \mathbb{C} \mathbb{G}$-pair — of

$$
G \curvearrowright \mathcal{O}^{\times}(G)
$$

maps surjectively [i.e., by forgetting " $\mathcal{O}^{\times}(G)$ "] onto the group of automorphisms of the topological group $G$, with kernel given by the [ $G$-linear] automorphisms of [the underlying topological module of] $\mathcal{O}^{\times}(G)$ determined by the natural action of $\widehat{\mathbb{Z}}^{\times}$[cf. [AbsTopIII], Proposition 3.3, (ii)]. 
Also, we observe that by the same proof involving the Kummer map as that given for (b) in [AbsTopIII], Proposition 3.3, (ii), it follows that

(c) the group of automorphisms of the underlying topological module equipped with a topological group action of

$$
G \curvearrowright \widehat{\mathcal{O}^{\mathrm{gp}}}(G)
$$

maps surjectively [i.e., by forgetting " $\widehat{\mathcal{O}} \widehat{\mathrm{gp}}(G)$ "] onto the group of automorphisms of the topological group $G$, with kernel given by the [ $G$-linear] automorphisms of [the underlying topological module of] $\mathcal{O}^{\widehat{g p}}(G)$ determined by the natural action of $\widehat{\mathbb{Z}}^{\times}$[or, equivalently, maps bijectively onto the group of automorphisms of the underlying topological module equipped with a topological group action of $G \curvearrowright \mathcal{O}^{\times}(G)$ - cf. (b)].

On the other hand, one verifies immediately that

(d) the underlying topological module of $\mathcal{O}^{\times \boldsymbol{\mu}}(G)$ is divisible, hence admits a natural action by $\mathbb{Q}_{p}$.

In particular, if, in (b), one replaces " $\mathcal{O}^{\times}$" by " $\mathcal{O}^{\times \boldsymbol{\mu}}$ ", then the resulting description of the kernel is false.

(ii) In the present series of papers, we shall primarily be interested in Corollary 1.11 in the case where

$$
\Gamma=\widehat{\mathbb{Z}}^{\times} .
$$

That is to say, allowing for a $\Gamma\left(=\widehat{\mathbb{Z}}^{\times}\right)$-multiple indeterminacy corresponds precisely to working, in the case of $G \curvearrowright \mathcal{O}^{\times}(G)$, with the underlying topological module equipped with topological group action $[\mathrm{cf} .(\mathrm{i}),(\mathrm{b})]$.

\section{Remark 1.11.2.}

(i) Observe that, in the context of the discussion of Remark 1.11.1, (i), (b), the natural action of $\widehat{\mathbb{Z}}^{\times}$on [the underlying topological module equipped with a topological group action of] $G \curvearrowright \mathcal{O}^{\times}(G)$ is compatible with pull-back via the composite of the natural surjection $\Pi \rightarrow \Pi / \Delta$ with any isomorphism $\Pi / \Delta \stackrel{\sim}{\rightarrow} G$ [cf. the notation of Example 1.8]. That is to say, one has a natural action of $\widehat{\mathbb{Z}}^{\times}$ on [the underlying topological module equipped with a topological group action of] the resulting pair $\Pi \curvearrowright \mathcal{O}^{\times}(G)$. Observe, moreover, that this action of $\widehat{\mathbb{Z}}^{\times}$fails to be compatible with the ring structure on $\mathcal{O}^{\times}(G) \otimes \mathbb{Q}$ [i.e., the ring structure determined by applying the $p$-adic logarithm]. That is to say, even though this ring structure on " $\mathcal{O}^{\times}$" may [unlike the case with $G$ !] be reconstructed from the topological group $\Pi$ [cf. [AbsTopIII], Theorem 1.9], the natural action of $\widehat{\mathbb{Z}}^{\times}$on $\Pi \curvearrowright \mathcal{O}^{\times}(G)$ fails to preserve the ring structure reconstructed from $\Pi$.

(ii) The observations of (i) are of interest in the context of understanding our adoption of " $G$ " as opposed to " $\Pi$ " in the construction of the " $\Theta$-link" between distinct $\Theta$-Hodge theaters given in [IUTchI], Corollary 3.7. That is to say, even if one 
tries to "force a preservation of arithmetic holomorphic structures" between distinct $\Theta$-Hodge theaters by working with " $\Pi \curvearrowright \mathcal{O}^{\times}(G)$ " instead of " $G \curvearrowright \mathcal{O}^{\times}(G)$ ", this does not result in the establishment of a consistent common arithmetic holomorphic structure for distinct $\Theta$-Hodge theaters, since the establishment of such a consistent common arithmetic holomorphic structure is already obstructed by the fact that distinct $\Theta$-Hodge theaters only share a common " $\mathcal{O}^{\times}$" [cf. [IUTchI], Corollary 3.7, (iii)] — on which $\widehat{\mathbb{Z}}^{\times}$acts [cf. (i)] — i.e., as opposed to a common "OOO $\triangleright$ ". Here, we recall that the establishment of a common " $\mathrm{O} \triangleright$ " is obstructed, in a quite essential manner, by the "valuative portion ${ }^{\dagger} \underline{\underline{\Theta}}_{\underline{v}} \mapsto^{\ddagger} \underline{\underline{q}}_{\underline{v}}$ " of the $\Theta$-link [cf. [IUTchI], Remark $3.8 .1,(\mathrm{i})]$.

\section{Remark 1.11.3.}

(i) In some sense, the starting point of any discussion of radial environments is the description of the radial functor, i.e., the specification of "which portion of the radial data one takes for one's coric data". From the point of view of the theory of [IUTchI], $\S 3$ [cf., especially, the portion at $\underline{v} \in \underline{\mathbb{V}}^{\text {bad }}$ of [IUTchI], Corollaries 3.7, 3.9], the coric data should, in particular, include the quotient $\Pi \rightarrow \Pi / \Delta \cong G$ of the topological group $\Pi$ isomorphic to $\Pi_{\underline{\underline{X}}_{k}}^{\mathrm{tp}}$ that appears in a $\Theta$-Hodge theater. On the other hand, in [IUTchIII], we shall ultimately be interested in applying the theory

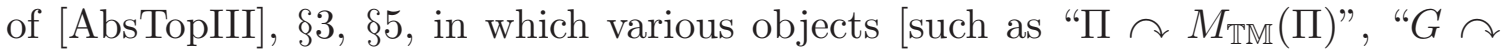
$\mathcal{O}^{\triangleright}(G)$ ", " $G \curvearrowright \mathcal{O}^{\times}(G)$ ", etc.] are constructed group-theoretically from $\Pi$ or $G$. One important aspect of the theory of [AbsTopIII], $\S 3, \S 5$, is that after these objects are constructed group-theoretically from $\Pi$ or $G$, one then proceeds to forget the "anabelian structure" of these objects, i.e., one forgets the data consisting of the way in which these objects [such as MLF-Galois $\mathbb{T M}$-pairs, MLF-Galois $\mathbb{T} \mathbb{C} \mathbb{G}$ pairs, etc.] are constructed from $\Pi$ or $G$. From the point of view of the issue of "specification of coric data", if one takes, for instance, " $G$ " to be a part of one's coric data, then any objects constructed group-theoretically from $G$ may also be regarded naturally as constituting a portion of the coric data - so long as one regards these objects as being equipped with the corresponding "anabelian structures" [i.e., the data that specifies the way in which they were constructed group-theoretically from $G]$. On the other hand, once one forgets these anabelian structures, it is no longer the case that such an object may also be regarded naturally as constituting a portion of the coric data. That is to say, once one forgets the anabelian structure of such an object, it is necessary to specify explicitly that such an object is to be regarded as a portion of the coric data, i.e., as a portion of the radial data that is to be subject to the "gluing", or "identification", discussed in Example 1.7, ( v).

(ii) In light of the "coricity of $\mathcal{O}^{\times}$" given in [IUTchI], Corollary 3.7, (iii), in addition to " $G$ ", it is possible take the underlying MLF-Galois $\mathbb{T} \mathbb{C} \mathbb{G}$-pair of

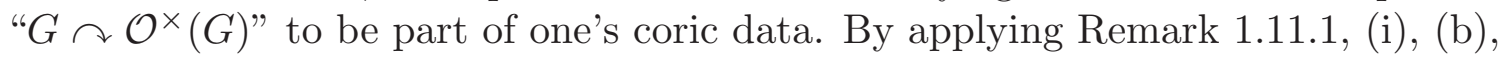
it follows that this amounts to working with " $G \curvearrowright \mathcal{O}^{\times}(G)$ " up to an (Aut $(G), \Gamma$ (= $\left.\widehat{\mathbb{Z}}^{\times}\right)$)-indeterminacy - where we recall from Remark 1.8.1 that the $p$-adic portion of the $\Gamma$-indeterminacy cannot be subsumed into the $\operatorname{Aut}(G)$-indeterminacy [i.e., which arises from the fact that $G$ is only known up to isomorphism as a topological group]. This situation is precisely the situation formulated in Example 1.8, (iii). On the 
other hand, as we saw already in Corollary 1.10 [cf. Remark 1.10.2], and as we shall see again in Corollary 1.12 below, in order to construct certain multiradially defined functors that will be of substantial importance in the development of the theory of the present series of papers, it is necessary to form the quotient of " $\mathrm{O}^{\times}(-)$" by its torsion subgroup " $\mathcal{O}^{\boldsymbol{\mu}}(-)$ ", i.e., to work with " $\mathcal{O}^{\times \boldsymbol{\mu}}(-)$ ", rather than " $\mathcal{O}^{\times}(-)$". As was discussed in Remark 1.11.1, (i), (d), one does not wish here to work solely with the underlying topological module equipped with topological group action determined by " $G \curvearrowright \mathcal{O}^{\times \boldsymbol{\mu}}(G)$ ". On the other hand, by applying [IUTchI], Corollary 3.7, (iii), together with Remark 1.11.1, (i), (b), one concludes that it is possible

to glue together, in a consistent fashion, the various " $G \curvearrowright \mathcal{O}^{\times \mu}(G)$ " $[c f$. Fig. 1.4] arising from distinct $\Theta$-Hodge theaters up to an $(\operatorname{Aut}(G), \Gamma(=$ $\left.\widehat{\mathbb{Z}}^{\times}\right)$)-indeterminacy

[where again we recall from Remark 1.8.1 that the $p$-adic portion of the $\Gamma$-indeterminacy cannot be subsumed into the Aut $(G)$-indeterminacy]. This sort of situation is formulated in the radial environments of Example 1.8, (v), (vi), (viii), (ix) [i.e., where one takes " $\Gamma^{\times \mu}$ " to be the image $\operatorname{Im}(\Gamma)$ of $\left.\Gamma\right]$. One important point in this context is that even if one only takes " $G \curvearrowright \mathcal{O}^{\times \mu}(G)$ " [i.e., as opposed to " $G \curvearrowright$

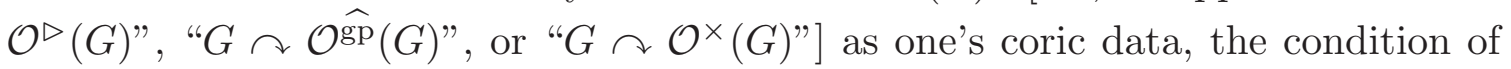
compatibility with respect to the natural maps

$$
\mathcal{O}^{\widehat{\mathrm{gp}}}(G) \hookleftarrow \mathcal{O}^{\times}(G) \rightarrow \mathcal{O}^{\times \boldsymbol{\mu}}(G)
$$

[cf. Example 1.8, (viii)] implies that

$$
\begin{aligned}
& \text { the }\left(\operatorname{Aut}(G), \Gamma\left(=\widehat{\mathbb{Z}}^{\times}\right)\right) \text {-indeterminacy on “ } G \curvearrowright \mathcal{O}^{\times \mu}(G) \text { " induces a } \\
& \left(\operatorname{Aut}(G), \Gamma\left(=\widehat{\mathbb{Z}}^{\times}\right)\right) \text {-indeterminacy on “ } G \curvearrowright \mathcal{O}^{\times}(G) \text { " and “ } G \curvearrowright \mathcal{O}^{\mathrm{gp}}(G) \text { " }
\end{aligned}
$$

— where one may think of the " $\Gamma$-indeterminacy on $\widehat{\mathcal{O}^{\mathrm{gp}}}(G)$ " as representing the " $\Gamma$-indeterminacy in the specification of the submonoid $\mathcal{O}^{\triangleright}(G) \subseteq \widehat{\mathcal{O}} \widehat{\text { gp }}(G)$ ". It is precisely these indeterminacies that induce the indeterminacies - i.e., "orbits" that appear in Corollary 1.11, (a), (b), in sharp contrast to the "strict cyclotomic rigidity" [i.e., without any indeterminacies] of Corollary 1.10 [cf. Remark 1.10.2].

(iii) Note that the algorithms applied to construct cyclotomic rigidity isomorphisms in Corollaries 1.10 and 1.11, (a), are obtained by composing with a group-theoretic construction algorithm an algorithm whose input data is "postanabelian" - i.e., consists of a type of mathematical object that arises upon forgetting the anabelian structure determined by the group-theoretic construction algorithm. More concretely, this post-anabelian input data consists of a system of mono-theta environments in the case of Corollary 1.10 and of an MLF-Galois TMpair in the case of Corollary 1.11, (a). As discussed in (ii), the indeterminacies that act on this post-anabelian input data induce the indeterminacies - i.e., "orbits" - that appear in Corollary 1.11, (a), (b). Put another way,

(a) the indeterminacies - i.e., "orbits" — that appear in Corollary 1.11, (a), (b), are a consequence of the highly nontrivial relationship [cf. the discussion of (ii)] between the input data " $\mathrm{O}^{\triangleright}(-)$ " of the cyclotomic rigidity algorithm involved and the coric data " $\mathrm{O}^{\times \boldsymbol{\mu}}(-)$ ". 
By contrast,

(b) the "strict cyclotomic rigidity" asserted in Corollary 1.10 is a consequence [cf. Remark 1.10.2] of the triviality of the homomorphism that relates the cyclotomic portion of " $\mathcal{O}^{\triangleright}(-)$ " — which is the only portion of "O $\mathrm{O}^{\triangleright}(-)$ " that appears in a mono-theta environment - to the coric data "Oㅅ $(-)$ ".

Here, it is important to note that although frequently in discussions of various "reconstruction algorithms" [such as group-theoretic reconstruction algorithms], emphasis is placed on the existence of "some" reconstruction algorithm. On the other hand, the present discussion of the multiradiality of cyclotomic rigidity isomorphisms in the context of Corollaries 1.10, 1.11 yields an important example of the phenomenon that sometimes not only the existence of "some" reconstruction algorithm, but also the content of the reconstruction algorithm [cf. the discussion of [IUTchIV], Example 3.5] is of crucial importance in the development of the theory.

(iv) Here, we note in passing that one may eliminate the $(\operatorname{Aut}(G), \Gamma)$-indeterminacy of Corollary 1.11, (a), (b), by working, in the fashion of Example 1.9, (iv), (b), with uniradially defined functors [and, in the case of Corollary 1.11, (a), (b), replacing " $G \curvearrowright \mathcal{O}^{\triangleright}(G)$ " by " $\Pi \curvearrowright M_{\mathbb{T M}}(\Pi)$ " and " $G$ " by " $\Pi / \Delta$ "].

(v) The reason [cf. the discussion of (iii)] that we wish to consider cyclotomic rigidity algorithms whose input data is post-anabelian is that we wish to be able to apply the same algorithms to input data that does not necessarily arise from a group-theoretic construction algorithm - e.g., to input data that arises from the [divisor and rational function] monoid portion of a Frobenioid, as in Proposition 1.3. In the context of Proposition 1.3, the exterior cyclotome of the mono-theta environment that appears in Corollary 1.10 and the cyclotome arising from " $\mathrm{O}^{\triangleright}(-)$ " that appears in Corollary 1.11, (a), both correspond to the same cyclotome " $\boldsymbol{\mu}_{N}(S)$ " [which arises from the monoid portion of the Frobenioid involved]. On the other hand, at the level of construction algorithms, in order to relate the exterior cyclotome " $\Pi_{\boldsymbol{\mu}}\left(\mathbb{M}_{*}^{\Theta}(\Pi)\right)$ " of Corollary 1.10 to the cyclotome " $\boldsymbol{\mu}_{\widehat{\mathbb{Z}}}\left(\mathcal{O}^{\times}(G)\right)$ " of Corollary 1.11, (a), it is necessary [cf. Proposition 1.3, (iii)] to pass through the cyclotome " $\left(l \cdot \Delta_{\Theta}\right)(\Pi)$ " by applying the cyclotomic rigidity isomorphisms of Corollaries 1.10, 1.11 - which, in the case of Corollary 1.11, results in various indeterminacies. Put another way, the Frobenioid-theoretic identification [i.e., via " $\boldsymbol{\mu}_{N}(S)$ "] of Proposition 1.3 between the cyclotomes " $\Pi_{\boldsymbol{\mu}}\left(\mathbb{M}_{*}^{\Theta}(\Pi)\right)$ ", " $\boldsymbol{\mu}_{\widehat{\mathbb{Z}}}\left(\mathcal{O}^{\times}(G)\right)$ " of Corollaries 1.10; 1.11, (a), may be thought of either as being only uniradially defined [cf. (iv)], or as multiradially defined, but only up to certain indeterminacies.

\section{Remark 1.11.4.}

(i) One way to understand the significance of the cyclotomic rigidity isomorphism obtained in Corollary 1.10 - i.e., of the triviality of the homomorphism that relates the cyclotomic portion of " $\mathrm{O}^{\triangleright}(-)$ " to the coric data " $\mathrm{O}^{\times \mu}(-)$ " [cf. Remark 1.11.3, (iii), (b)] — relative to the cyclotomic rigidity isomorphism of Corollary 1.11, which involves substantial indeterminacies arising from the highly nontrivial relationship between the input data " $\mathrm{O}^{\triangleright}(-)$ " of the cyclotomic rigidity algorithm involved and the coric data " $\mathrm{O}^{\times \boldsymbol{\mu}}(-)$ " [cf. Remark 1.11.3, (iii), (a)], is as a sort of 
splitting, or decoupling, that serves to separate the "purely radial data" that appears in the cyclotomic rigidity isomorphism of Corollary 1.10 from the "purely coric data" constituted by " $\mathcal{O}^{\times \mu}(-)$ ".

This point of view is discussed further in Remark 1.12.2, (vi), below.

(ii) From the point of view of the discussion of Remark 1.9.2, (iii), the "purely radial data" that appears in the cyclotomic rigidity isomorphism of Corollary 1.10 depends on the tautological collection of "labels of all possible arithmetic holomorphic structures". That is to say, the algorithms of Corollary 1.10 do not give rise to a "detailed, explicit description" of these labels in terms of the "purely coric data $\mathcal{O}^{\times \boldsymbol{\mu}}(-)$ ". On the other hand, one may also consider a modified version of Corollary 1.10 in which

(*) one replaces " $\mathcal{O}^{\times \mu}(-)$ " by " $\mathcal{O}^{\times}(-)$" [i.e., so that the crucial triviality discussed in Remark 1.11.3, (iii), (b), no longer holds!] and applies the tautological approach discussed in Example 1.9, (iv), (a), to constructing the cyclotomic rigidity isomorphism [without indeterminacies!] under consideration.

Now if one works with this modified version $(*)$, then the codomain of the cyclotomic rigidity isomorphism under consideration may be thought of as the submodule " $\mathrm{O}^{\mu}(-)$ " of the "purely coric data $\mathcal{O}^{\times}(-)$", equipped with a "certain rigidity" that depends on the choice of an element of the collection of "labels of all possible arithmetic holomorphic structures". That is to say, whereas Corollary 1.10 has the drawback of being "not entirely free of label-dependence", the significance of Corollary 1.10 [as stated!] relative to the tautological modified version $\left(^{*}\right)$ lies in the fact that the label-dependence inherent in Corollary 1.10 is confined to the "purely radial component" of the splitting, or decoupling, discussed in (i) - i.e., unlike the case with $\left(^{*}\right)$, the algorithms of Corollary 1.10 yield a "purely coric component" that is free of such "unwanted" label-dependent data. Thus, in summary, unlike the case with $\left(^{*}\right)$, the algorithms of Corollary 1.10 yield output data equipped with a splitting, or decoupling, into label-dependent [i.e., "purely radial"] and label-independent [i.e. "purely coric"] components.

Remark 1.11.5. Suppose that we are in the situation of Corollary 1.11.

(i) Recall the natural surjection

$$
H^{1}\left(G, \boldsymbol{\mu}_{\widehat{\mathbb{Z}}}(G)\right) \rightarrow \widehat{\mathbb{Z}}
$$

- which is constructed via a functorial group-theoretic algorithm in [AbsTopIII], Corollary 1.10, (b). That is to say, when $G=G_{k}$, this surjection is the surjection determined by the valuation of $k$ on the image of the natural Kummer map

$$
k^{\times} \hookrightarrow H^{1}\left(G_{k}, \boldsymbol{\mu}_{\widehat{\mathbb{Z}}}\left(G_{k}\right)\right)
$$

- where we recall that the image of this Kummer map is equal to the inverse image of $\mathbb{Z} \subseteq \widehat{\mathbb{Z}}$ via the surjection under consideration. In particular, the existence of this 
functorial group-theoretic algorithm implies that the data consisting of this natural surjection - hence, in particular, its kernel, i.e., " $\mathcal{O}_{k}^{\times}$" - may be formulated as a corically, hence, in particular, as a multiradially [cf. Example 1.7, (iv)], defined functor. [We leave the routine details to the reader.]

(ii) On the other hand, if one applies the isomorphisms $\left(*_{G, \triangleright}^{\mathrm{bs}-\mathrm{Gal}}\right)[\mathrm{cf}$. also the poly-isomorphism $\alpha_{\triangleright}$ of Example 1.8, (ii)] and $\left(*_{G, \Pi}^{\text {bs-Gal }}\right)$, of Corollary 1.11, then the natural surjection of (i) gives rise to natural surjections

$$
H^{1}\left(G, \boldsymbol{\mu}_{\widehat{\mathbb{Z}}}\left(M_{\mathbb{T M}}(\Pi)\right)\right) \rightarrow \widehat{\mathbb{Z}} ; \quad H^{1}\left(G,\left(l \cdot \Delta_{\Theta}\right)(\Pi)\right) \rightarrow \widehat{\mathbb{Z}}
$$

- which yield data that may be formulated either as a uniradially defined functor [cf. Remark 1.11.3, (iv)] or, when considered up to a $\widehat{\mathbb{Z}}^{\times}$-indeterminacy, as a multiradially defined functor [cf. Corollary 1.11]. In particular, the kernels of these natural surjections yield data that may be formulated as a multiradially defined functor. [We leave the routine details to the reader.]

Remark 1.11.6. The importance of cyclotomic rigidity in the theory of the present series of papers is interesting in light of the analogy between the ideas of the present series of papers and the $p$-adic Teichmüller theory of [ $p$ Teich] [cf. the discussion of [AbsTopIII], §I5]. Indeed, the proof of a fundamental absolute p-adic anabelian result concerning the canonical curves that arise in the theory of [ $p$ Teich] [cf. [CanLift], Theorem 3.6] depends, in an essential way, on a certain cyclotomic rigidity result proven in an earlier paper [cf. [AbsAnab], Lemma 2.5, (ii)]. In this context, we observe that one important theme that appears both in the present series of papers and in the theory of [CanLift], $\S 3$, is the idea that cyclotomes should be thought of as the "skeleta of arithmetic holomorphic structures" - cf. the relation of $\mathbb{S}^{1}$ to $\mathbb{C}^{\times}$in the complex archimedean theory.

We are now ready to discuss to main result of the present $\S 1$.

Corollary 1.12. (Multiradial Constant Multiple Rigidity) Write $(\mathcal{R}, \mathcal{C}, \Phi$ : $\mathcal{R} \rightarrow \mathcal{C})-i . e .$, in the notation of Example 1.8, (v), (vi),

$$
\left(\Pi \curvearrowright \Pi_{\boldsymbol{\mu}}\left(\mathbb{M}_{*}^{\Theta}(\Pi)\right) \otimes \mathbb{Q} / \mathbb{Z}, G \curvearrowright \mathcal{O}^{\times \boldsymbol{\mu}}(G), \alpha_{\boldsymbol{\mu}, \times \boldsymbol{\mu}}\right) \mapsto\left(G \curvearrowright \mathcal{O}^{\times \boldsymbol{\mu}}(G)\right)
$$

- for the multiradial environment discussed in Example 1.8, (v), (vi), where we take $\Gamma^{\times \mu} \stackrel{\text { def }}{=}$ Ism. Consider the functorial algorithm that associates to

$$
\Pi \mapsto
$$

the following commutative diagram $\left(\dagger_{\times \theta}\right)(\Pi)$

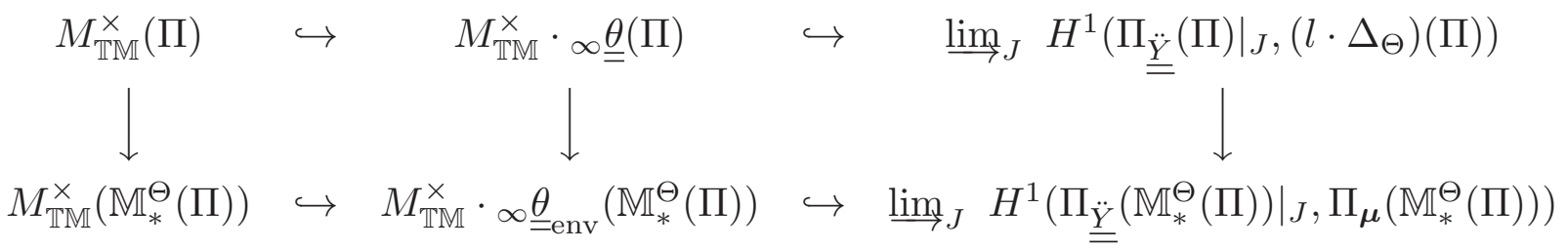


- where

(a) $J$ ranges over the open subgroups of $\Pi$; " $J$ " denotes the fiber product " $\times_{\Pi} J " ;$

(b) the right-hand vertical arrow is the isomorphism of modules induced by the cyclotomic rigidity isomorphism obtained via the functorial algorithm of Corollary 1.10;

(c) we recall that it follows from the definitions [cf. Example 1.8, (ii); [AbsTopIII], Definition 3.1, (vi); [IUTchI], Remark 3.1.2] that one has a natural inclusion $M_{\mathbb{T M}}^{\times}(\Pi) \hookrightarrow \varliminf_{J} H^{1}\left(J,\left(l \cdot \Delta_{\Theta}\right)(\Pi)\right)$, hence a natural inclusion of $M_{\mathbb{T M}}^{\times}(\Pi)$ into the inductive limit of the first line;

(d) we define $M_{\mathbb{T M}}^{\times}\left(\mathbb{M}_{*}^{\Theta}(\Pi)\right)$ and the left-hand vertical arrow to be the submodule and isomorphism of modules induced by the cyclotomic rigidity isomorphism of (b);

(e) we define $M_{\mathbb{T M}}^{\times} \cdot \infty \underline{\underline{\theta}}(\Pi) \stackrel{\text { def }}{=} M_{\mathbb{T M}}^{\times}(\Pi) \cdot \infty \underline{\underline{\theta}}(\Pi)$; here, $\infty \underline{\underline{\theta}}(\Pi)$ is obtained via the functorial algorithm of Proposition 1.4, applied to $\Pi$, and the "." is to be understood as being taken with respect to the module structure [i.e., which is usually denoted additively!] of the ambient cohomology module;

(f) we define $M_{\mathbb{T M}}^{\times} \cdot \infty_{\underline{\theta}}\left(\mathbb{M}_{*}^{\Theta}(\Pi)\right) \stackrel{\text { def }}{=} M_{\mathbb{T M}}^{\times}\left(\mathbb{M}_{*}^{\Theta}(\Pi)\right) \cdot{ }_{\infty} \underline{\underline{\theta}}_{\text {env }}\left(\mathbb{M}_{*}^{\Theta}(\Pi)\right)$; here, $\infty \underline{\underline{\theta}}_{\mathrm{env}}\left(\mathbb{M}_{*}^{\Theta}(\Pi)\right)$ is obtained via the functorial algorithm of Proposition 1.5, (iii), applied to $\mathbb{M}_{*}^{\Theta}(\Pi)$ [cf. Propositions 1.2, (i); 1.5, (i)]; the "." is as in (e); the middle vertical arrow is induced by the right-hand vertical arrow;

(g) the horizontal arrows " $\hookrightarrow$ " are the natural inclusions.

Also, let us write $M_{\mathbb{T M}}^{\times \boldsymbol{\mu}}(-) \stackrel{\text { def }}{=} M_{\mathbb{T M}}^{\times}(-) / M_{\mathbb{T M}}^{\mu}(-)$, where $M_{\mathbb{T M}}^{\boldsymbol{\mu}}(-) \subseteq M_{\mathbb{T M}}^{\times}(-)$denotes the submodule of torsion elements. Then:

(i) There is a functorial group-theoretic algorithm

$$
\Pi \quad \mapsto \quad\{(\iota, D)\}(\Pi)
$$

that assigns to the topological group $\Pi$ a collection of pairs $(\iota, D)-$ where $\Delta_{\ddot{\underline{Y}}}(\Pi) \stackrel{\text { def }}{=}$ $\Pi_{\underline{\underline{Y}}}(\Pi) \bigcap \Delta, \iota$ is a $\Delta_{\underline{\underline{Y}}}(\Pi)$-outer automorphism of $\Pi_{\underline{\underline{Y}}}(\Pi)$ [cf. Proposition $\overline{\overline{1.4}}$ ], and [by abuse of notation] $D \subseteq \Pi_{\underline{\underline{Y}}}(\Pi)$ is a $\Delta_{\underline{\underline{Y}}}(\Pi)$-conjugacy class of closed subgroups - with the property that when $\Pi=\Pi_{\underline{\underline{X}}_{k}}^{\mathrm{tp}}$, the resulting collection of pairs coincides with the collection of "pointed inversion automorphisms" of Remark 1.4.1, (ii). Here, each pair $(\iota, D)$ will be referred to as a pointed inversion automorphism. If $(\iota, D)$ is a pointed inversion automorphism, then we shall denote by a superscript " " the subset of $\iota$-invariants of any set that admits an induced action by $\iota$.

(ii) Let $(\iota, D)$ be a pointed inversion automorphism associated to $\Pi$ [cf. (i)]. Then restriction to the subgroup $D \subseteq \Pi_{\underline{\underline{\underline{Y}}}}(\Pi)$ determines [the horizontal arrows 
in] a commutative diagram

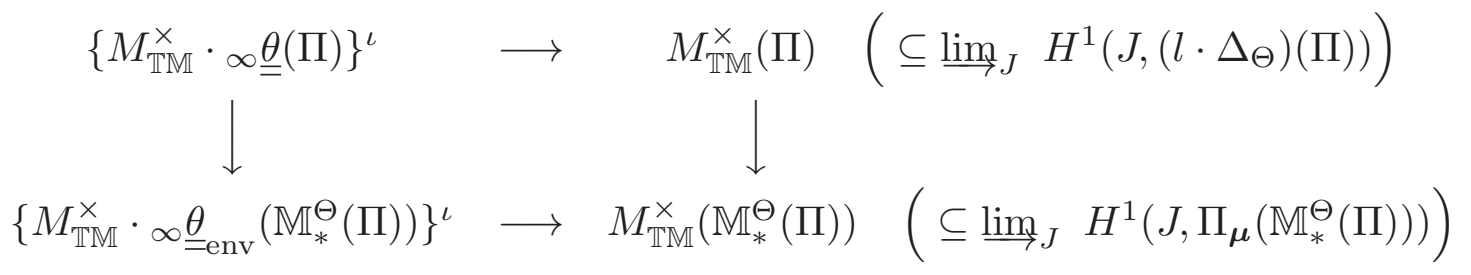

- where $J$ ranges over the open subgroups of $\Pi[c f .(a)]$; the vertical arrows are the isomorphisms induced by the cyclotomic rigidity isomorphism of Corollary 1.10 [cf. (b)]. Here, the inverse images of the submodules of torsion elements - i.e., [up to various natural isomorphisms] the submodules " $M_{\mathbb{T M}}^{\mu}(-)$ " - via the upper and lower horizontal arrows are given, respectively, by $\infty_{\underline{\theta}}(\Pi)^{\iota}$ and $\infty_{\underline{\underline{\theta}}}\left(\mathbb{M}_{*}^{\Theta}(\Pi)\right)^{\iota}$. In particular, we obtain a functorial algorithm [in the topological group $\Pi$ ] for constructing splittings

$$
\begin{gathered}
M_{\mathbb{T M}}^{\times \mu}(\Pi) \times\left\{\infty \underline{\underline{\theta}}(\Pi)^{\iota} / M_{\mathbb{T M}}^{\mu}(\Pi)\right\} ; \\
M_{\mathbb{T M}}^{\times \mu}\left(\mathbb{M}_{*}^{\Theta}(\Pi)\right) \times\left\{\infty \underline{\underline{\theta}}_{\mathrm{env}}\left(\mathbb{M}_{*}^{\Theta}(\Pi)\right)^{\iota} / M_{\mathbb{T M}}^{\mu}\left(\mathbb{M}_{*}^{\Theta}(\Pi)\right)\right\}
\end{gathered}
$$

- i.e., direct product decompositions inside the quotients of the inductive limits on the right-hand side of the diagram $\left(\dagger_{\times \theta}\right)(\Pi)$ by " $M_{\mathbb{T M}}^{\mu}(-)$ " - of the respective images of $\left\{M_{\mathbb{T M}}^{\times} \cdot \infty \underline{\underline{\theta}}(\Pi)\right\}^{\iota},\left\{M_{\mathbb{T M}}^{\times} \cdot \infty \underline{\underline{\theta}}_{\text {env }}\left(\mathbb{M}_{*}^{\Theta}(\Pi)\right)\right\}^{\iota}$ in these quotients.

(iii) Consider the assignment that associates to the data

$$
\left(\Pi \curvearrowright \Pi_{\boldsymbol{\mu}}\left(\mathbb{M}_{*}^{\Theta}(\Pi)\right) \otimes \mathbb{Q} / \mathbb{Z}, G \curvearrowright \mathcal{O}^{\times \boldsymbol{\mu}}(G), \alpha_{\boldsymbol{\mu}, \times \boldsymbol{\mu}}\right)
$$

the data consisting of

- $\mathbb{M}_{*}^{\Theta}(\Pi)$ - i.e., the projective systems of mono-theta environments of Propositions 1.2, (i); 1.5, (i);

- $\left(\dagger_{\times \theta}\right)(\Pi)-i . e .$, "subsets";

. $\left(\dagger_{\mu \theta}\right)(\Pi)-i . e .$, "splittings";

- the diagram

$$
\begin{aligned}
\Pi_{\boldsymbol{\mu}}\left(\mathbb{M}_{*}^{\Theta}(\Pi)\right) \otimes \mathbb{Q} / \mathbb{Z} & \stackrel{\sim}{\rightarrow} M_{\mathbb{T M}}^{\boldsymbol{\mu}}\left(\mathbb{M}_{*}^{\Theta}(\Pi)\right) \stackrel{\sim}{\rightarrow} M_{\mathbb{T M}}^{\boldsymbol{\mu}}(\Pi) \\
& \rightarrow M_{\mathbb{M} \mathbb{M}}^{\times \mu}(\Pi) \stackrel{\sim}{\rightarrow} \mathcal{O}^{\times \mu}(G)
\end{aligned}
$$

- where the first " $\stackrel{\sim}{\rightarrow}$ " is the isomorphism determined by the injection of Remark 1.5.2; the second " $\rightarrow$ " is the isomorphism determined by the vertical arrows of $\left(\dagger_{\times \theta}\right)(\Pi)$; the " $\rightarrow$ " is the trivial homomorphism; the final " $\underset{\rightarrow}{\rightarrow}$ " denotes the poly-isomorphism induced by the poly-isomorphism " $\alpha_{\times}$" of Example 1.8, (iii).

Then this assignment determines a functor $\mathcal{R} \rightarrow \mathcal{F}$ which arises from a functorial algorithm; denote the corresponding graph [cf. Example 1.9, (i)] by $\mathcal{R}^{\dagger}$. In 
particular, the resulting natural functor $\Psi_{\mathcal{R}}: \mathcal{R} \rightarrow \mathcal{R}^{\dagger}$ [cf. Example 1.9, (i)] is multiradially defined.

Proof. Assertion (i) follows immediately from the discussion of Remark 1.4.1 and the references quoted in this discussion. Assertion (ii) follows immediately from the structure of the objects under consideration, as described in [EtTh], Proposition 1.5, (ii), (iii) [cf. also the proofs of [EtTh], Theorems 1.6, 1.10]. Finally, the multiradiality of assertion (iii) follows immediately from the characteristic nature of the various torsion submodules " $M_{\mathbb{T M}}^{\mu}(-)$ " that appear [cf. the discussion of Remark 1.10.2; the discussion of Remark 1.12.2 below].

Remark 1.12.1. One verifies immediately that Corollaries 1.10, 1.11, and 1.12 admit "log-shell versions" [cf. Example 1.8, (ix)]. The various interpretations of these corollaries discussed in the remarks following the corollaries also apply to such "log-shell versions".

\section{Remark 1.12.2.}

(i) Modulo the multiradiality of the cyclotomic rigidity isomorphism of Corollary 1.10 [cf. Corollary 1.12, (b)], the essential content of the multiradiality of Corollary 1.12 lies in the

the functorial group-theoretic algorithm implicit in the proof of [EtTh], Theorem 1.10, (i), for constructing $\underline{\underline{\theta}}(\Pi)$ up to a $\boldsymbol{\mu}_{2 l}$-indeterminacy i.e., as opposed to only up to a " $\mathcal{O}_{k}^{\times}$-indeterminacy", as is done in the proof of [EtTh], Theorem 1.6, (iii) — together with the [elementary] observation that the submodule of [any isomorph of] $\mathcal{O}_{k}^{\times}$constituted by the $2 l$-torsion is characteristic [cf. the proof of Corollary 1.12, (iii)].

That is to say, it is this "essential content" that implies that the crucial splittings $\left(\dagger_{\boldsymbol{\mu} \theta}\right)(\Pi)$ are compatible with gluing together the various collections of coric data " $\left(G \curvearrowright \mathcal{O}^{\times \boldsymbol{\mu}}(G)\right)$ " that arise from distinct arithmetic holomorphic structures.

(ii) Here, we recall in passing [cf. also the discussion of Remark 1.4.1] that the functorial group-theoretic algorithm implicit in the proof of [EtTh], Theorem 1.10, (i), for constructing $\underline{\underline{\theta}}(\Pi)$ up to a $\boldsymbol{\mu}_{2 l}$-indeterminacy consists of

normalizing the étale theta functions under consideration by requiring that their values at points [cf. also the discussion of Remark 1.12.4 below] lying over the 2-torsion point " $\mu_{-}$" of [IUTchI], Example 4.4, (i), be $\in \boldsymbol{\mu}_{2 l}$

— i.e., of considering étale theta functions "of standard type" [cf. [EtTh], Definition 1.9, (ii); [EtTh], Theorem 1.10, (i); [EtTh], Definition 2.7]. Also, we recall from the proof of [EtTh], Theorem 1.10, (i), that the decomposition groups $\subseteq \Pi$ corresponding to these points lying over the 2-torsion point " $\mu_{-}$" are reconstructed precisely by applying the elliptic cuspidalizations reviewed in Proposition 1.6, (ii). 
(iii) By contrast, if, in the context of the discussion of (i), the normalization reviewed in (ii) consisted of the requirement that certain values of the étale theta function be equal, for instance, to

$$
2 \stackrel{\text { def }}{=} 1+1 \in \mathcal{O}_{k}^{\times} \subseteq\left(k^{\times}\right)^{\wedge}
$$

[where we recall that the residue characteristic of $k$ is assumed to be odd - cf. [IUTchI], Definition 3.1, (b)], i.e., an element of $\left(k^{\times}\right)^{\wedge}$ whose construction depends, in an essential way, on the ring structure relative to some specific $\Theta$-Hodge theater - i.e., some specific arithmetic holomorphic structure - then the normalization would fail to give rise to a multiradially defined functor, although [cf. [AbsTopIII], Corollary 1.10, (h); [IUTchI], Remark 3.1.2] it would nonetheless give rise to a uniradially defined functor [cf. the discussion of Example 1.9, (iv), (b); Remark 1.11.5, (ii)].

(iv) From the point of view of the further development of the theory of the present series of papers, the significance of obtaining "splittings up to a $\boldsymbol{\mu}$-indeterminacy" may be summarized as follows. Ultimately, we shall be interested, in [IUTchIII], in applying the theory of log-shells developed in [AbsTopIII] [cf. Remark 1.12.1]. From the point of view of log-shells, which may be thought of as being contained in $\mathcal{O}^{\times \boldsymbol{\mu}}(G)$, an indeterminacy up to some larger subgroup of $\mathcal{O}_{k}^{\times}$- such as, for instance, the subgroup generated by $2=1+1$, together with its $\operatorname{Aut}(G)$ conjugates [cf. the discussion of (iii)] — would imply that

one may only work, in an inconsistent fashion, with [for instance, the image of the log-shell in] the quotient of $\mathcal{O}^{\times \boldsymbol{\mu}}(G)$ by such a larger subgroup

- a situation which is unacceptable from the point of view of the further development of the theory of the present series of papers.

(v) The discussion in (i), (ii), and (iii) above of the multiradiality of the crucial splittings $\left(\dagger_{\boldsymbol{\mu} \theta}\right)(\Pi)$ of Corollary 1.12, (ii), yields another important example [cf. Remark 1.11.3, (iii)] of the phenomenon that sometimes not only the existence of a single reconstruction algorithm, but also the content of the reconstruction algorithm is of crucial importance in the development of the theory. Similar ideas, relative to the point of view of the theory of [EtTh], may also be seen in the discussion of [EtTh], Remarks 1.10.2, 1.10.4.

(vi) In general, multiradiality amounts to a sort of "surjectivity" [cf. the definition of a multiradial environment via a "fullness" condition in Example 1.7, (ii); the discussion of Example 1.7, (v)] of the radial data onto the coric data. From this point of view, the content of the multiradiality of the splittings $\left(\dagger_{\mu \theta}\right)(\Pi)$ of Corollary 1.12, (ii), may be thought of as consisting of a splitting of this "surjection of radial data onto coric data" into

(a) a "purely radial component" constituted by $\left\{\infty \underline{\underline{\theta}}(\Pi)^{\iota} / M_{\mathbb{T M}}^{\mu}(\Pi)\right\}$, $\left\{\infty \underline{\theta}_{\text {env }}\left(\mathbb{M}_{*}^{\Theta}(\Pi)\right)^{\iota} / M_{\mathbb{T M}}^{\mu}\left(\mathbb{M}_{*}^{\Theta}(\Pi)\right)\right\}$ and

(b) a "purely coric component" constituted by $M_{\mathbb{T M}}^{\times \mu}(\Pi), M_{\mathbb{T M}}^{\times \mu}\left(\mathbb{M}_{*}^{\Theta}(\Pi)\right)$. 
Remark 1.12.3. From the point of view of the discussion of Remark 1.11.3, it is useful to note that the subsets $M_{\mathbb{T M}}^{\times} \cdot \infty \underline{\underline{\theta}}(\Pi), M_{\mathbb{T M}}^{\times} \cdot{ }_{\infty} \underline{\underline{\theta}}_{\text {env }}\left(\mathbb{M}_{*}^{\Theta}(\Pi)\right)$ that appear in Corollary 1.12 may be thought of as the images, via the Kummer map, of a certain generating subset of the monoid of rational functions " $\mathcal{O}_{\mathcal{C}_{v}^{\ominus}}^{\triangleright}(-)$ " defined in [IUTchI], Example 3.2, (v), which is used to construct the underlying Frobenioid of the split Frobenioid " $\mathcal{F}_{v}^{\Theta}$ " — cf. also the discussion of Kummer classes in [EtTh], Proposition 5.2, (iii). Here, the splittings $\left(\dagger_{\mu \theta}\right)(\Pi)$ of Corollary 1.12, (ii), correspond to the splitting data of this split Frobenioid $\mathcal{F}_{\underline{v}}^{\Theta}$. Put another way,

this monoidal data that gives rise to the split Frobenioid

$$
\mathcal{F}_{\underline{v}}^{\Theta}
$$

may be thought of as the result of forgetting the "anabelian structure" of $M_{\mathbb{T M}}^{\times} \cdot \infty \underline{\underline{\theta}}(\Pi), M_{\mathbb{T M}}^{\times} \cdot \infty \underline{\underline{\theta}}_{\mathrm{env}}\left(\mathbb{M}_{*}^{\Theta}(\Pi)\right)$, and $\left(\dagger_{\boldsymbol{\mu} \theta}\right)(\Pi)$

— cf. the discussion of Remark 1.11.3, (i), (ii); the theory of $\S 3$ below, especially, Proposition 3.4. In particular, the specification of coric data " $\left(G \curvearrowright \mathcal{O}^{\times \boldsymbol{\mu}}(G)\right)$ " in the multiradial environment that appears in Corollary 1.12 arises naturally from the point of view of applying the "coricity of $\mathcal{O}^{\times}$" given in [IUTchI], Corollary 3.7, (iii), as in the discussion of Remark 1.11.3, (ii). Finally, we recall from the discussion of Remark 1.11.3, (ii), that this specification of coric data " $\left(G \curvearrowright \mathcal{O}^{\times \mu}(G)\right)$ " has the effect of inducing, in particular, an $\left(\operatorname{Aut}(G), \operatorname{Im}\left(\widehat{\mathbb{Z}}^{\times}\right)(\subseteq \operatorname{Ism})\right)$-indeterminacy on

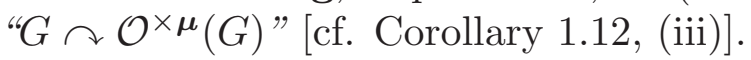

Remark 1.12.4. The fact that the "theta evaluation" functorial algorithm of Corollary 1.12, (ii), given by restriction to the decomposition groups associated to the point " $\mu_{-}$" involves only the topological group " $\Pi$ " as input data will be of crucial importance when we combine the theory developed in the present paper with the theory of log-shells [cf. [AbsTopIII]] in [IUTchIII]. At this point, it is useful to stop and consider to what extent this sort of "group-theoretic evaluation algorithm" is an inevitable consequence of various natural conditions. To this end, let us suppose that we are given some "mysterious evaluation algorithm"

$$
\text { (abstract theta function) } \mapsto \quad \text { (theta values) }
$$

- i.e., which is not necessarily given by restriction to the decomposition group associated to a closed point. Then [cf. [EtTh], Remark 1.10.4; the theory of the "log-wall", as discussed in [AbsTopIII], §I4] it is natural to require [cf., especially, the point of view of the discussion of Remark 1.12.3] that this algorithm be

compatible with forming Kummer classes by extracting $N$-th roots of the "abstract theta function" and the "theta values".

In particular, it is natural to require that this algorithm extend to coverings [e.g., Galois coverings] on both the input and output data of the algorithm. But then the natural requirement of functoriality with respect to the Galois groups on either 
side leads one [cf. Fig. 1.5 below], in effect, to the conclusion - which we shall refer to as the principle of Galois evaluation - that

the "mysterious evaluation algorithm" under consideration in fact arises from a section $G \rightarrow \Pi_{\underline{\underline{\underline{Y}}}}(\Pi)$ of the natural surjection $\Pi_{\underline{\underline{\underline{Y}}}}(\Pi) \rightarrow G$.

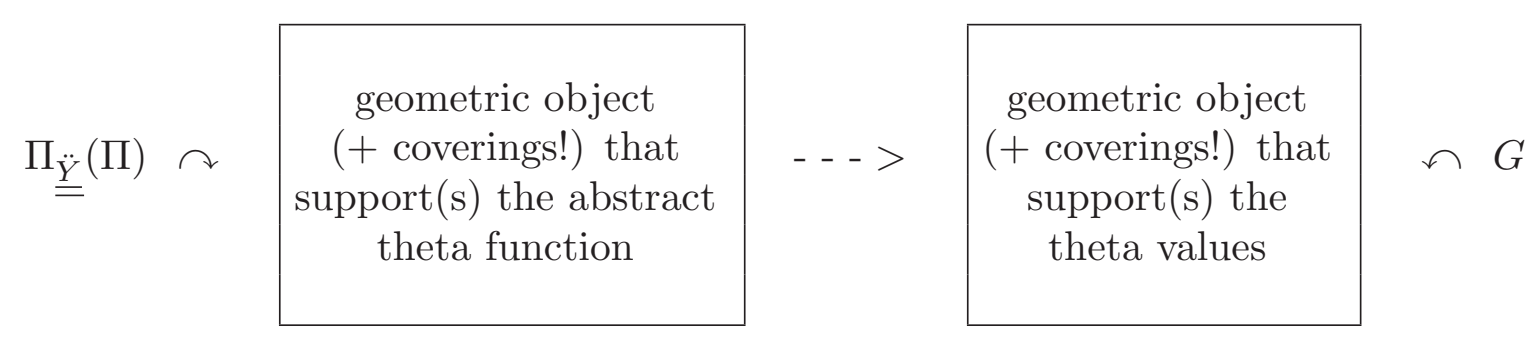

Fig. 1.5: Theta evaluation and Galois functoriality

Moreover, by the "Section Conjecture" of anabelian geometry, one expects that such [continuous] sections $G \rightarrow \Pi_{\underline{\underline{\underline{Y}}}}(\Pi)$ necessarily arise from geometric points. [Here, we pause to observe that this relation to the "Section Conjecture" is interesting in light of the discussion of [IUTchI], Example 4.5, (i); [IUTchI], Remark 2.5.1.] In this context, it is useful to recall that from the point of view of the theory of [AbsTopIII] [cf., e.g., the discussion of [AbsTopIII], §I5], the group-theoreticity of the evaluation algorithm may be thought of as a sort of abstract analogue of the condition, in the $p$-adic theory, that an operation involving various Frobenius crystals be compatible with the Frobenius crystal structures [i.e., connection and Frobenius action] on the input and output data of the operation.

\section{Remark 1.12.5.}

(i) Recall that the scheme-theoretic Hodge-Arakelov theory reviewed in [HASurI], [HASurII] may be thought of as a sort of scheme-theoretic version of the well-known classical computation of the Gaussian integral

$$
\int_{-\infty}^{\infty} e^{-x^{2}}=\sqrt{\pi}
$$

- i.e., by thinking of the square of this integral as an integral over the cartesian plane $\mathbb{R}^{2}$, which may be computed easily by applying a coordinate transformation into polar coordinates. That is to say [cf. the left-hand and middle columns of Fig. 1.6 below], the main theorem of scheme-theoretic Hodge-Arakelov theory is a certain comparison isomorphism [cf. [HASurI], Theorem A] between a "de Rham side" which consists of certain sections of an ample line on the universal extension of an elliptic curve - and an "étale side" — which consists of arbitrary functions on the set of $l$-torsion points of the elliptic curve [where $l$ is, say, some odd prime number]. Here, the module on the de Rham side is equipped with a natural Hodge filtration, while the module on the étale side is equipped with a natural Galois action by $G L_{2}\left(\mathbb{F}_{l}\right)$. The ordered, "step-like" structure of the Hodge filtration is reminiscent of the cartesian structure of the plane $\mathbb{R}^{2}$, i.e., regarded as an ordered collection 
[parametrized by one factor of $\mathbb{R}^{2}$ ] of lines [corresponding to the other factor of $\mathbb{R}^{2}$ ]. On the other hand, the $G L_{2}\left(\mathbb{F}_{l}\right)$-symmetry of the étale side is reminiscent of the rotational symmetry of the representation of the Gaussian integral on the plane via polar coordinates. Moreover, the function " $e^{-x^{2}}$ " itself appears in the Gaussian poles that appear in the de Rham side [cf. [HASurI], §1.1], while the " $\sqrt{\pi}$ " may be thought of as corresponding to the [negative] tensor powers of the sheaf " $\omega$ " of invariant differentials on the elliptic curve that appear in the subquotients of the Hodge filtration, which give rise to a Kodaira-Spencer isomorphism [cf. [HASurII], Theorems 2.8, 2.10] between $\omega^{\otimes 2}$ and the restriction to the base scheme of the sheaf of logarithmic differentials on the moduli stack of elliptic curves - i.e., between $\omega$ and the "square root" of this sheaf of logarithmic differentials. Finally, we recall that this relationship between the theory of [HASurI], [HASurII] and the classical Gaussian integral may be seen more explicitly when this theory is restricted to the archimedean primes of a number field via the "Hermite model" [cf. [HASurI], §1.1].

\begin{tabular}{|c|c|c|}
\hline $\begin{array}{c}\text { classical Gaussian } \\
\text { integral }\end{array}$ & $\begin{array}{c}\frac{\text { scheme-theoretic }}{\text { Hodge-Arakelov theory }} \\
\text { cartesian } \\
\text { coordinates }\end{array}$ & $\begin{array}{c}\text { inter-universal } \\
\text { Teichmüller theory }\end{array}$ \\
\hline $\begin{array}{c}\text { Hodge filtration } \\
\text { polar } \\
\text { coordinates }\end{array}$ & $\begin{array}{c}\text { Frobenius-like structures, } \\
\text { Frobenius-picture } \\
\text { action on torsion points }\end{array}$ & $\begin{array}{c}\text { étale-like structures, } \\
\text { étale-picture }\end{array}$ \\
\hline
\end{tabular}

Fig. 1.6: Analogy with the classical Gaussian integral

(ii) Just as the theory of [HASurI], [HASurII] may be thought of as a schemetheoretic version of the classical theory of the Gaussian integral,

the "inter-universal Teichmüller theory" developed in the present series of papers may be thought of as a sort of global arithmetic/Galoistheoretic version of the classical Gaussian integral

- cf. the right-hand column of Fig. 1.6. Indeed, the ordered, "step-like" nature of the cartesian representation of the Gaussian integral on the plane is reminiscent of the structure of the Frobenius-picture discussed in [IUTchI], Corollary 3.8; [IUTchI], Remark 3.8.1 - i.e., in particular, of the notion of a Frobenius-like mathematical structure that appears in the discussion of [FrdI], Introduction. On the other hand, the rotational symmetry of the representation of the Gaussian integral on the plane via polar coordinates is reminiscent of the étale-picture discussed in [IUTchI], Corollary 3.9, and the following remarks - i.e., in particular, of the notion of an étale-like mathematical structure that appears in the discussion of [FrdI], Introduction. The étale-picture that arises from the multiradially defined functor 
of Corollary 1.12 is depicted in Fig. 1.7 below. From the point of view of the classical series representation of a theta function - i.e., roughly speaking, the series " $\sum_{n \in \mathbb{Z}} q^{n^{2}} \cdot U^{n "}[$ cf. [EtTh], Proposition 1.4] -

this étale-picture of various copies of the Gaussian function " $q^{n^{2}}$ " defined on spokes emanating from a single common core

[cf. also the point of view of Remark 1.12.2, (vi)] is highly reminiscent of the polar coordinate representation of the Gaussian integral on the plane.

$$
\begin{aligned}
& \infty \underline{\underline{\theta}}\left({ }^{i} \Pi\right) \\
& \ldots \quad \downarrow
\end{aligned}
$$

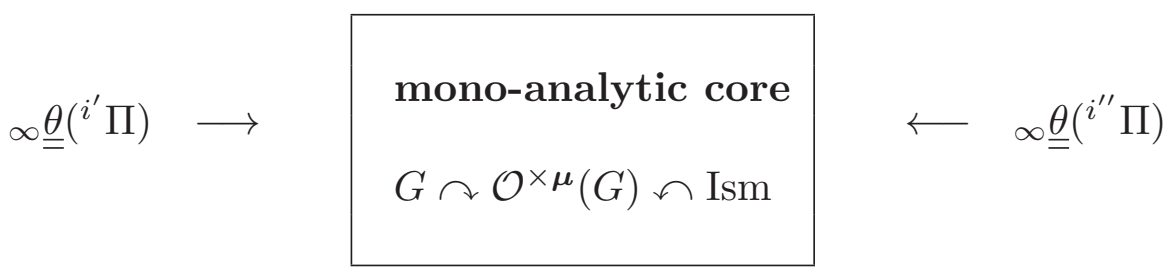

$$
\begin{aligned}
& \cdots \quad \uparrow_{\underline{\underline{\theta}}} \underbrace{\left.i^{\prime \prime \prime} \Pi\right)}
\end{aligned}
$$

Fig. 1.7: Multiradial étale theta functions

(iii) Just as the equivalence between cartesian and polar representations of the classical Gaussian integral is used effectively to compute the value of this Gaussian integral, the relationship between the Frobenius- and étale-pictures will play a central role [cf., especially, the computations of [IUTchIII], §3; [IUTchIV], §1] in the theory of the present series of papers. 


\section{Section 2: Galois-theoretic Theta Evaluation}

In the present $\S 2$, we develop the theory of group-theoretic algorithms surrounding the Hodge-Arakelov-theoretic evaluation of the étale theta function on l-torsion points. At a more technical level, this theory depends on a careful analysis of the issue of conjugate synchronization [cf. Remark 2.6.1] — i.e., of synchronizing conjugates of various copies of objects associated to the absolute Galois group of the base field that occur at the evaluation points - as well as on the computation, via the theory of [IUTchI], $§ 2$, of various conjugacy indeterminacies [cf. Corollaries 2.4, 2.5] that arise from the consideration of certain closed subgroups of various topological groups. In fact, these various technical issues arise, ultimately, as a consequence of the requirement of performing the HodgeArakelov-theoretic evaluation in question with respect to a single basepoint [cf. the discussions of Remark 1.12.4; [IUTchI], Remark 6.12.6]. This Hodge-Arakelovtheoretic evaluation will play a central role in the theory developed in the present series of papers.

In the present $\S 2$, we shall work mainly with the local portion at $\underline{v} \in \underline{\mathbb{V}}^{\text {bad }}$ of the various mathematical objects considered in [IUTchI], §3, $4, \S 5, \S 6$. In fact, however, many of the constructions carried out in the present $\S 2$ will be valid for strictly local data [as in $§ 1$ ], i.e., that does not necessarily arise from global data over a number field. Nevertheless, in order to keep the notation simple from the point of view of discussing the compatibility of the theory of the present $\S 2$ with the theory of [IUTchI], we shall carry out the discussion of the present $\S 2$ only for the localized objects that arise from the theory of [IUTchI], $3, \S 4, \S 5, \S 6$, leaving the routine details of a corresponding purely local theory to the interested reader.

Proposition 2.1. (Review of Certain Tempered Coverings) Let $\underline{v} \in \underline{\mathbb{V}}^{\text {bad }}$. Write

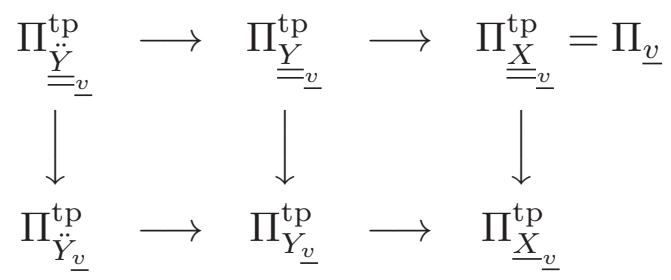

for the diagram of open injections of topological groups arising from the theory of [EtTh], §2 - where

(a) $\Pi_{\underline{X}_{\underline{v}}}^{\mathrm{tp}}, \Pi_{\underline{X}_{\underline{v}}}^{\mathrm{tp}}$ are the tempered fundamental groups determined by the hyperbolic [orbi]curves $\underline{X}_{\underline{v}}, \underline{\underline{X}}_{\underline{v}}$ of [IUTchI], Definition 3.1, (e);

(b) $\Pi_{\underline{\underline{Y}}_{\underline{v}}}^{\mathrm{tp}} \subseteq \Pi_{\underline{\underline{X}}_{\underline{v}}}^{\mathrm{tp}}, \Pi_{\underline{Y}_{\underline{v}}}^{\mathrm{tp}} \subseteq \Pi_{\underline{X}_{\underline{v}}}^{\mathrm{tp}}$ are the open subgroups corresponding to the tempered coverings $\underline{\underline{Y}}_{\underline{v}} \rightarrow \underline{\underline{X}}_{\underline{v}}, Y_{\underline{v}} \rightarrow \underline{X}_{\underline{\underline{v}}}$ determined by the objects " $\underline{Y}^{\log }$ ", " $Y$ log" in the discussion preceding [EtTh], Definition 2.7;

(c) $\Pi_{\underline{\underline{\underline{Y}}}_{\underline{v}}^{\mathrm{tp}}} \subseteq \Pi_{\underline{\underline{X}}_{\underline{v}}^{\mathrm{tp}}}^{\mathrm{t}}$ is the open subgroup determined by the tempered covering $\underline{\underline{Y}}_{\underline{v}} \rightarrow \underline{\underline{X}}_{\underline{v}}$ of [IUTchI], Example 3.2, (ii); $\Pi_{\ddot{Y}_{\underline{v}}}^{\mathrm{tp}} \subseteq \Pi_{\underline{X}_{\underline{v}}}^{\mathrm{tp}}$ is the open subgroup 
corresponding to the tempered covering $\ddot{Y}_{\underline{v}} \rightarrow \underline{X}_{\underline{v}}$ determined by the object "Ÿlog" in the discussion preceding [EtTh], Lemma 1.2;

(d) the arrows are the natural inclusions, and both squares are cartesian.

Then this diagram may be reconstructed via a functorial group-theoretic algorithm [cf. [EtTh], Proposition 2.4] from the [temp-slim! - cf., e.g., [SemiAnbd], Example 3.10] topological group $\Pi_{\underline{\underline{X}}}^{\mathrm{tp}}$.

Proof. The assertions of Proposition 2.1 follow immediately from the results of [EtTh], [SemiAnbd] that are quoted in the statements of these assertions.

Remark 2.1.1. In the notation of Proposition 2.1:

(i) Recall that the special fiber of any model of $\ddot{Y}_{v}$ that arises from a stable model of $\underline{X}_{\underline{v}}$ consists of a chain of copies of the projective line joined together at the points "0", " $\infty$ " [cf. the discussion preceding [EtTh], Proposition 1.1]. The set of irreducible components of this special fiber may be thought of as a torsor over the group $\underline{\mathbb{Z}}$. Moreover, the natural action of $\operatorname{Gal}\left(\ddot{Y}_{\underline{v}} / Y_{\underline{v}}\right) \cong\{ \pm 1\}$ on $\ddot{Y}_{\underline{v}}$ fixes each of the irreducible components of the special fiber of $\ddot{Y}_{\underline{v}}$ and fits into an exact sequence $1 \rightarrow \operatorname{Gal}\left(\ddot{Y}_{\underline{v}} / Y_{\underline{v}}\right) \rightarrow \operatorname{Gal}\left(\ddot{Y}_{\underline{v}} / \underline{X}_{v}\right) \rightarrow \operatorname{Gal}\left(Y_{\underline{v}} / \underline{X}_{v}\right) \rightarrow 1$, where $\operatorname{Gal}\left(Y_{\underline{v}} / \underline{X}_{v}\right)$ may be identified with the subgroup $l \cdot \underline{\mathbb{Z}} \subseteq \underline{\mathbb{Z}}$. Since the degree $l$ covering $\underline{\underline{X}}_{\underline{v}} \rightarrow \underline{X}_{\underline{v}}$ is totally ramified at the cusps, it thus follows that each of the maps

$$
\Gamma_{\underline{\underline{\underline{Y}}}} \rightarrow \Gamma_{\underline{\underline{Y}}} ; \quad \Gamma_{\ddot{Y}} \rightarrow \Gamma_{Y} ; \quad \Gamma_{\underline{\underline{\underline{Y}}}} \rightarrow \Gamma_{\ddot{Y}} ; \quad \Gamma_{\underline{\underline{Y}}} \rightarrow \Gamma_{Y} ; \quad \Gamma_{\underline{\underline{X}}} \rightarrow \Gamma_{\underline{X}}
$$

on dual graphs associated to the special fibers of stable models determined by the various coverings discussed in Proposition 2.1 induces a bijection on vertices.

(ii) Let $\iota_{\underline{\underline{X}}}, \iota_{\underline{\underline{Y}}}$ be as in Remark 1.4.1, where we take " $\underline{\underline{X}}_{k}$ " to be $\underline{\underline{X}}_{\underline{v}}$. Write $\iota_{\underline{X}}, \iota_{\ddot{Y}}$ for the automorphisms of $\underline{X_{\underline{v}}}, \ddot{Y}_{\underline{v}}$ induced by $\iota_{\underline{\underline{X}}}, \iota_{\underline{\underline{Y}}}$;

$$
\Gamma_{\underline{X}}^{\triangleright} \subseteq \Gamma_{\underline{X}}
$$

for the unique connected subgraph of $\Gamma_{\underline{X}}$ which is a tree that is stabilized by $\iota_{X}$ and contains every vertex of $\Gamma_{\underline{X}}$;

$$
\Gamma_{\underline{X}}^{\bullet} \subseteq \Gamma_{\underline{X}}^{\bullet}
$$

for the unique connected subgraph of $\Gamma_{\underline{X}}$ stabilized by $\iota_{\underline{X}}$ that contains precisely one vertex and no edges. Thus, if one thinks of the vertices of $\Gamma_{\underline{X}}$ as being labeled by elements $\in$

$$
\left\{-l^{*},-l^{*}+1, \ldots,-1,0,1, \ldots, l^{*}-1, l^{*}\right\}
$$

— where the vertex labeled 0 is fixed by $\iota_{\underline{X}}$ - then $\Gamma_{\underline{X}}$ is obtained from $\Gamma_{\underline{X}}$ by eliminating the unique edge joining the vertices with labels $\pm l^{*} ; \Gamma_{\underline{X}}^{\bullet}$ consists of the unique vertex 0 and no edges. In particular, by taking appropriate connected 
components of inverse images, one concludes $[\mathrm{cf} .(\mathrm{i})]$ that $\Gamma_{\underline{X}}^{\nabla}$ determines finite, connected subgraphs

$$
\Gamma_{\underline{\underline{X}}}^{\bullet} \subseteq \Gamma_{\underline{\underline{X}}}^{\triangleright} \subseteq \Gamma_{\underline{\underline{X}}}, \quad \Gamma_{\ddot{Y}}^{\bullet} \subseteq \Gamma_{\ddot{Y}}^{\triangleright} \subseteq \Gamma_{\ddot{Y}}, \quad \Gamma_{\underline{\underline{Y}}}^{\bullet} \subseteq \Gamma_{\underline{\underline{Y}}}^{\triangleright} \subseteq \Gamma_{\underline{\underline{Y}}}
$$

of the dual graphs corresponding to $\underline{\underline{X}}_{v}, \ddot{Y}_{\underline{v}}, \underline{\underline{Y}}_{v}$ which are stabilized by the respective "inversion automorphisms" $\iota_{\underline{\underline{X}}}, \iota_{\ddot{Y}}, \iota_{\underline{\underline{Y}}}$. Here, each subgraph $\Gamma_{(-)}^{\bullet}$ consists of precisely one vertex and no edges, while the set of vertices of each subgraph $\Gamma_{(-)}^{\nabla_{-}}$ maps bijectively to the set of vertices of $\Gamma_{\underline{X}}$.

Proposition 2.2. (Decomposition Groups Associated to Subgraphs) In the notation of Proposition 2.1, write

$$
\Pi_{\underline{v} \bullet} \subseteq \Pi_{\underline{v}} \subseteq \Pi_{\underline{v}}
$$

for the decomposition groups determined, respectively, by the subgraphs $\Gamma_{\underline{\underline{X}}}^{\bullet}$ and $\Gamma_{\underline{X}}^{\triangleright}$ - i.e., more precisely, the group " $\Pi_{X, \mathbb{H}}^{\mathrm{tp}}$ " of [IUTchI], Corollary 2.3, (iii), where we take "X" to be $\underline{\underline{X}}_{\underline{v}}$, " $\mathbb{H}$ " to be $\Gamma_{\underline{\underline{X}}}^{\bullet}$ or $\Gamma_{\underline{\underline{X}}}^{\underline{\underline{X}}}$, " $\Sigma$ " to be $\{l\}$, and " $\widehat{\Sigma}$ " to be Primes. Thus, $\Pi_{\underline{v}}$ is well- $\overline{\text { defined }}$ up to $\Pi_{\underline{v}}$-conjugacy; once one fixes $\Pi_{\underline{v}}$, then the subgroup $\Pi_{\underline{v}} \subseteq \Pi_{\underline{v}}$ is well-defined up to $\Pi_{\underline{v}}$-conjugacy; $\Pi_{\underline{v}} \subseteq \Pi_{Y_{\underline{v}}}^{\mathrm{tp}} \cap \Pi_{\underline{v}}=$ $\Pi_{\underline{\underline{Y}}}^{\text {tp }}$. Note, moreover, that we may assume that $\Pi_{\underline{v}}, \Pi_{\underline{v}}$, and $\iota \stackrel{\text { def }}{=} \iota_{\underline{\underline{Y}}}$ have been chosen so that some representative of $\iota$ stabilizes $\Pi_{\underline{v}} \bullet$ and $\Pi_{\underline{v}}$. Then:

(i) The collection of data $\left(\Pi_{\underline{v} \bullet} \subseteq \Pi_{\underline{v}} \subseteq \Pi_{\underline{v}}, \iota\right)$, regarded up to $\Pi_{\underline{v}}$-conjugacy, may be reconstructed via a functorial group-theoretic algorithm from the topological group $\Pi_{\underline{v}}$.

(ii) The functorial group-theoretic algorithms

$$
\Pi_{\underline{v}} \mapsto \underline{\underline{\theta}}\left(\Pi_{\underline{v}}\right) \subseteq \infty \underline{\underline{\theta}}\left(\Pi_{\underline{v}}\right) \subseteq \underset{J}{\lim _{J}} H^{1}\left(\left.\Pi_{\underline{\underline{Y}}}\left(\Pi_{\underline{v}}\right)\right|_{J},\left(l \cdot \Delta_{\Theta}\right)\left(\Pi_{\underline{v}}\right)\right)
$$

of Proposition 1.4 [i.e., where we take " $\Pi$ " to be $\Pi_{\underline{v}}$ ], together with the condition of invariance with respect to $\iota$ [cf. [EtTh], Proposition 1.4, (ii); the proof of [EtTh], Theorem 1.6], determines a specific $\boldsymbol{\mu}_{2 l^{-}}$(respectively, $\boldsymbol{\mu}\left(=M_{\mathbb{T M}}^{\boldsymbol{\mu}}(\Pi)\right)$-) orbit

$$
\underline{\underline{\theta}}^{\iota}\left(\Pi_{\underline{v}}\right) \subseteq \underline{\underline{\theta}}\left(\Pi_{\underline{v}}\right) \quad\left(\text { respectively, } \quad \infty \underline{\underline{\theta}}^{\iota}\left(\Pi_{\underline{v}}\right) \quad \subseteq \quad \infty \underline{\underline{\theta}}\left(\Pi_{\underline{v}}\right)\right)
$$

within the $\left\{(l \cdot \underline{\mathbb{Z}}) \times \boldsymbol{\mu}_{2 l}\right\}-($ respectively, $\{(l \cdot \underline{\mathbb{Z}}) \times \boldsymbol{\mu}\}-)$ orbit constituted by the set $\underline{\underline{\theta}}\left(\Pi_{\underline{v}}\right)$ (respectively, $\infty \underline{\underline{\theta}}\left(\Pi_{\underline{v}}\right)$ ) [cf. Proposition 1.4; Corollary 1.12, (ii)].

Proof. Assertion (i) follows immediately from the fact that dual graphs of stable models may be reconstructed via a functorial group-theoretic algorithm from the corresponding tempered fundamental group [cf., e.g., [SemiAnbd], Corollary 3.11, or, alternatively, [AbsTopI], Theorem 2.14, (i)]. Assertion (ii) follows immediately from the results of [EtTh] that are quoted in the statements of assertion (ii). 
Remark 2.2.1. In the notation of Proposition 2.2, we recall that since the subgroup $\Pi_{\underline{v}} \subseteq \Pi_{\underline{v}}$ is commensurably terminal [cf. [IUTchI], Corollary 2.3, (iv)], it follows that even when this subgroup is subject to a $\Pi_{\underline{\underline{v}}}$-conjugacy indeterminacy, the indeterminacy induced on any specific $\Pi_{\underline{\underline{v}}}$-conjugate of this subgroup $\Pi_{\underline{v}}$ is an indeterminacy with respect to inner automorphisms [i.e., of the specific $\Pi_{\underline{v}^{-}}$ conjugate of $\left.\Pi_{\underline{v}}\right]$.

\section{Definition 2.3.}

(i) In the notation of Proposition 2.2; [IUTchI], Definition 3.1, (e): Write $\Delta_{\underline{v}} \stackrel{\text { def }}{=} \Delta_{\underline{\underline{X}}}^{\mathrm{tp}}, \Delta_{\underline{v}}^{ \pm} \stackrel{\text { def }}{=} \Delta_{\underline{\underline{X}}}^{\mathrm{tp}}, \Pi_{\underline{v}}^{ \pm} \stackrel{\text { def }}{=} \Pi_{\underline{\underline{x}}}^{\mathrm{tp}}, \Delta_{\underline{v}}^{\text {cor }} \stackrel{\text { def }}{=} \Delta_{C_{\underline{v}}}^{\mathrm{tp}}, \Pi_{\underline{v}}^{\text {cor }} \stackrel{\text { def }}{=} \Pi_{C_{\underline{v}}}^{\mathrm{tp}}$; denote the respective profinite completions by means of a " $\wedge$ ". Thus, we have natural diagrams of outer inclusions of topological groups

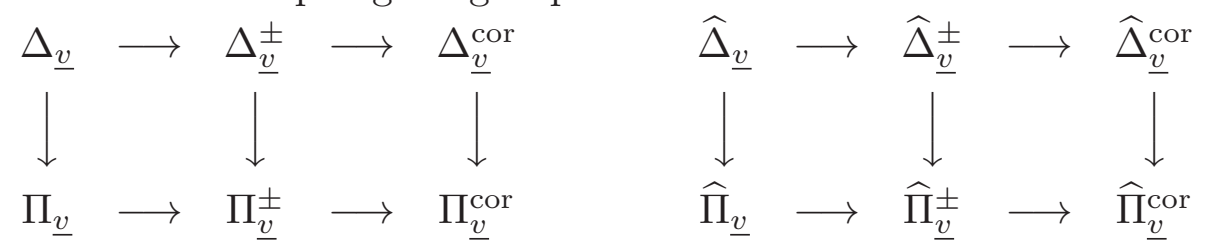

- where the left-hand diagram admits a natural outer inclusion into the righthand diagram, in the evident fashion. Here, we recall that $\widehat{\Delta}_{v}$ includes as a normal open subgroup of $\widehat{\Delta}_{\underline{v}}^{ \pm}$of index $l$ [cf. [EtTh], Proposition 2.2, (ii); [EtTh], Remark 2.6.1], that $\widehat{\Delta}_{\underline{v}}^{ \pm}$includes as a normal open subgroup of $\widehat{\Delta}_{\underline{v}}^{\text {cor }}$ of index $2 l$ [cf. the discussion preceding [EtTh], Definition 2.1], and that $\Pi_{\underline{v}}^{ \pm}$and $\Pi_{\underline{v}}^{\text {cor }}$ may be reconstructed group-theoretically from $\Pi_{\underline{v}}$ [cf. [EtTh], Proposition 2.4]. We shall use these diagrams to regard the various groups appearing in the diagrams as subgroups, well-defined up to $\widehat{\Pi}_{\underline{v}}^{\text {cor }}$-conjugacy, of $\widehat{\Pi}_{\underline{v}}^{\text {cor }}$. Recall the collection of data $\left(\Pi_{\underline{v}} \bullet \subseteq \Pi_{\underline{v}} \triangleright \Pi_{\underline{v}}, \iota\right)$, well-defined up to $\Pi_{\underline{\underline{v}}}-\overline{c o n j u g a c y}$, of Proposition 2.2, (i). Write

$$
\Pi_{\underline{v} \bullet}^{ \pm} \stackrel{\text { def }}{=} N_{\Pi_{\underline{v}}^{ \pm}}\left(\Pi_{\underline{v} \bullet}\right) \subseteq \Pi_{\underline{v} \triangleright}^{ \pm} \stackrel{\text { def }}{=} N_{\Pi_{\underline{v}}^{ \pm}}\left(\Pi_{\underline{v}}\right) \subseteq \Pi_{\underline{v}}^{ \pm}
$$

— so we have natural isomorphisms

$$
\Pi_{\underline{v} \bullet}^{ \pm} / \Pi_{\underline{v} \bullet} \stackrel{\sim}{\rightarrow} \Pi_{\underline{v}}^{ \pm} / \Pi_{\underline{v}} \stackrel{\sim}{\rightarrow} \Pi_{\underline{v}}^{ \pm} / \Pi_{\underline{v}} \stackrel{\sim}{\rightarrow} \widehat{\Delta}_{\underline{v}}^{ \pm} / \widehat{\Delta}_{\underline{v}} \stackrel{\sim}{\rightarrow} \operatorname{Gal}\left(\underline{\underline{X}}_{\underline{v}} / \underline{X}_{\underline{v}}\right)(\cong \mathbb{Z} / l \mathbb{Z})
$$

and equalities $\Pi_{\underline{v} \bullet}^{ \pm} \cap \Pi_{\underline{v}}=\Pi_{\underline{v} \bullet}, \Pi_{\underline{v}}^{ \pm} \cap \Pi_{\underline{v}}=\Pi_{\underline{v}}$ [cf. [IUTchI], Corollary 2.3, (iv)].

(ii) Let $\Pi_{\underline{D}}, \Pi_{\subseteq}$ be any of the topological groups $\Pi_{\underline{v}}, \Pi_{\underline{v}}^{ \pm}, \Pi_{\underline{v}}^{\text {cor }}, \widehat{\Pi}_{\underline{v}}, \widehat{\Pi}_{\underline{v}}^{ \pm}, \widehat{\Pi}_{\underline{v}}^{\text {cor of }}$ (i); suppose that $\Pi_{\subseteq} \subseteq \Pi_{\supseteq}$ relative to one of the natural outer inclusions $\overline{\text { discussed }}$ in (i). Then we recall that the cuspidal inertia groups of $\Pi_{\supseteq}$ may be reconstructed group-theoretically from the topological group $\Pi_{\supseteq}$ via the algorithms of $[$ AbsTopI], Lemma 4.5; [AbsTopI], Proposition 4.10, (vi), and that the cuspidal inertia groups of $\Pi_{\subseteq}$ may be obtained as the intersections with $\Pi_{\subseteq}$ of the cuspidal inertia groups of $\Pi_{\supseteq}$ [cf. [IUTchI], Corollary 2.5], while the cuspidal inertia groups of $\Pi_{\supseteq}$ may be obtained as the $\Pi_{\supseteq}$-conjugates of the commensurators [or, alternatively, the normalizers] in $\Pi_{\supseteq}$ of the cuspidal inertia groups of $\Pi_{\subseteq}[\mathrm{cf}$. [CombGC], Proposition 1.2 , (ii)]. 
(iii) Let $\Pi_{\subseteq}$ be any of the topological groups $\Pi_{\underline{v}}, \Pi_{\underline{v}}^{ \pm}, \widehat{\Pi}_{\underline{v}}, \widehat{\Pi}_{\underline{v}}^{ \pm}$of (i); if $\Pi_{\subseteq}$ is equal to $\Pi_{\underline{v}}$ or $\Pi_{\underline{v}}^{ \pm}$, then set $\Pi_{\underline{D}} \stackrel{\text { def }}{=} \Pi_{\underline{v}}^{ \pm}$; if $\Pi_{\underline{\subseteq}}$ is equal to $\widehat{\Pi}_{\underline{v}}$ or $\widehat{\Pi}_{\underline{v}}^{ \pm}$, then set $\Pi_{\supseteq} \stackrel{\text { def }}{=} \widehat{\Pi}_{v}^{ \pm}$. Thus, $\Pi_{\subseteq} \subseteq \Pi_{\supseteq}$. Then [cf. [IUTchI], Definition 6.1, (iii)] we define a \pm -label class of cusps of $\Pi_{\subseteq}$ to be the set of $\Pi_{\subseteq \text {-conjugacy classes of subgroups }}$ of $\Pi_{\subseteq}$ whose commensurators in $\Pi_{\supseteq}$ [cf. the discussion of (ii)] determine a single $\Pi_{\supseteq}$-conjugacy class of subgroups in $\Pi_{\supseteq}$; write

$$
\operatorname{LabCusp}^{ \pm}\left(\Pi_{\subseteq}\right)
$$

for the set of \pm -label classes of cusps of $\Pi_{\subseteq}$. Thus, when $\Pi_{\subseteq}=\Pi_{\underline{v}}$, if we set ${ }^{\dagger} \mathcal{D}_{\underline{v}} \stackrel{\text { def }}{=}$ $\mathcal{B}^{\text {temp }}\left(\Pi_{\subseteq}\right)^{0}$, then the set LabCusp ${ }^{ \pm}\left(\Pi_{\subseteq}\right)$ may be naturally identified with the set $\operatorname{LabCusp}^{ \pm}\left({ }^{\dagger} \mathcal{D}_{\underline{v}}\right)$ of $\left[\right.$ IUTchI], Definition 6.1 , (iii). In particular, LabCusp ${ }^{ \pm}\left(\Pi_{\underline{v}}\right)=$ $\operatorname{LabCusp}^{ \pm}\left({ }^{\dagger} \mathcal{D}_{\underline{v}}\right)$ admits a natural action by $\mathbb{F}_{l}^{\times}$, as well as a zero element

$$
{ }^{\dagger} \underline{\eta}_{\underline{v}}^{0} \in \operatorname{LabCusp}^{ \pm}\left(\Pi_{\underline{v}}\right)=\operatorname{LabCusp}^{ \pm}\left({ }^{\dagger} \mathcal{D}_{\underline{v}}\right)
$$

and a \pm -canonical element

$$
{ }^{\dagger} \underline{\eta}_{\underline{v}}^{ \pm} \in \operatorname{LabCusp}^{ \pm}\left(\Pi_{\underline{v}}\right)=\operatorname{LabCusp}^{ \pm}\left({ }^{\dagger} \mathcal{D}_{\underline{v}}\right)
$$

- well-defined up to multiplication by \pm 1 , which may be constructed solely from ${ }^{\dagger} \mathcal{D}_{\underline{\underline{v}}}[\mathrm{cf}$. [IUTchI], Definition 6.1, (iii)].

(iv) Let $t \in \operatorname{LabCusp}^{ \pm}\left(\Pi_{\underline{v}}\right)$. Then $t$ determines a unique vertex of $\Gamma_{\underline{\underline{X}}}[\mathrm{cf}$. [CombGC], Proposition 1.5, (i)]. Write $\Gamma_{\underline{X}}^{\bullet t} \subseteq \Gamma_{\underline{X}}$ for the connected subgraph with no edges whose unique vertex is the vertex determined by $t$. Then just as in the case of $\Gamma_{\underline{\underline{X}}}^{\bullet}$ [i.e., the case where $t$ is the zero element] discussed in Proposition 2.2, the subgraph $\Gamma_{\underline{\underline{X}}}^{\bullet t}$ determines - via a functorial group-theoretic algorithm - a decomposition group

$$
\Pi_{\underline{v} \bullet t} \subseteq \Pi_{\underline{v}} \triangleright \Pi_{\underline{v}}
$$

— which is well-defined up to $\Pi_{\underline{v}}$-conjugacy. Finally, we shall write $\Pi_{\underline{v} \bullet t}^{ \pm} \stackrel{\text { def }}{=}$ $N_{\Pi_{\underline{v}}^{ \pm}}\left(\Pi_{\underline{v} \bullet t}\right)\left[\right.$ cf. (i)]; thus, we have a natural isomorphism $\Pi_{\underline{v} \bullet t}^{ \pm} / \Pi_{\underline{v} \bullet t} \stackrel{\sim}{\rightarrow} \operatorname{Gal}\left(\underline{\underline{X}}_{\underline{v}} / \underline{X}_{\underline{v}}\right)$.

(v) Let $\Pi_{\subseteq}$ be either of the topological groups $\Pi_{\underline{v}}^{ \pm}, \widehat{\Pi}_{\underline{v}}^{ \pm}$of $(\mathrm{i})$; if $\Pi_{\subseteq}=\Pi_{\underline{v}}^{ \pm}$, then set $\Pi_{\underline{\supseteq}} \stackrel{\text { def }}{=} \Pi_{\underline{v}}^{\text {cor }}$; if $\Pi_{\subseteq}=\widehat{\Pi}_{\underline{v}}^{ \pm}$, then set $\Pi_{\underline{\supseteq}} \stackrel{\text { def }}{=} \widehat{\Pi}_{\underline{v}}^{\text {cor }}$. Then one verifies immediately that the images [via the natural outer injection $\Pi_{\underline{v}} \hookrightarrow \Pi_{\subseteq}$ ] in $\operatorname{LabCusp}^{ \pm}\left(\Pi_{\subseteq}\right)$ of the various structures on $\operatorname{LabCusp}^{ \pm}\left(\Pi_{\underline{v}}\right)$ reviewed in (iii) determine [in the notation and terminology of [IUTchI], Definition 6.1, (i)] a natural $\mathbb{F}_{l}^{ \pm}$-torsor structure on $\operatorname{LabCusp}^{ \pm}\left(\Pi_{\subseteq}\right)$. Moreover, the natural action of $\Pi_{\supseteq} / \Pi_{\subseteq}$ on $\Pi_{\subseteq}$ preserves this $\mathbb{F}_{l}^{ \pm}$-torsor structure, hence determines a natural outer isomorphism

$$
\Pi_{\supseteq} / \Pi_{\subseteq} \stackrel{\sim}{\rightarrow} \mathbb{F}_{l}^{\rtimes \pm}
$$

[cf. [IUTchI], Definition 6.1, (i)]. 
Remark 2.3.1. In the situation of (iii), suppose that the inclusion $\Pi_{\subseteq} \subseteq \Pi_{\supset}$ is strict. Then one verifies immediately that if $I \subseteq \Pi_{\supseteq}$ is a cuspidal inertia group of $\Pi_{\supseteq}$, then the cuspidal inertia group $I \cap \Pi_{\subseteq} \subseteq \Pi_{\subseteq}$ of $\Pi_{\subseteq}$ satisfies

$$
I \bigcap \Pi_{\subseteq}=I^{l}
$$

- where the superscript $l$ is relative to the group operation on $I$, written multiplicatively. In particular, [even though $\Pi_{\underline{v}}$ (respectively, $\widehat{\Pi}_{\underline{v}}$ ) fails to be normal in $\Pi_{\underline{v}}^{\text {cor }}$ (respectively, $\left.\left.\widehat{\Pi}_{\underline{v}}^{\text {cor }}\right)\right]$ it follows - since $\Pi_{\underline{v}}^{ \pm}$(respectively, $\left.\widehat{\Pi}_{\underline{v}}^{ \pm}\right)$is normal in $\Pi_{\underline{v}}^{\text {cor }}$ (respectively, $\widehat{\Pi}_{\underline{v}}^{\text {cor }}$ ) - that the cuspidal inertia groups of $\Pi_{\underline{v}}$ (respectively, $\widehat{\Pi}_{\underline{v}}$ ) are permuted by the conjugation action of $\Pi_{\underline{v}}^{\text {cor }}$ (respectively, $\widehat{\Pi}_{\underline{v}}^{\text {cor }}$ ).

The theta evaluation algorithm discussed in the following Corollaries 2.4, 2.5, 2.8 , and 2.9 is central to the theory of the present $\S 2$, and, indeed, of the present series of papers.

Corollary 2.4. $\quad\left(\mathbb{F}_{l}^{\rtimes \pm}\right.$-Symmetric Two-torsion Translates of Cusps) In the notation of Definition 2.3: Let $\square \in\{\bullet t, \bullet$. Write

$$
\begin{gathered}
\Delta_{\underline{v} \square} \stackrel{\text { def }}{=} \Delta_{\underline{v}} \bigcap \Pi_{\underline{v} \square}, \quad \Delta_{\underline{v} \square}^{ \pm} \stackrel{\text { def }}{=} \Delta_{\underline{v}}^{ \pm} \bigcap \Pi_{\underline{v} \square}^{ \pm} \\
\Pi_{\underline{v} \ddot{\square}} \stackrel{\text { def }}{=} \Pi_{\underline{v} \square} \bigcap \Pi_{\underline{\underline{\underline{Y}}}}^{\mathrm{tp}}, \quad \Delta_{\underline{v}} \ddot{\square} \stackrel{\text { def }}{=} \Delta_{\underline{v}} \bigcap \Pi_{\underline{v} \ddot{\square}}
\end{gathered}
$$

- so we have

$$
\begin{gathered}
{\left[\Pi_{\underline{v} \square}: \Pi_{\underline{v} \ddot{\square}}\right]=\left[\Delta_{\underline{v} \square}: \Delta_{\underline{v} \ddot{\square}}\right]=2, \quad\left[\Pi_{\underline{v} \square}^{ \pm}: \Pi_{\underline{v} \square}\right]=\left[\Delta_{\underline{v} \square}^{ \pm}: \Delta_{\underline{v} \square}\right]=l} \\
{\left[\Pi_{\underline{v} \square}^{ \pm}: \Pi_{\underline{v} \ddot{\square}}\right]=\left[\Delta_{\underline{v} \square}^{ \pm}: \Delta_{\underline{v}} \ddot{\square}\right]=2 l}
\end{gathered}
$$

[cf. Definition 2.3, (i), (iv)].

(i) (Inclusions and Conjugates) Let $t \in \operatorname{LabCusp}^{ \pm}\left(\Pi_{\underline{v}}\right), I_{t} \subseteq \Pi_{\underline{v}}$ a cuspidal inertia group that belongs to the class determined by $t$ such that $I_{t} \subseteq \Delta_{\underline{v}} \square$. Consider the sets of conjugacy classes of subgroups of $\widehat{\Pi}_{\underline{v}}^{ \pm}$

$$
\begin{gathered}
\left\{I_{t}^{\gamma_{1}}\right\}_{\gamma_{1} \in \widehat{\Pi}_{\underline{v}}^{ \pm}}=\left\{I_{t}^{\gamma_{1}}\right\}_{\gamma_{1} \in \widehat{\Delta}_{\underline{v}}^{ \pm}} \\
\left\{\Pi_{\underline{v} \square}^{\gamma_{2}}\right\}_{\gamma_{2} \in \widehat{\Pi}_{\underline{v}}^{ \pm}}=\left\{\Pi_{\underline{v} \square}^{\gamma_{2}}\right\}_{\gamma_{2} \in \widehat{\Delta}_{\underline{v}}^{ \pm}} ; \quad\left\{\left(\Pi_{\underline{v} \square}^{ \pm}\right)^{\gamma_{3}}\right\}_{\gamma_{3} \in \widehat{\Pi}_{\underline{v}}^{ \pm}}=\left\{\left(\Pi_{\underline{v} \square}^{ \pm}\right)^{\gamma_{3}}\right\}_{\gamma_{3} \in \widehat{\Delta}_{\underline{v}}^{ \pm}}
\end{gathered}
$$

- where the superscript " $\gamma$ 's" denotes conjugation [i.e., " $(-) \mapsto \gamma \cdot(-) \cdot \gamma^{-1}$ "] by $\gamma$. Then for $\gamma, \gamma^{\prime} \in \widehat{\Delta}_{\underline{v}}^{ \pm}$, the following three conditions are equivalent:
(a) $\gamma^{\prime} \in \Delta_{\underline{v} \square}^{ \pm}$
(b) $I_{t}^{\gamma \cdot \gamma^{\prime}} \subseteq \Pi_{\underline{v} \square}^{\gamma}$;
(c) $I_{t}^{\gamma \cdot \gamma^{\prime}} \subseteq\left(\Pi_{\underline{v} \square}^{ \pm}\right)^{\gamma}$.

(ii) (Two-torsion Translates of Cusps) In the situation of (i), if we write $\delta \stackrel{\text { def }}{=} \gamma \cdot \gamma^{\prime} \in \widehat{\Delta}_{\underline{v}}^{ \pm}$, then any inclusion

$$
I_{t}^{\delta}=I_{t}^{\gamma \cdot \gamma^{\prime}} \subseteq \Pi_{\underline{v} \square}^{\gamma}=\Pi_{\underline{v} \square}^{\delta}
$$


as in (i) completely determines the following data:

(a) a decomposition group $D_{t}^{\delta} \stackrel{\text { def }}{=} N_{\Pi_{\underline{v}}^{\delta}}\left(I_{t}^{\delta}\right) \subseteq \Pi_{\underline{v} \tilde{\square}}^{\delta}$ corresponding to the inertia group $I_{t}^{\delta}$;

(b) a decomposition group $D_{\mu_{-}}^{\delta} \subseteq \Pi_{\underline{v} \sim}^{\delta}$, well-defined up to $\left(\Pi_{\underline{v}}^{ \pm}\right)^{\delta}-[o r$, equivalently, $\left(\Delta_{\underline{v}}^{ \pm}\right)^{\delta}$-] conjugacy, corresponding to the torsion point " $\mu_{-}$" of Remark 1.4.1, (i), (ii), via the algorithm of [SemiAnbd], Corollary 3.11 [concerning the dual semi-graphs of the special fibers of stable models], applied to $\Delta_{\underline{v}}^{\delta} \subseteq \Pi_{\underline{v}}^{\delta}$;

(c) a decomposition group $D_{t, \mu_{-}}^{\delta} \subseteq \Pi_{v \ddot{\square}}^{\delta}$, well-defined up to $\left(\Pi_{\underline{v} \square}^{ \pm}\right)^{\delta}$ [or, equivalently, $\left(\Delta_{v \square}^{ \pm}\right)^{\delta}$-] conjugacy - i.e., the image of an evaluation section [cf. [IUTch $I]$, Example 4.4, (i)] - corresponding to the " $\mu_{--}$ translate of the cusp that gives rise to $I_{t}^{\delta}$, via the algorithm of [SemiAnbd], Theorem 6.8, (iii) [concerning the group-theoreticity of the decomposition groups of translates by torsion points of the cusps].

Moreover, the construction of the above data is compatible with conjugation by arbitrary $\delta \in \widehat{\Delta}_{\underline{v}}^{ \pm}$, as well as with the natural inclusion $\Pi_{\underline{v} \bullet t} \subseteq \Pi_{\underline{v}}$ of Definition 2.3, (iv), as one varies $\square \in\{\bullet t, \downarrow\}$.

(iii) $\left(\mathbb{F}_{l}^{\rtimes \pm}\right.$-Symmetry) Suppose that $\square=\bullet$. Then the construction of the data of (ii), (a), (c), is compatible with conjugation by arbitrary $\delta \in \widehat{\Pi}_{\underline{v}}^{\text {cor }}$ [cf. Remark 2.3.1]. Here, we recall from Definition 2.3, (v), that we have natural outer isomorphisms $\widehat{\Delta}_{\underline{v}}^{\text {cor }} / \widehat{\Delta}_{\underline{v}}^{ \pm} \stackrel{\sim}{\rightarrow} \widehat{\Pi}_{\underline{v}}^{\text {cor }} / \widehat{\Pi}_{\underline{v}}^{ \pm} \stackrel{\sim}{\rightarrow} \mathbb{F}_{l}^{\rtimes \pm}$.

Proof. First, we consider assertion (i). The implications (a) $\Longrightarrow(b)$ and (b) $\Longrightarrow(\mathrm{c})$ are immediate from the definitions [cf. also Remark 2.3.1]. Thus, it suffices to verify that $(\mathrm{c}) \Longrightarrow$ (a), i.e., that the condition $I_{t}^{\gamma \cdot \gamma^{\prime}} \subseteq\left(\Pi_{\underline{v} \square}^{ \pm}\right)^{\gamma}$ implies that $\gamma^{\prime} \in \Delta_{\underline{v} \square}^{ \pm}$; we may assume without loss of generality that $\gamma=1$. Then by [IUTchI], Corollary 2.5, the inclusion $I_{t}^{\gamma^{\prime}} \subseteq \Pi_{\underline{v} \square}^{ \pm} \subseteq \Pi_{\underline{v}}^{ \pm}$implies that $\gamma^{\prime} \in \Delta_{\underline{v}}^{ \pm}$. Now, by applying the equivalence of [IUTchI], Corollary 2.3, (vi) [cf. also [CombGC], Proposition 1.2, (ii)], to the various finite index open subgroups of $\Delta_{\underline{v}}^{ \pm}$, it follows that $\gamma^{\prime} \in \widehat{\Delta}_{\underline{v} \square}^{ \pm}$- where we use the notation " $\wedge$ " to denote the closure in $\widehat{\Delta}_{\underline{v}}^{ \pm}$[cf. Proposition 2.2 ; Definition 2.3, (iv); [IUTchI], Corollary 2.3, (ii)] - hence that $\gamma^{\prime} \in \Delta_{\underline{v} \square}^{ \pm}=\widehat{\Delta}_{\underline{v} \square}^{ \pm} \cap \Delta_{\underline{v}}^{ \pm}[\mathrm{cf}$. [IUTchI], Corollary 2.3, (v)]. This completes the proof of assertion (i). Assertions (ii) and (iii) follow immediately from the definitions and the references quoted in the statements of these assertions.

Remark 2.4.1. Note that by applying [IUTchI], Proposition 2.4, (i) [cf. the proof of [IUTchI], Corollary 2.5], one may replace " $I_{t}$ " in Corollary 2.4 by its maximal pro-l' subgroup for any $l^{\prime} \in \mathfrak{P r i m e s} \backslash\left\{p_{\underline{v}}\right\}$. The use of such maximal pro- $l^{\prime}$ subgroups sometimes results in the simplification of arguments involving intersections with other closed subgroups, since every closed subgroup of such a maximal pro- $l^{\prime}$ subgroup is either open or trivial. 
Corollary 2.5. (Group-theoretic Theta Evaluation) In the notation of Corollary 2.4:

(i) (Restriction of Subquotients to Subgraphs) Write

$$
\left(l \cdot \Delta_{\Theta}\right)\left(\Pi_{\underline{v} \ddot{\bullet}}\right)
$$

for the subquotient of $\Pi_{\underline{v}} \ddot{\forall}$ determined by the subquotient $\left(l \cdot \Delta_{\Theta}\right)\left(\Pi_{\underline{v}}\right)$ of $\Pi_{\underline{v}}$. Then

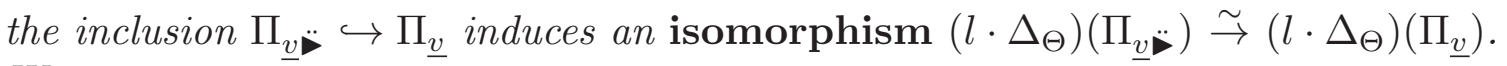
Write

$$
\Pi_{\underline{v}} \rightarrow G_{\underline{v}}\left(\Pi_{\underline{v}}\right), \quad \Pi_{\underline{v}} \ddot{\bullet} \rightarrow G_{\underline{v}}\left(\Pi_{\underline{v}} \ddot{\sim}\right)
$$

for the quotients determined by the natural surjection $\Pi_{v} \rightarrow G_{v}$. Then there exists a functorial group-theoretic algorithm for constructing these quotients from the topological group $\Pi_{\underline{v}}$ [cf., e.g., [AbsAnab], Lemma 1.3.8].

(ii) (Restriction of Étale Theta Functions to Subgraphs and Evaluation Points) Let

$$
I_{t}^{\delta}=I_{t}^{\gamma \cdot \gamma^{\prime}} \subseteq \Pi_{\underline{v}}^{\delta} \stackrel{\subseteq}{\subseteq} \Pi_{\underline{v}}^{\gamma}=\Pi_{\underline{v}}^{\delta}
$$

be an inclusion as in Corollary 2.4, (ii) [where we take $\square \stackrel{\text { def }}{=} \rightarrow$. Then restriction of the $\iota^{\gamma}$-invariant sets $\underline{\underline{\theta}}^{\iota}\left(\Pi_{\underline{v}}^{\gamma}\right), \infty \underline{\underline{\theta}}^{\iota}\left(\Pi_{\underline{v}}^{\gamma}\right)$ of Proposition 2.2, (ii), to the subgroup $\Pi_{\underline{v} \ddot{\sim}}^{\gamma} \subseteq \Pi_{\underline{\underline{Y}}}\left(\Pi_{\underline{v}}^{\gamma}\right) \quad\left(\subseteq \Pi_{\underline{v}}^{\gamma}\right)$ yields $\boldsymbol{\mu}_{2 l^{-}}, \boldsymbol{\mu}$-orbits of elements

$$
\underline{\theta}^{\iota}\left(\Pi_{\underline{v} \ddot{\bullet}}^{\gamma}\right) \subseteq \infty \underline{\theta}^{\iota}\left(\Pi_{\underline{v} \ddot{\bullet}}^{\gamma}\right) \subseteq \underline{\lim }_{\widehat{\widehat{J}}} H^{1}\left(\Pi_{\underline{v} \ddot{\nabla}}^{\gamma} \mid \widehat{\widehat{J}},\left(l \cdot \Delta_{\Theta}\right)\left(\Pi_{\underline{v} \ddot{\bullet}}^{\gamma}\right)\right)
$$

- where $\widehat{J} \subseteq \widehat{\Pi}_{\underline{v}}$ ranges over the open subgroups of $\widehat{\Pi}_{\underline{v}}$ - which, upon further restriction to the decomposition groups $D_{t, \mu_{-}}^{\delta}$ of Corollary 2.4, (ii), (c), yield $\boldsymbol{\mu}_{2 l^{-}}, \boldsymbol{\mu}$-orbits of elements

$$
\underline{\theta}^{t}\left(\Pi_{\underline{v} \ddot{\bullet}}^{\gamma}\right) \subseteq \infty \underline{\theta}^{t}\left(\Pi_{\underline{v} \ddot{\bullet}}^{\gamma}\right) \subseteq \underline{\lim }_{J_{G}} H^{1}\left(\left.G_{\underline{v}}\left(\Pi_{\underline{v} \ddot{\bullet}}^{\gamma}\right)\right|_{J_{G}},\left(l \cdot \Delta_{\Theta}\right)\left(\Pi_{\underline{v} \ddot{\bullet}}^{\gamma}\right)\right)
$$

for each $t \in \operatorname{LabCusp}^{ \pm}\left(\Pi_{\underline{v}}^{\gamma}\right) \stackrel{\sim}{\rightarrow} \operatorname{LabCusp}^{ \pm}\left(\Pi_{\underline{v}}\right)$ - where $J_{G} \subseteq G_{\underline{v}}\left(\Pi_{\underline{v}}^{\gamma}\right)$ ranges over the open subgroups of $G_{\underline{v}}\left(\Pi_{\underline{v}}^{\gamma}\right)$; the " $\stackrel{\sim}{\rightarrow}$ " is induced by conjugation by $\gamma$. Moreover, the sets $\underline{\underline{\theta}}^{t}\left(\Pi_{\underline{v} \ddot{\boldsymbol{v}}}^{\gamma}\right), \infty \underline{\underline{\theta}}^{t}\left(\Pi_{\underline{v} \ddot{\boldsymbol{\nu}}}^{\gamma}\right)$ depend only on the label $|t| \in\left|\mathbb{F}_{l}\right|$ determined by $t$ [cf. Definition 2.3, (iii); [IUTchI], Example 4.4, (i); [IUTchI], Definition 6.1, (iii)].

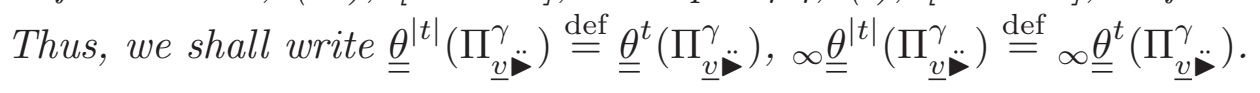

(iii) (Functorial Group-theoretic Evaluation Algorithm) If one starts with an arbitrary $\widehat{\Delta}_{\underline{v}}^{ \pm}$-conjugate $\Pi_{\underline{v}}^{\gamma}$ of $\Pi_{\underline{v}} \ddot{*}$, and one considers, as $t$ ranges over the elements of $\operatorname{LabCusp}^{ \pm}\left(\Pi_{\underline{v}}^{\gamma}\right) \stackrel{\sim}{\rightarrow} \operatorname{LabCusp}^{ \pm}\left(\Pi_{\underline{v}}\right)$ [where the $\stackrel{\sim}{\rightarrow}$ " is induced by conjugation by $\gamma]$, the resulting $\boldsymbol{\mu}_{2 l^{-}}, \boldsymbol{\mu}$-orbits $\underline{\underline{\theta}}^{|t|}\left(\Pi_{\underline{v}}^{\gamma}\right), \infty \underline{\underline{\theta}}^{|t|}\left(\Pi_{\underline{v}}^{\gamma}\right)$ arising from an arbitrary $\widehat{\Delta}_{\underline{v}}^{ \pm}$-conjugate $I_{t}^{\delta}$ of $I_{t}$ that is contained in $\Pi_{v}^{\gamma} \ddot{\vee}[c f$. (ii)], then one obtains a group-theoretic algorithm for constructing the collections of $\boldsymbol{\mu}_{2 l^{-}}, \boldsymbol{\mu}^{-}$ orbits

$$
\left\{\underline{\underline{\theta}}^{|t|}\left(\Pi_{\underline{v} \ddot{\sim}}^{\gamma}\right)\right\}_{|t| \in\left|\mathbb{F}_{l}\right|} ; \quad\left\{\infty \underline{\underline{\theta}}^{|t|}\left(\Pi_{\underline{\underline{v}}}^{\gamma}\right)\right\}_{|t| \in\left|\mathbb{F}_{l}\right|}
$$


which is functorial in the topological group $\Pi_{\underline{v}}$, and, moreover, compatible with the independent conjugacy actions of $\widehat{\Delta}_{\underline{v}}^{ \pm}$on the sets $\left\{I_{t}^{\gamma_{1}}\right\}_{\gamma_{1} \in \widehat{\Pi}_{\underline{v}}^{ \pm}}=\left\{I_{t}^{\gamma_{1}}\right\}_{\gamma_{1} \in \widehat{\Delta}_{\underline{v}}^{ \pm}}$ and $\left\{\Pi_{\underline{v}:}^{\gamma_{2}}\right\}_{\gamma_{2} \in \widehat{\Pi}_{\underline{v}}^{ \pm}}=\left\{\Pi_{\underline{v}}^{\gamma_{2}}\right\}_{\gamma_{2} \in \widehat{\Delta}_{\underline{v}}^{ \pm}}$[cf. the sets of Corollary 2.4, (i); Remark 2.2.1].

Proof. Assertions (i), (ii), and (iii) follow immediately from the definitions and the references quoted in the statements of these assertions. Here, in assertion (i), we observe that the fact that the inclusion $\Pi_{\underline{v}} \ddot{\hookrightarrow} \hookrightarrow \Pi_{\underline{v}}$ induces an isomorphism $\left(l \cdot \Delta_{\Theta}\right)\left(\Pi_{v} \ddot{\sim}\right) \stackrel{\sim}{\rightarrow}\left(l \cdot \Delta_{\Theta}\right)\left(\Pi_{\underline{v}}\right)$ follows immediately by considering the cuspidal inertia groups involved.

\section{Remark 2.5.1.}

(i) Recall from the discussion of [IUTchI], Example 4.4, (i), that relative to the "standard" cyclotomic rigidity isomorphism (* $\left.*^{\mathrm{bs}-\mathrm{Gal}}\right)$ of Proposition 1.3, (ii), and the resulting Kummer map

$$
K_{\underline{v}}^{\times} \hookrightarrow H^{1}\left(G_{\underline{v}}\left(\Pi_{\underline{v}} \ddot{\nabla}\right),\left(l \cdot \Delta_{\Theta}\right)\left(\Pi_{\underline{v} \ddot{\bullet}}\right)\right)
$$

[i.e., we take " $\delta$ " in Corollary 2.5, (ii), to be the identity — without loss of generality, in light of Remark 2.2.1], it follows immediately from the definition of the connected subgraph " $\Gamma_{\underline{\underline{X}}}^{\underline{\underline{X}}}$ " in Remark 2.1.1, (ii) [cf. also [IUTchI], Corollary 2.3, (vi)], that, for $j \in\left|\mathbb{F}_{l}\right|$, the set $\underline{\underline{\theta}}^{j}\left(\Pi_{\underline{v}} \ddot{)}\right)$ consists of precisely the $\boldsymbol{\mu}_{2 l}$-orbit of the "theta value"

$$
\underline{\underline{q} \underline{\underline{\underline{v}}}} \underline{\underline{\underline{v}}}
$$

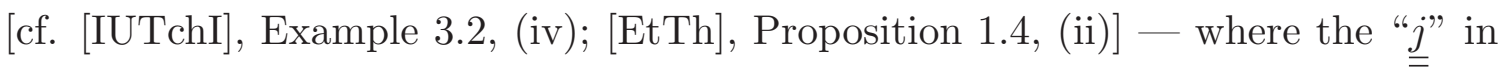
the exponent denotes the element $\in\{0,1, \ldots, l *\}$ determined by the given element $j \in\left|\mathbb{F}_{l}\right|$.

(ii) Note that [the $l$-th powers of] the theta values discussed in (i) are somewhat unusual among the various values

$$
\ddot{\Theta}(c)
$$

— where $c \in K_{\underline{v}}$ - attained by the theta series

$$
\ddot{\Theta}=\ddot{\Theta}(\ddot{U}) \stackrel{\text { def }}{=} q_{X}^{-\frac{1}{8}} \cdot \sum_{n \in \mathbb{Z}}(-1)^{n} \cdot q_{X}^{\frac{1}{2}\left(n+\frac{1}{2}\right)^{2}} \cdot \ddot{U}^{2 n+1}
$$

discussed in [EtTh], Proposition 1.4 [cf. the notation of loc. cit.] in that they satisfy the following crucial property [cf. the discussion of Remark 1.12.2]:

the ratio $\ddot{\Theta}(c) / \ddot{\Theta}\left(c^{\prime}\right)$ is a root of unity, for any $c^{\prime} \in K_{\underline{v}}$ [corresponding to a point of $\ddot{Y}_{\underline{v}}$ ] that occurs as the result of applying an automorphism of $\Pi_{\underline{v}}$ to [the point of $\ddot{Y}_{\underline{v}}$ correspond to] $c$ such that $c^{\prime} / c$ is a unit.

That is to say, [the $l$-th powers of] the theta values discussed in (i) correspond to the values $\underline{\underline{\Theta}}\left( \pm \sqrt{-1} \cdot q_{\bar{X}}^{\overline{\underline{j}}}\right)$, where $\underline{\underline{j}} \in\left\{0,1, \ldots, l^{*}\right\}$, i.e., the values at points 
separated by periods [i.e., the " $q_{X}^{\bar{j}} / 2$ "] from the point " $\pm \sqrt{-1}$ ". These values may be computed easily from the "functional equations" given in [EtTh], Proposition 1.4, (ii).

(iii) Note that, in the context of the $\mathbb{F}_{l}^{\rtimes \pm}$-symmetry discussed in Corollary 2.4, (iii),

the various $\boldsymbol{\mu}_{2 l}$-multiple indeterminacies that occur, for various $j \in\left|\mathbb{F}_{l}\right|$, in the $\boldsymbol{\mu}_{2 l}$-orbit $\underline{\underline{\theta}}^{j}\left(\Pi_{\underline{v}} \ddot{\sim}\right)$ are independent.

That is to say, these indeterminacies are not "synchronized" so as to arise from a single indeterminacy that is independent of $j$. Indeed, each of these $\boldsymbol{\mu}_{2 l}$-multiple indeterminacies arises as a consequence of the action of $\left(\Delta_{v \bullet t}^{ \pm} / \Delta_{\underline{v}} \ddot{\bullet} t\right)^{\delta}$, where we recall from Corollary 2.4 that $\left[\Delta_{v \bullet t}^{ \pm}: \Delta_{\underline{v} \bullet t}\right]=2 l$, on the decomposition groups " $D_{t, \mu_{-}}^{\delta} \subseteq \Pi_{v}^{\delta}$." of Corollary 2.4, (ii), (c), hence is induced by the $\widehat{\Delta}_{\underline{v}}^{ \pm}$-outer nature of the action of $\widehat{\Delta}_{\underline{v}}^{\text {cor }} / \widehat{\Delta}_{\underline{v}}^{ \pm} \stackrel{\sim}{\rightarrow} \mathbb{F}_{l}^{\rtimes \pm}$ that appears in Corollary 2.4 , (iii) - cf. the discussion of Remarks $2 . \overline{5} .2,2.6 .2$ below.

\section{Remark 2.5.2.}

(i) If one thinks of the

$$
\begin{gathered}
\text { "set }\left\{I_{t}^{\gamma_{1}}\right\}_{\gamma_{1} \in \widehat{\Pi}_{\underline{v}}^{ \pm}}=\left\{I_{t}^{\gamma_{1}}\right\}_{\gamma_{1} \in \widehat{\Delta}_{\underline{v}}^{ \pm}} \text {regarded up to } \widehat{\Delta}_{\underline{v}}^{ \pm} \text {-conjugacy" } \\
\text { (respectively, "set }\left\{\Pi_{\underline{v}:{ }^{2}}^{\gamma_{2}}\right\}_{\gamma_{2} \in \widehat{\Pi}_{\underline{v}}^{ \pm}}=\left\{\Pi_{\underline{v}}^{\gamma_{2}}\right\}_{\gamma_{2} \in \widehat{\Delta}_{\underline{v}}^{ \pm}} \text {regarded up to } \widehat{\Delta}_{\underline{v}}^{ \pm} \text {-conjugacy") }
\end{gathered}
$$

[cf. Corollary 2.5, (iii)] as a sort of quotient by $\widehat{\Delta}_{\underline{v}}^{ \pm}$, then one may think of the various inclusion morphisms $I_{t}^{\gamma_{1}} \hookrightarrow \Pi_{v}^{\gamma_{2}}$ as a sort of morphism between quotients

$$
\left(\widehat{\Delta}_{\underline{v}}^{ \pm} \curvearrowright\left\{I_{t}^{\gamma_{1}}\right\}_{\gamma_{1} \in \widehat{\Delta}_{\underline{v}}^{ \pm}}\right) / \widehat{\Delta}_{\underline{v}}^{ \pm} \rightarrow\left(\widehat{\Delta}_{\underline{v}}^{ \pm} \curvearrowright\left\{\Pi_{\underline{v} \dot{\bullet}}^{\gamma_{2}}\right\}_{\gamma_{2} \in \widehat{\Delta}_{\underline{v}}^{ \pm}}\right) / \widehat{\Delta}_{\underline{v}}^{ \pm}
$$

which induces a morphism between quotients

$$
\left(\widehat{\Delta}_{\underline{v}}^{ \pm} \curvearrowright\left\{D_{t, \mu_{-}}^{\gamma_{1}}\right\}_{\gamma_{1} \in \widehat{\Delta}_{\underline{v}}^{ \pm}}\right) / \widehat{\Delta}_{\underline{v}}^{ \pm} \rightarrow\left(\widehat{\Delta}_{\underline{v}}^{ \pm} \curvearrowright\left\{\Pi_{\underline{v}}^{\gamma_{2}}\right\}_{\gamma_{2} \in \widehat{\Delta}_{\underline{v}}^{ \pm}}\right) / \widehat{\Delta}_{\underline{v}}^{ \pm}
$$

— cf. Corollary 2.4, (ii); the discussion of [IUTchI], Remark 4.5.1, (i), (iii). Since all of the inclusions involved occur within a single "ambient container" — namely, $\widehat{\Pi}_{\underline{v}}^{ \pm}$, regarded up to $\widehat{\Pi}_{\underline{v}}^{ \pm}$-conjugacy — the evaluation algorithm discussed in Corollary $2 . \overline{5}$, (iii), may be thought of as a sort of "nested" version of the principle of "Galois evaluation" discussed in Remark 1.12.4. Here, we note that unlike the situation discussed in Remark 1.12.4, in which the subgroup $\Pi_{\underline{\underline{\underline{Y}}}}(\Pi) \subseteq \Pi$ is normal, the subgroups $\Pi_{\underline{v}}, \Pi_{\underline{v}} \approx \widehat{\Pi}_{\underline{v}}^{ \pm}$are far from being normal!

(ii) In the notation of [IUTchI], Definition 3.1, (d) [cf. also the notation of [IUTchI], Definition 6.1, (v)], write

$$
\Pi^{\odot \pm} \stackrel{\text { def }}{=} \Pi_{\underline{X}_{K}} ; \quad \Delta^{\odot \pm} \stackrel{\text { def }}{=} \Delta_{\underline{X}}
$$


— so $\Delta^{\odot \pm}$ may be naturally identified, up to inner automorphism, with $\widehat{\Delta}_{\underline{v}}^{ \pm}$. Then note that unlike the tempered fundamental groups $\Delta_{\underline{v}}, \Delta_{\underline{v}}^{ \pm}, \Delta_{\underline{v}}, \Delta_{\underline{v}}(\stackrel{\text { def }}{=}$ $\Pi_{\underline{v}} \sim \Delta_{\underline{v}}$ ) or the local Galois groups $\Pi_{\underline{v}} / \Delta_{\underline{v}}, \Pi_{\underline{v}}^{ \pm} / \Delta_{\underline{v}}^{ \pm}, \Pi_{\underline{v}} / \Delta_{\underline{v}}, \Pi_{\underline{v}} / \Delta_{\underline{v}}$ of which depend, in a quite essential way, on $\underline{v}-$ the topological group $\Delta^{\odot \pm} \cong \widehat{\Delta}_{\underline{v}}^{ \pm}$ is independent of $\underline{v}$, and, moreover, may be recovered directly from the global portion "广 $\mathcal{D} \odot \pm$ " of a $\mathcal{D}$ - $\Theta^{\text {ell }}$-bridge [cf. [IUTchI], Definition 6.4, (ii); [AbsAnab], Lemma 1.1.4, (i)]. On the other hand, $\Delta^{\odot \pm} \cong \widehat{\Delta}_{\underline{v}}^{ \pm}$also serves as an "ambient container" for the $\widehat{\Delta}_{\underline{v}}^{ \pm}$-conjugates of both $I_{t}$ and $\underline{\Delta}_{\underline{v}}$. That is to say,

$\Delta^{\odot \pm}\left(\cong \widehat{\Delta}_{\underline{v}}^{ \pm}\right)$serves as a sort of "common bridge" between local data [such as $\Delta_{\underline{v}}^{-}$] and global data such as the labels

$$
t \in \operatorname{LabCusp}^{ \pm}\left(\Pi^{\odot \pm}\right) \quad\left(\stackrel{\sim}{\rightarrow} \operatorname{LabCusp}^{ \pm}\left(\Pi_{\underline{v}}^{\gamma}\right) \stackrel{\sim}{\rightarrow} \operatorname{LabCusp}^{ \pm}\left(\Pi_{\underline{v}}\right)\right)
$$

[where we write $\operatorname{LabCusp}^{ \pm}\left(\Pi^{\odot \pm}\right) \stackrel{\text { def }}{=} \operatorname{LabCusp}^{ \pm}\left(\mathcal{B}\left(\Pi^{\odot \pm}\right)^{0}\right)$ - cf. [IUTchI], Definition 6.1, (vi)], in the form of conjugacy classes of $I_{t}$.

(iii) On the other hand, if, in the discussion of (ii), one passes - as in the theory of [IUTchI], $\S 6$ - between distinct $\underline{v}$ via this "global bridge" $\Delta^{\odot \pm}$, then one must take into account the fact that, unlike the labels $t$ [i.e., conjugacy classes of $\left.I_{t}\right]$, the groups $\Pi_{\underline{v}}$ do not admit globalizations or extensions to multiple $\underline{v}$ 's. This is precisely the reason for

the independence of the $\widehat{\Delta}_{\underline{v}}^{ \pm}\left(\cong \Delta^{\odot \pm}\right)$ - [or, equivalently, $\widehat{\Pi}_{\underline{v}}^{ \pm}-$] conjugacy indeterminacies that act on the conjugates of $I_{t}$ and $\Pi_{\underline{v}}$

[cf. the "quotient interpretation" of (i) above; the statement of Corollary 2.5, (iii)]. Here, we observe that since [in the notation of [IUTchI], Definition 3.1] neither of the natural surjections $\widehat{\Pi}_{\underline{v}}^{ \pm} \rightarrow G_{\underline{v}}, \Pi^{\odot \pm} \rightarrow G_{K}$ admits a section that simultaneously normalizes the subgroups $I_{t}$, as $t$ ranges over the elements of $\operatorname{LabCusp}^{ \pm}\left(\Pi^{\odot \pm}\right) \stackrel{\sim}{\rightarrow}$ $\operatorname{LabCusp}^{ \pm}\left(\Pi_{\underline{v}}^{\gamma}\right) \stackrel{\sim}{\rightarrow} \operatorname{LabCusp}^{ \pm}\left(\Pi_{\underline{v}}\right)$ [cf., e.g., [AbsSect], Theorem 1.3, (ii); $p$ GC], Theorem C], it follows that any $G_{\underline{v}^{-}}$(respectively, $G_{K^{-}}$) conjugacy indeterminacy necessarily results in a $\widehat{\Delta}_{\underline{v}}^{ \pm} \cong \Delta^{\odot \pm}$-conjugacy indeterminacy acting on the various $I_{t}$, i.e.,

$$
\begin{aligned}
G_{\underline{v}} \text {-conjugacy indeterminacy } & \Longrightarrow \widehat{\Delta}_{\underline{v}}^{ \pm} \text {-conjugacy indeterminacy, } \\
G_{K} \text {-conjugacy indeterminacy } & \Longrightarrow \Delta^{\odot \pm} \text {-conjugacy indeterminacy. }
\end{aligned}
$$

Since, moreover, the natural surjection $\widehat{\Delta}_{\underline{v}}^{\text {cor }} \rightarrow \widehat{\Delta}_{\underline{v}}^{\text {cor }} / \widehat{\Delta}_{\underline{v}}^{ \pm}$does not admit a splitting, it follows that the $\widehat{\Delta}_{\underline{v}}^{ \pm}$-outer action of $\widehat{\Delta}_{\underline{v}}^{\text {cor }} / \widehat{\Delta}_{\underline{v}}^{ \pm} \stackrel{\widetilde{T}}{\rightarrow} \mathbb{F}_{l}^{\rtimes \pm}$ of Corollary 2.4, (iii), induces

independent $\widehat{\Delta}_{\underline{v}}^{ \pm} \cong \Delta^{\odot \pm}$-conjugacy indeterminacies on the subgroups $I_{t}$, for distinct $t$. 
In a similar vein, since $G_{v}$ does not determine a direct summand of $G_{K}$ - cf. [NSW], Corollary 12.1.3; the phenomenon of the non-splitting of "prime decomposition trees" discussed in [IUTchI], Remark 4.3.1, (ii) — it follows that any $G_{K}$-conjugacy indeterminacy [which, as just discussed, gives rise to $\Delta^{\odot \pm}$-conjugacy indeterminacy] induces independent $G_{v}$-conjugacy indeterminacies on the various $G_{K}$-conjugates of $G_{\underline{v}}$ [hence also, as just discussed, independent $\widehat{\Delta}_{\underline{v}}^{ \pm}$-conjugacy indeterminacies] i.e.,

\section{$G_{K}$-conjugacy indeterminacy $\Longrightarrow$ independent $G_{\underline{\underline{v}}}$-conjugacy indeterminacies}

— cf. the discussion of [IUTchI], Remark 4.5.1, (iii).

(iv) One way to visualize the independent conjugacy indeterminacies of the discussion of (iii) above is via the illustration given in Fig. 2.1 below. That is to say, one thinks of the upper and lower lines of Fig. 2.1 as being equipped with independent actions by groups of horizontal translations [i.e., each of which is isomorphic to $\mathbb{Z}$ ]; one thinks of each of the "o's" in the upper line as representing a $\Delta^{\odot \pm} \cong \widehat{\Delta}_{v}^{ \pm}$-conjugate of $I_{t}$ and of each of the "• $\longrightarrow \bullet$ 's" in the lower line as representing a $\Delta^{\odot \pm} \cong \widehat{\Delta}_{v}^{ \pm}$-conjugate of $\Pi_{v} \ddot{\bullet}$. Thus, since the translation actions on the upper and lower lines are not synchronized with one another [cf. the discussion of (iii)],

there is no way to separate - i.e., in a fashion that is compatible with the indeterminacy arising from both translation actions - the inclusion

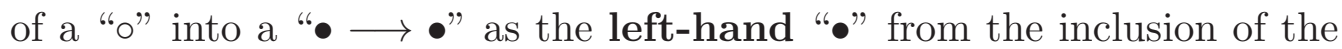

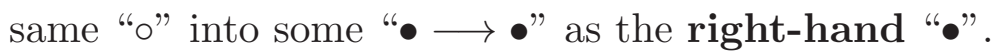

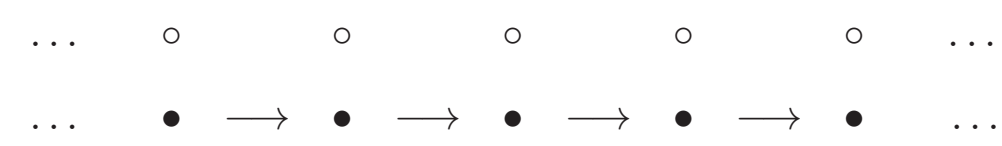

Fig. 2.1: Independent conjugacy indeterminacies

Corollary 2.6. $\quad$ (Splittings Defined on Subgraphs) In the notation of Corollary 2.5, (ii):

(i) (" $M_{\mathbb{T M}}^{\times}$" Defined on Subgraphs) The $\gamma$-conjugate of the quotient $\Pi_{\underline{v}} \ddot{\bullet} \rightarrow$ $G_{\underline{v}}\left(\Pi_{\underline{v}} \ddot{)}\right)$ of Corollary 2.5, (i), determines submodules

$$
\begin{aligned}
& M_{\mathbb{T M}}^{\times}\left(\Pi_{\underline{v} \ddot{\bullet}}^{\gamma}\right) \subseteq \varliminf_{J_{G}} H^{1}\left(J_{G},\left(l \cdot \Delta_{\Theta}\right)\left(\Pi_{\underline{v}}^{\gamma}\right)\right) \subseteq M_{\mathbb{T M}}^{\times} \cdot \underline{\theta}^{\iota}\left(\Pi_{\underline{v}}^{\gamma} \ddot{\sim}\right) \\
& \subseteq \quad M_{\mathbb{T M}}^{\times} \cdot \infty \underline{\underline{\theta}}^{\iota}\left(\Pi_{\underline{v} \ddot{\boldsymbol{\nu}}}^{\gamma}\right) \subseteq \underline{\lim }_{\widehat{J}} H^{1}\left(\Pi_{\underline{v} \ddot{\bullet}}^{\gamma} \mid \widehat{J},\left(l \cdot \Delta_{\Theta}\right)\left(\Pi_{\underline{v} \ddot{\boldsymbol{\nu}}}^{\gamma}\right)\right)
\end{aligned}
$$

- where $J_{G} \subseteq G_{\underline{v}}\left(\Pi_{\underline{v}} \ddot{\sim}\right), \widehat{J} \subseteq \widehat{\Pi}_{\underline{v}}$ range over the open subgroups of $G_{\underline{v}}\left(\Pi_{\underline{v}} \ddot{\sim}\right), \widehat{\Pi}_{\underline{v}}$, respectively; $M_{\mathbb{T M}}^{\times} \cdot \underline{\underline{\theta}}^{\iota}(-) \stackrel{\text { def }}{=} M_{\mathbb{T M}}^{\times}(-) \cdot \underline{\underline{\theta}}^{\iota}(-), M_{\mathbb{T M}}^{\times} \cdot \infty \underline{\underline{\theta}}^{\iota}(-) \stackrel{\text { def }}{=} M_{\mathbb{T M}}^{\times}(-) \cdot \infty \underline{\underline{\theta^{\iota}}}(-)$ [cf. Corollary 1.12, (a), (c), (e); Remark 1.11.5, (i), (ii)]. Also, [cf. Corollary 1.12] let us write

$$
M_{\mathbb{T M}}^{\times \mu}\left(\Pi_{\underline{v} \ddot{\boldsymbol{\Sigma}}}^{\gamma}\right) \stackrel{\text { def }}{=} M_{\mathbb{T M}}^{\times}\left(\Pi_{\underline{v} \ddot{\boldsymbol{\nu}}}^{\gamma}\right) / M_{\mathbb{T M}}^{\boldsymbol{\mu}}\left(\Pi_{\underline{v} \ddot{\boldsymbol{\varpi}}}^{\gamma}\right)
$$


- where $M_{\mathbb{T M}}^{\mu}\left(\Pi_{\underline{v} \ddot{\sim}}^{\gamma}\right) \subseteq M_{\mathbb{T M}}^{\times}\left(\Pi_{\underline{v}}^{\gamma} \ddot{\sim}\right)$ denotes the submodule of torsion elements.

(ii) (Splittings at Zero-labeled Evaluation Points) In the situation of Corollary 2.5, (ii), suppose that $t$ is taken to be the zero element. Then the set $\underline{\underline{\theta}}^{t}\left(\Pi_{v \ddot{\boldsymbol{\Sigma}}}^{\gamma}\right)$ (respectively, $\left.\infty \underline{\underline{\theta}}^{t}\left(\Pi_{v \ddot{\boldsymbol{*}}}^{\gamma}\right)\right)$ is equal to the $\boldsymbol{\mu}_{2 l}$ - (respectively, $\left.\boldsymbol{\mu}^{-}\right)$orbit of the identity element [i.e., the zero element of cohomology module in question, if one denotes the module structure additively]. In particular, if one considers the quotient of the diagram of the first display of (i) by $M_{\mathbb{T M}}^{\boldsymbol{\mu}}\left(\Pi_{v}^{\gamma}\right.$ : $)$, then restriction to the decomposition groups $D_{t, \mu_{-}}^{\delta}$ of Corollary 2.4, (ii), (c), determines splittings

$$
M_{\mathbb{T M}}^{\times \boldsymbol{\mu}}\left(\Pi_{\underline{v} \ddot{\boldsymbol{\nu}}}^{\gamma}\right) \times\left\{\infty \underline{\underline{\theta}}^{\iota}\left(\Pi_{\underline{v} \ddot{\bullet}}^{\gamma}\right) / M_{\mathbb{T M}}^{\boldsymbol{\mu}}\left(\Pi_{\underline{v} \ddot{\bullet}}^{\gamma}\right)\right\}
$$

of $M_{\mathbb{T M}}^{\times} \cdot \infty \underline{\underline{\theta}}^{\iota}\left(\Pi_{\underline{v} \ddot{\nu}}^{\gamma}\right) / M_{\mathbb{T M}}^{\mu}\left(\Pi_{\underline{v} \ddot{\sim}}^{\gamma}\right)$ which are compatible - relative to the first restriction operation discussed in Corollary 2.5, (ii) - with the splittings of Corollary 1.12, (ii).

Proof. Assertions (i) and (ii) follow immediately from the definitions and the references quoted in the statements of these assertions.

\section{Remark 2.6.1.}

(i) One of the most central properties, from the point of view of the theory of the present series of papers, of the evaluation algorithm of Corollary 2.5, (iii), consists of the observation that this algorithm is performed

relative to a single basepoint - i.e., from a more geometric point of view, relative to the "fundamental group" $\Pi_{v: \text { corresponding to the connected }}^{\gamma}$ subgraph $\Gamma_{\underline{\underline{X}}}^{\nabla} \subseteq \Gamma_{\underline{\underline{X}}}[$ cf. Remark 2.1.1, (ii)].

In particular, despite the fact that we are ultimately interested in [not a single, but rather] a plurality of theta values, associated to the various $|t| \in\left|\mathbb{F}_{l}\right|$, these theta values

$$
\underline{\theta}^{|t|}\left(\Pi_{\underline{v} \ddot{\sim}}^{\gamma}\right) \subseteq H^{1}\left(G_{\underline{v}}\left(\Pi_{\underline{v} \ddot{\sim}}^{\gamma}\right),\left(l \cdot \Delta_{\Theta}\right)\left(\Pi_{\underline{v} \ddot{\sim}}^{\gamma}\right)\right)
$$

for various $|t| \in\left|\mathbb{F}_{l}\right|$ are all computed relative to the single copy [i.e., which is independent of $|t| !]$ of the Galois group $G_{\underline{v}}\left(\Pi_{v \ddot{v}}^{\gamma}\right)$ and the single cyclotome $\left(l \cdot \Delta_{\Theta}\right)\left(\Pi_{v}^{\gamma}\right)$ [i.e., which is independent of $|t| !$ ] arising from $\Pi_{v}^{\gamma}$ - - i.e., arising from the "single basepoint" under consideration. We shall refer to this phenomenon by the term conjugate synchronization. This conjugate synchronization is necessary in order to perform Kummer theory [cf. the discussion of Galois evaluation in Remark 1.12.4], as we shall do in $§ 3$.

(ii) Put another way, the significance of conjugate synchronization in the context of Kummer theory - especially, in the context of the theory of Gaussian Frobenioids, to be developed in $\S 3$ below - may be understood as arising from the requirement that the collection of theta values, for $|t| \in \mathbb{F}_{l}^{*}$, be treated as 
a single unified entity, whose Kummer theory may be described by considering the action of a single Galois group in the context of the simultaneous extraction of $N$-th roots of all theta values, relative to a single cyclotome [i.e., copy of the module of $N$-th roots of unity] that acts simultaneously on the $N$-th roots of all of the theta values, and in a fashion that is compatible with the Kummer theory of the "base field" [i.e., arising from the quotient $\left.\Pi_{\underline{v} \ddot{\bullet}}^{\gamma} \rightarrow G_{\underline{v}}\left(\Pi_{\underline{v}}^{\gamma}\right)\right]$.

This point of view may only be realized by means of a "single basepoint" of a suitable category of coverings of a geometric object that consists of a single connected component [cf. the discussion of Galois evaluation in Remark 1.12.4; the discussion of [EtTh], Remark 1.10.4]. Also, we recall [cf. the discussion of Galois evaluation in Remark 1.12.4] that this "Kummer-theoretic representation" of the ["Frobenioid-theoretic"] monoid generated by the ["Frobenioid-theoretic"] theta function satisfies the crucial property of being compatible [unlike the various ring structures involved!] with the "log-wall" [cf. the theory of [AbsTopIII]]. This crucial property will play a fundamental role in the theory to be developed in [IUTchIII].

\section{Remark 2.6.2.}

(i) In the context of the discussion of conjugate synchronization in Remark 2.6.1, it is useful to recall the theory of $\mathcal{D}-\Theta^{ \pm \text {ell }}$-Hodge theaters

$$
{ }^{\dagger} \mathcal{H} \mathcal{T}^{\mathcal{D}-\Theta^{ \pm \mathrm{ell}}}=\left({ }^{\dagger} \mathfrak{D}_{\succ} \stackrel{{ }^{\dagger} \phi_{ \pm}^{\Theta^{ \pm}}}{\longleftarrow}{ }^{\dagger} \mathfrak{D}_{T} \stackrel{{ }^{\dagger} \dot{\phi}_{ \pm}^{\Theta^{\mathrm{ell}}}}{\longrightarrow}{ }^{\dagger} \mathcal{D}^{\odot \pm}\right)
$$

[cf. [IUTchI], Definition 6.4, (iii)] developed in [IUTchI], §6. That is to say, from the point of view of the theory of $\mathcal{D}-\Theta^{ \pm \text {ell }}$-Hodge theaters, it is natural to think

(a) of the topological group $\Pi_{\underline{v}}$ that appears in Corollaries 2.4, 2.5, and 2.6 as the tempered fundamental group of $\dagger^{\dagger} \mathfrak{D}_{\succ, \underline{v}}$,

(b) of the topological group $\widehat{\Pi}_{v}^{ \pm}$that appears in Corollaries 2.4, 2.5, and 2.6 as the commensurator of the closure of $\Pi_{\underline{v}}$ [i.e., relative to the interpretation of (a)] inside the profinite fundamental group of ${ }^{\dagger} \mathcal{D}^{\odot \pm}$ relative to the composite poly-morphism

$$
\dagger \mathfrak{D}_{\succ, \underline{v}} \stackrel{{ }^{\dagger}{\left.\stackrel{\phi_{\underline{v}}}{\Theta^{ \pm}}\right)^{-1}}^{\longrightarrow}}{\dagger} \mathfrak{D}_{\underline{v}_{0}} \stackrel{{ }^{\dagger} \underline{\phi}_{\underline{v}_{0}}^{\text {ell }}}{\longrightarrow} \dagger \mathcal{D}^{\odot \pm}
$$

determined by the portions of ${ }^{\dagger} \phi_{ \pm}^{\Theta^{ \pm}},{ }^{\dagger} \phi_{ \pm}^{\Theta^{\text {ell }}}$ labeled by $0 \in T, \underline{v} \in \underline{\mathbb{V}}[\mathrm{cf}$. the discussions of [IUTchI], Examples 6.2, (i); 6.3, (i)], and

(c) of the $\widehat{\Delta}_{\underline{v}}^{ \pm}$-outer action of $\widehat{\Delta}_{\underline{v}}^{\text {cor }} / \widehat{\Delta}_{\underline{v}}^{ \pm} \stackrel{\sim}{\rightarrow} \mathbb{F}_{l}^{\rtimes \pm}$ that appears in Corollary 2.4, (iii), as corresponding to the $\mathbb{F}_{l}^{\rtimes \overline{ \pm}}$-symmetry of [IUTchI], Proposition 6.8 , (i).

Relative to the interpretation of (a), (b), and (c), one has the following fundamental observation concerning the discussion of Remark 2.6.1: 
the single basepoint that underlies the conjugate synchronization discussed in Remark 2.6.1 is compatible with the single basepoint that underlies the label synchronization discussed in [IUTchI], Remark 6.12.4.

That is to say, both of these basepoints may be thought of as arising from a single basepoint that gives rise to the various topological groups $\Pi_{\underline{v}}, \widehat{\Pi}_{\underline{v}}^{ \pm}$, etc. that appear in Corollaries 2.4, 2.5, and 2.6. In particular,

the conjugate synchronization discussed in Remark 2.6.1 is compatible with the $\mathbb{F}_{l}^{\rtimes \pm}$-symmetry of [IUTchI], Proposition 6.8, (i) [cf. also Remark 3.8.3 below].

Indeed, this compatibility is essentially the content of Corollary 2.4, (iii) [cf. (c) above].

(ii) Note that the compatibility of basepoints discussed in (i) contrasts sharply with the incompatibility of the conjugate synchronization basepoint of Remark 2.6.1 with the $\mathbb{F}_{l}^{*}$-symmetry of [IUTchI], Proposition 4.9, (i), in the case of $\mathcal{D}$ - $\Theta N F$-Hodge theaters. At a more concrete level, this difference between $\mathbb{F}_{l}^{\rtimes \pm}$ - and $\mathbb{F}_{l}^{*}$-symmetries may be understood as a consequence of the fact that whereas the $\mathbb{F}_{l}^{\rtimes \pm}$-symmetry is defined relative to a single copy of a local geometric object at $\underline{v}$ - i.e., " $\widehat{\Pi}_{\underline{v}}^{ \pm}$" [cf. (a), (b), (c) above] - the $\mathbb{F}_{l}^{*}$-symmetry involves permuting multiple copies of local geometric objects in such a way that one may only identify these multiple copies with one another at the expense of allowing the phenomenon of "label crushing" [cf. the discussions of [IUTchI], Remark 4.9.2, (i), (ii); 6.12.6, (i), (ii), (iii)].

(iii) Another important property of the $\mathbb{F}_{l}^{\rtimes \pm}$-symmetry — which is not satisfied by the $\mathbb{F}_{l}^{*}$-symmetry! — is that the $\mathbb{F}_{l}^{\rtimes \pm}$-symmetry allows comparison with the label zero [cf. the discussion of [IUTchI], Remark 6.12.5], hence, in particular, comparison with the copies of " $\mathcal{O}_{\bar{k}}^{\times}$" [cf. the discussion of Remark 1.12.2] that occur in the splittings of Corollary 1.12, (ii), that give rise to the crucial constant multiple rigidity of the étale theta function. This important property is precisely the content of Corollary 2.6.

\section{Remark 2.6.3.}

(i) The discussion of independent conjugacy indeterminacies in Remark 2.5.2 and of "single basepoints" that are compatible with the $\mathbb{F}_{l}^{\rtimes \pm}$-symmetry of [IUTchI], $\S 6$, in Remarks 2.6.1, 2.6.2 imply rather severe restrictions concerning the subgraph " $\Gamma_{\ddot{Y}} \subseteq \Gamma_{\ddot{Y}}$ " of Remark 2.1.1, (ii). That is to say, suppose that one attempts to develop the theory of the present $\S 2$ for another subgraph $\Gamma^{\prime}$ of the graph $\Gamma_{\ddot{Y}}$. Recall from the discussion of Remark 2.1.1, (i), that the graph $\Gamma_{\ddot{Y}}$ may be thought of as a "copy of the real line $\mathbb{R}$ ", in which the integers $\mathbb{Z} \subseteq \mathbb{R}$ are taken to be the vertices, and the line segments joining the integers are taken to be the edges. Then the discussion of "single basepoints" [cf. Remark 2.6.1] implies, first of all, that

(a) this subgraph $\Gamma^{\prime}$ must be connected. 
Since, moreover, one wishes to consider the crucial splittings of Corollary 2.6, (ii) [cf. Remark 2.6.2, (iii)], it follows that

(b) this subgraph $\Gamma^{\prime}$ must contain the vertex of $\Gamma_{\ddot{Y}}$ labeled " 0 ".

The conditions (a) and (b) already impose substantial restrictions on $\Gamma^{\prime}$ and hence on the collection of values of the étale theta function that may arise by restricting to the $\mu_{-}$-translates of the cusps that lie in $\Gamma^{\prime}$ [cf. Remark 2.5.1, (ii)] - i.e., on the collection of

$$
\underline{\underline{q}} \underline{\underline{\underline{j}}} \underline{\underline{\underline{v}}}
$$

obtained by allowing $\underline{\underline{j}} \in \mathbb{Z}$ to range [relative to the identification of the vertices of $\Gamma_{\ddot{Y}}$ with the integral points of the real line] over the "vertices" of $\Gamma^{\prime}$ [cf. Remark 2.5.1, (i)].

(ii) By abuse of notation, let us write " $\underline{\underline{j}} \in \Gamma^{\prime \prime}$ for "vertices" $\underline{\underline{j}} \in \mathbb{Z}$ that lie in $\Gamma^{\prime}$. Also, for simplicity, let us assume that the subgraph $\Gamma^{\prime}$ is $\overline{\bar{f}}$ inite [cf. (iii) below]. Then ultimately, in the theory of [IUTchIV], when we consider various height inequalities, we shall be concerned with the issue of maximizing the quantity

$$
\left\|\Gamma^{\prime}\right\| \stackrel{\text { def }}{=}\left|\Gamma^{\prime}\right|^{-1} \cdot \sum_{j \in \mathbb{F}_{l}^{*}} \min _{\underline{j} \in j \bigcap \Gamma^{\prime}}\left\{\underline{j}^{2}\right\}
$$

— where we write $\left|\Gamma^{\prime}\right|$ for the cardinality of the image in $\mathbb{F}_{l}^{*}$ of the nonzero elements of $\Gamma^{\prime}$; we regard the "min" over an empty set as being equal to zero; we think of the various $j \in \mathbb{F}_{l}^{*}$ as corresponding to the subsets of $\mathbb{Z}$ determined by the fibers of the natural projection $\mathbb{Z} \rightarrow\left|\mathbb{F}_{l}\right|\left(\supseteq \mathbb{F}_{l}^{*}\right)$. Here, we observe that

(c) the set of " $\underline{\underline{j}}$ 's" that occur in the "min" ranging over " $\underline{\underline{j}}$ " [i.e., not over " $j " !]$ that appears in the definition of $\left\|\Gamma^{\prime}\right\|$ is always equal to a fiber of the restriction to the set of vertices of $\Gamma^{\prime}$ of the natural projection $\mathbb{Z} \rightarrow\left|\mathbb{F}_{l}\right|$.

In fact, this observation (c) is, in essence, a consequence of the phenomenon discussed in Remark 2.5.2 of independent conjugacy indeterminacies [cf., especially, Remark 2.5.2, (iv)] — i.e., roughly speaking, that

one cannot restrict the étale theta function to "one $\underset{\underline{j}}{\underline{\underline{y}}} \in \Gamma^{\prime}$ " without also restricting the étale theta function to the various "other $\underline{\underline{j}} \in \Gamma^{\prime}$ " that lie in the same fiber over $\left|\mathbb{F}_{l}\right|$.

Next, let us make the [easily verified - cf. (a), (b)!] observation that if one thinks of $\left\|\Gamma^{\prime}\right\|$ as a function of $\left|\Gamma^{\prime}\right|$, then as $\left|\Gamma^{\prime}\right|$ ranges over the positive integers, it holds that

(d) the function of $\left|\Gamma^{\prime}\right|$ constituted by $\left\|\Gamma^{\prime}\right\|$ - which may be thought of as a sort of average - is a monotone increasing [but not strictly increasing!] 
function of $\left|\Gamma^{\prime}\right|$ valued in the positive rational numbers which attains its maximum when $\left|\Gamma^{\prime}\right|=l^{*}$ and is constant for $\left|\Gamma^{\prime}\right| \geq l^{*}$.

Now it follows formally from (d) that, as $\left|\Gamma^{\prime}\right|$ ranges over the positive integers, the quantity ||$\Gamma^{\prime}||$ attains its maximum when $\left|\Gamma^{\prime}\right|=l^{*}$ — hence, in particular, when $\Gamma^{\prime}$ is taken to be $\Gamma_{\ddot{Y}}$. Thus, from the point of view of the issue of maximizing this quantity $\left\|\Gamma^{\prime}\right\|$, there is "no loss of generality" in assuming that $\Gamma^{\prime}=\Gamma_{\ddot{Y}}[$ cf. also the discussion of (iv) below].

(iii) Although in the discussion of (ii) above we assumed that $\Gamma^{\prime}$ is finite, this assumption does not in fact result in any loss of generality. Indeed, one verifies immediately that $\left\|\Gamma^{\prime}\right\|$ is defined, finite, and satisfies the evident analogue of (d) even for infinite $\Gamma^{\prime}$. Thus, the case of inifnite $\Gamma^{\prime}$ may be excluded without loss of generality.

(iv) Ultimately, in $\S 4$ of the present paper, we shall be concerned with the issue of globalizing, via the construction of various global realified Frobenioids, the monoids determined by the theta values at $\underline{v} \in \underline{\mathbb{V}}^{\text {bad }}$ that appear in the present $\S 2$. This globalization will be achieved, in effect, by imposing the condition that the product formula be satisfied. On the other hand, the indeterminacies discussed in (ii) above [cf., especially, (ii), (c)] that arise when a fiber of $\Gamma^{\prime}$ over $\left|\mathbb{F}_{l}\right|$ contains more than one element are easily seen to be fundamentally incompatible with the product formula. In particular, from the point of view of the issue of maximizing the quantity $\left\|\Gamma^{\prime}\right\|$, in fact the only choice for $\Gamma^{\prime}$ that is compatible with the "globalization via the product formula" to be performed in $\S 4$ is $\Gamma_{\ddot{Y}}$.

(v) One may summarize the discussion of (i), (ii), (iii), and (iv) as follows:

the collection of values " $\underline{\underline{q}} \underline{\underline{\underline{v}}} \stackrel{\underline{\underline{v}}}{\text { " }}$ of the étale theta function determined by the subgraph $\Gamma_{\ddot{Y}}^{\longrightarrow}$ is of a highly distinguished nature

- and, indeed, is essentially determined [cf. the discussion at the end of (ii); the discussion of (iv)] by the requirement of maximizing the quantity " ||$\Gamma^{\prime}||$ " in a fashion compatible with the global product formula, together with various qualitative considerations that arise from Corollaries 2.4, 2.5, 2.6; the discussion of Remarks 2.5.1, 2.5.2, 2.6.1, 2.6.2.

Definition 2.7. In the notation of Definition 2.3: Let

$$
\mathbb{M}_{*}^{\Theta}=\left\{\ldots \rightarrow \mathbb{M}_{M^{\prime}}^{\Theta} \rightarrow \mathbb{M}_{M}^{\Theta} \rightarrow \ldots\right\}
$$

be a projective system of mono-theta environments as in Proposition 1.5, such that $\Pi_{\underline{\underline{X}}}\left(\mathbb{M}_{*}^{\Theta}\right) \cong \Pi_{\underline{v}}$.

(i) Write 
for the inverse limit of the induced projective system of topological groups $\{\ldots \rightarrow$ $\left.\Pi_{\mathbb{M}_{M^{\prime}}^{\Theta}} \rightarrow \Pi_{\mathbb{M}_{M}^{\Theta}}^{\Theta} \rightarrow \ldots\right\}$. Thus, [in the notation of Proposition 1.5] we have a natural homomorphism of topological groups

$$
\Pi_{\mathbb{M}_{*}^{\Theta}} \rightarrow \Pi_{\underline{\underline{X}}}\left(\mathbb{M}_{*}^{\Theta}\right)
$$

whose kernel may be identified with the exterior cyclotome $\Pi_{\boldsymbol{\mu}}\left(\mathbb{M}_{*}^{\Theta}\right)$, and whose image is the subgroup of $\Pi_{\underline{\underline{X}}}\left(\mathbb{M}_{*}^{\Theta}\right) \cong \Pi_{\underline{v}}$ determined by $\Pi_{\underline{\underline{Y}}}^{\text {tp }}$.

(ii) Write

$$
\Pi_{\mathbb{M}_{* \bullet}^{\Theta}} \subseteq \Pi_{\mathbb{M}_{* \triangleright}^{\Theta}} \subseteq \Pi_{\mathbb{M}_{*}^{\Theta}}
$$

for the respective inverse images of $\Pi_{\underline{v}} \ddot{\sim} \subseteq \Pi_{\underline{v}} \subseteq \Pi_{\underline{v}} \cong \Pi_{\underline{\underline{X}}}\left(\mathbb{M}_{*}^{\Theta}\right)$ in $\Pi_{\mathbb{M}_{*}^{\Theta}}$;

$$
\Pi_{\boldsymbol{\mu}}\left(\mathbb{M}_{* \ddot{\bullet}}^{\Theta}\right), \quad\left(l \cdot \Delta_{\Theta}\right)\left(\mathbb{M}_{* \ddot{\nabla}}^{\Theta}\right), \quad \Pi_{\underline{v}} \ddot{\bullet}\left(\mathbb{M}_{* \ddot{\bullet}}^{\Theta}\right), \quad G_{\underline{v}}\left(\mathbb{M}_{* \ddot{\bullet}}^{\Theta}\right)
$$

for the subquotients of $\Pi_{\mathbb{M}_{* \bullet}^{\Theta}}$ determined by the subquotient $\Pi_{\boldsymbol{\mu}}\left(\mathbb{M}_{*}^{\Theta}\right)$ of $\Pi_{\underline{\underline{X}}}\left(\mathbb{M}_{*}^{\Theta}\right)$ and the subquotients $\left(l \cdot \Delta_{\Theta}^{*}\right)\left(\Pi_{\underline{\underline{X}}}\left(\mathbb{M}_{*}^{\Theta}\right)\right), \Pi_{\underline{v}}$, and $G_{\underline{v}}\left(\Pi_{\underline{\underline{X}}}\left(\mathbb{M}_{*}^{\Theta}\right)\right)$ of $\Pi_{\underline{v}} \cong \Pi_{\underline{\underline{X}}}\left(\mathbb{M}_{*}^{\Theta}\right)$. Thus, we obtain a cyclotomic rigidity isomorphism

$$
\left(l \cdot \Delta_{\Theta}\right)\left(\mathbb{M}_{* \ddot{\sim}}^{\Theta}\right) \stackrel{\sim}{\rightarrow} \Pi_{\boldsymbol{\mu}}\left(\mathbb{M}_{* \ddot{\bullet}}^{\Theta}\right)
$$

- i.e., by restricting the cyclotomic rigidity isomorphism $\left(l \cdot \Delta_{\Theta}\right)\left(\mathbb{M}_{*}^{\Theta}\right) \stackrel{\sim}{\rightarrow} \Pi_{\boldsymbol{\mu}}\left(\mathbb{M}_{*}^{\Theta}\right)$ of Proposition 1.5, (iii), to $\Pi_{\mathbb{M}_{* \bullet}^{\Theta}}$.

Corollary 2.8. (Mono-theta-theoretic Theta Evaluation) In the notation of Definition 2.7: Suppose that we are in the situation of Proposition 2.2, (ii); Corollary 2.5, (ii); to simplify notation, we assume that $\Pi_{\underline{\underline{X}}}\left(\mathbb{M}_{*}^{\Theta}\right)=\Pi_{\underline{v}}$, and we use the notation for objects constructed from " $\Pi_{\underline{v}}$ " to denote the corresponding objects constructed from $\Pi_{\underline{\underline{X}}}\left(\mathbb{M}_{*}^{\Theta}\right)$. Also, let us write

$$
\left(\mathbb{M}_{*}^{\Theta}\right)^{\gamma}
$$

for the projective system of mono-theta environments obtained via transport of structure from the isomorphism $\Pi_{\underline{v}} \stackrel{\sim}{\rightarrow} \Pi_{\underline{v}}^{\gamma}$ determined by conjugation by $\gamma$.

(i) (Restriction of Étale Theta Functions to Subgraphs and Evaluation Points) In the situation of Proposition 2.2, (ii); Corollary 2.5, (ii), let us apply the cyclotomic rigidity isomorphisms

$$
\left(l \cdot \Delta_{\Theta}\right)\left(\left(\mathbb{M}_{* \ddot{\boldsymbol{\nu}}}^{\Theta}\right)^{\gamma}\right) \stackrel{\sim}{\rightarrow} \Pi_{\boldsymbol{\mu}}\left(\left(\mathbb{M}_{* \ddot{\boldsymbol{\sim}}}^{\Theta}\right)^{\gamma}\right) ; \quad\left(l \cdot \Delta_{\Theta}\right)\left(\left(\mathbb{M}_{*}^{\Theta}\right)^{\gamma}\right) \stackrel{\sim}{\rightarrow} \Pi_{\boldsymbol{\mu}}\left(\left(\mathbb{M}_{*}^{\Theta}\right)^{\gamma}\right)
$$

[cf. Definition 2.7, (ii), applied to $\left(\mathbb{M}_{*}^{\Theta}\right)^{\gamma}$ ] to replace " $\left(l \cdot \Delta_{\Theta}\right)(-)$ " by " $\Pi_{\boldsymbol{\mu}}(-)$ ". Then the $\iota^{\gamma}$-invariant subsets $\underline{\underline{\theta}}^{\iota}\left(\Pi_{\underline{v}}^{\gamma}\right) \subseteq \underline{\underline{\theta}}\left(\Pi_{\underline{v}}^{\gamma}\right), \infty \underline{\underline{\theta}}^{\iota}\left(\Pi_{\underline{v}}^{\gamma}\right) \subseteq \infty \underline{\underline{\theta}}\left(\Pi_{\underline{v}}^{\gamma}\right)$ [cf. Proposition 2.2, (ii); Corollary 2.5, (ii)] determine $\iota^{\gamma}$-invariant subsets

$$
\underline{\underline{\theta}}_{=}^{\iota}\left(\left(\mathbb{M}_{*}^{\Theta}\right)^{\gamma}\right) \subseteq \quad \underline{\theta}_{\mathrm{env}}\left(\left(\mathbb{M}_{*}^{\Theta}\right)^{\gamma}\right) ; \quad \infty \underline{\underline{\theta}}_{\mathrm{env}}\left(\left(\mathbb{M}_{*}^{\Theta}\right)^{\gamma}\right) \subseteq \infty \underline{\underline{e}}_{\mathrm{env}}\left(\left(\mathbb{M}_{*}^{\Theta}\right)^{\gamma}\right)
$$


[cf. Proposition 1.5, (iii), applied to $\left.\left(\mathbb{M}_{*}^{\Theta}\right)^{\gamma}\right]$; restriction of these subsets $\underline{\underline{\theta}}_{\mathrm{env}}\left(\left(\mathbb{M}_{*}^{\Theta}\right)^{\gamma}\right)$, $\infty \underline{\underline{\theta}}_{\text {env }}^{\iota}\left(\left(\mathbb{M}_{*}^{\Theta}\right)^{\gamma}\right)$ to $\Pi_{\underline{v}} \ddot{\sim}\left(\left(\mathbb{M}_{* \ddot{\bullet}}^{\Theta}\right)^{\gamma}\right)$ yields $\boldsymbol{\mu}_{2 l^{-}}, \boldsymbol{\mu}$-orbits of elements

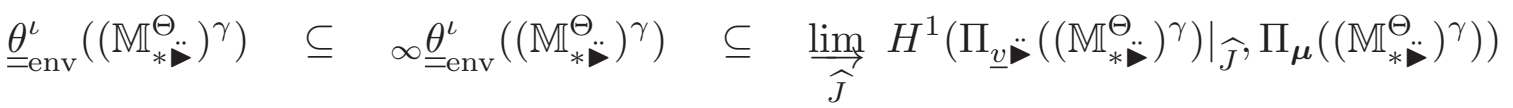

- where $\widehat{J} \subseteq \widehat{\Pi}_{\underline{v}}$ ranges over the open subgroups of $\widehat{\Pi}_{\underline{v}}$ - which, upon further restriction to the decomposition groups $D_{t, \mu_{-}}^{\delta}$ of Corollary 2.4, (ii), (c), yield $\boldsymbol{\mu}_{2 l^{-}}, \boldsymbol{\mu}$-orbits of elements

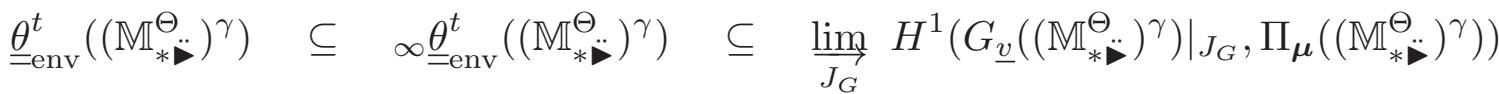

for each $t \in \operatorname{LabCusp}^{ \pm}\left(\Pi_{\underline{v}}^{\gamma}\right) \stackrel{\sim}{\rightarrow} \operatorname{LabCusp}^{ \pm}\left(\Pi_{\underline{v}}\right)$ - where $J_{G} \subseteq G_{\underline{v}}\left(\left(\mathbb{M}_{* \ddot{*}}^{\Theta}\right)^{\gamma}\right)$ ranges over the open subgroups of $G_{\underline{v}}\left(\left(\mathbb{M}_{* \ddot{*}}^{\Theta}\right)^{\gamma}\right)$; the " $\stackrel{\sim}{\rightarrow}$ " is induced by conjugation by $\gamma$. Moreover, the sets $\underline{\theta}_{\text {env }}^{t}\left(\left(\mathbb{M}_{* \ddot{*}}^{\Theta}\right)^{\gamma}\right), \infty \underline{\underline{\theta}}_{\text {env }}^{t}\left(\left(\mathbb{M}_{* \ddot{*}}^{\Theta}\right)^{\gamma}\right)$ depend only on the label $|t| \in\left|\mathbb{F}_{l}\right|$ determined by $t$ [cf. Corollary 2.5, (ii)]. Thus, we shall write $\underline{\theta}_{=\mathrm{env}}^{|t|}\left(\left(\mathbb{M}_{* \ddot{\boldsymbol{\nabla}}}^{\Theta}\right)^{\gamma}\right) \stackrel{\text { def }}{=}$ $\underline{\theta}_{=}^{t}\left(\left(\mathbb{M}_{* \ddot{\bullet}}^{\Theta}\right)^{\gamma}\right), \infty \underline{\theta}_{\text {env }}^{|t|}\left(\left(\mathbb{M}_{* \ddot{\bullet}}^{\Theta}\right)^{\gamma}\right) \stackrel{\text { def }}{=} \infty \underline{\theta}_{\text {env }}^{t}\left(\left(\mathbb{M}_{* \ddot{\bullet}}^{\Theta}\right)^{\gamma}\right)$.

(ii) (Functorial Group-theoretic Evaluation Algorithm) If one starts with an arbitrary $\widehat{\Delta}_{\underline{v}}^{ \pm}$-conjugate $\Pi_{\underline{v}} \ddot{\boldsymbol{\sim}}\left(\left(\mathbb{M}_{* \ddot{*}}^{\Theta}\right)^{\gamma}\right)$ of $\Pi_{\underline{v}} \ddot{\sim}\left(\mathbb{M}_{* \ddot{*}}^{\Theta}\right)$, and one considers, as $t$ ranges over the elements of $\operatorname{LabCusp}^{ \pm}\left(\Pi_{\underline{v}}^{\gamma}\right) \stackrel{\sim}{\rightarrow} \operatorname{LabCusp}^{ \pm}\left(\Pi_{\underline{v}}\right)$ [where the " $\stackrel{\sim}{\rightarrow}$ " is induced by conjugation by $\gamma]$, the resulting $\boldsymbol{\mu}_{2 l^{-}}, \boldsymbol{\mu}$-orbits $\underline{\theta}_{=}^{|t|}\left(\left(\mathbb{M}_{*}^{\Theta}\right)^{\gamma}\right)$, $\infty \underline{\theta}_{\text {env }}^{|t|}\left(\left(\mathbb{M}_{* \ddot{*}}^{\Theta}\right)^{\gamma}\right)$ arising from an arbitrary $\widehat{\Delta}_{\underline{v}}^{ \pm}$-conjugate $I_{t}^{\delta}$ of $I_{t}$ that is contained in $\Pi_{\underline{v}} \ddot{(}\left(\left(\mathbb{M}_{* \ddot{*}}^{\Theta}\right)^{\gamma}\right)$ [cf. (i)], then one obtains an algorithm for constructing the collections of $\boldsymbol{\mu}_{2 l^{-}}, \boldsymbol{\mu}$-orbits

$$
\left\{\underline{\underline{\theta}}_{\mathrm{env}}^{|t|}\left(\left(\mathbb{M}_{* \ddot{\bullet}}^{\Theta}\right)^{\gamma}\right)\right\}|t| \in\left|\mathbb{F}_{l}\right| ; \quad\left\{\infty \underline{\underline{\theta}}_{\mathrm{env}}^{|t|}\left(\left(\mathbb{M}_{* \ddot{\bullet}}^{\Theta}\right)^{\gamma}\right)\right\}|t| \in\left|\mathbb{F}_{l}\right|
$$

which is functorial in the projective system of mono-theta environments $\mathbb{M}_{*}^{\Theta}$, and, moreover, compatible with the independent conjugacy actions of $\widehat{\Delta}_{\underline{v}}^{ \pm}$on the $\operatorname{sets}\left\{I_{t}^{\gamma_{1}}\right\}_{\gamma_{1} \in \widehat{\Pi}_{\underline{v}}^{ \pm}}=\left\{I_{t}^{\gamma_{1}}\right\}_{\gamma_{1} \in \widehat{\Delta}_{\underline{v}}^{ \pm}}$and $\left\{\Pi_{\underline{v} \ddot{\bullet}}\left(\left(\mathbb{M}_{* \ddot{\bullet}}^{\Theta}\right)^{\gamma_{2}}\right)\right\}_{\gamma_{2} \in \widehat{\Pi}_{\underline{v}}^{ \pm}}=\left\{\Pi_{\underline{v}} \ddot{\boldsymbol{\Sigma}}\left(\left(\mathbb{M}_{* \ddot{*}}^{\Theta}\right)^{\bar{\gamma}_{2}}\right)\right\}_{\gamma_{2} \in \widehat{\Delta}_{\underline{v}}^{ \pm}}$ [cf. the sets of Corollary 2.4, (i); Remark 2.2.1].

(iii) (Splittings at Zero-labeled Evaluation Points) In the situation of (i), suppose that $t$ is taken to be the zero element. Then, by applying the cyclotomic rigidity isomorphisms of (i) to replace " $\left(l \cdot \Delta_{\Theta}\right)(-)$ " by " $\Pi_{\boldsymbol{\mu}}(-)$ " — an operation that, when applied to " $M_{\mathbb{T} M}^{? ?}(-)$ ", we shall denote by replacing the notation " $\Pi_{\underline{v} \boldsymbol{\bullet}}^{\gamma}$ " by “ $\left(\mathbb{M}_{*}^{\Theta}{ }_{*}\right)^{\gamma}$ " - in Corollary 2.6, (ii), the second restriction operation discussed in (i) determines splittings [cf. Corollary 2.6, (ii)]

$$
M_{\mathbb{T M}}^{\times \mu}\left(\left(\mathbb{M}_{* \ddot{\bullet}}^{\Theta}\right)^{\gamma}\right) \times\left\{\infty \underline{\underline{\theta}}_{\mathrm{env}}^{\iota}\left(\left(\mathbb{M}_{* \ddot{\bullet}}^{\Theta}\right)^{\gamma}\right) / M_{\mathbb{T} \mathbb{M}}^{\mu}\left(\left(\mathbb{M}_{* \ddot{\bullet}}^{\Theta}\right)^{\gamma}\right)\right\}
$$

of $M_{\mathbb{T M}}^{\times} \cdot \infty \underline{\theta}_{\text {env }}^{\iota}\left(\left(\mathbb{M}_{* \ddot{*}}^{\Theta}\right)^{\gamma}\right) / M_{\mathbb{T M}}^{\mu}\left(\left(\mathbb{M}_{* \ddot{*}}^{\Theta}\right)^{\gamma}\right)$ which are compatible - relative to the first restriction operation discussed in (i) - with the splittings of Corollary 1.12, (ii) [i.e., relative to any isomorphism $\mathbb{M}_{*}^{\Theta} \stackrel{\sim}{\rightarrow} \mathbb{M}_{*}^{\Theta}\left(\Pi_{\underline{v}}\right)-c f$. Proposition 1.2, (i); Proposition 1.5, (i); Remarks 2.8.1, 2.8.2 below]. 
Proof. Assertions (i), (ii), and (iii) follow immediately from the definitions and the references quoted in the statements of these assertions.

Remark 2.8.1. One may regard Corollaries 2.5, 2.6 as a special case of Corollary 2.8 , i.e., the case where the projective system of mono-theta environments $\mathbb{M}_{*}^{\Theta}$ arises from the topological group $\Pi_{v}$ by applying the functorial group-theoretic algorithm of Proposition 1.2, (i) [cf. also Proposition 1.5, (i)].

Remark 2.8.2. The significance of the mono-theta-theoretic version of Corollaries 2.5, 2.6 constituted by Corollary 2.8 lies in the fact that this mono-thetatheoretic version allows one to relate the group-theoretic theta evaluation theory of the present $\S 2$ to the theory of Frobenioid-theoretic theta functions associated to tempered Frobenioids [cf. [EtTh], \$5], i.e., by considering the case where $\mathbb{M}_{*}^{\Theta}$ arises from a tempered Frobenioid [cf. Proposition 1.2, (ii)]. For instance, by considering the case where $\mathbb{M}_{*}^{\Theta}$ arises from a tempered Frobenioid, one may treat the Frobenioid-theoretic cyclotomes [i.e., cyclotomes that arise from the units of the Frobenioid] of Proposition 1.3, (i), in the context of the theory of the present $\S 2$.

\section{Remark 2.8.3.}

(i) The use of the archimedean line segment $\Gamma_{\underline{X}}^{\nabla} \subseteq \Gamma_{\underline{X}}$ [cf. Remark 2.1.1, (ii)] to single out the elements $\in\left\{-l^{*},-l^{*}+1, \ldots,-1,0,1, \ldots, l^{*}-1, l^{*}\right\}-$ i.e., the elements with absolute value $\leq l^{*}$ — within the nonarchimedean congruence classes modulo $l$ constituted by an element $\in \mathbb{F}_{l}^{*}$ is reminiscent of the computation of the set of global sections of an arithmetic line bundle on a number field [cf., e.g., [Szp], pp. 13-14], as well as of the arithmetic inherent in the graph theory associated to the loop $\Gamma_{\underline{X}}$ [cf. [SemiAnbd], Remark 1.5.1].

(ii) The sort of argument discussed in (i) involving the connected, "archimedean" line segment $\Gamma_{\underline{X}}^{\boldsymbol{\nabla}} \subseteq \Gamma_{\underline{X}}[\mathrm{cf}$. Remark 2.6 .1 for more on the importance of this connectedness] depends, in an essential way, on the discreteness of $\underline{\mathbb{Z}}(\cong \mathbb{Z})$. Put another way, this sort of argument may be thought of as an application of the discrete rigidity that forms one of the central themes of [EtTh]. Note, moreover, that in the context of Corollary 2.8, this application of discrete rigidity is closely related to the application of cyclotomic rigidity. This is perhaps not so surprising, since discrete rigidity - in the form of the discreteness of squares of elements of $\underline{\mathbb{Z}}$, i.e., in effect, the quotient of $\underline{\mathbb{Z}}$ by the action of $\{ \pm 1\}$ - may be thought of as a sort of dual property to the cyclotomic rigidity of " $\left(l \cdot \Delta_{\Theta}\right)$ ". Indeed, one may think of this duality as being embodied in the very structure and values of the étale theta function [cf. [EtTh], Proposition 1.4, (ii), (iii); [EtTh], Proposition 1.5, (ii)].

In a similar vein, one may also consider the theory of group-theoretic theta evaluation developed in the present $\S 2$ in the context of the cyclotomic rigidity isomorphism " $\boldsymbol{\mu}_{\widehat{\mathbb{Z}}}\left(G_{k}\right) \stackrel{\sim}{\rightarrow} \boldsymbol{\mu}_{\widehat{\mathbb{Z}}}\left(\Pi_{X}\right)$ " of [AbsTopIII], Corollary 1.10, (c) [cf. also Proposition 1.3, (ii); Corollary 1.11, (b)].

Corollary 2.9. (Theta Evaluation via Base-field-theoretic Cyclotomes) Suppose that we are in the situation of Proposition 2.2, (ii); Corollary 2.5, (ii). 
Also, let us write

$$
\boldsymbol{\mu}_{\widehat{\mathbb{Z}}}\left(G_{\underline{v}}\left(\Pi_{\underline{v}}\right)\right) \stackrel{\sim}{\rightarrow}\left(l \cdot \Delta_{\Theta}\right)\left(\Pi_{\underline{v}}\right) ; \quad \boldsymbol{\mu}_{\widehat{\mathbb{Z}}}\left(G_{\underline{v}}\left(\Pi_{\underline{v}}^{\gamma} \ddot{\sim}\right)\right) \stackrel{\sim}{\rightarrow}\left(l \cdot \Delta_{\Theta}\right)\left(\Pi_{\underline{v}}^{\gamma}\right)
$$

for the cyclotomic rigidity isomorphism “ $\boldsymbol{\mu}_{\widehat{\mathbb{Z}}}\left(G_{k}\right) \stackrel{\sim}{\rightarrow} \boldsymbol{\mu}_{\widehat{\mathbb{Z}}}\left(\Pi_{X}\right)$ " of [AbsTopIII], Corollary 1.10, (c) [cf. also Proposition 1.3, (ii); Corollary 1.11, (b)] and its restriction to $\Pi_{\underline{v}}^{\gamma}$.

(i) (Restriction of Étale Theta Functions to Subgraphs and Evaluation Points) In the situation of Proposition 2.2, (ii); Corollary 2.5, (ii), let us apply the above cyclotomic rigidity isomorphisms to replace " $\left(l \cdot \Delta_{\Theta}\right)(-)$ " by " $\mu_{\widehat{\mathbb{Z}}}\left(G_{\underline{v}}(-)\right) "$. Then the $\iota^{\gamma}$-invariant subsets $\underline{\underline{\theta}}^{\iota}\left(\Pi_{\underline{v}}^{\gamma}\right) \subseteq \underline{\underline{\theta}}\left(\Pi_{\underline{v}}^{\gamma}\right), \infty \underline{\underline{\theta}}^{\iota}\left(\Pi_{\underline{v}}^{\gamma}\right) \subseteq \infty \underline{\underline{\theta}}\left(\Pi_{\underline{v}}^{\gamma}\right)$ [cf. Proposition 2.2, (ii); Corollary 2.5, (ii)] $\overline{\text { determine }} \iota^{\gamma}$-invariant subsets

$$
\underline{\theta}_{\mathrm{bs}}^{\iota}\left(\Pi_{\underline{v}}^{\gamma}\right) \subseteq \underline{\theta}_{\mathrm{bs}}\left(\Pi_{\underline{v}}^{\gamma}\right) ; \quad \infty \underline{\underline{\theta}}_{\mathrm{bs}}^{\iota}\left(\Pi_{\underline{v}}^{\gamma}\right) \subseteq \infty \underline{\underline{\theta}}_{\mathrm{bs}}\left(\Pi_{\underline{v}}^{\gamma}\right)
$$

- where one may think of the "bs" as an abbreviation of the term "base-fieldtheoretic"; restriction of these subsets $\underline{\underline{\theta}}_{\mathrm{bs}}^{\iota}\left(\Pi_{\underline{v}}^{\gamma}\right), \infty \underline{\theta}_{\mathrm{bs}}^{\iota}\left(\Pi_{\underline{v}}^{\gamma}\right)$ to $\Pi_{\underline{v}}^{\gamma}$ yields $\boldsymbol{\mu}_{2 l^{-}}$, $\boldsymbol{\mu}$-orbits of elements

$$
\underline{\theta}_{\mathrm{bs}}^{\iota}\left(\Pi_{\underline{v} \ddot{\boldsymbol{}}}^{\gamma}\right) \subseteq \infty \underline{\theta}_{\mathrm{bs}}^{\iota}\left(\Pi_{\underline{v} \ddot{\boldsymbol{\nu}}}^{\gamma}\right) \subseteq \underline{\lim }_{\widehat{\widehat{J}}} H^{1}\left(\Pi_{\underline{v} \ddot{\boldsymbol{\nu}}}^{\gamma} \mid \widehat{\widehat{J}}, \boldsymbol{\mu}_{\widehat{\mathbb{Z}}}\left(G_{\underline{v}}\left(\Pi_{\underline{\underline{v}}}^{\gamma}\right)\right)\right)
$$

- where $\widehat{J} \subseteq \widehat{\Pi}_{\underline{v}}$ ranges over the open subgroups of $\widehat{\Pi}_{\underline{v}}$ - which, upon further restriction to the decomposition groups $D_{t, \mu_{-}}^{\delta}$ of Corollary 2.4, (ii), (c), yield $\boldsymbol{\mu}_{2 l^{-}}, \boldsymbol{\mu}$-orbits of elements

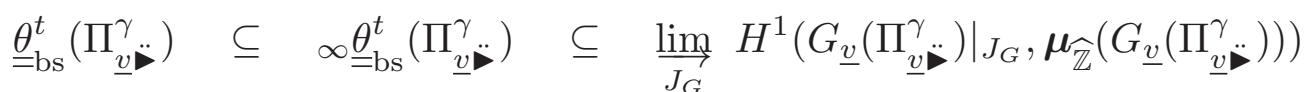

for each $t \in \operatorname{LabCusp}^{ \pm}\left(\Pi_{\underline{v}}^{\gamma}\right) \stackrel{\sim}{\rightarrow} \operatorname{LabCusp}^{ \pm}\left(\Pi_{\underline{v}}\right)$ - where $J_{G} \subseteq G_{\underline{v}}\left(\Pi_{\underline{v}}^{\gamma}\right)$ ranges over the open subgroups of $G_{\underline{v}}\left(\Pi_{\underline{v}}^{\gamma}\right)$; the " $\stackrel{\sim}{\rightarrow}$ " is induced by conjugation by $\gamma$. Moreover, the sets $\underline{\theta}_{\mathrm{bs}}^{t}\left(\Pi_{\underline{v} \ddot{\sim}}^{\gamma}\right), \infty \underline{\theta}_{\mathrm{bs}}^{t}\left(\Pi_{\underline{v}}^{\bar{\nu}}\right)$ depend only on the label $|t| \in\left|\mathbb{F}_{l}\right|$ determined by $t$ [cf. Corollary 2.5, (ii)]. Thus, we shall write $\underline{\theta}_{\mathrm{\theta} s}^{|t|}\left(\Pi_{\underline{v}}^{\gamma}\right) \stackrel{\text { def }}{=} \underline{\underline{\theta}}_{\mathrm{bs}}^{t}\left(\Pi_{\underline{v} \ddot{\boldsymbol{v}}}^{\gamma}\right), \infty \underline{\theta}_{\mathrm{bs}}^{|t|}\left(\Pi_{\underline{v}}^{\gamma}\right) \stackrel{\text { def }}{=}$ $\infty \underline{\theta}_{\mathrm{bs}}^{t}\left(\Pi_{\underline{v}}^{\gamma}\right)$.

(ii) (Functorial Group-theoretic Evaluation Algorithm) If one starts with an arbitrary $\widehat{\Delta}_{\underline{v}}^{ \pm}$-conjugate $\Pi_{\underline{v}}^{\gamma}$ : of $\Pi_{\underline{v}} \ddot{\boldsymbol{\sim}}$, and one considers, as $t$ ranges over the elements of $\operatorname{LabCusp}^{ \pm}\left(\Pi_{\underline{v}}^{\gamma}\right) \stackrel{\sim}{\rightarrow} \operatorname{LabCusp}^{ \pm}\left(\Pi_{\underline{v}}\right)$ [where the $\stackrel{\sim}{\rightarrow}$ " is induced by conjugation by $\gamma]$, the resulting $\boldsymbol{\mu}_{2 l^{-}}, \boldsymbol{\mu}$-orbits $\underline{\underline{\theta}}_{=\mathrm{bs}}^{|t|}\left(\Pi_{\underline{v}}^{\gamma}\right), \infty \underline{\theta}_{\mathrm{bs}}^{|t|}\left(\Pi_{\underline{v}}^{\gamma}\right)$ arising from an arbitrary $\widehat{\Delta}_{\underline{v}}^{ \pm}$-conjugate $I_{t}^{\delta}$ of $I_{t}$ that is contained in $\Pi_{\underline{v}}^{\gamma}$. [cf. (i)], then one obtains an algorithm for constructing the collections of $\boldsymbol{\mu}_{2 l^{-}}, \boldsymbol{\mu}$-orbits

$$
\left\{\underline{\underline{\theta}}_{\mathrm{bs}}^{|t|}\left(\Pi_{\underline{v} \ddot{\nabla}}^{\gamma}\right)\right\}|t| \in\left|\mathbb{F}_{l}\right| ; \quad\left\{\infty \underline{\underline{\theta}}_{\mathrm{bs}}^{|t|}\left(\Pi_{\underline{v} \ddot{\boldsymbol{\nu}}}^{\gamma}\right)\right\}|t| \in\left|\mathbb{F}_{l}\right|
$$

which is functorial in the topological group $\Pi_{\underline{v}}$, and, moreover, compatible with the independent conjugacy actions of $\widehat{\Delta}_{\underline{v}}^{ \pm}$on the sets $\left\{I_{t}^{\gamma_{1}}\right\}_{\gamma_{1} \in \widehat{\Pi}_{\underline{v}}^{ \pm}}=\left\{I_{t}^{\gamma_{1}}\right\}_{\gamma_{1} \in \widehat{\Delta}_{\underline{v}}^{ \pm}}$ and $\left\{\Pi_{\underline{v} \dot{*}}^{\gamma_{2}}\right\}_{\gamma_{2} \in \widehat{\Pi}_{\underline{v}}^{ \pm}}=\left\{\Pi_{\underline{v}}^{\gamma_{2}}\right\}_{\gamma_{2} \in \widehat{\Delta}_{\underline{v}}^{ \pm}}$[cf. the sets of Corollary 2.4, (i) ; Remark 2.2.1]. 
(iii) (Splittings at Zero-labeled Evaluation Points) In the situation of (i), suppose that $t$ is taken to be the zero element. Then, by applying the cyclotomic rigidity isomorphisms reviewed above to replace " $\left(l \cdot \Delta_{\Theta}\right)(-)$ " by " $\boldsymbol{\mu}_{\widehat{\mathbb{Z}}}\left(G_{\underline{v}}(-)\right)$ " - an operation that, when applied to " $M_{\mathbb{T M}}^{? ?}(-)$ ", we shall denote by means of a subscript "bs" - in Corollary 2.6, (ii), the second restriction operation discussed in (i) determines splittings [cf. Corollary 2.6, (ii)]

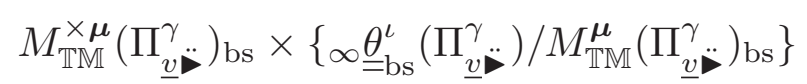

of $M_{\mathbb{T M}}^{\times} \cdot \infty_{\underline{b s}}^{\iota}\left(\Pi_{\underline{v} \ddot{\bullet}}^{\gamma}\right) / M_{\mathbb{T M}}^{\boldsymbol{\mu}}\left(\Pi_{\underline{v} \ddot{\boldsymbol{\nu}}}^{\gamma}\right)_{\mathrm{bs}}$ which are compatible - relative to the first restriction operation discussed in (i) and the cyclotomic rigidity isomorphisms reviewed above - with the splittings of Corollary 1.12, (ii).

Proof. Assertions (i), (ii), and (iii) follow immediately from the definitions and the references quoted in the statements of these assertions.

\section{Remark 2.9.1.}

(i) Let us recall that [the cyclotomic rigidity isomorphisms involving] the cyclotomes " $\Pi_{\boldsymbol{\mu}}(-)$ " that appear in Corollary 2.8 admit a multiradial formulation [cf. Corollary 1.10]. By contrast, at least relative to the point of view of Remark 1.11.3, (iv), [the cyclotomic rigidity isomorphisms involving] the cyclotomes " $\boldsymbol{\mu}_{\widehat{\mathbb{Z}}}\left(G_{\underline{v}}(-)\right)$ " that appear in Corollary 2.9 only admit a uniradial formulation - i.e., unless one is willing to sacrifice the crucial cyclotomic rigidity under consideration as in the formulation of Corollary 1.11.

(ii) On the other hand, the use of [the cyclotomic rigidity isomorphisms involving] the cyclotomes " $\boldsymbol{\mu}_{\widehat{\mathbb{Z}}}\left(G_{\underline{v}}(-)\right)$ " has the crucial advantage that it allows one to apply the [not multiradially (!), but rather] uniradially defined natural surjection

$$
H^{1}\left(G_{\underline{v}}(-), \boldsymbol{\mu}_{\widehat{\mathbb{Z}}}\left(G_{\underline{v}}(-)\right)\right) \rightarrow \widehat{\mathbb{Z}}
$$

of Remark 1.11.5, (i), (ii).

(iii) One immediate consequence of the discussion of (i) is the observation that, at least relative to the point of view of Remark 1.11.3, (iv), the algorithms of Corollary 2.9, (ii), (iii), only give rise to a uniradially defined functor. On the other hand, one important consequence of the theory to be developed in [IUTchIII] is the result that,

by applying the theory of log-shells [cf. [AbsTopIII]], one may modify these algorithms in such a way as to obtain algorithms that [yield functors which] are manifestly multiradially defined

— albeit at the cost of allowing for certain [relatively mild!] indeterminacies. 


\section{Section 3: Tempered Gaussian Frobenioids}

In the present $\S 3$, we relate the theory of group-theoretic algorithms surrounding the Hodge-Arakelov-theoretic evaluation of the étale theta function on $l$-torsion points developed in $\S 1, \S 2$ to the local portion at bad primes [i.e., at $\left.\underline{v} \in \underline{\mathbb{V}}^{\mathrm{bad}}\right]$ of the various Frobenioids considered in [IUTchI], $33, \S 4, \S 5, \S 6$. In particular, we shall discuss how the various multiradial formulations developed in $\S 1$ and the theory of conjugate synchronization developed in $\S 2$ may be applied in the context of the "tempered Gaussian Frobenioids" that arise from the Hodge-Arakelov-theoretic evaluation of the étale theta function on $l$-torsion points.

In the present $\S 3$, we shall continue to use the notation of $\S 2$. In particular, our discussion concerns the local portion at $\underline{v} \in \underline{\mathbb{V}}^{\text {bad }}$ of the various mathematical objects considered in [IUTchI], $33, \S 4, \S 5, \S 6$.

\section{Proposition 3.1. (Mono-theta-theoretic Theta Monoids) Let}

$$
\mathbb{M}_{*}^{\Theta}=\left\{\ldots \rightarrow \mathbb{M}_{M^{\prime}}^{\Theta} \rightarrow \mathbb{M}_{M}^{\Theta} \rightarrow \ldots\right\}
$$

be a projective system of mono-theta environments [cf. Proposition 1.5, Corollary 2.8] such that $\Pi_{\underline{\underline{X}}}\left(\mathbb{M}_{*}^{\Theta}\right) \cong \Pi_{\underline{v}}$. In the following, to simplify the notation, we shall denote a " $\Pi_{\underline{\underline{X}}}\left(\mathbb{M}_{*}^{\overline{\bar{\Theta}}}\right)$ " in parenthesis by means of the abbreviated notation "M $\mathbb{M}_{*}^{\Theta} "$

(i) (Split Theta Monoids) By applying the constructions of Proposition 1.5, (iii); Corollary 2.8, (i), one obtains a functorial algorithm

$$
\begin{aligned}
\mathbb{M}_{*}^{\Theta} \mapsto\left\{M_{\mathbb{T M}}^{\times}\left(\mathbb{M}_{*}^{\Theta}\right),\right. & \underline{\theta}_{\mathrm{env}}^{\iota}\left(\mathbb{M}_{*}^{\Theta}\right), \infty \underline{\underline{\theta}}_{\mathrm{env}}\left(\mathbb{M}_{*}^{\Theta}\right) \\
& \left.\subseteq M_{\mathbb{T M}^{\times}}^{\times} \infty \underline{\underline{\theta}}_{\mathrm{env}}^{\iota}\left(\mathbb{M}_{*}^{\Theta}\right) \subseteq \underline{\lim _{J}} H^{1}\left(\left.\Pi_{\underline{\underline{\underline{Y}}}}\left(\mathbb{M}_{*}^{\Theta}\right)\right|_{J}, \Pi_{\boldsymbol{\mu}}\left(\mathbb{M}_{*}^{\Theta}\right)\right)\right\}_{\iota}
\end{aligned}
$$

- where $J$ ranges over the finite index open subgroups of $\Pi_{\underline{X}}\left(\mathbb{M}_{*}^{\Theta}\right)$, and $\iota$ ranges over the inversion automorphisms of Proposition 2.2, (i) - $\bar{f}_{\text {or }}$ constructing various subsets of the direct limit of cohomology modules in the above display; this collection of subsets is equipped with a natural conjugation action by $\Pi_{\underline{X}}\left(\mathbb{M}_{*}^{\Theta}\right)$. In particular, one obtains a functorial algorithm for constructing the data

$$
\begin{gathered}
\Psi_{\text {env }}\left(\mathbb{M}_{*}^{\Theta}\right) \stackrel{\text { def }}{=}\left\{\Psi_{\text {env }}^{\iota}\left(\mathbb{M}_{*}^{\Theta}\right)=M_{\mathbb{T M}}^{\times}\left(\mathbb{M}_{*}^{\Theta}\right) \cdot \underline{\underline{\theta}}_{\text {env }}^{\iota}\left(\mathbb{M}_{*}^{\Theta}\right)^{\mathbb{N}}\right\}_{\iota} ; \\
\infty \Psi_{\text {env }}\left(\mathbb{M}_{*}^{\Theta}\right) \stackrel{\text { def }}{=}\left\{\infty \Psi_{\text {env }}^{\iota}\left(\mathbb{M}_{*}^{\Theta}\right)=M_{\mathbb{T M}}^{\times}\left(\mathbb{M}_{*}^{\Theta}\right) \cdot \infty_{\underline{\theta}_{\text {env }}^{\iota}}\left(\mathbb{M}_{*}^{\Theta}\right)^{\mathbb{N}}\right\}_{\iota}
\end{gathered}
$$

consisting of the submonoids $\left\{\Psi_{\mathrm{env}}^{\iota}\left(\mathbb{M}_{*}^{\Theta}\right)\right\}_{\iota},\left\{\infty \Psi_{\mathrm{env}}^{\iota}\left(\mathbb{M}_{*}^{\Theta}\right)\right\}_{\iota}$. of the direct limit of cohomology modules in the above display] generated, respectively, by the subsets " $M_{\mathbb{T M}}^{\times} \cdot \underline{\underline{\theta}}_{\text {env }}^{\iota}\left(\mathbb{M}_{*}^{\Theta}\right)$ ", " $M_{\mathbb{T M}}^{\times} \cdot \infty \underline{\underline{\theta}}_{\text {env }}\left(\mathbb{M}_{*}^{\Theta}\right)$ ", as well as a functorial algorithm for constructing the splittings up to torsion determined by the subsets " $M_{\mathbb{T M}}^{\times}\left(\mathbb{M}_{*}^{\Theta}\right)$ ", " $\underline{\theta}_{\mathrm{env}}^{\iota}\left(\mathbb{M}_{*}^{\Theta}\right) ", " \infty \underline{\theta}_{\mathrm{env}}^{\iota}\left(\mathbb{M}_{*}^{\Theta}\right)$ " [cf. Corollary 2.8, (iii)]. We shall refer to each $\Psi_{\mathrm{env}}^{\iota}\left(\mathbb{M}_{*}^{\Theta}\right)$, $\infty \Psi_{\mathrm{env}}^{\iota}\left(\mathbb{M}_{*}^{\Theta}\right)$ as a theta monoid. 
(ii) (Constant Monoids) By applying the cyclotomic rigidity isomorphisms of Corollaries 2.8, (i); 2.9, and considering the inverse image of $\mathbb{Z} \subseteq \widehat{\mathbb{Z}}$ via the surjection of Remark 1.11.5, (i), applied to $G_{\underline{v}}\left(\mathbb{M}_{*}^{\Theta}\right)\left(=G_{\underline{v}}\left(\Pi_{\underline{X}}\left(\mathbb{M}_{*}^{\Theta}\right)\right)\right)$ [cf. the notation of Corollary 2.5, (i)], one obtains a functorial algorithm

$$
\mathbb{M}_{*}^{\Theta} \mapsto \Psi_{\mathrm{cns}}\left(\mathbb{M}_{*}^{\Theta}\right) \stackrel{\text { def }}{=} M_{\mathbb{T M}}\left(\mathbb{M}_{*}^{\Theta}\right) \subseteq \underline{\lim _{J}} H^{1}\left(\left.\Pi_{\underline{\underline{\underline{Y}}}}\left(\mathbb{M}_{*}^{\Theta}\right)\right|_{J}, \Pi_{\boldsymbol{\mu}}\left(\mathbb{M}_{*}^{\Theta}\right)\right)
$$

[where $J$ is as in (i)] for constructing a "monoid of constants" - i.e., which is naturally isomorphic to $\mathcal{O}{\frac{F_{v}}{v}}$ [cf. Example 1.8, (ii)] - equipped with a natural conjugation action by $\Pi_{\underline{\underline{X}}}^{-}\left(\mathbb{M}_{*}^{\Theta}\right)$. We shall refer to $\Psi_{\mathrm{cns}}\left(\mathbb{M}_{*}^{\Theta}\right)$ as a constant monoid.

Proof. Assertions (i) and (ii) follow immediately from the definitions and the references quoted in the statements of these assertions.

Before proceeding, we pause to review the theory of tempered Frobenioids discussed in [IUTchI], Example 3.2.

\section{Example 3.2. Theta Monoids Constructed from Tempered Frobenioids.} In the situation of of [IUTchI], Example 3.2:

(i) Recall the tempered Frobenioid $\underline{\underline{\mathcal{F}}}_{v}$ of [IUTchI], Example 3.2, (i). Then, in the notation of loc. cit., the choice of a Frobenioid-theoretic theta function

$$
\underline{\underline{\Theta}}_{\underline{v}} \in \mathcal{O}^{\times}\left(\mathbb{T}_{\underline{\underline{\dot{Y}}}_{\underline{v}}^{\dot{\ddots}}}^{\dot{v}}\right)
$$

—i.e., among the $\boldsymbol{\mu}_{2 l}\left(\mathbb{T}_{\underline{\underline{\dot{Y}}}_{\underline{v}}}^{\dot{\dot{\sigma}}}\right)$-multiples of the $\operatorname{Aut}_{\mathcal{D}_{\underline{v}}}\left(\underline{\underline{Y}}_{\underline{v}}\right)$-conjugates of $\underline{\underline{\Theta}}_{\underline{v}}$ - determines a monoid $\mathcal{O}_{\mathcal{C}_{\underline{v}}^{\ominus}}^{\triangleright}(-)$ on $\mathcal{D}_{\underline{\underline{v}}}^{\Theta}$. Now suppose, for simplicity, that the topological group $\Pi_{\underline{v}}$ arises from a basepoint, i.e., more concretely, from a "universal covering pro-object" $A_{\infty}^{\Theta}$ of $\mathcal{D}_{\underline{v}}$ [i.e., a pro-object determined by a cofinal projective system of Galois objects of $\overline{\mathcal{D}}_{\underline{v}}$. Then by evaluating $\mathcal{O}_{\mathcal{C}_{\underline{\underline{v}}}^{\ominus}}^{\triangleright}(-)$ on [the "universal covering pro-object" of $\mathcal{D}_{\underline{v}}^{\Theta}$ determined by] $A_{\infty}^{\Theta}$, we obtain submonoids [in the usual sense]

$$
\begin{aligned}
& \Psi_{\mathcal{F}_{\underline{v}}^{\Theta}, \mathrm{id}} \stackrel{\text { def }}{=} \mathcal{O}_{\mathcal{C}_{\underline{v}}^{\Theta}}^{\triangleright}\left(A_{\infty}^{\Theta}\right)=\left.\mathcal{O}_{\mathcal{C}_{\underline{v}}^{\Theta}}^{\times}\left(A_{\infty}^{\Theta}\right) \cdot \underline{\underline{\Theta}} \underline{\underline{\Theta}}\right|_{A_{\infty}^{\Theta}} ^{\mathbb{N}} \\
& \left.\subseteq \infty \Psi_{\underline{\mathcal{F}_{v}^{\Theta}}, \text { id }} \stackrel{\text { def }}{=} \mathcal{O}_{\mathcal{C}_{\underline{v}}^{\Theta}}^{\times}\left(A_{\infty}^{\Theta}\right) \cdot \underline{\underline{\Theta}}_{\underline{\underline{v}}}^{\mathbb{Q} \geq 0}\right|_{A_{\infty}^{\Theta}} \subseteq \mathcal{O}^{\times}\left(\mathbb{T}_{\dot{A}_{\infty}^{\Theta}}^{\dot{\Theta}}\right)
\end{aligned}
$$

— where the superscript " $\mathbb{Q} \geq 0$ " denotes the set of elements for which some [positive integer] power is equal to a [positive integer] power of $\left.\underline{\underline{\Theta}}_{\underline{v}}\right|_{A_{\infty}^{\Theta}}$. In a similar vein, by considering [cf. [IUTchI], Remark 3.2.3, (i)] the various conjugates $\underline{\underline{\Theta}}_{\underline{v}}^{\alpha}$ of $\underline{\underline{\Theta}}_{\underline{v}}$, for $\alpha \in \operatorname{Aut}_{\mathcal{D}_{\underline{v}}}\left(\underline{\underline{\underline{Y}}}_{\underline{v}}\right)$, we also obtain submonoids $\Psi_{\mathcal{F}_{\underline{v}}^{\Theta}, \alpha} \subseteq \infty \Psi_{\mathcal{F}_{\underline{v}}^{\Theta}, \alpha} \subseteq \mathcal{O}^{\overline{\times}}\left(\mathbb{T}_{\dot{A}_{\infty}^{\Theta}} \dot{\bar{\omega}}^{\Theta}\right)$. 
Moreover, one has a natural surjection $\Pi_{\underline{v}} \rightarrow \operatorname{Aut}_{\mathcal{D}_{\underline{v}}}\left(\underline{\underline{\underline{Y}}}_{\underline{v}}\right)$, as well as a natural conjugation action of $\Pi_{\underline{v}}$ on the collections of submonoids

$$
\Psi_{\mathcal{F}_{\underline{v}}^{\Theta}} \stackrel{\text { def }}{=}\left\{\Psi_{\mathcal{F}_{\underline{v}}^{\Theta}, \alpha}\right\}_{\alpha \in \Pi_{\underline{v}}} ; \quad \infty \Psi_{\mathcal{F}_{\underline{v}}^{\Theta}} \stackrel{\text { def }}{=}\left\{\infty \Psi_{\mathcal{F}_{\underline{v}}^{\Theta}, \alpha}\right\}_{\alpha \in \Pi_{\underline{v}}}
$$

- i.e., where, by abuse of notation, we think of the subscripted " $\alpha$ 's" as denoting the image of " $\alpha$ " via the surjection $\Pi_{\underline{v}} \rightarrow \operatorname{Aut}_{\mathcal{D}_{\underline{v}}}\left(\underline{\underline{Y}}_{\underline{v}}\right)$. Also, we recall from loc. cit. that $\left.\underline{\underline{\Theta}}_{\underline{v}}^{\mathbb{Q} \geq 0}\right|_{A_{\infty}^{\Theta}}$ determines characteristic splittings, up to torsion, of the monoids $\Psi_{\mathcal{F}_{v}^{\Theta}, \alpha}\left[\right.$ cf. the " $\tau_{\underline{v}}^{\Theta}$ " of [IUTchI], Example 3.2, (v)], $\Psi_{\mathcal{F}_{v}^{\Theta}, \alpha}$ which are compatible with the action of $\Pi_{\underline{v}}$. Finally, we recall that the collection of data

$$
\Pi_{\underline{v}} \curvearrowright \quad \Psi_{\mathcal{F}_{\underline{v}}^{\Theta}}=\left\{\Psi_{\mathcal{F}_{\underline{v}}^{\Theta}, \alpha}\right\}_{\alpha \in \Pi_{\underline{v}}}, \quad \infty \Psi_{\mathcal{F}_{\underline{v}}^{\Theta}}=\left\{\infty \Psi_{\mathcal{F}_{\underline{v}}^{\Theta}, \alpha}\right\}_{\alpha \in \Pi_{\underline{v}}}
$$

- i.e., consisting of two collections of submonoids of the group of units [namely, $\left.\mathcal{O}^{\times}\left(\mathbb{T}_{\dot{A_{\infty}}}^{\ominus}\right)\right]$ associated to the birationalization of a certain characteristic pro-object of $\underline{\underline{F}}_{\underline{v}}$, equipped with the conjugation action by an automorphism group of a certain characteristic pro-object of $\mathcal{D}_{\underline{v}}$ - as well as the characteristic splittings, up to torsion, just discussed, may be reconstructed category-theoretically from $\underline{\underline{\mathcal{F}}}_{v}$ [cf. [IUTchI], Example 3.2, (vi), (e)], up to an indeterminacy arising from the inner automorphisms of $\Pi_{\underline{v}}$.

(ii) In a similar, but somewhat simpler, vein, the Frobenioid structure on the subcategory $\mathcal{C}_{\underline{v}} \subseteq \underline{\underline{\mathcal{F}}}_{\underline{v}}$ - i.e., the "base-theoretic hull" of the tempered Frobenioid $\underline{\underline{\mathcal{F}}}_{\underline{v}}$ [cf. [IUTchI], Example 3.2, (iii)] - determines, via the general theory of Frobenioids [cf. [FrdI], Proposition 2.2], a monoid $\mathcal{O}_{\mathcal{C}_{\underline{v}}}^{\triangleright}(-)$ on $\mathcal{D}_{\underline{v}}$. Then by evaluating $\mathcal{O}_{\mathcal{C}_{\underline{v}}}^{\triangleright}(-)$ on $A_{\infty}^{\Theta}$, we obtain a monoid [in the usual sense]

$$
\Psi_{\mathcal{C}_{\underline{v}}} \stackrel{\text { def }}{=} \quad \mathcal{O}_{\mathcal{C}_{\underline{v}}}^{\triangleright}\left(A_{\infty}^{\Theta}\right)
$$

which is equipped with a natural action by $\Pi_{\underline{v}}$. Finally, we recall that the collection of data

$$
\Pi_{\underline{v}} \curvearrowright \Psi_{\mathcal{C}_{\underline{v}}}
$$

— i.e., consisting of a submonoid of the group of units [namely, $\left.\mathcal{O}^{\times}\left(\mathbb{T}_{\dot{\dot{A}_{\infty}}}^{\dot{\Theta}}\right)\right]$ associated to the birationalization of a certain characteristic pro-object of $\underline{\underline{\mathcal{F}}}_{\underline{v}}$, equipped with the conjugation action by an automorphism group of a certain characteristic pro-object of $\mathcal{D}_{\underline{v}}$ - may be reconstructed category-theoretically from $\underline{\underline{\mathcal{F}}}_{\underline{v}}$ [cf. [IUTchI], Example 3.2, (iii); [IUTchI], Example 3.2, (vi), (d); [FrdI], Theorem 3.4, (iv); [FrdII], Theorem 1.2, (i); [FrdII], Example 1.3, (i)], up to an indeterminacy arising from the inner automorphisms of $\Pi_{\underline{v}}$.

Proposition 3.3. (Frobenioid-theoretic Theta Monoids) Suppose, in the situation of Proposition 3.1, that $\mathbb{M}_{*}^{\Theta}$ arises [cf. Proposition 1.2, (ii)] from a tempered Frobenioid ${ }^{\dagger} \underline{\underline{\mathcal{F}}}_{\underline{v}}-i . e .$,

$$
\mathbb{M}_{*}^{\Theta}=\mathbb{M}_{*}^{\Theta}\left(\underline{\underline{\mathcal{F}}}_{\underline{v}}\right)
$$


- that appears in a $\Theta$-Hodge theater ${ }^{\dagger} \mathcal{H} \mathcal{T}^{\Theta}=\left(\left\{\underline{\underline{F}}_{\underline{\underline{F}}}\right\}_{\underline{v} \in \underline{\mathbb{V}}},{ }^{\dagger} \mathfrak{F}_{\text {mod }}^{\vdash}\right)$ [cf. [IUTchI],

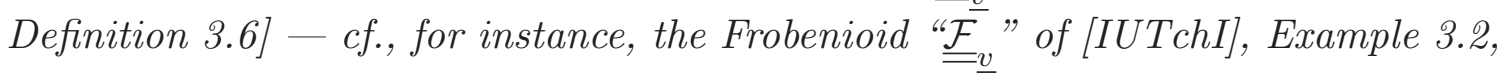

(i). Observe that by applying the category-theoretic constructions of Example 3.2, (i), (ii), to ${ }^{\dagger} \underline{\underline{\mathcal{F}}}_{\underline{v}}$, one obtains data

$$
\begin{gathered}
\Pi_{\underline{\underline{X}}}\left(\mathbb{M}_{*}^{\Theta}\right) \curvearrowright \quad \Psi_{\dagger \mathcal{F}_{\underline{v}}^{\Theta}}=\left\{\Psi_{\dagger \mathcal{F}_{\underline{v}}^{\Theta}, \alpha}\right\}_{\alpha \in \Pi_{\underline{\underline{X}}}\left(\mathbb{M}_{*}^{\Theta}\right)}, \quad \infty \Psi_{\mathcal{F}_{\underline{v}}^{\Theta}}=\left\{\infty \Psi_{\mathcal{F}_{\underline{v}}^{\Theta}, \alpha}\right\}_{\alpha \in \Pi_{\underline{\underline{X}}}\left(\mathbb{M}_{*}^{\Theta}\right)} ; \\
\Pi_{\underline{\underline{X}}}\left(\mathbb{M}_{*}^{\Theta}\right) \curvearrowright \Psi_{\dagger \mathcal{C}_{\underline{v}}} \curvearrowright
\end{gathered}
$$

as well as splittings, up to torsion, of each of the monoids $\Psi_{\dagger \mathcal{F}_{\underline{v}}^{\Theta}, \alpha}, \infty \Psi_{\dagger \mathcal{F}_{\underline{v}}^{\Theta}, \alpha}$.

(i) (Split Theta Monoids) By forming Kummer classes relative to the Frobenioid structure of ${ }^{\dagger} \underline{\underline{\mathcal{F}}}_{\underline{v}}-i . e$. , in essence, by considering the Galois cohomology classes that arise when one extracts $N$-th roots of unity for $N \in \mathbb{N}_{\geq 1}$ [cf. [FrdII], Definition 2.1, (ii); [IUTchI], Remark 3.2.3, (ii); the discussion of [EtTh], $\S 5]$ - and applying the description given in Proposition 1.3, (i), of the exterior cyclotome of a mono-theta environment that arises from a tempered Frobenioid, one obtains, for an appropriate correspondence of indices $\alpha \mapsto \iota$, collections of isomorphisms of monoids

$$
\Psi_{\dagger \mathcal{F}_{\underline{v}}^{\Theta}, \alpha} \stackrel{\sim}{\rightarrow} \quad \Psi_{\mathrm{env}}^{\iota}\left(\mathbb{M}_{*}^{\Theta}\right) ; \quad \infty \Psi_{\dagger \mathcal{F}_{\underline{v}}^{\Theta}, \alpha} \stackrel{\sim}{\rightarrow} \infty \Psi_{\mathrm{env}}^{\iota}\left(\mathbb{M}_{*}^{\Theta}\right)
$$

- each of which is well-defined up to composition with an inner automorphism [cf. the discussion of Example 3.2, (i)] and compatible with both the respective conjugation actions by $\Pi_{\underline{X}}\left(\mathbb{M}_{*}^{\Theta}\right)$ and the splittings up to torsion on the monoids under consideration. We shall denote these collections of isomorphisms by means of the notation

$$
\Psi_{\dagger \mathcal{F}_{\underline{v}}^{\Theta}} \stackrel{\sim}{\rightarrow} \quad \Psi_{\mathrm{env}}\left(\mathbb{M}_{*}^{\Theta}\right) ; \quad \infty \Psi_{\dagger \mathcal{F}_{\underline{\underline{v}}}^{\Theta}} \stackrel{\sim}{\rightarrow} \infty \Psi_{\mathrm{env}}\left(\mathbb{M}_{*}^{\Theta}\right)
$$

[cf. the notation of Proposition 3.1, (i); Example 3.2, (i)].

(ii) (Constant Monoids) By forming Kummer classes relative to the Frobenioid structure of ${ }^{\dagger} \underline{\underline{\mathcal{F}}}_{\underline{v}}$ - i.e., in essence, by considering the Galois cohomology classes that arise when one extracts $N$-th roots of unity for $N \in \mathbb{N}_{\geq 1}$ [cf. [FrdII], Definition 2.1, (ii); [IUTchI], Remark 3.2.3, (ii); [FrdII], Theorem 2.4] - and applying the description given in Proposition 1.3, (i), of the exterior cyclotome of a mono-theta environment that arises from a tempered Frobenioid, one obtains an isomorphism of monoids

$$
\Psi_{\dagger \mathcal{C}_{\underline{v}}} \stackrel{\sim}{\rightarrow} \Psi_{\mathrm{cns}}\left(\mathbb{M}_{*}^{\Theta}\right)
$$

- which is well-defined up to composition with an inner automorphism [cf. the discussion of Example 3.2, (ii)] and compatible with the respective conjugation actions by $\Pi_{\underline{\underline{X}}}\left(\mathbb{M}_{*}^{\Theta}\right)$.

Proof. Assertions (i) and (ii) follow immediately from the definitions and the references quoted in the statements of these assertions. 
Proposition 3.4. (Group-theoretic Theta Monoids) Let ${ }^{\dagger} \underline{\underline{\mathcal{F}}}_{\underline{v}}$ be a tempered Frobenioid as in Proposition 3.3. Consider the full poly-isomorphism

$$
\mathbb{M}_{*}^{\Theta}\left(\Pi_{\underline{v}}\right) \stackrel{\sim}{\rightarrow} \mathbb{M}_{*}^{\Theta}\left(\underline{\underline{\underline{\mathcal{F}}}}^{\dagger}\right)
$$

- where $\mathbb{M}_{*}^{\Theta}\left(\Pi_{\underline{v}}\right)$ is the projective system of mono-theta environments arising from the algorithm of Proposition 1.2, (i) [cf. also Proposition 1.5, (i)] - of projective systems of mono-theta environments.

(i) (Multiradiality of Split Theta Monoids) Each isomorphism of projective systems of mono-theta environments $\mathbb{M}_{*}^{\Theta}\left(\Pi_{\underline{v}}\right) \stackrel{\sim}{\rightarrow} \mathbb{M}_{*}^{\Theta}\left({ }^{\dagger} \underline{\underline{\mathcal{F}}}_{\underline{v}}\right)$ induces compatible [in the evident sense] collections of isomorphisms

$$
\begin{aligned}
& \Pi_{\underline{v}} \stackrel{\sim}{\rightarrow} \Pi_{\underline{\underline{X}}}\left(\mathbb{M}_{*}^{\Theta}\left(\Pi_{\underline{v}}\right)\right) \quad \stackrel{\sim}{\rightarrow} \quad \Pi_{\underline{\underline{X}}}\left(\mathbb{M}_{*}^{\Theta}\left({ }^{\dagger} \underline{\underline{\mathcal{F}}}_{\underline{v}}\right)\right) \quad=\quad \Pi_{\underline{\underline{X}}}\left(\mathbb{M}_{*}^{\Theta}\left({ }^{\dagger} \underline{\underline{\mathcal{F}}}_{\underline{v}}\right)\right)
\end{aligned}
$$

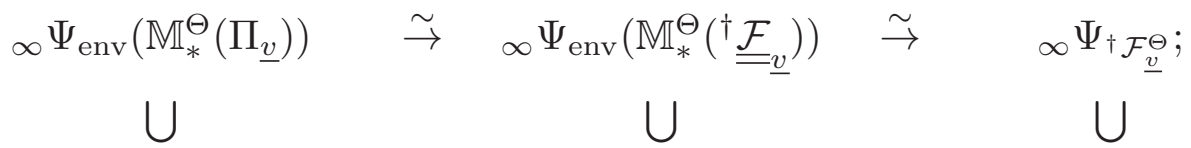

$$
\begin{aligned}
& \Psi_{\mathrm{env}}\left(\mathbb{M}_{*}^{\Theta}\left(\Pi_{\underline{v}}\right)\right) \quad \stackrel{\sim}{\rightarrow} \quad \Psi_{\mathrm{env}}\left(\mathbb{M}_{*}^{\Theta}\left(\underline{\underline{\underline{\mathcal{F}}}}_{\underline{v}}\right)\right) \quad \stackrel{\sim}{\rightarrow} \quad \Psi_{\dagger} \mathcal{F}_{\underline{\underline{v}}}
\end{aligned}
$$

and

$$
\begin{array}{rlrrr}
G_{\underline{v}} \stackrel{\sim}{\rightarrow} G_{\underline{v}}\left(\mathbb{M}_{*}^{\Theta}\left(\Pi_{\underline{v}}\right)\right) & \stackrel{\sim}{\rightarrow} & G_{\underline{v}}\left(\mathbb{M}_{*}^{\Theta}\left({ }^{\dagger} \underline{\underline{\mathcal{F}}}_{\underline{v}}\right)\right) & = & G_{\underline{v}}\left(\mathbb{M}_{*}^{\Theta}\left({ }^{\dagger} \underline{\underline{\mathcal{F}}}_{\underline{v}}\right)\right) \\
\curvearrowright & & \curvearrowright & \curvearrowright \\
\Psi_{\mathrm{env}}\left(\mathbb{M}_{*}^{\Theta}\left(\Pi_{\underline{v}}\right)\right)^{\times} & \stackrel{\sim}{\rightarrow} & \Psi_{\mathrm{env}}\left(\mathbb{M}_{*}^{\Theta}\left({ }^{\dagger} \underline{\underline{\underline{\mathcal{F}}}}_{\underline{v}}\right)\right)^{\times} & \stackrel{\sim}{\rightarrow} & \left(\Psi_{\dagger \mathcal{F}_{\underline{\underline{v}}}^{\Theta}}\right)^{\times}
\end{array}
$$

- where the upper horizontal isomorphisms in each diagram are isomorphisms of topological groups; the lower/middle horizontal isomorphisms in each diagram are isomorphisms of [topological] monoids; the lower horizontal isomorphisms in the first diagram are compatible with the respective splittings up to torsion; the lefthand square in each diagram arises from the functoriality of the algorithms involved, relative to isomorphisms of projective systems of mono-theta environments; the right-hand square in each diagram arises from the inverses of the isomorphisms of the second display of Proposition 3.3, (i); the superscript " $\times$ " denotes the submonoid of units. Finally, if we write $\left(\Psi_{\dagger \mathcal{F}_{\nu}^{\Theta}}\right)^{\times \boldsymbol{\mu}}$ for the topological monoid obtained by forming the quotient of $\left(\Psi_{\dagger \mathcal{F}_{\underline{v}}^{\Theta}}\right)^{\times}$by its torsion subgroup, then the functorial algorithms

$$
\Pi_{\underline{v}} \mapsto \Psi_{\mathrm{env}}\left(\mathbb{M}_{*}^{\Theta}\left(\Pi_{\underline{v}}\right)\right) ; \quad \Pi_{\underline{v}} \mapsto{ }_{\infty} \Psi_{\mathrm{env}}\left(\mathbb{M}_{*}^{\Theta}\left(\Pi_{\underline{v}}\right)\right)
$$

- where we think of $\Psi_{\mathrm{env}}\left(\mathbb{M}_{*}^{\Theta}\left(\Pi_{\underline{v}}\right)\right), \infty \Psi_{\mathrm{env}}\left(\mathbb{M}_{*}^{\Theta}\left(\Pi_{\underline{v}}\right)\right)$ as being equipped with their natural $\Pi_{\underline{v}}$-actions and splittings up to torsion [cf. Proposition 3.1, (i)] - obtained by composing the algorithms of Propositions 1.2, (i); 3.1, (i), are compatible, relative to the above displayed diagrams, with arbitrary automorphisms of the underlying pair, consisting of a topological monoid equipped with the action of a topological group, determined by] the pair

$$
G_{\underline{v}}\left(\mathbb{M}_{*}^{\Theta}\left(\underline{\underline{\mathcal{F}}}_{\underline{v}}\right)\right) \curvearrowright\left(\Psi_{\dagger \mathcal{F}_{\underline{v}}^{\Theta}}\right)^{\times \mu}
$$


which arise from Ism-multiples of automorphisms of [the underlying pair, consisting of a topological monoid equipped with the action of a topological group, determined by] the pair $G_{\underline{v}}\left(\mathbb{M}_{*}^{\Theta}\left({ }^{\dagger} \underline{\underline{\mathcal{F}}}_{\underline{v}}\right)\right) \curvearrowright\left(\Psi_{\dagger} \mathcal{F}_{\underline{\underline{v}}}^{\Theta}\right)^{\times}$[cf. Example 1.8, (iv); Remark 1.8.1; Remark 1.11.1, (i), (b) $\bar{j}$ — in the sense that the natural functor " $\Psi_{\mathcal{R}}$ " of Corollary 1.12, (iii), is multiradially defined.

(ii) (Uniradiality of Constant Monoids) Each isomorphism of projective systems of mono-theta environments $\mathbb{M}_{*}^{\Theta}\left(\Pi_{\underline{v}}\right) \stackrel{\widetilde{\sim}}{\rightarrow} \mathbb{M}_{*}^{\Theta}\left({ }^{\dagger} \underline{\underline{\mathcal{F}}}_{\underline{v}}\right)$ induces compatible collections of isomorphisms

$$
\begin{array}{rlccc}
\Pi_{\underline{v}} \stackrel{\sim}{\rightarrow} \Pi_{\underline{\underline{X}}}\left(\mathbb{M}_{*}^{\Theta}\left(\Pi_{\underline{v}}\right)\right) & \stackrel{\sim}{\rightarrow} & \Pi_{\underline{\underline{X}}}\left(\mathbb{M}_{*}^{\Theta}\left({ }^{\dagger} \underline{\underline{\mathcal{F}}}_{\underline{v}}\right)\right) & = & \Pi_{\underline{\underline{X}}}\left(\mathbb{M}_{*}^{\Theta}\left({ }^{\dagger} \underline{\underline{\mathcal{F}}}_{\underline{v}}\right)\right) \\
\curvearrowright & & \curvearrowright & \curvearrowright \\
\Psi_{\mathrm{cns}}\left(\mathbb{M}_{*}^{\Theta}\left(\Pi_{\underline{\underline{v}}}\right)\right) & \stackrel{\sim}{\rightarrow} & \Psi_{\mathrm{cns}}\left(\mathbb{M}_{*}^{\Theta}\left(\underline{\underline{\underline{\mathcal{F}}}}_{\underline{v}}\right)\right) & \stackrel{\sim}{\rightarrow} & \Psi_{\dagger \mathcal{C}_{\underline{v}}}
\end{array}
$$

and

$$
\begin{aligned}
& G_{\underline{v}} \stackrel{\sim}{\rightarrow} G_{\underline{v}}\left(\mathbb{M}_{*}^{\Theta}\left(\Pi_{\underline{v}}\right)\right) \quad \stackrel{\sim}{\rightarrow} \quad G_{\underline{v}}\left(\mathbb{M}_{*}^{\Theta}\left(\underline{\underline{F}}_{\underline{\underline{\mathcal{F}}}}\right)\right) \quad=\quad G_{\underline{\underline{v}}}\left(\mathbb{M}_{*}^{\Theta}\left({ }^{\dagger} \underline{\underline{\mathcal{F}}} \underline{\underline{v}}\right)\right) \\
& \Psi_{\mathrm{cns}}\left(\mathbb{M}_{*}^{\Theta}\left(\Pi_{\underline{v}}\right)\right)^{\times} \stackrel{\sim}{\rightarrow} \quad \Psi_{\mathrm{cns}}\left(\mathbb{M}_{*}^{\Theta}\left(\underline{\underline{F}}_{\underline{\underline{\mathcal{F}}}}\right)\right)^{\times} \stackrel{\sim}{\rightarrow} \quad\left(\Psi_{\dagger \mathcal{C}_{\underline{v}}}\right)^{\times}
\end{aligned}
$$

- where the upper horizontal isomorphisms in each diagram are isomorphisms of topological groups; the lower horizontal isomorphisms in each diagram are isomorphisms of [topological] monoids; the second diagram may be naturally identified with the second displayed commutative diagram of (i); the left-hand square in each diagram arises from the functoriality of the algorithms involved, relative to isomorphisms of projective systems of mono-theta environments; the right-hand square in each diagram arises from the inverse of the displayed isomorphism of Proposition 3.3, (ii); the superscript " $\times$ " denotes the submonoid of units. Finally, if we write $\left(\Psi_{{ }_{C_{\underline{C}}}}\right)^{\times \boldsymbol{\mu}}$ for the topological monoid obtained by forming the quotient of $\left(\Psi_{{ }_{\mathcal{C}_{\underline{v}}}}\right)^{\times}$by its torsion subgroup, then the functorial algorithm

$$
\Pi_{\underline{v}} \mapsto \Psi_{\mathrm{cns}}\left(\mathbb{M}_{*}^{\Theta}\left(\Pi_{\underline{v}}\right)\right)
$$

- where we think of $\Psi_{\mathrm{cns}}\left(\mathbb{M}_{*}^{\Theta}\left(\Pi_{\underline{v}}\right)\right)$ as being equipped with its natural $\Pi_{\underline{\underline{v}}}$-action [cf. Proposition 3.1, (ii)] — obtained by composing the algorithms of Proposition 1.2, (i); 3.1, (ii), depends on the cyclotomic rigidity isomorphism of Corollary 1.11, (b) [cf. Remark 1.11.5, (ii); the use of the surjection of Remark 1.11.5, (i), in the algorithm of Proposition 3.1, (ii)], hence fails to be compatible, relative to the above displayed diagrams, with automorphisms of [the underlying pair, consisting of a topological monoid equipped with the action of a topological group, determined by] the pair

$$
G_{\underline{v}}\left(\mathbb{M}_{*}^{\Theta}\left(\underline{\underline{\underline{\mathcal{F}}}}_{\underline{v}}\right)\right) \curvearrowright\left(\Psi_{\dagger \mathcal{C}_{\underline{v}}}\right)^{\times \mu}
$$

which arise from automorphisms of [the underlying pair, consisting of a topological monoid equipped with the action of a topological group, determined by] the pair 
$G_{\underline{v}}\left(\mathbb{M}_{*}^{\Theta}\left({ }^{\dagger} \underline{\underline{\mathcal{F}}}_{\underline{v}}\right)\right) \curvearrowright\left(\Psi_{\dagger_{\mathcal{C}_{\underline{v}}}}\right) \times$ [cf. Remarks 1.11.1, (i), (b); 1.8.1] - in the sense that this algorithm, as given, only admits a uniradial formulation [cf. Remarks 1.11.3, (iv); 1.11.5, (ii)].

Proof. Assertions (i) and (ii) follow immediately from the definitions and the references quoted in the statements of these assertions.

\section{Remark 3.4.1.}

(i) Note that the pairs

$$
" G_{\underline{v}}\left(\mathbb{M}_{*}^{\Theta}\left({ }^{\dagger} \underline{\underline{\mathcal{F}}}_{\underline{v}}\right)\right) \curvearrowright\left(\Psi_{\dagger \mathcal{F}_{\underline{v}}^{\Theta}}\right) \times \boldsymbol{\mu} " \text { and } " G_{\underline{v}}\left(\mathbb{M}_{*}^{\Theta}\left({ }^{\dagger} \underline{\underline{\mathcal{F}}}_{\underline{v}}\right)\right) \curvearrowright\left(\Psi_{\dagger \mathcal{C}_{\underline{v}}}\right) \times \boldsymbol{\mu} "
$$

that appear in Proposition 3.4, (i), (ii), correspond to the pair " $G \curvearrowright \mathcal{O}^{\times \mu}(G)$ " that appears in the discussion of Remark 1.11.3, (ii) - i.e., the data that arises by replacing the " $\mathcal{O}^{\times}$" that appears in the $\Theta$-link of [IUTchI], Corollary 3.7, (iii), by " $\mathcal{O}^{\times \mu}$ ". That is to say, from the point of view of the present series of papers, the significance of Proposition 3.4 lies in the point of view that

the multiradiality (respectively, uniradiality) asserted in Proposition 3.4, (i) (respectively, (ii)), may be thought of as a statement of the compatibility (respectively, incompatibility) of the algorithm in question with the " $\mathrm{O}^{\times \boldsymbol{\mu}}$-version" of the $\Theta$-link of [IUTchI], Corollary 3.7, (iii).

(ii) One important consequence of the theory to be developed in [IUTchIII] [cf. Remark 2.9.1, (iii)] is the result that,

by applying the theory of log-shells [cf. [AbsTopIII]], one may construct certain algorithms related to the algorithm of Proposition 3.4, (ii), that [yield functors which] are manifestly multiradially defined

— albeit at the cost of allowing for certain [relatively mild!] indeterminacies.

The following two corollaries will play a fundamental role in the present series of papers.

Corollary 3.5. (Mono-theta-theoretic Gaussian Monoids) Let $\mathbb{M}_{*}^{\Theta}$ be as in Proposition 3.1 [cf. also Corollary 2.8, in the case where $\gamma=1$; Remark 3.5.1 below]. For $t \in \operatorname{LabCusp}^{ \pm}\left(\Pi_{\underline{\underline{X}}}\left(\mathbb{M}_{*}^{\Theta}\right)\right)$, we shall denote copies labeled by $t$ of various objects functorially constructed from $\mathbb{M}_{*}^{\Theta}$ by means of a subscript " $t$ ". Also, we shall write

$$
\begin{aligned}
& \Pi_{\underline{\underline{X}}}\left(\mathbb{M}_{*}^{\Theta}\right) \subseteq \Pi_{\underline{X}}\left(\mathbb{M}_{*}^{\Theta}\right) \subseteq \Pi_{C}\left(\mathbb{M}_{*}^{\Theta}\right) \\
& \Delta_{\underline{\underline{X}}}\left(\mathbb{M}_{*}^{\Theta}\right) \subseteq \Delta_{\underline{X}}\left(\mathbb{M}_{*}^{\Theta}\right) \subseteq \Delta_{C}\left(\mathbb{M}_{*}^{\Theta}\right)
\end{aligned}
$$

for the inclusions - which may be functorially constructed from $\Pi_{\underline{\underline{X}}}\left(\mathbb{M}_{*}^{\Theta}\right)$ - corresponding to the inclusions $\Pi_{\underline{v}} \subseteq \Pi_{\underline{v}}^{ \pm} \subseteq \Pi_{\underline{v}}^{\text {cor }}, \Delta_{\underline{v}} \subseteq \Delta_{\underline{v}}^{ \pm} \subseteq \Delta_{\underline{v}}^{\text {cor of Definition 2.3, }}$ (i). 
(i) (Labels, $\mathbb{F}_{l}^{\rtimes \pm}$-Symmetries, and Conjugate Synchronization) If we think of the cuspidal inertia groups $\subseteq \Pi_{\underline{\underline{X}}}\left(\mathbb{M}_{*}^{\Theta}\right)$ corresponding to $t$ as subgroups of cuspidal inertia groups of $\Pi_{\underline{X}}\left(\mathbb{M}_{*}^{\Theta}\right)$ [cf. Remark 2.3.1], then the $\Delta_{\underline{X}}\left(\mathbb{M}_{*}^{\Theta}\right)$-outer action of $\mathbb{F}_{l}^{\rtimes \pm} \cong \Delta_{C}\left(\mathbb{M}_{*}^{\Theta}\right) / \Delta_{X}\left(\mathbb{M}_{*}^{\Theta}\right)$ on $\Pi_{\underline{X}}\left(\mathbb{M}_{*}^{\Theta}\right)$ [cf. Corollary 2.4, (iii)] induces isomorphisms between the pairs

$$
G_{\underline{v}}\left(\mathbb{M}_{* \ddot{\sim}}^{\Theta}\right)_{t} \curvearrowright \Psi_{\mathrm{cns}}\left(\mathbb{M}_{*}^{\Theta}\right)_{t}
$$

- consisting of a labeled topological monoid equipped with the action of a labeled topological group [cf. Proposition 3.1, (ii)] - for distinct $t \in \operatorname{LabCusp}^{ \pm}\left(\Pi_{\underline{X}}\left(\mathbb{M}_{*}^{\Theta}\right)\right)$. We shall refer to these isomorphisms as $\left[\mathbb{F}_{l}^{\rtimes \pm}\right.$-] symmetrizing isomorphisms [cf. Remark 3.5.2 below]. We shall denote by means of a subscript " $|t| \in\left|\mathbb{F}_{l}\right|$ " the result of identifying copies labeled by $t,-t$ via a suitable symmetrizing isomorphism. We shall denote by means of a subscript " || $\mathbb{F}_{l}|\rangle$ " (respectively, " $\left|\mathbb{F}_{l}^{*}\right\rangle$ ") the diagonal embedding, determined by suitable symmetrizing isomorphisms, inside the direct product of copies labeled by $|t| \in\left|\mathbb{F}_{l}\right|$ (respectively, $|t| \in \mathbb{F}_{l}^{*}$ ). In particular, by restricting the monoid $\Psi_{\mathrm{cns}}\left(\mathbb{M}_{*}^{\Theta}\right)$ of Proposition 3.1, (ii), via the restriction operations [i.e., to " $\Pi_{\mathbb{M}^{\Theta}}$ " " and " $D_{t, \mu_{-}}^{\delta}$ "] described in detail in Corollary 2.8, (i), (ii), one obtains a collection of compatible morphisms

$$
\begin{array}{rlcc}
\left(\Pi_{\underline{\underline{X}}}\left(\mathbb{M}_{*}^{\Theta}\right) \hookleftarrow\right) \quad \Pi_{\underline{v}}\left(\mathbb{M}_{* \ddot{\bullet}}^{\Theta}\right) & \rightarrow & G_{\underline{v}}\left(\mathbb{M}_{* \ddot{\bullet}}^{\Theta}\right)\left\langle\left|\mathbb{F}_{l}\right|\right\rangle \\
\curvearrowright & \curvearrowright \\
\Psi_{\mathrm{cns}}\left(\mathbb{M}_{*}^{\Theta}\right) & \stackrel{\sim}{\rightarrow} & \Psi_{\mathrm{cns}}\left(\mathbb{M}_{*}^{\Theta}\right)\left\langle\left|\mathbb{F}_{l}\right|\right\rangle
\end{array}
$$

- where the notation " $\curvearrowright$ " denotes the natural actions; the bottom horizontal arrow is an isomorphism of monoids - which are compatible with the various symmetrizing isomorphisms and well-defined up to composition with an inner automorphism of $\Pi_{\underline{\underline{X}}}\left(\mathbb{M}_{*}^{\Theta}\right)$ [i.e., up to composition with the conjugation action by $\Pi_{\underline{\underline{X}}}\left(\mathbb{M}_{*}^{\Theta}\right)$ on the pair $\left.\Pi_{\underline{v} \ddot{\sim}}\left(\mathbb{M}_{* \ddot{*}}^{\Theta}\right) \curvearrowright \Psi_{\mathrm{cns}}\left(\mathbb{M}_{*}^{\Theta}\right)\right]$. Put another way, this inner automorphism indeterminacy - which, a priori, depends on the index $|t|-i s$, in fact, independent of $|t| \in\left|\mathbb{F}_{l}\right|$.

(ii) (Gaussian Monoids) We shall refer to an element of the set

$$
\left.\left.\stackrel{\theta^{\mathbb{F}_{l}^{*}}}{=}\left(\mathbb{M}_{* \text { env }}^{\Theta}\right)\right) \stackrel{\text { def }}{=} \prod_{|t| \in \mathbb{F}_{l}^{*}} \underline{\theta}_{\text {env }}^{|t|}\left(\mathbb{M}_{* \ddot{\bullet}}^{\Theta}\right)\right) \subseteq \prod_{|t| \in \mathbb{F}_{l}^{*}} \Psi_{\mathrm{cns}}\left(\mathbb{M}_{*}^{\Theta}\right)_{|t|}
$$

[cf. the notation of Corollary 2.8, (ii)] - which is of cardinality $(2 l)^{l^{*}}$ - as a value-profile. Then by applying [the various objects constructed from] the symmetrizing isomorphisms of (i), together with the functorial algorithm [for restricting elements of $\left.\underline{\underline{\theta}}_{\text {env }}^{\iota}\left(\mathbb{M}_{*}^{\Theta}\right), \infty \underline{\underline{\theta}}_{\text {env }}^{\iota}\left(\mathbb{M}_{*}^{\Theta}\right)\right]$ of Corollary 2.8, (ii), one obtains a functorial algorithm for constructing two collections of submonoids

$$
\begin{aligned}
& \mathbb{M}_{*}^{\Theta} \mapsto \\
& \Psi_{\text {gau }}\left(\mathbb{M}_{*}^{\Theta}\right) \stackrel{\text { def }}{=}\left\{\Psi_{\xi}\left(\mathbb{M}_{*}^{\Theta}\right) \stackrel{\text { def }}{=} \Psi_{\text {cns }}^{\times}\left(\mathbb{M}_{*}^{\Theta}\right)_{\left\langle\mathbb{F}_{l}^{*}\right\rangle} \cdot \xi^{\mathbb{N}} \subseteq \prod_{|t| \in \mathbb{F}_{l}^{*}} \Psi_{\text {cns }}\left(\mathbb{M}_{*}^{\Theta}\right)_{|t|}\right\}_{\xi} \\
& \infty \Psi_{\text {gau }}\left(\mathbb{M}_{*}^{\Theta}\right) \stackrel{\text { def }}{=}\left\{\infty \Psi_{\xi}\left(\mathbb{M}_{*}^{\Theta}\right) \stackrel{\text { def }}{=} \Psi_{\text {cns }}^{\times}\left(\mathbb{M}_{*}^{\Theta}\right)_{\left\langle\mathbb{F}_{l}^{*}\right\rangle} \cdot \xi^{\mathbb{Q} \geq 0} \subseteq \prod_{|t| \in \mathbb{F}_{l}^{*}} \Psi_{\text {cns }}\left(\mathbb{M}_{*}^{\Theta}\right)_{|t|}\right\}_{\xi}
\end{aligned}
$$


- where the superscript " $\times$ " denotes the submonoid of units; $\xi$ ranges over the value-profiles; " $\xi \mathbb{Q} \geq 0$ " denotes the submonoid generated by the $N$-th roots [for $N \in$ $\mathbb{N}_{\geq 1}$ ] of $\xi$ [which are uniquely determined, up to multiplication by an element of the $N$-torsion subgroup of $\Psi_{\mathrm{cns}}^{\times}\left(\mathbb{M}_{*}^{\Theta}\right)_{\left\langle\mathbb{F}_{l}^{*}\right\rangle}$ !] that arise by restricting elements of $\infty \underline{\theta}_{\text {env }}^{\iota}\left(\mathbb{M}_{*}^{\Theta}\right)$; each $\Psi_{\xi}\left(\mathbb{M}_{*}^{\Theta}\right)$ is equipped with a natural action by $G_{\underline{v}}\left(\mathbb{M}_{*}^{\Theta}\right)_{\left\langle\mathbb{F}_{l}^{*}\right\rangle}$. We shall refer to each $\Psi_{\xi}\left(\mathbb{M}_{*}^{\Theta}\right)$ or ${ }_{\infty} \Psi_{\xi}\left(\mathbb{M}_{*}^{\Theta}\right)$ as a Gaussian monoid. Here, the submonoid of the $\Psi_{2 l \cdot \xi}\left(\mathbb{M}_{*}^{\Theta}\right) \subseteq \Psi_{\xi}\left(\mathbb{M}_{*}^{\Theta}\right)$ generated by $\Psi_{\mathrm{cns}}^{\times}\left(\mathbb{M}_{*}^{\Theta}\right)_{\left\langle\mathbb{F}_{l}^{*}\right\rangle}$ and $\xi^{2 l \cdot \mathbb{N}}$ is independent of the value-profile $\xi$. Finally, the restriction operations described in detail in Corollary 2.8, (i), (ii), determine a collection of compatible [in the evident sense] morphisms [cf. Remark 3.6.1 below]

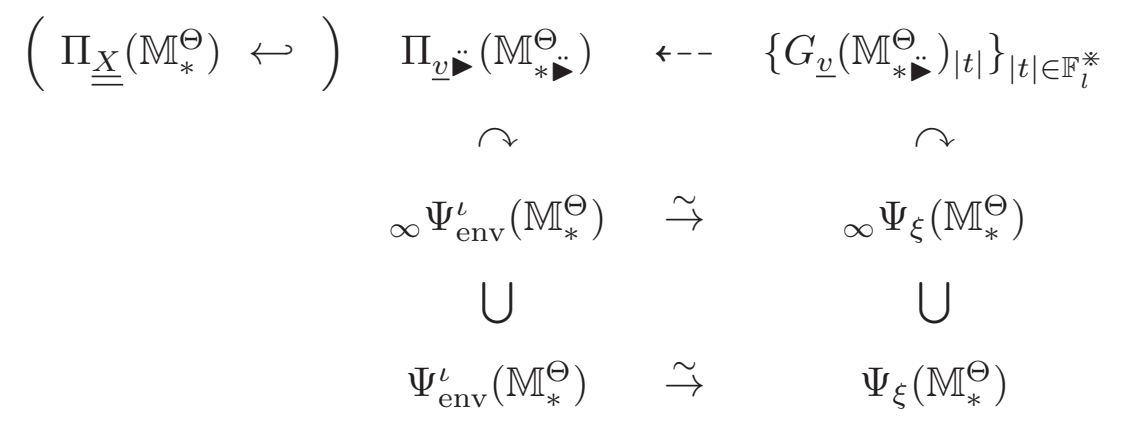

- where the " $\leftarrow--$ " in the first line denotes the compatibility of the action [denoted by the second " $\curvearrowright$ " in the second line] of $G_{\underline{v}}\left(\mathbb{M}_{*}^{\Theta} \Theta_{|t|}\right.$ on the factor labeled " $t \mid$ " of the direct product containing ${ }_{\infty} \Psi_{\xi}\left(\mathbb{M}_{*}^{\Theta}\right)$ [cf. the definition of ${ }_{\infty} \Psi_{\xi}\left(\mathbb{M}_{*}^{\Theta}\right)$ ] with the inclusions $G_{\underline{v}}\left(\mathbb{M}_{*}^{\Theta}\right) \hookrightarrow \Pi_{\underline{v}} \ddot{(}\left(\mathbb{M}_{* \ddot{*}}^{\Theta}\right)$ determined by the various choices of the " $D_{t, \mu_{-}}^{\delta}$ " [cf. Corollary 2.8, (i), (ii)] that gave rise to the value-profile $\xi$; the first " $\curvearrowright$ " in the second line denotes the natural action; the lower/middle horizontal arrows are isomorphisms of monoids - which are well-defined up to composition with an inner automorphism of $\Pi_{\underline{X}}\left(\mathbb{M}_{*}^{\Theta}\right)$ and compatible [in the evident sense] with the equalities of submonoids $\overline{\Psi_{2 l} \cdot \xi_{1}}\left(\mathbb{M}_{*}^{\Theta}\right)=\Psi_{2 l \cdot \xi_{2}}\left(\mathbb{M}_{*}^{\Theta}\right)$ for distinct value-profiles $\xi_{1}$, $\xi_{2}$. For simplicity, we shall use the notation

$$
\Psi_{\mathrm{env}}\left(\mathbb{M}_{*}^{\Theta}\right) \stackrel{\sim}{\rightarrow} \Psi_{\mathrm{gau}}\left(\mathbb{M}_{*}^{\Theta}\right) ; \quad \infty \Psi_{\mathrm{env}}\left(\mathbb{M}_{*}^{\Theta}\right) \stackrel{\sim}{\rightarrow} \infty \Psi_{\mathrm{gau}}\left(\mathbb{M}_{*}^{\Theta}\right)
$$

to denote these collections of compatible morphisms induced by restriction.

(iii) (Constant Monoids and Splittings) Denote the zero element of $\left|\mathbb{F}_{l}\right|$ by $0 \in\left|\mathbb{F}_{l}\right|$. Then [in the notation of $(i)$ ] the diagonal submonoid $\Psi_{\mathrm{cns}}\left(\mathbb{M}_{*}^{\Theta}\right)_{\left\langle\left|\mathbb{F}_{l}\right|\right\rangle}$ determines - i.e., may be thought of as the graph of - an isomorphism of monoids

$$
\Psi_{\mathrm{cns}}\left(\mathbb{M}_{*}^{\Theta}\right)_{0} \stackrel{\sim}{\rightarrow} \Psi_{\mathrm{cns}}\left(\mathbb{M}_{*}^{\Theta}\right)_{\left\langle\mathbb{F}_{l}^{*}\right\rangle}
$$

that is compatible with the respective labeled $G_{\underline{v}}\left(\mathbb{M}_{* \ddot{*}}^{\Theta}\right)$-actions. Moreover, the restriction operations to zero-labeled evaluation points described in detail in Corollary 2.8, (i), (ii), (iii), determine a splitting up to torsion of each of the Gaussian monoids

$$
\Psi_{\xi}\left(\mathbb{M}_{*}^{\Theta}\right)=\Psi_{\mathrm{cns}}^{\times}\left(\mathbb{M}_{*}^{\Theta}\right)_{\left\langle\mathbb{F}_{l}^{*}\right\rangle} \cdot \xi^{\mathbb{N}}, \quad \infty \Psi_{\xi}\left(\mathbb{M}_{*}^{\Theta}\right)=\Psi_{\mathrm{cns}}^{\times}\left(\mathbb{M}_{*}^{\Theta}\right)_{\left\langle\mathbb{F}_{l}^{*}\right\rangle} \cdot \xi^{\mathbb{Q}_{\geq 0}}
$$

[cf. the definition of $\Psi_{\xi}\left(\mathbb{M}_{*}^{\Theta}\right),{ }_{\infty} \Psi_{\xi}\left(\mathbb{M}_{*}^{\Theta}\right)$ in (ii)] which is compatible, relative to the restriction isomorphisms of the third display of (ii), with the splittings up to torsion of Proposition 3.1, (i). 
Proof. The various assertions of Corollary 3.5 follow immediately from the definitions and the references quoted in the statements of these assertions.

\section{Remark 3.5.1.}

(i) Note that in Corollary 3.5, unlike the situation of Corollary 2.8, we took $\gamma$ to be $=1$. This was done primarily to simplify the notation and does not result in any substantive loss of generality. Indeed, one may always simply take the " $\mathbb{M}_{*}^{\Theta}$ " of Corollary 3.5 to be the " $\left(\mathbb{M}_{*}^{\Theta}\right)^{\gamma}$ " of Corollary 2.8. Alternatively, one may observe that the " $\delta$ " that appears in the " $D_{t, \mu_{-}}^{\delta}$ " that occurs in the various restriction operations invoked in Corollary 3.5 [cf. Corollary 2.8, (i), (ii)] is arbitrary, i.e., it is subject to the independent conjugation indeterminacies discussed in Corollary 2.5, (iii); Remark 2.5.2.

(ii) In the present context, it is useful to recall that from the point of view of the discussion of [IUTchI], Remark 3.2.3, (i), the various $\Pi_{\underline{X}}\left(\mathbb{M}_{*}^{\Theta}\right)$-conjugacy indeterminacies that appear in Corollary 3.5 are applied, in the context of the theory of the present series of papers, to identify the various $\Pi_{\underline{\underline{X}}}\left(\mathbb{M}_{*}^{\Theta}\right)$-conjugates

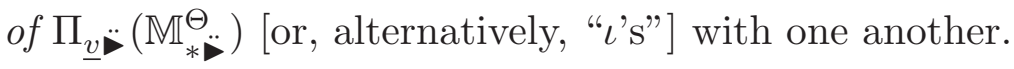

Remark 3.5.2. Before proceeding, it is useful to pause to consider the significance of the symmetrizing isomorphisms of Corollary 3.5, (i).

(i) We begin by discussing a simple combinatorial model of the phenomenon of interest. Consider the totally ordered set $E=\{0,1\}$ whose ordering is completely determined by the inequality

$$
0<1
$$

— which we shall denote, in the following discussion, by the notation " $\prec$ ". Then one may consider labeled copies

$$
\prec_{0}, \quad \prec_{1}
$$

of $\prec$. Now suppose that one attempts to identify these labeled copies $\prec_{0}, \prec_{1}$ by simply forgetting the labels. This amounts, in effect, to sending the two distinct elements of $E$

$$
E \ni 0,1 \mapsto *
$$

to a single point "*". In particular, this naive approach to identifying the labeled copies $\prec_{0}, \prec_{1}$ fails to be compatible - in a sense that we shall examine in more detail in the discussion to follow - with operations that require one to distinguish the two labels $0,1 \in E$. Now if, to avoid confusion, one writes $S$ for the underlying set of $E$ [i.e., obtained from $E$ by forgetting the ordering on $E$ ], then one has a natural Aut $(S)$-orbit of bijections

$$
E \stackrel{\sim}{\rightarrow} S \curvearrowleft \operatorname{Aut}(S)
$$

— where $\operatorname{Aut}(S) \cong \mathbb{Z} / 2 \mathbb{Z}$. Next, let us suppose that we are given an object $F(\prec)$ functorially constructed from [the "totally ordered set of cardinality two"] $\prec$. Then 
any "factorization" of the functorial construction $F(-)$ [i.e., on "totally ordered sets of cardinality two"] through a functorial construction

$$
F^{\mathrm{sym}}(S) \curvearrowleft \operatorname{Aut}(S)
$$

on unordered sets of cardinality two [i.e., relative to the "forgetful functor" that associates to an ordered set the underlying unordered set] may be thought of as a collection of "symmetrizing isomorphisms" [cf. the discussion of (ii) below; Corollary 3.5, (i)], or, alternatively, as "descent data" for $F(-)$ from $E$ to the "orbiset quotient" of $S$ by $\operatorname{Aut}(S)$. Moreover, this "descent data" satisfies the crucial property that it allows one to perform this "descent to the orbiset quotient" in such a way that one is

never required to violate the bijective relationship - albeit via an indeterminate bijection! - between $E$ and $S$.

By contrast, the "naive approach" discussed above may be thought of as corresponding to working with the "coarse set-theoretic quotient" $Q$ of $S$ by $\operatorname{Aut}(S)$ — which we shall think of as consisting of a single point $* \stackrel{\text { def }}{=}\{0,1\} \in Q=\{*\}$. Now suppose, for instance, in the case $F(\prec) \stackrel{\text { def }}{=} \prec$, that one attempts to regard $F(\prec)_{(-)} \stackrel{\text { def }}{=} \prec_{(-)}$[where $\left.(-) \in S\right]$ as an object "pulled back" from a copy $\prec_{Q}$ [i.e., " $0_{Q}<1_{Q}$ "] of $\prec$ over $Q$. On the other hand, if one wishes to relate each point $s \in S$ to one or more points $\in E_{Q} \stackrel{\text { def }}{=}\left\{0_{Q}, 1_{Q}\right\}$ via an $\operatorname{Aut}(S)$-equivariant assignment in such a way that every point of $E_{Q}$ appears in the image of this assignment, then one has no choice but to assign to each point $s \in S$ the collection of all points $\in E_{Q}$. Put another way, one must contend with an independent indeterminacy

$$
s \mapsto 0_{Q} ? \quad 1_{Q} ?
$$

for each $s \in S$ - i.e., if we write $S=\left\{0_{S}, 1_{S}\right\}$, then these indeterminacies give rise to a total of 4 possibilities

$$
\begin{array}{llll}
0_{S} & \mapsto & 0_{Q} ? & 1_{Q} ? \\
1_{S} & \mapsto & 0_{Q} ? & 1_{Q} ?
\end{array}
$$

for the desired assignment, certain of which [i.e., $0_{S}, 1_{S} \mapsto 0_{Q}$ and $0_{S}, 1_{S} \mapsto 1_{Q}$ ] fail to be bijective. Here, it is useful to note that to synchronize these indeterminacies amounts, tautologically, to the requirement of an "automorphism of $\prec_{Q}$ that induces the unique nontrivial automorphism of the set $E_{Q}=\left\{0_{Q}, 1_{Q}\right\}$ ". On the other hand, by the definition of an "inequality", it is a tautology that such an automorphism of $\prec_{Q}$ cannot exist. Finally, in this context, it is useful to recall that this difference between "crushing the set E to a single point" and "symmetrizing without violating the bijective relationship to $E$ " is precisely the topic of the discussion of [IUTchI], Remark 4.9.2, (i); [IUTchI], Remark 6.12.4, (i) — cf., especially, [IUTchI], Fig. 4.5.

(ii) The starting point of the theory surrounding the symmetrizing isomorphisms of Corollary 3.5, (i), is the connectedness - or "single basepoint" observed in the discussion of Remark 2.6.1, (i), together with the compatibility of 
this connectedness with a certain $\mathbb{F}_{l}^{\rtimes \pm}$-symmetry, as discussed in Remark 2.6.2, (i). These symmetrizing isomorphisms may be applied to labeled copies of various objects constructed from $\mathbb{M}_{*}^{\Theta}$ - e.g., $\Psi_{\text {cns }}\left(\mathbb{M}_{*}^{\Theta}\right), G_{\underline{v}}\left(\mathbb{M}_{*}^{\Theta}\right), \Pi_{\mu}\left(\mathbb{M}_{*}^{\Theta}\right)$ - cf. the discussion of "conjugate synchronization" in Remark 2.6.1, (i). Note that in the absence of the $\mathbb{F}_{l}^{\rtimes \pm}$-symmetry involved, the "single basepoint" under consideration has a rigidifying effect not only on the various conjugates involved, but also on the labels under consideration. That is to say, a priori, it is quite possible that

the desired rigidity of the conjugates involved depends on the rigidity of the labels under consideration.

Indeed, this is precisely what happens when the data that one wishes to synchronize - i.e., such as monoids, absolute Galois groups, or cyclotomes - consists, for instance, of an arrow from one label to another, as was [essentially] the case in the discussion of the combinatorial model of (i). Put another way,

the significance of the $\mathbb{F}_{l}^{\rtimes \pm}$-symmetry under consideration lies precisely in the observation that this symmetry serves to eliminate this unwanted "a priori" possibility.

This is in some sense the central principle illustrated by the combinatorial model of (i). Put in another words, this "central principle" discussed in (i) may be summarized, in the situation of Corollary 3.5, as follows: the $\mathbb{F}_{l}^{\rtimes \pm}$-symmetry under consideration allows one to construct

\section{(a) symmetrizing isomorphisms [cf. Corollary 3.5, (i)]}

in a fashion that is compatible with maintaining a

(b) bijective link with the set of labels $\operatorname{LabCusp}^{ \pm}\left(\Pi_{\underline{\underline{X}}}\left(\mathbb{M}_{*}^{\Theta}\right)\right)$

- which is necessary in order to construct the Gaussian monoids [i.e., which involve distinct values at distinct labels!] in Corollary 3.5, (ii) — all relative to

(c) a single basepoint [i.e., which gives rise to the single topological group $\Pi_{\underline{X}}\left(\mathbb{M}_{*}^{\Theta}\right)$ - cf. the discussion of Remark 2.6.2, (i)]

— which is necessary in order to establish conjugate synchronization.

(iii) In the context of Corollary 3.5, (i), one essential aspect of the $\mathbb{F}_{l}^{\rtimes \pm}$ symmetry under consideration is that this symmetry arises from a $\Delta_{\underline{X}}\left(\mathbb{M}_{*}^{\Theta}\right)$-outer action of $\Delta_{C}\left(\mathbb{M}_{*}^{\Theta}\right) / \Delta_{X}\left(\mathbb{M}_{*}^{\Theta}\right) \stackrel{\sim}{\rightarrow} \mathbb{F}_{l}^{\rtimes \pm}$ [cf. the discussion of Remark 2.6.2, (i)]. That is to say, the fact that this action may be formulated entirely in terms of conjugation by elements of geometric [i.e., " $\Delta$ "] fundamental groups - that is to say, as opposed to arithmetic [i.e., "П"] fundamental groups - plays a crucial role in establishing the conjugate synchronization of the various copies of " $G_{\underline{v}}\left(\mathbb{M}_{*}^{\Theta}\right)$ " [and objects constructed from " $G_{\underline{v}}\left(\mathbb{M}_{*}^{\Theta}\right)$ "] under consideration [cf. the discussion of [IUTchI], Remark 6.12.6, (ii)]. 
(iv) If one thinks of the $\mathbb{F}_{l}^{\rtimes \pm}$-symmetries that appear in the conjugate synchronization of Corollary 3.5, (i), as "connecting" the various copies of objects at distinct evaluation points, then it is perhaps natural to regard the "conjugate synchronization via symmetry" of Corollary 3.5, (i), as a sort of nonarchimedean version of the "conjugate synchronization via connectedness" discussed in Remark 2.6.1, (i), which may be thought of as being based on the "archimedean" connectedness of the subgraph $\Gamma_{\underline{\underline{X}}} \subseteq \Gamma_{\underline{\underline{X}}}$ [cf. the discussion of Remarks 2.6.1, (i); 2.8.3].

(v) In $\S 4$ below, we shall generalize the ideas discussed in the present Remark 3.5.2 concerning conjugate synchronization in the case of $\underline{v} \in \underline{\mathbb{V}}^{\text {bad }}$ to the global portion, as well as to the portion at good $\underline{v} \in \underline{\mathbb{V}}^{\text {good }}$, of a $\mathcal{D}$ - $\Theta^{ \pm \text {ell }}$-Hodge theater [cf. the discussions of Remark 2.6.2, (i); Remark 3.8.2 below].

Remark 3.5.3. The delicacy and subtlety of the theory surrounding Corollary 3.5, (i), may be thought of as a consequence of the requirement of simultaneously satisfying the conditions (a), (b), (c) discussed in Remark 3.5.2, (ii). On the other hand, if one is willing to eliminate condition (c) from one's arguments, then one may obtain symmetrizing isomorphisms by simply applying the functors of [IUTchI], Proposition 6.8, (i), (ii), (iii); [IUTchI], Proposition 6.9, (i), (ii) — i.e., by passing to $\mathcal{D}$ - $\Theta^{\text {ell }}$-bridges or [holomorphic or mono-analytic] capsules or processions. Here, we observe that this "multi-basepoint" approach to constructing symmetrizing isomorphisms is compatible with the single basepoint $\mathbb{F}_{l}^{\rtimes \pm}$-symmetric approach of Corollary 3.5, (i), relative to the evident "forgetful functors". We leave the routine details to the reader.

Corollary 3.6. (Frobenioid-theoretic Gaussian Monoids) Suppose that we are in the situation of Proposition 3.3, i.e., that

$$
\mathbb{M}_{*}^{\Theta}=\mathbb{M}_{*}^{\Theta}\left(\underline{\underline{\mathcal{F}}}_{\underline{v}}\right)
$$

- where ${ }^{\dagger} \underline{\underline{\mathcal{F}}}_{\underline{v}}$ is a tempered Frobenioid. We continue to use the conventions introduced in Corollary 3.5 concerning subscripted labels.

(i) (Labels, $\mathbb{F}_{l}^{\rtimes \pm}$-Symmetries, and Conjugate Synchronization) The isomorphism of Proposition 3.3, (ii), determines, for each $t \in \operatorname{LabCusp}^{ \pm}\left(\Pi_{\underline{\underline{X}}}\left(\mathbb{M}_{*}^{\Theta}\right)\right)$, a collection of compatible morphisms

$$
\begin{aligned}
\left(\Pi_{\underline{\underline{X}}}\left(\mathbb{M}_{*}^{\Theta}\right)_{t} \rightarrow\right) \quad G_{\underline{\underline{v}}}\left(\mathbb{M}_{*}^{\Theta}\right)_{t} & \stackrel{\sim}{\rightarrow} G_{\underline{\underline{v}}}\left(\mathbb{M}_{* \ddot{\bullet}}^{\Theta}\right)_{t} \\
\curvearrowright & \curvearrowright \\
\left(\Psi_{\mathcal{C}_{\underline{\underline{v}}}}\right)_{t} & \stackrel{\sim}{\rightarrow} \Psi_{\mathrm{cns}}\left(\mathbb{M}_{*}^{\Theta}\right)_{t}
\end{aligned}
$$

- which are well-defined up to composition with an inner automorphism of $\Pi_{\underline{\underline{X}}}\left(\mathbb{M}_{*}^{\Theta}\right)$ which is independent of $t \in \operatorname{LabCusp}^{ \pm}\left(\Pi_{\underline{\underline{X}}}\left(\mathbb{M}_{*}^{\Theta}\right)\right)$ - as well as $\left[\mathbb{F}_{l}^{\rtimes \pm}{ }_{-}\right.$ ]symmetrizing isomorphisms, induced by the $\left.\Delta_{\underline{X}} \overline{(\mathbb{M}}_{*}^{\Theta}\right)$-outer action of $\mathbb{F}_{l}^{\rtimes \pm} \cong$ $\Delta_{C}\left(\mathbb{M}_{*}^{\Theta}\right) / \Delta_{\underline{X}}\left(\mathbb{M}_{*}^{\Theta}\right)$ on $\Pi_{\underline{X}}\left(\mathbb{M}_{*}^{\Theta}\right)$ [cf. Corollary 3.5, (i)], between the data indexed by distinct $t \in \operatorname{LabCusp}^{ \pm}\left(\Pi_{\underline{X}}\left(\mathbb{M}_{*}^{\Theta}\right)\right)$. 
(ii) (Gaussian Monoids) For each value-profile $\xi$ [cf. Corollary 3.5, (ii)], write

$$
\Psi_{\mathcal{F}_{\xi}}\left({ }^{\dagger} \underline{\underline{\mathcal{F}}}_{\underline{v}}\right) \subseteq \infty \Psi_{\mathcal{F}_{\xi}}\left({ }^{\dagger} \underline{\underline{\mathcal{F}}}_{\underline{v}}\right) \subseteq \prod_{|t| \in \mathbb{F}_{i}^{*}}\left(\Psi_{\dagger \mathcal{C}_{\underline{v}}}\right)|t|
$$

for the submonoids determined, respectively, via the isomorphisms $\left(\Psi_{{ }_{\mathcal{C}_{\underline{v}}}}\right)_{|t|} \stackrel{\sim}{\rightarrow}$ $\Psi_{\mathrm{cns}}\left(\mathbb{M}_{*}^{\Theta}\right)_{|t|}$ of (i), by the monoids $\Psi_{\xi}\left(\mathbb{M}_{*}^{\Theta}\right), \infty \Psi_{\xi}\left(\mathbb{M}_{*}^{\Theta}\right)$ of Corollary 3.5, (ii), and

$$
\Psi_{\mathcal{F}_{\text {gau }}}\left(\underline{\underline{\mathcal{F}}}_{\underline{\underline{v}}}\right) \stackrel{\text { def }}{=}\left\{\Psi_{\mathcal{F}_{\xi}}\left(\mathbb{M}_{*}^{\Theta}\right)\right\}_{\xi}, \quad \infty \Psi_{\mathcal{F}_{\text {gau }}}\left({ }^{\dagger} \underline{\underline{\mathcal{F}}}_{\underline{v}}\right) \stackrel{\text { def }}{=}\left\{\infty \Psi_{\mathcal{F}_{\xi}}\left(\mathbb{M}_{*}^{\Theta}\right)\right\}_{\xi}
$$

- where $\xi$ ranges over the value-profiles. Thus, each monoid $\Psi_{\mathcal{F}_{\xi}}\left({ }^{\dagger} \underline{\underline{\mathcal{F}}}_{\underline{\underline{v}}}\right)$ is equipped with a natural action by $G_{\underline{v}}\left(\mathbb{M}_{*}^{\Theta}\right)_{\left\langle\mathbb{F}_{l}^{*}\right\rangle}$. Then by composing the Kummer isomorphisms discussed in (i) above and Proposition 3.3, (i), (ii), with the restriction isomorphisms of Corollary 3.5, (ii), one obtains a diagram of compatible morphisms

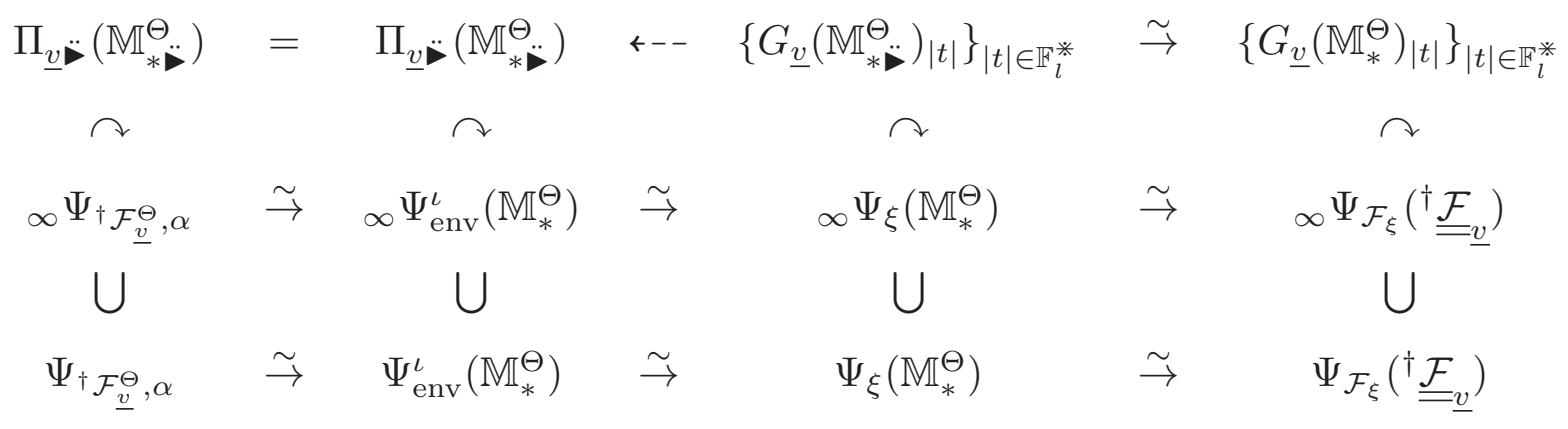

- where the " +-- " in the first line [cf. also the third and fourth " $\curvearrowright$ " in the second line] is as in Corollary 3.5, (ii); we recall the natural inclusion $\Pi_{\underline{v}} \ddot{\sim}\left(\mathbb{M}_{* \ddot{*}}^{\Theta}\right) \hookrightarrow$ $\Pi_{\underline{\underline{X}}}\left(\mathbb{M}_{*}^{\Theta}\right)$ - which are well-defined up to composition with an inner automorphism of $\Pi_{\underline{X}}\left(\mathbb{M}_{*}^{\Theta}\right)$ and compatible [in the evident sense] with the equalities of submonoids involving " $\Psi_{2 l \cdot \xi}(-)$ " [cf. Corollary 3.5, (ii)]. For simplicity, we shall use the notation

$$
\begin{aligned}
& \Psi_{\dagger \mathcal{F}_{\underline{v}}^{\Theta}} \stackrel{\sim}{\rightarrow} \Psi_{\mathrm{env}}\left(\mathbb{M}_{*}^{\Theta}\right) \stackrel{\sim}{\rightarrow} \Psi_{\mathrm{gau}}\left(\mathbb{M}_{*}^{\Theta}\right) \stackrel{\sim}{\rightarrow} \Psi_{\mathcal{F}_{\mathrm{gau}}}\left(\underline{\underline{\underline{\mathcal{F}}}}_{\underline{\underline{v}}}\right) ; \\
& \infty \Psi_{\dagger \mathcal{F}_{\underline{v}}^{\Theta}} \stackrel{\sim}{\rightarrow} \infty \Psi_{\text {env }}\left(\mathbb{M}_{*}^{\Theta}\right) \stackrel{\sim}{\rightarrow} \infty \Psi_{\text {gau }}\left(\mathbb{M}_{*}^{\Theta}\right) \stackrel{\sim}{\rightarrow} \infty \Psi_{\mathcal{F}_{\text {gau }}}\left({ }^{\dagger} \underline{\underline{\mathcal{F}}}_{\underline{v}}\right)
\end{aligned}
$$

to denote these collections of compatible morphisms.

(iii) (Constant Monoids and Splittings) Relative to the notational conventions adopted thus far [cf. also Corollary 3.5, (iii)], the diagonal submonoid $\left(\Psi_{{ }_{\mathcal{C}_{\underline{v}}}}\right)_{\left\langle\left|\mathbb{F}_{l}\right|\right\rangle}$ determines - i.e., may be thought of as the graph of - an isomorphism of monoids

$$
\left(\Psi_{\dagger \mathcal{C}_{\underline{v}}}\right)_{0} \stackrel{\sim}{\rightarrow} \quad\left(\Psi_{\dagger \mathcal{C}_{\underline{v}}}\right)_{\left\langle\mathbb{F}_{l}^{*}\right\rangle}
$$

that is compatible with the respective labeled $G_{\underline{v}}\left(\mathbb{M}_{*}^{\Theta}\right)$-actions. Moreover, the splittings of Corollary 3.5, (iii), determine splittings up to torsion of each of the ["Frobenioid-theoretic"] Gaussian monoids

$$
\Psi_{\mathcal{F}_{\xi}}\left({ }^{\dagger} \underline{\underline{\mathcal{F}}}_{\underline{v}}\right)=\left(\Psi_{{ }^{\dagger} \underline{C}_{\underline{v}}}^{\times}\right)_{\left\langle\mathbb{F}_{l}^{*}\right\rangle} \cdot \operatorname{Im}(\xi)^{\mathbb{N}}, \quad \infty \Psi_{\mathcal{F}_{\xi}}\left({ }^{\dagger} \underline{\underline{\mathcal{F}}}_{\underline{v}}\right)=\left(\Psi_{{ }^{\dagger} \underline{C}_{\underline{v}}}^{\times}\right)_{\left\langle\mathbb{F}_{l}^{*}\right\rangle} \cdot \operatorname{Im}(\xi)^{\mathbb{Q}_{\geq 0}}
$$


- where $\operatorname{Im}(\xi)$ " denotes the image of $\xi$ via the isomorphisms discussed in (ii) which are compatible, relative to the various isomorphisms of the third display of (ii), with the splittings up to torsion of Proposition 3.1, (i); Proposition 3.3, (i); Corollary 3.5, (iii).

Proof. The various assertions of Corollary 3.6 follow immediately from the definitions and the references quoted in the statements of these assertions.

Remark 3.6.1. The "Galois compatibility" denoted by the " $\star--^{-}$" in the third display of Corollaries 3.5, (ii); 3.6, (ii) - involving the monoids " $\infty_{\infty} \Psi$ " [i.e., not just the monoids " $\Psi$ "!] — corresponds precisely to the "Galois functoriality" [cf. Fig. 1.5] of the discussion of Remark 1.12.4.

Remark 3.6.2. The diagram in the third display of Corollary 3.6, (ii) which may be thought of a sort of concrete realization of the principle of Galois evaluation discussed in Remark 1.12.4 [cf. also Remark 3.6.1] — will play a central role in the theory of the present series of papers. Thus, it is of interest to pause here to discuss various aspects of the significance of this diagram.

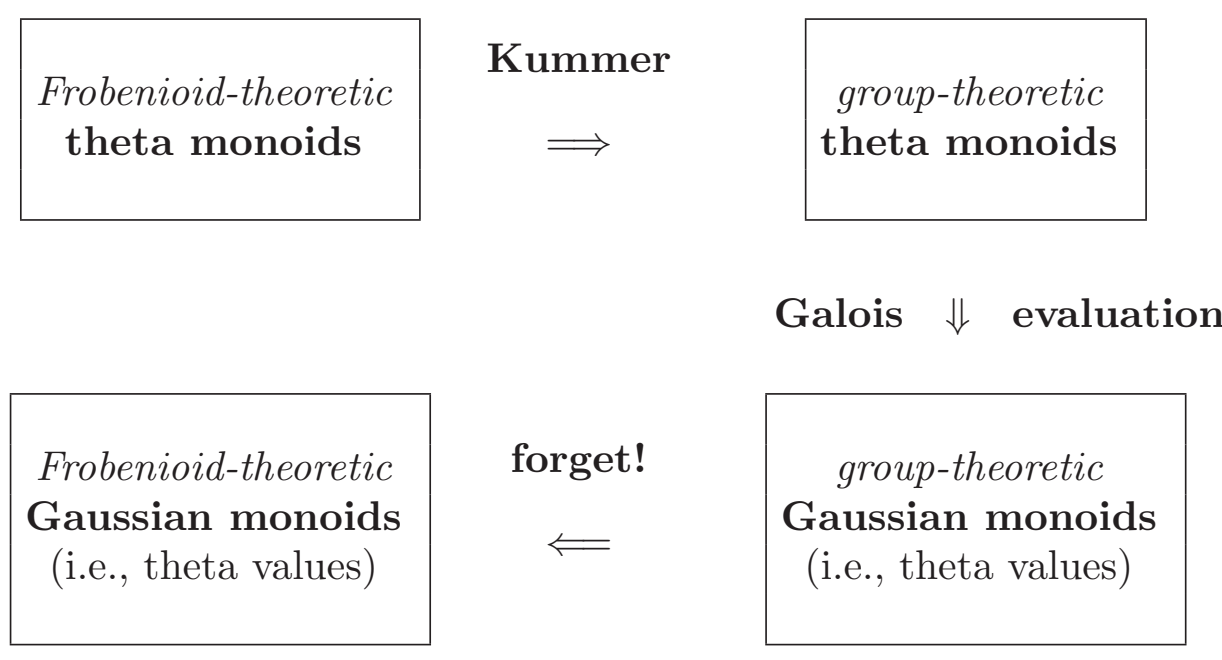

Fig. 3.1: Kummer theory and Galois evaluation

(i) The left-hand, central, and right-hand portions of this diagram are summarized, at a more conceptual level, in Fig. 3.1 - that is to say, if one thinks of the mono-theta environments " $\mathbb{M}_{*}^{\Theta}$ " involved as arising group-theoretically [which is, of course, always the case up to isomorphism! - cf. the situation discussed in Corollary 3.7, (i), below], then these portions correspond, respectively, to the

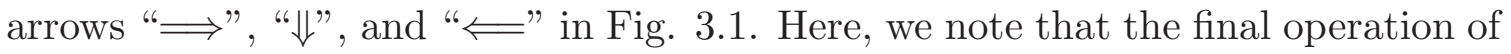
"forgetting" [i.e., "£"] may be thought of as the operation of forgetting the grouptheoretic - i.e., "anabelian" - construction of the Gaussian monoids, so as to obtain "abstract monoids stripped of any information concerning the group-theoretic algorithms used to construct them" - which we refer to as "post-anabelian" [cf. the discussion of Remark 1.11.3, (iii); Corollary 3.7, (i), below; the constructions of Definition 3.8 below]. On the other hand, the composite of the arrows “ $\Longrightarrow$ " and " $\Downarrow$ " may be thought of as a sort of 
comparison isomorphism between "Frobenius-like" [i.e., "Frobenioidtheoretic"] and "étale-like" [i.e., "group-theoretic"] structures

— cf. the discussion of [FrdI], Introduction; [IUTchI], Corollaries 3.8, 3.9. In this context, it is useful to recall that the comparison isomorphism of the "classical" scheme-theoretic version of Hodge-Arakelov theory [cf. [HASurI], Theorem A] is obtained precisely by evaluating theta functions and their derivatives at certain torsion points of an elliptic curve.

(ii) The existence of both "Frobenius-like" and "étale-like" structures in the theory of the present series of papers, together with the somewhat complicated theory of comparison isomorphisms as discussed above in (i), prompts the following question:

What are the various merits and demerits of "Frobenius-like" and "étalelike" structures that require one to avail oneself of both types of structure in the theory of the present series of papers [cf. Fig. 3.2 below]?

On the one hand, unlike Frobenius-like structures, étale-like structures — in the form of étale or tempered fundamental groups [such as Galois groups] - have the crucial advantage of being functorial or invariant with respect to various nonring/scheme-theoretic filters between distinct ring/scheme theories. In the context of the present series of papers, the main examples of this phenomenon consist of the $\Theta$-link [cf., e.g., [IUTchI], Corollary 3.7] and the log-wall [cf. [AbsTopIII], §I1, §I4; this theory will be incorporated into the present series of papers in [IUTchIII]]. Another important characteristic of the étale-like structures constituted by étale or tempered fundamental group is their "remarkable rigidity" - a property that is exhibited explicitly [cf., e.g., the theory of [EtTh]; [AbsTopIII]] by various anabelian algorithms that may be applied to construct, in a "purely group-theoretic fashion", various structures motivated by conventional scheme theory. By contrast, the Frobenius-like structures constituted by various abstract monoids - which typically give rise to various Frobenioids — satisfy the crucial property of not being subject to such rigidifying anabelian algorithms that relate various étale-like structures to conventional scheme theory. It is precisely this property of such abstract monoids that allows one use these abstract monoids to construct such non-scheme-theoretic filters as the $\Theta$-link [cf. [IUTchI], Corollary 3.7] or the $\mathfrak{l o g}$-wall of the theory of [AbsTopIII]. Here, it is interesting to observe that

these merits/demerits of étale-like and Frobenius-like structures play somewhat complementary roles with respect to binding/not binding the structures under consideration to conventional scheme theory.

Finally, we note that Kummer theory serves the crucial role [cf. the discussion of (i)] of relating [via various comparison isomorphisms — cf. (i)] — within a given Hodge theater - potentially non-scheme-theoretic Frobenius-like structures to étale-like structures which are subject to anabelian rigidifications that bind them to conventional scheme theory. 
(iii) If one composes the correspondence " $\underline{\underline{q}} \underline{\underline{\Theta}} \mapsto \underline{\underline{\Theta}} \underline{\underline{v}}$ " [cf. the discussion of [IUTchI], Remark 3.8.1, (i)] constituted by the $\bar{\Theta}$-link - i.e., which relates the " $(n+1)$-th generation q-parameter" to the " $n$-th generation $\Theta$-function" - with

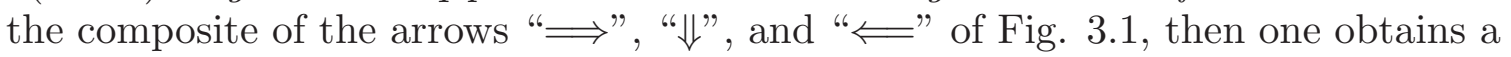
correspondence

$$
\left.\underline{\underline{q}} \underline{v} \mapsto \quad \mapsto \underline{\underline{q}}_{\underline{v}}^{j^{2}}\right\}_{1 \leq j \leq l}
$$

[cf. Remark 2.5.1, (i)]. In fact, in the theory of the present series of papers, it is ultimately this "modified version of the $\Theta$-link" — i.e., which takes into account the Hodge-Arakelov-theoretic evaluation theory developed so far in $\S 2$ and the present $\S 3$ - that will be of interest to us. The theory of this "modified version of the $\Theta$-link" will constitute one of the main topics treated in $\S 4$ below. Here, we observe that the above correspondence may be thought of as a sort of "abstract, combinatorial Frobenius lifting" — i.e., as a sort of "homotopy" between

• the identity $\underline{\underline{q}} \underline{\underline{v}} \mapsto \underline{\underline{q}} \underline{\underline{v}}$ [i.e., which corresponds to "characteristic zero"]

and

- the purely monoid-theoretic/highly non-scheme-theoretic correspondence $\underline{\underline{q}} \underline{\underline{v}} \mapsto \underline{\underline{q}}_{\underline{\underline{v}}}^{(l *)^{2}}$ [i.e., which corresponds to the "positive characteristic Frobenius morphism"].

Moreover, we recall [cf. the discussion of Remark 2.6.3] that the collection of exponents $\left\{j^{2}\right\}_{1 \leq j \leq l *}$ that appear in this "abstract, combinatorial Frobenius lifting" is highly distinguished - hence, in particular, far from arbitrary!

\begin{tabular}{|c|c|}
\hline $\begin{array}{c}\text { étale-like structures } \\
\text { functoriality/invariance } \\
\text { with respect to } \\
\text { log-wall, } \Theta \text {-link }\end{array}$ & Frobenius-like structures \\
\hline $\begin{array}{c}\text { rigidified relationship via } \\
\text { Kummer theory } \\
+ \text { anabelian geom. } \\
\text { to conventional arith. geom. }\end{array}$ & \\
\hline & $\begin{array}{c}\text { lack of rigification allows construction } \\
\text { of non-scheme-theoretic filters, } \\
\text { such as log-wall, } \Theta \text {-link }\end{array}$ \\
\hline
\end{tabular}

Fig. 3.2: Étale-like versus Frobenius-like structures 
(iv) In the context of the discussion of (i), it is of interest to recall that various "Grothendieck Conjecture-type results" in anabelian geometry [e.g., over p-adic local fields and finite fields] - i.e., which may be thought of as comparison isomorphisms between polynomial-function-theoretic and group-theoretic collections of morphisms - are obtained precisely by considering the "Galois evaluation" via Kummer theory of polynomial functions or differential forms at various rational points cf. the theory of $[p \mathrm{GC}]$; [Cusp], $\S 2$.

Remark 3.6.3. Before proceeding, we make some observations concerning basepoints in the context of the "non-ring/scheme-theoretic filters" discussed in Remark 3.6.2.

(i) First, let us recall from the elementary theory of étale fundamental groups that the fiber functor associated to a basepoint is defined by considering the points of a finite étale covering valued in some separably closed field that lie over a fixed point [valued in the same separably closed field] of the base scheme over which the covering is given. Thus, for instance, when this base scheme is the spectrum of a field, the finite set of points associated by the fiber functor to a finite étale covering is obtained by considering the various ring homomorphisms from this field into some separably closed field. In particular, it follows that

the conventional scheme-theoretic definition of a basepoint [in the form of a fiber functor] depends, in an essential fashion, on the ring/scheme structure of the rings or schemes under consideration.

One immediate consequence of these elementary considerations - which is of central importance in the theory of the present series of papers - is the following $o b$ servation concerning the "non-ring/scheme-theoretic filters" discussed in Remark 3.6.2, which relate one ring to another in a fashion that is incompatible with the respective ring structures:

The distinct ring structures on either side of one of the "non-ring/ scheme-theoretic filters" discussed in Remark 3.6.2 — i.e., the log-wall of [AbsTopIII] and the $\Theta$-link of [IUTchI], Corollary 3.7 - give rise to distinct, unrelated basepoints [cf. the discussion of [AbsTopIII], Remark $3.7 .7,(\mathrm{i})]$.

In some sense, the above discussion may be thought of as an "expanded, leisurely version" of an observation made at the beginning of the discussion of [AbsTopIII], Remark 3.7.7, (i)].

(ii) The observations of (i) also apply to the "N-th power morphisms" [where $N>1]$ - i.e., "morphisms of Frobenius type" - that appear in the theory of Frobenioids [cf. [FrdI], [FrdII], [EtTh]]. That is to say, in the context of the tempered Frobenioids that appear in the theory of [EtTh], $\S 5$, such "morphisms of Frobenius type" [i.e., " $N$-th power morphisms" regarded as morphisms contained in the underlying categories associated to these tempered Frobenioids] induce " $N$-th power morphisms" between various monoids [arising from the Frobenioid structure] isomorphic to $\mathcal{O}_{K_{\underline{v}}}^{\triangleright}$. In particular, 
these $N$-th power morphisms of monoids fail [since $N>1$ ] to preserve the ring structure of $K_{\underline{v}}$, hence give rise to distinct, unrelated basepoints on the domain and codomain objects of the original "morphism of Frobenius type" [cf. the discussion of (i)].

On the other hand, let us observe that unlike the situations considered in the discussion of (i), the considerations of the present discussion involving $N$-th power morphisms take place in a fashion that is compatible with the projection functor to the base category of the Frobenioid. One important consequence of this last observation is that unlike the situations discussed in (i) involving the log-wall and the $\Theta$-link in which one must consider arbitrary isomorphisms of topological groups between the étale [or tempered] fundamental groups that arise in the domain and the codomain of the operation under consideration,

in the situation of the present discussion of $N$-th power morphisms, the "distinct, unrelated basepoints" that arise only give rise to inner automorphisms of the topological group determined by [i.e., roughly speaking, the "fundamental group" of] the base category.

This phenomenon may be thought of as a reflection of the fact that the application of an $N$-th power morphism is somewhat "milder" than the log-wall or $\Theta$-link considered in (i) in that it only involves an operation - i.e., raising to the $N$-th power - that is "algebraic", in the sense that it is defined with respect to the ring structure of the ring [e.g., $K_{\underline{v}}$ ] involved. This somewhat "milder nature" of an $N$-th power morphism allows one to consider $N$-th power morphisms within a single category [namely, the tempered Frobenioid under consideration] which can be defined in terms of [formal] flat $\mathcal{O}_{K_{\underline{v}}}$-schemes [cf. the point of view of [EtTh], $\S 1$. By contrast, the operation inherent in the log-wall or $\Theta$-link considered in (i) is much more drastic and arithmetic [i.e., "non-algebraic"] in nature, and it is difficult to see how to fit such an operation into a single category that somehow "extends" the tempered Frobenioid under consideration in a fashion that "lies over" the same base category as the tempered Frobenioid — cf., e.g., Remark 1.11.2, (ii), in the case of the $\Theta$-link; the discussion of [AbsTopIII], Remark 3.7.7, in the case of the log-wall. Put another way,

the highly nontrivial study of the mathematical structures "generated by the log-wall and $\Theta$-link" is, in some sense, one of the main themes of the theory of the present series of papers

— cf., especially, the theory of [IUTchIII]!

Remark 3.6.4. Since the theory of mono-theta environments developed in [EtTh] plays a fundamental role in the theory of the present paper - cf., e.g., Corollaries 1.12, 2.8, 3.5, 3.6 - it is of interest to pause to review the relationship of the theory of [EtTh] to the theory developed so far in the present paper.

(i) The various remarks following [EtTh], Corollary 5.12, discuss the significance of the various rigidity properties of a mono-theta environment that are verified 
in [EtTh]. The logical starting point of this discussion is the situation considered in [EtTh], Remark 5.12.1, consisting of an abstract category which is only known up to isomorphism [i.e., up to an indeterminate equivalence of categories], and in which each of the objects is only known up to isomorphism. The main example of such a category, in the context of the theory of [EtTh], is a tempered Frobenioid of the sort considered in Propositions 3.3, 3.4; Corollary 3.6. The situation of [EtTh], Remark 5.12.1, in which each of the objects in the category is only known up to isomorphism, contrasts sharply with the notion of a system, or tower, of [specific!] coverings - e.g., of the sort that appears in Kummer theory, in which the coverings are related by [specific!] $N$-th power morphisms. Indeed, the various rigidity properties verified in [EtTh] are of interest precisely because

they yield effective reconstruction algorithms for reconstructing the various structures of interest in a fashion that is invariant with respect to the indeterminacies that arise from a situation in which each of the objects in the category is only known up to isomorphism.

This prompts the following question:

What is the fundamental reason, in the context of the theory of the present series of papers, that one must work under the assumption that each of the objects in the category is only known up to isomorphism, thus requiring one to avail oneself of the rigidity theory of [EtTh]?

To understand the answer to this question, let us first observe that Kummer towers involving [specific!] $N$-th power morphisms are constructed by using the multiplicative structure of the "rational functions" [such as the $p_{\underline{v}}$-adic local field $K_{\underline{v}}$ ] under consideration. That is to say, the $N$-th power morphisms are compatible with the multiplicative structure, but not the additive structure of such rational functions. On the other hand, ultimately,

when, in [IUTchIII], we consider the theory of the log-wall [cf. [AbsTopIII]], it will be of crucial importance to consider, within each Hodge theater, the ring structure [i.e., both the multiplicative and additive structures] of the fields $K_{\underline{v}}$.

That is to say, without the ring structure on $K_{\underline{v}}$, one cannot even define the $p_{\underline{v}^{-}}$ adic logarithm! Put another way, the $N$-th power morphisms that appear in a Kummer tower may be thought of as "Frobenius morphisms of a sort" that relate distinct ring structures - i.e., since the $N$-th power morphism fails to be compatible with addition! In particular, the distinct ring structures that exist in the domain and codomain of such a "Frobenius morphism" necessarily give rise to distinct, unrelated basepoints [cf. the discussion of Remark 3.6.3, (ii)] - i.e., at an abstract category-theoretic level, to objects which are only known up to isomorphism! This is what requires one to contend with the indeterminacies discussed in [EtTh], Remark 5.12.1.

(ii) The theory of [EtTh] may be summarized as asserting that one may reconstruct various structures of interest from a mono-theta environment without 
sacrificing certain fundamental rigidity properties, even in a situation subject to certain indeterminacies [cf. (i)]. Moreover, mono-theta environments serve as a sort of bridge [cf. [EtTh], Remark 5.10.1] between tempered Frobenioids - i.e., "Frobenius-like structures" [cf. Remark 3.6.2] — as in Propositions 3.3, 3.4; Corollary 3.6, on the one hand, and tempered fundamental groups [cf. Proposition 3.4] — i.e., "étale-like structures [cf. Remark 3.6.2] — on the other.

(iii) One central feature of the theory of [EtTh] is an explanation of the special role played by the first power of the [reciprocal of the $l$-th root of the] theta function, a role which is reflected in the theory of cyclotomic rigidity developed in [EtTh] [cf. [EtTh], Introduction]. Note that the operation of Galois evaluation is necessarily linear [cf. the discussion of Remark 1.12.4]. This linearity may be

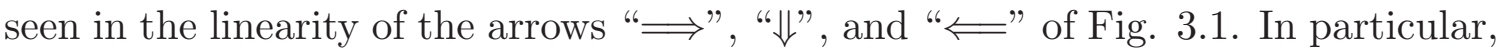
these arrows are compatible with the ring str. on the constants [i.e., " $K_{\underline{v}}$ "] — a property that will be of crucial importance when, in [IUTchIII], we consider the theory of the log-wall [cf. the discussion of (i) above]. Moreover, this linearity property of the operation of Galois evaluation implies that

the first power of the theta values of the [reciprocal of the l-th root of the] theta function "inherits", so to speak, the special role played by the first power of the [reciprocal of the $l$-th root of the] theta function.

This observation is interesting in light of the discussions of Remarks 2.6.3; 3.6.2, (iii).

(iv) In the context of (iii), we note that the various theta monoids discussed in Propositions 3.1, 3.3, as well as the various Gaussian monoids discussed in Corollaries 3.5, 3.6, involve arbitrary powers/roots of the [reciprocal of the $l$-th root of the] theta function. Nevertheless, it is important to remember that

in order to apply the $\Theta$-link — which requires one to work with "Frobenius-like structures" [cf. the discussion of Remark 3.6.2, (ii)] — it is necessary to consider the operation of Galois evaluation summarized in Fig. 3.1 applied to the first power of the [reciprocal of the $l$-th root of the] Frobenioid-theoretic theta function in order to avail oneself of the cyclotomic rigidity furnished by the delicate bridge constituted by the mono-theta environment

— cf. (ii) above. That is to say, the "narrow bridge" afforded by the mono-theta environment between the worlds of "Frobenius-like" and "étale-like" structures may only be crossed by the first power of the [reciprocal of the $l$-th root of the] theta function and its theta values. Put another way,

from the point of view of the étale-like portion [i.e., "group-theoretic"] of the operation of Galois evaluation summarized in Fig. 3.1, the $N$-th power of the [reciprocal of the $l$-th root of the] Frobenioid-theoretic theta

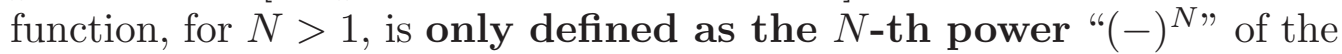
first power of the [reciprocal of the $l$-th root of the] Frobenioid-theoretic theta function. 
That is to say, from the point of view of the étale-like portion of the operation of Galois evaluation summarized in Fig. 3.1, the $N$-th power of the [reciprocal of the $l$-th root of the] Frobenioid-theoretic theta function, for $N>1$ - hence, in particular, the $\Theta$-link - may only be calculated by forming the $N$-th power " $(-)^{N}$ " of the first power of the [reciprocal of the $l$-th root of the] Frobenioidtheoretic theta function.

(v) The necessity of working with "Frobenius-like structures" [cf. the discussion of (iv)] may also be thought of as the necessity of working with the various postanabelian monoids arising from the group-theoretic "anabelian" algorithms that appear in the operation of Galois evaluation [cf. the discussion of Remark 3.6.2, (i)]. In the context of this observation, it is useful to recall that from the point of view of the theory of $\S 1$,

the "narrow bridge" furnished by [for instance, the cyclotomic rigidity of] a mono-theta environment satisfies the crucial property of multiradiality [cf. Corollaries 1.10, 1.12] — i.e., of being "horizontal" with respect to the "connection structure" determined by the formulation of this multiradiality [cf. the point of view discussed in Remarks 1.7.1, 1.9.2].

Put another way, to work with powers other than the first power of the [reciprocal of the $l$-th root of the] theta function or its theta values gives rise to structures with are "not horizontal" with respect to this "connection structure". This point of view is consistent with the point of view of Remark 3.6.5, (iii), below. A similar observation concerning multiradiality will also apply to the "multiradial versions of the Gaussian monoids" that will be constructed in [IUTchIII] [cf. Remark 3.7.1 below].

Remark 3.6.5. In light of the central role played by mono-theta-theoretic cyclotomic rigidity in the discussion of Remark 3.6.4, we pause to make some observations - of a somewhat more philosophical nature - concerning this topic.

(i) First of all, we observe that

a cyclotome may be thought of as a sort of "skeleton of the arithmetic holomorphic structure" under consideration

— cf. the discussion of Remark 1.11.6. Indeed, this point of view may be thought of as being motivated by the situation at archimedean primes, where the circle " $\mathbb{S}$ " may be thought of as a sort of "representative skeleton of $\mathbb{C}^{\times}$". This point of view will play a central role in the remainder of the discussion of the present Remark 3.6.5, as well as in the discussion of Remark 3.8.3 below.

(ii) In the theory of [EtTh],

(a) the commutator structure $[-,-]$ of the theta group plays a central role in the theory of mono-theta-theoretic cyclotomic rigidity 
- cf. [EtTh], Introduction; [EtTh], Remark 2.19.2. On the other hand, in the classical theory of algebraic theta functions

(b) the commutator structure $[-,-]$ of the theta group plays a central role in the theory via the observation that this commutator structure implies the irreducibility of certain representations of the theta group.

At first glance, these two applications (a), (b) of the commutator structure $[-,-]$ of the theta group may appear to be unrelated. In fact, however, they may both be understood as examples of the following phenomenon:

(c) the commutator structure [-,-] of the theta group may be thought of as a sort of concrete embodiment of the "coherence of holomorphic structures".

Indeed, as discussed in [EtTh], Introduction, from the point of view of the schemetheoretic Hodge-Arakelov theory of [HASurI], [HASurII], the irreducible representations that appear in the classical theory of algebraic theta functions as submodules of the module of all set-theoretic functions on the $l$-torsion points of an elliptic curve [cf. (b)] may be thought of, for instance, when $l$ is large, as discrete analogues of the submodule of "holomorphic functions" within the module of all real analytic functions. On the other hand, if one thinks of cyclotomes as "skeleta of arithmetic holomorphic structures" [cf. (i)], then the theory of conjugate synchronization [cf. Remark 3.5.2, as well as Remark 3.8.3 below] - applied, for instance, in the case of cyclotomes - may be thought of as a sort of "discretely parametrized" [in the sense that it is indexed by torsion points] coherence of arithmetic holomorphic structures, which is obtained by working with the connected subgraph $\Gamma_{\underline{\underline{X}}} \subseteq \Gamma_{\underline{\underline{X}}}[$ cf. Remark 2.6.1, (i)]. In this context, mono-theta-theoretic cyclotomic rigidity [cf. (a)] may be thought of as a sort of "continuously parametrized version" [i.e., supported on $\underline{\underline{\underline{Y}}}_{\underline{v}}$, as opposed to a finite set of torsion points] of this coherence of arithmetic holomorphic structures. Finally, we recall that the interaction - i.e., via restriction operations - between these "discrete" and "continuous" versions of the "coherence of arithmetic holomorphic structures" plays a central role in the theory of Galois evaluation given in Corollaries 2.8, (i); 3.5, (ii); 3.6, (ii).

(iii) If one thinks of cyclotomes at localizations [say, at $\left.\underline{v} \in \underline{\mathbb{V}}^{\text {bad }}\right]$ of a number field [i.e., $K$ ] as local skeleta of the arithmetic holomorphic structure [cf. (i)], then

the mono-theta-theoretic cyclotomic rigidity may be thought of as a sort of "local uniformization" of a number field [cf. the exterior cyclotome of a mono-theta environment that arises from a tempered Frobenioid, as in Proposition 1.3, (i)] via a local portion [cf. the interior cyclotome in the situation of Proposition 1.3, (i)] of the geometric tempered fundamental group $\Delta_{\underline{v}}$ associated to a certain covering of the once-punctured elliptic curve $X_{F}^{-}$[cf. Definition 2.3, (i); [IUTchI], Definition 3.1, (e)].

Since the cyclotomic rigidity isomorphism arising from mono-theta-theoretic cyclotomic rigidity may be thought of as the "cyclotomic portion" of the theta function, mono-theta-theoretic cyclotomic rigidity may be interpreted as the statement that 
the theta function constructed from a mono-theta environment is free of any $\widehat{\mathbb{Z}}^{\times}$power indeterminacies. Moreover, if one takes this point of view, then

constant multiple rigidity may be thought of as the statement that the above "local uniformization" is sufficiently rigid as to be free of any constant multiple indeterminacies.

Here, it is useful to recall that the once-punctured elliptic curve $X_{F}$ on the number field $F$ that occurs in the theory of the present series of papers may be thought of as being analogous to the nilpotent ordinary indigenous bundles on a hyperbolic curve in positive characteristic in p-adic Teichmüller theory [cf. the discussion of [AbsTopIII], §I5]. That it to say, from this point of view, the "local uniformizations" of the above discussion may be thought of as corresponding to the local uniformizations via canonical coordinates of $p$-adic Teichmüller theory [cf., e.g., $[p$ Teich], $\S 0.9]$, which are also "sufficiently rigid" as to be free of any $\widehat{\mathbb{Z}}^{\times}$-power or constant multiple indeterminacies. Here, mono-theta-theoretic cyclotomic rigidity may be thought of as corresponding to the Kodaira-Spencer isomorphism [associated to the Hodge section of the canonical indigenous bundle], which, in some sense, may be thought of as the "skeleton" of the local uniformizations of $p$-adic Teichmüller theory. Also, it is useful to recall in this context that the canonical coordinates of $p$-adic Teichmüller theory are constructed by considering invariants with respect to certain canonical Frobenius liftings. Put another way, the technique of considering Frobenius-invariants allows one to pass, in a canonical way, from objects defined modulo $p$ to objects defined modulo higher powers of $p$. Since the various $\Theta$-links of the Frobenius-picture may be regarded as corresponding to the various transitions from " $\bmod p^{n}$ to $\bmod p^{n+1} "[$ where $n \in \mathbb{N}$ ] in the theory of Witt vectors [cf. the discussion of [IUTchI], §I4; [IUTchIII], Remark 1.4.1, (iii)], it is natural to regard, in the context of the canonical splittings furnished by the étale-picture [cf. the discussion of [IUTchI], §I1],

the multiradiality of the formulation of mono-theta-theoretic cyclotomic rigidity and constant multiple rigidity given in Corollary 1.12 as corresponding to the Frobenius-invariant nature of the canonical coordinates of $p$-adic Teichmüller theory.

Finally, in this context, we observe that it is perhaps natural to think of the discrete rigidity of the theory of [EtTh] as corresponding to the fact that the canonical coordinates of $p$-adic Teichmüller theory, which a priori may only be constructed as PD-formal power series, may in fact be constructed as power series in the usual sense, i.e., elements of the completion $\widehat{\mathcal{O}}$ of the local ring at the point under consideration. Indeed, the discrete rigidity of [EtTh] implies that one may restrict oneself to working with the usual theta function, canonical multiplicative coordinates [i.e., " $U$ "], and $q$-parameters on appropriate tempered coverings of the Tate curve, all of which, like the power series arising from canonical parameters in $p$-adic Teichmüller theory, give rise to "functions on suitable formal schemes" in the sense of classical scheme theory. By contrast, if this discrete rigidity were to fail, then one would be obliged to work in an "a priori profinite" framework that involves, for in-

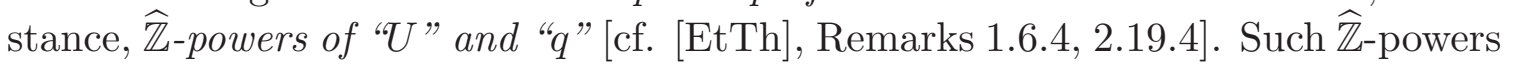


appear naturally in the $\widehat{\mathbb{Z}}$-modules that arise [e.g., as cohomology modules] in the Kummer theory of the theta function and may be thought of as corresponding to PD-formal power series in the sense that arbitrary $\widehat{\mathcal{O}}$-powers of canonical parameters [say, for simplicity, at non-cuspidal ordinary points of a canonical curve], which arise naturally when one considers such parameters additively [cf. the discussion of "canonical affine coordinates" in [ $p$ Ord], Chapter III], cannot be defined if one restricts oneself to working with conventional power series - i.e., such $\widehat{\mathcal{O}}$-powers may only be defined if one allows oneself to work with $P D$-formal power series.

Corollary 3.7. (Group-theoretic Gaussian Monoids and Uniradiality) Suppose that we are in the situation of Proposition 3.4, i.e., in the following, we consider the full poly-isomorphism

$$
\mathbb{M}_{*}^{\Theta}\left(\Pi_{\underline{v}}\right) \stackrel{\sim}{\rightarrow} \quad \mathbb{M}_{*}^{\Theta}\left(\underline{\underline{F}}_{\underline{v}}^{\dagger}\right)
$$

- where $\mathbb{M}_{*}^{\Theta}\left(\Pi_{\underline{v}}\right)$ is the projective system of mono-theta environments arising from the algorithm of Proposition 1.2, (i) [cf. also Proposition 1.5, (i)]; ${ }^{\dagger} \underline{\underline{\mathcal{F}}}_{\underline{v}}$ is a tempered Frobenioid as in Proposition 3.3 - of projective systems of monotheta environments. When " $\mathbb{M}_{*}^{\Theta}$ " is taken to be $\mathbb{M}_{*}^{\Theta}\left({ }^{\dagger} \underline{\underline{\mathcal{F}}}_{\underline{v}}\right)$, we shall denote the resulting " $\mathbb{M}_{* \bullet}^{\Theta}$ " by $\mathbb{M}_{* \dot{*}}^{\Theta}\left({ }^{\dagger} \underline{\underline{\mathcal{F}}}_{\underline{v}}\right)$ [cf. Definition 2.7, (ii)]. When " $\mathbb{M}_{*}^{\Theta}$ " is taken to be $\mathbb{M}_{*}^{\Theta}\left(\Pi_{\underline{v}}\right)$, we shall identify $\Pi_{\underline{v} \ddot{\bullet}}\left(\mathbb{M}_{* \ddot{*}}^{\Theta}\right)$ and $G_{\underline{v}}\left(\mathbb{M}_{* \ddot{*}}^{\Theta}\right)$ [cf. Definition 2.7, (ii)]

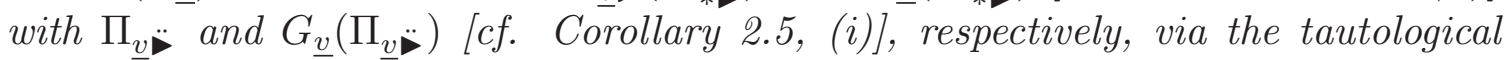

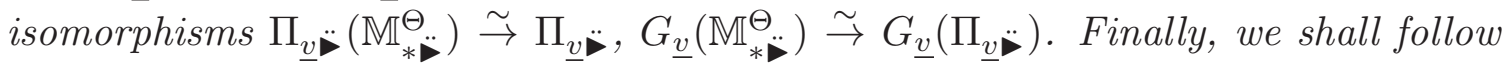
the notational conventions of Corollaries 3.5, $3 . \overline{6}$ with regard to the subscripts "|t|", for $|t| \in\left|\mathbb{F}_{l}\right|$, and " $\left\langle\mathbb{F}_{l}^{*}\right\rangle "$.

(i) (From Group-theoretic to Post-anabelian Gaussian Monoids) Each isomorphism of projective systems of mono-theta environments $\mathbb{M}_{*}^{\Theta}\left(\Pi_{\underline{v}}\right) \stackrel{\sim}{\rightarrow} \mathbb{M}_{*}^{\Theta}\left({ }^{\dagger} \underline{\underline{\mathcal{F}}}_{\underline{v}}\right)$ induces compatible [in the evident sense] collections of isomorphisms

$$
\begin{aligned}
& \Pi_{\underline{v} \ddot{\bullet} \quad \leftarrow-} \quad\left\{G_{\underline{v}}\left(\Pi_{\underline{v}} \ddot{\bullet}\right)|t|\right\}_{|t| \in \mathbb{F}_{i}^{*}} \\
& \curvearrowright \curvearrowright \curvearrowright \\
& \infty \Psi_{\mathrm{env}}^{\iota}\left(\mathbb{M}_{*}^{\Theta}\left(\Pi_{\underline{v}}\right)\right) \stackrel{\sim}{\rightarrow} \quad \infty \Psi_{\xi}\left(\mathbb{M}_{*}^{\Theta}\left(\Pi_{\underline{v}}\right)\right) \\
& \bigcup \\
& \Psi_{\mathrm{env}}^{\iota}\left(\mathbb{M}_{*}^{\Theta}\left(\Pi_{\underline{v}}\right)\right) \stackrel{\sim}{\rightarrow} \quad \Psi_{\xi}\left(\mathbb{M}_{*}^{\Theta}\left(\Pi_{\underline{v}}\right)\right) \\
& \stackrel{\sim}{\rightarrow}\left\{G_{\underline{v}}\left(\mathbb{M}_{* \ddot{\bullet}}^{\Theta}\left(\underline{\underline{F}}_{\underline{\underline{\mathcal{F}}}}\right)\right)_{|t|}\right\}_{|t| \in \mathbb{F}_{l}^{*}} \stackrel{\sim}{\rightarrow} \quad\left\{G_{\underline{v}}\left(\mathbb{M}_{*}^{\Theta}\left(\underline{\underline{\underline{\mathcal{F}}}}_{\underline{v}}\right)\right)_{|t|}\right\}_{|t| \in \mathbb{F}_{l}^{*}} \\
& \stackrel{\sim}{\rightarrow} \quad \infty \Psi_{\xi}\left(\mathbb{M}_{*}^{\Theta}\left({ }^{\dagger} \underline{\underline{\mathcal{F}}}_{\underline{v}}\right)\right) \quad \stackrel{\sim}{\rightarrow} \quad \infty \Psi_{\mathcal{F}_{\xi}}\left({ }^{\dagger} \underline{\underline{\mathcal{F}}}_{\underline{v}}\right) \\
& \text { U } U \\
& \stackrel{\sim}{\rightarrow} \quad \Psi_{\xi}\left(\mathbb{M}_{*}^{\Theta}\left({ }^{\dagger} \underline{\underline{\mathcal{F}}}_{\underline{v}}\right)\right) \quad \stackrel{\sim}{\rightarrow} \quad \Psi_{\mathcal{F}_{\xi}}\left({ }^{\dagger} \underline{\underline{\mathcal{F}}}_{\underline{v}}\right)
\end{aligned}
$$


and

$$
\begin{aligned}
& G_{\underline{v}}\left(\Pi_{\underline{v}} \ddot{\boldsymbol{\varpi}}\right) \quad \stackrel{\sim}{\rightarrow} \quad G_{\underline{v}}\left(\Pi_{\underline{v}} \ddot{\bullet}\right)_{\left\langle\mathbb{F}_{l}^{*}\right\rangle} \\
& \curvearrowright \curvearrowright \curvearrowright \\
& \Psi_{\text {env }}^{\iota}\left(\mathbb{M}_{*}^{\Theta}\left(\Pi_{\underline{v}}\right)\right)^{\times} \stackrel{\sim}{\rightarrow} \quad \Psi_{\xi}\left(\mathbb{M}_{*}^{\Theta}\left(\Pi_{\underline{v}}\right)\right)^{\times} \\
& \stackrel{\sim}{\rightarrow} G_{\underline{v}}\left(\mathbb{M}_{*}^{\Theta}\left({ }^{\dagger} \underline{\underline{\mathcal{F}}}_{\underline{v}}\right)\right)_{\left\langle\mathbb{F}_{l}^{*}\right\rangle} \stackrel{\sim}{\rightarrow} \quad G_{\underline{v}}\left(\mathbb{M}_{*}^{\Theta}\left({ }^{\dagger} \underline{\underline{\mathcal{F}}}_{\underline{v}}\right)\right)_{\left\langle\mathbb{F}_{l}^{*}\right\rangle} \\
& \curvearrowright \curvearrowright \curvearrowright \\
& \stackrel{\sim}{\rightarrow} \quad \Psi_{\xi}\left(\mathbb{M}_{*}^{\Theta}\left(\underline{\underline{\underline{\mathcal{F}}}}_{\underline{v}}\right)\right)^{\times} \quad \stackrel{\sim}{\rightarrow} \quad \Psi_{\mathcal{F}_{\xi}}\left({ }^{\dagger} \underline{\underline{\mathcal{F}}}_{\underline{v}}\right)^{\times}
\end{aligned}
$$

- where the upper left-hand portion of the first display [involving " $\leftarrow--"]$ is obtained by applying the third display [involving " +-- "] of Corollary 3.5, (ii), in the case where " $\mathbb{M}_{*}^{\Theta}$ " is taken to be $\mathbb{M}_{*}^{\Theta}\left(\Pi_{\underline{v}}\right)$; the isomorphisms that relate the upper left-hand portion of the first display to the lower right-hand portion of the first display arise from the functoriality of the algorithms involved, relative to isomorphisms of projective systems of mono-theta environments; the lower righthand portion of the first display is obtained by applying the right-hand portion of the third display of Corollary 3.6, (ii), in the case where " $\mathbb{M}_{*}^{\Theta}$ " is taken to be $\mathbb{M}_{*}^{\Theta}\left({ }^{\dagger} \underline{\underline{\mathcal{F}}}_{\underline{v}}\right)$; the second display is obtained from the first display by considering the units [denoted by means of a superscript " $\times$ "].

(ii) (Uniradiality of Gaussian Monoids) If we write $\Psi_{\mathcal{F}_{\xi}}\left({ }^{\dagger} \underline{\underline{\mathcal{F}}}_{\underline{v}}\right){ }^{\times \mu}$ for the topological monoid obtained by forming the quotient of $\Psi_{\mathcal{F}_{\xi}}\left(\underline{\underline{\mathcal{F}}}_{\underline{v}}\right)^{\times}$by its torsion subgroup, then the functorial algorithms

$$
\Pi_{\underline{v}} \mapsto \Psi_{\text {gau }}\left(\mathbb{M}_{*}^{\Theta}\left(\Pi_{\underline{v}}\right)\right) ; \quad \Pi_{\underline{v}} \mapsto \infty \Psi_{\text {gau }}\left(\mathbb{M}_{*}^{\Theta}\left(\Pi_{\underline{v}}\right)\right)
$$

- where we think of $\Psi_{\text {gau }}\left(\mathbb{M}_{*}^{\Theta}\left(\Pi_{\underline{v}}\right)\right), \infty \Psi_{\text {gau }}\left(\mathbb{M}_{*}^{\Theta}\left(\Pi_{\underline{v}}\right)\right)$ as being equipped with their natural splittings up to torsion [cf. Corollary 3.5, (iii)] and, in the case of $\Psi_{\text {gau }}\left(\mathbb{M}_{*}^{\Theta}\left(\Pi_{\underline{v}}\right)\right)$, the natural $G_{\underline{v}}\left(\Pi_{\underline{v}} \ddot{\sim}\right)$-action [cf. Corollary 3.5, (ii)] - obtained by composing the algorithms of Proposition 1.2, (i); Corollary 3.5, (ii), (iii), depend on the cyclotomic rigidity isomorphism of Corollary 1.11, (b) [cf. Remark 1.11.5, (ii); the use of the surjection of Remark 1.11.5, (i), in the algorithms of Proposition 3.1, (ii), and Corollary 3.5, (ii)], hence fails to be compatible, relative to the displayed diagrams of (i), with automorphisms of [the underlying pair, consisting of a topological monoid equipped with the action of a topological group, determined by] the pair

$$
G_{\underline{v}}\left(\mathbb{M}_{*}^{\Theta}\left(\underline{\underline{F}}_{\underline{v}}^{\dagger}\right)\right)_{\left\langle\mathbb{F}_{l}^{*}\right\rangle} \curvearrowright \Psi_{\mathcal{F}_{\xi}}\left({ }^{\dagger} \underline{\underline{\mathcal{F}}}_{\underline{v}}\right)^{\times \mu}
$$

which arise from automorphisms of [the underlying pair, consisting of a topological monoid equipped with the action of a topological group, determined by] the pair $G_{\underline{v}}\left(\mathbb{M}_{*}^{\Theta}\left({ }^{\dagger} \underline{\underline{\mathcal{F}}}_{\underline{v}}\right)\right)_{\left\langle\mathbb{F}_{l}^{*}\right\rangle} \curvearrowright \Psi_{\mathcal{F}_{\xi}}\left({ }^{\dagger} \underline{\underline{\mathcal{F}}}_{\underline{v}}\right)^{\times} \quad[c f . \quad$ Remarks 1.11.1, (i), (b); 1.8.1] - in the sense that this algorithm, as given, only admits a uniradial formulation [cf. Remarks 1.11.3, (iv); 1.11.5, (ii)]. 
Proof. The various assertions of Corollary 3.7 follow immediately from the definitions and the references quoted in the statements of these assertions.

Remark 3.7.1. One central consequence of the theory to be developed in [IUTchIII] [cf. Remarks 2.9.1, (iii); 3.4.1, (ii)] is the result that,

by applying the theory of log-shells [cf. [AbsTopIII]], one may modify the algorithms of Corollary 3.7, (ii), in such a way as to obtain algorithms for computing the Gaussian monoids that [yield functors which] are manifestly multiradially defined

— albeit at the cost of allowing for certain [relatively mild!] indeterminacies.

The following definition in some sense summarizes the theory of the present $\S 3$.

Definition 3.8. Many of the "monoids equipped with a Galois action" that appear in the discussion of the present $\S 3$ may be thought of as giving rise to Frobenioids, as follows.

(i) Each of the monoids equipped with a $\Pi_{\underline{\underline{X}}}\left(\mathbb{M}_{*}^{\Theta}\right)$-action

$$
\Pi_{\underline{\underline{X}}}\left(\mathbb{M}_{*}^{\Theta}\right) \curvearrowright \quad \Psi_{\mathrm{cns}}\left(\mathbb{M}_{*}^{\Theta}\right) ; \quad \Pi_{\underline{\underline{X}}}\left(\mathbb{M}_{*}^{\Theta}\right) \curvearrowright \quad \Psi_{\dagger \underline{\mathcal{C}_{\underline{v}}}}
$$

of Propositions 3.1, (ii); 3.3, (ii), gives rise to a $p_{\underline{v}}$-adic Frobenioid of monoid type $\mathbb{Z}[$ cf. [FrdII], Example 1.1, (ii)]

$$
\mathcal{F}_{\mathrm{cns}}\left(\mathbb{M}_{*}^{\Theta}\right) ; \quad \mathcal{F}_{\dagger \mathcal{C}_{\underline{v}}}
$$

whose divisor monoid associates to every object of $\mathcal{B}^{\text {temp }}\left(\Pi_{\underline{X}}\left(\mathbb{M}_{*}^{\Theta}\right)\right)^{0}$ a monoid isomorphic to $\mathbb{Q}_{>0}$. It follows immediately from the construction of the data " $\Pi_{\underline{\underline{X}}}\left(\mathbb{M}_{*}^{\Theta}\right) \curvearrowright \bar{\Psi}_{{ }_{\mathcal{C}_{\underline{v}}}}$ " [cf. Example 3.2, (ii)] that one has a tautological isomorphism of Frobenioids

$$
{ }^{\dagger} \mathcal{C}_{\underline{v}} \stackrel{\sim}{\rightarrow} \mathcal{F}_{\dagger} \mathcal{C}_{\underline{v}}
$$

[cf. the discussion of [IUTchI], Example 3.2, (iii), (iv)], which we shall use to identify these two Frobenioids. Thus, the isomorphism of monoids of Proposition 3.3, (ii), may be interpreted as an isomorphism of Frobenioids

$$
{ }^{\dagger} \mathcal{C}_{\underline{v}} \stackrel{\sim}{\rightarrow} \mathcal{F}_{\mathrm{cns}}\left(\mathbb{M}_{*}^{\Theta}\right)
$$

— which also admits [indeed, induces] a "mono-analytic version" ${ }^{\dagger} \mathcal{C}_{\underline{v}}^{\vdash} \stackrel{\sim}{\rightarrow} \quad \mathcal{F}_{\text {cns }}^{\vdash}\left(\mathbb{M}_{*}^{\Theta}\right)$ [cf. the category " $\mathcal{C}_{\underline{v}}^{+}$" of [IUTchI], Example 3.2, (iv)]. This mono-analytic version admits a "labeled version" [cf. Remark 3.8.1 below]

$$
\left({ }^{\dagger} \mathcal{C}_{\underline{v}}^{\vdash}\right)_{|t|} \stackrel{\sim}{\rightarrow}\left(\mathcal{F}_{\mathrm{cns}}^{+}\left(\mathbb{M}_{*}^{\Theta}\right)\right)_{|t|}
$$


— cf. Corollary 3.6, (i). Finally, one has Frobenioid-theoretic interpretations

$$
\begin{aligned}
&\left(\mathcal{F}_{\mathrm{cns}}^{\vdash}\left(\mathbb{M}_{*}^{\Theta}\right)\right)_{\left\langle\left|\mathbb{F}_{l}\right|\right\rangle} ; \quad\left(\mathcal{F}_{\mathrm{cns}}^{\vdash}\left(\mathbb{M}_{*}^{\Theta}\right)\right)_{0} \stackrel{\sim}{\rightarrow}\left(\mathcal{F}_{\mathrm{cns}}^{\vdash}\left(\mathbb{M}_{*}^{\Theta}\right)\right)_{\left\langle\mathbb{F}_{l}^{*}\right\rangle} \\
&\left({ }^{\dagger} \mathcal{C}_{\underline{v}}^{\vdash}\right)_{\left\langle\left|\mathbb{F}_{l}\right|\right\rangle} ; \quad\left({ }^{\dagger} \mathcal{C}_{\underline{v}}^{\vdash}\right)_{0} \stackrel{\sim}{\rightarrow}\left({ }^{\dagger} \mathcal{C}_{\underline{v}}^{\vdash}\right)_{\left\langle\mathbb{F}_{l}^{*}\right\rangle}
\end{aligned}
$$

of the constructions of Corollary 3.5, (iii); 3.6, (iii).

(ii) Each of the monoids equipped with a topological group action

$$
\begin{aligned}
& G_{\underline{v}}\left(\mathbb{M}_{* \ddot{*}}^{\Theta}\right) \curvearrowright \Psi_{\text {env }}^{\iota}\left(\mathbb{M}_{*}^{\Theta}\right) ; \quad G_{\underline{v}}\left(\mathbb{M}_{* \ddot{\bullet}}^{\Theta}\right) \curvearrowright \Psi_{\dagger \mathcal{F}_{\underline{v}}^{\Theta}, \alpha} \\
& G_{\underline{v}}\left(\mathbb{M}_{* \ddot{\nabla}}^{\Theta}\right)_{\left\langle\mathbb{F}_{l}^{*}\right\rangle} \curvearrowright \Psi_{\xi}\left(\mathbb{M}_{*}^{\Theta}\right) ; \quad G_{\underline{v}}\left(\mathbb{M}_{*}^{\Theta}\right)_{\left\langle\mathbb{F}_{l}^{*}\right\rangle} \curvearrowright \Psi_{\mathcal{F}_{\xi}}\left({ }^{\dagger} \underline{\underline{F}}_{\underline{v}}\right)
\end{aligned}
$$

[cf. Proposition 3.1, (i); Proposition 3.3, (i); Corollary 3.5, (ii); Corollary 3.6, (ii)] gives rise to a $p_{\underline{v}}$-adic Frobenioid of monoid type $\mathbb{Z}$ [cf. [FrdII], Example 1.1, (ii)]

$$
\mathcal{F}_{\text {env }}^{\iota}\left(\mathbb{M}_{*}^{\Theta}\right) ; \quad \mathcal{F}_{\dagger \mathcal{F}_{\underline{v}}^{\Theta}, \alpha} ; \quad \mathcal{F}_{\xi}\left(\mathbb{M}_{*}^{\Theta}\right) ; \quad \mathcal{F}_{\mathcal{F}_{\xi}}\left({ }^{\dagger} \underline{\underline{\mathcal{F}}}_{\underline{v}}\right)
$$

whose divisor monoid associates to every object of $\mathcal{B}^{\text {temp }}\left(G_{\underline{v}}(-)\right)^{0}$ [where " $(-)$ " is $\mathbb{M}_{* \dot{\sim}}^{\Theta}$ or $\left.\mathbb{M}_{*}^{\Theta}\right]$ a monoid isomorphic to $\mathbb{N}$. Moreover, each of these Frobenioids is equipped with a collection of splittings [cf. Proposition 3.1, (i); Proposition 3.3, (i); Corollary 3.5, (iii); Corollary 3.6, (iii)]. Also, we shall write

$$
\begin{gathered}
\mathcal{F}_{\text {env }}\left(\mathbb{M}_{*}^{\Theta}\right) \stackrel{\text { def }}{=}\left\{\mathcal{F}_{\text {env }}^{\iota}\left(\mathbb{M}_{*}^{\Theta}\right)\right\}_{i} ; \quad \mathcal{F}_{\dagger \mathcal{F}_{\underline{v}}^{\Theta}} \stackrel{\text { def }}{=}\left\{\mathcal{F}_{\dagger \mathcal{F}_{\underline{v}}^{\Theta}, \alpha}\right\}_{\alpha} \\
\mathcal{F}_{\text {gau }}\left(\mathbb{M}_{*}^{\Theta}\right) \stackrel{\text { def }}{=}\left\{\mathcal{F}_{\xi}\left(\mathbb{M}_{*}^{\Theta}\right)\right\}_{\xi} ; \quad \mathcal{F}_{\mathcal{F}_{\text {gau }}}\left({\left.\stackrel{\dagger}{\underline{\mathcal{F}}_{\underline{v}}}\right)}^{\underline{\text { def }}}=\left\{\mathcal{F}_{\mathcal{F}_{\xi}}\left({ }^{\dagger} \underline{\underline{\mathcal{F}}}_{\underline{v}}\right)\right\}_{\xi}\right.
\end{gathered}
$$

[cf. the notation of Proposition 3.1, (i); Proposition 3.3, (i); Corollary 3.5, (ii); Corollary 3.6, (ii)]. It follows immediately from the construction of the data "G $G_{\underline{v}}\left(\mathbb{M}_{* \ddot{\bullet}}^{\Theta}\right) \curvearrowright \Psi_{\dagger \mathcal{F}_{\underline{v}}^{\Theta}, \alpha}$ " [cf. Example 3.2, (i)] that one has a tautological isomorphism of Frobenioids

$$
{ }^{\dagger} \mathcal{C}_{\underline{v}}^{\Theta} \stackrel{\sim}{\rightarrow} \mathcal{F}_{\dagger \mathcal{F}_{\underline{v}}^{\Theta}, \alpha}
$$

which is compatible with the associated splittings [cf. the discussion of [IUTchI], Example 3.2, (v)], and which we shall use to identify these two split Frobenioids. Thus, the isomorphisms of monoids in the third display of Corollary 3.6, (ii), may be interpreted as isomorphisms of split Frobenioids

$$
\mathcal{F}_{\dagger \mathcal{F}_{\underline{v}}^{\Theta}, \alpha} \stackrel{\sim}{\rightarrow} \mathcal{F}_{\text {env }}^{\iota}\left(\mathbb{M}_{*}^{\Theta}\right) \quad \stackrel{\sim}{\rightarrow} \quad \mathcal{F}_{\xi}\left(\mathbb{M}_{*}^{\Theta}\right) \stackrel{\sim}{\rightarrow} \quad \mathcal{F}_{\mathcal{F}_{\xi}}\left({ }^{\dagger} \underline{\underline{\mathcal{F}}}_{\underline{v}}\right)
$$

[cf. Proposition 3.3, (i); Corollary 3.5, (iii); Corollary 3.6, (iii)] which are compatible with the subcategories

$$
\mathcal{F}_{2 l \cdot \xi}\left(\mathbb{M}_{*}^{\Theta}\right) \quad \subseteq \quad \mathcal{F}_{\xi}\left(\mathbb{M}_{*}^{\Theta}\right) ; \quad \mathcal{F}_{\mathcal{F}_{2 l \cdot \xi}}\left(\underline{\underline{\underline{\mathcal{F}}}}_{\underline{v}}\right) \subseteq \mathcal{F}_{\mathcal{F}_{\xi}}\left({ }^{\dagger} \underline{\underline{\mathcal{F}}}_{\underline{v}}\right)
$$

determined by the submonoids " $\Psi_{2 l \cdot \xi}(-)$ " [cf. Corollaries 3.5, (ii); 3.6, (ii)] and which yield isomorphisms of collections of split Frobenioids

$$
\mathcal{F}_{\dagger \mathcal{F}_{\underline{\underline{v}}}^{\Theta}} \stackrel{\sim}{\rightarrow} \mathcal{F}_{\text {env }}\left(\mathbb{M}_{*}^{\Theta}\right) \quad \stackrel{\sim}{\rightarrow} \quad \mathcal{F}_{\text {gau }}\left(\mathbb{M}_{*}^{\Theta}\right) \stackrel{\sim}{\rightarrow} \quad \mathcal{F}_{\mathcal{F}_{\text {gau }}}\left(\underline{\underline{\mathcal{F}}}_{\underline{\underline{v}}}\right)
$$


[cf. the fourth display of Corollary 3.6, (ii)].
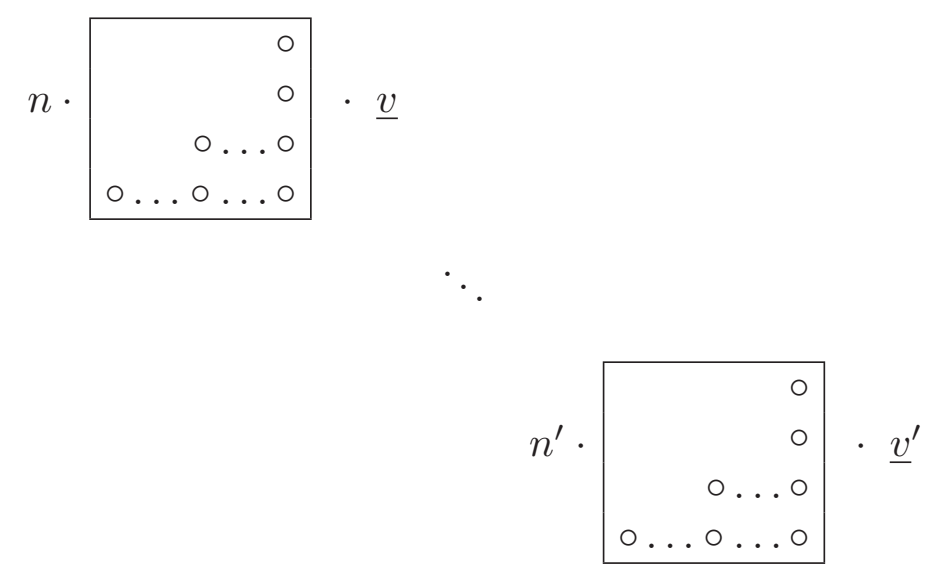

Fig. 3.3: Gaussian distribution

(iii) The direct products in which the submonoids $\Psi_{\xi}\left(\mathbb{M}_{*}^{\Theta}\right)$ and $\Psi_{\mathcal{F}_{\xi}}\left(\underline{\underline{\mathcal{F}}}_{\underline{\underline{\mathcal{F}}}}\right)$ are constructed [cf. the second display of Corollary 3.5, (ii); the first display of Corollary 3.6, (ii)] determine natural embeddings of categories [cf. Remark 3.8.1 below]

$$
\mathcal{F}_{\xi}\left(\mathbb{M}_{*}^{\Theta}\right) \hookrightarrow \prod_{|t| \in \mathbb{F}_{l}^{*}} \mathcal{F}_{\mathrm{cns}}^{\vdash}\left(\mathbb{M}_{*}^{\Theta}\right)_{|t|} ; \quad \mathcal{F}_{\mathcal{F}_{\xi}}\left({ }^{\dagger} \underline{\underline{\mathcal{F}}}_{\underline{v}}\right) \hookrightarrow \prod_{|t| \in \mathbb{F}_{l}^{*}}\left({ }^{\dagger} \mathcal{C}_{\underline{v}}^{\vdash}\right)_{|t|}
$$

which coincide on the subcategories $\mathcal{F}_{2 l \cdot \xi}\left(\mathbb{M}_{*}^{\Theta}\right) \subseteq \mathcal{F}_{\xi}\left(\mathbb{M}_{*}^{\Theta}\right), \mathcal{F}_{\mathcal{F}_{2 l \cdot \xi}}\left(\underline{\underline{\mathcal{F}}}_{\underline{v}}\right) \subseteq \mathcal{F}_{\mathcal{F}_{\xi}}\left({ }^{\dagger} \underline{\underline{\mathcal{F}}}_{\underline{v}}\right)$. We shall write [cf. Remark 3.8.1 below]

$$
\begin{aligned}
& \mathcal{F}_{\text {gau }}\left(\mathbb{M}_{*}^{\Theta}\right) \hookrightarrow \mathcal{F}_{\text {cns }}^{+}\left(\mathbb{M}_{*}^{\Theta}\right)_{\mathbb{F}_{l}^{*}} \stackrel{\text { def }}{=} \prod_{|t| \in \mathbb{F}_{l}^{*}} \mathcal{F}_{\text {cns }}^{+}\left(\mathbb{M}_{*}^{\Theta}\right)_{|t|} \\
& \mathcal{F}_{\mathcal{F}_{\text {gau }}}\left({ }^{\dagger} \underline{\underline{\mathcal{F}}}_{\underline{v}}\right) \hookrightarrow \quad\left({ }^{\dagger} \underline{C}_{\underline{v}}^{\vdash}\right)_{\mathbb{F}_{l}^{*}} \stackrel{\text { def }}{=} \prod_{|t| \in \mathbb{F}_{l}^{*}}\left({ }^{\dagger} \mathcal{C}_{\underline{v}}^{\vdash}\right)|t|
\end{aligned}
$$

for the collections of embeddings of categories obtained by allowing $\xi$ to vary. These embeddings may be thought of as "Gaussian distributions" and are depicted in Fig. 3.3 above. In this context, it is useful to observe that we also have natural diagonal embeddings of categories, i.e., "constant distributions" [cf. Remark 3.8.1 below]

$$
\mathcal{F}_{\mathrm{cns}}^{\vdash}\left(\mathbb{M}_{*}^{\Theta}\right) \stackrel{\sim}{\rightarrow} \mathcal{F}_{\mathrm{cns}}^{\vdash}\left(\mathbb{M}_{*}^{\Theta}\right)_{\left\langle\mathbb{F}_{l}^{*}\right\rangle} \hookrightarrow \mathcal{F}_{\mathrm{cns}}^{\vdash}\left(\mathbb{M}_{*}^{\Theta}\right)_{\mathbb{F}_{l}^{*}}=\prod_{|t| \in \mathbb{F}_{l}^{*}} \mathcal{F}_{\mathrm{cns}}^{\vdash}\left(\mathbb{M}_{*}^{\Theta}\right)_{|t|}
$$




$$
{ }^{\dagger} \mathcal{C}_{\underline{v}}^{\vdash} \stackrel{\sim}{\rightarrow}\left({ }^{\dagger} \mathcal{C}_{\underline{v}}^{\vdash}\right)_{\left\langle\mathbb{F}_{l}^{*}\right\rangle} \hookrightarrow \quad\left({ }^{\dagger} \mathcal{C}_{\underline{v}}^{\vdash}\right)_{\mathbb{F}_{i}^{*}}=\prod_{|t| \in \mathbb{F}_{l}^{*}}\left({ }^{\dagger} \mathcal{C}_{\underline{v}}^{\vdash}\right)_{|t|}
$$

— where the " $\stackrel{\sim}{\rightarrow}$ 's" denote the tautological isomorphisms - cf. the discussion [and notational conventions!] of [IUTchI], Example 5.4, (i); [IUTchI], Fig. 5.1.

Remark 3.8.1. In the present series of papers, we follow the convention [cf. [IUTchI], §0] that an "isomorphism of categories" is to be understood as an isomorphism class of equivalences of categories. On the other hand, in the context of the discussion of Frobenioids in Definition 3.8, in order to obtain a precise "Frobenioidtheoretic translation" of the results obtained so far [in the language of monoids] that involve the phenomenon of conjugate synchronization [cf. Remark 3.5.2; the discussion of Remark 3.8.3 below], one is obliged to consider the various Frobenioids indexed by a subscript " $|t| \in\left|\mathbb{F}_{l}\right|$ " as being determined up to an isomorphism of the identity functor - i.e., corresponding to an "inner automorphism" in the context of Corollaries 3.5, (i); 3.6, (i) — which is independent of $|t| \in\left|\mathbb{F}_{l}\right|$. In particular, when there is a danger of confusion, perhaps the simplest approach is to resort to the original "monoid-theoretic formulations" of Corollaries 3.5, 3.6.

Remark 3.8.2. At this point, it is of interest to pause to discuss the relationship between the theory of the present $\S 3$ and the theories of $\mathbb{F}_{l}^{\rtimes \pm}$-symmetry [cf. [IUTchI], §6] and $\mathbb{F}_{l}^{*}$-symmetry [cf. [IUTchI], §4, §5] developed in [IUTchI].

(i) First of all, the construction algorithms for the Gaussian monoids discussed in Corollaries 3.5, (ii); 3.6, (ii), as well as for the closely relating splittings discussed in Corollaries 3.5, (iii); 3.6, (iii), involve restriction to the decomposition groups of torsion points indexed [via a functorial algorithm] by profinite conjugacy classes of cusps [cf. Corollary 2.4, (ii)] which are subject to a certain $\mathbb{F}_{l}^{\rtimes \pm}$-symmetry [cf. Corollary 2.4, (iii)]. This $\mathbb{F}_{l}^{\rtimes \pm}$-symmetry may be thought of as the restriction, to the portion labeled by the valuation $\underline{v} \in \underline{\mathbb{V}}^{\text {bad }}$ under consideration, of the $\mathbb{F}_{l}^{\rtimes \pm}$-symmetry [cf. [IUTchI], Proposition 6.8, (i)] associated to a $\mathcal{D}-\Theta^{ \pm e l l}-$ Hodge theater $[\mathrm{cf}$. Remark 2.6.2, (i)]. From the point of view of the issue of "which portion of the original once-punctured elliptic curve over a number field $X_{F}$ [cf. [IUTchI], Definition 3.1] is involved", this theory of split Gaussian monoids revolves around various labeled [i.e., by elements of copies of $\mathbb{F}_{l}$ or $\left|\mathbb{F}_{l}\right|$ ] copies of the local Frobenioids at $\underline{v}$ of the mono-analyticizations of the $\mathcal{F}$-prime-strips that appear in a $\mathcal{D}$ - $\Theta^{ \pm \text {ell }}$-Hodge theater - cf. the various natural embeddings discussed in Definition 3.8, (iii) - i.e., more concretely, copies of the portion of the pair " $G_{\underline{v}}\left(\Pi_{\underline{v}}\right) \curvearrowright \mathcal{O} \frac{\triangleright}{F_{\underline{v}}}$ " determined by a certain submonoid of $\mathcal{O}{\stackrel{\triangleright}{F_{\underline{v}}}}_{\text {. Finally, we recall }}$ that after one executes these construction algorithms for split Gaussian monoids and observes the $\mathbb{F}_{l}^{\rtimes \pm}$-symmetry discussed above, one may then form holomorphic or mono-analytic processions, indexed by subsets of $\left|\mathbb{F}_{l}\right|$, as discussed in [IUTchI], Proposition 6.9, (i), (ii).

(ii) On the other hand, by applying the algorithm of [IUTchI], Proposition 6.7, one may pass to the local portion at $\underline{v} \in \underline{\mathbb{V}}^{\text {bad }}$ of a $\mathcal{D}$ - $\Theta N F-H o d g e$ theater. At the level of labels, this amounts to removing the label $0 \in\left|\mathbb{F}_{l}\right|$ and identifying this label with the complement of 0 in $\left|\mathbb{F}_{l}\right|$, i.e., with $\mathbb{F}_{l}^{*}$ - cf. the assignment

$$
\text { " } 0, \succ \mapsto>\text { " }
$$


of $\mathcal{D}$-prime-strips discussed in [IUTchI], Proposition 6.7. At the level of local Frobenioids at $\underline{v} \in \underline{\mathbb{V}}^{\text {bad }}$ [i.e., copies of the pair " $\Pi_{\underline{v}} \curvearrowright \mathcal{O} \frac{\triangleright}{\bar{F}_{v}}$ "] corresponding to these labels, this assignment may be thought of as corresponding to the isomorphisms of monoids " $\Psi_{\mathrm{cns}}\left(\mathbb{M}_{*}^{\Theta}\right)_{0} \stackrel{\sim}{\rightarrow} \Psi_{\mathrm{cns}}\left(\mathbb{M}_{*}^{\Theta}\right)_{\left\langle\mathbb{F}_{l}^{*}\right\rangle}$ " and " $\left(\Psi_{\dagger \mathcal{C}_{\underline{v}}}\right)_{0} \stackrel{\sim}{\rightarrow}\left(\Psi_{\dagger \mathcal{C}_{\underline{v}}}\right)_{\left\langle\mathbb{F}_{l}^{*}\right\rangle}$ " discussed in the first displays of Corollaries 3.5, (iii); 3.6, (iii). This newly obtained situation involving the local portion at $\underline{v} \in \underline{\mathbb{V}}^{\text {bad }}$ of a $\mathcal{D}$ - $\Theta$ NF-Hodge theater admits a $\mathbb{F}_{l}^{*}$-symmetry [cf. [IUTchI], Proposition 4.9, (i)] — cf. the discussion of the $\mathbb{F}_{l}^{\rtimes \pm}$ symmetry in the situation of (i). As we shall see in $\S 4$ below, at least at the level of value groups, this newly obtained situation involving $\mathbb{F}_{l}^{*}$-symmetries is well-suited to relating the theory of the present $\S 3$ at $\underline{v} \in \underline{\mathbb{V}}^{\text {bad }}$ to the valuations $\in \underline{\mathbb{V}}^{\text {good }}$, as well as to the global theory of [IUTchI], $\S 5$. This global theory satisfies the crucial property that it allows one to relate the multiplicative and additive structures of a global number field [cf. the discussion of [IUTchI], Remark 4.3.2; [IUTchI], Remark 6.12.5, (ii)]. Finally, starting from this newly obtained situation, one may proceed to form holomorphic or mono-analytic processions, indexed by subsets of $\mathbb{F}_{l}^{*}$, as discussed in [IUTchI], Proposition 4.11, (i), (ii), which are compatible [cf. [IUTchI], Proposition 6.9, (iii)] with the "|F $\mathbb{F}_{l} \mid$-processions" discussed in (i).

Remark 3.8.3. One central theme of the theory of the present $\S 3$ is the application of the phenomenon of conjugate synchronization [cf. Remark 3.5.2], which plays a fundamental role in the theory of the group-theoretic version of Hodge-Arakelov-theoretic evaluation of the theta function developed in $\S 2$. Thus, it is of interest to pause to discuss precisely what was gained in the present $\S 3$ by applying the conjugate synchronization obtained in $\S 2$.

(i) We begin our discussion by reviewing the following direct technical consequences of the conjugate synchronization discussed in Remark 3.5.2:

(a) the isomorphisms of monoids

$\Psi_{\mathrm{cns}}\left(\mathbb{M}_{*}^{\Theta}\right)_{\left|t_{1}\right|} \stackrel{\sim}{\rightarrow} \Psi_{\mathrm{cns}}\left(\mathbb{M}_{*}^{\Theta}\right)_{\left|t_{2}\right|} ; \quad\left(\Psi_{\dagger \mathcal{C}_{\underline{v}}}\right)_{\left|t_{1}\right|} \stackrel{\sim}{\rightarrow}\left(\Psi_{\dagger \mathcal{C}_{\underline{v}}}\right)_{\left|t_{2}\right|} ; \quad\left(\Psi_{\dagger \mathcal{C}_{\underline{v}}}\right)_{|t|} \stackrel{\sim}{\rightarrow} \Psi_{\mathrm{cns}}\left(\mathbb{M}_{*}^{\Theta}\right)_{|t|}$

- where $|t|,\left|t_{1}\right|,\left|t_{2}\right| \in\left|\mathbb{F}_{l}\right|$; the third isomorphism is well-defined up to an inner automorphism indeterminacy that is independent of $|t|$ — discussed in Corollaries 3.5, (i); 3.6, (i);

(b) the construction of well-defined diagonal submonoids

$$
\Psi_{\mathrm{cns}}\left(\mathbb{M}_{*}^{\Theta}\right)_{\left\langle\left|\mathbb{F}_{l}\right|\right\rangle} \subseteq \prod_{|t| \in\left|\mathbb{F}_{l}\right|} \Psi_{\mathrm{cns}}\left(\mathbb{M}_{*}^{\Theta}\right)_{|t|} ; \quad \Psi_{\mathrm{cns}}\left(\mathbb{M}_{*}^{\Theta}\right)_{\left\langle\mathbb{F}_{l}^{*}\right\rangle} \subseteq \prod_{|t| \in \mathbb{F}_{l}^{*}} \Psi_{\mathrm{cns}}\left(\mathbb{M}_{*}^{\Theta}\right)_{|t|}
$$

in Corollary 3.5, (i), and the corresponding diagonal embeddings of categories — i.e., "constant distributions" — discussed in Definition 3.8, (iii);

(c) the well-defined isomorphisms of monoids

$$
\Psi_{\mathrm{cns}}\left(\mathbb{M}_{*}^{\Theta}\right)_{0} \stackrel{\sim}{\rightarrow} \Psi_{\mathrm{cns}}\left(\mathbb{M}_{*}^{\Theta}\right)_{\left\langle\mathbb{F}_{l}^{*}\right\rangle} ; \quad\left(\Psi_{\dagger \mathcal{C}_{\underline{v}}}\right)_{0} \stackrel{\sim}{\rightarrow}\left(\Psi_{\dagger \mathcal{C}_{\underline{v}}}\right)_{\left\langle\mathbb{F}_{l}^{*}\right\rangle}
$$

of Corollaries 3.5, (iii); 3.6, (iii); 
(d) the restriction to the units of the [composite] isomorphism of monoids

$$
\Psi_{\dagger \mathcal{F}_{\underline{\underline{v}}}^{\Theta}, \alpha} \stackrel{\sim}{\rightarrow} \Psi_{\mathcal{F}_{\xi}}\left(\underline{\underline{\underline{\mathcal{F}}}}_{\underline{v}}\right)
$$

that appears in the third display of Corollary 3.6, (ii) [cf. also Fig. 3.1; the discussion of Remark 3.6.2, (i)].

Here, we observe that (b) and (c) may be thought of as formal consequences of (a), while (d) may be thought of as an alternate formulation of the portion of (a) concerning the units in the case of $|t| \in \mathbb{F}_{l}^{*}$. Moreover, as discussed in Remark 3.6.2, (iii), ultimately, in the present series of papers, we shall be interested in composing

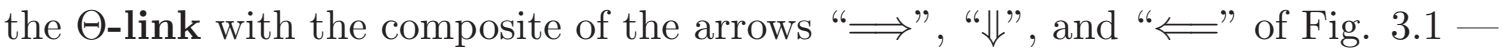
i.e., with the isomorphism of monoids that appears in the display of (d). Indeed, from the point of view of the theory of the present series of papers,

our main application [cf. $\S 4$ below] of the conjugate synchronization discussed in Remark 3.5.2 will consist precisely of the isomorphism of units of $(d)$, in the context of composition with the $\Theta$-link - cf. the "coricity of $\mathcal{O}^{\times}$" given in [IUTchI], Corollary 3.7, (iii).

Finally, in this context, we recall that the isomorphisms of monoids that appear in the $\Theta$-link or in the third display of Corollary 3.6, (ii), only make sense if one works with post-anabelian abstract monoids/Frobenioids — i.e., with "Frobenius-like" structures [cf. the discussion of Remark 3.6.2, (i), (ii)].

(ii) In [IUTchIII], it will be of central importance to consider the theory of the present paper in the context of the log-wall [i.e., the situation considered in [AbsTopIII]]. In the context of the log-wall, it will be of fundamental importance to construct versions of the various Frobenioid-theoretic theta and Gaussian monoids that appeared in the discussion at the end of (i) that are capable of "penetrating the log-wall" [cf. the discussion of [AbsTopIII], §I4] — i.e., to construct étale-like versions of these Frobenioid-theoretic theta and Gaussian monoids, by availing oneself of the right-hand portion of Fig. 3.1. Now to pass from these Frobenioid-theoretic monoids to their étale-like counterparts, one must apply Kummer theory — cf.

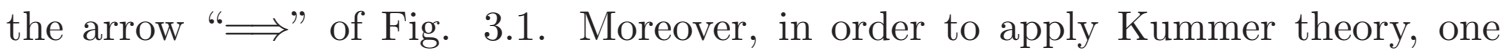
must avail oneself of the cyclotomes contained in [i.e., the torsion subgroups of] the various groups of units of the relevant monoids. It is at this point that it is necessary to apply, in the fashion discussed in (i), the conjugate synchronization discussed in Remark 3.5.2 in an essential way. That is to say, if one is in a situation in which one cannot avail oneself of this conjugate synchronization, then it follows from the distinct, unrelated nature of the basepoints on either side of the log-wall [cf. the discussion of Remark 3.6.3, (i)] that

one may only construct diagonal embeddings of either submonoids of Galoisinvariants or sets of Galois-orbits of the various constant monoids [i.e., " $\Psi_{\text {cns }}$ "] involved.

On the other hand, such Galois-invariants or Galois-orbits are clearly insufficient for conducting Kummer theory [cf. [IUTchIII], Remark 1.5.1, (ii), for a discussion 
of a related topic]. Moreover, the operation of passing to sets of Galois-orbits fails to be compatible with the ring structure - e.g., the additive structure - on [the formal union with " $\{0\}$ " of $]$ the various constant monoids. Such an incompatibility is unacceptable in the context of the theory of the present series of papers since it is impossible to develop the theory of the log-wall [cf. [AbsTopIII]; [IUTchIII]] without applying the ring structure within each Hodge theater [cf. the discussion of Remark 3.6.4, (i)].

(iii) As discussed at the beginning of $\S 1$, the problem of giving an explicit description of what one arithmetic holomorphic structure looks like from the point of view of a distinct arithmetic holomorphic structure that is only related to the orginal arithmetic holomorphic structure via some mono-analytic core is one of the central themes of the theory of the present series of papers. The phenomenon of conjugate synchronization as discussed in (i) and (ii) above, as well as the closely related phenomenon of mono-theta-theoretic cyclotomic rigidity [cf. the discussion of Remark 3.6.5, (ii)], may be thought of as particular instances of this general theme. Indeed, from the point of view of classical discusssions of scheme-theoretic arithmetic geometry,

the "natural isomorphisms" that exist between various cyclotomes that appear in a discussion are typically taken for granted

- i.e., typically no attention is given to the issue of devising explicit, intrinsic reconstruction algorithms for these "natural isomorphisms" between cyclotomes. 


\section{Section 4: Global Gaussian Frobenioids}

In the present $\S 4$, we generalize the theory of Gaussian monoids, developed in $\S 3$ in the case of bad $\underline{v} \in \underline{\mathbb{V}}^{\text {bad }}$, first to the case of nonarchimedean and archimedean good $\underline{v} \in \underline{\mathbb{V}}^{\text {good }}$ and then to the global case. One important feature of these generalizations, especially in the global case, is the theme of compatibility with the theory of $\Theta N F$ - (respectively, $\Theta^{ \pm \text {ell }}$-) Hodge theaters - and, in particular, the $\mathbb{F}_{l}^{*}$ - (respectively, $\mathbb{F}_{l}^{\rtimes \pm}$-) symmetries of such Hodge theaters - developed in [IUTchI], §4, §5 (respectively, [IUTchI], §6).

In the following discussion, we assume that we have been given initial $\Theta$ data as in [IUTchI], Definition 3.1. We begin our discussion by considering good nonarchimedean $\underline{v} \in \underline{\mathbb{V}}^{\text {good }} \cap \underline{\mathbb{V}}^{\text {non }}$.

Proposition 4.1. (Group-theoretic Gaussian Monoids at Good Nonarchimedean Primes) Let $\underline{v} \in \underline{\mathbb{V}}^{\text {good }} \cap \underline{\mathbb{V}}^{\text {non }}$. In the notation of [IUTchI], Definition 3.1, (e), (f), write

$$
\Pi_{\underline{v}} \stackrel{\text { def }}{=} \Pi_{\underline{X}_{\underline{v}}} \subseteq \Pi_{\underline{v}}^{ \pm} \stackrel{\text { def }}{=} \Pi_{\underline{X}_{\underline{v}}} \subseteq \Pi_{\underline{v}} \stackrel{\text { cor }}{ } \stackrel{\text { def }}{=} \Pi_{C_{\underline{v}}}
$$

[cf. Definition 2.3, (i), in the case of $\left.\underline{v} \in \underline{\mathbb{V}}^{\text {bad }}\right]-$ so $\Pi_{\underline{v}}^{ \pm} / \Pi_{\underline{v}} \cong \mathbb{Z} / l \mathbb{Z}[c f$. the discussion preceding [IUTchI], Definition 1.1], $\Pi_{\underline{v}}^{\text {cor }} / \Pi_{\underline{v}}^{ \pm} \cong \overline{\mathbb{F}}_{l}^{\rtimes \pm}$;

$$
\Pi_{\underline{v}} \rightarrow G_{\underline{v}}\left(\Pi_{\underline{v}}\right), \quad \Pi_{\underline{v}}^{ \pm} \rightarrow G_{\underline{v}}\left(\Pi_{\underline{v}}^{ \pm}\right), \quad \Pi_{\underline{v}}^{\text {cor }} \rightarrow G_{\underline{v}}\left(\Pi_{\underline{v}}^{\text {cor }}\right)
$$

for the quotients - which admit natural isomorphisms $G_{\underline{v}}\left(\Pi_{\underline{v}}\right) \stackrel{\sim}{\rightarrow} G_{\underline{v}}\left(\Pi_{\underline{v}}^{ \pm}\right) \stackrel{\sim}{\rightarrow}$ $G_{\underline{v}}\left(\Pi_{\underline{v}}^{\text {cor }}\right) \stackrel{\sim}{\rightarrow} G_{\underline{v}}$ - determined by the natural surjections to $G_{\underline{v}} ; \Delta_{\underline{v}}, \Delta_{\underline{v}}^{ \pm}, \Delta_{\underline{v}}^{\text {cor }}$ for the respective kernels of these quotients. Also, we recall that $\Pi_{\underline{v}}^{ \pm}, \Pi_{\underline{v}}^{\text {cor }}, G_{\underline{v}}^{-}\left(\Pi_{\underline{v}}\right)$, $G_{\underline{v}}\left(\Pi_{\underline{v}}^{ \pm}\right)$, and $G_{\underline{v}}\left(\Pi_{\underline{v}}^{\text {cor }}\right)$ may be reconstructed algorithmically [c $\bar{f}$. [IUTchI], Corollary 1.2, and its proof; [AbsAnab], Lemma 1.3.8] from the topological group $\Pi_{\underline{v}}$.

(i) (Constant Monoids) The functorial group-theoretic algorithm of [AbsTopIII], Corollary 1.10, (b) [cf. also the discussion of Remark 1.11.5, (i), in the case of $\underline{v} \in \underline{\mathbb{V}}^{\text {bad }}$ ] yields a functorial group-theoretic algorithm in the topological group $G_{\underline{v}}$ for constructing the topological submonoid [which is naturally isomorphic to $\left.\overline{\mathcal{O}} \underline{\bar{F}}_{\underline{v}}\right]$

$$
\Psi_{\mathrm{cns}}\left(G_{\underline{v}}\right) \subseteq \underset{J}{\lim _{J}} H^{1}\left(J, \boldsymbol{\mu}_{\widehat{\mathbb{Z}}}\left(G_{\underline{v}}\right)\right)
$$

- where $J$ ranges over the open subgroups of $G_{\underline{v}} ; \boldsymbol{\mu}_{\widehat{\mathbb{Z}}}\left(G_{\underline{v}}\right)$ is as in [AbsTopIII], Corollary 1.10, (b) - equipped with its natural $G_{\underline{\underline{v}}}$-action. In particular, we obtain a functorial group-theoretic algorithm in the topological group $\Pi_{\underline{v}}$ for constructing the topological submonoid

$$
\begin{aligned}
\Psi_{\mathrm{cns}}\left(\Pi_{\underline{v}}\right) & \stackrel{\text { def }}{=} \Psi_{\mathrm{cns}}\left(G_{\underline{v}}\left(\Pi_{\underline{v}}\right)\right) \subseteq \varliminf_{J} H^{1}\left(\left.G_{\underline{v}}\left(\Pi_{\underline{v}}\right)\right|_{J}, \boldsymbol{\mu}_{\widehat{\mathbb{Z}}}\left(G_{\underline{v}}\left(\Pi_{\underline{v}}\right)\right)\right) \\
& \subseteq \varliminf_{J} H^{1}\left(\left.\Pi_{\underline{v}}^{ \pm}\right|_{J}, \boldsymbol{\mu}_{\widehat{\mathbb{Z}}}\left(G_{\underline{v}}\left(\Pi_{\underline{v}}\right)\right)\right) \subseteq \underset{J}{\subseteq} H^{1}\left(\left.\Pi_{\underline{v}}\right|_{J}, \boldsymbol{\mu}_{\widehat{\mathbb{Z}}}\left(G_{\underline{v}}\left(\Pi_{\underline{v}}\right)\right)\right)
\end{aligned}
$$


- where $J$ ranges over the open subgroups of $G_{\underline{v}}\left(\Pi_{\underline{v}}\right)$ - equipped with its natural $G_{\underline{v}}\left(\Pi_{\underline{v}}\right)$-action [cf. Proposition 3.1, (ii), in the case of $\underline{v} \in \underline{\mathbb{V}}^{\text {bad }}$ ].

(ii) (Mono-analytic Semi-simplifications) The functorial algorithm discussed in [IUTchI], Example 3.5, (iii), for constructing " $\left(\mathbb{R}_{\geq 0}^{\vdash}\right)_{\underline{v}}$ " [cf. also [AbsTopIII], Proposition 5.8, (iii)] yields a functorial group-theoretic algorithm in the topological group $G_{\underline{v}}$ for constructing a topological monoid $\mathbb{R}_{\geq 0}\left(G_{\underline{v}}\right)$ equipped with a natural isomorphism

$$
\Psi_{\mathrm{cns}}^{\mathbb{R}}\left(G_{\underline{\underline{v}}}\right) \stackrel{\text { def }}{=}\left(\Psi_{\mathrm{cns}}\left(G_{\underline{v}}\right) / \Psi_{\mathrm{cns}}\left(G_{\underline{v}}\right)^{\times}\right)^{\mathrm{rlf}} \stackrel{\sim}{\rightarrow} \mathbb{R}_{\geq 0}\left(G_{\underline{v}}\right)
$$

- where the superscript " $\times$ " denotes the submonoid of units; the superscript "rlf" denotes the realification [which is isomorphic to $\mathbb{R}_{\geq 0}$ ] of the monoid in parentheses [which is isomorphic to $\mathbb{Q}_{\geq 0}$ ] — and a distinguished element

$$
\log ^{G_{\underline{v}}}\left(p_{\underline{v}}\right) \in \mathbb{R}_{\geq 0}\left(G_{\underline{v}}\right)
$$

- i.e., the element $\log _{\Phi}^{\mathcal{D}}\left(p_{\underline{v}}\right)$ " of [IUTchI], Example 3.5, (iii). Write

$$
\Psi_{\mathrm{cns}}^{\mathrm{ss}}\left(G_{\underline{v}}\right) \stackrel{\text { def }}{=} \Psi_{\mathrm{cns}}\left(G_{\underline{v}}\right)^{\times} \times \mathbb{R}_{\geq 0}\left(G_{\underline{v}}\right)
$$

- which we shall think of as a sort of "semi-simplified version" of $\Psi_{\mathrm{cns}}\left(G_{\underline{v}}\right)$. Also, just as in (i), we shall abbreviate notation that denotes a dependence on " $G_{\underline{v}}\left(\Pi_{\underline{v}}\right)$ " [e.g., a " $G_{\underline{v}}\left(\Pi_{\underline{v}}\right)$ " in parentheses] by means of notation that denotes a dependence on " $\Pi_{\underline{v}}$ ".

(iii) (Labels, $\mathbb{F}_{l}^{\rtimes \pm}$-Symmetries, and Conjugate Synchronization) Let $t \in \operatorname{LabCusp}^{ \pm}\left(\Pi_{\underline{v}}\right) \stackrel{\text { def }}{=} \operatorname{LabCusp}^{ \pm}\left(\mathcal{B}\left(\Pi_{\underline{v}}\right)^{0}\right)$ [cf. [IUTchI], Definition 6.1, (iii)]. In the following, we shall use analogous conventions to the conventions introduced in Corollary 3.5 concerning subscripted labels. Then if we think of the cuspidal inertia groups $\subseteq \Pi_{\underline{v}}$ corresponding to $t$ as subgroups of cuspidal inertia groups of $\Pi_{\underline{v}}^{ \pm}$[cf. Remark 2.3.1, in the case of $\left.\underline{v} \in \underline{\mathbb{V}}^{\text {bad }}\right]$, then the $\Delta_{\underline{v}}^{ \pm}$-outer action of $\mathbb{F}_{l}^{\rtimes \pm} \cong \Delta_{\underline{v}}^{\text {cor }} / \Delta_{\underline{v}}^{ \pm}$on $\Pi_{\underline{v}}^{ \pm}$[cf. Corollary 2.4, (iii), in the case of $\left.\underline{v} \in \underline{\mathbb{V}}^{\text {bad }}\right]$ induces isomorphisms between the pairs

$$
G_{\underline{v}}\left(\Pi_{\underline{v}}\right)_{t} \curvearrowright \Psi_{\mathrm{cns}}\left(\Pi_{\underline{v}}\right)_{t}
$$

- consisting of a labeled topological monoid equipped with the action of a labeled topological group - for distinct $t \in \operatorname{LabCusp}^{ \pm}\left(\Pi_{\underline{v}}\right)$. We shall refer to these isomorphisms as $\left[\mathbb{F}_{l}^{\rtimes \pm}\right.$-] symmetrizing isomorphisms [cf. Remark 3.5.2, in the case of $\underline{v} \in \underline{\mathbb{V}}^{\text {bad }}$. These symmetrizing isomorphisms determine diagonal submonoids

$$
\Psi_{\mathrm{cns}}\left(\Pi_{\underline{v}}\right)_{\left\langle\left|\mathbb{F}_{l}\right|\right\rangle} \subseteq \prod_{|t| \in\left|\mathbb{F}_{l}\right|} \Psi_{\mathrm{cns}}\left(\Pi_{\underline{v}}\right)_{|t|} ; \quad \Psi_{\mathrm{cns}}\left(\Pi_{\underline{v}}\right)_{\left\langle\mathbb{F}_{l}^{*}\right\rangle} \subseteq \prod_{|t| \in \mathbb{F}_{l}^{*}} \Psi_{\mathrm{cns}}\left(\Pi_{\underline{v}}\right)_{|t|}
$$

of the respective product monoids compatible with the respective actions by subscripted versions of $G_{\underline{v}}\left(\Pi_{\underline{v}}\right)$ [cf. the discussion of Corollary 3.5, (i), in the case of $\left.\underline{v} \in \underline{\mathbb{V}}^{\mathrm{bad}}\right]$, as well as an isomorphism of topological monoids

$$
\Psi_{\mathrm{cns}}\left(\Pi_{\underline{v}}\right)_{0} \stackrel{\sim}{\rightarrow} \quad \Psi_{\mathrm{cns}}\left(\Pi_{\underline{v}}\right)_{\left\langle\mathbb{F}_{l}^{*}\right\rangle}
$$


compatible with the respective actions by subscripted versions of $G_{\underline{v}}\left(\Pi_{\underline{v}}\right)$ [cf. Corollary 3.5, (iii), in the case of $\left.\underline{v} \in \underline{\mathbb{V}}^{\text {bad }}\right]$.

(iv) (Theta and Gaussian Monoids) Write

$$
\Psi_{\mathrm{env}}\left(\Pi_{\underline{v}}\right) \stackrel{\text { def }}{=} \Psi_{\mathrm{cns}}\left(\Pi_{\underline{v}}\right)^{\times} \times\left\{\mathbb{R}_{\geq 0} \cdot \log ^{\Pi_{\underline{v}}}\left(p_{\underline{v}}\right) \cdot \log \Pi^{\underline{v}}(\underline{\underline{\Theta}})\right\}
$$

- where the notation " $\log ^{\Pi_{\underline{v}}}\left(p_{\underline{v}}\right) \cdot \log ^{\Pi_{\underline{v}}}(\underline{\underline{\Theta}})$ " is to be understood as a formal symbol [cf. the discussion of [IUTchI], Example 3.3, (ii)] — and

$$
\begin{aligned}
\Psi_{\text {gau }}\left(\Pi_{\underline{v}}\right) & \stackrel{\text { def }}{=} \Psi_{\mathrm{cns}}\left(\Pi_{\underline{v}}\right)_{\left\langle\mathbb{F}_{l}^{*}\right\rangle}^{\times} \times\left\{\mathbb{R}_{\geq 0} \cdot\left(\ldots, j^{2} \cdot \log \Pi_{\underline{v}}\left(p_{\underline{v}}\right), \ldots\right)\right\} \\
& \subseteq \prod_{j \in \mathbb{F}_{l}^{*}} \Psi_{\mathrm{cns}}^{\mathrm{ss}}\left(\Pi_{\underline{v}}\right)_{j}=\prod_{j \in \mathbb{F}_{l}^{*}} \Psi_{\mathrm{cns}}\left(\Pi_{\underline{v}}\right)_{j}^{\times} \times \mathbb{R}_{\geq 0}\left(\Pi_{\underline{v}}\right)_{j}
\end{aligned}
$$

- where, by abuse of notation, we also write " $j$ " for the natural number $\in\left\{1, \ldots, l^{*}\right\}$ determined by an element $j \in \mathbb{F}_{l}^{*}$. In particular, [cf. (i), (ii), (iii)] we obtain a functorial group-theoretic algorithm in the topological group $\Pi_{\underline{v}}$ for constructing the theta monoid $\Psi_{\text {env }}\left(\Pi_{\underline{v}}\right)$ and the Gaussian monoid $\Psi_{\text {gau }}\left(\Pi_{\underline{v}}\right)$, equipped with their [evident] natural splittings, as well as the formal evaluation isomorphism [cf. Corollary 3.5, (ii), in the case of $\underline{v} \in \underline{\mathbb{V}}^{\text {bad }}$ ]

$$
\begin{aligned}
\Psi_{\text {env }}\left(\Pi_{\underline{v}}\right) & \stackrel{\sim}{\rightarrow} \Psi_{\text {gau }}\left(\Pi_{\underline{v}}\right) \\
\log \Pi_{\underline{v}}\left(p_{\underline{v}}\right) \cdot \log \Pi_{\underline{v}}(\underline{\underline{\Theta}}) & \mapsto\left(\ldots, j^{2} \cdot \log ^{\Pi_{\underline{v}}}\left(p_{\underline{v}}\right), \ldots\right)
\end{aligned}
$$

- which restricts to the identity on the respective copies of " $\Psi_{\mathrm{cns}}\left(\Pi_{\underline{v}}\right)^{\times}$" and is compatible with the respective natural actions of $G_{\underline{v}}\left(\Pi_{\underline{v}}\right)$ as well as with the natural splittings on the domain and codomain.

Proof. The various assertions of Proposition 4.1 follow immediately from the definitions and the references quoted in the statements of these assertions.

\section{Remark 4.1.1.}

(i) Proposition 4.1 may be thought of as a sort of "easy" formal generalization of much of the theory of $\S 2, \S 3$ - more precisely, the portion constituted by Proposition 3.1 and Corollaries 2.4, 3.5 - to the case of $\underline{v} \in \underline{\mathbb{V}}^{\text {good }} \cap \underline{\mathbb{V}}^{\text {non }}$. By comparison to the corresponding portion of the theory of $\S 2, \S 3$, this generalization is somewhat tautological and, for the most part, "vacuous". As we shall see later, the reason for considering this formal generalization to $\underline{v} \in \underline{\mathbb{V}}^{\text {good }} \cap \underline{\mathbb{V}}^{\text {non }}$ is that it allows one to "globalize" the theory of $\S 2, \S 3$, i.e., by gluing together the theories at $\underline{v} \in \underline{\mathbb{V}}^{\text {bad }}$ and $\underline{v} \in \underline{\mathbb{V}}^{\text {good }}$.

(ii) The symmetrizing isomorphisms of Proposition 4.1, (iii), constitute the analogue at $\underline{v} \in \underline{\mathbb{V}}^{\text {good }} \cap \underline{\mathbb{V}}^{\text {non }}$ of the conjugate synchronization at $\underline{v} \in \underline{\mathbb{V}}^{\text {bad }}$ discussed in Corollary 3.5, (i); Remark 3.5.2. In this context, it is perhaps most natural to think of the "copies of $G_{\underline{v}}\left(\Pi_{\underline{v}}\right)$ labeled by $t \in \operatorname{LabCusp}^{ \pm}\left(\Pi_{\underline{v}}\right)$ " as the quotients

$$
D_{t} / I_{t}
$$


- where $I_{t}$ is a cuspidal inertia group $\subseteq \Pi_{\underline{v}}$ corresponding to $t ; D_{t}$ is the corresponding decomposition group $\subseteq \Pi_{\underline{v}}$ [i.e., the normalizer, or, equivalently, the commensurator, of $I_{t}$ in $\Pi_{\underline{v}}$ - cf., e.g., [AbsSect], Theorem 1.3, (ii)]; we think of $D_{t} / I_{t}$ as being equipped with the isomorphism $D_{t} / I_{t} \stackrel{\sim}{\rightarrow} G_{\underline{v}}\left(\Pi_{\underline{v}}\right)$ induced by the natural surjection $\Pi_{\underline{v}} \rightarrow G_{\underline{v}}\left(\Pi_{\underline{v}}\right)$.

(iii) One may also formulate an easy tautological formal analogue at $\underline{v} \in$ $\underline{\mathbb{V}}^{\text {good }} \cap \underline{\mathbb{V}}^{\text {non }}$ of the multiradiality and uniradiality assertions of Proposition 3.4, Corollary 3.7 at $\underline{v} \in \underline{\mathbb{V}}$. For instance,

(a) the construction of the monoids $\Psi_{\text {cns }}\left(\Pi_{\underline{v}}\right)$ [cf. Proposition 4.1, (i)] is uniradial [cf. Proposition 3.4, (ii)], while

(b) the construction of the monoids $\Psi_{\mathrm{cns}}^{\mathrm{ss}}\left(\Pi_{\underline{v}}\right), \Psi_{\mathrm{env}}\left(\Pi_{\underline{v}}\right)$, and $\Psi_{\mathrm{gau}}\left(\Pi_{\underline{v}}\right)[\mathrm{cf}$. Proposition 4.1, (ii), (iv)], as well as of the isomorphism $\Psi_{\text {env }}\left(\Pi_{\underline{v}}\right) \stackrel{\sim}{\rightarrow} \Psi_{\text {gau }}\left(\Pi_{\underline{v}}\right)$ [cf. Proposition 4.1, (iv)], is multiradial.

We leave the routine details to the reader. Ultimately, in the present series of papers [cf., especially, the theory of [IUTchIII]], we shall be interested in a global analogue of the theory of multiradiality and uniradiality developed in $\S 1, \S 3$ at $\underline{v} \in \underline{\mathbb{V}}^{\text {bad }}$. This global analogue will "specialize" to the theory of $\S 1, \S 3$ at $\underline{v} \in \underline{\mathbb{V}}^{\text {bad }}$ and to the formal analogue just discussed [i.e., (a), (b)] at $\underline{v} \in \underline{\mathbb{V}}^{\text {good }} \cap \underline{\mathbb{V}}^{\text {non }}$.

Proposition 4.2. (Frobenioid-theoretic Gaussian Monoids at Good Nonarchimedean Primes) We continue to use the notation of Proposition 4.1. Let ${ }^{\dagger} \underline{\underline{\mathcal{F}}}_{v}$ be a $\boldsymbol{p}_{\underline{v}}$-adic Frobenioid that appears in a $\Theta$-Hodge theater ${ }^{\dagger} \mathcal{H} \mathcal{T}^{\Theta}=$ $\left(\left\{\underline{\underline{F}}_{\underline{v}}\right\}_{\underline{v}} \in \underline{\underline{V}},{ }^{\dagger} \mathfrak{F}_{\text {mod }}^{\vdash}\right)[c f$. [IUTchI], Definition 3.6] - cf., for instance, the Frobenioid $\underline{\underline{F}}_{v}=\mathcal{C}_{\underline{v}}$ " of [IUTchI], Example 3.3, (i); here, we assume [for simplicity] that the base category of ${ }^{\dagger} \underline{\underline{\mathcal{F}}}_{\underline{v}}$ is equal to $\mathcal{B}^{\text {temp }}\left({ }^{\dagger} \Pi_{\underline{v}}\right)^{0}$, and we denote by means of a "†" the various topological groups associated to ${ }^{\dagger} \Pi_{\underline{v}}$ that correspond to the topological groups associated to $\Pi_{\underline{v}}$ in Proposition 4.1. Write

$$
G_{\underline{v}}\left(\Pi_{\underline{v}}\right) \curvearrowright \Psi_{\underline{\mathcal{F}}_{\underline{\mathcal{F}}}}
$$

for the topological monoid $\Psi_{\dagger} \underline{\underline{\mathcal{F}}}_{\underline{v}}$ equipped with a continuous $G_{\underline{v}}\left({ }^{\dagger} \Pi_{\underline{v}}\right)$-action determined, up to inner automorphism [i.e., up to an automorphism arising from an element of $\left.{ }^{\dagger} \Pi_{\underline{v}}\right]$, by ${ }^{\dagger} \underline{\underline{\mathcal{F}}}_{\underline{v}}$ [cf. the construction of " $\Psi_{\mathcal{C}_{\underline{v}}}$ " in Example 3.2, (ii), in the case of $\underline{v} \in \underline{\mathbb{V}}^{\text {bad }}$; the discussion of [AbsTopIII], Remark 3.1.1] and

$$
{ }^{\dagger} G_{\underline{\underline{v}}} \curvearrowright \Psi_{\dagger \mathcal{F}_{\underline{v}}^{\vdash}}
$$

for the topological monoid $\Psi_{\dagger \mathcal{F}_{v}}$ equipped with a continuous ${ }^{\dagger} G_{\underline{v}^{-}}$action determined,

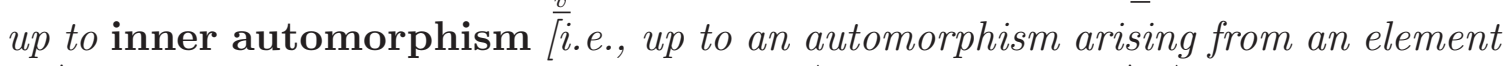
of $\left.{ }^{\dagger} G_{\underline{v}}\right]$, by the portion indexed by $\underline{v}$ of the $\mathcal{F}^{\vdash}$-prime-strip $\left\{{ }^{\dagger} \mathcal{F}_{\underline{w}}^{\vdash}\right\}_{\underline{w} \in \underline{\mathbb{V}}}$ determined by the $\Theta$-Hodge theater ${ }^{\dagger} \mathcal{H} \mathcal{T}^{\Theta}$ [cf. [IUTchI], Definition 3.6; [I $\bar{U}$ TchI], Definition 5.2, (ii)]. 
(i) (Constant Monoids) There exists a unique $G_{v}\left({ }^{\dagger} \Pi_{v}\right)$-equivariant isomorphism of monoids [cf. Proposition 3.3, (ii), in the case of $\underline{v} \in \underline{\mathbb{V}}^{\text {bad }}$ ]

$$
\Psi_{\underline{\underline{\mathcal{F}}}_{\underline{v}}} \stackrel{\sim}{\rightarrow} \quad \Psi_{\mathrm{cns}}\left({ }^{\dagger} \Pi_{\underline{v}}\right)
$$

- cf. Remark 1.11.1, (i), (a); [AbsTopIII], Proposition 3.2, (iv).

(ii) (Mono-analytic Semi-simplifications) There exists a unique ${ }^{\dagger} G_{\underline{v}^{-}}$ equivariant $\widehat{\mathbb{Z}}^{\times}$-orbit of isomorphisms of topological groups

$$
\Psi_{\dagger \mathcal{F}_{\underline{v}}}^{\times} \stackrel{\sim}{\rightarrow} \Psi_{\mathrm{cns}}\left({ }^{\dagger} G_{\underline{v}}\right)^{\times}
$$

- cf. Remark 1.11.1, (i), (b); [AbsTopIII], Proposition 3.3, (ii) - as well as a unique isomorphism of monoids

$$
\Psi_{\dagger \mathcal{F}_{\underline{v}}^{\vdash}}^{\mathbb{R}} \stackrel{\text { def }}{=}\left(\Psi_{\dagger \mathcal{F}_{\underline{v}}} / \Psi_{\dagger \mathcal{F}_{\underline{v}}^{\vdash}}^{\times}\right)^{\mathrm{rlf}} \stackrel{\sim}{\rightarrow} \quad \Psi_{\mathrm{cns}}^{\mathbb{R}}\left({ }^{\dagger} G_{\underline{v}}\right)
$$

that maps the distinguished element of $\Psi_{\dagger}^{\mathbb{R} \mathcal{F}_{\underline{v}}^{\vdash}}$ determined by the unique generator of $\Psi_{\dagger \mathcal{F}_{\underline{v}}^{\vdash}} / \Psi_{\Psi_{\mathcal{F}_{\underline{v}}}^{\vdash}}^{\times}$to the distinguished element of $\Psi_{\mathrm{cns}}^{\mathbb{R}}\left({ }^{\dagger} G_{\underline{v}}\right)$ determined by $\log ^{\dagger} G_{\underline{v}}\left(p_{\underline{v}}\right) \in \mathbb{R}_{\geq 0}\left({ }^{\dagger} G_{\underline{v}}\right)$ [cf. Proposition 4.1, (ii)]. In particular, one may define $a$ "semi-simplified version" $\Psi_{\dagger \mathcal{F}_{\underline{v}}^{\vdash}}^{\text {ss }} \stackrel{\text { def }}{=} \Psi_{\dagger \mathcal{F}_{\underline{v}}}^{\times} \times \Psi_{\dagger \mathcal{F}_{\underline{v}}^{\vdash}}^{\mathbb{R}}$ of $\Psi_{\dagger \mathcal{F}_{\underline{v}}^{\vdash}}$; the isomorphisms discussed above determine a natural poly-isomorphism of topological monoids

$$
\Psi_{\dagger \mathcal{F}_{\underline{v}}}^{\mathrm{ss}} \stackrel{\sim}{\rightarrow} \quad \Psi_{\mathrm{cns}}^{\mathrm{ss}}\left({ }^{\dagger} G_{\underline{v}}\right)
$$

[cf. Proposition 4.1, (ii)] that is compatible with the natural splittings on the domain and codomain. Write $\Psi_{\Psi_{\underline{\underline{\mathcal{F}}}}^{\mathrm{ss}}}^{\mathrm{ss}} \stackrel{\text { def }}{=} \Psi_{+\mathcal{F}_{\underline{v}}^{\vdash}}^{\mathrm{ss}}$; thus, it follows from the definitions that we have a natural isomorphism $\Psi_{\underline{\underline{\mathcal{F}}}_{\underline{v}}^{\mathrm{ss}}}^{\mathrm{ss}} \stackrel{\sim}{\rightarrow} \Psi_{\mathfrak{F}_{\underline{v}}^{\vdash}}^{\mathrm{ss}}$.

(iii) (Labels, $\mathbb{F}_{l}^{\rtimes \pm}$-Symmetries, and Conjugate Synchronization) The isomorphism of $(i)$ determines, for each $t \in \operatorname{LabCusp}^{ \pm}\left({ }^{\dagger} \Pi_{\underline{v}}\right)$, a collection of compatible isomorphisms

$$
\left(\Psi_{\underline{\mathcal{F}}_{\underline{\underline{\mathcal{F}}}}}\right)_{t} \stackrel{\sim}{\rightarrow} \quad \Psi_{\mathrm{cns}}\left({ }^{\dagger} \Pi_{\underline{v}}\right)_{t}
$$

- which are well-defined up to composition with an inner automorphism of ${ }^{\dagger} \Pi_{\underline{v}}$ which is independent of $t \in \operatorname{LabCusp}^{ \pm}\left({ }^{\dagger} \Pi_{\underline{v}}\right)$ [cf. Corollary 3.6, (i), in the case of $\left.\underline{v} \in \underline{\mathbb{V}}^{\text {bad }}\right]$ - as well as $\left[\mathbb{F}_{l}^{\rtimes \pm}\right.$-] symmetrizing isomorphisms, induced by the ${ }^{\dagger} \Delta_{\underline{v}}^{ \pm}$-outer action of $\mathbb{F}_{l}^{\rtimes \pm} \cong{ }^{\dagger} \Delta_{\underline{v}}^{\text {cor }} /{ }^{\dagger} \Delta_{\underline{v}}^{ \pm}$on ${ }^{\dagger} \Pi_{\underline{v}}^{ \pm}$[cf. Corollary 2.4, (iii), in the case of $\left.\underline{v} \in \underline{\mathbb{V}}^{\text {bad }}\right]$, between the data indexed by distinct $t \in \operatorname{LabCusp}^{ \pm}\left({ }^{\dagger} \Pi_{\underline{v}}\right)$. Moreover, these symmetrizing isomorphisms determine [various diagonal submonoids, as well as] an isomorphism of topological monoids

$$
\left(\Psi_{\underline{\mathcal{F}}_{\underline{\underline{\mathcal{F}}}}}\right)_{0} \quad \stackrel{\sim}{\rightarrow} \quad\left(\Psi_{\underline{\underline{\mathcal{F}}}_{\underline{v}}}\right)_{\left.\mathbb{F}_{l}^{*}\right\rangle}
$$


compatible with the respective actions by subscripted versions of $G_{\underline{v}}\left({ }^{\dagger} \Pi_{\underline{v}}\right)$ [cf. Corollary 3.6, (iii), in the case of $\left.\underline{v} \in \underline{\mathbb{V}}^{\text {bad }}\right]$.

(iv) (Theta and Gaussian Monoids) Write

$$
\Psi_{\dagger \mathcal{F}_{\underline{\underline{v}}}^{\Theta}}, \quad \Psi_{\mathcal{F}_{\text {gau }}}\left({ }^{\dagger} \underline{\underline{\mathcal{F}}}_{\underline{v}}\right)
$$

for the monoids equipped with $G_{\underline{v}}\left({ }^{\dagger} \Pi_{\underline{v}}\right)$-actions and natural splittings determined, respectively - via the isomorphisms of (i), (ii), and (iii) - by the monoids $\Psi_{\mathrm{env}}\left({ }^{\dagger} \Pi_{\underline{v}}\right), \Psi_{\mathrm{gau}}\left({ }^{\dagger} \Pi_{\underline{v}}\right)$, Galois actions, and splittings of Proposition 4.1, (iv). Then the definition of the various monoids involved, together with the formal evaluation isomorphism of Proposition 4.1, (iv), gives rise to a collection of natural isomorphisms [cf. Corollary 3.6, (ii), in the case of $\underline{v} \in \underline{\mathbb{V}}^{\text {bad }}$ ]

$$
\Psi_{\dagger \mathcal{F}_{\underline{v}}^{\Theta}} \stackrel{\sim}{\rightarrow} \quad \Psi_{\mathrm{env}}\left({ }^{\dagger} \Pi_{\underline{v}}\right) \quad \stackrel{\sim}{\rightarrow} \quad \Psi_{\mathrm{gau}}\left({ }^{\dagger} \Pi_{\underline{\underline{v}}}\right) \quad \stackrel{\sim}{\rightarrow} \quad \Psi_{\mathcal{F}_{\mathrm{gau}}}\left({ }^{\dagger} \underline{\underline{\mathcal{F}}}_{\underline{v}}\right)
$$

- which restrict to the identity or to the [restriction to " $(-)^{\times}$" of the] isomorphism of (i) [or its inverse] on the various copies of $\Psi_{\underline{\dagger}_{\underline{\underline{F}}}^{\times}}^{\times}, "$ " $\Psi_{\mathrm{cns}}\left({ }^{\dagger} \Pi_{\underline{v}}\right)^{\times}$" and are compatible with the various natural actions of $G_{\underline{v}}\left({ }^{\dagger} \Pi_{\underline{v}}\right)$ and natural splittings.

Proof. The various assertions of Proposition 4.2 follow immediately from the definitions and the references quoted in the statements of these assertions.

\section{Remark 4.2.1.}

(i) In the case of $\underline{v} \in \underline{\mathbb{V}}^{\text {bad }}$ treated in $\S 3$, we did not discuss an analogue of the "mono-analytic semi-simplification" $\Psi_{\mathrm{cns}}^{\mathrm{ss}}\left({ }^{\dagger} G_{\underline{v}}\right)$ of Proposition 4.1, (ii). On the other hand, one verifies immediately that one may define, in the case of $\underline{v} \in \underline{\mathbb{V}}^{\text {bad }}$ via the same group-theoretic algorithms as those applied in Proposition 4.1, (i), (ii) - topological monoids $\Psi_{\mathrm{cns}}^{\mathrm{ss}}\left({ }^{\dagger} G_{\underline{v}}\right), \mathbb{R}_{\geq 0}\left({ }^{\dagger} G_{\underline{v}}\right)$ equipped with natural ${ }^{\dagger} G_{\underline{\underline{v}}}$-actions, a distinguished element $\log ^{\dagger} G_{\underline{v}}\left(p_{\underline{v}}\right) \in \mathbb{R}_{\geq 0}\left({ }^{\dagger} G_{\underline{v}}\right)$, and a tautological splitting

$$
\Psi_{\mathrm{cns}}^{\mathrm{ss}}\left({ }^{\dagger} G_{\underline{v}}\right)=\Psi_{\mathrm{cns}}^{\mathrm{ss}}\left({ }^{\dagger} G_{\underline{v}}\right)^{\times} \times \mathbb{R}_{\geq 0}\left({ }^{\dagger} G_{\underline{v}}\right)
$$

[cf. Proposition 4.1, (ii)]. Moreover, if we write

$$
\Psi_{\mathrm{cns}}\left(\Pi_{\underline{v}}\right) \stackrel{\text { def }}{=} \Psi_{\mathrm{cns}}\left(\mathbb{M}_{*}^{\Theta}\left(\Pi_{\underline{v}}\right)\right)
$$

— where the latter " $\Psi_{\text {cns }}(-)$ " is as in Proposition 3.1, (ii) — then, by applying the cyclotomic rigidity isomorphisms of Definition 1.1, (ii), and the discussion at the beginning of Corollary 2.9, one obtains a functorial group-theoretic [i.e., in the topological group $\left.\Pi_{\underline{v}}\right] \Pi_{\underline{v}}$-equivariant isomorphism

$$
\Psi_{\mathrm{cns}}\left(\Pi_{\underline{v}}\right)^{\times} \stackrel{\sim}{\rightarrow} \Psi_{\mathrm{cns}}^{\mathrm{ss}}\left(G_{\underline{v}}\left(\Pi_{\underline{v}}\right)\right)^{\times}
$$

— cf. the discussion of " $\Psi_{\mathrm{cns}}^{\mathrm{ss}}(-)$ " in the case of $\underline{v} \in \underline{\mathbb{V}}^{\text {good }} \cap \underline{\mathbb{V}}^{\text {non }}$ in Proposition 4.1, (ii). Finally, we observe that, relative to the above notation, one has analogues 
of " $\Psi_{\uparrow \mathcal{F}_{\underline{v}}^{\vdash}}^{\mathrm{ss}}$ " and of Proposition 4.2, (i), (ii), in the case of $\underline{v} \in \underline{\mathbb{V}}^{\text {bad }}$. We leave the routine $\overline{\text { details }}$ to the reader.

(ii) Note that in the case of $\underline{v} \in \underline{\mathbb{V}}^{\text {good }} \cap \underline{\mathbb{V}}^{\text {non }}$, the monoids $\Psi_{\text {env }}\left(\Pi_{\underline{v}}\right), \Psi_{\text {gau }}\left(\Pi_{\underline{v}}\right)$ of Proposition 4.1, (iv), are already divisible. Thus, it is natural, in the case of $\underline{v} \in \underline{\mathbb{V}}^{\text {good }} \cap \underline{\mathbb{V}}^{\text {non }}$, to simply set

$$
\begin{array}{rlllll}
\infty \Psi_{\text {env }}\left(\Pi_{\underline{v}}\right) & \stackrel{\text { def }}{=} & \Psi_{\text {env }}\left(\Pi_{\underline{v}}\right) ; & \infty \Psi_{\text {gau }}\left(\Pi_{\underline{v}}\right) & \stackrel{\text { def }}{=} & \Psi_{\text {gau }}\left(\Pi_{\underline{v}}\right) \\
\infty \Psi_{\dagger \mathcal{F}_{\underline{v}}^{\Theta}} & \stackrel{\text { def }}{=} & \Psi_{\dagger \mathcal{F}_{\underline{v}}^{\Theta}} ; & \infty \Psi_{\mathcal{F}_{\text {gau }}}\left({ }^{\dagger} \underline{\underline{\mathcal{F}}}_{\underline{v}}\right) & \stackrel{\text { def }}{=} & \Psi_{\mathcal{F}_{\text {gau }}}\left({ }^{\dagger} \underline{\underline{\mathcal{F}}}\right)
\end{array}
$$

— cf. the various monoids " $\infty \Psi(-)$ " that appeared in the discussion of $\S 3$.

(iii) In the situation of (ii), if one regards the pairs $G_{\underline{v}}\left(\Pi_{\underline{v}}\right) \curvearrowright \Psi_{\text {env }}\left(\Pi_{\underline{v}}\right)$, $G_{\underline{v}}\left(\Pi_{\underline{v}}\right) \curvearrowright \Psi_{\text {gau }}\left(\Pi_{\underline{v}}\right), G_{\underline{v}}\left(\Pi_{\underline{v}}\right) \curvearrowright \infty \Psi_{\text {env }}\left(\Pi_{\underline{v}}\right), G_{\underline{v}}\left(\Pi_{\underline{v}}\right) \curvearrowright \infty \Psi_{\text {gau }}\left(\Pi_{\underline{v}}\right)$ up to an indeterminacy with respect to $\Pi_{v}$-inner automorphisms, then one obtains data which we shall denote by means of the notation

$\Psi_{\text {env }}\left(\mathcal{B}^{\text {temp }}\left(\Pi_{\underline{v}}\right)^{0}\right), \quad \Psi_{\text {gau }}\left(\mathcal{B}^{\text {temp }}\left(\Pi_{\underline{v}}\right)^{0}\right), \quad \infty \Psi_{\text {env }}\left(\mathcal{B}^{\text {temp }}\left(\Pi_{\underline{v}}\right)^{0}\right), \quad \infty \Psi_{\text {gau }}\left(\mathcal{B}^{\text {temp }}\left(\Pi_{\underline{v}}\right)^{0}\right)$

- i.e., since $\Pi_{\underline{v}}$ may only be reconstructed from $\mathcal{B}^{\text {temp }}\left(\Pi_{\underline{v}}\right)^{0}$ up to an inner automorphism indeterminacy [cf. the discussion of [IUTchI], §0].

(iv) Suppose that $\underline{v} \in \underline{\mathbb{V}}^{\text {bad }}$. Then the above discussion motivates the following notational conventions. First, let us write

$$
\begin{aligned}
& \Psi_{\mathrm{env}}\left(\Pi_{\underline{v}}\right) \stackrel{\text { def }}{=} \Psi_{\mathrm{env}}\left(\mathbb{M}_{*}^{\Theta}\left(\Pi_{\underline{v}}\right)\right), \quad \Psi_{\mathrm{gau}}\left(\Pi_{\underline{v}}\right) \stackrel{\text { def }}{=} \Psi_{\mathrm{gau}}\left(\mathbb{M}_{*}^{\Theta}\left(\Pi_{\underline{v}}\right)\right) \\
& \infty \Psi_{\mathrm{env}}\left(\Pi_{\underline{v}}\right) \stackrel{\text { def }}{=} \infty \Psi_{\mathrm{env}}\left(\mathbb{M}_{*}^{\Theta}\left(\Pi_{\underline{v}}\right)\right), \quad \infty \Psi_{\text {gau }}\left(\Pi_{\underline{v}}\right) \stackrel{\text { def }}{=}{ }_{\infty} \Psi_{\text {gau }}\left(\mathbb{M}_{*}^{\Theta}\left(\Pi_{\underline{v}}\right)\right)
\end{aligned}
$$

- cf. (ii) above; the notation of Corollary 3.5, (ii). When these monoids equipped with various topological group actions are considered only up to a $\Pi_{\underline{v}}$-inner automorphism indeterminacy, we shall denote the resulting data by means of the notation

$\Psi_{\text {env }}\left(\mathcal{B}^{\text {temp }}\left(\Pi_{\underline{v}}\right)^{0}\right), \quad \Psi_{\text {gau }}\left(\mathcal{B}^{\text {temp }}\left(\Pi_{\underline{v}}\right)^{0}\right), \quad \infty \Psi_{\text {env }}\left(\mathcal{B}^{\text {temp }}\left(\Pi_{\underline{v}}\right)^{0}\right), \quad \infty \Psi_{\text {gau }}\left(\mathcal{B}^{\text {temp }}\left(\Pi_{\underline{v}}\right)^{0}\right)$

— cf. (iii) above.

Next, we consider [good] archimedean $\underline{v} \in \underline{\mathbb{V}}^{\text {arc }}\left(\subseteq \underline{\mathbb{V}}^{\text {good }}\right)$.

Proposition 4.3. (Aut-holomorphic-space-theoretic Gaussian Monoids at Archimedean Primes) Let $\underline{v} \in \underline{\mathbb{V}}^{\text {arc }}\left(\subseteq \underline{\mathbb{V}}^{\text {good }}\right)$. Recall the Aut-holomorphic orbispaces of [IUTchI], Example 3.4, (i),

$$
\mathbb{U}_{\underline{v}} \stackrel{\text { def }}{=} \underline{\mathbb{X}}_{\underline{v}} \rightarrow \mathbb{U}_{\underline{v}}^{ \pm} \stackrel{\text { def }}{=} \underline{\mathbb{X}}_{\underline{v}} \subseteq \mathbb{U}_{\underline{v}}^{\text {cor }} \stackrel{\text { def }}{=} \mathbb{C}_{\underline{v}}
$$

- so $\operatorname{Gal}\left(\mathbb{U}_{\underline{v}}^{ \pm} / \mathbb{U}_{\underline{v}}\right) \cong \mathbb{Z} / l \mathbb{Z}$ [cf. the discussion preceding [IUTchI], Definition 1.1], $\operatorname{Gal}\left(\mathbb{U}_{\underline{v}}^{\text {cor }} / \mathbb{U}_{\underline{v}}^{ \pm}\right) \cong \mathbb{F}_{l}^{\rtimes \pm}$; we shall apply the notation " $\overline{\mathcal{A}}_{\square}$ ", “A $\mathcal{A}_{\square}$ " of [IUTchI], Example 3.4, (i), to these Aut-holomorphic orbispaces. Also, we shall write $\mathcal{A}_{\square} \subseteq$ 
$\mathcal{A}_{\square} \subseteq \overline{\mathcal{A}}_{\square}$ for the topological monoid of nonzero elements of absolute value $\leq 1$ of the complex archimedean field $\overline{\mathcal{A}}_{\square}$ [cf. the slightly different notation of [Abs TopIII], Corollary 4.5, (ii)]. Finally, we recall the object $\mathcal{D}_{\underline{v}}^{\vdash}$ of the category "TTM" ${ }^{\vdash}$ " of split topological monoids discussed in [IUTchI], Example 3.4, (ii); we shall write $\mathcal{D}_{\underline{v}}^{-}\left(\mathbb{U}_{\underline{v}}\right)$ when we wish to regard $\mathcal{D}_{\underline{v}}^{\vdash}$ as an object algorithmically constructed from $\mathbb{U}_{\underline{v}}$.

(i) (Constant Monoids) There is a functorial algorithm in the Autholomorphic space $\mathbb{U}_{\underline{v}}$ for constructing the topological monoid

$$
\Psi_{\text {cns }}\left(\mathbb{U}_{\underline{v}}\right) \stackrel{\text { def }}{=} \mathcal{A}_{\mathbb{U}_{\underline{v}}}^{\triangleright}
$$

- cf. [IUTchI], Example 3.4, (i); [AbsTopIII], Definition 4.1, (i); [AbsTopIII], Corollary 2.7, (e). Moreover, if we write $\Psi_{\mathrm{cns}}\left(\mathcal{D}_{\underline{v}}^{+}\right)$for the underlying topological monoid of $\mathcal{D}_{\underline{v}}^{+}$, then we have a tautological isomorphism of topological monoids

$$
\Psi_{\mathrm{cns}}\left(\mathbb{U}_{\underline{v}}\right) \stackrel{\sim}{\rightarrow} \quad \Psi_{\mathrm{cns}}\left(\mathcal{D}_{\underline{v}}^{\vdash}\left(\mathbb{U}_{\underline{v}}\right)\right)
$$

[cf. [IUTchI], Example 3.4, (ii)] — which we shall use to identify these two topological monoids.

(ii) (Mono-analytic Semi-simplifications) The functorial algorithm discussed in [IUTchI], Example 3.5, (iii), for constructing " $\left(\mathbb{R}_{\geq_{0}}^{\vdash}\right)_{\underline{v}}$ " [cf. also [AbsTopIII], Proposition 5.8, (vi)] yields a functorial algorithm in the object $\mathcal{D}_{\underline{v}}^{\vdash}$ of $\mathbb{T M}^{\vdash}$ for constructing a topological monoid $\mathbb{R}_{\geq 0}\left(\mathbb{U}_{\underline{v}}\right)$ equipped with a distinguished element

$$
\log ^{\mathcal{D}_{\underline{v}}^{\vdash}}\left(p_{\underline{v}}\right) \in \mathbb{R}_{\geq 0}\left(\mathcal{D}_{\underline{v}}^{\vdash}\right)
$$

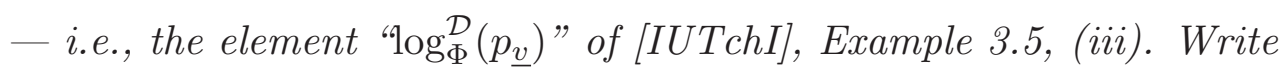

$$
\Psi_{\mathrm{cns}}^{\mathrm{ss}}\left(\mathcal{D}_{\underline{v}}^{\vdash}\right) \stackrel{\text { def }}{=} \Psi_{\mathrm{cns}}\left(\mathcal{D}_{\underline{v}}^{\vdash}\right)^{\times} \times \mathbb{R}_{\geq 0}\left(\mathcal{D}_{\underline{v}}^{\vdash}\right)
$$

- where the superscript "X" denotes the submonoid of units - which we shall think of as a sort of "semi-simplified version" of $\Psi_{\mathrm{cns}}\left(\mathcal{D}_{\underline{v}}^{\vdash}\right)$. We shall abbreviate notation that denotes a dependence on " $\mathcal{D}_{\underline{v}}^{\vdash}\left(\mathbb{U}_{\underline{v}}\right)$ " [e.g., a " $\mathcal{D}_{\underline{v}}^{\vdash}\left(\mathbb{U}_{\underline{v}}\right)$ " in parentheses] by means of notation that denotes a dependence on " $\mathbb{U}_{\underline{v}}$ ". Finally, there is a functorial algorithm in the Aut-holomorphic space $\mathbb{U}_{\underline{v}}$ for constructing the natural isomorphism [which arises immediately from the definitions]

$$
\Psi_{\mathrm{cns}}^{\mathbb{R}}\left(\mathbb{U}_{\underline{v}}\right) \stackrel{\text { def }}{=} \Psi_{\mathrm{cns}}\left(\mathbb{U}_{\underline{v}}\right) / \Psi_{\mathrm{cns}}\left(\mathbb{U}_{\underline{v}}\right)^{\times} \stackrel{\sim}{\rightarrow} \mathbb{R}_{\geq 0}\left(\mathbb{U}_{\underline{v}}\right)
$$

- cf. [IUTchI], Example 3.4, (i).

(iii) (Labels, $\mathbb{F}_{l}^{\rtimes \pm}$-Symmetries, and Conjugate Synchronization) Let $t \in \operatorname{LabCusp}^{ \pm}\left(\mathbb{U}_{\underline{v}}\right)$ [cf. [IUTchI], Definition 6.1, (iii)]. In the following, we shall use analogous conventions to the conventions introduced in Corollary 3.5 concerning subscripted labels. Then the action of $\mathbb{F}_{l}^{\rtimes \pm} \cong \operatorname{Gal}\left(\mathbb{U}_{\underline{v}}^{ \pm} / \mathbb{U}_{\underline{v}}^{\text {cor }}\right)$ on the various $\operatorname{Gal}\left(\mathbb{U}_{\underline{v}} / \mathbb{U}_{\underline{v}}^{ \pm}\right)$-orbits of cusps of $\mathbb{U}_{\underline{v}}\left[c f\right.$. the definition of "LabCusp ${ }^{ \pm}(-)$" in 
[IUTchI], Definition 6.1, (iii)] induces isomorphisms between the labeled topological monoids

$$
\Psi_{\mathrm{cns}}\left(\mathbb{U}_{\underline{v}}\right)_{t}
$$

for distinct $t \in \operatorname{LabCusp}^{ \pm}\left(\mathbb{U}_{\underline{v}}\right)$. We shall refer to these isomorphisms as $\left[\mathbb{F}_{l}^{\rtimes \pm}\right.$ ]symmetrizing isomorphisms [cf. Remark 3.5.2, in the case of $\underline{v} \in \underline{\mathbb{V}}^{\text {bad }}$ ]. These symmetrizing isomorphisms determine diagonal submonoids

$$
\Psi_{\mathrm{cns}}\left(\mathbb{U}_{\underline{v}}\right)_{\left\langle\left|\mathbb{F}_{l}\right|\right\rangle} \subseteq \prod_{|t| \in\left|\mathbb{F}_{l}\right|} \Psi_{\mathrm{cns}}\left(\mathbb{U}_{\underline{v}}\right)_{|t|} ; \quad \Psi_{\mathrm{cns}}\left(\mathbb{U}_{\underline{\underline{v}}}\right)_{\left\langle\mathbb{F}_{l}^{*}\right\rangle} \subseteq \prod_{|t| \in \mathbb{F}_{l}^{*}} \Psi_{\mathrm{cns}}\left(\mathbb{U}_{\underline{v}}\right)_{|t|}
$$

of the respective product monoids [cf. the discussion of Corollary 3.5, (i), in the case of $\underline{v} \in \underline{\mathbb{V}}^{\text {bad }}$, as well as an isomorphism of topological monoids

$$
\Psi_{\mathrm{cns}}\left(\mathbb{U}_{\underline{v}}\right)_{0} \stackrel{\sim}{\rightarrow} \Psi_{\mathrm{cns}}\left(\mathbb{U}_{\underline{v}}\right)_{\left\langle\mathbb{F}_{l}^{*}\right\rangle}
$$

[cf. Corollary 3.5, (iii), in the case of $\underline{v} \in \underline{\mathbb{V}}^{\text {bad }}$ ].

(iv) (Theta and Gaussian Monoids) Write

$$
\Psi_{\text {env }}\left(\mathbb{U}_{\underline{v}}\right) \stackrel{\text { def }}{=} \Psi_{\text {cns }}\left(\mathbb{U}_{\underline{v}}\right)^{\times} \times\left\{\mathbb{R}_{\geq 0} \cdot \log ^{\mathbb{U}_{\underline{v}}}\left(p_{\underline{v}}\right) \cdot \log ^{\mathbb{U}_{\underline{v}}}(\underline{\underline{\Theta}})\right\}
$$

- where the notation $" \log ^{\mathbb{U}_{\underline{v}}}\left(p_{\underline{v}}\right) \cdot \log ^{\mathbb{U}_{\underline{v}}}(\underline{\Theta})$ " is to be understood as a formal symbol [cf. the discussion of [IUTchI], Example 3.4, (iii)] - and

$$
\begin{aligned}
\Psi_{\text {gau }}\left(\mathbb{U}_{\underline{v}}\right) & \left.\stackrel{\text { def }}{=} \Psi_{\mathrm{cns}}\left(\mathbb{U}_{\underline{v}}\right)_{\left\langle\mathbb{F}_{l}^{*}\right\rangle}^{\times} \times\left\{\mathbb{R}_{\geq 0} \cdot\left(\ldots, j^{2} \cdot \log _{\underline{\underline{v}}}^{\mathbb{U}_{\underline{v}}}\right), \ldots\right)\right\} \\
& \subseteq \prod_{j \in \mathbb{F}_{l}^{*}} \Psi_{\mathrm{cns}}^{\mathrm{ss}}\left(\mathbb{U}_{\underline{v}}\right)_{j}=\prod_{j \in \mathbb{F}_{l}^{*}} \Psi_{\mathrm{cns}}\left(\mathcal{D}_{\underline{v}}^{\vdash}\right)_{j}^{\times} \times \mathbb{R}_{\geq 0}\left(\mathcal{D}_{\underline{v}}^{\vdash}\right)_{j}
\end{aligned}
$$

- where, by abuse of notation, we also write " $j$ " for the natural number $\in\left\{1, \ldots, l^{*}\right\}$ determined by an element $j \in \mathbb{F}_{l}^{*}$. In particular, [cf. (i), (ii), (iii)] we obtain a functorial algorithm in the Aut-holomorphic space $\mathbb{U}_{\underline{v}}$ for constructing the theta monoid $\Psi_{\text {env }}\left(\mathbb{U}_{\underline{v}}\right)$ and the Gaussian monoid $\Psi_{\text {gau }}\left(\mathbb{U}_{\underline{v}}\right)$, equipped with their levident] natural splittings, as well as the formal evaluation isomorphism [cf. Corollary 3.5, (ii), in the case of $\left.\underline{v} \in \underline{\mathbb{V}}^{\text {bad }}\right]$

$$
\begin{aligned}
\Psi_{\mathrm{env}}\left(\mathbb{U}_{\underline{v}}\right) & \stackrel{\sim}{\rightarrow} \Psi_{\mathrm{gau}}\left(\mathbb{U}_{\underline{v}}\right) \\
\log ^{\mathbb{U}_{\underline{v}}}\left(p_{\underline{v}}\right) \cdot \log ^{\mathbb{U}_{\underline{v}}}(\underline{\underline{\Theta}}) & \mapsto\left(\ldots, j^{2} \cdot \log ^{\mathbb{U}_{\underline{v}}}\left(p_{\underline{v}}\right), \ldots\right)
\end{aligned}
$$

- which restricts to the identity on the respective copies of " $\Psi_{\mathrm{cns}}\left(\mathbb{U}_{\underline{v}}\right)^{\times}$" and is compatible with the natural splittings on the domain and codomain.

Proof. The various assertions of Proposition 4.3 follow immediately from the definitions and the references quoted in the statements of these assertions.

Remark 4.3.1. Analogous observations to the observations made in Remark 4.1.1, (i), (ii), (iii), may be made in the present case of $\underline{v} \in \underline{\mathbb{V}}^{\text {arc }}$. We leave the routine details to the reader. In this context, we note that the cuspidal decomposition 
groups that appear in the discussion of Remark 4.1.1, (ii), may be thought of as corresponding to the " $\mathcal{A}_{p}$ " that appear in [AbsTopIII], Corollary 2.7, (e) - i.e., in the construction of $\overline{\mathcal{A}}_{\mathbb{U}_{\underline{v}}}$ - in the case of points $p$ that belong to "sufficiently small" neighborhoods of the cusps that correspond to an element $t \in \operatorname{LabCusp}^{ \pm}\left(\mathbb{U}_{\underline{v}}\right)$.

Proposition 4.4. (Frobenioid-theoretic Gaussian Monoids at Archimedean Primes) We continue to use the notation of Proposition 4.3. Let $\underline{\underline{\mathcal{F}}}_{\underline{\underline{v}}}=$ $\left({ }^{\dagger} \mathcal{C}_{\underline{v}},{ }^{\dagger} \mathcal{D}_{\underline{v}},{ }^{\dagger} \kappa_{\underline{v}}\right)$ be the collection of data indexed by $\underline{v} \in \underline{\mathbb{V}}^{\text {arc }}$ of a $\Theta$-Hodge theater ${ }^{\dagger} \mathcal{H} \mathcal{T}^{\Theta}=\left(\left\{\underline{\underline{\mathcal{F}}}_{\underline{v}}\right\}_{\underline{v}} \in \underline{\mathbb{V}},{ }^{\dagger} \mathfrak{F}_{\text {mod }}^{\vdash}\right)$ [cf. [IUTchI], Definition 3.6; [IUTchI], Example 3.4, (i)]. Write ${ }^{\dagger} \overline{\mathcal{F}}_{\underline{v}}^{\vdash}=\left({ }^{\dagger} \mathcal{C}_{\underline{v}}^{\vdash},{ }^{\dagger} \mathcal{D}_{\underline{v}},{ }^{\dagger} \tau_{\underline{v}}^{\vdash}\right)$ for the data indexed by $\underline{v}$ [cf. the discussion of [IUTchI], Example 3.4, (ii)] of the $\mathcal{F}^{\vdash}$-prime-strip determined by the $\Theta$-Hodge theater ${ }^{\dagger} \mathcal{H} \mathcal{T}^{\Theta}$ [cf. [IUTchI], Definition 3.6; [IUTchI], Definition 5.2, (ii)]. Also, let us write ${ }^{\dagger} \mathbb{U}_{\underline{v}} \stackrel{\text { def }}{=} \dagger \mathcal{D}_{\underline{v}}$ and ${ }^{\dagger} \mathbb{U}_{\underline{v}}^{ \pm},{ }^{\dagger} \mathbb{U}_{\underline{v}}^{\text {cor }}$ for the Aut-holomorphic orbispaces associated

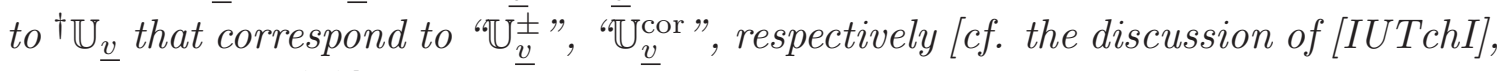
Definition 6.1, (ii)].

(i) (Constant Monoids) In the notation of [IUTchI], Definition 3.6; [IUTchI], Example 3.4, (i), the Kummer structure

$$
{ }^{\dagger} \kappa_{\underline{\underline{v}}}: \Psi_{\Psi_{\dagger} \underline{\mathcal{F}}_{\underline{v}}} \stackrel{\text { def }}{=} \mathcal{O}^{\triangleright}\left({ }^{\dagger} \mathcal{C}_{\underline{v}}\right) \hookrightarrow \mathcal{A}_{\dagger} \mathcal{D}_{\underline{v}}
$$

on the category ${ }^{\dagger} \mathcal{C}_{\underline{v}}$, together with the tautological equality ${ }^{\dagger} \mathcal{D}_{\underline{v}}={ }^{\dagger} \mathbb{U}_{\underline{v}}$ of Autholomorphic spaces, determine a unique isomorphism

$$
\Psi_{\underline{\underline{\mathcal{F}}}_{\underline{v}}} \stackrel{\sim}{\rightarrow} \quad \Psi_{\mathrm{cns}}\left({ }^{\dagger} \mathbb{U}_{\underline{v}}\right)
$$

of topological monoids.

(ii) (Mono-analytic Semi-simplifications) Write $\Psi_{\dagger \mathcal{F}_{\underline{v}}^{\vdash}} \stackrel{\text { def }}{=} \mathcal{O}^{\triangleright}\left({ }^{\dagger} \mathcal{C}_{\underline{v}}^{\vdash}\right)[c f$. [IUTchI], Example 3.4, (ii)]. Then there exists a unique $\{ \pm 1\}$-orbit of isomorphisms of topological groups

$$
\Psi_{\dagger \mathcal{F}_{\underline{v}}^{\vdash}}^{\times} \stackrel{\sim}{\rightarrow} \quad \Psi_{\mathrm{cns}}\left({ }^{\dagger} \mathcal{D}_{\underline{\underline{v}}}^{\vdash}\right)^{\times}
$$

as well as a unique isomorphism of monoids

$$
\Psi_{\dagger \mathcal{F}_{\underline{v}}^{\vdash}}^{\mathbb{R}} \stackrel{\text { def }}{=} \Psi_{\dagger \mathcal{F}_{\underline{v}}^{\vdash}} / \Psi_{\dagger \mathcal{F}_{\underline{v}}^{\vdash}}^{\times} \stackrel{\sim}{\rightarrow} \quad \Psi_{\text {cns }}^{\mathbb{R}}\left({ }^{\dagger} \mathcal{D}_{\underline{v}}^{\vdash}\right)
$$

that maps the distinguished element of $\Psi_{\dagger_{\mathcal{F}_{\underline{v}}}^{\vdash}}^{\mathbb{R}}$ determined by $p_{\underline{v}}=e=2.71828 \ldots$ [i.e., the element of the complex archimedean field that gives rise to $\Psi_{\underline{+\mathcal{F}}_{\underline{v}}}$ whose natural logarithm is equal to 1$]$ to the distinguished element of $\Psi_{\mathrm{cns}}^{\mathbb{R}}\left({ }^{\dagger} \mathcal{D}_{\underline{v}}^{+}\right)^{\underline{v}}$ determined by $\log ^{\dagger} \mathcal{D}_{\underline{v}}^{\vdash}\left(p_{\underline{v}}\right) \in \mathbb{R}_{\geq 0}\left({ }^{\dagger} \mathcal{D}_{\underline{v}}^{\vdash}\right)$ [cf. the isomorphism of the final display of Proposition 4.3, (ii)]. In particular, if we write $\Psi_{\dagger \mathcal{F}_{\underline{v}}^{\vdash}}^{\mathrm{ss}} \stackrel{\text { def }}{=} \Psi_{\dagger \mathcal{F}_{\underline{v}}^{\vdash}}^{\times} \times \Psi_{\dagger \mathcal{F}_{\underline{v}}^{\vdash}}^{\mathbb{R}}$ for the 
"semi-simplified version" of $\Psi_{\dagger \mathcal{F}_{\underline{v}}^{\vdash}}$, then the former distinguished element, together with the poly-isomorphism of the first display of the present (ii), determine a natural poly-isomorphism of topological monoids

$$
\Psi_{\dagger \mathcal{F}_{\underline{v}}^{\vdash}}^{\mathrm{ss}} \stackrel{\sim}{\rightarrow} \Psi_{\mathrm{cns}}^{\mathrm{ss}}\left({ }^{\dagger} \mathcal{D}_{\underline{v}}^{\vdash}\right)
$$

[cf. Proposition 4.3, (ii)] that is compatible with the natural splittings on the domain and codomain. Write $\Psi_{\Psi_{\underline{\mathcal{F}}}^{\mathrm{F}}}^{\mathrm{ss}} \stackrel{\text { def }}{=} \Psi_{+\mathcal{F}_{\underline{v}}}^{\mathrm{ss}}$; thus, it follows from the definitions that we

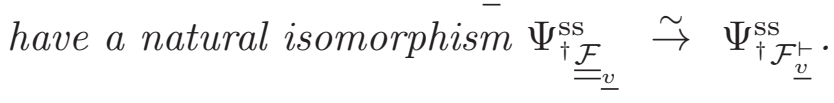

(iii) (Labels, $\mathbb{F}_{l}^{\rtimes \pm}$-Symmetries, and Conjugate Synchronization) The isomorphism of $(i)$ determines, for each $t \in \mathrm{LabCusp}^{ \pm}\left({ }^{\dagger} \mathbb{U}_{\underline{v}}\right)$, a collection of compatible isomorphisms

$$
\left(\Psi_{\underline{\mathcal{F}}_{\underline{v}}}\right)_{t} \quad \stackrel{\sim}{\rightarrow} \quad \Psi_{\mathrm{cns}}\left({ }^{\dagger} \mathbb{U}_{\underline{v}}\right)_{t}
$$

[cf. Corollary 3.6, (i), in the case of $\left.\underline{v} \in \underline{\mathbb{V}}^{\text {bad }}\right]$, as well as $\left[\mathbb{F}_{l}^{\rtimes \pm}\right.$-]symmetrizing isomorphisms, induced by the action of $\mathbb{F}_{l}^{\rtimes \pm} \cong \operatorname{Gal}\left({ }^{\dagger} \mathbb{U}_{\underline{v}}^{ \pm} / \dagger \mathbb{U}_{\underline{v}}^{\text {cor }}\right)$ on the various $\mathrm{Gal}\left({ }^{\dagger} \mathbb{U}_{\underline{v}} /{ }^{\dagger} \mathbb{U}_{\underline{v}}^{ \pm}\right)$-orbits of cusps of $\dagger^{\dagger} \mathbb{U}_{\underline{v}}$ [cf. the definition of "LabCusp ${ }^{ \pm}(-)$" in [IUTchI], Definition 6.1, (iii)], between the data indexed by distinct $t \in \operatorname{LabCusp}^{ \pm}\left({ }^{\dagger} \mathbb{U}_{\underline{v}}\right)$. Moreover, these symmetrizing isomorphisms determine [various diagonal submonoids, as well as] an isomorphism of topological monoids

$$
\left(\Psi_{\underline{\underline{F}}_{\underline{\underline{F}}}}\right)_{0} \stackrel{\sim}{\rightarrow} \quad\left(\Psi_{\underline{\underline{F}}_{\underline{\underline{F}}}}\right)_{\left\langle\mathbb{F}_{l}^{*}\right\rangle}
$$

[cf. Corollary 3.6, (iii), in the case of $\underline{v} \in \underline{\mathbb{V}}^{\text {bad }}$ ].

(iv) (Theta and Gaussian Monoids) Write

$$
\Psi_{\dagger \mathcal{F}_{\underline{v}}^{\Theta}}, \quad \Psi_{\mathcal{F}_{\text {gau }}}\left({ }^{\dagger} \underline{\underline{\mathcal{F}}}_{\underline{v}}\right)
$$

for the topological monoids equipped with natural splittings determined, respectively - via the isomorphisms of (i), (ii), and (iii) - by the monoids $\Psi_{\mathrm{env}}\left({ }^{\dagger} \mathbb{U}_{\underline{v}}\right)$, $\Psi_{\text {gau }}\left(\dagger \mathbb{U}_{\underline{v}}\right)$ and splittings of Proposition 4.3, (iv). Then the definition of the various monoids involved, together with the formal evaluation isomorphism of Proposition 4.3, (iv), gives rise to a collection of natural isomorphisms [cf. Corollary 3.6, (ii), in the case of $\left.\underline{v} \in \underline{\mathbb{V}}^{\text {bad }}\right]$

$$
\Psi_{\dagger \mathcal{F}_{\underline{v}}^{\Theta}} \stackrel{\sim}{\rightarrow} \quad \Psi_{\text {env }}\left({ }^{\dagger} \mathbb{U}_{\underline{v}}\right) \quad \stackrel{\sim}{\rightarrow} \quad \Psi_{\text {gau }}\left({ }^{\dagger} \mathbb{U}_{\underline{v}}\right) \quad \stackrel{\sim}{\rightarrow} \quad \Psi_{\mathcal{F}_{\text {gau }}}\left({ }^{\dagger} \underline{\underline{\mathcal{F}}}_{\underline{v}}\right)
$$

- which restrict to the identity or to the [restriction to " $(-)^{\times}$" of the] isomor-

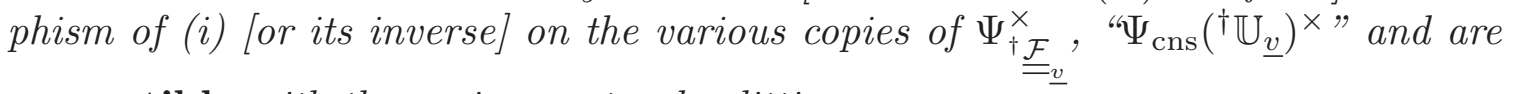
compatible with the various natural splittings.

Proof. The various assertions of Proposition 4.4 follow immediately from the definitions and the references quoted in the statements of these assertions. 
Remark 4.4.1. In the case of $\underline{v} \in \underline{\mathbb{V}}^{\text {arc }}$, one verifies immediately that one can make a remark analogous to Remark 4.2.1, (ii).

Corollary 4.5. (Group-theoretic Monoids Associated to Base- $\Theta^{ \pm e l l}$ Hodge Theaters) Let

$$
{ }^{\dagger} \mathcal{H} \mathcal{T}^{\mathcal{D}-\Theta^{ \pm \mathrm{ell}}}=\left({ }^{\dagger} \mathfrak{D}_{\succ} \stackrel{{ }^{\dagger} \phi_{ \pm}^{\Theta \pm}}{\longleftarrow} \mathfrak{D}_{T} \stackrel{{ }^{\dagger} \phi_{ \pm}^{\Theta^{\mathrm{ell}}}}{\longrightarrow}{ }^{\dagger} \mathcal{D}^{\odot \pm}\right)
$$

be a $\mathcal{D}-\Theta^{ \pm e l l}$-Hodge theater [relative to the given initial $\Theta$-data $-c f$. [IUTchI], Definition 6.4, (iii)] and

$$
\ddagger \mathfrak{D}=\left\{{ }^{\ddagger} \mathcal{D}_{\underline{v}}\right\}_{\underline{v}} \in \underline{\mathbb{V}}
$$

a D-prime-strip; here, we assume [for simplicity] that ${ }^{\ddagger} \mathcal{D}_{\underline{v}}=\mathcal{B}^{\text {temp }}\left({ }^{\ddagger} \Pi_{\underline{v}}\right)^{0}$ for $\underline{v} \in$ $\underline{V}^{\text {non }}$. Also, we shall denote the $\mathcal{D}^{\vdash}$-prime-strip associated to - i.e., the monoanalyticization of - a D-prime-strip [cf. [IUTchI], Definition 4.1, (iv)] by means of a superscript "q " and assume [for simplicity] that ${ }^{\ddagger} \mathcal{D}_{\underline{v}}^{\vdash}=\mathcal{B}^{\text {temp }}\left({ }^{\ddagger} G_{\underline{v}}\right)^{0}$ for $\underline{v} \in \underline{\mathbb{V}}^{\text {non }}$.

(i) (Constant Monoids) There is a functorial algorithm in the $\mathcal{D}$-primestrip $\ddagger_{\mathfrak{D}}$ for constructing the assignment $\Psi_{\mathrm{cns}}\left({ }^{\ddagger} \mathfrak{D}\right)$ given by

$$
\begin{aligned}
& \underline{\mathbb{V}}^{\text {non }} \ni \underline{v} \mapsto \Psi_{\text {cns }}\left({ }^{\ddagger} \mathfrak{D}\right)_{\underline{v}} \stackrel{\text { def }}{=}\left\{G_{\underline{v}}\left({ }^{\ddagger} \Pi_{\underline{v}}\right) \curvearrowright \Psi_{\text {cns }}\left({ }^{\ddagger} \Pi_{\underline{v}}\right)\right\} \\
& \underline{\mathbb{V}}^{\text {arc }} \ni \underline{v} \mapsto \Psi_{\text {cns }}\left({ }^{\ddagger} \mathfrak{D}\right)_{\underline{v}} \stackrel{\text { def }}{=} \Psi_{\text {cns }}\left({ }^{\ddagger} \mathcal{D}_{\underline{v}}\right)
\end{aligned}
$$

- where the data in brackets " $\{-\}$ " is to be regarded as being well-defined only up to $a{ }^{\ddagger} \Pi_{\underline{v}}$-conjugacy indeterminacy — cf. Remark 4.2.1, (i), and Propositions 3.1, (ii); 4.1, (i); 4.3, (i).

(ii) (Mono-analytic Semi-simplifications) There is a functorial algorithm in the $\mathcal{D}^{\vdash}$-prime-strip $\ddagger \mathfrak{D}^{\vdash}$ for constructing the assignment $\Psi_{\mathrm{cns}}^{\mathrm{ss}}\left({ }^{\ddagger} \mathfrak{D}^{\vdash}\right)$ given by

$$
\begin{aligned}
& \underline{\mathbb{V}}^{\text {non }} \ni \underline{v} \mapsto \Psi_{\text {cns }}^{\mathrm{ss}}\left({ }^{\ddagger} \mathfrak{D}^{\vdash}\right)_{\underline{v}} \stackrel{\text { def }}{=}\left\{{ }^{\ddagger} G_{\underline{v}} \curvearrowright \Psi_{\mathrm{cns}}^{\mathrm{ss}}\left({ }^{\ddagger} G_{\underline{v}}\right)\right\} \\
& \underline{\mathbb{V}}^{\mathrm{arc}} \ni \underline{v} \mapsto \Psi_{\mathrm{cns}}^{\mathrm{ss}}\left({ }^{\ddagger} \mathfrak{D}^{\vdash}\right)_{\underline{v}} \stackrel{\text { def }}{=} \Psi_{\mathrm{cns}}^{\mathrm{ss}}\left({ }^{\ddagger} \mathcal{D}_{\underline{v}}^{\vdash}\right)
\end{aligned}
$$

- where the data in brackets " $\{-\}$ " is to be regarded as being well-defined only up to $a \ddagger G_{\underline{v}}$-conjugacy indeterminacy; each " $\Psi_{\mathrm{cns}}^{\mathrm{ss}}(-)$ " is equipped with a splitting, i.e., a direct product decomposition

$$
\Psi_{\mathrm{cns}}^{\mathrm{ss}}\left({ }^{\ddagger} \mathfrak{D}^{\vdash}\right)_{\underline{v}}=\Psi_{\mathrm{cns}}^{\mathrm{ss}}\left({ }^{\ddagger} \mathfrak{D}^{\vdash}\right)_{\underline{v}}^{\times} \times \mathbb{R}_{\geq 0}\left({ }^{\ddagger} \mathfrak{D}^{\vdash}\right)_{\underline{v}}
$$

as the product of the submonoid of units and a submonoid with no nontrivial units [each of which is equipped with the action of a topological group when $\underline{v} \in \underline{\mathbb{V}}^{\text {non }}$ ]; each submonoid $\mathbb{R}_{\geq 0}\left({ }^{\ddagger} \mathfrak{D}^{\vdash}\right)_{\underline{v}}$ is equipped with a distinguished element

$$
\log ^{\ddagger} \mathfrak{D}^{\vdash}\left(p_{\underline{v}}\right) \in \mathbb{R}_{\geq 0}\left({ }^{\ddagger} \mathfrak{D}^{\vdash}\right)_{\underline{v}}
$$

- cf. Remark 4.2.1, (i); Propositions 4.1, (ii), and 4.3, (ii). Here, if we regard $\ddagger \mathfrak{D}^{\vdash}$ as functorially constructed from $\ddagger \mathfrak{D}$, then there is a functorial algorithm in 
the $\mathcal{D}$-prime-strip $¥ \mathfrak{D}$ for constructing isomorphisms lof topological abelian groups, equipped with the action of a topological group when $\left.\underline{v} \in \underline{\mathbb{V}}^{\text {non }}\right]$

$$
\Psi_{\mathrm{cns}}\left({ }^{\ddagger} \mathfrak{D}\right)_{\underline{v}}^{\times} \stackrel{\sim}{\rightarrow} \quad \Psi_{\mathrm{cns}}^{\mathrm{ss}}\left({ }^{\ddagger} \mathfrak{D}^{\vdash}\right)_{\underline{v}}^{\times}
$$

for each $\underline{v} \in \underline{\mathbb{V}}-c f$. Remark 4.2.1, (i); Propositions 4.1, (i), and 4.3, (i). Finally, there is a functorial algorithm in the $\mathcal{D}^{\vdash}$-prime-strip ${ }^{\ddagger} \mathfrak{D}^{\vdash}$ for constructing a Frobenioid

$$
\mathcal{D}^{\Vdash}\left({ }^{\ddagger} \mathfrak{D}^{\vdash}\right)
$$

[cf. the Frobenioid " $\mathcal{D}_{\bmod }^{\Vdash}$ " of [IUTchI], Example 3.5, (iii)] isomorphic to the Frobenioid "C ${ }_{\bmod }^{\Vdash}$ " of [IUTchI], Example 3.5, (i), equipped with a bijection

$$
\operatorname{Prime}\left(\mathcal{D}^{\Vdash}\left({ }^{\ddagger} \mathfrak{D}^{\vdash}\right)\right) \stackrel{\sim}{\rightarrow} \underline{\mathbb{V}}
$$

- where we write "Prime(-)" for the set of primes associated to the divisor monoid of the Frobenioid in parentheses [cf. the discussion of [IUTchI], Example 3.5, (i)] — and, for each $\underline{v} \in \underline{\mathbb{V}}$, an isomorphism of topological monoids ${ }^{\ddagger} \rho_{\mathcal{D}^{\Vdash,}, \underline{v}}: \Phi_{\mathcal{D}^{\Vdash}\left(\mathfrak{D}^{\vdash}\right), \underline{v}} \stackrel{\sim}{\rightarrow} \mathbb{R}_{\geq 0}\left({ }^{\ddagger} \mathfrak{D}^{\vdash}\right)_{\underline{v}}$, where we write $" \Phi_{\mathcal{D}^{\Vdash}\left({ }^{\ddagger} \mathcal{D}^{\vdash}\right), \underline{v}}$ " for the submonoid [isomorphic to $\mathbb{R}_{\geq 0}$ ] of the divisor monoid of $\mathcal{D}^{\Vdash}\left({ }^{\ddagger} \mathfrak{D}^{\vdash}\right)$ associated to $\underline{v}$ [cf. the isomorphism " $\rho_{\underline{v}}^{\mathcal{D}}$ " of [IUTchI], Example 3.5, (iii)].

(iii) (Labels, $\mathbb{F}_{l}^{\rtimes \pm}$-Symmetries, and Conjugate Synchronization) Write

$$
{ }^{\dagger} \zeta_{\succ}: \operatorname{LabCusp}^{ \pm}\left({ }^{\dagger} \mathfrak{D}_{\succ}\right) \stackrel{\sim}{\rightarrow} T
$$

for the bijection ${ }^{\dagger} \zeta_{ \pm} \circ{ }^{\dagger} \zeta_{0}^{\Theta^{\mathrm{ell}}} \circ\left({ }^{\dagger} \zeta_{0}^{\Theta^{ \pm}}\right)^{-1}$ arising from the bijections discussed in [IUTchI], Proposition 6.5, (i), (ii), (iii). Let $t \in \operatorname{LabCusp}^{ \pm}\left({ }^{\dagger} \mathfrak{D}_{\succ}\right)$. In the following, we shall use analogous conventions to the conventions introduced in Corollary 3.5 concerning subscripted labels. Then the various local $\mathbb{F}_{l}^{\rtimes \pm}$-actions discussed in Corollary 3.5, (i), and Propositions 4.1, (iii); 4.3, (iii), induce isomorphisms between the labeled data

$$
\Psi_{\mathrm{cns}}\left({ }^{\dagger} \mathfrak{D}_{\succ}\right)_{t}
$$

[cf. (i)] for distinct $t \in \operatorname{LabCusp}^{ \pm}\left({ }^{\dagger} \mathfrak{D}_{\succ}\right)$. We shall refer to these isomorphisms as $\left[\mathbb{F}_{l}^{\rtimes \pm}\right.$ - $]$ symmetrizing isomorphisms. These symmetrizing isomorphisms are compatible, relative to ${ }^{\dagger} \zeta_{\succ}$, with the $\mathbb{F}_{l}^{\rtimes \pm}$-symmetry of the associated $\mathcal{D}$ - $\Theta^{\text {ell }}$ bridge [cf. [IUTchI], Proposition 6.8, (i)] and determine diagonal submonoids

$$
\Psi_{\mathrm{cns}}\left({ }^{\dagger} \mathfrak{D}_{\succ}\right)_{\left\langle\left|\mathbb{F}_{l}\right|\right\rangle} \subseteq \prod_{|t| \in\left|\mathbb{F}_{l}\right|} \Psi_{\mathrm{cns}}\left({ }^{\dagger} \mathfrak{D}_{\succ}\right)_{|t|} ; \quad \Psi_{\mathrm{cns}}\left({ }^{\dagger} \mathfrak{D}_{\succ}\right)_{\left\langle\mathbb{F}_{l}^{*}\right\rangle} \subseteq \prod_{|t| \in \mathbb{F}_{l}^{*}} \Psi_{\mathrm{cns}}\left({ }^{\dagger} \mathfrak{D}_{\succ}\right)_{|t|}
$$

- where the " $\subseteq$ 's" denote the various local inclusions of diagonal submonoids of Corollary 3.5, (i), and Propositions 4.1, (iii); 4.3, (iii) - as well as an isomorphism

$$
\Psi_{\mathrm{cns}}\left({ }^{\dagger} \mathfrak{D}_{\succ}\right)_{0} \stackrel{\sim}{\rightarrow} \Psi_{\mathrm{cns}}\left({ }^{\dagger} \mathfrak{D}_{\succ}\right)_{\left\langle\mathbb{F}_{l}^{*}\right\rangle}
$$

constituted by the various corresponding local isomorphisms of Corollary 3.5, (iii), and Propositions 4.1, (iii); 4.3, (iii). 
(iv) (Local Theta and Gaussian Monoids) There is a functorial algorithm in the $\mathcal{D}$-prime-strip ${ }^{\dagger} \mathfrak{D}_{\succ}$ for constructing assignments $\Psi_{\mathrm{env}}\left({ }^{\dagger} \mathfrak{D}_{\succ}\right), \Psi_{\text {gau }}\left({ }^{\dagger} \mathfrak{D}_{\succ}\right)$, $\infty \Psi_{\mathrm{env}}\left({ }^{\dagger} \mathfrak{D}_{\succ}\right), \infty \Psi_{\mathrm{gau}}\left({ }^{\dagger} \mathfrak{D}_{\succ}\right)$

$$
\begin{gathered}
\underline{\mathbb{V}} \ni \underline{v} \mapsto \Psi_{\mathrm{env}}\left({ }^{\dagger} \mathfrak{D}_{\succ}\right)_{\underline{v}} \stackrel{\text { def }}{=} \Psi_{\mathrm{env}}\left({ }^{\dagger} \mathcal{D}_{\succ, \underline{v}}\right) ; \quad \underline{\mathbb{V}} \ni \underline{v} \mapsto \Psi_{\mathrm{gau}}\left({ }^{\dagger} \mathfrak{D}_{\succ}\right)_{\underline{v}} \stackrel{\text { def }}{=} \Psi_{\mathrm{gau}}\left({ }^{\dagger} \mathcal{D}_{\succ, \underline{v}}\right) \\
\underline{\mathbb{V}} \ni \underline{v} \mapsto \infty \Psi_{\mathrm{env}}\left({ }^{\dagger} \mathfrak{D}_{\succ}\right)_{\underline{v}} \stackrel{\text { def }}{=} \infty \Psi_{\mathrm{env}}\left({ }^{\dagger} \mathcal{D}_{\succ, \underline{v}}\right) \\
\underline{\mathbb{V}} \ni \underline{v} \mapsto \infty \Psi_{\mathrm{gau}}\left({ }^{\dagger} \mathfrak{D}_{\succ}\right)_{\underline{\underline{v}}} \stackrel{\text { def }}{=} \infty \Psi_{\mathrm{gau}}\left({ }^{\dagger} \mathcal{D}_{\succ, \underline{v}}\right)
\end{gathered}
$$

- where the various local data are equipped with actions by topological groups when $\underline{v} \in \underline{\mathbb{V}}^{\text {non }}$ and splittings [for all $\underline{v} \in \mathbb{V}$ ], as described in detail in Corollary 3.5, (ii), (iii), and Propositions 4.1, (iv); 4.3, (iv) [cf. also Remarks 4.2.1, (ii), (iii), (iv); 4.4.1] — as well as compatible evaluation isomorphisms

$$
\Psi_{\text {env }}\left({ }^{\dagger} \mathfrak{D}_{\succ}\right) \quad \stackrel{\sim}{\rightarrow} \quad \Psi_{\text {gau }}\left({ }^{\dagger} \mathfrak{D}_{\succ}\right) ; \quad \infty \Psi_{\text {env }}\left({ }^{\dagger} \mathfrak{D}_{\succ}\right) \quad \stackrel{\sim}{\rightarrow} \quad \infty \Psi_{\text {gau }}\left({ }^{\dagger} \mathfrak{D}_{\succ}\right)
$$

as described in detail in Corollary 3.5, (ii), and Propositions 4.1, (iv); 4.3, (iv).

(v) (Global Theta and Gaussian Monoids) There is a functorial algorithm in the $\mathcal{D}^{\vdash}$-prime-strip ${ }^{\dagger} \mathfrak{D}_{\succ}^{\vdash}$ for constructing a Frobenioid

$$
\mathcal{D}_{\text {env }}^{\Vdash}\left({ }^{\dagger} \mathfrak{D}_{\succ}^{\vdash}\right)
$$

- namely, as a copy of the Frobenioid " $\mathcal{D}^{\Vdash}\left({ }^{\dagger} \mathfrak{D}_{\succ}^{\vdash}\right)$ " of (ii) above, multiplied by a formal symbol " $\log ^{\dagger} \mathfrak{D}_{\succ}^{+}(\underline{\underline{\Theta}})$ " [cf. the constructions of Propositions 4.1, (iv); 4.3, (iv)] - isomorphic to the Frobenioid "C ${ }_{\bmod }^{\Vdash}$ " of [IUTchI], Example 3.5, (i), equipped with a bijection Prime $\left(\mathcal{D}_{\text {env }}^{\vdash}\left({ }^{\dagger} \mathfrak{D}_{\succ}^{\vdash}\right)\right) \stackrel{\sim}{\rightarrow} \underline{\mathbb{V}}[c f . \quad$ (ii) above] and, for each $\underline{v} \in \underline{\mathbb{V}}$, an isomorphism of topological monoids

$$
\Phi_{\mathcal{D}_{\text {env }}^{\Vdash}\left(\mathfrak{D}_{\succ}^{\vdash}\right), \underline{v}} \stackrel{\sim}{\rightarrow} \Psi_{\mathrm{env}}\left({ }^{\dagger} \mathfrak{D}_{\succ}\right)_{\underline{v}}^{\mathbb{R}}
$$

- where we write " $\Phi_{\mathcal{D}_{\text {env }}^{\Vdash}}\left(\mathfrak{D}_{\succ}^{\leftarrow}\right), \underline{v}$ " for the submonoid [isomorphic to $\mathbb{R}_{\geq 0}$ ] of the divisor monoid of $\mathcal{D}_{\text {env }}^{\Vdash}\left({ }^{\dagger} \mathfrak{D}_{\succ}^{\vdash}\right)$ associated to $\underline{v}$; we write $\Psi_{\mathrm{env}}\left({ }^{\dagger} \mathfrak{D}_{\succ}\right)_{\underline{v}}^{\mathbb{R}}$ for the data obtained from $\Psi_{\mathrm{env}}\left({ }^{\dagger} \mathfrak{D}_{\succ}\right)_{\underline{v}}$ [cf. (iv) above] by replacing the topological monoid portion of $\Psi_{\mathrm{env}}\left({ }^{\dagger} \mathfrak{D}_{\succ}\right)_{\underline{v}}$ by the realification of the quotient of this topological monoid by its submonoid of units. There is a functorial algorithm in the $\mathcal{D}^{\vdash}$-prime-strip ${ }^{\dagger} \mathfrak{D}_{\succ}^{\leftarrow}$ for constructing a subcategory, equipped with a Frobenioid structure,

$$
\mathcal{D}_{\text {gau }}^{\Vdash}\left({ }^{\dagger} \mathfrak{D}_{\succ}^{\vdash}\right) \subseteq \prod_{j \in \mathbb{F}_{l}^{*}} \mathcal{D}^{\Vdash}\left({ }^{\dagger} \mathfrak{D}_{\succ}^{\vdash}\right)_{j}
$$

- [cf. Remark 4.5.2, (i), below concerning the subscript "j's"] whose divisor and rational function monoids are determined [relative to the divisor and rational function monoids of each factor in the product category of the display] by the "vector of ratios"

$$
\left(\ldots, j^{2} \cdot \ldots\right)
$$


whose components are indexed by $j \in \mathbb{F}_{i}^{*}$ [cf. the notational conventions of Propositions 4.1, (iv); 4.3, (iv)] — equipped with a bijection Prime $\left(\mathcal{D}_{\text {gau }}^{\Vdash}\left({ }^{\dagger} \mathfrak{D}_{\succ}^{\vdash}\right)\right) \stackrel{\sim}{\rightarrow} \mathbb{V}$ [cf. (ii) above] and, for each $\underline{v} \in \underline{\mathbb{V}}$, an isomorphism of topological monoids

$$
\Phi_{\mathcal{D}_{\text {gau }}^{\Vdash}\left(\mathfrak{D}_{\succ}^{\vdash}\right), \underline{v}} \stackrel{\sim}{\rightarrow} \Psi_{\text {gau }}\left({ }^{\dagger} \mathfrak{D}_{\succ}\right)_{\underline{v}}^{\mathbb{R}}
$$

- where we write " $\Phi_{\mathcal{D}_{\text {gau }}}\left(\dagger^{\circ} \mathfrak{D}_{\succ}^{\leftarrow}\right), \underline{v}$ " for the submonoid [isomorphic to $\mathbb{R}_{\geq 0}$ ] of the divisor monoid of $\mathcal{D}_{\text {gau }}^{\Vdash}\left({ }^{\dagger} \mathfrak{D}_{\succ}^{\vdash}\right)$ associated to $\underline{v}$; we write $\Psi_{\text {gau }}\left({ }^{\dagger} \mathfrak{D}_{\succ}\right)_{\underline{v}}^{\mathbb{R}}$ for the data obtained from $\Psi_{\text {gau }}\left({ }^{\dagger} \mathfrak{D}_{\succ}\right)_{\underline{v}}$ [cf. (iv) above] by replacing the topological monoid portion of $\Psi_{\text {gau }}\left({ }^{\dagger} \mathfrak{D}_{\succ}\right)_{\underline{v}}$ by the realification of the quotient of this topological monoid by its submonoid of units. Finally, there is a functorial algorithm in the $\mathcal{D}^{\vdash}$ prime-strip ${ }^{\dagger} \mathfrak{D}_{\succ}^{\vdash}$ for constructing a global formal evaluation isomorphism of Frobenioids

$$
\mathcal{D}_{\text {env }}^{\vdash}\left({ }^{\dagger} \mathfrak{D}_{\succ}^{\vdash}\right) \quad \stackrel{\sim}{\rightarrow} \quad \mathcal{D}_{\text {gau }}^{\Vdash}\left({ }^{\dagger} \mathfrak{D}_{\succ}^{\vdash}\right)
$$

which is compatible, relative to the bijections and local isomorphisms of topological monoids associated to these Frobenioids, with the local evaluation isomorphisms of (iv) above.

Proof. The various assertions of Corollary 4.5 follow immediately from the definitions and the references quoted in the statements of these assertions.

\section{Remark 4.5.1.}

(i) Just as was done in Definition 3.8, one may interpret the various collections of monoids constructed in Corollary 4.5, (i), (iv) as collections of Frobenioids. That is to say, the collection of monoids discussed in Corollary 4.5, (i), gives rise to an $\mathcal{F}$ prime-strip, hence also to an associated $\mathcal{F}^{\vdash}$-prime-strip. In a similar vein, the theta and Gaussian monoids of Corollary 4.5, (iv), give rise to a well-defined $\mathcal{F}^{\vdash}$-primestrip - up to an indeterminacy, at the $\underline{v} \in \underline{\mathbb{V}}^{\text {bad }}$ [corresponding to the various "value-profiles"], relative to automorphisms of the split Frobenioid at such $\underline{v} \in \underline{\mathbb{V}}^{\text {bad }}$ that induce the identity automorphism on the subcategory of isometries [cf. [FrdI], Theorem 5.1, (iii)] of the underlying category of the split Frobenioid — cf. Remark 4.10.1 below. On the other hand, as discussed in Remark 3.8.1, this Frobenioidtheoretic formulation is - by comparison to the original monoid-theoretic formulation - technically ill-suited to discussions of conjugate synchronization.

(ii) On the other hand, such technical complications do not occur if one restricts oneself to discussions of realifications - cf., e.g., the objects " $\mathbb{R}_{\geq 0}\left({ }^{\ddagger} \mathfrak{D}^{\vdash}\right)_{\underline{v}}$ ", " $\mathcal{D}^{\Vdash}\left({ }^{\ddagger} \mathfrak{D}^{\vdash}\right)$ " discussed in Corollary 4.5, (ii). In general, Frobenioid-theoretic formulations are typically technically easier to work with than monoid-theoretic formulations when the associated "Picard groups Pic $c_{\Phi}(-)$ " [cf. [FrdI], Theorem 5.1; [FrdI], Theorem 6.4, (i); [IUTchI], Remark 3.1.5] contain nontorsion elements - i.e., at a more intuitive level, when there is a nontrivial notion of the "degree" of a line bundle. Examples of such Frobenioids include global arithmetic Frobenioids such as the Frobenioid " $\mathcal{D}^{\Vdash}\left({ }^{\ddagger} \mathfrak{D}^{\vdash}\right)$ " of Corollary 4.5, (ii), as well as the tempered Frobenioids that appeared in Propositions 3.3 and 3.4; Corollary 3.6. 


\section{Remark 4.5.2.}

(i) One may also construct symmetrizing isomorphisms as in Corollary 4.5, (iii), for versions labeled by $t \in \operatorname{LabCusp}^{ \pm}\left({ }^{\dagger} \mathfrak{D}_{\succ}\right)$ of the semi-simplifications $\Psi_{\mathrm{cns}}^{\mathrm{ss}}\left({ }^{\dagger} \mathfrak{D}_{\succ}^{\vdash}\right)$, equipped with splittings and distinguished elements, and the global realified Frobenioids $\mathcal{D}^{\Vdash}\left({ }^{\dagger} \mathfrak{D}_{\succ}^{\vdash}\right)$, equipped with bijections and local isomorphisms of topological monoids, as discussed in Corollary 4.5, (iii). We leave the routine details to the reader.

(ii) Just as was discussed in Remark 3.5.3, one may also consider "multibasepoint" versions of the symmetrizing isomorphisms of Corollary 4.5, (iii) [cf. also the discussion of (i) above] - i.e., by passing to $\mathcal{D}$ - $\Theta^{\text {ell }}$-bridges or [holomorphic or mono-analytic] capsules or processions [cf. [IUTchI], Proposition 6.8, (i), (ii), (iii); [IUTchI], Proposition 6.9, (i), (ii)]. We leave the routine details to the reader.

Remark 4.5.3. Before proceeding, we pause to review the significance of the $\mathbb{F}_{l}^{\rtimes \pm}$-symmetry that gives rise to the symmetrizing isomorphisms of Corollary 4.5, (iii) [cf. Remark 3.5.2].

(i) First, we recall that the crucial conjugate synchronization established in Corollaries 3.5, (i); 4.5, (iii) [cf. also Propositions 4.1, (iii); 4.3, (iii)], is possible in the case of the $\mathbb{F}_{l}^{\rtimes \pm}$-symmetry — but not in the case of the $\mathbb{F}_{l}^{*}$-symmetry! precisely because of the connectedness, at each $\underline{v} \in \underline{\mathbb{V}}$, of the local components involved - cf. the discussion of Remarks 2.6.1, (i); 2.6.2, (i); 3.5.2, (ii), as well as [IUTchI], Remark 6.12.4, (i), (ii). Here, we note in passing that although these remarks essentially only concern $\underline{v} \in \underline{\mathbb{V}}^{\text {bad }}$, similar [but, in some sense, easier!] remarks hold at $\underline{v} \in \underline{\mathbb{V}}^{\text {good }}$. A related property of the $\mathbb{F}_{l}^{\rtimes \pm}$-symmetry - which, again, is not satisfied by the $\mathbb{F}_{l}^{*}$-symmetry! — is the "geometric" nature of the automorphisms that give rise to this symmetry [cf. Remark 3.5.2, (iii)].

(ii) One way to understand the significance of the "single basepoint" symmetrizing isomorphisms arising from the $\mathbb{F}_{l}^{\rtimes \pm}$-symmetry is to compare these symmetrizing isomorphisms with the symmetrizing isomorphisms that arise from the various "multi-basepoint" [i.e., "multi-connected component"] symmetries discussed in Remarks 3.5.3; 4.5.2, (ii). That is to say:

(a) By comparison to the symmetries that arise from mono-analytic capsules/processions: the ring structure - i.e., "arithmetic holomorphic structure" - that remains intact in the case of the symmetrizing isomorphisms of Corollary 4.5, (iii), will play an essential role in the theory of the log-wall [cf. the discussion of Remark 3.6.4, (i)], which we shall apply in [IUTchIII].

(b) By comparison to the symmetries that arise from holomorphic capsules/processions: the "single basepoint" that remains intact in the case of the symmetrizing isomorphisms of Corollary 4.5, (iii), is used not only to establish conjugate synchronization, but also to maintain a bijective link with the set of labels in "LabCusp ${ }^{ \pm}(-)$" [cf. the discussion of Remark 3.5.2]. Both conjugate synchronization and the bijective link with the set of labels play crucial roles in the theory of Galois-theoretic theta 
evaluation developed in $\S 3$ [cf. the various remarks following Corollaries 3.5, 3.6; Remark 3.8.3].

(c) By comparison to the symmetries that arise from the $\mathbb{F}_{l}^{\rtimes \pm}$-symmetries of $\mathcal{D}-\Theta^{\text {ell }}$-bridges: Although the structure of an $\mathcal{D}-\Theta^{\text {ell }}$-bridge allows one to maintain a bijective link with the set of labels in "LabCusp ${ }^{ \pm}(-)$" [cf. the discussion of [IUTchI], Remark 4.9.2, (i); [IUTchI], Remark 6.12.4, (i)], the multi-basepoint nature of the $\mathbb{F}_{l}^{\rtimes \pm}$-symmetries of $\mathcal{D}$ - $\Theta^{\text {ell }}$-bridges does not allow one to establish conjugate synchronization [cf. (b)].

(iii) Note that in order to glue together the various local $\mathbb{F}_{l}^{\rtimes \pm}$-symmetries of Corollary 3.5, (i), and Propositions 4.1, (iii); 4.3, (iii), so as to obtain the global $\mathbb{F}_{l}^{\rtimes \pm}$-symmetry of Corollary 4.5, (iii), it is necessary to make use of the global portion "† $\mathcal{D}^{\odot \pm " ~ o f ~ t h e ~} \mathcal{D}$ - $\Theta^{ \pm \text {ell }}$-Hodge theater under consideration - i.e., by applying the theory of [IUTchI], Proposition 6.5 [cf. also [IUTchI], Remark 6.12.4, (iii)]. That is to say, the global portion of the $\mathcal{D}-\Theta^{ \pm \text {ell }}-$ Hodge theater under consideration plays, in particular, the role of

synchronizing the \pm -indeterminacies at each $\underline{v} \in \underline{\mathbb{V}}$.

Indeed, in some sense, this is precisely the content of [IUTchI], Proposition 6.5. In particular, the essential role played in this context by "† $\mathcal{D}^{\odot \pm}$ " in synchronizing, or coordinating, the various local \pm -indeterminacies is one important underlying

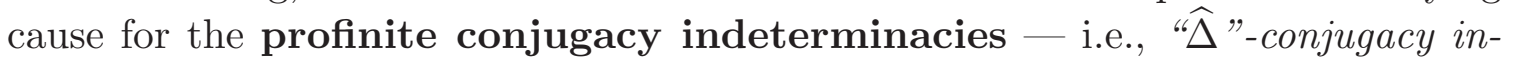
determinacies - that occur in Corollaries 2.4, 2.5 — cf. the discussion of Remark 2.5.2. Thus, in summary, these local \pm -indeterminacies constitute one important reason for the need to apply the "complements on tempered coverings" developed in [IUTchI], $\S 2$, in the proof of Corollary 2.4 of the present paper.

Corollary 4.6. (Frobenioid-theoretic Monoids Associated to $\Theta^{ \pm e l l}$ Hodge Theaters) Let

$$
{ }^{\dagger} \mathcal{H} \mathcal{T}^{\Theta^{ \pm \mathrm{ell}}}=\left({ }^{\dagger} \mathfrak{F}_{\succ} \stackrel{{ }^{\dagger} \psi^{\psi_{ \pm}}}{\longleftarrow} \quad{ }^{\dagger} \mathfrak{F}_{T} \stackrel{{ }^{\dagger}}{\stackrel{\psi_{ \pm}^{\Theta^{\mathrm{ell}}}}{\longrightarrow}}{ }^{\dagger} \mathcal{D}^{\odot \pm}\right)
$$

be a $\Theta^{ \pm \text {ell }}$-Hodge theater [relative to the given initial $\Theta$-data - cf. [IUTchI], Definition 6.11, (iii)] and

$$
{ }^{\ddagger} \mathfrak{F}=\left\{{ }^{\ddagger} \mathcal{F}_{\underline{v}}\right\}_{\underline{v} \in \underline{\mathbb{V}}}
$$

an $\mathcal{F}$-prime-strip; here, we assume [for simplicity] that the $\mathcal{D}$ - $\Theta^{ \pm \text {ell }}$-Hodge theater associated to ${ }^{\dagger} \mathcal{H} \mathcal{T}^{\Theta^{ \pm e l l}}$ [cf. [IUTchI], Definition 6.11, (iii)] is the $\mathcal{D}-\Theta^{ \pm \text {ell }}$-Hodge theater ${ }^{\dagger} \mathcal{H} \mathcal{T}^{\mathcal{D}-\Theta^{ \pm \text {ell }}}$ of Corollary 4.5, and that the $\mathcal{D}$-prime-strip associated to ${ }^{\ddagger} \mathfrak{F}$ [cf. [IUTchI], Remark 5.2.1, (i)] is the $\mathcal{D}$-prime-strip $\ddagger_{\mathfrak{D}}$ of Corollary 4.5. Also, we shall denote the $\mathcal{F}^{\vdash}$-prime-strip associated to - i.e., the mono-analyticization of - an $\mathcal{F}$-prime-strip [cf. [IUTchI], Definition 5.2.1, (ii)] by means of a superscript "-".

(i) (Constant Monoids) There is a functorial algorithm in the $\mathcal{F}$-primestrip ${ }^{\ddagger} \mathfrak{F}$ for constructing the assignment $\Psi_{\text {cns }}\left({ }^{\ddagger} \mathfrak{F}\right)$ given by

$$
\begin{aligned}
& \underline{\mathbb{V}}^{\text {non }} \ni \underline{v} \mapsto \Psi_{\text {cns }}\left({ }^{\ddagger} \mathfrak{F}\right)_{\underline{v}} \stackrel{\text { def }}{=}\left\{G_{\underline{v}}\left({ }^{\ddagger} \Pi_{\underline{v}}\right) \curvearrowright \Psi_{\ddagger \mathcal{F}_{\underline{v}}}\right\} \\
& \underline{\mathbb{V}}^{\operatorname{arc}} \ni \underline{v} \mapsto \Psi_{\text {cns }}\left({ }^{\ddagger} \mathfrak{F}\right)_{\underline{v}} \stackrel{\text { def }}{=} \Psi_{\ddagger} \mathcal{F}_{\underline{v}}
\end{aligned}
$$


- where the data in brackets " $\{-\}$ " is to be regarded as being well-defined only up to $a{ }^{\ddagger} \Pi_{\underline{v}}$-conjugacy indeterminacy - cf. [IUTchI], Definition 5.2, (i); Propositions 3.3, (ii) [i.e., where we take " $\mathcal{C}_{\underline{v}}$ " to be ${ }^{\ddagger} \mathcal{F}_{\underline{v}}$ ]; 4.2, (i); 4.4, (i). We shall write

$$
\Psi_{\mathrm{cns}}\left({ }^{\ddagger} \mathfrak{F}\right) \stackrel{\sim}{\rightarrow} \Psi_{\mathrm{cns}}\left({ }^{\ddagger} \mathfrak{D}\right)
$$

for the collection of isomorphisms of data indexed by $\underline{v} \in \underline{\mathbb{V}}$ determined by the "Kummer-theoretic" isomorphisms of Propositions 3.3, (ii) [i.e., where we take " $\mathcal{C}_{\underline{\underline{v}}}$ " to be ${ }^{\ddagger} \mathcal{F}_{\underline{v}}$ and apply the conventions discussed in Remark 4.2.1., (i)]; 4.2, (i); $4.4,(i)$.

(ii) (Mono-analytic Semi-simplifications) There is a functorial algorithm in the $\mathcal{F}^{\vdash}$-prime-strip ${ }^{\ddagger} \mathfrak{F} \vdash$ for constructing the assignment $\Psi_{\text {cns }}^{\text {ss }}\left({ }^{\ddagger} \mathfrak{F}{ }^{\vdash}\right)$ given by

$$
\underline{\mathbb{V}} \ni \underline{v} \quad \mapsto \quad \Psi_{\text {cns }}^{\mathrm{ss}}\left({ }^{\ddagger} \mathfrak{F}^{\vdash}\right)_{\underline{v}} \stackrel{\text { def }}{=} \Psi_{\ddagger \mathcal{F}_{\underline{v}}}^{\mathrm{ss}}
$$

- where we regard each " $\Psi_{\ddagger \mathcal{F}_{v}^{\vdash}}^{\mathrm{ss}}$ " as being equipped with its natural splitting and,

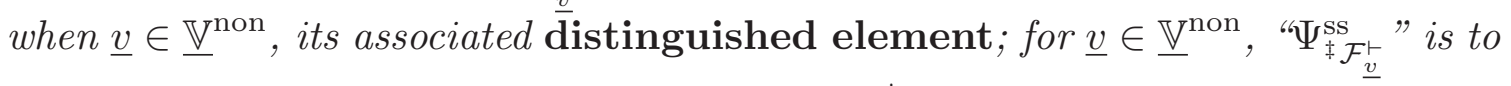
be regarded as being well-defined only up to a $G_{\underline{v}}\left({ }^{\ddagger} \Pi_{\underline{v}}\right)$-conjugacy indeterminacy - cf. Remark 4.2.1, (i), and Propositions 4.2, (ii); 4.4, (ii). We shall write

$$
\Psi_{\text {cns }}^{\mathrm{ss}}\left({ }^{\ddagger} \mathfrak{F}^{\vdash}\right) \stackrel{\sim}{\rightarrow} \Psi_{\text {cns }}^{\mathrm{ss}}\left({ }^{\ddagger} \mathfrak{D}^{\vdash}\right)
$$

for the collection of isomorphisms of data indexed by $\underline{v} \in \underline{\mathbb{V}}$ determined by the "Kummer-theoretic" isomorphisms of Propositions 4.2, (ii); 4.4, (ii) - cf. also Remark 4.2.1, (i); Corollary 4.5, (ii). Now recall the $\mathcal{F}^{\Vdash}$-prime-strip

$$
{ }^{\ddagger} \mathfrak{F}^{\Vdash}=\left({ }^{\ddagger} \mathcal{C}^{\Vdash}, \operatorname{Prime}\left({ }^{\ddagger} \mathcal{C}^{\Vdash}\right) \stackrel{\sim}{\rightarrow} \underline{\mathbb{V}},{ }^{\ddagger} \mathfrak{F},\left\{{ }^{\ddagger} \rho_{\underline{v}}\right\}_{\underline{v} \in \underline{\mathbb{V}}}\right)
$$

associated to ${ }^{\ddagger} \mathfrak{F}$ in [IUTchI], Remark 5.2.1, (ii). Then, in the notation of Corollary 4.5, (ii); [IUTchI], Remark 5.2.1, (ii), there is an isomorphism of Frobenioids

$$
{ }^{\ddagger} \mathcal{C}^{\Vdash} \stackrel{\sim}{\rightarrow} \mathcal{D}^{\Vdash}\left({ }^{\ddagger} \mathfrak{D}^{\vdash}\right)
$$

that is uniquely determined by the condition that it be compatible with the respective bijections Prime $(-) \stackrel{\sim}{\rightarrow} \underline{\mathbb{V}}$ and local isomorphisms of topological monoids for each $\underline{v} \in \underline{\mathbb{V}}$, relative to the above collection of isomorphisms $\Psi_{\mathrm{cns}}^{\mathrm{ss}}\left({ }^{\ddagger} \mathfrak{F}^{\vdash}\right) \stackrel{\sim}{\rightarrow} \Psi_{\mathrm{cns}}^{\mathrm{ss}}\left({ }^{\ddagger} \mathfrak{D}^{\vdash}\right)$. Finally, there is a functorial algorithm for constructing from the $\mathcal{F}^{\Vdash}$-prime-strip ${ }^{\ddagger} \mathfrak{F}^{\Vdash}$ [recalled above] the isomorphism ${ }^{\ddagger} \mathcal{C}^{\Vdash} \stackrel{\sim}{\rightarrow} \mathcal{D}^{\Vdash}\left({ }^{\ddagger} \mathfrak{D}^{\vdash}\right.$ ) [of the preceding display] and the [necessarily compatible] collection of isomorphisms $\Psi_{\mathrm{cns}}^{\mathrm{ss}}\left({ }^{\ddagger} \mathfrak{F}^{\vdash}\right) \stackrel{\sim}{\rightarrow} \Psi_{\mathrm{cns}}^{\mathrm{ss}}\left({ }^{\ddagger} \mathfrak{D}^{\vdash}\right)$ [cf. Remark 4.6 .1 below].

(iii) (Labels, $\mathbb{F}_{l}^{\rtimes \pm}$-Symmetries, and Conjugate Synchronization) In the notation of Corollary 4.5, (iii), the collection of isomorphisms of (i) determines, for each $t \in \operatorname{LabCusp}^{ \pm}\left({ }^{\dagger} \mathfrak{D}_{\succ}\right)$, a collection of compatible isomorphisms

$$
\Psi_{\mathrm{cns}}\left({ }^{\dagger} \mathfrak{F}_{\succ}\right)_{t} \stackrel{\sim}{\rightarrow} \Psi_{\mathrm{cns}}\left({ }^{\dagger} \mathfrak{D}_{\succ}\right)_{t}
$$

- where the ${ }^{\dagger} \Pi_{\underline{\underline{v}}}$-conjugacy indeterminacy at each $\underline{v} \in \underline{\mathbb{V}}^{\text {non }}[c f . \quad$ (i)] is independent of $t \in \operatorname{LabCusp}^{ \pm}\left({ }^{\dagger} \mathfrak{D}_{\succ}\right)$ - as well as $\left[\mathbb{F}_{l}^{\rtimes \pm}\right.$-]symmetrizing isomorphisms, induced by the various local $\mathbb{F}_{l}^{\rtimes \pm}$-actions discussed in Corollary 3.6, 
(i), and Propositions 4.2, (iii); 4.4, (iii), between the data indexed by distinct $t \in \operatorname{LabCusp}^{ \pm}\left({ }^{\dagger} \mathfrak{D}_{\succ}\right)$. Moreover, these symmetrizing isomorphisms are compatible, relative to ${ }^{\dagger} \zeta_{\succ}\left[c f\right.$. Corollary 4.5, (iii)], with the $\mathbb{F}_{l}^{\rtimes \pm}$-symmetry of the associated $\mathcal{D}-\Theta^{\text {ell }}$-bridge [cf. [IUTchI], Proposition 6.8, (i)] and determine [various diagonal submonoids, as well as] an isomorphism

$$
\Psi_{\mathrm{cns}}\left({ }^{\dagger} \mathfrak{F}_{\succ}\right)_{0} \stackrel{\sim}{\rightarrow} \quad \Psi_{\mathrm{cns}}\left({ }^{\dagger} \mathfrak{F}_{\succ}\right)_{\left\langle\mathbb{F}_{l}^{*}\right\rangle}
$$

constituted by the various corresponding local isomorphisms of Corollary 3.6, (iii), and Propositions 4.2, (iii); 4.4, (iii).

(iv) (Local Theta and Gaussian Monoids) Let

$$
\left({ }^{\dagger} \mathfrak{F}_{J} \stackrel{{ }^{\dagger} \psi_{\text {*ै }}^{\ominus}}{\longrightarrow}{ }^{\dagger} \mathfrak{F}_{>} \quad \rightarrow \quad \rightarrow{ }^{\dagger} \mathcal{H} \mathcal{T}^{\Theta}\right)
$$

be a $\Theta$-bridge [relative to the given initial $\Theta$-data - cf. [IUTchI], Definition 5.5, (ii)] which is glued to the $\Theta^{ \pm}$-bridge associated to the $\Theta^{ \pm \text {ell }}$-Hodge theater ${ }^{\dagger} \mathcal{H} \mathcal{T}^{\Theta^{ \pm e l l}}$ via the functorial algorithm of [IUTchI], Proposition 6.7 [so $\mathrm{J}=T^{*}$ ] - cf. the discussion of [IUTchI], Remark 6.12.2, (i). Then there is a functorial algorithm in the $\Theta$-bridge of the above display, equipped with its gluing to the $\Theta^{ \pm}$-bridge associated to ${ }^{\dagger} \mathcal{H} \mathcal{T}^{\Theta^{ \pm e l l}}$, for constructing assignments $\Psi_{\mathcal{F}_{\text {env }}}\left({ }^{\dagger} \mathcal{H} \mathcal{T}^{\Theta}\right)$, $\Psi_{\mathcal{F}_{\text {gau }}}\left({ }^{\dagger} \mathcal{H} \mathcal{T}^{\Theta}\right), \infty \Psi_{\mathcal{F}_{\text {env }}}\left({ }^{\dagger} \mathcal{H} \mathcal{T}^{\Theta}\right), \infty \Psi_{\mathcal{F}_{\text {gau }}}\left({ }^{\dagger} \mathcal{H} \mathcal{T}^{\Theta}\right)$ [where we make a slight abuse of the notation " $\mathcal{H} \mathcal{T}^{\Theta}$ "]

$$
\begin{aligned}
& \underline{\mathbb{V}} \ni \underline{v} \mapsto \Psi_{\mathcal{F}_{\text {env }}}\left({ }^{\dagger} \mathcal{H} \mathcal{T}^{\Theta}\right)_{\underline{\underline{v}}} \stackrel{\text { def }}{=} \Psi_{\dagger \mathcal{F}_{\underline{\underline{\Theta}}}} ; \quad \underline{\mathbb{V}} \ni \underline{v} \mapsto \Psi_{\mathcal{F}_{\text {gau }}}\left({ }^{\dagger} \mathcal{H} \mathcal{T}^{\Theta}\right)_{\underline{v}} \stackrel{\text { def }}{=} \Psi_{\mathcal{F}_{\text {gau }}}\left({ }^{\dagger} \underline{\underline{\mathcal{F}}}_{\underline{v}}\right) \\
& \underline{\mathbb{V}} \ni \underline{v} \mapsto \Phi_{\mathcal{F}_{\text {env }}}\left({ }^{\dagger} \mathcal{H} \mathcal{T}^{\Theta}\right)_{\underline{v}} \stackrel{\text { def }}{=} \infty \Psi_{\dagger} \mathcal{F}_{\underline{v}}^{\Theta} \\
& \underline{\mathbb{V}} \ni \underline{v} \mapsto \infty \Psi_{\mathcal{F}_{\text {gau }}}\left({ }^{\dagger} \mathcal{H} \mathcal{T}^{\Theta}\right)_{\underline{\underline{v}}} \stackrel{\text { def }}{=} \infty \Psi_{\mathcal{F}_{\text {gau }}}\left(\stackrel{\dagger}{\underline{\mathcal{F}}}_{\underline{v}}\right)
\end{aligned}
$$

- where the various local data are equipped with actions by topological groups when $\underline{v} \in \underline{\mathbb{V}}^{\text {non }}$ and splittings [for all $\underline{v} \in \mathbb{V}$ ], as described in detail in Corollary 3.6, (ii), (iii), and Propositions 4.2, (iv); 4.4, (iv) [cf. also Remarks 4.2.1, (ii); 4.4.1] - as well as compatible evaluation isomorphisms

$$
\begin{aligned}
& \Psi_{\mathcal{F}_{\text {env }}}\left({ }^{\dagger} \mathcal{H} \mathcal{T}^{\Theta}\right) \stackrel{\sim}{\rightarrow} \Psi_{\text {env }}\left({ }^{\dagger} \mathfrak{D}_{>}\right) \stackrel{\sim}{\rightarrow} \Psi_{\text {gau }}\left({ }^{\dagger} \mathfrak{D}_{>}\right) \stackrel{\sim}{\rightarrow} \Psi_{\mathcal{F}_{\text {gau }}}\left({ }^{\dagger} \mathcal{H} \mathcal{T}^{\Theta}\right) ; \\
& \infty \Psi_{\mathcal{F}_{\text {env }}}\left({ }^{\dagger} \mathcal{H} \mathcal{T}^{\Theta}\right) \stackrel{\sim}{\rightarrow} \infty \Psi_{\text {env }}\left({ }^{\dagger} \mathfrak{D}_{>}\right) \stackrel{\sim}{\rightarrow} \infty \Psi_{\text {gau }}\left({ }^{\dagger} \mathfrak{D}_{>}\right) \stackrel{\sim}{\rightarrow} \infty \Psi_{\mathcal{F}_{\text {gau }}}\left({ }^{\dagger} \mathcal{H} \mathcal{T}^{\Theta}\right)
\end{aligned}
$$

as described in detail in Corollary 3.6, (ii) [cf. also Remark 4.2.1, (iv); the upper left-hand portion of the first display of Proposition 3.4, (i); the first display of Proposition 3.7, (i)], and Propositions 4.2, (iv); 4.4, (iv) [cf. also Corollary 4.5, (iv)].

(v) (Global Theta and Gaussian Monoids) By applying - i.e., in the fashion of the constructions of Propositions 4.2, (iv); 4.4, (iv) — both labeled [as in (iii) - cf. Remark 4.6.2, (ii), below] and non-labeled versions of the isomorphism “\$ $\mathcal{C}^{\Vdash} \stackrel{\sim}{\rightarrow} \mathcal{D}^{\Vdash}\left({ }^{\ddagger} \mathfrak{D}^{\vdash}\right)$ " of (ii) to the global Frobenioids " $\mathcal{D}_{\text {env }}^{\Vdash}\left({ }^{\dagger} \mathfrak{D}_{\succ}^{\vdash}\right)$ ", " $\mathcal{D}_{\text {gau }}^{\Vdash}\left({ }^{\dagger} \mathfrak{D}_{\succ}^{\vdash}\right)$ " constructed in Corollary 4.5, (v), one obtains a functorial algorithm in the $\Theta$ bridge of the first display of (iv), equipped with its gluing to the $\Theta^{ \pm}$-bridge associated to ${ }^{\dagger} \mathcal{H} \mathcal{T}^{\Theta^{ \pm e l l}}$, for constructing Frobenioids

$$
\mathcal{C}_{\text {env }}^{\Vdash}\left({ }^{\dagger} \mathcal{H} \mathcal{T}^{\Theta}\right), \quad \mathcal{C}_{\text {gau }}^{\Vdash}\left({ }^{\dagger} \mathcal{H} \mathcal{T}^{\Theta}\right)
$$


- where again we make a slight abuse of the notation "† $\mathcal{H} \mathcal{T}^{\Theta}$ "; we note in passing that the construction of " $\mathrm{C}_{\mathrm{env}}^{\Vdash}\left({ }^{\dagger} \mathcal{H} \mathcal{T}^{\Theta}\right)$ " is essentially similar to the construction of "C $\mathrm{Cht}^{\Vdash}$ " in [IUTchI], Example 3.5, (ii) - together with bijections $\operatorname{Prime}\left(\mathcal{C}_{\text {env }}^{\Vdash}\left({ }^{\dagger} \mathcal{H} \mathcal{T}^{\Theta}\right)\right)$ $\stackrel{\sim}{\rightarrow} \underline{\mathbb{V}}$, Prime $\left(\mathcal{C}_{\text {gau }}^{\Vdash}\left({ }^{\dagger} \mathcal{H} \mathcal{T}^{\Theta}\right)\right) \stackrel{\sim}{\rightarrow} \underline{\mathbb{V}}$ and isomorphisms of topological monoids

$$
\Phi_{\mathcal{C}_{\text {env }}^{\Vdash}}\left(\dagger \mathcal{H} \mathcal{T}^{\ominus}\right), \underline{v} \stackrel{\sim}{\rightarrow} \Psi_{\mathcal{F}_{\text {env }}}\left({ }^{\dagger} \mathcal{H} \mathcal{T}^{\Theta}\right)_{\underline{v}}^{\mathbb{R}} ; \quad \Phi_{\mathcal{C}_{\text {gau }}^{\Vdash}\left(\dagger \mathcal{H} \mathcal{T}^{\ominus}\right), \underline{v}} \stackrel{\sim}{\rightarrow} \Psi_{\mathcal{F}_{\text {gau }}}\left({ }^{\dagger} \mathcal{H} \mathcal{T}^{\Theta}\right)_{\underline{v}}^{\mathbb{R}}
$$

[cf. the notational conventions of Corollary 4.5, (v)] for each $\underline{v} \in \underline{\mathbb{V}}$, as well as evaluation isomorphisms

$$
\mathcal{C}_{\text {env }}^{\Vdash}\left({ }^{\dagger} \mathcal{H} \mathcal{T}^{\Theta}\right) \quad \stackrel{\sim}{\rightarrow} \quad \mathcal{D}_{\text {env }}^{\Vdash}\left({ }^{\dagger} \mathfrak{D}_{>}^{\vdash}\right) \quad \stackrel{\sim}{\rightarrow} \quad \mathcal{D}_{\text {gau }}^{\Vdash}\left({ }^{\dagger} \mathfrak{D}_{>}^{\vdash}\right) \quad \stackrel{\sim}{\rightarrow} \quad \mathcal{C}_{\text {gau }}^{\Vdash}\left({ }^{\dagger} \mathcal{H} \mathcal{T}^{\Theta}\right)
$$

- i.e., in the fashion of the constructions of Propositions 4.2, (iv); 4.4, (iv), by "conjugating" the evaluation isomorphism of Corollary 4.5, (v), by the isomorphism " $\mathcal{C}^{\Vdash} \stackrel{\sim}{\rightarrow} \mathcal{D}^{\Vdash}\left({ }^{\ddagger} \mathfrak{D}^{\vdash}\right)$ " of (ii) — which are compatible, relative to the local isomorphisms of topological monoids for each $\underline{v} \in \underline{\mathbb{V}}$ discussed above, with the local evaluation isomorphisms of (iv).

Proof. The various assertions of Corollary 4.6 follow immediately from the definitions and the references quoted in the statements of these assertions.

Remark 4.6.1. One verifies easily that, in the case of $\underline{v} \in \underline{\mathbb{V}}^{\text {non }}$, the polyisomorphism $\Psi_{\mathfrak{T}_{\underline{\mathcal{F}^{v}}}}^{\mathrm{ss}} \stackrel{\sim}{\rightarrow} \Psi_{\mathrm{cns}}^{\mathrm{ss}}\left({ }^{\dagger} G_{\underline{v}}\right.$ ) of Proposition 4.2, (ii) [cf. also Remark 4.2.1, (i)], may be reconstructed algorithmically from ${ }^{\dagger} \mathcal{F}_{\underline{v}}^{\vdash}$. By contrast, in the case of $\underline{v} \in \underline{\mathbb{V}}^{\text {arc }}$, it is not possible to reconstruct algorithmically [the non-unit portion of] the corresponding poly-isomorphism $\Psi_{\dagger \mathcal{F}_{\underline{v}}^{\vdash}}^{\mathrm{ss}} \stackrel{\sim}{\rightarrow} \Psi_{\mathrm{cns}}^{\mathrm{ss}}\left(\mathcal{D}_{\underline{v}}^{\vdash}\right)$ of Proposition 4.4, (ii), from ${ }^{\dagger} \mathcal{F}_{\underline{v}}^{\vdash}$. That is to say, in the case of $\underline{v} \in \underline{\mathbb{V}}^{\text {arc }}$, the distinguished element of $\Psi_{\dagger \mathcal{F}_{\underline{v}}^{\vdash}}^{\mathrm{ss}}$ is not preserved by arbitrary automorphisms of ${ }^{\dagger} \mathcal{F}_{\underline{\underline{v}}}^{\vdash}$. On the other hand, in the context of Corollary 4.6, (ii), if one reconstructs both $\Psi_{\text {cns }}^{\text {ss }}\left({ }^{\ddagger} \mathfrak{F}^{\vdash}\right) \stackrel{\sim}{\rightarrow} \Psi_{\text {cns }}^{\text {ss }}\left({ }^{\ddagger} \mathfrak{D}^{\vdash}\right)$ and ${ }^{\ddagger} \mathcal{C}^{\Vdash} \stackrel{\sim}{\rightarrow} \mathcal{D}^{\Vdash}\left({ }^{\ddagger} \mathfrak{D}^{\vdash}\right)$ in a compatible fashion, then the distinguished elements at $\underline{v} \in \underline{\mathbb{V}}^{\text {arc }}$ may be computed [in the evident fashion] from the distinguished elements at $\underline{v} \in \underline{\mathbb{V}}^{\text {non }}$, together with the structure of the global Frobenioids ${ }^{\ddagger} \mathcal{C}^{\Vdash}, \mathcal{D}^{\Vdash}\left({ }^{\ddagger} \mathfrak{D}^{\vdash}\right)$, i.e., by thinking of these global Frobenioids as "devices for currency exchange" between the various "local currencies" constituted by the divisor monoids at the various $\underline{v} \in \underline{\mathbb{V}}[$ cf. [IUTchI], Remark 3.5.1, (ii)].

\section{Remark 4.6.2.}

(i) Similar observations to the observations made in Remark 4.5.1, (i), concerning the content of Corollary 4.5, (i), (iv), may be made in the case of Corollary 4.6, (i), (iv).

(ii) Similar observations to the observations made in Remark 4.5.2, (i), (ii), concerning the content of Corollary 4.5, (iii), may be made in the case of Corollary 4.6, (iii). 
Corollary 4.7. (Group-theoretic Monoids Associated to Base- $\Theta N F-$ Hodge Theaters) Let

$$
{ }^{\dagger} \mathcal{H} \mathcal{T}^{\mathcal{D}-\Theta \mathrm{NF}}=\left({ }^{\dagger} \mathcal{D}^{\odot} \stackrel{{ }^{\dagger} \phi_{*}^{\mathrm{NF}}}{\longleftarrow} \quad \dagger \mathfrak{D}_{J} \stackrel{{ }^{\dagger} \phi_{*}^{\Theta}}{\longrightarrow} \dagger \mathfrak{D}_{>}\right)
$$

be a D-ONF-Hodge theater [cf. [IUTchI], Definition 4.6, (iii)] which is glued to the $\mathcal{D}-\Theta^{ \pm \text {ell }}$-Hodge theater ${ }^{\dagger} \mathcal{H} \mathcal{T}^{\mathcal{D}-\Theta^{ \pm \text {ell }}}$ of Corollary 4.5 via the functorial algorithm of [IUTchI], Proposition 6.7 [so $J=T^{*}$ ] - cf. the discussion of [IUTchI], Remark 6.12.2, (i), (ii).

(i) (Non-realified Global Structures) There is a functorial algorithm in the category ${ }^{\dagger} \mathcal{D}^{\odot}$ for constructing the morphism

$$
{ }^{\dagger} \mathcal{D}^{\odot} \rightarrow{ }^{\dagger} \mathcal{D}^{\circledast}
$$

[i.e., a "category-theoretic version" of the natural morphism of hyperbolic orbicurves $\left.\underline{C}_{K} \rightarrow C_{F_{\mathrm{mod}}}\right]$ of [IUTchI], Example 5.1, (i), the monoid and field equipped with natural $\pi_{1}\left({ }^{\dagger} \mathcal{D}^{\circledast}\right)$-actions

$$
\pi_{1}\left({ }^{\dagger} \mathcal{D}^{\circledast}\right) \curvearrowright \mathbb{M}^{\odot}\left({ }^{\dagger} \mathcal{D}^{\odot}\right) ; \quad \pi_{1}\left({ }^{\dagger} \mathcal{D}^{\circledast}\right) \curvearrowright \overline{\mathbb{M}}^{\odot}\left({ }^{\dagger} \mathcal{D}^{\odot}\right)
$$

- which are well-defined up to $a \pi_{1}\left({ }^{\dagger} \mathcal{D}^{\circledast}\right)$-conjugacy indeterminacy - of [IUTchI], Example 5.1, (i), the submonoid and subfield of $\pi_{1}\left({ }^{\dagger} \mathcal{D}^{\circledast}\right)$-invariants

$$
\mathbb{M}_{\bmod }^{\odot}\left({ }^{\dagger} \mathcal{D}^{\odot}\right) \subseteq \mathbb{M}^{\odot}\left({ }^{\dagger} \mathcal{D}^{\odot}\right), \quad \overline{\mathbb{M}}_{\text {mod }}^{\odot}\left({ }^{\dagger} \mathcal{D}^{\odot}\right) \subseteq \overline{\mathbb{M}}^{\odot}\left({ }^{\dagger} \mathcal{D}^{\odot}\right)
$$

[cf. [IUTchI], Example 5.1, (i)], the sets of collections of $\pi_{1}\left({ }^{\dagger} \mathcal{D}^{\circledast}\right)$-orbits of $\mathbb{M}^{\odot}\left({ }^{\dagger} \mathcal{D}^{\odot}\right), \overline{\mathbb{M}}^{\odot}\left({ }^{\dagger} \mathcal{D}^{\odot}\right)$

$$
\mathbb{M}_{\text {orb }}^{\odot}\left({ }^{\dagger} \mathcal{D}^{\odot}\right), \quad \overline{\mathbb{M}}_{\text {orb }}^{\odot}\left({ }^{\dagger} \mathcal{D}^{\odot}\right)
$$

[i.e., sets of subsets of $\mathbb{M}^{\odot}\left({ }^{\dagger} \mathcal{D}^{\odot}\right), \overline{\mathbb{M}}^{\odot}\left({ }^{\dagger} \mathcal{D}^{\odot}\right)$ stabilized by $\left.\pi_{1}\left({ }^{\dagger} \mathcal{D}^{\circledast}\right)\right]$, the ["corresponding"] Frobenioids

$$
\mathcal{F}_{\bmod }^{\odot}\left({ }^{\dagger} \mathcal{D}^{\odot}\right) \quad \subseteq \quad \mathcal{F}^{\circledast}\left({ }^{\dagger} \mathcal{D}^{\odot}\right) \quad \supseteq \mathcal{F}^{\odot}\left({ }^{\dagger} \mathcal{D}^{\odot}\right)
$$

- where we write $\mathcal{F}_{\bmod }^{\odot}\left({ }^{\dagger} \mathcal{D}^{\odot}\right), \mathcal{F}^{\odot}\left({ }^{\dagger} \mathcal{D}^{\odot}\right)$ for the subcategories "† $\mathcal{F}_{\bmod }^{\odot}$ ", “† $\mathcal{F}^{\odot}$ " obtained in [IUTchI], Example 5.1, (iii), by taking the "i $\mathcal{F}^{\circledast}$ " of loc. cit. to be $\mathcal{F}^{\circledast}\left({ }^{\dagger} \mathcal{D}^{\odot}\right)$; by abuse of notation, we regard this Frobenioid $\mathcal{F}^{\circledast}\left({ }^{\dagger} \mathcal{D}^{\odot}\right)$ as being equipped with a natural bijection

$$
\operatorname{Prime}\left(\mathcal{F}_{\bmod }^{\odot}\left({ }^{\dagger} \mathcal{D}^{\odot}\right)\right) \stackrel{\sim}{\rightarrow} \underline{\mathbb{V}}
$$

[cf. the final portion of [IUTchI], Example 5.1, (v)] — of [IUTchI], Example 5.1, (ii), (iii), and the natural realification functor

$$
\mathcal{F}_{\text {mod }}^{\odot}\left({ }^{\dagger} \mathcal{D}^{\odot}\right) \rightarrow \mathcal{F}_{\bmod }^{\odot \mathbb{R}}\left({ }^{\dagger} \mathcal{D}^{\odot}\right)
$$

[cf. [IUTchI], Example 5.1, (vii); [FrdI], Proposition 5.3]. 
(ii) (Labels and $\mathbb{F}_{l}^{*}$-Symmetry) Recall the bijection

$$
{ }^{\dagger} \zeta_{*}: \operatorname{LabCusp}\left({ }^{\dagger} \mathcal{D}^{\odot}\right) \stackrel{\sim}{\rightarrow} J \quad\left(\stackrel{\sim}{\rightarrow} \mathbb{F}_{l}^{*}\right)
$$

of [IUTchI], Proposition 4.7, (iii). In the following, we shall use analogous conventions to the conventions applied in Corollary 4.5 concerning subscripted labels. Let $j \in \operatorname{LabCusp}\left({ }^{\dagger} \mathcal{D}^{\odot}\right)$. Then there is a functorial algorithm in the category ${ }^{\dagger} \mathcal{D}^{\odot}$ for constructing an $\mathcal{F}$-prime-strip

$$
\left.\mathcal{F}^{\odot}\left({ }^{\dagger} \mathcal{D}^{\odot}\right)\right|_{j}
$$

- which is well-defined up to isomorphism - from $\mathcal{F}^{\circledast}\left({ }^{\dagger} \mathcal{D}^{\odot}\right)-c f . \quad$ [IUTchI], Example 5.4, (iv) [where we take the " $\delta$ " of loc. cit. to be j]. Moreover, the natural poly-action of $\mathbb{F}_{l}^{*}$ on ${ }^{\dagger} \mathcal{D}^{\odot}$ [cf. [IUTchI], Example 4.3, (iv)] induces isomorphisms between the labeled data

$$
\begin{gathered}
\left.\mathcal{F}^{\odot}\left({ }^{\dagger} \mathcal{D}^{\odot}\right)\right|_{j} ; \quad \mathbb{M}_{\text {mod }}^{\odot}\left({ }^{\dagger} \mathcal{D}^{\odot}\right)_{j} ; \quad \overline{\mathbb{M}}_{\text {mod }}^{\odot}\left({ }^{\dagger} \mathcal{D}^{\odot}\right)_{j} ; \quad \mathbb{M}_{\text {orb }}^{\odot}\left({ }^{\dagger} \mathcal{D}^{\odot}\right)_{j} ; \quad \overline{\mathbb{M}}_{\text {orb }}^{\odot}\left({ }^{\dagger} \mathcal{D}^{\odot}\right)_{j} \\
\mathcal{F}_{\text {mod }}^{\odot}\left({ }^{\dagger} \mathcal{D}^{\odot}\right)_{j} \rightarrow \mathcal{F}_{\bmod }^{\odot \mathbb{R}}\left({ }^{\dagger} \mathcal{D}^{\odot}\right)_{j}
\end{gathered}
$$

[cf. (i)] for distinct $j \in \operatorname{LabCusp}\left({ }^{\dagger} \mathcal{D}^{\odot}\right)$ [cf. Remark 4.7.2 below]. We shall refer to these isomorphisms as $\left[\mathbb{F}_{l}^{*}-\right]$ symmetrizing isomorphisms. These symmetrizing isomorphisms are compatible, relative to ${ }^{\dagger} \zeta_{*}$, with the $\mathbb{F}_{l}^{*}$-symmetry of the associated D-NF-bridge [cf. [IUTchI], Proposition 4.9, (i)] and determine diagonal $\mathcal{F}$-prime-strips/submonoids/subfields/sets of subsets/subcategories [cf. Remark 4.7.2 below]

$$
(-)_{\left\langle\mathbb{F}_{i}^{*}\right\rangle} \subseteq \prod_{j \in \mathbb{F}_{i}^{*}}(-)_{j}
$$

- where "(-)..." may be taken to be $\mathcal{F}^{\odot}\left({ }^{\dagger} \mathcal{D}^{\odot}\right) \mid \ldots$ [cf. the discussion of [IUTchI], Example 5.4, $(i)], \quad \mathbb{M}_{\text {mod }}^{\odot}\left({ }^{\dagger} \mathcal{D}^{\odot}\right)_{\ldots}, \quad \overline{\mathbb{M}}_{\text {mod }}^{\odot}\left({ }^{\dagger} \mathcal{D}^{\odot}\right)_{\ldots}, \quad \mathbb{M}_{\text {orb }}^{\odot}\left({ }^{\dagger} \mathcal{D}^{\odot}\right)_{\ldots}, \quad \overline{\mathbb{M}}_{\text {orb }}^{\odot}\left({ }^{\dagger} \mathcal{D}^{\odot}\right)_{\ldots}$, $\mathcal{F}_{\text {mod }}^{\odot}\left({ }^{\dagger} \mathcal{D}^{\odot}\right)_{\ldots}$, or $\mathcal{F}_{\bmod }^{\odot \mathbb{R}}\left({ }^{\dagger} \mathcal{D}^{\odot}\right)_{\text {... }}$ [cf. the discussion of [IUTchI], Example 5.1, (vii)].

(iii) (Localization Functors and Realified Global Structures) Let $j \in$ LabCusp $\left({ }^{\dagger} \mathcal{D}^{\odot}\right)$. Then there is a functorial algorithm in the category ${ }^{\dagger} \mathcal{D}^{\odot}$ for constructing [1-]compatible collections of "localization" functors [up to isomorphism]

$$
\left.\mathcal{F}_{\bmod }^{\odot}\left({ }^{\dagger} \mathcal{D}^{\odot}\right)_{j} \quad \rightarrow \quad \mathcal{F}^{\odot}\left({ }^{\dagger} \mathcal{D}^{\odot}\right)\right|_{j} ; \quad \mathcal{F}_{\bmod }^{\odot \mathbb{R}}\left({ }^{\dagger} \mathcal{D}^{\odot}\right)_{j} \quad \rightarrow \quad\left(\left.\mathcal{F}^{\odot}\left({ }^{\dagger} \mathcal{D}^{\odot}\right)\right|_{j}\right)^{\mathbb{R}}
$$

- where the superscript "R" denotes the realification - as in the discussion of [IUTchI], Example 5.4, (vi), together with a natural isomorphism of Frobenioids

$$
\mathcal{D}^{\Vdash}\left(\left(\left.\mathcal{F}^{\odot}\left({ }^{\dagger} \mathcal{D}^{\odot}\right)\right|_{j}\right)^{\mathcal{D}^{\vdash}}\right) \stackrel{\sim}{\rightarrow} \quad \mathcal{F}_{\bmod }^{\odot \mathbb{R}}\left({ }^{\dagger} \mathcal{D}^{\odot}\right)_{j}
$$

- where we denote the $\mathcal{D}^{\vdash}$-prime-strip associated [cf. [IUTchI], Definition 4.1, (iv); [IUTchI], Remark 5.2.1, (i)] to an $\mathcal{F}$-prime-strip by means of a superscript 
" $\mathcal{D}^{\vdash "}$ [cf. also the notation of Corollary 4.5, (ii); Remark 4.7.1 below] - and, for each $\underline{v} \in \underline{\mathbb{V}}$, a natural isomorphism of topological monoids

$$
\mathbb{R}_{\geq 0}\left(\left(\left.\mathcal{F}^{\odot}\left({ }^{\dagger} \mathcal{D}^{\odot}\right)\right|_{j}\right)^{\mathcal{D}^{\dagger}}\right)_{\underline{v}} \stackrel{\sim}{\rightarrow} \Psi_{\left(\left.\mathcal{F} \odot(\dagger \mathcal{D} \odot)\right|_{j}\right)^{\mathbb{R}}, \underline{v}}
$$

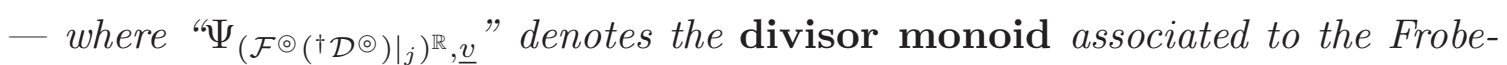
nioid that constitutes $\left(\left.\mathcal{F}^{\odot}\left({ }^{\dagger} \mathcal{D}^{\odot}\right)\right|_{j}\right)^{\mathbb{R}}$ at $\underline{v}$ - which are compatible with the respective bijections involving "Prime (-)" and the respective local isomorphisms of topological monoids $\left[\right.$ cf. the arrow $\mathcal{F}_{\bmod }^{\odot \mathbb{R}}\left({ }^{\dagger} \mathcal{D}^{\odot}\right)_{j} \rightarrow\left(\left.\mathcal{F}^{\odot}\left({ }^{\dagger} \mathcal{D}^{\odot}\right)\right|_{j}\right)^{\mathbb{R}}$ discussed above; Corollary 4.5, (ii)]. Finally, all of these structures are compatible with the respective $\mathbb{F}_{l}^{*}$-symmetrizing isomorphisms [cf. (ii)].

Proof. The various assertions of Corollary 4.7 follow immediately from the definitions and the references quoted in the statements of these assertions.

Remark 4.7.1. Similar observations to the observations made in Remark 4.5.2, (i), (ii), concerning the $\mathbb{F}_{l}^{\rtimes \pm}$-symmetrizing isomorphisms of Corollary 4.5, (iii), may be made in the case of the $\mathbb{F}_{l}^{*}$-symmetrizing isomorphisms of Corollary 4.7, (ii).

Remark 4.7.2. In the context of Corollary 4.7, (ii), we recall from Remarks 3.5.2, (iii); 4.5.3, (i), that unlike the case with $\mathbb{F}_{l}^{\rtimes \pm}$-symmetry, in the case of $\mathbb{F}_{l}^{*}$ symmetry, it is not possible to establish the sort of conjugate synchronization given in Corollary 4.5, (iii), since the $\mathbb{F}_{l}^{*}$-symmetry involves - i.e., more precisely, arises from conjugation by elements with nontrivial image in - the arithmetic portion [i.e., the absolute Galois group of the base field] of the global arithmetic fundamental groups involved — cf. the discussion of how " $G_{K}$-conjugacy indeterminacies give rise to $G_{\underline{\underline{v}}}$-conjugacy indeterminacies" in Remark 2.5 .2 , (iii). This is precisely why, in Corollary 4.7, (ii), we work with

(a) $\mathcal{F}$-prime-strips, as opposed to the corresponding topological monoids with Galois actions as in Corollary 4.5, (iii), and with

(b) the various objects introduced in Corollary 4.7, (i), that are equipped with a subscript "mod" — corresponding to " $F_{\bmod }$ " — or a subscript "orb", as opposed to the objects not equipped with such subscripts, which correspond to "F"'.

That is to say, both (a) and (b) allow one to ignore the various independent i.e., non-synchronizable — conjugacy indeterminacies that occur at the various distinct labels as a consequence of the single basepoint with respect to which one considers both the labels and the labeled objects [cf. the discussion of Remark 3.5.2, (ii)]. Here, it is also useful to observe that by working with the various objects introduced in Corollary 4.7, (i), that are equipped with a subscript "mod" - i.e., on which the various conjugacy indeterminacies involved act trivially - it follows that at least the base category portions of the various diagonal subcategories associated to the corresponding Frobenioids may be defined — just as in the case of realified Frobenioids [cf. the discussion of Remark 4.5.1, (ii)] — in a fashion in which one is not obliged to contend with the technical subtleties that arise 
from independent conjugacy indeterminacies at distinct labels [cf. the discussion of "Galois-invariants/Galois-orbits" in Remark 3.8.3, (ii)]. In [IUTchIII], the ring structure on these objects equipped with a subscript "mod" will be applied as a sort of translation apparatus between “ $\boxplus$-line bundles" [i.e., arithmetic line bundles thought of as additive modules with additional structure] and " $\square$-line bundles" [i.e., arithmetic line bundles thought of "multiplicatively" or "idèlically", as in the theory of Frobenioids] — cf. [AbsTopIII], Definition 5.3, (i), (ii).

Remark 4.7.3. At this point, it is of interest to review the significance of the $\mathbb{F}_{l}^{\rtimes \pm}$ - and $\mathbb{F}_{l}^{*}$-symmetries in the context of the theory of the present $\S 4$.

(i) First, we recall that, in the context of the present series of papers, the " $\mathbb{F}_{l}$ " that appears in the notation $" \mathbb{F}_{l}^{\rtimes \pm " ~}$ and " $\mathbb{F}_{l}^{*}$ " is to be thought of - since $l$ is "large" - as a sort of finite approximation of the ring of rational integers $\mathbb{Z}$ [cf. the discussion of [IUTchI], Remark 6.12.3, (i)]. That is to say, the $\mathbb{F}_{l}^{\rtimes \pm}$-symmetry corresponds to the additive structure of $\mathbb{Z}$, while the $\mathbb{F}_{l}^{*}$-symmetry corresponds to the multiplicative structure of $\mathbb{Z}$. Since the " $\mathbb{F}_{l}$ " under consideration arises from the torsion points of an elliptic curve, it is natural - especially in light of the central role played in the present series of papers by $\underline{v} \in \underline{\mathbb{V}}^{\text {bad }}$ — to think of the " $\mathbb{Z}$ " under consideration as the Galois group "Z्Z" of the universal combinatorial covering of the Tate curves that appear at $\underline{v} \in \underline{\mathbb{V}}$ [cf. the discussion at the beginning of [EtTh], §1]. In particular, in light of the theory of Tate curves, it is natural to think of this " $\mathbb{Z}$ " as representing a sort of universal version of the value group associated to a local field that occurs at a $\underline{v} \in \underline{\mathbb{V}}^{\text {bad }}$, and to think of the element $0 \in \mathbb{Z}$ - hence, the label

$$
0 \in\left|\mathbb{F}_{l}\right|
$$

— as representing the units.

(ii) Perhaps the most fundamental difference between the $\mathbb{F}_{l}^{\rtimes \pm}$ - and $\mathbb{F}_{l}^{*}$-symmetries is that the $\mathbb{F}_{l}^{\rtimes \pm}$-symmetry involves the zero label $0 \in\left|\mathbb{F}_{l}\right|$ [cf. the discussion of [IUTchI], Remark 6.12.5]. In particular, the $\mathbb{F}_{l}^{\rtimes \pm}$-symmetry is suited to application to the "units" - i.e., to the various local " $\mathcal{O}^{\times}$" and " $\mathcal{O}^{\times \mu}$ " that appear in the theory. At a more technical level, this relationship between the $\mathbb{F}_{l}^{\rtimes \pm}$ symmetry and " $\mathrm{O}^{\times}$" may be seen in the theory of $\S 3$ [cf. also Corollaries 4.5, (iii); 4.6, (iii)]. That is to say, in $\S 3$ [cf. the discussion of Remark 3.8.3], the $\mathbb{F}_{l}^{\rtimes \pm}$ symmetry is applied precisely to establish conjugate synchronization, which, in turn, will be applied eventually to establish the crucial coricity of " $\mathcal{O} \times \boldsymbol{\mu}$ " in the context of the $\Theta_{\text {gau }}^{\times \mu}$-link [cf. Corollary 4.10 below]. Here, let us observe that the conjugate synchronization, established by means of the $\mathbb{F}_{l}^{\rtimes \pm}$-symmetry, of copies of the absolute Galois group of the local base field at various $\underline{v} \in \underline{\mathbb{V}}^{\text {non }}$ is a very delicate property that depends quite essentially on the "arithmetic holomorphic structure" of the Hodge theaters under consideration. That is to say, from the point of view of the theory of $\S 1$, conjugate synchronization in one Hodge theater fails to be compatible with conjugate synchronization in another Hodge theater with a distinct arithmetic holomorphic structure. Put another way, from the point of view of the theory of $\S 1$, conjugate synchronization can only be naturally formulated in a uniradial fashion. This uniradiality may also be seen at a purely combinatorial level, as we shall discuss in Remark 4.7 .4 below. On the other hand, if one 
passes to mono-analyticizations - e.g., to mono-analytic processions - then the mono-analytic " $\mathcal{O} \times \boldsymbol{\mu}$ " that appears in the $\Theta_{\text {gau }}^{\times \mu}$-link [cf. Corollary 4.10 below] is, by contrast, coric. That is to say, by relating the zero label, which is common to distinct arithmetic holomorphic structures, to the various nonzero labels, which belong to a single fixed arithmetic holomorphic structure, the condition of invariance with respect to the $\mathbb{F}_{l}^{\rtimes \pm}$-symmetry may - e.g., in the case of the mono-analytic "O $\times$ $\times \mu "$ - amount to a condition of coricity. In particular, in the case of the mono-analytic " $\mathcal{O}^{\times \mu}$ ",

$\mathbb{F}_{l}^{\rtimes \pm}$-symmetry plays the role of establishing the coric pieces - i.e., components which are "uniform" with respect to all of the distinct arithmetic holomorphic structures involved - of the apparatus to be established in the present series of papers.

This dual role - i.e., consisting of both uniradial and coric aspects - played by the $\mathbb{F}_{l}^{\rtimes \pm}$-symmetry is to be considered in contrast to the strictly multiradial role [cf. (iii) below] played by the $\mathbb{F}_{l}^{*}$-symmetry.

(iii) The significance of the $\mathbb{F}_{l}^{*}$-symmetry lies, in a word, in the fact that it allows one to separate the zero label from the nonzero labels. From the point of view of the theory of the present series of papers, this property makes the $\mathbb{F}_{l}^{*}$ symmetry well-suited for the construction/description of the internal structure of the Gaussian monoids, which are, in effect, "distributions" or "functions" of a parameter $j \in \mathbb{F}_{l}^{*}$ [cf. Corollaries 4.5, (iv), (v); 4.6, (iv), (v)]. Here, we note that this separation of the zero label — which parametrizes coric data that is common to distinct arithmetic holomorphic structures - from the nonzero labels — which parametrize the Gaussian monoid associated to a particular arithmetic holomorphic structure - is crucial from the point of view of describing the Gaussian monoid associated to a particular arithmetic holomorphic structure in terms that may be understood from the point of view of some "alien" arithmetic holomorphic structure. Put another way, from the point of view of the theory of $\S 1$, the $\mathbb{F}_{l}^{*}$-symmetry admits a natural multiradial formulation. This multiradiality may also be seen at a purely combinatorial level, as we shall discuss in Remark 4.7.4 below. In this context, it is important to note that if one thinks of the coric constant distribution, labeled by zero, as embedded via the diagonal embedding into the various products parametrized by $j \in \mathbb{F}_{l}^{*}$ that appear in the construction of the Gaussian monoids [cf. the isomorphisms that appear in the final displays of Corollaries 4.5, (iii); 4.6, (iii)], then it is natural to think of the volumes computed at each $j \in \mathbb{F}_{l}^{*}$ as being assigned a weight $1 / l^{*}$ — i.e., so that the diagonal embedding of the constant distribution is compatible with taking the constant distribution to be of weight 1 [cf. the discussion of [IUTchI], Remark 5.4.2]. Put another way, from the point of view of "computation of weighted volumes", the various nonzero $j \in \mathbb{F}_{l}^{*}$ are "subordinate" to $0 \in\left|\mathbb{F}_{l}\right|$ - i.e., $\mathbb{F}_{l}^{*} \ni j \lll 0$. In particular, to symmetrize, in the context of the internal structure of the Gaussian monoids, the zero and nonzero labels [i.e., as in the case of the $\mathbb{F}_{l}^{\rtimes \pm}$-symmetry!] amounts to allowing a relation

$$
\text { "0 «0" }
$$

— which is absurd [i.e., in the sense that it fails to be compatible with weighted volume computations]! 


\section{Remark 4.7.4.}

(i) One way to understand the underlying combinatorial structure of the uniradiality of the $\mathbb{F}_{l}^{\rtimes \pm}$-symmetry and the multiradiality of the $\mathbb{F}_{l}^{*}$-symmetry [cf. the discussion of Remark 4.7.3, (ii), (iii)] is to consider these symmetries — which are defined relative to some given arithmetic holomorphic structure [or, at a more technical level, some given $\Theta^{ \pm e l l} N F$-Hodge theater — cf. [IUTchI], Definition 6.13, (i) - in the context of the étale-pictures that arise from each of these symmetries [cf. [IUTchI], Corollaries 4.12, 6.10]. In the case of the $\mathbb{F}_{l}^{\rtimes \pm}$ - (respectively, $\mathbb{F}_{l}^{*}$-) symmetry, this étale-picture consists of a collection of copies of $\mathbb{F}_{l}$ (respectively, $\left.\left|\mathbb{F}_{l}\right|=\mathbb{F}_{l}^{*} \bigcup\{0\}\right)$, each copy corresponding to a single arithmetic holomorphic structure, which are glued together at the coric label $0 \in \mathbb{F}_{l}$ (respectively, $0 \in\left|\mathbb{F}_{l}\right|$ ). In Fig. 4.1 (respectively, 4.2) below, an illustration is given of such an étale-picture, in which the notation " \pm " (respectively, "*”) is used to denote the various elements of $\mathbb{F}_{l} \backslash\{0\}$ (respectively, $\mathbb{F}_{l}^{*}$ ) in each copy of $\mathbb{F}_{l}$ (respectively, $\left|\mathbb{F}_{l}\right|$ ). Moreover, on each copy of $\mathbb{F}_{l}$ (respectively, $\left|\mathbb{F}_{l}\right|$ ) - labeled, say, by some spoke $\alpha$ [corresponding to a single arithmetic holomorphic structure] — one has a natural action of a "corresponding copy" of $\mathbb{F}_{l}^{\rtimes \pm}$ (respectively, $\mathbb{F}_{l}^{*}$ ).

(ii) The fundamental difference between the simple combinatorial models of the étale-pictures considered in (i) lies in the fact that whereas

(a) in the case of the $\mathbb{F}_{l}^{\rtimes \pm}$-symmetry, the $\mathbb{F}_{l}^{\rtimes \pm}$-actions on distinct spokes fail to commute with one another,

(b) in the case of the $\mathbb{F}_{i}^{*}$-symmetry, the $\mathbb{F}_{l}^{*}$-actions on distinct spokes commute with one another, and, moreover, are compatible with the permutations of spokes discussed in [IUTchI], Corollary 4.12, (iii).

Indeed, the noncommutativity, or "incompatibility with simultaneous execution at distinct spokes" [cf. Remark 1.9.1], of (a) is a direct consequence of the inclusion of the zero label in the $\mathbb{F}_{l}^{\rtimes \pm}$-symmetry and may be thought of as a sort of prototypical combinatorial representation of the phenomenon of uniradiality. By contrast, the commutativity, or "compatibility with simultaneous execution at distinct spokes" [cf. Remark 1.9.1], of (b) is a direct consequence of the exclusion of the zero label from the $\mathbb{F}_{l}^{*}$-symmetry and may be thought of as a sort of prototypical combinatorial representation of the phenomenon of multiradiality. Note that in the case of the $\mathbb{F}_{l}^{\rtimes \pm}$-symmetry, it is also a direct consequence of the inclusion of the zero label that the condition of invariance with respect to the $\mathbb{F}_{l}^{\rtimes \pm}$-actions on all of the spokes may be thought of as a condition of "uniformity" among the elements of the copies of $\mathbb{F}_{l}$ at the various spokes, hence as a sort of coricity [cf. the discussion of Remark 4.7.3, (ii)].

(iii) Although the combinatorial versions of uniradiality and multiradiality discussed in (ii) above are not formulated in terms of the formalism of uniradial and multiradial environments developed in $\S 1$ [cf. Example 1.7, (ii)], it is not difficult to produce such a formulation. For instance, one may take the coric data to consist objects of the form " $0_{\alpha}$ " - i.e., the zero label, subscripted by the label $\alpha$ associated to some spoke. For any two spokes $\alpha, \beta$, we define the set of arrows

$$
0_{\alpha} \rightarrow 0_{\beta}
$$


to consist of precisely one element $(\alpha, \beta)$. We then take, in the case of the $\mathbb{F}_{l}^{\rtimes \pm}$ (respectively, $\mathbb{F}_{l}^{*}$ ) symmetry, the radial data to consist of a copy $\left(\mathbb{F}_{l}\right)_{\alpha}$ (respectively, $\left|\mathbb{F}_{l}\right|_{\alpha}$ ) of $\mathbb{F}_{l}$ (respectively, $\left|\mathbb{F}_{l}\right|$ ) subscripted by the label $\alpha$ associated to some spoke. For any two spokes $\alpha, \beta$, we define the set of arrows

$$
\left.\left(\mathbb{F}_{l}\right)_{\alpha} \rightarrow\left(\mathbb{F}_{l}\right)_{\beta} \quad \text { (respectively, }\left|\mathbb{F}_{l}\right|_{\alpha} \rightarrow\left|\mathbb{F}_{l}\right|_{\beta}\right)
$$

to consist of precisely one element if the actions $\left(\mathbb{F}_{l}^{\rtimes \pm}\right)_{\gamma} \curvearrowright\left(\mathbb{F}_{l}\right)_{\gamma}$ (respectively, $\left.\left(\mathbb{F}_{l}^{*}\right)_{\gamma} \curvearrowright\left(\left|\mathbb{F}_{l}\right|\right)_{\gamma}\right)$, for $\gamma=\alpha, \beta$, determine an action of

$$
\left(\mathbb{F}_{l}^{\rtimes \pm}\right)_{\alpha} \times\left(\mathbb{F}_{l}^{\rtimes \pm}\right)_{\beta} \quad\left(\text { respectively, }\left(\mathbb{F}_{l}^{*}\right)_{\alpha} \times\left(\mathbb{F}_{l}^{*}\right)_{\beta}\right)
$$

on the co-product $\left(\mathbb{F}_{l}\right)_{\alpha} \coprod_{0}\left(\mathbb{F}_{l}\right)_{\beta}$ (respectively, $\left.\left(\left|\mathbb{F}_{l}\right|\right)_{\alpha} \coprod_{0}\left(\left|\mathbb{F}_{l}\right|\right)_{\beta}\right)$ obtained by identifying the respective zero labels $0_{\alpha}, 0_{\beta}$, and to equal the empty set if such an action does not exist. Then one has a natural radial functor $\left(\mathbb{F}_{l}\right)_{\alpha} \mapsto 0_{\alpha}$ (respectively, $\left|\mathbb{F}_{l}\right|_{\alpha} \mapsto 0_{\alpha}$ ) that associates coric data to radial data. Moreover, the resulting radial environment is easily seen to be uniradial (respectively, multiradial). We leave the routine details to the reader. Finally, we note in passing that the formulation involving products given above is reminiscent both of the discussion of the switching functor in Example 1.7, (iii), and of the discussion of parallel transport via connections in Remark 1.7.1.

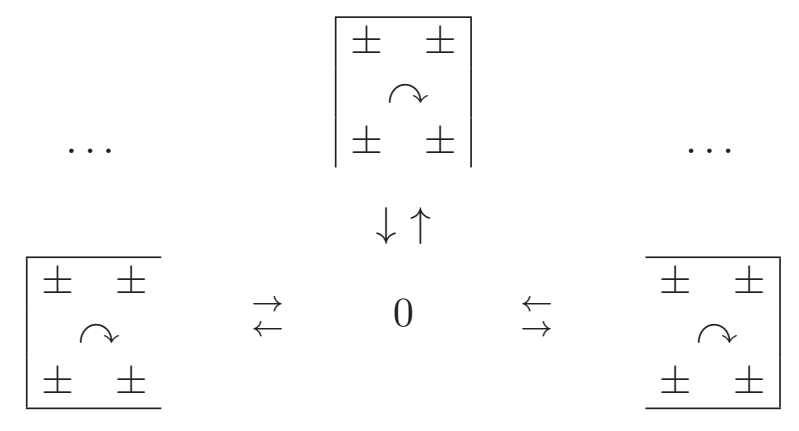

Fig. 4.1: Étale-picture of $\mathbb{F}_{l}^{\rtimes \pm}$-symmetries

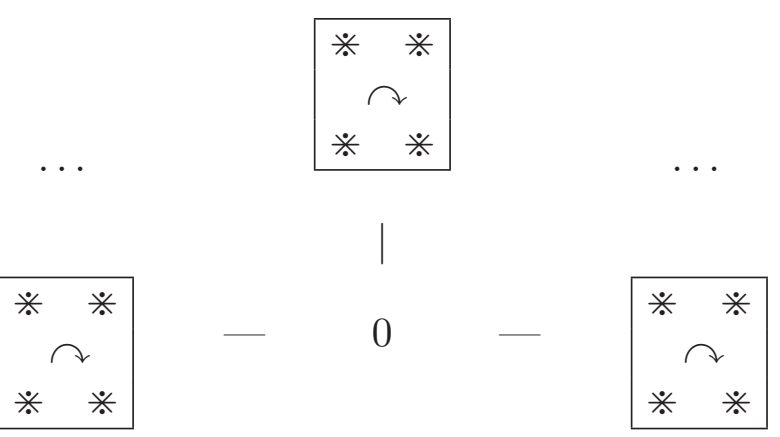

Fig. 4.2: Étale-picture of $\mathbb{F}_{l}^{*}$-symmetries 
Remark 4.7.5. In the context of the discussion of the combinatorial models of the $\mathbb{F}_{l}^{\rtimes \pm}$ - and $\mathbb{F}_{l}^{*}$-symmetries in Remark 4.7 .4 , it is useful to recall that the $\mathbb{F}_{l}^{\rtimes \pm}$ - and $\mathbb{F}_{l}^{*}$-symmetries correspond, respectively, to the additive and multiplicative structures of the field $\mathbb{F}_{l}$ - which [cf. Remark 4.7.3, (i)] we wish to think of as a sort of finite approximation of the ring $\mathbb{Z}$. That is to say, from the point of view of the theory of the present series of papers,

(a) the $\mathbb{F}_{l}^{\rtimes \pm}$ - and $\mathbb{F}_{l}^{*}$-symmetries correspond, respectively, to the two combinatorial dimensions - i.e., addition and multiplication - of a ring [cf. the discussion of [AbsTopIII], §I3].

Moreover, in the context of the discussion of Remark 4.7.3, (i), concerning units and value groups, it is useful to recall that these two combinatorial dimensions may be thought of as corresponding to

(b) the units and value group of a mixed-characteristic nonarchimedean or complex archimedean local field [cf. the discussion of [AbsTopIII], §I3]

or, alternatively, to

(c) the two cohomological dimensions of the absolute Galois group of a mixed-characteristic nonarchimedean local field or the two underlying real dimensions of a complex archimedean local field [cf. the discussion of [AbsTopIII], §I3].

Finally, the hierarchical structure of these two dimensions - i.e., the way in which "one dimension [i.e., multiplication] is piled on top of the other [i.e., addition]" is reflected in the

(d) subordination structure "«", relative to the computation of weighted volumes, of nonzero labels with respect to the zero label [cf. the discussion of Remark 4.7.3, (iii)].

as well as in the fact that

(e) the $\mathbb{F}_{l}^{\rtimes \pm}$-symmetry arises from the conjugation action of the geometric fundamental group [cf. Remarks 3.5.2, (iii); 4.5.3, (i)], whereas the $\mathbb{F}_{l}^{*}$ symmetry arises from the conjugation action of the absolute Galois group of the global base field [cf. Remark 4.7.2]

- i.e., where we recall that the arithmetic fundamental groups involved may be thought of as having a natural hierarchical structure constituted by their extension structure [corresponding to the natural outer action of the absolute Galois group of the base field on the geometric fundamental group].

Remark 4.7.6. One important observation in the context of Corollary 4.7, (i), is that it only makes sense to consider non-realified global Frobenioids [corresponding, e.g., to " $F$ mod"] in the case of the $\mathbb{F}_{l}^{*}$-symmetry. Indeed, in order to consider the field " $F_{\text {mod" }}$ " from an anabelian, or Galois-theoretic, point of view, 
it is necessary to consider the full profinite group $\Pi_{C_{F}}$ - i.e., not just the open subgroups $\Pi_{\underline{C}_{K}}, \Pi_{\underline{X}_{K}}$ of $\Pi_{C_{F}}$ which give rise, respectively, to the global portions of the $\mathbb{F}_{l}^{*}$ - and $\mathbb{F}_{l}^{\rtimes \pm}$-symmetries [cf. [IUTchI], Definition 4.1, (v); [IUTchI], Definition $6.1,(\mathrm{v})]$. On the other hand, to work with the abstract topological group $\Pi_{C_{F}}$ means that the subgroups $\Pi_{\underline{C}_{K}}$, $\Pi_{\underline{X}_{K}}$ of $\Pi_{C_{F}}$ are only well-defined up to $\Pi_{C_{F}}$-conjugacy. That is to say, in this context, the subgroups $\Pi_{\underline{C}_{K}}, \Pi_{\underline{X}_{K}}$ are only well-defined up to automorphisms arising from their normalizers in $\Pi_{C_{F}}$ [cf. the discussion of [IUTchI], Remark 6.12.6, (iii), (iv)]. In particular, in the present context, one is obliged to regard these groups $\Pi_{\underline{C}_{K}}, \Pi_{\underline{X}_{K}}$ as being subject to indeterminacies arising from the natural $\mathbb{F}_{l}^{*}$-poly-actions [i.e., actions by a group that surjects naturally onto $\mathbb{F}_{l}^{*}$ - cf. [IUTchI], Example 4.3, (iv)] on these groups - that is to say, subject to indeterminacies arising from the natural $\mathbb{F}_{l}^{*}$-symmetries of these groups. Here, it is important to note that one cannot simply "form the quotient by the indeterminacy constituted by these $\mathbb{F}_{l}^{*}$-symmetries" since this would give rise to "label-crushing", i.e., to identifying to a single point the distinct labels $j \in \mathbb{F}_{l}^{*}$, which play a crucial role in the construction of the Gaussian monoids [cf. the discussion of [IUTchI], Remark 4.7.1]. But then the $\mathbb{F}_{l}^{*}$-symmetries of $\Pi_{\underline{C}_{K}}$, $\Pi_{\underline{X}_{K}}$ that one must contend with necessarily involve conjugation by elements of the absolute Galois groups of the global base fields involved, hence are fundamentally incompatible with the establishment of conjugate synchronization [cf. the discussion of Remark 4.7.2]. That is to say, just as it is necessary to

(a) isolate the $\mathbb{F}_{l}^{\rtimes \pm}$-symmetry from the $\mathbb{F}_{l}^{*}$-symmetry in order to establish conjugate synchronization [cf. the discussion of Remark 4.7.2],

it is also necessary to

(b) isolate the $\mathbb{F}_{l}^{*}$-symmetry from the $\mathbb{F}_{l}^{\rtimes \pm}$-symmetry in order to work with Galois-theoretic representations of the global base field $F_{\bmod }$.

Indeed, in this context, it is useful to recall that one of the fundamental themes of the theory of the present series of papers consists precisely of the dismantling of the two [a priori intertwined!] combinatorial dimensions of a ring [cf. Remark 4.7.5; [AbsTopIII], §I3].

Remark 4.7.7. The theory of "tempered versus profinite conjugates" developed in [IUTchI], $\S 2$, is applied in the proof of Corollary 2.4, (i), in a setting which ultimately [cf. Remark 2.6.2, (i); Corollary 4.5, (iii)] is seen to amount to a certain local portion $\left[\right.$ at $\left.\underline{v} \in \underline{\mathbb{V}}^{\text {bad }}\right]$ of a $[\mathcal{D}$ - $] \Theta^{ \pm \text {ell }}$-Hodge theater - i.e., a setting in which one considers the $\mathbb{F}_{l}^{\rtimes \pm}$-symmetry. On the other hand, in [IUTchI], Remark 4.5.1, (iii), a discussion is given in which this theory of "tempered versus profinite conjugates" developed in [IUTchI], $\S 2$, is applied in a setting which constitutes a certain local portion $\left[\right.$ at $\left.\underline{v} \in \underline{\mathbb{V}}^{\text {bad }}\right]$ of a $[\mathcal{D}-] \Theta N F-H o d g e$ theater. In this context, it is useful to note that the point of view of this discussion given in [IUTchI], Remark 4.5.1, (iii), may be regarded as "implicit" in the point of view of the theory of the present $\S 4$ in the following sense: The profinite conjugacy indeterminacies that occur in an $[\mathcal{D}$-] $\Theta N F-H o d g e$ theater [cf. [IUTchI], Remark 4.5.1, (iii)] are linked via the gluing operation discussed in [IUTchI], Remark 6.12.2, (i), (ii) — cf. 
Corollaries 4.6, (iv); 4.7 - to the profinite conjugacy indeterminacies that occur in an $[\mathcal{D}-] \Theta^{ \pm \text {ell }}$-Hodge theater [cf. Remarks 2.5.2, (ii), (iii); 2.6.2, (i); 4.5.3, (iii)], i.e., to the profinite conjugacy indeterminacies that are "resolved" in the proof of Corollary 2.4, (i), by applying the theory of [IUTchI], $§ 2$.

Corollary 4.8. (Frobenioid-theoretic Monoids Associated to $\Theta N F-$ Hodge Theaters) Let

$$
{ }^{\dagger} \mathcal{H} \mathcal{T}^{\Theta N F}=\left({ }^{\dagger} \mathcal{F}^{\circledast} \quad \leftarrow--\quad \dagger \mathcal{F}^{\odot} \stackrel{{ }^{\dagger} \psi_{*}^{\mathrm{NF}}}{\longleftarrow} \quad \dagger \mathfrak{F}_{J} \stackrel{{ }^{\dagger} \psi_{*}^{\Theta}}{\longleftarrow} \quad \dagger \mathfrak{F}_{>} \quad-\rightarrow \quad \dagger \mathcal{H} \mathcal{T}^{\Theta}\right)
$$

be a $\Theta \mathrm{NF}-\mathrm{Hodge}$ theater [cf. [IUTchI], Definition 5.5, (iii)] which lifts the $\mathcal{D}$ $\Theta \mathrm{NF}-$ Hodge theater ${ }^{\dagger} \mathcal{H} \mathcal{T}^{\mathcal{D}-\Theta \mathrm{NF}}$ of Corollary 4.7 and is glued to the $\Theta^{ \pm \text {ell }}$-Hodge theater ${ }^{\dagger} \mathcal{H} \mathcal{T}^{\Theta^{ \pm e l l}}$ of Corollary 4.6 via the functorial algorithm of [IUTchI], Proposition 6.7 [so $\left.J=T^{*}\right]$ - cf. the discussion of [IUTchI], Remark 6.12.2, (i), (ii).

(i) (Non-realified Global Structures) There is a functorial algorithm in the category ${ }^{\dagger} \mathcal{F}^{\odot}$ [or in the category ${ }^{\dagger} \mathcal{F}^{\circledast}$ ] for constructing [cf. the notation of Corollary 4.7, (i)] a compatible collection of isomorphisms of Frobenioids

$$
\begin{gathered}
{ }^{\dagger} \mathcal{F}^{\odot} \stackrel{\sim}{\rightarrow} \mathcal{F}^{\odot}\left({ }^{\dagger} \mathcal{D}^{\odot}\right) ; \quad{ }^{\dagger} \mathcal{F}^{\circledast} \stackrel{\sim}{\rightarrow} \mathcal{F}^{\circledast}\left({ }^{\dagger} \mathcal{D}^{\odot}\right) \\
{ }^{\dagger} \mathcal{F}_{\text {mod }}^{\odot} \stackrel{\sim}{\rightarrow} \mathcal{F}_{\bmod }^{\odot}\left({ }^{\dagger} \mathcal{D}^{\odot}\right) ; \quad{ }^{\dagger} \mathcal{F}_{\text {mod }}^{\odot \mathbb{R}} \stackrel{\sim}{\rightarrow} \mathcal{F}_{\bmod }^{\odot \mathbb{R}}\left({ }^{\dagger} \mathcal{D}^{\odot}\right)
\end{gathered}
$$

- cf. the discussion of [IUTchI], Example 5.1, (v), (vi). In particular, this functorial algorithm gives rise to natural "Kummer-theoretic" isomorphisms

$$
\begin{aligned}
&\left\{\pi_{1}\left({ }^{\dagger} \mathcal{D}^{\circledast}\right)\right.\left.\curvearrowright{ }^{\dagger} \mathbb{M}^{\odot}\right\} \stackrel{\sim}{\rightarrow}\left\{\pi_{1}\left({ }^{\dagger} \mathcal{D}^{\circledast}\right) \curvearrowright \mathbb{M}^{\odot}\left({ }^{\dagger} \mathcal{D}^{\odot}\right)\right\} \\
&\left\{\pi_{1}\left({ }^{\dagger} \mathcal{D}^{\circledast}\right)\right.\left.\curvearrowright{ }^{\dagger} \overline{\mathbb{M}}^{\odot}\right\} \stackrel{\sim}{\rightarrow}\left\{\pi_{1}\left({ }^{\dagger} \mathcal{D}^{\circledast}\right) \curvearrowright \overline{\mathbb{M}}^{\odot}\left({ }^{\dagger} \mathcal{D}^{\odot}\right)\right\} \\
&{ }^{\dagger} \mathbb{M}_{\text {mod }}^{\odot} \stackrel{\sim}{\rightarrow} \mathbb{M}_{\text {mod }}^{\odot}\left({ }^{\dagger} \mathcal{D}^{\odot}\right) ; \quad{ }^{\dagger} \overline{\mathbb{M}}_{\text {mod }}^{\odot} \stackrel{\sim}{\rightarrow} \overline{\mathbb{M}}_{\text {mod }}^{\odot}\left({ }^{\dagger} \mathcal{D}^{\odot}\right) \\
&{ }^{\dagger} \mathbb{M}_{\text {orb }}^{\odot} \stackrel{\sim}{\rightarrow} \mathbb{M}_{\text {orb }}^{\odot}\left({ }^{\dagger} \mathcal{D}^{\odot}\right) ; \quad{ }^{\dagger} \overline{\mathbb{M}}_{\text {orb }}^{\odot} \stackrel{\sim}{\rightarrow} \overline{\mathbb{M}}_{\text {orb }}^{\odot}\left({ }^{\dagger} \mathcal{D}^{\odot}\right)
\end{aligned}
$$

between the field equipped with a $\pi_{1}\left({ }^{\dagger} \mathcal{D}^{\circledast}\right)$-action [cf. the codomain of the arrow in the second line of the above display] of Corollary 4.7, (i), together with its respective submonoids/subfield/sets of $\pi_{1}\left({ }^{\dagger} \mathcal{D}^{\circledast}\right)$-orbit-collections [i.e., the codomains of the various displayed isomorphisms], and the corresponding objects [i.e., the domains of the various displayed isomorphisms] arising from the Frobenioid structure of ${ }^{\dagger} \mathcal{F}^{\circledast}$ [cf. the discussion of [IUTchI], Example 5.1, (v)].

(ii) (Labels and $\mathbb{F}_{l}^{*}$-Symmetry) In the notation of Corollary 4.7, (ii), the collection of isomorphisms of Corollary 4.6, (i) [applied to the $\mathcal{F}$-prime-strips of the capsule $\left.{ }^{\dagger} \mathfrak{F}_{J}\right]$, together with the isomorphisms of (i) above, determine, for each $j \in \operatorname{LabCusp}\left({ }^{\dagger} \mathcal{D}^{\odot}\right)(\stackrel{\sim}{\rightarrow} J)\left[c f\right.$. the bijection ${ }^{\dagger} \zeta_{*}$ of Corollary 4.7, (ii)], a collection of isomorphisms

$$
\begin{gathered}
\left.{ }^{\dagger} \mathfrak{F}_{j} \stackrel{\sim}{\rightarrow} \mathcal{F}^{\odot}\left({ }^{\dagger} \mathcal{D}^{\odot}\right)\right|_{j} ; \quad\left({ }^{\dagger} \mathcal{F}_{\mathrm{mod}}^{\odot}\right)_{j} \stackrel{\sim}{\rightarrow} \mathcal{F}_{\text {mod }}^{\odot}\left({ }^{\dagger} \mathcal{D}^{\odot}\right)_{j} ; \quad\left({ }^{\dagger} \mathcal{F}_{\bmod }^{\odot \mathbb{R}}\right)_{j} \stackrel{\sim}{\rightarrow} \mathcal{F}_{\bmod }^{\odot \mathbb{R}}\left({ }^{\dagger} \mathcal{D}^{\odot}\right)_{j} \\
\left({ }^{\dagger} \mathbb{M}_{\mathrm{mod}}^{\odot}\right)_{j} \stackrel{\sim}{\rightarrow} \mathbb{M}_{\mathrm{mod}}^{\odot}\left({ }^{\dagger} \mathcal{D}^{\odot}\right)_{j} ; \quad\left({ }^{\dagger} \overline{\mathbb{M}}_{\text {mod }}^{\odot}\right)_{j} \stackrel{\sim}{\rightarrow} \overline{\mathbb{M}}_{\text {mod }}^{\odot}\left({ }^{\dagger} \mathcal{D}^{\odot}\right)_{j}
\end{gathered}
$$




$$
\left({ }^{\dagger} \mathbb{M}_{\text {orb }}^{\odot}\right)_{j} \stackrel{\sim}{\rightarrow} \mathbb{M}_{\text {orb }}^{\odot}\left({ }^{\dagger} \mathcal{D}^{\odot}\right)_{j} ; \quad\left({ }^{\dagger} \overline{\mathbb{M}}_{\text {orb }}^{\odot}\right)_{j} \stackrel{\sim}{\rightarrow} \overline{\mathbb{M}}_{\text {orb }}^{\odot}\left({ }^{\dagger} \mathcal{D}^{\odot}\right)_{j}
$$

as well as $\left[\mathbb{F}_{l}^{*}-\right]$ symmetrizing isomorphisms, induced by the natural poly-action of $\mathbb{F}_{l}^{*}$ on ${ }^{\dagger} \mathcal{F}^{\odot}$ [cf. [IUTchI], Example 4.3, (iv); [IUTchI], Corollary 5.3, (i)], between the data indexed by distinct $j \in \operatorname{LabCusp}\left({ }^{\dagger} \mathcal{D}^{\odot}\right)$. Moreover, these symmetrizing isomorphisms are compatible, relative to ${ }^{\dagger} \zeta_{*}[c f$. Corollary 4.7, (ii)], with the $\mathbb{F}_{l}^{*}$-symmetry of the associated NF-bridge [cf. [IUTchI], Proposition 4.9, (i)] and determine various diagonal $\mathcal{F}$-prime-strips/submonoids/subfields/sets of subsets/subcategories [cf. Remark 4.7.2]

$$
(-)_{\left\langle\mathbb{F}_{l}^{*}\right\rangle} \subseteq \prod_{j \in \mathbb{F}_{i}^{*}}(-)_{j}
$$

- cf. the discussion of Corollary 4.7, (ii).

(iii) (Localization Functors and Realified Global Structures) Let $j \in$ LabCusp $\left({ }^{\dagger} \mathcal{D}^{\odot}\right)$. Then there is a functorial algorithm in the NF-bridge $\left({ }^{\dagger} \mathfrak{F}_{J} \rightarrow\right.$ $\left.\dagger \mathcal{F}^{\odot}-\rightarrow \dagger \mathcal{F}^{\circledast}\right)$ for constructing mutually [1-]compatible collections of "localization" functors [up to isomorphism]

$$
\left({ }^{\dagger} \mathcal{F}_{\bmod }^{\odot}\right)_{j} \rightarrow{ }^{\dagger} \mathfrak{F}_{j} ; \quad\left({ }^{\dagger} \mathcal{F}_{\text {mod }}^{\odot \mathbb{R}}\right)_{j} \quad \rightarrow \quad{ }^{\dagger} \mathfrak{F}_{j}^{\mathbb{R}}
$$

as in the discussion of [IUTchI], Example 5.4, (vi) - which are compatible, relative to the various ["Kummer-theoretic"] isomorphisms of (i), (ii), with the collections of functors of Corollary 4.7, (iii) - together with a natural isomorphism of Frobenioids

$$
{ }^{\dagger} \mathcal{C}_{j}^{\Vdash} \quad \stackrel{\sim}{\rightarrow} \quad\left({ }^{\dagger} \mathcal{F}_{\bmod }^{\odot \mathbb{R}}\right)_{j}
$$

[cf. the notation of Corollary 4.6, (ii); [IUTchI], Remark 5.2.1, (ii), applied to the $\mathcal{F}$-prime-strip ${ }^{\dagger} \mathfrak{F}_{j}$; Remark 4.8 .3 below] which is compatible with the respective bijections involving "Prime $(-)$ ", the respective local isomorphisms of topological monoids [cf. the arrow $\left({ }^{\dagger} \mathcal{F}_{\bmod }^{\odot \mathbb{R}}\right)_{j} \rightarrow{ }^{\dagger} \mathfrak{F}_{j}^{\mathbb{R}}$ discussed above; [IUTchI], Remark 5.2.1, (ii)], the isomorphisms of Corollary 4.7, (iii), and the various ["Kummertheoretic"] isomorphisms of (i), (ii) [cf. also Corollary 4.6, (ii)]. Finally, all of these structures are compatible with the respective $\mathbb{F}_{l}^{*}$-symmetrizing isomorphisms [cf. (ii)].

Proof. The various assertions of Corollary 4.8 follow immediately from the definitions and the references quoted in the statements of these assertions.

\section{Remark 4.8.1.}

(i) The Frobenioid $\mathcal{C}_{\text {gau }}^{\Vdash}\left({ }^{\dagger} \mathcal{H} \mathcal{T}^{\Theta}\right)$ of Corollary 4.6, (v), is constructed as a subcategory of a product over $j \in \mathbb{F}_{i}^{*}$ of copies ${ }^{\dagger} \mathcal{C}_{j}^{\Vdash}$ of the category ${ }^{\dagger} \mathcal{C}^{\Vdash}$. In particular, one may apply the isomorphism ${ }^{\dagger} \mathcal{C}_{j}^{\Vdash} \stackrel{\sim}{\rightarrow}\left({ }^{\dagger} \mathcal{F}_{\bmod }^{\odot \mathbb{R}}\right)_{j}$ of Corollary 4.8 , (iii), to regard this Frobenioid $\mathcal{C}_{\text {gau }}^{\Vdash}\left({ }^{\dagger} \mathcal{H} \mathcal{T}^{\Theta}\right)$ as a subcategory

$$
\mathcal{C}_{\mathrm{gau}}^{\Vdash}\left({ }^{\dagger} \mathcal{H} \mathcal{T}^{\Theta}\right) \hookrightarrow \prod_{j \in \mathbb{F}_{l}^{*}}\left({ }^{\dagger} \mathcal{F}_{\bmod }^{\odot \mathbb{R}}\right)_{j}
$$


of the product over $j \in \mathbb{F}_{l}^{*}$ of the $\left({ }^{\dagger} \mathcal{F}_{\bmod }^{\odot \mathbb{R}}\right)_{j}$.

(ii) In a similar vein, the local data at $\underline{v} \in \underline{\mathbb{V}}$ of the objects $\Psi_{\mathcal{F}_{\text {gau }}}\left({ }^{\dagger} \mathcal{H} \mathcal{T}^{\Theta}\right)$ constructed in Corollary 4.6, (iv), give rise to [the local data at $\underline{v}$ of a $\mathcal{F}^{\vdash}$-primestrip, i.e., in particular, to] split Frobenioids $\mathcal{F}_{\text {gau }}\left({ }^{\dagger} \mathcal{H} \mathcal{T}^{\Theta}\right)_{\underline{v}}$ [cf. Definition 3.8, (ii), in the case of $\left.\underline{v} \in \underline{\mathbb{V}}^{\text {bad }}\right]$. Write $\mathcal{F}_{\text {gau }}\left({ }^{\dagger} \mathcal{H} \mathcal{T}^{\Theta}\right)$ for the $\mathcal{F}^{\vdash}$-prime-strip determined by this local data $\mathcal{F}_{\text {gau }}\left({ }^{\dagger} \mathcal{H} \mathcal{T}^{\Theta}\right)_{\underline{v}}$ at $\underline{v}$, for $\underline{v} \in \underline{\mathbb{V}}$, and

$$
\mathcal{F}_{\text {gau }}\left({ }^{\dagger} \mathcal{H} \mathcal{T}^{\Theta}\right)^{\mathbb{R}}
$$

for the object obtained by forming, at each $\underline{v} \in \underline{\mathbb{V}}$, the realification of the underlying Frobenioid of $\mathcal{F}_{\text {gau }}\left({ }^{\dagger} \mathcal{H} \mathcal{T}^{\Theta}\right)$ at $\underline{v}$. Then it follows from the construction discussed in Corollary 4.6, (iv), that one may think of the realified Frobenioid, at each $\underline{v} \in \underline{\mathbb{V}}$, of $\mathcal{F}_{\text {gau }}\left({ }^{\dagger} \mathcal{H} \mathcal{T}^{\Theta}\right)^{\mathbb{R}}$ as being naturally embedded

$$
\mathcal{F}_{\text {gau }}\left({ }^{\dagger} \mathcal{H} \mathcal{T}^{\Theta}\right)^{\mathbb{R}} \hookrightarrow \prod_{j \in \mathbb{F}_{l}^{*}}\left({ }^{\dagger} \mathfrak{F}_{>}^{\mathbb{R}}\right)_{j}
$$

[where we use this notation to denote the collection of embeddings indexed by $\underline{v} \in \mathbb{V}$ ] in the product of copies of realifications of [the underlying Frobenioids of] the $\mathcal{F}$-prime-strip ${ }^{\dagger} \mathfrak{F}_{>}$labeled by $j \in \mathbb{F}_{l}^{*}$. Moreover, by applying the full polyisomorphisms $\left({ }^{\dagger} \mathfrak{F}_{>}\right)_{j} \stackrel{\sim}{\rightarrow}{ }^{\dagger} \mathfrak{F}_{j}$ — which are tautologically compatible with the labels $j \in \mathbb{F}_{l}^{*}$ ! - we may think of $\mathcal{F}_{\text {gau }}\left({ }^{\dagger} \mathcal{H} \mathcal{T}^{\Theta}\right)^{\mathbb{R}}$ as being naturally embedded

$$
\mathcal{F}_{\text {gau }}\left({ }^{\dagger} \mathcal{H} \mathcal{T}^{\Theta}\right)^{\mathbb{R}} \hookrightarrow \prod_{j \in \mathbb{F}_{l}^{*}}{ }^{\dagger} \mathfrak{F}_{j}^{\mathbb{R}}
$$

[where we use this notation to denote the collection of embeddings indexed by $\underline{v} \in \mathbb{V}$ ] in the product associated to the realifications of [the underlying Frobenioids of] the $\mathcal{F}$-prime-strips ${ }^{\dagger} \mathfrak{F}_{j}$.

(iii) Thus, by applying the various embeddings considered in (i), (ii), one may think of the "realified localization" functors

$$
\left({ }^{\dagger} \mathcal{F}_{\bmod }^{\odot \mathbb{R}}\right)_{j} \rightarrow \dagger \mathfrak{F}_{j}^{\mathbb{R}}
$$

of Corollary 4.8, (iii), as inducing a "realified localization" functor [up to isomorphism]

$$
\mathcal{C}_{\text {gau }}^{\Vdash}\left({ }^{\dagger} \mathcal{H} \mathcal{T}^{\Theta}\right) \rightarrow \mathcal{F}_{\text {gau }}\left({ }^{\dagger} \mathcal{H} \mathcal{T}^{\Theta}\right)^{\mathbb{R}}
$$

- which [as one verifies immediately] is compatible [cf. the various compatibilities discussed in Corollary 4.8, (iii)] with the realified localization isomorphisms $\Phi_{\mathcal{C}_{\text {gau }}\left(\dagger \mathcal{H} \mathcal{T}^{\Theta}\right), \underline{v}} \stackrel{\sim}{\rightarrow} \Psi_{\mathcal{F}_{\text {gau }}}\left({ }^{\dagger} \mathcal{H} \mathcal{T}^{\Theta}\right)_{\underline{v}}^{\mathbb{R}}$, for $\underline{v} \in \underline{\mathbb{V}}$, considered in Corollary 4.6, (v).

\section{Remark 4.8.2.}

(i) The realified localization functor discussed in Remark 4.8.1, (iii), only concerns the realification of the Frobenioid-theoretic version $\mathcal{F}_{\text {gau }}\left({ }^{\dagger} \mathcal{H} \mathcal{T}^{\Theta}\right)$ of the Gaussian monoids. The unit portion of the Gaussian monoids will be used, in 
the context of the theory involving the log-wall that will be developed in [IUTchIII], not in its capacity as a "multiplicative object", but rather - i.e., by applying the operation "log" to the units at the various $\underline{v} \in \underline{\mathbb{V}}$, as in the theory of [AbsTopIII] - as an "additive object". In this theory, the non-realified global Frobenioids of Corollary 4.8, (i), will appear in the context of localization functors/morphisms i.e., as a sort of translation apparatus between $\nabla$ - and $\boxplus$-line bundles [cf. the discussion of Remark 4.7.2] — that relate these [multiplicative!] non-realified global Frobenioids to the [additive!] images via "log" of the units. Note that this sort of construction - i.e., in which the localization operations involving units and value groups differ by a shift via the operation "log" — depends, in an essential way [cf. the discussion of Remark 1.12.2, (iv)], on the natural splittings with which the Gaussian monoids are equipped [cf. Corollary 4.6, (iv)].

(ii) In the context of (i), it is useful to observe that, although the non-realified global Frobenioids of Corollary 4.8, (i), may only be considered in the context of the $\mathbb{F}_{l}^{*}$-symmetry [cf. the discussion of Remark 4.7.6], this does not yield any obstacles, relative to the discussion in (i) of Gaussian monoids, since Gaussian monoids are most naturally considered as "functions" of a parameter $j \in \mathbb{F}_{i}^{*}$ [cf. the discussion of Remark 4.7.3, (iii)].

(iii) From the point of view of the analogy of the theory of the present series of papers with $p$-adic Teichmüller theory [cf. the discussion of [AbsTopIII], §I5], it is of interest to note that the construction discussed in (i) involving the use of the natural splittings of Gaussian monoids to consider "log-shifted units" together with "non-log-shifted value groups" may be thought of as corresponding to the situation that frequently occurs in $p$-adic Teichmüller theory in which an indigenous bundle $\left(\mathcal{E}, \nabla_{\mathcal{E}}\right)$ equipped with a Hodge filtration $0 \rightarrow \omega \rightarrow \mathcal{E} \rightarrow \tau \rightarrow 0$ on a hyperbolic curve in positive characteristic is represented, in the context of local Frobenius liftings modulo higher powers of $p$, as a direct sum

$$
\Phi^{*} \tau \oplus \omega
$$

- where $\Phi$ denotes the Frobenius morphism on the curve, which, as may be recalled from the discussion of [AbsTopIII], §I5, corresponds, relative to the analogy under consideration, to the operation "log" studied in [AbsTopIII].

Remark 4.8.3. Similar observations to the observations made in Remark 4.5.2, (i), (ii), concerning the $\mathbb{F}_{l}^{\rtimes \pm}$-symmetrizing isomorphisms of Corollary 4.5, (iii), may be made in the case of the $\mathbb{F}_{l}^{*}$-symmetrizing isomorphisms of Corollary 4.8, (ii).

\section{Definition 4.9.}

(i) Let $\mathcal{C}$ be an arbitrary Frobenioid. Write $\mathcal{D}$ for the base category of $\mathcal{C}$. Suppose that $\mathcal{D}$ is isomorphic to the category of connected finite étale coverings of the spectrum of an $M L F$ or a $C A F$. Let $A$ be a "universal covering pro-object" of $\mathcal{D}$ [cf. the discussion of Example 3.2, (i), (ii)]. Write $G \stackrel{\text { def }}{=} \operatorname{Aut}(A)$ [so $G$ is isomorphic to the absolute Galois group of an MLF or a CAF]. Now by evaluating the monoid " $\mathrm{O}^{\triangleright}(-)$ " on $\mathcal{D}$ that arises from the general theory of Frobenioids [cf. 
[FrdI], Proposition 2.2] at $A$, we thus obtain a monoid [in the usual sense] equipped with a natural action by $G$

$$
G \curvearrowright \mathcal{O}^{\triangleright}(A)
$$

[cf. the discussion of Example 3.2, (ii)]. If $N$ is a positive integer, then we shall write

$$
\boldsymbol{\mu}_{N}(A) \subseteq \mathcal{O}^{\boldsymbol{\mu}}(A) \subseteq \mathcal{O}^{\times}(A)
$$

for the subgroups of $N$-torsion elements [cf. [FrdII], Definition 2.1, (i)] and torsion elements of arbitrary order;

$$
\mathcal{O}^{\times}(A) \quad \rightarrow \quad \mathcal{O}^{\times \boldsymbol{\mu}}(A) \quad \rightarrow \quad \mathcal{O}^{\times \boldsymbol{\mu}_{N}}(A)
$$

for the respective quotients of the submonoid of units $\mathcal{O}^{\times}(A) \subseteq \mathcal{O}^{\triangleright}(A)$ by $\mathcal{O}^{\boldsymbol{\mu}}(A)$, $\boldsymbol{\mu}_{N}(A)$. Thus, $\mathcal{O}^{\triangleright}(A), \mathcal{O}^{\times}(A), \mathcal{O}^{\times \boldsymbol{\mu}}(A), \mathcal{O}^{\times \boldsymbol{\mu}_{N}}(A), \mathcal{O}^{\boldsymbol{\mu}}(A)$, and $\boldsymbol{\mu}_{N}(A)$ are all equipped with natural $G$-actions. Next, let us suppose that $G$ is nontrivial [i.e., arises from an MLF]. Recall the group-theoretic algorithms " $G \mapsto\left(G \curvearrowright \mathcal{O}^{\times}(G)\right)$ " and " $G \mapsto\left(G \curvearrowright \mathcal{O}^{\times \boldsymbol{\mu}}(G)\right)$ " discussed in Example 1.8, (iii), (iv). We define a $\times$-Kummer structure (respectively, $\times \boldsymbol{\mu}$-Kummer structure) on $\mathcal{C}$ to be a $\widehat{\mathbb{Z}}^{\times}$(respectively, Ism- [cf. Example 1.8, (iv)]) orbit of isomorphisms

$$
\left.\mathcal{O}^{\times}(G) \stackrel{\sim}{\rightarrow} \mathcal{O}^{\times}(A) \quad \text { (respectively, } \quad \mathcal{O}^{\times \boldsymbol{\mu}}(G) \stackrel{\sim}{\rightarrow} \mathcal{O}^{\times \boldsymbol{\mu}}(A)\right)
$$

of topological $G$-modules. Note that since any two "universal covering pro-objects" of $\mathcal{D}$ are isomorphic, it follows immediately that the definition of a $\times$ - (respectively, $\times \boldsymbol{\mu}$-) Kummer structure is independent of the choice of $A$. Next, let us recall from Remark 1.11.1, (b), that

\section{any $\times$-Kummer structure on $\mathcal{C}$ is unique.}

In the case of $\times \boldsymbol{\mu}$-Kummer structures, let us observe that a $\times \boldsymbol{\mu}$-Kummer structure $\kappa^{\times \mu}$ on $\mathcal{C}$ determines, for each open subgroup $H \subseteq G$, a submodule

$$
\mathcal{I}_{H}^{\kappa}(A) \stackrel{\text { def }}{=} \operatorname{Im}\left(\mathcal{O}^{\times}(G)^{H}\right) \subseteq \mathcal{O}^{\times \boldsymbol{\mu}}(A)
$$

- namely, the image via $\kappa^{\times \boldsymbol{\mu}}$ of the image of $\mathcal{O}^{\times}(G)^{H}$ in $\mathcal{O}^{\times \boldsymbol{\mu}}(G)^{H}$ [where the superscript " $H$ 's" denote the submodules of $H$-invariants]. Conversely, it is essentially a tautology [cf. the definition of "Ism" given in Example 1.8, (iv)!] that the $\times \boldsymbol{\mu}$-Kummer structure $\kappa^{\times \boldsymbol{\mu}}$ on $\mathcal{C}$ is completely determined by the submodules $\left\{\mathcal{I}_{H}^{\kappa}(A) \subseteq \mathcal{O}^{\times \boldsymbol{\mu}}(A)\right\}_{H}$ [where $H$ ranges over the open subgroups of $G$ ], namely, as the unique Ism-orbit of $G$-equivariant isomorphisms $\mathcal{O}^{\times \boldsymbol{\mu}}(G) \stackrel{\sim}{\rightarrow} \mathcal{O}^{\times \boldsymbol{\mu}}(A)$ that maps $\mathcal{O}^{\times}(G)^{H}$ onto $\mathcal{I}_{H}^{\kappa}(A)$ for each open subgroup $H \subseteq G$. That is to say,

a $\times \boldsymbol{\mu}$-Kummer structure $\kappa^{\times \boldsymbol{\mu}}$ on $\mathcal{C}$ may be thought of as - i.e., in the sense that it determines and is uniquely determined by - the collection of submodules $\left\{\mathcal{I}_{H}^{\kappa}(A) \subseteq \mathcal{O}^{\times \boldsymbol{\mu}}(A)\right\}_{H}$ [where $H$ ranges over the open subgroups of $G]$.

Finally, we shall refer to as a $[\times-, \times \boldsymbol{\mu}$ - $]$ Kummer Frobenioid any Frobenioid equipped with a $\left[\times-, \times \boldsymbol{\mu}^{-}\right]$Kummer structure. We shall refer to as a split- $[\times-, \times \boldsymbol{\mu}$-]Kummer Frobenioid any split Frobenioid equipped with a $[\times-, \times \boldsymbol{\mu}$ - $]$ Kummer structure. 
(ii) Let

$$
{ }^{\ddagger} \mathfrak{F}^{\vdash}=\left\{\mathcal{F}_{\underline{v}}^{\vdash}\right\}_{\underline{v} \in \underline{\mathbb{V}}}
$$

be an $\mathcal{F}^{\vdash}$-prime-strip; $\underline{w} \in \underline{\mathbb{V}}^{\text {bad }}$. Write $\ddagger^{\ddagger} \mathfrak{D}^{\vdash}=\left\{{ }^{\ddagger} \mathcal{D}_{v}^{\vdash}\right\}_{\underline{v} \in \underline{\mathbb{V}}}$ for the $\mathcal{D}^{\vdash}$-primestrip associated to ${ }^{\ddagger} \mathfrak{F}^{\vdash}$ [cf. [IUTchI], Remark 5.2.1, (i)]. Thus, ${ }^{\ddagger} \mathcal{F}_{\underline{w}}^{\vdash}$ is a split Frobenioid [cf. [IUTchI], Definition 5.2, (ii), (a); [IUTchI], Example 3.2, (v)], with base category ${ }^{\ddagger} \mathcal{D}_{\underline{w}}^{\vdash}$. Let $\ddagger A$ be a "universal covering pro-object" of $\ddagger \mathcal{D}_{\underline{w}}^{\vdash}[$ cf. the discussion of (i)]. Write ${ }^{\ddagger} G \stackrel{\text { def }}{=} \operatorname{Aut}\left({ }^{\ddagger} A\right)$ [so ${ }^{\ddagger} G$ is a profinite group isomorphic to $\left.G_{\underline{w}}\right]$. Then the 2 -torsion subgroup $\boldsymbol{\mu}_{2 l}\left({ }^{\ddagger} A\right) \subseteq \mathcal{O}^{\times}\left({ }^{\ddagger} A\right)$ of the submonoid of units $\mathcal{O}^{\overline{\times}}\left({ }^{\ddagger} A\right) \subseteq \mathcal{O}^{\triangleright}\left({ }^{\ddagger} A\right)$ of $\mathcal{O}^{\triangleright}\left({ }^{\ddagger} A\right)$, together with the images of the splittings with which $\ddagger \mathcal{F}_{\underline{w}}^{\vdash}$ is equipped, generate a submonoid $\mathcal{O}^{\perp}\left({ }^{\ddagger} A\right) \subseteq \mathcal{O}^{\triangleright}\left({ }^{\ddagger} A\right)$, whose quotient by $\boldsymbol{\mu}_{2 l}\left({ }^{\ddagger} A\right)$ we denote by

$$
\mathcal{O}^{\triangleright}\left({ }^{\ddagger} A\right) \quad \supseteq \quad \mathcal{O}^{\perp}\left({ }^{\ddagger} A\right) \quad \rightarrow \quad \mathcal{O}^{\triangleright}\left({ }^{\ddagger} A\right) \quad \stackrel{\text { def }}{=} \quad \mathcal{O}^{\perp}\left({ }^{\ddagger} A\right) / \boldsymbol{\mu}_{2 l}\left({ }^{\ddagger} A\right)
$$

[so we have a natural isomorphism $\left.\mathcal{O}^{\triangleright}\left({ }^{\ddagger} A\right) / \mathcal{O}^{\times}\left({ }^{\ddagger} A\right) \stackrel{\sim}{\rightarrow} \mathcal{O} \triangleright\left({ }^{\ddagger} A\right)\right]$. Write

$$
\mathcal{O} \triangleright \times \boldsymbol{\mu}\left({ }^{\ddagger} A\right) \quad \stackrel{\text { def }}{=} \quad \mathcal{O} \triangleright\left({ }^{\ddagger} A\right) \times \mathcal{O}^{\times \boldsymbol{\mu}}\left({ }^{\ddagger} A\right)
$$

for the direct product monoid. Thus, $\mathcal{O}^{\triangleright}\left({ }^{\ddagger} A\right), \mathcal{O}^{\perp}\left({ }^{\ddagger} A\right), \mathcal{O}^{\triangleright}\left({ }^{\ddagger} A\right), \mathcal{O}^{\times}\left({ }^{\ddagger} A\right), \mathcal{O}^{\times \mu}\left({ }^{\ddagger} A\right)$, $\mathcal{O}^{\boldsymbol{\mu}}\left({ }^{\ddagger} A\right)$, and $\mathcal{O}^{\triangleright \times \boldsymbol{\mu}\left({ }^{\ddagger} A\right)}$ are all equipped with natural ${ }^{\ddagger} G$-actions. Next, we consider the group-theoretic algorithms " $G \mapsto\left(G \curvearrowright \mathcal{O}^{\times}(G)\right)$ " and " $G \mapsto\left(G \curvearrowright \mathcal{O}^{\times \boldsymbol{\mu}}(G)\right)$ " discussed in Example 1.8, (iii), (iv). If we apply the first of these algorithms to ${ }^{\ddagger} G$, then it follows from Remark 1.11.1, (b), that there exists a unique $\widehat{\mathbb{Z}}^{\times}$-orbit of isomorphisms

$$
{ }^{\ddagger} \kappa_{\underline{w}}^{\vdash \times}: \quad \mathcal{O}^{\times}\left({ }^{\ddagger} G\right) \stackrel{\sim}{\rightarrow} \quad \mathcal{O}^{\times}\left({ }^{\ddagger} A\right)
$$

of topological modules equipped with ${ }^{\ddagger} G$-actions. Moreover, ${ }^{\ddagger} \kappa_{\underline{w}}^{\vdash \times}$ induces an Ismorbit [cf. Example 1.8, (iv)] of isomorphisms

$$
{ }^{\ddagger} \kappa_{\underline{w}}^{\vdash \times \mu}: \quad \mathcal{O}^{\times \mu}\left({ }^{\ddagger} G\right) \quad \stackrel{\sim}{\rightarrow} \quad \mathcal{O}^{\times \mu}\left({ }^{\ddagger} A\right)
$$

— i.e., by forming the quotient by "Oํ $\mathrm{O}^{\mu}(-)$ ".

(iii) In the notation of (ii), the [rational function monoid determined by the groupification of the] monoid with ${ }^{\ddagger} G$-action $\mathcal{O} \triangleright \times \boldsymbol{\mu}\left({ }^{\ddagger} A\right)$, together with the divisor monoid of [the underlying Frobenioid of] ${ }^{\ddagger} \mathcal{F}_{\underline{w}}^{\leftarrow}$, determines a "model Frobenioid" [cf. [FrdI], Theorem 5.2, (ii)] equipped with a splitting, i.e., the splitting arising from the definition of $\mathcal{O} \triangleright \times \boldsymbol{\mu}\left({ }^{\ddagger} A\right)$ as a direct product. Thus, the ${ }^{\ddagger} G$-module obtained by evaluating at $\ddagger A$ the group of units " $\mathcal{O}^{\times}(-)$" (respectively, the monoid " $\mathcal{O}^{\triangleright}(-)$ ") associated to this Frobenioid may be naturally identified with $\mathcal{O}^{\times \boldsymbol{\mu}}\left({ }^{\ddagger} A\right)$ (respectively, $\left.\mathcal{O} \triangleright \times \boldsymbol{\mu}\left(\ddagger^{\ddagger} A\right)\right)$. In particular, the Ism-orbit of isomorphisms $\kappa_{\underline{w}}^{\vdash} \times \boldsymbol{\mu}$ determines a $\times \boldsymbol{\mu}$-Kummer structure on this Frobenioid. We shall write

$$
\ddagger \mathcal{F}_{\underline{w}}^{\vdash \triangleright \times \mu}
$$

for the resulting split-Kummer Frobenioid and — by abuse of notation! -

$$
{ }^{\ddagger} \mathcal{F}_{\underline{w}}^{\vdash}
$$


for the split-Kummer Frobenioid determined by the split Frobenioid ${ }^{\ddagger} \mathcal{F}_{\underline{w}}^{\vdash}$ equipped with the $\times$-Kummer structure determined by ${ }^{\ddagger} \kappa_{\underline{w}}^{\vdash \times}$. Here, we remark that the primary justification for this abuse of notation lies in the uniqueness of $\times$-Kummer structures discussed in (i) above.

(iv) Let ${ }^{\ddagger} \mathfrak{F}^{\vdash}$ be as in (ii); $\underline{w} \in \underline{\mathbb{V}}^{\text {good }} \cap \underline{\mathbb{V}}^{\text {non }}$. Thus, ${ }^{\ddagger} \mathcal{F}_{\underline{w}}^{\vdash}$ is a split Frobenioid [cf. [IUTchI], Definition 5.2, (ii), (a); [IUTchI], Example 3.3, (i], with base category ${ }^{\ddagger} \mathcal{D}_{\underline{w}}^{\vdash}$. Let $\ddagger A$ be a "universal covering pro-object" of $\ddagger \mathcal{D}_{\underline{w}}^{\vdash}[$ cf. the discussion of (i)]. Write ${ }^{\ddagger} G \stackrel{\text { def }}{=} \operatorname{Aut}\left({ }^{\ddagger} A\right)\left[\right.$ so ${ }^{\ddagger} G$ is a profinite group isomorphic to $\left.G_{\underline{w}}\right]$. Then the image of the splitting with which $\ddagger \mathcal{F}_{\underline{w}}^{\vdash}$ is equipped determines a submonoid $\mathcal{O}^{\perp}\left({ }^{\ddagger} A\right) \subseteq \mathcal{O}^{\triangleright}\left({ }^{\ddagger} A\right)$. Write $\mathcal{O} \triangleright\left({ }^{\ddagger} A\right) \stackrel{\text { def }}{=} \mathcal{O}^{\perp}\left({ }^{\ddagger} A\right)$,

$$
\mathcal{O} \triangleright \times \boldsymbol{\mu}\left({ }^{\ddagger} A\right) \quad \stackrel{\text { def }}{=} \quad \mathcal{O} \triangleright\left({ }^{\ddagger} A\right) \times \mathcal{O}^{\times \boldsymbol{\mu}}\left({ }^{\ddagger} A\right)
$$

for the direct product monoid. Thus, the monoids $\mathcal{O}^{\triangleright}\left({ }^{\ddagger} A\right), \mathcal{O}^{\perp}\left({ }^{\ddagger} A\right), \mathcal{O}^{\triangleright}\left({ }^{\ddagger} A\right)$, $\mathcal{O}^{\times}\left({ }^{\ddagger} A\right), \mathcal{O}^{\times \boldsymbol{\mu}}\left({ }^{\ddagger} A\right), \mathcal{O}^{\boldsymbol{\mu}}\left({ }^{\ddagger} A\right)$, and $\mathcal{O}^{\triangleright \times \boldsymbol{\mu}\left({ }^{\ddagger} A\right)}$ are all equipped with natural ${ }^{\ddagger} G$ actions. Next, we consider the group-theoretic algorithms " $G \mapsto\left(G \curvearrowright \mathcal{O}^{\times}(G)\right)$ " and " $G \mapsto\left(G \curvearrowright \mathcal{O}^{\times \boldsymbol{\mu}}(G)\right)$ " discussed in Example 1.8, (iii), (iv). If we apply the first of these algorithms to ${ }^{\ddagger} G$, then it follows from Remark 1.11.1, (b), that there exists a unique $\widehat{\mathbb{Z}}^{\times}$-orbit of isomorphisms

$$
{ }^{\ddagger} \kappa_{\underline{w}}^{\vdash \times}: \quad \mathcal{O}^{\times}\left({ }^{\ddagger} G\right) \stackrel{\sim}{\rightarrow} \quad \mathcal{O}^{\times}\left({ }^{\ddagger} A\right)
$$

of topological modules equipped with ${ }^{\ddagger} G$-actions. Moreover, ${ }^{\ddagger} \kappa_{\underline{w}}^{\vdash \times}$ induces an Ismorbit [cf. Example 1.8, (iv)] of isomorphisms

$$
{ }_{\underline{w}}^{\ddagger} \kappa^{\vdash \mu}: \quad \mathcal{O}^{\times \mu}\left({ }^{\ddagger} G\right) \quad \stackrel{\sim}{\rightarrow} \quad \mathcal{O}^{\times \mu}\left({ }^{\ddagger} A\right)
$$

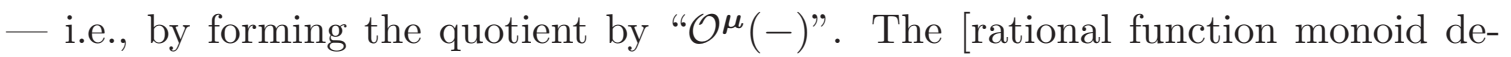
termined by the groupification of the] monoid with ${ }^{\ddagger} G$-action $\mathcal{O}^{\vee} \times \boldsymbol{\mu}\left({ }^{\ddagger} A\right)$, together with the divisor monoid of [the underlying Frobenioid of] ${ }^{\ddagger} \mathcal{F}_{\underline{w}}^{\vdash}$, determines a "model Frobenioid" [cf. [FrdI], Theorem 5.2, (ii)] equipped with a splitting, i.e., the splitting arising from the definition of $\mathcal{O} \triangleright \times \boldsymbol{\mu}\left({ }^{\ddagger} A\right)$ as a direct product. Thus, the ${ }^{\ddagger} G$-module obtained by evaluating at $\ddagger A$ the group of units " $\mathcal{O}^{\times}(-)$" (respectively, the monoid "O⿱㇒冋 ${ }^{\triangleright}(-)$ ") associated to this Frobenioid may be naturally identified with $\mathcal{O}^{\times \boldsymbol{\mu}}\left({ }^{\ddagger} A\right)$ (respectively, $\mathcal{O}^{\vee} \times \boldsymbol{\mu}\left({ }^{\ddagger} A\right)$ ). In particular, the Ism-orbit of isomorphisms ${ }^{\ddagger} \kappa_{\underline{w}}^{\vdash \times \boldsymbol{\mu}}$ determines a $\times \boldsymbol{\mu}$-Kummer structure on this Frobenioid. We shall write

$$
\ddagger \mathcal{F}_{\underline{w}}^{\vdash \triangleright \times \mu}
$$

for the resulting split-Kummer Frobenioid and - by abuse of notation! [cf. the discussion of (iii) above] —

$$
\ddagger \mathcal{F}_{\underline{w}}^{\vdash}
$$

for the split-Kummer Frobenioid determined by the split Frobenioid ${ }^{\ddagger} \mathcal{F}_{\underline{w}}^{\vdash}$ equipped with the $\times$-Kummer structure determined by ${ }^{\ddagger} \kappa_{\underline{w}}^{\vdash \times}$.

(v) Let ${ }^{\ddagger} \mathfrak{F} \vdash$ be as in (ii); $\underline{w} \in \underline{\mathbb{V}}^{\text {arc }}$. Then we shall write

$$
\ddagger \mathcal{F}_{\underline{w}}^{\vdash \triangleright \times \mu}
$$


for the collection of data obtained by replacing the split Frobenioid that appears in the collection of data ${ }^{\ddagger} \mathcal{F}_{w}^{\vdash}$ [cf. [IUTchI], Definition 5.2, (ii), (b); [IUTchI], Example 3.4, (ii)] by the inductive system, indexed by any ["multiplicatively"] cofinal subset of the multiplicative monoid $\mathbb{N}_{\geq 1}$, of split Frobenioids obtained [in the evident fashion] from ${ }^{\ddagger} \mathcal{F}_{\underline{w}}^{\vdash}$ by forming the quotients by the $N$-torsion, for $N \in \mathbb{N}_{\geq 1}$. Here, we identify [in the evident fashion] the inductive systems arising from distinct cofinal subsets of $\mathbb{N}_{\geq 1}$. Thus, [cf. the notation of (i)] the units of the split Frobenioids of this inductive system give rise to an inductive system

$$
\ldots \quad \rightarrow \mathcal{O}^{\times \boldsymbol{\mu}_{N}}(A) \quad \rightarrow \quad \ldots \quad \rightarrow \quad \mathcal{O}^{\times \boldsymbol{\mu}_{N \cdot N^{\prime}}}(A) \quad \rightarrow \quad \ldots
$$

[where $N, N^{\prime} \in \mathbb{N}_{\geq 1}$ ]. Now recall that ${ }^{\ddagger} \mathcal{D}_{\underline{w}}^{\vdash}$ is an object of the category $\mathbb{T M}^{\vdash}[\mathrm{cf}$. [IUTchI], Definition 4.1, (iii), (b)]. In particular, the units $\left({ }^{\ddagger} \mathcal{D}_{\underline{w}}^{\vdash}\right)^{\times}$of this object of $\mathbb{T M}^{\vdash}$ form a topological group [noncanonically isomorphic to $\mathbb{S}^{1}$ ], which we think of as being related to the above inductive system of units via a system of compatible surjections

$$
\left({ }^{\ddagger} \mathcal{D}_{\underline{w}}^{\vdash}\right)^{\times} \rightarrow \mathcal{O}^{\times \boldsymbol{\mu}_{N}}(A)
$$

[i.e., where the kernel of the displayed surjection is the subgroup of $N$-torsion]. This system of compatible surjections is well-defined up to an indeterminacy given by composition with the unique nontrivial automorphism of $\left({ }^{\ddagger} \mathcal{D}_{\underline{w}}^{\vdash}\right)^{\times}$. When considered up to this indeterminacy, this system of compatible surjections may be thought of as a sort of Kummer structure on ${ }^{\ddagger} \mathcal{F}_{w}^{\vdash} \times \boldsymbol{\mu}$ [which may be algorithmically reconstructed from the collection of data $\left.{ }^{\ddagger} \mathcal{F}_{\underline{w}}^{\vdash} \times \bar{\mu}\right]$.

(vi) Write

$$
{ }^{\ddagger} \mathfrak{F} \vdash \times \mu=\left\{{ }^{\ddagger} \mathcal{F}_{\underline{v}}^{\vdash \bullet \times \mu}\right\}_{\underline{v} \in \underline{\mathbb{V}}}
$$

for the collection of data indexed by $\underline{\mathbb{V}}$ obtained as follows: (a) if $\underline{v} \in \underline{\mathbb{V}}^{\text {bad }}$, then we take $\mathcal{F}_{\underline{v}}^{+} \times \boldsymbol{\mu}$ to be the split-Kummer Frobenioid constructed in (iii); (b) if $\underline{v} \in$ $\underline{\mathbb{V}}^{\text {good }} \cap \underline{\mathbb{V}}^{\text {non }}$, then we take ${ }^{\ddagger} \mathcal{F}_{\underline{v}}^{+} \times \boldsymbol{\mu}$ to be the split-Kummer Frobenioid constructed in (iv); (c) if $\underline{v} \in \underline{\mathbb{V}}^{\text {arc }}$, then we take $\mathcal{F}_{\underline{v}}^{\vdash} \times \boldsymbol{\mu}$ to be the collection of data constructed in $(\mathrm{v})$. Moreover, by replacing the various split Frobenioids of $\ddagger \mathfrak{F}^{\vdash}$ (respectively, $\ddagger \mathfrak{F} \bullet \times \boldsymbol{\mu}$ ) with the split Frobenioids — i.e., equipped with trivial splittings! obtained by considering the subcategories [of the underlying categories associated to these Frobenioids] determined by the isometries [i.e., roughly speaking, the "units" — cf. [FrdI], Theorem 5.1, (iii), in the case of $\underline{v} \in \underline{\mathbb{V}}^{\text {non }}$; [FrdII], Example 3.3, (iii), in the case of $\left.\underline{v} \in \underline{\mathbb{V}}^{\text {arc }}\right]$, one obtains a collection of data

$$
\left.{ }^{\ddagger} \mathfrak{F}^{\vdash \times}=\left\{{ }^{\ddagger} \mathcal{F}_{\underline{v}}^{\vdash \times}\right\}_{\underline{v} \in \mathbb{V}} \quad \text { (respectively, }{ }^{\ddagger} \mathfrak{F}^{\vdash \times \boldsymbol{\mu}}=\left\{{ }^{\ddagger} \mathcal{F}_{\underline{v}}^{\vdash \times \mu}\right\}_{\underline{v} \in \underline{\mathbb{V}}}\right)
$$

indexed by $\underline{\mathbb{V}}$. Thus, for each $\underline{v} \in \underline{\mathbb{V}}^{\text {non }},{ }^{\ddagger} \mathcal{F}_{\underline{v}}^{\vdash \times}$ (respectively, ${ }^{\ddagger} \mathcal{F}_{\underline{v}}^{\vdash \times \boldsymbol{\mu}}$ ) is a split- $\times$ (respectively, split- $\times \boldsymbol{\mu}$-Kummer) Frobenioid.

(vii) Let $\vdash \square \in\{\vdash \times, \vdash \times \boldsymbol{\mu}, \vdash \vdash \times \boldsymbol{\mu}\}$. Then we define an $\mathcal{F}^{\vdash} \square$-prime-strip to be a collection of data

$$
\mathfrak{F}^{\vdash} \square=\left\{{ }^{*} \mathcal{F}_{\underline{v}}^{\vdash} \square\right\}_{\underline{v} \in \underline{\mathbb{V}}}
$$


such that for each $\underline{v} \in \underline{\mathbb{V}},{ }^{*} \mathcal{F}_{\underline{v}}^{\vdash} \square$ is a collection of data that is isomorphic to ${ }^{\ddagger} \mathcal{F}_{\underline{v}}^{\vdash}$ [cf. (vi)]. A morphism of $\mathcal{F}^{\vdash} \overline{-}$-prime-strips is defined to be a collection of isomorphisms, indexed by $\underline{\mathbb{V}}$, between the various constituent objects of the prime-strips [cf. [IUTchI], Definition 5.2, (iii)].

(viii) We define an $\mathcal{F}^{\Vdash \triangleright \times \boldsymbol{\mu}}$-prime-strip to be a collection of data

$$
{ }^{*} \mathfrak{F}^{\Vdash \triangleright \times \boldsymbol{\mu}}=\left({ }^{*} \mathcal{C}^{\Vdash}, \operatorname{Prime}\left({ }^{*} \mathcal{C}^{\Vdash}\right) \stackrel{\sim}{\rightarrow} \underline{\mathbb{V}},{ }^{*} \mathfrak{F}^{\vdash} \times \boldsymbol{\mu},\left\{{ }^{*} \rho_{\underline{v}}\right\}_{\underline{v} \in \underline{\mathbb{V}}}\right)
$$

satisfying the conditions (a), (b), (c), (d), (e), (f) of [IUTchI], Definition 5.2, (iv), for an $\mathcal{F}^{\Vdash}$-prime-strip, where the portion of the collection of data constituted by

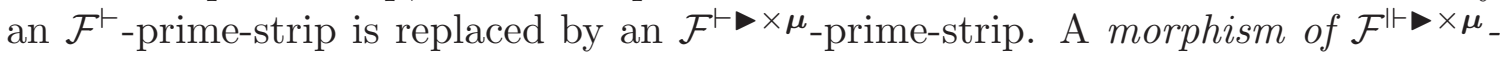
prime-strips is defined to be an isomorphism between collections of data as discussed above.

We conclude the present paper with the following two results, which may be thought of as enhanced versions of [IUTchI], Corollaries 3.7, 3.8, 3.9 - i.e., versions that reflect the various enhancements made to the theory in [IUTchI], $44, \S 5, \S 6$, as well as in the present paper.

Corollary 4.10. (Frobenioid-pictures of $\Theta^{ \pm \text {ell }}$ NF-Hodge Theaters) Fix a collection of initial $\Theta$-data $\left(\bar{F} / F, X_{F}, l, \underline{C}_{K}, \underline{\mathbb{V}}, \underline{\epsilon}\right)$ as in [IUTchI], Definition 3.1. Let

$$
{ }^{\dagger} \mathcal{H} \mathcal{T}^{\Theta^{ \pm \mathrm{ell}} \mathrm{NF}} ; \quad \ddagger \mathcal{H} \mathcal{T}^{\Theta^{ \pm \mathrm{ell}} \mathrm{NF}}
$$

be $\Theta^{ \pm \text {ell }}$ NF-Hodge theaters [relative to the given initial $\Theta$-data] - cf. [IUTchI], Definition 6.13, (i). Write ${ }^{\dagger} \mathcal{H} \mathcal{T}^{\mathcal{D}-\Theta^{ \pm \mathrm{ell}} \mathrm{NF}},{ }^{\ddagger} \mathcal{H} \mathcal{T}^{\mathcal{D}-\Theta^{ \pm \mathrm{ell}} \mathrm{NF}}$ for the associated $\mathcal{D}$ $\Theta^{ \pm \text {ell }}$ NF-Hodge theaters - cf. [IUTchI], Definition 6.13, (ii). Then:

(i) (Constant Prime-Strips) Let us apply the constructions of Corollary 4.6, (i), (iii), to the underlying $\Theta^{ \pm \mathrm{ell}}$-Hodge theater of ${ }^{\dagger} \mathcal{H} \mathcal{T}^{\Theta^{ \pm \mathrm{ell}} \mathrm{NF}}$. Then, for each $t \in \operatorname{LabCusp}^{ \pm}\left({ }^{\dagger} \mathfrak{D}_{\succ}\right)$, the collection of data $\Psi_{\mathrm{cns}}\left({ }^{\dagger} \mathfrak{F}_{\succ}\right)_{t}$ determines, in a natural way, an $\mathcal{F}$-prime-strip [cf. Remark 4.6.2, (i)]. Let us identify the collections of data

$$
\Psi_{\mathrm{cns}}\left({ }^{\dagger} \mathfrak{F}_{\succ}\right)_{0} \text { and } \Psi_{\mathrm{cns}}\left({ }^{\dagger} \mathfrak{F}_{\succ}\right)_{\left\langle\mathbb{F}_{l}^{*}\right\rangle}
$$

via the isomorphism of the second display of Corollary 4.6, (iii), and denote by

$$
{ }^{\dagger} \mathfrak{F}_{\triangle}^{\Vdash}=\left({ }^{\dagger} \mathcal{C}_{\triangle}^{\Vdash}, \operatorname{Prime}\left({ }^{\dagger} \mathcal{C}_{\triangle}^{\Vdash}\right) \stackrel{\sim}{\rightarrow} \underline{\mathbb{V}},{ }^{\dagger} \mathfrak{F}_{\triangle}^{\vdash},\left\{{ }^{\dagger} \rho_{\triangle, \underline{v}}\right\}_{\underline{v} \in \underline{\mathbb{V}}}\right)
$$

the resulting $\mathcal{F}^{\Vdash}$-prime-strip determined by the constructions discussed in [IUTchI], Remark 5.2.1, (ii) [which, as is easily verified, are compatible with the $\mathbb{F}_{l}^{\rtimes \pm_{-}}$ symmetrizing isomorphisms of Corollary 4.6, (iii)]. Thus, [it follows immediately from the constructions involved that] one has a natural identification isomorphism of $\mathcal{F}^{\Vdash}$-prime-strips ${ }^{\dagger} \mathfrak{F}_{\triangle}^{\Vdash} \stackrel{\sim}{\rightarrow} \mathfrak{F}_{\text {mod }}^{\Vdash}$ between ${ }^{\dagger} \mathfrak{F}_{\triangle}^{\Vdash}$ and the collection of data ${ }^{\dagger} \mathfrak{F}_{\text {mod }}^{\Vdash}$ associated to the underlying $\Theta$-Hodge theater of ${ }^{\dagger} \mathcal{H} \mathcal{T}^{\Theta^{ \pm \mathrm{ell}} \mathrm{NF}}$ [cf. [IUTchI], Definition 3.6, (c)] — cf. the discussion of the assignment " $0, \succ \mapsto>$ " in Remark 3.8.2, (ii). 
(ii) (Theta and Gaussian Prime-Strips) Let us apply the constructions of Corollary 4.6, (iv), (v), to the underlying $\Theta$-bridge and $\Theta^{ \pm \text {ell }}$-Hodge theater of ${ }^{\dagger} \mathcal{H} \mathcal{T}^{\Theta^{ \pm e l l} \mathrm{NF}}$. Then the collection of data $\Psi_{\mathcal{F}_{\text {env }}}\left({ }^{\dagger} \mathcal{H} \mathcal{T}^{\Theta}\right)$ [cf. Corollary 4.6, (iv)], the global realified Frobenioid ${ }^{\dagger} \mathcal{C}_{\mathrm{env}}^{\Vdash} \stackrel{\text { def }}{=} \mathcal{C}_{\mathrm{env}}^{\Vdash}\left({ }^{\dagger} \mathcal{H} \mathcal{T}^{\Theta}\right)$ [cf. Corollary 4.6, (v)], and the local isomorphisms $\Phi_{\mathcal{C}_{\text {env }}^{\Vdash}\left({ }^{\dagger} \mathcal{H} \mathcal{T}^{\Theta}\right), \underline{v}} \stackrel{\sim}{\rightarrow} \Psi_{\mathcal{F}_{\text {env }}}\left({ }^{\dagger} \mathcal{H} \mathcal{T}^{\Theta}\right)_{\underline{v}}^{\mathbb{R}}$ for $\underline{v} \in \underline{\mathbb{V}}$ [cf. Corollary 4.6, (v)] give rise, in a natural fashion, to an $\mathcal{F}^{\Vdash}$-prime-strip

$$
{ }^{\dagger} \mathfrak{F}_{\text {env }}^{\Vdash}=\left({ }^{\dagger} \mathcal{C}_{\text {env }}^{\Vdash}, \operatorname{Prime}\left({ }^{\dagger} \mathcal{C}_{\text {env }}^{\Vdash}\right) \stackrel{\sim}{\rightarrow} \underline{\mathbb{V}},{ }^{\dagger} \mathfrak{F}_{\text {env }}^{\vdash},\left\{{ }^{\dagger} \rho_{\text {env }, \underline{v}}\right\}_{\underline{v} \in \underline{\mathbb{V}}}\right)
$$

[so, in particular, ${ }^{\dagger} \mathfrak{F}_{\text {env }}^{\vdash}$ is the $\mathcal{F}^{\vdash}$-prime-strip determined by $\Psi_{\mathcal{F}_{\text {env }}}\left({ }^{\dagger} \mathcal{H} \mathcal{T}^{\Theta}\right)-c f$. Remark 4.6.2, (i); Remark 4.10.1 below]. Thus, [it follows immediately from the constructions involved that] there is a natural identification isomorphism of $\mathcal{F}^{\Vdash}$-prime-strips ${ }^{\dagger} \mathfrak{F}_{\text {env }}^{\Vdash} \stackrel{\sim}{\rightarrow}{ }^{\dagger} \mathfrak{F}_{\text {tht }}^{\Vdash}$ between ${ }^{\dagger} \mathfrak{F}_{\text {env }}^{\vdash}$ and the collection of data ${ }^{\dagger} \mathfrak{F}_{\text {tht }}^{\vdash}$ associated to the underlying $\Theta$-Hodge theater of ${ }^{\dagger} \mathcal{H} \mathcal{T}^{\Theta^{ \pm \mathrm{ell}} \mathrm{NF}}$ [cf. [IUTchI], Definition 3.6, (c)]. In a similar vein, the collection of data $\Psi_{\mathcal{F}_{\text {gau }}}\left({ }^{\dagger} \mathcal{H} \mathcal{T}^{\Theta}\right)$ [cf. Corollary 4.6, (iv)], the global realified Frobenioid ${ }^{\dagger} \mathcal{C}_{\text {gau }}^{\Vdash} \stackrel{\text { def }}{=} \mathcal{C}_{\text {gau }}^{\Vdash}\left({ }^{\dagger} \mathcal{H} \mathcal{T}^{\Theta}\right)$ [cf. Corollary 4.6, $(v)]$, and the local isomorphisms $\Phi_{\mathcal{C}_{\text {gau }}^{\Vdash}}\left(\dagger \mathcal{H} \mathcal{T}^{\ominus}\right), \underline{v} \stackrel{\sim}{\rightarrow} \Psi_{\mathcal{F}_{\text {gau }}}\left({ }^{\dagger} \mathcal{H} \mathcal{T}^{\Theta}\right)_{\underline{v}}^{\mathbb{R}}$ for $\underline{v} \in \underline{\mathbb{V}}$ [cf. Corollary 4.6, (v)] give rise, in a natural fashion, to an $\mathcal{F}^{\Vdash}$-prime-strip

$$
{ }^{\dagger} \mathfrak{F}_{\text {gau }}^{\Vdash}=\left({ }^{\dagger} \mathcal{C}_{\text {gau }}^{\Vdash}, \operatorname{Prime}\left({ }^{\dagger} \mathcal{C}_{\text {gau }}^{\Vdash}\right) \stackrel{\sim}{\rightarrow} \underline{\mathbb{V}},{ }^{\dagger} \mathfrak{F}_{\text {gau }}^{\vdash},\left\{{ }^{\dagger} \rho_{\text {gau }, \underline{v}}\right\}_{\underline{v} \in \underline{\mathbb{V}}}\right)
$$

[so, in particular, ${ }^{\dagger} \mathfrak{F}_{\text {gau }}^{\vdash}$ is the $\mathcal{F}^{\vdash}$-prime-strip determined by $\Psi_{\mathcal{F}_{\text {gau }}}\left({ }^{\dagger} \mathcal{H} \mathcal{T}^{\Theta}\right)-c f$. Remark 4.6.2, (i); Remark 4.10.1 below]. Finally, the evaluation isomorphisms of Corollary 4.6, (iv), (v), determine an evaluation isomorphism

$$
{ }^{\dagger} \mathfrak{F}_{\text {env }}^{\vdash} \stackrel{\sim}{\rightarrow} \dagger \mathfrak{F}_{\text {gau }}^{\vdash}
$$

of $\mathcal{F}^{\Vdash}$-prime-strips.

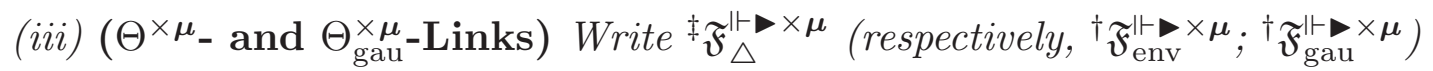

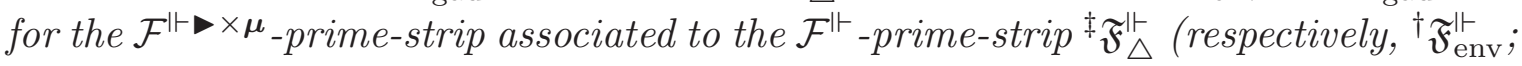
$\dagger \mathfrak{F}_{\text {gau }}^{\Vdash}$ ) [cf. Definition 4.9, (viii); the functorial algorithm described in Definition 4.9, (vi)]. Then the functoriality of this algorithm induces maps

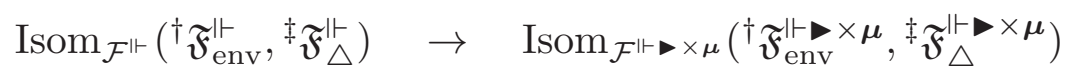

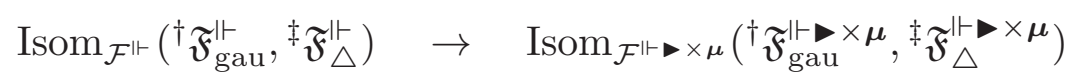

from [nonempty!] sets of isomorphisms of $\mathcal{F}^{\Vdash}$-prime-strips to [nonempty!] sets

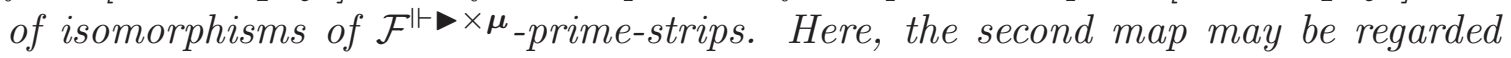
as being obtained from the first map via composition [in the case of the domain "Isom $\mathcal{F}^{\Vdash}(-,-)$ "] with the evaluation isomorphism ${ }^{\dagger} \mathfrak{F}_{\text {env }}^{\vdash} \stackrel{\sim}{\rightarrow} \dagger \mathfrak{F}_{\text {gau }}^{\vdash}$ of (ii) and composition [in the case of the codomain " $\operatorname{som}_{\mathcal{F}} \bullet \times \mu(-,-)$ "] with the isomorphism $\dagger \mathfrak{F}_{\text {env }}^{\Vdash \bullet} \times \boldsymbol{\mu} \stackrel{\sim}{\rightarrow} \dagger \mathfrak{F}_{\text {gau }}^{\mapsto \rightarrow \times \mu}$ functorially obtained from this isomorphism of (ii). We shall

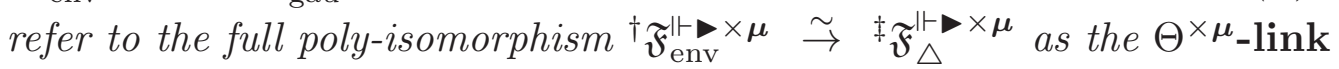

$$
{ }^{\dagger} \mathcal{H} \mathcal{T}^{\Theta^{ \pm e l l}} \mathrm{NF} \quad \stackrel{\Theta^{\times \mu}}{\longrightarrow} \ddagger \mathcal{H} \mathcal{T}^{\Theta^{ \pm e l l} \mathrm{NF}}
$$




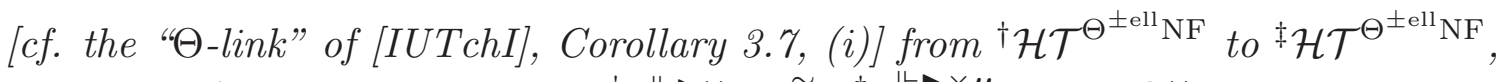

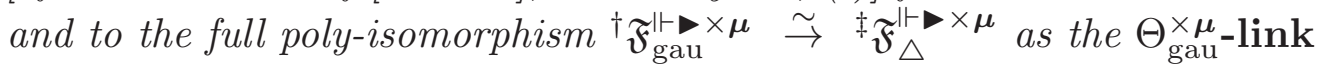

$$
\dagger \mathcal{H} \mathcal{T}^{\Theta^{ \pm \mathrm{ell}} \mathrm{NF}} \stackrel{\Theta_{\text {gau }}^{\times \mu}}{\longrightarrow} \ddagger \mathcal{H} \mathcal{T}^{\Theta^{ \pm \mathrm{ell}} \mathrm{NF}}
$$

from ${ }^{\dagger} \mathcal{H} \mathcal{T}^{\Theta^{ \pm e l l} \mathrm{NF}}$ to ${ }^{\ddagger} \mathcal{H} \mathcal{T}^{\Theta^{ \pm e l l} \mathrm{NF}}$.

(iv) (Coric $\mathcal{F}^{\vdash \times \mu}$-Prime-Strips) The definition of the unit portion of the theta and Gaussian monoids involved [cf. Corollaries 3.5, (ii); 3.6, (ii); 4.1, (iv); 4.2, (iv); 4.3, (iv); 4.4, (iv)] gives rise to natural isomorphisms

$$
\dagger \mathfrak{F}_{\triangle}^{\vdash \times \mu} \stackrel{\sim}{\rightarrow} \dagger \mathfrak{F}_{\text {env }}^{\vdash \times \mu} \stackrel{\sim}{\rightarrow} \dagger \mathfrak{F}_{\text {gau }}^{\vdash \times \mu}
$$

- where we write ${ }^{\dagger} \mathfrak{F}_{\triangle}^{\vdash \times \boldsymbol{\mu}},{ }^{\dagger} \mathfrak{F}_{\text {env }}^{\vdash \times \boldsymbol{\mu}},{ }^{\dagger} \mathfrak{F}_{\text {gau }}^{\vdash \times \boldsymbol{\mu}}$ for the $\mathcal{F}^{\vdash \times \boldsymbol{\mu}}$-prime-strips associated to the $\mathcal{F}^{\vdash}$-prime-strips ${ }^{\dagger} \mathfrak{F}_{\triangle}^{\vdash},{ }^{\dagger} \mathfrak{F}_{\text {env }}^{\vdash},{ }^{\dagger} \mathfrak{F}_{\text {gau }}^{\vdash}$, respectively [cf. the functorial algorithm described in Definition 4.9, (vi)]. Moreover, by composing these natural isomorphisms with the poly-isomorphisms induced on the respective $\mathcal{F}^{\vdash \times \boldsymbol{\mu}}$-prime-strips by the $\Theta^{\times \boldsymbol{\mu}}$ - and $\Theta_{\mathrm{gau}}^{\times \boldsymbol{\mu}}$-links of (iii), one obtains a poly-isomorphism

$$
{ }^{\dagger} \mathfrak{F}_{\triangle}^{\vdash \times \mu} \stackrel{\sim}{\rightarrow}{ }^{\ddagger} \mathfrak{F}_{\triangle}^{\vdash} \times \mu
$$

which coincides with the full poly-isomorphism between these two $\mathcal{F}^{\vdash \times \boldsymbol{\mu}}$-primestrips - that is to say, “(-) $\mathfrak{F}_{\triangle}^{\vdash} \times \boldsymbol{\mu}$ ” is an invariant of both the $\Theta^{\times \boldsymbol{\mu}}$ - and $\Theta_{\mathrm{gau}}^{\times \boldsymbol{\mu}}$-links. Finally, this full poly-isomorphism induces [cf. Definition 4.9, (vii); [IUTchI], Remark 5.2.1, (i)] the full poly-isomorphism

$$
\dagger \mathfrak{D}_{\triangle}^{\vdash} \stackrel{\sim}{\rightarrow} \ddagger \mathfrak{D}_{\triangle}^{\vdash}
$$

between the associated $\mathcal{D}^{\vdash}$-prime-strips; we shall refer to this poly-isomorphism as the $\mathcal{D}$ - $\Theta^{ \pm \text {ell }} \mathrm{NF}$-link from ${ }^{\dagger} \mathcal{H} \mathcal{T}^{\mathcal{D}-\Theta^{ \pm \mathrm{ell}} \mathrm{NF}}$ to ${ }^{\ddagger} \mathcal{H} \mathcal{T}^{\mathcal{D}-\Theta^{ \pm e l l} \mathrm{NF}}$.

(v) (Coric Realified Global Frobenioids) The full poly-isomorphism ${ }^{\dagger} \mathfrak{D}_{\triangle}^{\vdash}$ $\stackrel{\sim}{\rightarrow} \ddagger \mathfrak{D}_{\triangle}^{\vdash}$ of (iv) induces [cf. Corollary 4.5, (ii)] an isomorphism of collections of data

$$
\begin{aligned}
\left(\mathcal{D}^{\Vdash}\left({ }^{\dagger} \mathfrak{D}_{\triangle}^{\vdash}\right),\right. & \left.\operatorname{Prime}\left(\mathcal{D}^{\Vdash}\left({ }^{\dagger} \mathfrak{D}_{\triangle}^{\vdash}\right)\right) \stackrel{\sim}{\rightarrow} \underline{\mathbb{V}}, \quad\left\{{ }^{\dagger} \rho_{\mathcal{D}^{\Vdash}, \underline{v}}\right\}_{\underline{v} \in \underline{\mathbb{V}}}\right) \\
& \stackrel{\sim}{\rightarrow} \quad\left(\mathcal{D}^{\Vdash}\left({ }^{\ddagger} \mathfrak{D}_{\triangle}^{\vdash}\right), \operatorname{Prime}\left(\mathcal{D}^{\Vdash}\left({ }^{\ddagger} \mathfrak{D}_{\triangle}^{\vdash}\right)\right) \stackrel{\sim}{\rightarrow} \underline{\mathbb{V}}, \quad\left\{{ }^{\ddagger} \rho_{\mathcal{D}^{\Vdash}, \underline{v}}\right\}_{\underline{v}} \in \underline{\mathbb{V}}\right)
\end{aligned}
$$

- i.e., consisting of a Frobenioid, a bijection, and a collection of isomorphisms of topological monoids indexed by $\underline{\mathbb{V}}$. Moreover, this isomorphism of collections of data is compatible, relative to the $\Theta^{\times \mu_{-}}$and $\Theta_{\text {gau }}^{\times \mu}$-links of (iii), with the $\mathbb{R}_{>0}$-orbits of the isomorphisms of collections of data

$$
\begin{aligned}
\left({ }^{\dagger} \mathcal{C}_{\triangle}^{\Vdash},\right. & \left.\operatorname{Prime}\left({ }^{\dagger} \mathcal{C}_{\triangle}^{\Vdash}\right) \stackrel{\sim}{\rightarrow} \underline{\mathbb{V}},\left\{{ }^{\dagger} \rho_{\triangle, \underline{v}}\right\}_{\underline{v} \in \underline{\mathbb{V}}}\right) \\
& \stackrel{\sim}{\rightarrow}\left(\mathcal{D}^{\Vdash}\left({ }^{\dagger} \mathfrak{D}_{\triangle}^{\vdash}\right), \operatorname{Prime}\left(\mathcal{D}^{\Vdash}\left({ }^{\dagger} \mathfrak{D}_{\triangle}^{\vdash}\right)\right) \stackrel{\sim}{\rightarrow} \underline{\mathbb{V}},\left\{{ }^{\dagger} \rho_{\mathcal{D}^{\Vdash}, \underline{v}}\right\}_{\underline{v} \in \underline{\mathbb{V}}}\right) \\
\left({ }^{\ddagger} \mathcal{C}_{\triangle}^{\Vdash},\right. & \left.\operatorname{Prime}\left({ }^{\ddagger} \mathcal{C}_{\triangle}^{\Vdash}\right) \stackrel{\sim}{\rightarrow} \underline{\mathbb{V}},\left\{{ }^{\ddagger} \rho_{\triangle, \underline{v}}\right\}_{\underline{v} \in \mathbb{V}}\right) \\
& \stackrel{\sim}{\rightarrow}\left(\mathcal{D}^{\Vdash}\left({ }^{\ddagger} \mathfrak{D}_{\triangle}^{\vdash}\right), \operatorname{Prime}\left(\mathcal{D}^{\Vdash}\left({ }^{\ddagger} \mathfrak{D}_{\triangle}^{\vdash}\right)\right) \stackrel{\sim}{\rightarrow} \underline{\mathbb{V}},\left\{{ }^{\ddagger} \rho_{\mathcal{D}^{\Vdash}, \underline{v}}\right\}_{\underline{v}} \in \underline{\mathbb{V}}\right)
\end{aligned}
$$


obtained by applying the functorial algorithm discussed in the final portion of Corollary 4.6, (ii). Here, the " $\mathbb{R}_{>0}$-orbits" are defined relative to the natural $\mathbb{R}_{>0}$ actions on the Frobenioids involved obtained by multiplying the "arithmetic degrees" by a given element $\in \mathbb{R}_{>0}$ [cf. [FrdI], Example 6.3; [FrdI], Theorem 6.4, (ii); [IUTchI], Remark 3.1.5].

(vi) (Frobenius-pictures) Let $\left\{{ }^{n} \mathcal{H} \mathcal{T}^{\Theta^{ \pm \mathrm{ell}} \mathrm{NF}}\right\}_{n \in \mathbb{Z}}$ be a collection of distinct $\Theta^{ \pm \text {ell }} \mathbf{N F - H o d g e}$ theaters indexed by the integers. Then by applying the $\Theta^{\times \boldsymbol{\mu}_{-}}$and $\Theta_{\mathrm{gau}}^{\times \mu}$-links of (iii), we obtain infinite chains

$$
\begin{aligned}
& \ldots \stackrel{\Theta^{\times \mu}}{\longrightarrow}(n-1) \mathcal{H} \mathcal{T}^{\Theta^{ \pm \mathrm{ell}} \mathrm{NF}} \stackrel{\Theta^{\times \mu}}{\longrightarrow}{ }^{n} \mathcal{H} \mathcal{T}^{\Theta^{ \pm \mathrm{ell}} \mathrm{NF}} \stackrel{\Theta^{\times \mu}}{\longrightarrow}{ }^{(n+1)} \mathcal{H} \mathcal{T}^{\Theta^{ \pm \mathrm{ell}} \mathrm{NF}} \stackrel{\Theta^{\times \mu}}{\longrightarrow} \ldots \\
& \ldots \stackrel{\Theta_{\text {gau }}^{\times \mu}}{\longrightarrow}(n-1) \mathcal{H} \mathcal{T}^{\Theta^{ \pm e l l}} \mathrm{NF} \stackrel{\Theta_{\text {gau }}^{\times \mu}}{\longrightarrow} n \mathcal{H} \mathcal{T}^{\Theta^{ \pm e l l}} \mathrm{NF} \stackrel{\Theta_{\text {gau }}^{\times \mu}}{\longrightarrow}(n+1) \mathcal{H} \mathcal{T}^{\Theta^{ \pm e l l}} \mathrm{NF} \stackrel{\Theta_{\text {gau }}^{\times \mu}}{\longrightarrow} \ldots
\end{aligned}
$$

of $\Theta^{\times \boldsymbol{\mu}_{-}} / \Theta_{\mathrm{gau}}^{\times \boldsymbol{\mu}}$-linked $\Theta$-Hodge theaters. Either of these infinite chains may be represented symbolically as an oriented graph $\vec{\Gamma}$ [cf. [AbsTopIII], §O]

- i.e., where the arrows correspond to either the " $\stackrel{\Theta^{\times \mu}}{\longrightarrow}$ 's" or the " $\stackrel{\Theta_{\text {gau }}^{\times \mu}}{\longrightarrow}$ 's", and

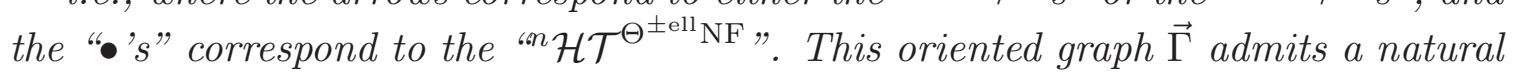
action by $\mathbb{Z}$ - i.e., a translation symmetry - but it does not admit arbitrary permutation symmetries. For instance, $\vec{\Gamma}$ does not admit an automorphism that switches two adjacent vertices, but leaves the remaining vertices fixed - cf. the discussion of [IUTchI], Corollary 3.8; [IUTchI], Remark 3.8.1.

Proof. The various assertions of Corollary 4.10 follow immediately from the definitions and the references quoted in the statements of these assertions.

Remark 4.10.1. Strictly speaking [cf. Remark 4.6.2, (i)], the $\mathcal{F}^{\vdash}$-prime-strips constructed, in Corollary 4.10, (ii), from the theta and Gaussian monoids of Corollary 4.6, (iv), are only well-defined up to an indeterminacy, at the $\underline{v} \in \underline{\mathbb{V}}^{\text {bad }}$, relative to automorphisms of the split Frobenioid at such $\underline{v} \in \underline{\mathbb{V}}^{\text {bad }}$ that induce the identity automorphism on the associated $\mathcal{F}^{\vdash \times}$-prime-strip. On the other hand, such indeterminacies may, in essence, be ignored, since they are "absorbed" in the full poly-isomorphisms that appear in the $\Theta^{\times \mu_{-}}$and $\Theta_{\text {gau- }}^{\times \mu}$-links of Corollary 4.10, (iii).

\section{Remark 4.10.2.}

(i) Although both the $\Theta^{\times \mu_{-}}$-and $\Theta_{\text {gau }}^{\times \mu}$-links are treated, in essence, on an equal footing in Corollary 4.10, in the remainder of the present series of papers, we shall ultimately mainly be interested in [a further enhanced version of] the $\Theta_{\text {gau }}^{\times \mu}$-link. On

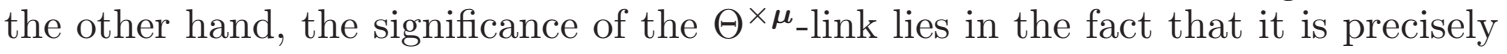
by thinking of the $\Theta_{\text {gau }}^{\times \boldsymbol{\mu}}$-link as being constructed as the composite of the $\Theta^{\times \boldsymbol{\mu}_{-}}$ link with the operation of Galois evaluation that one may establish conjugate synchronization [cf. the discussion of Remark 2.6.1]. 
(ii) At $\underline{v} \in \underline{\mathbb{V}}^{\text {bad }}$, the $\Theta^{\times \boldsymbol{\mu}}$-link may be thought of as a sort of equivalence between the split theta monoids of Proposition 3.1, (i) [cf. also Corollary 1.12, (ii)] and certain submonoids of the constant monoids of Proposition 3.1, (ii), equipped with the splittings that arise from the $q$-parameter " $\underline{\underline{q}}_{v}$ " [cf. the discussion of " $\tau_{\underline{v}}^{\vdash}$ " in [IUTchI], Example 3.2, (iv)]. On the other hand, it is important to note in this context that unlike the case with the splittings that occur in the case of the theta monoids, the splittings that occur in the case of the constant monoids do not arise from the operation of Galois evaluation - i.e., from a splitting " $H \hookrightarrow G_{v}$ " at the level of Galois groups of some surjection $G_{\underline{v}} \rightarrow H$. In particular, the splittings in the case of the constant monoids do not admit a natural multiradial formulation [cf. Remark 1.11.5; Proposition 3.4, (ii)], as in the case of the theta monoids [cf. Corollary 1.12, (iii)], that allows one to decouple the monoids into "purely radial" and "purely coric" components [cf. discussion of Remarks 1.11.4, (i); 1.12.2, (vi)].

\section{Remark 4.10.3.}

(i) The "coricity of $\mathcal{F}^{\vdash \times \boldsymbol{\mu}}$-prime-strips"

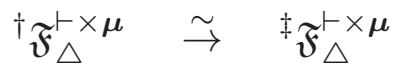

discussed in Corollary 4.10, (iv), amounts, in essence, to the "coricity of $\mathcal{D}^{\vdash}$-primestrips" $\dagger \mathfrak{D}_{\triangle}^{\vdash} \stackrel{\sim}{\rightarrow} \ddagger \mathfrak{D}_{\triangle}^{\vdash}$ [cf. Corollary 4.10, (iv)], together with the "coricity of [various quotients by torsion of] the units $\mathcal{O}^{\times}(-)$" of the Frobenioids involved cf. [IUTchI], Corollary 3.7, (ii), (iii). In [IUTchIII], this coricity of the units will play a central role when we apply the theory of the log-wall [cf. [AbsTopIII]]. In particular, this coricity of the units will allow us to compare volumes on either side of the $\Theta^{\times \boldsymbol{\mu}}$-link.

(ii) Unlike the units [cf. the discussion of (i)!], the "divisor monoid", or "value group", portion of the Frobenioids involved is by no means preserved by the $\Theta^{\times \boldsymbol{\mu}_{-}}$ link! Indeed, this "value group" portion of the $\Theta^{\times} \boldsymbol{\mu}$-link may be thought of as a sort of "Frobenius morphism" - cf. the discussion of Remark 3.6.2, (iii), as well as Remark 4.11 .1 below. Alternatively, from the point of view of the analogy between [complex or $p$-adic] Teichmüller theory and the theory of the present series of papers, this portion of the $\Theta^{\times \boldsymbol{\mu}}$-link may be thought of as a sort of Teichmüller deformation [cf. the discussion of [IUTchI], Remark 3.9.3, (ii)]. Indeed, the computation of the "volume distortion" arising from this "arithmetic Teichmüller deformation" may, in some sense, be regarded as the ultimate goal of the present series of papers.

(iii) In the context of the discussion of (ii), it is interesting to note that if one restricts the value group portion of the $\Theta^{\times \mu_{-}}$link - i.e.,

$$
\underline{\underline{q}} \underline{\underline{v}} \mapsto\left\{\underline{\underline{q}}_{\underline{\underline{v}}}^{j^{2}}\right\}_{1 \leq j \leq l *}
$$

[cf. Remark 3.6.2, (iii)] — to the label $j=1$, then the resulting correspondence

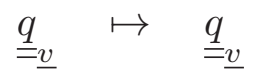


may be naturally identified with the "identity" - cf. the discussion of Remark 3.6.2, (iii). Put another way, the restriction to the label $j=1$ of the Gaussian distribution may be identified, for instance at the level of realifications, with the pivotal distribution discussed in [IUTchI], Example 5.4, (vii). On the other hand, in this context, it is important to observe that the operation of restriction to various proper subsets of the set of all labels $\left|\mathbb{F}_{l}\right|$ fails, in general, to be compatible with the crucial $\mathbb{F}_{l}^{\rtimes \pm}$ - and $\mathbb{F}_{l}^{*}$-symmetries of Corollaries 4.5, (iii); 4.6, (iii); 4.7, (ii); 4.8, (ii) [cf. also the discussion of Remark 2.6.3].

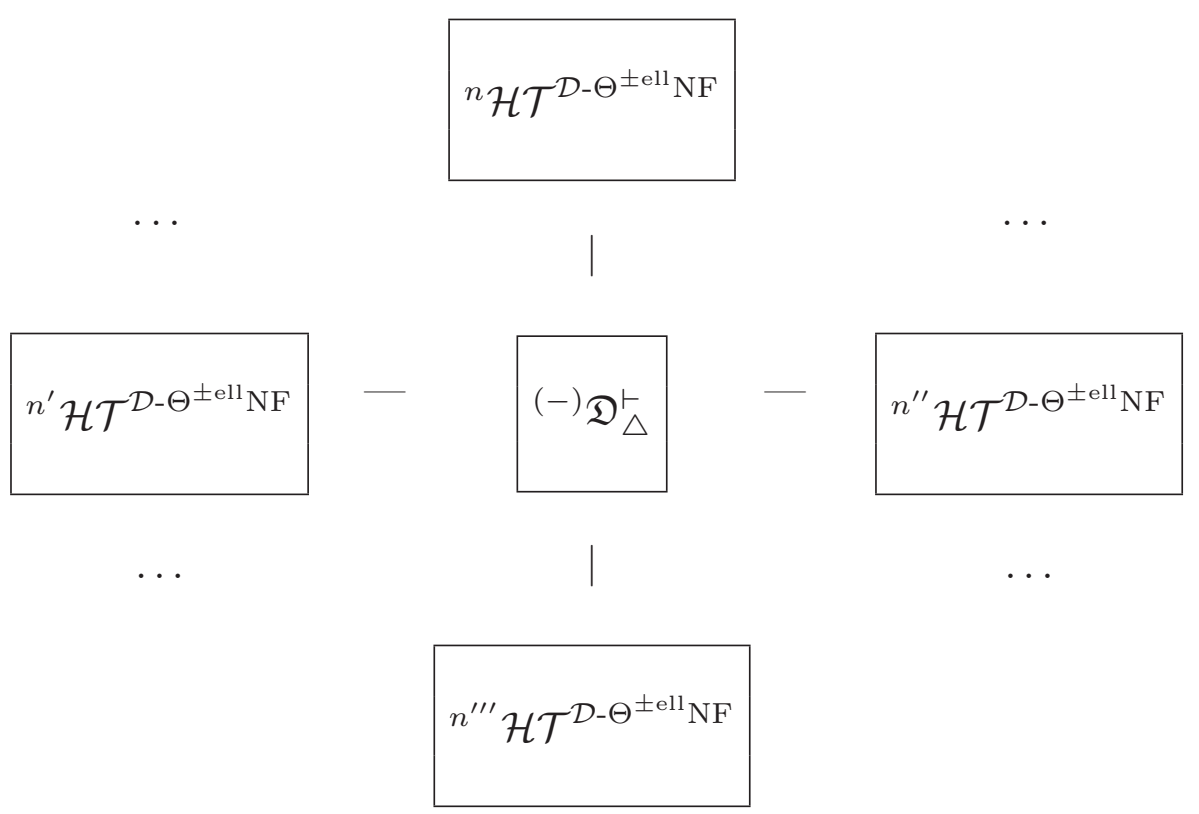

Fig. 4.3: Étale-picture of $\mathcal{D}-\Theta^{ \pm \text {ell }} \mathrm{NF}$-Hodge Theaters

Corollary 4.11. (Étale-pictures of Base- $\Theta^{ \pm \text {ell }}$ NF-Hodge Theaters) Suppose that we are in the situation of Corollary 4.10, (vi).

(i) Write

$$
\quad \stackrel{\mathcal{D}}{\longrightarrow} n^{n} \mathcal{H} \mathcal{T}^{\mathcal{D}-\Theta^{ \pm \mathrm{ell}} \mathrm{NF}} \stackrel{\mathcal{D}}{\longrightarrow} \quad{ }^{(n+1)} \mathcal{H} \mathcal{T}^{\mathcal{D}-\Theta^{ \pm \mathrm{ell}} \mathrm{NF}} \stackrel{\mathcal{D}}{\longrightarrow} \quad \ldots
$$

- where $n \in \mathbb{Z}$ - for the infinite chain of $\mathcal{D}-\Theta^{ \pm \text {ell }} \mathbf{N F}$-linked $\mathcal{D}$ - $\Theta^{ \pm \text {ell }} \mathbf{N F}$ Hodge theaters [cf. Corollary 4.10, (iv), (vi)] induced by either of the infinite chains of Corollary 4.10, (vi). Then this infinite chain induces a chain of full poly-isomorphisms

$$
\ldots \stackrel{\sim}{\rightarrow} n \mathfrak{D}_{\triangle}^{\vdash} \stackrel{\sim}{\rightarrow}(n+1) \mathfrak{D}_{\triangle}^{\vdash} \stackrel{\sim}{\rightarrow} \ldots
$$

[cf. Corollary 4.10, (iv)]. That is to say, “(-) $\mathfrak{D}_{\triangle}^{\vdash}$ " forms a constant invariant [cf. the discussion of [IUTchI], Remark 3.8.1, (ii)] - i.e., a mono-analytic core [cf. the situation discussed in [IUTchI], Remark 3.9.1] - of the above infinite chain.

(ii) If we regard each of the $\mathcal{D}-\Theta^{ \pm \mathrm{ell}} N F$-Hodge theaters of the chain of (i) as a spoke emanating from the mono-analytic core “(-) $\mathfrak{D}_{\triangle}^{\vdash}$ " discussed in (i), then we 
obtain a diagram - i.e., an étale-picture of $\mathcal{D}-\Theta^{ \pm \text {ell }} \mathbf{N F}-$ Hodge theaters - as in Fig. 4.3 above [cf. the situation discussed in [IUTchI], Corollaries 4.12, 6.10]. Thus, each spoke may be thought of as a distinct "arithmetic holomorphic structure" on the mono-analytic core. Finally, [cf. the situation discussed in [IUTchI], Corollaries 4.12, 6.10] this diagram satisfies the important property of admitting arbitrary permutation symmetries among the spokes [i.e., the labels $n \in \mathbb{Z}$ of the $\mathcal{D}-\Theta^{ \pm \text {ell }}$ NF-Hodge theaters].

(iii) The constructions of (i) and (ii) are compatible, in the evident sense, with the constructions of [IUTChI], Corollaries 4.12, 6.10, relative to the natural identification isomorphisms ${ }^{(-)} \mathfrak{D}_{\triangle}^{\vdash} \stackrel{\sim}{\rightarrow}{ }^{(-)} \mathfrak{D}_{>}^{\vdash}$ [cf. Corollary 4.10, (i); the discussion preceding [IUTchI], Example 5.4] and the operation of passing to the underlying $\mathcal{D}-\Theta N F-\left[\right.$ in the case of [IUTchI], Corollary 4.12] and $\mathcal{D}-\Theta^{ \pm \text {ell }}-$ Hodge theaters [in the case of [IUTchI], Corollary 6.10].

Proof. The various assertions of Corollary 4.11 follow immediately from the definitions and the references quoted in the statements of these assertions.

Remark 4.11.1. The $\Theta_{\text {gau }}^{\times \mu}$-link of Corollary 4.10, (iii), may be thought of, roughly, as a sort of transformation

$$
\underline{\underline{q}} \mapsto \underline{\underline{q}}\left(\begin{array}{c}
1^{2} \\
\vdots \\
(l *)^{2}
\end{array}\right)
$$

— cf. the discussion of Remark 3.6.2, (iii). From this point of view, the infinite chain of the Frobenius-picture discussed in Corollary 4.10, (vi), may be represented as an infinite iteration

$$
\left.\underline{\underline{q}} \mapsto\left(\begin{array}{c}
1^{2} \\
\vdots \\
\underline{\underline{q}} \\
\left(l^{*}\right)^{2}
\end{array}\right)\right)\left(\begin{array}{c}
1^{2} \\
\vdots \\
\left(l^{*}\right)^{2}
\end{array}\right) \quad \cdots
$$

of this transformation. By contrast, the associated étale-picture discussed in Corollary 4.11 corresponds to a sort of commutativity involving these "theta exponents"

$$
\underline{\underline{q}} \mapsto \underline{\underline{q}}\left(\begin{array}{c}
1^{2} \\
\vdots \\
\left(l^{*}\right)^{2}
\end{array}\right) \cdot\left(\begin{array}{c}
1^{2} \\
\vdots \\
\left(l^{*}\right)^{2}
\end{array}\right) \cdot\left(\begin{array}{c}
1^{2} \\
\vdots \\
(l *)^{2}
\end{array}\right) \quad \ldots
$$

— cf. the "arbitrary permutation symmetries" discussed in Corollary 4.11, (ii). In this context, it is useful to recall the analogy between the classical Gaussian integral and the theory of the present series of papers [cf. Remark 1.12.5] — an analogy in which the "order-conscious" Frobenius-picture corresponds to the cartesian coordinate representation of the Gaussian integral, while the "permutationsymmetric" étale-picture corresponds to the polar coordinate representation of the Gaussian integral. Finally, from the point of view of the discussion of Remark 
4.7.4, the $l$-torsion that occurs as the index set of the various " $\underline{\underline{j}}^{j}$ ' $\mathrm{s}$ " that appear in the Gaussian monoid of each $\Theta^{ \pm \text {ell }} \mathrm{NF}$-Hodge theater may be thought of as a sort of multiradial combinatorial representation of the distinct "arithmetic holomorphic structures" corresponding to the various $\Theta^{ \pm \text {ell } N F-H o d g e ~ t h e a t e r s . ~}$

Remark 4.11.2. At this point, we pause to review the theory developed so far in the present series of papers.

(i) The notion of a $\Theta^{ \pm \text {ell }} N F$-Hodge theater [cf. [IUTchI], Definition 6.13, (i)] is intended as a model of conventional scheme-theoretic arithmetic geometry - i.e., more precisely, of the conventional scheme-theoretic arithmetic geometry surrounding the theta function at primes of bad reduction $\in \underline{\mathbb{V}}^{\text {bad }}$ of the elliptic curve over a number field under consideration. At a more technical level, a $\Theta^{ \pm \text {ell }} \mathrm{NF}$ Hodge theater may be thought of as an apparatus that allows one to construct a sort of bridge between the number field and theta functions [at $\underline{v} \in \underline{\mathbb{V}}^{\text {bad }}$ ] under consideration. From a more concrete point of view, this bridge is realized by the Gaussian distribution - i.e., a globalized version of the theta values

$$
\left\{\underline{\underline{q}}_{\underline{\underline{v}}}^{j^{2}}\right\}_{1 \leq j \leq l}
$$

at l-torsion points [cf. Remark 3.6.2, (iii)]. Here, we remark that the term "Gaussian distribution" is intended as an intuitive expression that includes the more technical notions of "Gaussian monoids" and "Gaussian Frobenioids". The Gaussian distribution also plays the crucial role of allowing the construction of the [non-

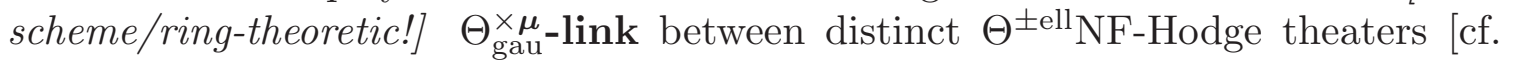
Corollary 4.10, (iii)] — i.e., between distinct models of conventional schemetheoretic arithmetic geometry.

(ii) Within a single $\Theta^{ \pm \text {ell } N F-H o d g e ~ t h e a t e r, ~ t h e ~ t h e o r y ~ o f ~ e ́ t a l e ~ a n d ~ F r o b e n i o i d-~}$ theoretic theta functions developed in [EtTh] is applied to construct a single connected geometric "Kummer theory-compatible theater for evaluation of the theta function", whose étale-theoretic realization admits a multiradial formulation [cf. the theory of $\S 1$, especially Corollary 1.12], and whose connectedness allows one to establish conjugate synchronization [cf. the discussion of Remark 2.6.1] between the various copies of the absolute Galois group of the base field at the various $l$-torsion points at which the theta function is evaluated. Moreover, this conjugate synchronization satisfies the crucial property of compatibility with the $\mathbb{F}_{l}^{\rtimes \pm}$-symmetry [cf. the discussion of Remark 3.5.2, as well as Corollaries 4.5, (iii); 4.6, (iii)] of the underlying $\mathcal{D}$ - $\Theta^{\text {ell-bridge }}$ [cf. [IUTchI], Proposition 6.8, (i)] of the $\Theta^{ \pm \text {ell }}$ NF-Hodge theater under consideration. Conjugate synchronization plays an essential role in establishing the coricity of the units [cf. Corollary 4.10, (iv)] in a fashion which is compatible with both the étale-theoretic - i.e., "anabelian" - and abstract monoid/Frobenioid-theoretic - i.e., "post-anabelian" - representations of the Gaussian monoids [cf. the discussion of Remark 3.8.3]. Here, we recall that the "post-anabelian" representation of the Gaussian monoids is necessary to construct the $\Theta_{\text {gau }}^{\times \mu}$-link of Corollary 4.10, (iii) [cf. Remarks 3.6.2, (ii); 3.8.3, (i)]. On the other hand, the "anabelian" representation of the Gaussian monoids will play an essential role when we apply the theory of the log-wall [cf. 
[AbsTopIII]] in [IUTchIII] [cf. Remark 3.8.3, (ii)]. Another important aspect of the theory of Gaussian distibutions, at $\underline{v} \in \underline{\mathbb{V}}^{\text {bad }}$, is the canonical splittings of the monoids involved into "unit" and "value group" components. These splittings may be thought of, in the context of the $\Theta_{\text {gau }}^{\times \boldsymbol{\mu}}$-link, as corresponding to the "nondeformed" [cf. the "coricity of the units"] and "Teichmüller-dilated" [cf. the "value group" portion of the Gaussian distribution] real dimensions that appear in classical complex Teichmüller theory [cf. the discussion of Remark 4.10.3, (i), (ii)]. Finally, these splittings will play a crucial role in the theory of log-shells [cf. [AbsTopIII]], which we shall apply in [IUTchIII].

(iii) By contrast, the number fields that appear in the underlying $\Theta N F$ Hodge theater of the $\Theta^{ \pm \text {ell }} \mathrm{NF}$-Hodge theater under consideration [cf. the theory of [IUTchI], §5] will ultimately, in [IUTchIII], in the context of log-shells, play the role of relating — via the ring structure of these number fields - $\triangle$-line bundles [i.e., "idèlic" or "Frobenioid-theoretic" line bundles] to " $\boxplus$-line bundles" [i.e., line bundles thought of as modules] — cf. the discussion of Remark 4.7.2. Such relationships are only possible if one considers all of the primes of the number fields involved [cf. [AbsTopIII], Remark 5.10.2, (iv)]. Constructions associated to these number fields satisfy the property of being compatible with the $\mathbb{F}_{l}^{*}$-symmetry [cf. [IUTchI], Proposition 4.9, (i)] of the underlying NF-bridge of the $\Theta^{ \pm \text {ell }}$ NF-Hodge theater under consideration. Unlike the $\mathbb{F}_{l}^{\rtimes \pm}$-symmetry discussed in (ii), which is combinatorially uniradial in nature and may be thought of, in the context of the splittings discussed in (ii), as being associated with the "units", the $\mathbb{F}_{l}^{*}$-symmetry is combinatorially multiradial in nature and may be thought of, in the context of the splittings discussed in (ii), as being associated with the "value groups" [cf. the discussion of Remarks 4.7.3, 4.7.4, 4.7.5]. On the other hand, [cf. the discussion of (ii)] the $\mathbb{F}_{l}^{\rtimes \pm}$-symmetry satisfies the crucial property of being compatible with conjugate synchronization - a property which may only be established after one isolates the prime-strips under consideration from the conjugacy indeterminacies inherent in the global structure of the absolute Galois group of a number field [cf. Remark 4.7.2]. Put another way, conjugate synchronization may only be established once the prime-strips under consideration are treated as objects which are free of any combinatorial constraints arising from the "prime-trees" associated to a number field [cf. the discussion of [IUTchI], Remark 4.3.1]. On the other hand, one important property shared by both the $\mathbb{F}_{l}^{\rtimes \pm}$ - and $\mathbb{F}_{l}^{*}$-symmetries is the connectedness of the global objects that appear in the $\left[\Theta^{\text {ell }}-/\right.$ NF-bridges of $]$ these symmetries. This connectedness plays an essential role in the bookkeeping operations involving the labels of the evaluation points [cf. the discussion of Remarks 3.5.2 and 4.5.3, (iii), as well as [IUTchI], Remark 4.9.2, (i)]. In particular, such bookkeeping operations cannot be implemented if, for instance, instead of working with a global number field, one attempts to take as one's "global objects" formal products of the local objects at the various primes of the number field under consideration [cf. the discussion of [AbsTopIII], Remark 3.7.6, (v)]. Finally, we recall that the essential role played by these "global bookkeeping operations" gives rise, in light of the profinite nature of the global geometric étale fundamental groups involved, to a situation in which one must apply the "complements on tempered coverings" developed in [IUTchI], §2 [cf. Remark 4.5.3, (iii)].

(iv) One way to summarize the above discussion is as follows. The bridge 
constituted by the Gaussian distribution of a $\Theta^{ \pm \text {ell }}$ NF-Hodge theater between theta functions and number fields may be thought of as being constructed by dismantling those aspects of the "characteristic topography" of the theta functions and number fields involved that constitute an obstruction to relating theta functions to number fields. In the case of theta functions, the main obstruction to constructing such a link to the number field under consideration is constituted by the geometric dimension of the tempered coverings of elliptic curves [at $\underline{v} \in \underline{\mathbb{V}}^{\text {bad }}$ ] on which the theta functions are defined. This obstruction is resolved by means of the operation of evaluation at the l-torsion points. Thus, from the point of view of the schemetheoretic Hodge-Arakelov theory of [HASurI], [HASurII], one may think of these l-torsion points as a sort of "rough finite approximation" of the tempered coverings of elliptic curves under consideration [cf. the discussion of [HASurI], §1.3.4]. By contrast, in the case of number fields, the main obstruction to constructing such a link to the theta functions under consideration is the "prime-trees" arising from the global structure of the number field, which give rise to the conjugacy indeterminacies that obstruct the establishment of conjugacy synchronization [cf. the discussion of (iii) above]. This obstruction is resolved by dismantling the global prime-tree structure of the number fields involved by working with various prime-strips labeled by elements $\in \mathbb{F}_{l}^{*}$ [cf. the discussion of [IUTchI], Remark 4.3.1]. Thus, one may think of these collections of prime-strips labeled by elements $\in \mathbb{F}_{l}^{*}$ as "rough finite approximations" of the infinite prime-trees associated to the number fields involved. At a more combinatorial level [cf. the discussion of Remark 4.7.5], this dismantling process may be thought of as the process of dismantling the ring structure of $\mathbb{F}_{l}$ — which we think of as a "rough finite approximation" of $\mathbb{Z}$ [cf. [IUTchI], Remark 6.12.3, (i)] — into its additive and multiplicative components, which correspond, respectively, to the $\mathbb{F}_{l}^{\rtimes \pm}$ - and $\mathbb{F}_{l}^{*}$ symmetries.

Remark 4.11.3. In the context of the discussion of Remark 4.11.2, it is interesting to observe that, whereas, from the point of view of the combinatorics of the $\mathbb{F}_{l}^{\rtimes \pm}$ and $\mathbb{F}_{l}^{*}$-symmetries, one has correspondences

$$
\Theta^{\mathrm{ell}} \longleftrightarrow \boxplus, \quad \mathrm{NF} \longleftrightarrow \otimes
$$

- i.e., the $\Theta^{\text {ell }}$-bridge corresponds to the additive $\mathbb{F}_{l}^{\rtimes \pm}$-symmetry, while the NFbridge corresponds to the multiplicative $\mathbb{F}_{l}^{*}$-symmetry — at the level of line bundles, one has correspondences

$$
\Theta^{\text {ell }} \longleftrightarrow \otimes, \quad \mathrm{NF} \longleftrightarrow \boxplus
$$

- i.e., the arithmetic line bundles under consideration are treated multiplicatively, via monoids or Frobenioids, in the context of the $\Theta^{\text {ell }}$-bridge, while the equivalence of such $\triangle$-line bundles with $\boxplus$-line bundles may only be realized in the context of the global ring structure of the number fields associated, via the theory of [IUTchI], $\S 5$, to the NF-bridge. This "juggling of $\boxplus$ and $\square$ " is reminiscent of the theory of the log-wall developed in [AbsTopIII] [cf., e.g., the discussion of [AbsTopIII], §I3] and, indeed, may be thought of as a sort of combinatorial counterpart to the "juggling of $\boxplus$ and $\square$ " that occurs in the theory of the log-wall. 


\section{Remark 4.11.4.}

(i) From the point of view of scheme-theoretic Hodge-Arakelov theory, the ltorsion points of an elliptic curve may be thought of as a "rough finite approximation" of the two real dimensions of the underlying real analytic manifold of a one-dimensional complex torus [cf. the discussion of [HASurI], §1.3.4]. In schemetheoretic Hodge-Arakelov theory, one considers spaces of functions on these $l$-torsion points. The two dimensions mentioned above then correspond to a "holomorphic dimension" and a "one-dimensional deformation of this holomorphic dimension" [cf. the discussion of [HASurI], §1.4.2]. In the context of the theory of the present series of papers, we work, in effect, with an elliptic curve which is isogenous to the given elliptic curve via an isogeny of degree $l$ - i.e., with " $\underline{X}$ " as opposed to " $X$ " - so that we may neglect the "holomorphic dimension" mentioned above and concentrate instead on the deformations of this "holomorphic dimension" [cf. the discussion of the Introduction to [EtTh]]. In particular, the various possible values at the various $l$-torsion points at which the theta function is evaluated in the theory of the present series of papers may be thought of as various possible deformations of the holomorphic structure, while the specific values of the theta function may be thought of as a specific deformation of the holomorphic structure. Here, we note that the parameter " $0 \neq t \in \operatorname{LabCusp}^{ \pm}(-)$" that indexes these values - which, like the tangent space to the original elliptic curve, is linear which respect to the group structure of the elliptic curve - descends naturally [especially in the context of $\Theta \mathrm{NF}$-Hodge theater!] to the parameter " $j \in \mathbb{F}_{l}^{*}$ " — which may be thought of as the "square $\left(\mathbb{F}_{l}^{\times}\right)^{2}$ " of $\mathbb{F}_{l}^{\times}$, hence, like the square of the tangent space of the elliptic curve, which is naturally isomorphic to the tangent space to the moduli space of elliptic curves at the point determined by the elliptic curve in question, is quadratic in its dependence on the linear group structure of the elliptic curve. Finally, this point of view concerning the values of the theta function is reminiscent of the point of view of Remark 3.6.2, (iii), in which we observe that, in the context of the $\Theta_{\mathrm{gau}^{-}}^{\times \boldsymbol{\mu}^{-}}$ link, these values of the theta function may be thought of as a sort of "deformation between the identity and a Frobenius morphism". The theta function involved may then be thought of as a sort of continuous version [i.e., as opposed to a "rough finite approximation"] of such a deformation.

(ii) From the point of view of the analogy between the theory of the present series of papers and p-adic Teichmüller theory [cf. [AbsTopIII], §I5], the portion of the infinite chain of $\Theta^{\times \mu}$-links of Corollary 4.10, (vi), parametrized by $n \leq 0$

$$
\ldots \stackrel{\Theta^{\times \mu}}{\longrightarrow}(n-1) \mathcal{H} \mathcal{T}^{\Theta^{ \pm \mathrm{ell}}} \mathrm{NF} \stackrel{\Theta^{\times \mu}}{\longrightarrow} n \mathcal{H} \mathcal{T}^{\Theta^{ \pm \mathrm{ell}} \mathrm{NF}} \stackrel{\Theta^{\times \mu}}{\longrightarrow} \ldots \stackrel{\Theta^{\times \mu}}{\longrightarrow}{ }^{0} \mathcal{H} \mathcal{T}^{\Theta^{ \pm \mathrm{ell}} \mathrm{NF}}
$$

may be thought of as corresponding to the canonical liftings of $p$-adic Teichmüller theory. That is to say, each $\Theta^{ \pm \text {ell }} \mathrm{NF}$-Hodge theater — which one may think of as representing the conventional scheme theory surrounding the given number field equipped with an elliptic curve - corresponds to a hyperbolic curve in positive characteristic equipped with a nilpotent ordinary indigenous bundle [cf. the discussion of [AbsTopIII], §I5]. The theta functions that give rise to the $\Theta^{\times \boldsymbol{\mu}}$-links may be thought of as specifying the specific canonical deformation [cf. the discussion of (i)] that gives rise to this "canonical lifting". The canonical Frobenius lifting on this canonical lifting may be thought of as corresponding to the theory 
to be developed in [IUTchIII]. From this point of view, the passage

\section{theta functions, number fields $\rightsquigarrow$ Gaussian distributions}

[cf. the discussion of Remark 4.11.2] effected in the theory of the present series of papers presented thus far - i.e., at a more concrete level, the passage, via Hodge-Arakelov-theoretic evaluation, from the above semi-infinite chain to the corresponding semi-infinite chain

$$
\ldots \stackrel{\Theta_{\text {gau }}^{\times \mu}}{\longrightarrow}(n-1) \mathcal{H} \mathcal{T}^{\Theta^{ \pm e l l}} \mathrm{NF} \stackrel{\Theta_{\text {gau }}^{\times \mu}}{\longrightarrow} n \mathcal{H} \mathcal{T}^{\Theta^{ \pm e l l}} \mathrm{NF} \stackrel{\Theta_{\text {gau }}^{\times \mu}}{\longrightarrow} \ldots \stackrel{\Theta_{\text {gau }}^{\times \mu}}{\longrightarrow} \mathcal{H}^{\Theta^{\Theta^{ \pm e l l}} \mathrm{NF}}
$$

of $\Theta_{\mathrm{gau}}^{\times \boldsymbol{\mu}}$-links - may be thought of as corresponding to the passage

$$
\mathcal{M F}^{\nabla} \text {-objects } \rightsquigarrow \text { Galois representations }
$$

in the case of the canonical indigenous bundles that occur in $p$-adic Teichmüller theory - cf. the discussion of [ $p$ Teich], Introduction, $\S 1.3, \S 1.7$; the discussion in [HASurI], $\S 1.3, \S 1.4$, of the relationship between such canonical indigenous bundles in the case of the moduli stack of elliptic curves and the scheme-theoretic HodgeArakelov theory of [HASurI], [HASurII]. Put another way, it corresponds to the passage from thinking of the "canonical lifting" as a curve equipped with the $\mathcal{M F}^{\nabla}$ object constituted by a canonical Frobenius-invariant indigenous bundle to thinking of the "canonical lifting" as a curve equipped with a canonical Galois representation, i.e., a canonical crystalline representation [that is to say, a representation that happens to arise from an $\mathcal{M F}^{\nabla}$-object] of the arithmetic fundamental group of the generic fiber of the curve into $P G L_{2}\left(\mathbb{Z}_{p}\right)$.

(iii) The analogy between the theory of the present series of papers and $p$-adic Teichmüller theory may also be seen, at a more technical level, in the following correspondences between various aspects of the theory presented thus far in the present series of papers and various aspects of the theory of [CanLift], $§ 3$ [cf. also Remark 4.11 .5 below]:

(a) The discussion of (ii) above is reminiscent of the important role played by the canonical Galois representation in the absolute p-adic anabelian theory of [CanLift], $\S 3$ [cf. the proof of [CanLift], Lemma 3.5].

(b) In light of the important role played, in the present series of papers, by mono-theta-theoretic cyclotomic rigidity [which was reviewed in Definition 1.1, (ii)], it is perhaps of interest to recall [cf. Remark 1.11.6] the important role played by cyclotomic rigidity isomorphisms in the theory of [CanLift], §3, via the theory of [AbsAnab], §2 [cf., especially, [AbsAnab], Lemmas 2.5, 2.6]. On the other hand, at the level of direct correspondences between the theory of the present series of papers and $p$-adic Teichmüller theory, it is perhaps better to think of mono-theta-theoretic cyclotomic rigidity as corresponding to the local uniformizations arising from the canonical indigenous bundle [cf. the discussion of Remark 3.6.5, (iii)].

(c) The important role played, in the present series of papers, by the "twodimensional symmetry" constituted by the $\mathbb{F}_{l}^{\rtimes \pm}$ - and $\mathbb{F}_{l}^{*}$-symmetries 
- whose two-dimensionality may be thought of as corresponding to the two real dimensions of the complex upper half-plane [cf. the discussion of [IUTchI], Remark 6.12.3, (iii)] — is reminiscent of the important role played in the theory of [CanLift], $\S 3$, in effect, by the vanishing of the zero-th group cohomology module

$$
H^{0}(\operatorname{Ad}(-))
$$

of the canonical Galois representation associated to the canonical indigenous bundle - cf. the various geometric conditions over the ordinary locus and at the supersingular points of the $\bmod p$ representations considered in [CanLift], Lemma 3.2. That is to say, the $\mathbb{F}_{l}^{\rtimes \pm}$-symmetry may be regarded as corresponding to the unipotent monodromy over the ordinary locus

$$
\left\{\left(\begin{array}{ll}
1 & * \\
0 & 1
\end{array}\right)\right\} \stackrel{\sim}{\rightarrow} \mathbb{F}_{p}
$$

— which is isomorphic to the additive group underlying $\mathbb{F}_{p}$ - while the $\mathbb{F}_{l}^{*}$-symmetry may be regarded as corresponding to the toral monodromy at the supersingular points

$$
\left\{\left(\begin{array}{cc}
* & 0 \\
0 & *^{-1}
\end{array}\right)\right\} \stackrel{\sim}{\rightarrow} \mathbb{F}_{p}^{\times}
$$

- which is isomorphic to the multiplicative group $\mathbb{F}_{p}^{\times}$and arises from extracting a $(p-1)$-th root of the Hasse invariant. Moreover, the "intuitive, conventional" nature of the theory over any single connected component of the ordinary locus - a theory which allows one, for instance, to construct q-parameters - is reminiscent of the uniradial nature of the $\mathbb{F}_{l}^{\rtimes \pm}$ symmetry, while the fact that supersingular points lie simultaneously on irreducible components obtained as closures of distinct connected components of the ordinary locus is reminiscent of the multiradiality - i.e., compatibility with simultaneous execution in distinct Hodge theaters of the $\mathbb{F}_{l}^{*}$-symmetry [cf. the discussion of Remark 4.7.4]. The above discussion is summarized, at the level of keywords, in Fig. 4.4 below.

(d) The important role played, in the present series of papers, by conjugate synchronization at the various evaluation points of the theta function - which gives rise, in the form of the Gaussian distribution, to the links between the various $\Theta^{ \pm e l l} \mathrm{NF}$-Hodge theaters in the second semiinfinite chain that appeared in the discussion of (ii) - is reminiscent of the important role played in the theory of [CanLift], $\S 3$, by the description given in [CanLift], Lemma 3.4, of the first group cohomology module

$$
H^{1}(\operatorname{Ad}(-))
$$

of the canonical Galois representation associated to the canonical indigenous bundle — whose "slope -1 portion" may be thought of as governing 
the "links" between the " $\bmod p^{n "}$ and "mod $p^{n+1 "}$ portions of the canonical Galois representation, as it is considered in the proof of [CanLift], Lemma 3.5. Here, we note that this description may be summarized, in effect, as asserting that the slope -1 portion in question is, up to tensor product with an unramified Galois representation, isomorphic to a direct product of $3 g-3+r$ copies of $\mathbb{F}_{p}(-1)$ [where the " $(-1)$ " denotes a Tate twist] - a situation that is reminiscent of the $l^{*}$ synchronized copies of cyclotomes that occur in the context of the evaluation of the theta function considered in the present series of papers. Moreover, the deformations of the canonical Galois representation parametrized by this module " $H^{1}(\operatorname{Ad}(-))$ " may be thought of as corresponding, in the theory of the present series of papers, to the "independent $\operatorname{Aut}\left(G_{\underline{v}}\right)$-indeterminacies" [i.e., for $\underline{v} \in \underline{\mathbb{V}}^{\text {non }}$ ] that occur at each label $\in \mathbb{F}_{l}$ when one consider multiradial representations of Gaussian monoids - cf. the theory of [IUTchIII], $\S 3$; [IUTchIII], Remark 3.12.4, (iii).

Finally, we observe, with regard to (d), that the description in question that appears in [CanLift], Lemma 3.4, may be thought of as a reflection of the ordinarity [i.e., as opposed to just admissibility] of the positive characteristic nilpotent indigenous bundle under consideration, hence is reminiscent of the discussion of [AbsTopIII], Remark 5.10.3, (ii), of the correspondence between ordinarity in $p$-adic Teichmüller theory and the theory of the étale theta function developed in [EtTh].

\begin{tabular}{|c|c|}
\hline $\mathbb{F}_{l}^{\rtimes \pm}$-symmetry & $\mathbb{F}_{l}^{*}$-symmetry \\
\hline additive & multiplicative \\
\hline uniradial & multiradial \\
\hline $\begin{array}{c}\text { monodromy over the } \\
\text { ordinary locus }\end{array}$ & $\begin{array}{c}\text { monodromy at the } \\
\text { supersingular points }\end{array}$ \\
\hline
\end{tabular}

Fig. 4.4: Correspondence of symmetries with $p$-adic Teichmüller theory

Remark 4.11.5. We take this opportunity to correct a few notational errors in the statement of the condition $\left(\dagger_{M}\right)$ of [CanLift], Lemma 3.4, which, however, do not affect the proof of this lemma in any substantive way. The subquotient " $\mathbb{G}^{2}(M)$ " (respectively, "G্G ${ }^{-1}$ ") should have been denoted " $\mathbb{G}^{-2}(M)$ " (respectively, " $\mathbb{G}^{1 ")}$. The subquotient $\mathbb{G}^{-2}(M)$ (respectively, $\mathbb{G}^{1}$ ) is isomorphic to the tensor product of an unramified module with a Tate twist $\mathbb{F}_{p}(-2)$ (respectively, $\mathbb{F}_{p}(1)$ ). That is to say, there is a sign error in the Tate twists stated in $\left(\dagger_{M}\right)$. Finally, in 
order to obtain the desired dimensions over $\mathbb{F}_{p}$, one must replace the cohomology module

$$
\text { " } M \stackrel{\text { def }}{=} H^{1}\left(\Delta_{X} \log , \operatorname{Ad}\left(V_{\mathbb{F}_{p}}\right)\right) "
$$

by the submodule of this module consisting of elements whose restriction to each of the cuspidal inertia groups of $\Delta_{X^{\log }}$ is upper triangular with respect to the filtration determined by the nilpotent monodromy action on $V_{\mathbb{F}_{p}}$ [i.e., by the cuspidal inertia group in question]. That is to say, an elementary computation shows that the operation of restriction to this submodule has the effect of lowering the dimension of $\mathbb{G}^{-2}(M)$ from $3 g-3+2 r$ to $3 g-3+r$, as desired. 


\section{Bibliography}

[Lehto] O. Lehto, Univalent Functions and Teichmüller Spaces, Graduate Texts in Mathematics 109, Springer, 1987.

[pOrd] S. Mochizuki, A Theory of Ordinary p-adic Curves, Publ. Res. Inst. Math. Sci. 32 (1996), pp. 957-1151.

[pTeich] S. Mochizuki, Foundations of p-adic Teichmüller Theory, AMS/IP Studies in Advanced Mathematics 11, American Mathematical Society/International Press (1999).

$[p \mathrm{GC}]$ S. Mochizuki, The Local Pro- $p$ Anabelian Geometry of Curves, Invent. Math. 138 (1999), pp. 319-423.

[HASurI] S. Mochizuki, A Survey of the Hodge-Arakelov Theory of Elliptic Curves I, Arithmetic Fundamental Groups and Noncommutative Algebra, Proceedings of Symposia in Pure Mathematics 70, American Mathematical Society (2002), pp. 533-569.

[HASurII] S. Mochizuki, A Survey of the Hodge-Arakelov Theory of Elliptic Curves II, Algebraic Geometry 2000, Azumino, Adv. Stud. Pure Math. 36, Math. Soc. Japan (2002), pp. 81-114.

[AbsAnab] S. Mochizuki, The Absolute Anabelian Geometry of Hyperbolic Curves, Galois Theory and Modular Forms, Kluwer Academic Publishers (2004), pp. 77-122.

[CanLift] S. Mochizuki, The Absolute Anabelian Geometry of Canonical Curves, Kazuya Kato's fiftieth birthday, Doc. Math. 2003, Extra Vol., pp. 609-640.

[AbsSect] S. Mochizuki, Galois Sections in Absolute Anabelian Geometry, Nagoya Math. J. 179 (2005), pp. 17-45.

[SemiAnbd] S. Mochizuki, Semi-graphs of Anabelioids, Publ. Res. Inst. Math. Sci. 42 (2006), pp. 221-322.

[CombGC] S. Mochizuki, A combinatorial version of the Grothendieck conjecture, Tohoku Math. J. 59 (2007), pp. 455-479.

[Cusp] S. Mochizuki, Absolute anabelian cuspidalizations of proper hyperbolic curves, J. Math. Kyoto Univ. 47 (2007), pp. 451-539.

[FrdI] S. Mochizuki, The Geometry of Frobenioids I: The General Theory, Kyushu J. Math. 62 (2008), pp. 293-400.

[FrdII] S. Mochizuki, The Geometry of Frobenioids II: Poly-Frobenioids, Kyushu J. Math. 62 (2008), pp. 401-460.

[EtTh] S. Mochizuki, The Étale Theta Function and its Frobenioid-theoretic Manifestations, Publ. Res. Inst. Math. Sci. 45 (2009), pp. 227-349.

[AbsTopI] S. Mochizuki, Topics in Absolute Anabelian Geometry I: Generalities, RIMS Preprint 1624 (March 2008).

[AbsTopII] S. Mochizuki, Topics in Absolute Anabelian Geometry II: Decomposition Groups, RIMS Preprint 1625 (March 2008). 
[AbsTopIII] S. Mochizuki, Topics in Absolute Anabelian Geometry III: Global Reconstruction Algorithms, RIMS Preprint 1626 (March 2008).

[IUTchI] S. Mochizuki, Inter-universal Teichmüller Theory I: Construction of Hodge Theaters, preprint.

[IUTchIII] S. Mochizuki, Inter-universal Teichmüller Theory III: Canonical Splittings of the Log-theta-lattice, preprint.

[IUTchIV] S. Mochizuki, Inter-universal Teichmüller Theory IV: Log-volume Computations and Set-theoretic Foundations, preprint.

[NSW] J. Neukirch, A. Schmidt, K. Wingberg, Cohomology of number fields, Grundlehren der Mathematischen Wissenschaften 323, Springer-Verlag (2000).

[Szp] L. Szpiro, Degrés, intersections, hauteurs in Astérisque 127 (1985), pp. 11-28. 